\title{
Construction of Axially Chiral Arylborons via Atroposelective Miyaura
}

\section{Borylation}

Kai Yang, ${ }^{1}$ Yanfei Mao, ${ }^{1}$ Jie Xu, ${ }^{1}$ Hao Wang, ${ }^{1}$ Yong He, ${ }^{1}$ Wangyang $\mathrm{Li}^{1}$ and Qiuling Song $*{ }^{1,2}$

${ }^{1}$ Key Laboratory of Molecule Synthesis and Function Discovery, Fujian Province University, College of Chemistry at Fuzhou University, Fuzhou, Fujian, 350108 (China)

${ }^{2}$ Institute of Next Generation Matter Transformation, College of Materials Science Engineering at Huaqiao University 668 Jimei Boulevard, Xiamen, Fujian, 361021 (China)

*E-mail: qsong@ @qu.edu.cn

\section{Contents}

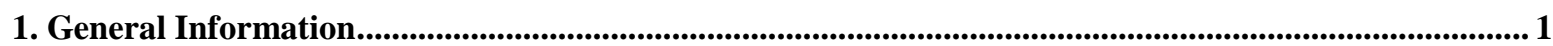

2. Synthesis of Substituted pinB-Bdan ....................................................................................................................... 1

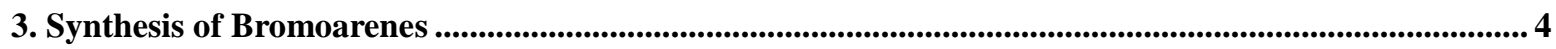

4. General Procedure for the Atroposelective Miyaura Borylation Reaction.................................................. 12

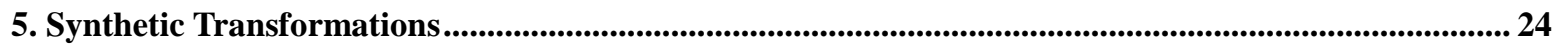

6. Application of atropisomeric mono-phosphine $7 \mathrm{c}$ or $7 \mathrm{~d}$ as ligand................................................................ 26

7. Rotational Barriers of Axially Chiral Arylborons .................................................................................................. 26

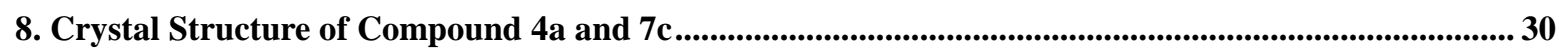

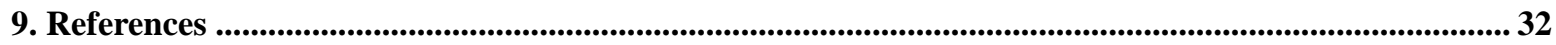

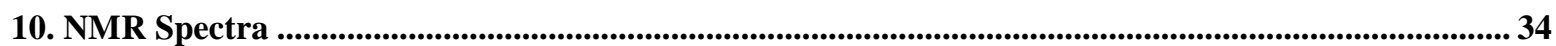

11. Chiral HPLC Charts ...................................................................................................................................... 112 


\section{General Information}

Unless otherwise noted, materials obtained from commercial suppliers were used without further purification. Flash column chromatography was performed over silica gel (200-300 mesh). ${ }^{1} \mathrm{H}$ NMR and ${ }^{13} \mathrm{C}$ NMR spectra were recorded at ambient temperature using Bruker 400M and JEOL 500M spectrometers, chemical shifts (in ppm) were referenced to $\mathrm{CDCl}_{3}(\delta=7.26 \mathrm{ppm})$ as internal standards. ${ }^{13} \mathrm{C}$ NMR spectra were obtained by using the same NMR spectrometers and were calibrated with $\mathrm{CDCl}_{3}(\delta=77.0 \mathrm{ppm})$. Data for ${ }^{1} \mathrm{H}$ NMR are recorded as following abbreviations: multiplicity $(\mathrm{s}=$ singlet, $\mathrm{d}=$ doublet, $\mathrm{t}=$ triplet, $\mathrm{q}=$ quarter, $\mathrm{m}=$ multiplet $)$, coupling constant $(J, \mathrm{~Hz})$. High resolution mass spectroscopy (HRMS) analysis was performed at an Exactive Plus (Thermo Scientific) or Agilent 8890-7250. Enantioselectivities were recorded on Waters or Agilent HPLC. The X-ray crystal structure was measured on Bruker D8 venture.

\section{Synthesis of Substituted pinB-Bdan}
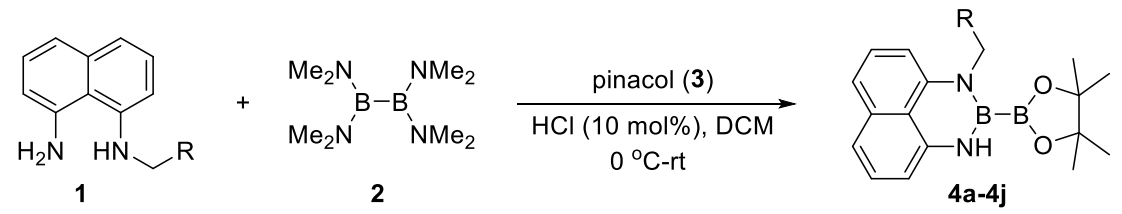

By analogy to a modified literature procedure, ${ }^{1}$ in air, a $10 \mathrm{~mL}$ schlenk tube was charged with 1 (1 mmol, 1 equiv) and pinacol ( 1 mmol, 1 equiv). The tube was evacuated and filled with argon for three cycles. Then, 3 $\mathrm{mL}$ of DCM and tetrakis(dimethylamino)diborane ( $1 \mathrm{mmol}, 1$ equiv) were added under argon. At $0{ }^{\circ} \mathrm{C}, 4 \mathrm{M}$ dioxane solution of hydrogen chloride $(0.025 \mathrm{~mL}, 0.1 \mathrm{mmol})$ was added dropwise to the mixture and the mixture was stirred at $0{ }^{\circ} \mathrm{C}$ for $30 \mathrm{~min}$. The reaction was allowed to stir at $\mathrm{rt}$ for 36 hours. Upon completion, proper amount of silica gel was added to the reaction mixture. After removal of the solvent, the crude reaction mixture was purified on short column chromatography (petroleum ether and ethyl acetate) to afford the crude products. Finally, the crude product was recrystallized (petroleum ether and ethyl acetate) to obtain a pure product.

1-ethyl-2-(4,4,5,5-tetramethyl-1,3,2-dioxaborolan-2-yl)-2,3-dihydro-1H-naphtho[1,8-de][1,3,2]diazabori nine (4a)

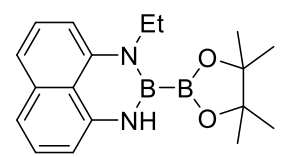

White solid, mp: $131-133^{\circ} \mathrm{C}$. Yield: $45 \% . \mathrm{R}_{\mathrm{f}}=0.25$ (silica gel, PE:EtOAc $=20: 1$ ).

${ }^{1} \mathbf{H}$ NMR $\left(\mathbf{5 0 0} \mathbf{M H z}, \mathbf{C D C l}_{3}\right) \delta 7.19(\mathrm{t}, J=8.0 \mathrm{~Hz}, 1 \mathrm{H}), 7.09(\mathrm{dd}, J=8.1,7.4 \mathrm{~Hz}, 1 \mathrm{H}), 7.02(\mathrm{dd}, J=11.4,4.4$ $\mathrm{Hz}, 2 \mathrm{H}), 6.43(\mathrm{~d}, J=7.7 \mathrm{~Hz}, 1 \mathrm{H}), 6.32(\mathrm{dd}, J=7.3,1.0 \mathrm{~Hz}, 2 \mathrm{H}), 3.81(\mathrm{q}, J=7.0 \mathrm{~Hz}, 2 \mathrm{H}), 1.30(\mathrm{~s}, 12 \mathrm{H}), 1.26$ $(\mathrm{t}, J=7.0 \mathrm{~Hz}, 3 \mathrm{H}) .{ }^{13} \mathbf{C}$ NMR (126 MHz, $\left.\mathbf{C D C l}_{3}\right) \delta 142.0,140.2,136.5,127.5,127.1,121.6,117.5,117.4$, 105.2, 103.0, 83.2, 42.6, 25.0, 14.0. ${ }^{11} \mathbf{B}$ NMR (128 $\left.\mathbf{~ M H z , ~ C D C l}\right)_{3} \delta$ 32.5. HRMS (ESI) calcd for $\mathrm{C}_{18} \mathrm{H}_{25} \mathrm{~B}_{2} \mathrm{~N}_{2} \mathrm{O}_{2}[\mathrm{M}+\mathrm{H}]^{+}:$323.2097, found: 323.2098.

1-(cyclopropylmethyl)-2-(4,4,5,5-tetramethyl-1,3,2-dioxaborolan-2-yl)-2,3-dihydro-1H-naphtho[1,8-de][ $1,3,2]$ diazaborinine $(4 b)$

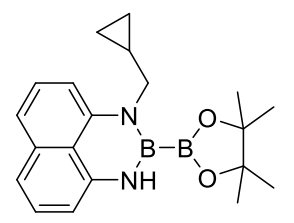


White solid, mp: $95-97{ }^{\circ} \mathrm{C}$. Yield: $36 \% . \mathrm{R}_{\mathrm{f}}=0.3$ (silica gel, PE: EtOAc $=20: 1$ ).

${ }^{1} \mathbf{H}$ NMR $\left(\mathbf{5 0 0} \mathbf{M H z}, \mathbf{C D C l}_{3}\right) \delta 7.21(\mathrm{t}, J=8.0 \mathrm{~Hz}, 1 \mathrm{H}), 7.10(\mathrm{dd}, J=8.2,7.3 \mathrm{~Hz}, 1 \mathrm{H}), 7.08-7.01(\mathrm{~m}, 2 \mathrm{H})$, $6.61(\mathrm{~d}, J=7.7 \mathrm{~Hz}, 1 \mathrm{H}), 6.36-6.30(\mathrm{~m}, 2 \mathrm{H}), 3.67(\mathrm{~d}, J=6.6 \mathrm{~Hz}, 2 \mathrm{H}), 1.43-1.33(\mathrm{~m}, 1 \mathrm{H}), 1.29(\mathrm{~s}, 12 \mathrm{H})$, $0.52-0.42(\mathrm{~m}, 2 \mathrm{H}), 0.42-0.33$ (m, 2H). ${ }^{13} \mathbf{C}$ NMR (126 MHz, $\left.\mathbf{C D C l}_{3}\right) \delta 142.5,140.2,136.5,127.5,127.2$, 121.5, 117.6, 117.4, 105.2, 103.7, 83.3, 51.5, 25.0, 9.6, 3.7. ${ }^{11} \mathbf{B}$ NMR (128 $\left.\mathbf{~ M H z}, \mathbf{C D C l}_{3}\right) \delta 32.6$. HRMS (ESI) calcd for $\mathrm{C}_{20} \mathrm{H}_{27} \mathrm{~B}_{2} \mathrm{~N}_{2} \mathrm{O}_{2}[\mathrm{M}+\mathrm{H}]^{+}$: 349.2253, found: 349.2255 .

1-(cyclopentylmethyl)-2-(4,4,5,5-tetramethyl-1,3,2-dioxaborolan-2-yl)-2,3-dihydro-1H-naphtho[1,8-de][1 $, 3,2]$ diazaborinine $(4 \mathrm{c})$

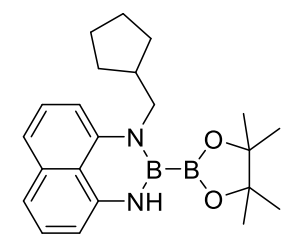

White solid, mp: $120-121{ }^{\circ} \mathrm{C}$. Yield: $33 \% . \mathrm{R}_{\mathrm{f}}=0.4$ (silica gel, PE: EtOAc $=20: 1$ ).

${ }^{1} \mathbf{H}$ NMR $\left(500 \mathrm{MHz}, \mathbf{C D C l}_{3}\right) \delta 7.18(\mathrm{t}, J=8.0 \mathrm{~Hz}, 1 \mathrm{H}), 7.09(\mathrm{t}, J=7.7 \mathrm{~Hz}, 1 \mathrm{H}), 7.02(\mathrm{dd}, J=10.7,8.3 \mathrm{~Hz}$, 2H), $6.44(\mathrm{~d}, J=7.7 \mathrm{~Hz}, 1 \mathrm{H}), 6.34(\mathrm{~s}, 1 \mathrm{H}), 6.32(\mathrm{~d}, J=7.3 \mathrm{~Hz}, 1 \mathrm{H}), 3.70(\mathrm{~d}, J=7.1 \mathrm{~Hz}, 2 \mathrm{H}), 2.54-2.39(\mathrm{~m}$, 1H), $1.75-1.63(\mathrm{~m}, 4 \mathrm{H}), 1.57-1.47$ (m, 2H), $1.35-1.28$ (m, 14H). ${ }^{\mathbf{1 3}} \mathbf{C}$ NMR (126 MHz, $\left.\mathbf{C D C l}_{3}\right) \delta 142.2$, $140.2,136.5,127.4,127.1,121.6,117.6,117.3,105.2,103.8,83.2,52.6,35.6,30.1,25.3,25.0 .{ }^{11} \mathbf{B}$ NMR (128 $\mathbf{M H z}, \mathbf{C D C l}_{3}$ ) $\delta$ 30.2. HRMS (ESI) calcd for $\mathrm{C}_{22} \mathrm{H}_{31} \mathrm{~B}_{2} \mathrm{~N}_{2} \mathrm{O}_{2}[\mathrm{M}+\mathrm{H}]^{+}:$377.2566, found: 377.2561.

1-benzyl-2-(4,4,5,5-tetramethyl-1,3,2-dioxaborolan-2-yl)-2,3-dihydro-1H-naphtho[1,8-de][1,3,2]diazabor inine (4d)

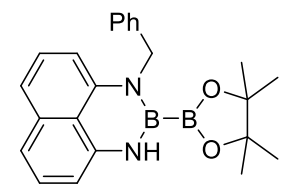

White solid, mp: $122-123{ }^{\circ} \mathrm{C}$. Yield: $19 \% . \mathrm{R}_{\mathrm{f}}=0.3$ (silica gel, PE:EtOAc $\left.=20: 1\right) .{ }^{1} \mathbf{H}$ NMR $\left(\mathbf{5 0 0} \mathbf{~ M H z}, \mathbf{C D C l}_{\mathbf{3}}\right.$ ) $\delta 7.35(\mathrm{dd}, J=7.9,0.7 \mathrm{~Hz}, 2 \mathrm{H}), 7.32-7.28(\mathrm{~m}, 2 \mathrm{H}), 7.21(\mathrm{t}, J=7.2 \mathrm{~Hz}, 1 \mathrm{H}), 7.13(\mathrm{dd}, J=8.1,7.4 \mathrm{~Hz}, 1 \mathrm{H})$, $7.07-6.97(\mathrm{~m}, 3 \mathrm{H}), 6.46(\mathrm{~s}, 1 \mathrm{H}), 6.40(\mathrm{dd}, J=7.3,1.0 \mathrm{~Hz}, 1 \mathrm{H}), 6.26(\mathrm{dd}, J=7.1,1.5 \mathrm{~Hz}, 1 \mathrm{H}), 5.02(\mathrm{~s}, 2 \mathrm{H})$, 1.23 (s, 12H). ${ }^{13} \mathbf{C}$ NMR (126 MHz, $\left.\mathbf{C D C l}_{3}\right) \delta 142.0,139.9,139.1,136.2,128.3,127.4,127.1,126.5,126.5$, 121.5, 117.9, 117.7, 105.5, 105.1, 83.3, 52.0, 24.9. ${ }^{11} \mathbf{B}$ NMR (128 $\left.\mathbf{~ M H z , ~} \mathbf{C D C l}_{3}\right) \delta$ 32.5.HRMS (ESI) calcd for $\mathrm{C}_{23} \mathrm{H}_{27} \mathrm{~B}_{2} \mathrm{~N}_{2} \mathrm{O}_{2}[\mathrm{M}+\mathrm{H}]^{+}$: 385.2253, found: 385.2255 .

1-(2-methylbenzyl)-2-(4,4,5,5-tetramethyl-1,3,2-dioxaborolan-2-yl)-2,3-dihydro-1H-naphtho[1,8-de][1,3, 2]diazaborinine (4e)

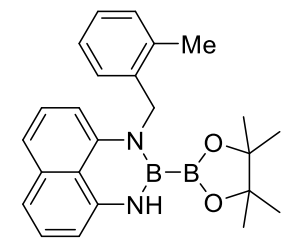

White solid, mp: $98-100{ }^{\circ} \mathrm{C}$. Yield: $52 \% . \mathrm{R}_{\mathrm{f}}=0.3$ (silica gel, PE:EtOAc $=20: 1$ ).

${ }^{1}$ H NMR (500 MHz, CDCl $_{3}$ ) $\delta 7.21-7.09(\mathrm{~m}, 4 \mathrm{H}), 7.08-7.00(\mathrm{~m}, 4 \mathrm{H}), 6.45(\mathrm{~s}, 1 \mathrm{H}), 6.40(\mathrm{dd}, J=7.3,1.0$ $\mathrm{Hz}, 1 \mathrm{H}), 6.10(\mathrm{dd}, J=5.9,2.9 \mathrm{~Hz}, 1 \mathrm{H}), 4.95(\mathrm{~s}, 2 \mathrm{H}), 2.44(\mathrm{~s}, 3 \mathrm{H}), 1.16(\mathrm{~s}, 12 \mathrm{H}),{ }^{13} \mathbf{C}$ NMR $\left(\mathbf{1 2 6} \mathbf{~ M H z}, \mathbf{C D C l}_{\mathbf{3}}\right)$ 
$\delta 142.3,139.9,136.5,136.3,135.1,129.8,127.6,127.2,126.1,125.9,125.0,121.3,117.9,117.7,105.6,104.6$, 83.2, 49.6, 24.8, 19.1. ${ }^{11} \mathbf{B}$ NMR (128 $\left.\mathbf{~ M H z , ~} \mathbf{C D C l}_{3}\right) \delta 32.3$. HRMS (ESI) calcd for $\mathrm{C}_{24} \mathrm{H}_{24} \mathrm{BN}_{2} \mathrm{O}_{2}[\mathrm{M}+\mathrm{H}]^{+}$: 399.2410, found: 399.2408 .

1-(3-methylbenzyl)-2-(4,4,5,5-tetramethyl-1,3,2-dioxaborolan-2-yl)-2,3-dihydro-1H-naphtho[1,8-de][1,3, 2]diazaborinine (4f)

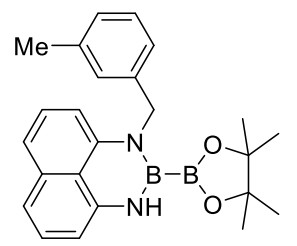

White solid, mp: $197-198^{\circ} \mathrm{C}$. Yield: $50 \% . \mathrm{R}_{\mathrm{f}}=0.3$ (silica gel, PE:EtOAc $=20: 1$ ).

${ }^{1}$ H NMR (500 MHz, CDCl $) \delta 7.20-7.08(\mathrm{~m}, 4 \mathrm{H}), 7.07-6.94(\mathrm{~m}, 4 \mathrm{H}), 6.44(\mathrm{~s}, 1 \mathrm{H}), 6.38(\mathrm{~d}, J=7.2 \mathrm{~Hz}$, 1H), $6.26(\mathrm{~d}, J=7.5 \mathrm{~Hz}, 1 \mathrm{H}), 4.95$ (s, 2H), 2.30 (s, 3H), 1.22 (s, 12H). ${ }^{13} \mathbf{C}$ NMR (126 MHz, $\left.\mathbf{C D C l}_{3}\right) \delta$ 142.1, 139.9, 139.1, 137.9, 136.2, 128.2, 127.5, 127.3, 127.2, 127.1, 123.6, 121.5, 117.9, 117.6, 105.5, 105.1, 83.3, 52.1, 24.9, 21.5. ${ }^{11} \mathbf{B}$ NMR (128 $\left.\mathbf{M H z}, \mathbf{C D C l}_{3}\right) \delta$ 32.9. HRMS (ESI) calcd for $\mathrm{C}_{24} \mathrm{H}_{29} \mathrm{~B}_{2} \mathrm{~N}_{2} \mathrm{O}_{2}[\mathrm{M}+\mathrm{H}]^{+}$: 399.2410, found: 399.2413 .

1-(4-methylbenzyl)-2-(4,4,5,5-tetramethyl-1,3,2-dioxaborolan-2-yl)-2,3-dihydro-1H-naphtho[1,8-de][1,3, 2]diazaborinine $(4 \mathrm{~g})$

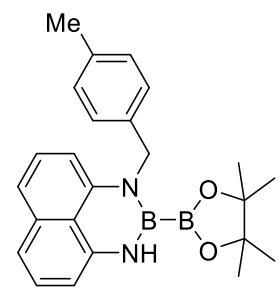

White solid, mp: $115-118^{\circ} \mathrm{C}$. Yield: $40 \% . \mathrm{R}_{\mathrm{f}}=0.3$ (silica gel, PE:EtOAc $=20: 1$ ).

${ }^{1} \mathbf{H}$ NMR $\left(\mathbf{5 0 0 ~ M H z}, \mathbf{C D C l}_{3}\right) \delta 7.23(\mathrm{~d}, J=8.0 \mathrm{~Hz}, 2 \mathrm{H}), 7.14-7.07(\mathrm{~m}, 3 \mathrm{H}), 7.05-6.97(\mathrm{~m}, 3 \mathrm{H}), 6.44(\mathrm{~s}$, $1 \mathrm{H}), 6.38(\mathrm{dd}, J=7.3,0.9 \mathrm{~Hz}, 1 \mathrm{H}), 6.26(\mathrm{dd}, J=7.4,1.1 \mathrm{~Hz}, 1 \mathrm{H}), 4.97(\mathrm{~s}, 2 \mathrm{H}), 2.31(\mathrm{~s}, 3 \mathrm{H}), 1.23(\mathrm{~s}, 12 \mathrm{H}) .{ }^{13} \mathrm{C}$ NMR (126 MHz, $\left.\mathbf{C D C l}_{3}\right) \delta 142.0,139.9,136.2,136.0,129.0,127.4,127.1,126.5,121.5,117.9,117.6,105.4$, 105.1, 83.3, 51.8, 24.9, 21.1. ${ }^{11} \mathbf{B}$ NMR (128 $\left.\mathbf{~ M H z , ~} \mathbf{C D C l}_{3}\right) \delta 31.1$. HRMS (ESI) calcd for $\mathrm{C}_{24} \mathrm{H}_{29} \mathrm{~B}_{2} \mathrm{~N}_{2} \mathrm{O}_{2}$ $[\mathrm{M}+\mathrm{H}]^{+}:$399.2410, found: 399.2404 .

1-(4-fluorobenzyl)-2-(4,4,5,5-tetramethyl-1,3,2-dioxaborolan-2-yl)-2,3-dihydro-1H-naphtho[1,8-de][1,3,2 ]diazaborinine (4h)

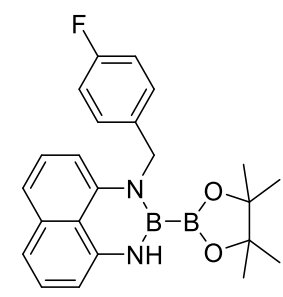

White solid, mp: $124-126^{\circ} \mathrm{C}$. Yield: $33 \% . \mathrm{R}_{\mathrm{f}}=0.3$ (silica gel, PE:EtOAc $=20: 1$ ).

${ }^{1} \mathbf{H}$ NMR (500 MHz, $\left.\mathbf{C D C l}_{\mathbf{3}}\right) \delta 7.33-7.27(\mathrm{~m}, 2 \mathrm{H}), 7.12(\mathrm{dd}, J=8.2,7.4 \mathrm{~Hz}, 1 \mathrm{H}), 7.06-6.94(\mathrm{~m}, 5 \mathrm{H}), 6.44$ (s, 1H), $6.39(\mathrm{dd}, J=7.4,1.1 \mathrm{~Hz}, 1 \mathrm{H}), 6.20(\mathrm{dd}, J=6.9,1.8 \mathrm{~Hz}, 1 \mathrm{H}), 4.96(\mathrm{~s}, 2 \mathrm{H}), 1.22(\mathrm{~s}, 1 \mathrm{H}) .{ }^{13} \mathbf{C} \mathbf{N M R}$ 
$\left(126 \mathbf{M H z}_{\mathbf{C}} \mathbf{C D C l}_{3}\right) \delta 152.2(\mathrm{~d}, J=244.4 \mathrm{~Hz}), 141.8,139.8,136.2,134.7(\mathrm{~d}, J=3.0 \mathrm{~Hz}), 127.9(\mathrm{~d}, J=8.1 \mathrm{~Hz})$, 127.4, 127.2, 121.4, 118.1, 117.7, 115.3, 115.1, 105.6, 104.9, 83.4, 51.4, 24.9. ${ }^{19} \mathbf{F}$ NMR (471 $\left.\mathbf{~ M H z , ~} \mathbf{C D C l}_{3}\right) \delta$ -116.6. ${ }^{11} \mathbf{B}$ NMR (128 MHz, $\left.\mathbf{C D C l}_{3}\right) \delta$ 31.6. HRMS (ESI) calcd for $\mathrm{C}_{23} \mathrm{H}_{26} \mathrm{~B}_{2} \mathrm{FN}_{2} \mathrm{O}_{2}[\mathrm{M}+\mathrm{H}]^{+}: 403.2159$, found: 403.2158 .

2-(4,4,5,5-tetramethyl-1,3,2-dioxaborolan-2-yl)-1-(thiophen-3-ylmethyl)-2,3-dihydro-1H-naphtho[1,8-de ]$[1,3,2]$ diazaborinine (4i)

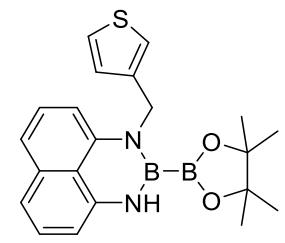

White solid, mp: $116-118{ }^{\circ} \mathrm{C}$. Yield: $60 \% . \mathrm{R}_{\mathrm{f}}=0.2$ (silica gel, PE:EtOAc $=20: 1$ ).

${ }^{1}$ H NMR (500 MHz, CDCl $\left.{ }_{3}\right) \delta 7.24(\mathrm{dd}, J=5.0,3.0 \mathrm{~Hz}, 1 \mathrm{H}), 7.14-7.06(\mathrm{~m}, 4 \mathrm{H}), 7.05-7.00(\mathrm{~m}, 2 \mathrm{H}), 6.41$ (s, 1H), $6.39-6.35$ (m, 2H), 4.98 (s, 2H), 1.24 (s, 12H). ${ }^{13} \mathbf{C}$ NMR (126 MHz, CDCl $\mathbf{~}_{3} \delta$ 142.0, 140.5, 139.8, 136.3, 127.4, 127.1, 126.8, 125.5, 121.4, 120.9, 118.0, 117.7, 105.5, 104.5, 83.4, 47.9, 25.0. ${ }^{11}$ B NMR (128 MHz, $\mathbf{C D C l}_{3}$ ) $\delta$ 31.1. HRMS (ESI) calcd for $\mathrm{C}_{21} \mathrm{H}_{25} \mathrm{~B}_{2} \mathrm{~N}_{2} \mathrm{O}_{2} \mathrm{~S}[\mathrm{M}+\mathrm{H}]^{+}:$391.1817, found: 391.1811.

1-(naphthalen-2-ylmethyl)-2-(4,4,5,5-tetramethyl-1,3,2-dioxaborolan-2-yl)-2,3-dihydro-1H-naphtho[1,8de][1,3,2]diazaborinine $(4 \mathbf{j})$

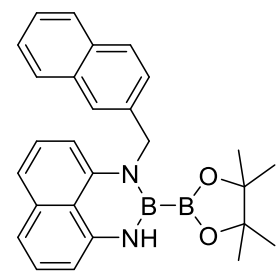

Pink solid, mp: $158-160{ }^{\circ} \mathrm{C}$. Yield: $20 \% . \mathrm{R}_{\mathrm{f}}=0.2$ (silica gel, PE:EtOAc $=20: 1$ ).

${ }^{1} \mathbf{H}$ NMR (500 MHz, $\left.\mathbf{C D C l}_{3}\right) \delta 7.83-7.72(\mathrm{~m}, 4 \mathrm{H}), 7.51-7.38(\mathrm{~m}, 3 \mathrm{H}), 7.14(\mathrm{dd}, J=8.2,7.4 \mathrm{~Hz}, 1 \mathrm{H}), 7.04$ $(\mathrm{dd}, J=8.2,1.0 \mathrm{~Hz}, 1 \mathrm{H}), 7.01-6.94(\mathrm{~m}, 2 \mathrm{H}), 6.49(\mathrm{~s}, 1 \mathrm{H}), 6.42(\mathrm{dd}, J=7.4,1.0 \mathrm{~Hz}, 1 \mathrm{H}), 6.28(\mathrm{dd}, J=5.9$, $2.8 \mathrm{~Hz}, 1 \mathrm{H}), 5.17$ (s, 2H), 1.20 (s, 12H). ${ }^{13} \mathbf{C}$ NMR (126 MHz, $\left.\mathbf{C D C l}_{3}\right) \delta$ 142.0, 139.9, 136.7, 136.2, 133.5, 132.6, 128.1, 127.7, 127.6, 127.5, 127.1, 125.8, 125.3, 124.9, 124.8, 121.5, 118.0, 117.7, 105.6, 105.3, 83.4, 52.3, 24.9. ${ }^{11} \mathbf{B}$ NMR (128 MHz, $\left.\mathbf{C D C l}_{3}\right) \delta$ 33.4. HRMS (ESI) calcd for $\mathrm{C}_{27} \mathrm{H}_{29} \mathrm{~B}_{2} \mathrm{~N}_{2} \mathrm{O}_{2}[\mathrm{M}+\mathrm{H}]^{+}:$435.2410, found: 435.2406 .

\section{Synthesis of Bromoarenes}

\section{1-Bromo-2-isopropoxynaphthalene $(5 b)$}

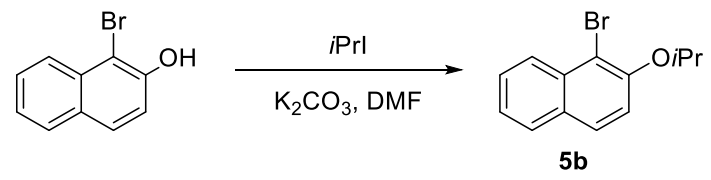

By analogy to a modified literature procedure, ${ }^{2}$ to a solution of 1-bromo-2-naphthol $(1.10 \mathrm{~g}, 5 \mathrm{mmol}, 1$ equiv) in $10 \mathrm{~mL}$ DMF was charged with $\mathrm{K}_{2} \mathrm{CO}_{3}$ (1.38g, $10 \mathrm{mmol}, 2$ equiv) and isopropyl iodide (1.67g, 10 mmol, 2 equiv). The mixture was then agitated at $100{ }^{\circ} \mathrm{C}$ for 12 hours. The reaction was cooled down to $\mathrm{rt}$ and $30 \mathrm{~mL}$ water was added, then extracted with EtOAc. The combined organic layers were washed with brine and dried over anhydrous $\mathrm{Na}_{2} \mathrm{SO}_{4}$. After removal of the solvent, the crude reaction mixture was purified on 
column chromatography (petroleum ether and ethyl acetate) to afford the desired product $\mathbf{5 b}(1.12 \mathrm{~g}, 85 \%$ yield) as a colorless oil. $\mathrm{R}_{\mathrm{f}}=0.6$ (silica gel, PE: EtOAc $=20: 1$ ).

${ }^{1}$ H NMR $\left(500\right.$ MHz, $\left.\mathbf{C D C l}_{3}\right) \delta 8.37(\mathrm{~d}, J=8.6 \mathrm{~Hz}, 1 \mathrm{H}), 7.86-7.81(\mathrm{~m}, 1 \mathrm{H}), 7.78(\mathrm{~d}, J=8.9 \mathrm{~Hz}, 1 \mathrm{H}), 7.68-$ $7.61(\mathrm{~m}, 1 \mathrm{H}), 7.51-7.43(\mathrm{~m}, 1 \mathrm{H}), 7.25(\mathrm{~d}, J=9.0 \mathrm{~Hz}, 1 \mathrm{H}), 4.75-4.63(\mathrm{~m}, 1 \mathrm{H}), 1.49(\mathrm{~d}, J=6.1 \mathrm{~Hz}, 6 \mathrm{H})$.

Known compound.

\section{2-(Benzyloxy)-1-bromonaphthalene (5c)}

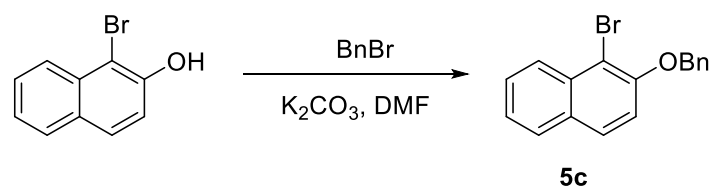

By analogy to a modified literature procedure, ${ }^{2}$ to a solution of 1-bromo-2-naphthol $(1.115 \mathrm{~g}, 5 \mathrm{mmol}, 1$ equiv) in $10 \mathrm{~mL}$ DMF was charged with $\mathrm{K}_{2} \mathrm{CO}_{3}(1.38 \mathrm{~g}, 10 \mathrm{mmol}, 2$ equiv) and benzyl bromide $(1.71 \mathrm{~g}, 10$ mmol, 2 equiv). The mixture was then agitated at $100{ }^{\circ} \mathrm{C}$ for 12 hours. The reaction was cooled down to $\mathrm{rt}$ and $30 \mathrm{~mL}$ water was added, then extracted with EtOAc. The combined organic layers were washed with brine and dried over anhydrous $\mathrm{Na}_{2} \mathrm{SO}_{4}$. After removal of the solvent, the crude reaction mixture was purified on column chromatography (petroleum ether and ethyl acetate) to afford the desired product 5c (1.341 g, $86 \%$ yield) as a white solid. $\mathrm{R}_{\mathrm{f}}=0.5$ (silica gel, PE:EtOAc $=20: 1$ ).

${ }^{1} \mathbf{H}$ NMR (500 MHz, $\left.\mathbf{C D C l}_{3}\right) \delta 8.26(\mathrm{~d}, J=8.6 \mathrm{~Hz}, 1 \mathrm{H}), 7.80-7.74$ (m, 2H), $7.61-7.51$ (m, 3H), $7.45-7.37$ $(\mathrm{m}, 3 \mathrm{H}), 7.36-7.31(\mathrm{~m}, 1 \mathrm{H}), 7.28(\mathrm{~d}, J=9.0 \mathrm{~Hz}, 1 \mathrm{H}), 5.31(\mathrm{~s}, 2 \mathrm{H})$. Known compound.

\section{1-bromo-2-(methoxymethoxy)naphthalene (5d)}<smiles>Oc1ccc2ccccc2c1Br</smiles><smiles>O=[R]OC(=O)OCc1ccccc1</smiles><smiles>COc1ccc2ccccc2c1Br</smiles>

5d

By analogy to a modified literature procedure, ${ }^{3}$ to a solution of 1-bromo-2-naphthol $(1.115 \mathrm{~g}, 5 \mathrm{mmol}, 1$ equiv) in $10 \mathrm{~mL}$ DMF was charged with $\mathrm{K}_{2} \mathrm{CO}_{3}(1.38 \mathrm{~g}, 10 \mathrm{mmol}, 2$ equiv) and chloromethyl methyl ether $\left(0.8 \mathrm{~g}, 10 \mathrm{mmol}, 2\right.$ equiv). The mixture was then agitated at $100{ }^{\circ} \mathrm{C}$ for 12 hours. The reaction was cooled down to rt and $30 \mathrm{~mL}$ water was added, then extracted with EtOAc. The combined organic layers were washed with brine and dried over anhydrous $\mathrm{Na} 2 \mathrm{SO} 4$. After removal of the solvent, the crude reaction mixture was purified on column chromatography (petroleum ether and ethyl acetate) to afford the desired product 5d $\left(0.891 \mathrm{~g}, 67 \%\right.$ yield) as a colorless clear liquid. $\mathrm{R}_{\mathrm{f}}=0.4$ (silica gel, PE:EtOAc $\left.=20: 1\right)$.

${ }^{1} \mathbf{H}$ NMR $\left(\mathbf{5 0 0} \mathbf{M H z}, \mathbf{C D C l}_{3}\right) \delta 8.25(\mathrm{dd}, J=8.6,0.9 \mathrm{~Hz}, 1 \mathrm{H}), 7.81-7.76(\mathrm{~m}, 2 \mathrm{H}), 7.62-7.53(\mathrm{~m}, 1 \mathrm{H}), 7.46$ $-7.40(\mathrm{~m}, 2 \mathrm{H}), 5.37$ (s, 2H), 3.58 (s, 3H). Known compound.

\section{5-bromo-6-methoxy-2-naphthaldehyde (5e)}

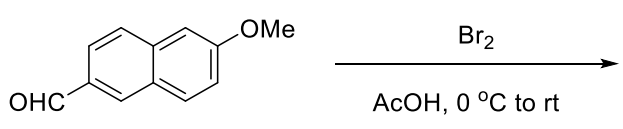<smiles>COc1ccc2cc(C=O)ccc2c1Br</smiles>

By analogy to a modified literature procedure, ${ }^{4}$ to a solution of 6-methoxy-2-naphthaldehyde $(0.931 \mathrm{~g}, 5$ $\mathrm{mmol})$ in $\mathrm{AcOH}(12 \mathrm{~mL})$ at $0{ }^{\circ} \mathrm{C}$ was added $\mathrm{Br}_{2}(0.26 \mathrm{~mL}, 5 \mathrm{mmol})$ over a period of $10 \mathrm{~min}$. The mixture was stirred at the same temperature for $1 \mathrm{~h}$ and then allowed to warm to room temperature and stirred for 10 hours. 
The mixture was diluted with saturated $\mathrm{Na}_{2} \mathrm{~S}_{2} \mathrm{O}_{3}$ solution and stirred for $10 \mathrm{~min}$. The resulting precipitate was filtered, dried under reduced pressure and purified by column chromatography (petroleum ether and ethyl acetate) to afford the desired product $5 \mathbf{e}(1.19 \mathrm{~g}, 90 \%)$ as a white solid. $\mathrm{R}_{\mathrm{f}}=0.2$ (silica gel, PE:EtOAc $\left.=5: 1\right)$.

${ }^{1} \mathbf{H}$ NMR $\left(\mathbf{5 0 0} \mathbf{M H z}, \mathbf{C D C l}_{3}\right) \delta 10.13(\mathrm{~s}, 1 \mathrm{H}), 8.30(\mathrm{~d}, J=9.0 \mathrm{~Hz}, 1 \mathrm{H}), 8.26(\mathrm{~d}, J=1.4 \mathrm{~Hz}, 1 \mathrm{H}), 8.00(\mathrm{dd}, J=$ 9.0, 1.6 Hz, 1H), 7.98 (d, $J=9.0 \mathrm{~Hz}, 1 \mathrm{H}), 7.35$ (d, $J=8.9 \mathrm{~Hz}, 1 \mathrm{H}), 4.07$ (s, 3H). Known compound.

\section{5-Bromo-6-methoxy-2-naphthonitrile (5f)}

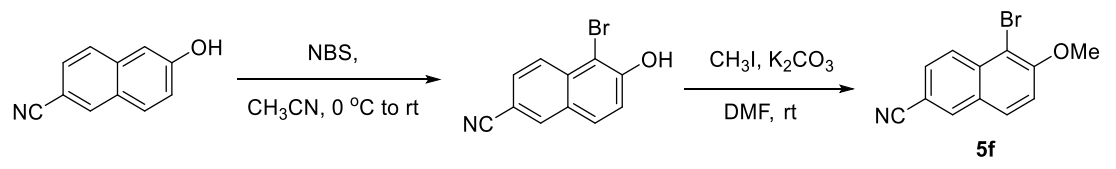

By analogy to a modified literature procedure, ${ }^{2}$ to a solution of 6-hydroxy-2-naphthonitrile $(0.846 \mathrm{~g}, 5$ mmol, 1 equiv) in $\mathrm{CH}_{3} \mathrm{CN}(15 \mathrm{~mL})$ was added NBS (0.89 g, $5 \mathrm{mmol}, 1$ equiv) dissolved in $\mathrm{CH}_{3} \mathrm{CN}(15 \mathrm{~mL})$ over 3 hours at $0{ }^{\circ} \mathrm{C}$. It was stirred at $\mathrm{rt}$ for 1 hour. Water was added, and the mixture was extracted with EtOAc. The combined organic phase was washed with brine and dried over $\mathrm{Na}_{2} \mathrm{SO}_{4}$. After removal of the solvent, the crude reaction mixture was purified on column chromatography (petroleum ether and ethyl acetate) to afford the desired product 5-bromo-6-hydroxy-2-naphthonitrile (1.19 g, 96\%) as a white solid.

To a solution of 5-bromo-6-hydroxy-2-naphthonitrile $(0.744 \mathrm{~g}, 3 \mathrm{mmol}, 1$ equiv) in DMF (10 $\mathrm{mL})$ was added iodomethane $\left(0.23 \mathrm{~mL}, 3.6 \mathrm{mmol}, 1.2\right.$ equiv) and $\mathrm{K}_{2} \mathrm{CO}_{3}(0.621 \mathrm{~g}, 4.5 \mathrm{mmol}, 1.5 \mathrm{equiv})$ at room temperature. The reaction mixture was stirred at $\mathrm{rt}$ for 8 hours. The solution was diluted with water $(40 \mathrm{~mL})$ and extracted with EtOAc. The combined organic layer was washed with water and dried over $\mathrm{Na}_{2} \mathrm{SO}_{4}$. After removal of the solvent, the crude reaction mixture was purified on column chromatography (petroleum ether and ethyl acetate) to afford the desired product $\mathbf{5 f}(0.473 \mathrm{~g}, 61 \%)$ as a white solid. $\mathrm{R}_{\mathrm{f}}=0.2$ (silica gel, PE:EtOAc $=5: 1)$.

${ }^{1}$ H NMR (500 MHz, $\left.\mathbf{C D C l}_{3}\right) \delta 8.30(\mathrm{~d}, J=8.9 \mathrm{~Hz}, 1 \mathrm{H}), 8.18(\mathrm{~s}, 1 \mathrm{H}), 7.89(\mathrm{~d}, J=9.1 \mathrm{~Hz}, 1 \mathrm{H}), 7.68(\mathrm{dd}, J=$ 9.0, 1.1 Hz, 1H), 7.39 (d, $J=9.1 \mathrm{~Hz}, 1 \mathrm{H}), 4.08$ (s, 3H). Known compound.

1-(5-Bromo-6-methoxynaphthalen-2-yl)ethan-1-one (5g)<smiles>COc1ccc2cc(C(C)(C)C)ccc2c1</smiles>

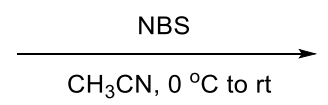

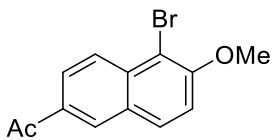

$5 \mathrm{~g}$

By analogy to a modified literature procedure, ${ }^{5}$ to a solution of 1-(6-methoxynaphthalen-2-yl)ethan-1-one $(1.0 \mathrm{~g}, 5 \mathrm{mmol})$ and in $\mathrm{CH}_{3} \mathrm{CN}(15 \mathrm{~mL})$ was added NBS $\left(0.89 \mathrm{~g}, 5 \mathrm{mmol}, 1.0\right.$ equiv) dissolved in $\mathrm{CH}_{3} \mathrm{CN}(15$ $\mathrm{mL}$ ) over 3 hours at $0{ }^{\circ} \mathrm{C}$. It was stirred at room temperature for 1 hour. Water was added, and the mixture was extracted with EtOAc. The combined organic phase was washed with brine and dried over $\mathrm{Na}_{2} \mathrm{SO}_{4}$. After removal of the solvent, the crude reaction mixture was purified on column chromatography (petroleum ether and ethyl acetate) to afford the desired product $\mathbf{5 g}(1.12 \mathrm{~g}, 80 \%)$ as a white solid. $\mathrm{R}_{\mathrm{f}}=0.2$ (silica gel, PE:EtOAc $=5: 1)$.

${ }^{1}$ H NMR $\left(\mathbf{5 0 0}\right.$ MHz, $\left.\mathbf{C D C l}_{3}\right) \delta 8.34(\mathrm{~d}, J=1.5 \mathrm{~Hz}, 1 \mathrm{H}), 8.20(\mathrm{~d}, J=9.0 \mathrm{~Hz}, 1 \mathrm{H}), 8.04(\mathrm{dd}, J=9.0,1.8 \mathrm{~Hz}$, 2H), $7.89(\mathrm{~d}, J=9.1 \mathrm{~Hz}, 1 \mathrm{H}), 7.28(\mathrm{~d}, J=9.0 \mathrm{~Hz}, 1 \mathrm{H}), 4.04(\mathrm{~s}, 3 \mathrm{H}), 2.69$ (s, 3H). Known compound. 
Methyl 5-bromo-6-methoxy-2-naphthoate (5h)

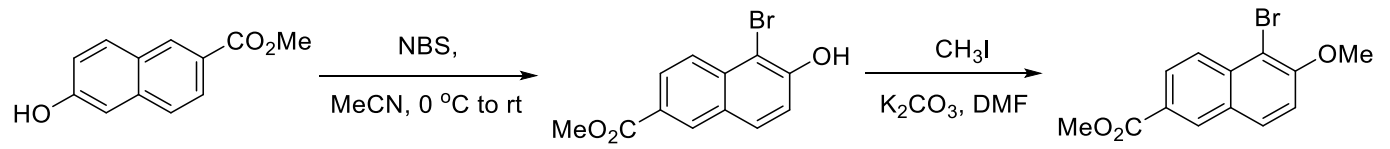

$5 \mathrm{~h}$

By analogy to a modified literature procedure, ${ }^{2}$ to a solution of methyl 6-hydroxy-2-naphthoate ( $2.03 \mathrm{~g}, 10$ mmol, 1 equiv) in MeCN (60 ml) was added dropwise a solution of NBS (1.76 g, $10 \mathrm{mmol}, 1$ equiv) in MeCN (40 ml) at $0{ }^{\circ} \mathrm{C}$ over $30 \mathrm{~min}$. The reaction was let to warm up to room temperature and stirred for 18 hours. After completion, water was added and the product was extracted with EtOAc, dried over $\mathrm{Na}_{2} \mathrm{SO}_{4}$. After removal of the solvent, the crude reaction mixture was purified on column chromatography (petroleum ether and ethyl acetate) to afford the desired product methyl 5-bromo-6-hydroxy-2-naphthoate (1.81 g, 65\% yield).

To a solution of methyl 5-bromo-6-hydroxy-2-naphthoate (1.115 g, $5 \mathrm{mmol}, 1$ equiv) in $10 \mathrm{~mL}$ DMF was charged with $\mathrm{K}_{2} \mathrm{CO}_{3}(1.38 \mathrm{~g}, 10 \mathrm{mmol}, 2$ equiv) and iodomethane (1.42g, $10 \mathrm{mmol}, 2$ equiv). The mixture was then agitated at $100{ }^{\circ} \mathrm{C}$ for 12 hours. The reaction was cooled down to $\mathrm{rt}$ and $30 \mathrm{~mL}$ water was added, then extracted with EtOAc. The combined organic layers were washed with brine and dried over anhydrous $\mathrm{Na}_{2} \mathrm{SO}_{4}$. After removal of the solvent, the crude reaction mixture was purified on column chromatography (petroleum ether and ethyl acetate) to afford the desired product $\mathbf{5 g}\left(1.18 \mathrm{~g}, 80 \%\right.$ yield) as a white solid. $\mathrm{R}_{\mathrm{f}}=$ 0.25 (silica gel, PE:EtOAc $=20: 1$ ).

${ }^{1}$ H NMR (500 MHz, CDCl $) \delta 8.52(\mathrm{~d}, J=1.7 \mathrm{~Hz}, 1 \mathrm{H}), 8.25-8.20(\mathrm{~m}, 1 \mathrm{H}), 8.10(\mathrm{dd}, J=9.0,1.7 \mathrm{~Hz}, 1 \mathrm{H})$, $7.90(\mathrm{~d}, J=9.0 \mathrm{~Hz}, 1 \mathrm{H}), 7.30(\mathrm{~d}, J=9.0 \mathrm{~Hz}, 1 \mathrm{H}), 4.05$ (s, 3H), 3.97 (s, 3H). Known compound.

1-Bromo-2-methoxy-6-phenylnaphthalene (5i)<smiles>Oc1ccc2cc(-c3ccccc3)ccc2c1</smiles>

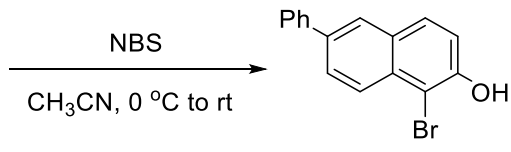

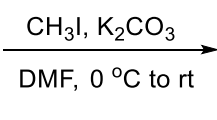<smiles>COc1ccc2cc(-c3ccccc3)ccc2c1Br</smiles>

By analogy to a modified literature procedure, ${ }^{6}$ to a solution of 6-phenylnaphthalen-2-ol (1.10 g, 5 mmol) in $\mathrm{CH}_{3} \mathrm{CN}(15 \mathrm{~mL})$ was added NBS $\left(0.890 \mathrm{~g}, 5 \mathrm{mmol}, 1\right.$ equiv) dissolved in $\mathrm{CH}_{3} \mathrm{CN}(15 \mathrm{~mL})$ over 3 hours at 0 ${ }^{\circ} \mathrm{C}$. It was stirred at room temperature for 1 hour. Water was added, and the mixture was extracted with EtOAc. The combined organic phase was washed with brine and dried over $\mathrm{Na}_{2} \mathrm{SO}_{4}$. After filtration the solvent was removed by evaporation, and the resulting mixture was purified by column chromatography on silica gel (petroleum ether and ethyl acetate) to afford the desired product 1-bromo-6-phenylnaphthalen-2-ol $(1.07 \mathrm{~g}, 72 \%$ yield) as a white solid.

To a solution of 1-bromo-6-phenylnaphthalen-2-ol (0.894 g, 3 mmol, 1 equiv) in DMF (10 mL) was added iodomethane $\left(0.23 \mathrm{~mL}, 3.6 \mathrm{mmol}, 1.2\right.$ equiv) and $\mathrm{K}_{2} \mathrm{CO}_{3}(0.621 \mathrm{~g}, 4.5 \mathrm{mmol}, 1.5$ equiv) at rt. The reaction mixture was stirred at $\mathrm{rt}$ for 8 hours. The solution was diluted with water and extracted with EtOAc. The combined organic layer was washed with water and dried over $\mathrm{Na}_{2} \mathrm{SO}_{4}$. After removal of the solvent, the crude reaction mixture was purified on column chromatography (petroleum ether and ethyl acetate) to afford the desired product $5 \mathbf{i}\left(0.571 \mathrm{~g}, 61 \%\right.$ yield) as a white solid. $\mathrm{R}_{\mathrm{f}}=0.4$ (silica gel, PE:EtOAc $=20: 1$ ).

${ }^{1} \mathbf{H}$ NMR $\left(\mathbf{5 0 0} \mathbf{~ M H z}, \mathbf{C D C l}_{\mathbf{3}}\right) \delta 8.29(\mathrm{~d}, J=8.9 \mathrm{~Hz}, 1 \mathrm{H}), 7.99(\mathrm{~d}, J=1.9 \mathrm{~Hz}, 1 \mathrm{H}), 7.87(\mathrm{~d}, J=8.9 \mathrm{~Hz}, 1 \mathrm{H})$, $7.84(\mathrm{dd}, J=8.8,1.9 \mathrm{~Hz}, 1 \mathrm{H}), 7.73-7.70(\mathrm{~m}, 2 \mathrm{H}), 7.52-7.47(\mathrm{~m}, 2 \mathrm{H}), 7.41-7.37$ (m, 1H), 7.30 (d, $J=9.0$ $\mathrm{Hz}, 1 \mathrm{H}), 4.05$ (s, 3H). Known compound. 
1-Bromo-2-methoxy-6-methylnaphthalene (5j)

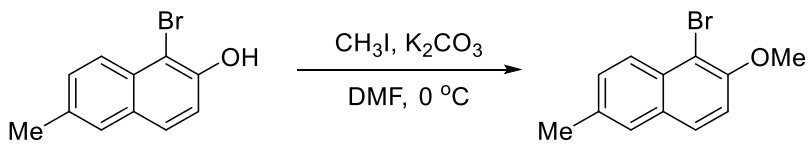

5j

By analogy to a modified literature procedure, ${ }^{6}$ to a solution of 1-bromo-6-methylnaphthalen-2-ol (0.944 g, $4 \mathrm{mmol})$ in DMF $(10 \mathrm{~mL})$ was added iodomethane $\left(0.30 \mathrm{~mL}, 4.9 \mathrm{mmol}, 1.2\right.$ equiv) and $\mathrm{K}_{2} \mathrm{CO}_{3}(0.828 \mathrm{~g}, 6$ mmol, 1.5 equiv) at $\mathrm{rt}$. The reaction mixture was stirred at $\mathrm{rt}$ for 8 hours. The solution was diluted with water and extracted with EtOAc. The combined organic layer was washed with water $(35 \mathrm{~mL})$ and dried over $\mathrm{Na}_{2} \mathrm{SO}_{4}$. After removal of the solvent, the crude reaction mixture was purified on column chromatography (petroleum ether and ethyl acetate) to afford the desired product $5 \mathbf{j}$ ( $0.94 \mathrm{~g}, 94 \%$ yield) as a white solid. $\mathrm{R}_{\mathrm{f}}=0$. 5 (silica gel, PE:EtOAc $=20: 1$ ).

${ }^{1}$ H NMR (500 MHz, CDCl $) \delta 8.11(\mathrm{~d}, J=8.7 \mathrm{~Hz}, 1 \mathrm{H}), 7.73(\mathrm{~d}, J=8.9 \mathrm{~Hz}, 1 \mathrm{H}), 7.55(\mathrm{~s}, 1 \mathrm{H}), 7.40(\mathrm{dd}, J=$ $8.7,1.7 \mathrm{~Hz}, 1 \mathrm{H}), 7.24(\mathrm{~d}, J=9.0 \mathrm{~Hz}, 1 \mathrm{H}), 4.02(\mathrm{~s}, 3 \mathrm{H}), 2.50$ (s, 3H). Known compound.

\section{1-Bromo-2,6-dimethoxynaphthalene (5k)}<smiles>COc1ccc2cc(OC)ccc2c1</smiles><smiles>COc1ccc2c(Br)c(OC)ccc2c1</smiles>

$5 \mathbf{k}$

By analogy to a modified literature procedure, ${ }^{5}$ to a solution of 2,6-dimethoxynaphthalene $(0.94 \mathrm{~g}, 5.0$ mmol) in $\mathrm{CH}_{3} \mathrm{CN}(15 \mathrm{~mL})$ was added NBS $\left(0.890 \mathrm{~g}, 5 \mathrm{mmol}, 1\right.$ equiv) dissolved in $\mathrm{CH}_{3} \mathrm{CN}(15 \mathrm{~mL})$ over 3 hours at $0{ }^{\circ} \mathrm{C}$. It was stirred at room temperature for 1 hour. Water was added, and the mixture was extracted with EtOAc. The combined organic phase was washed with brine and dried over $\mathrm{Na}_{2} \mathrm{SO}_{4}$. After removal of the solvent, the crude reaction mixture was purified on column chromatography (petroleum ether and ethyl acetate) to afford the desired $\mathbf{5 k}\left(0.665 \mathrm{~g}, 50 \%\right.$ yield) as a white solid. $\mathrm{R}_{\mathrm{f}}=0.35$ (silica gel, PE:EtOAc $\left.=20: 1\right)$.

${ }^{1} \mathbf{H}$ NMR $\left(\mathbf{5 0 0} \mathbf{M H z}, \mathbf{C D C l}_{3}\right) \delta 8.13(\mathrm{~d}, J=9.3 \mathrm{~Hz}, 1 \mathrm{H}), 7.70(\mathrm{~d}, J=8.9 \mathrm{~Hz}, 1 \mathrm{H}), 7.25-7.21(\mathrm{M}, 2 \mathrm{H}), 7.09$ (d, $J=2.6 \mathrm{~Hz}, 1 \mathrm{H}), 4.00$ (s, 3H), 3.91 (s, 3H). Known compound.

\section{Methyl (S)-2-(5-bromo-6-methoxynaphthalen-2-yl)propanoate (5l)}

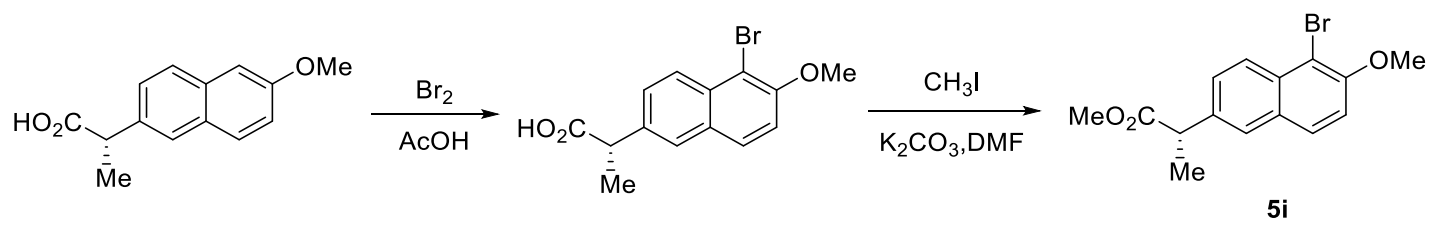

By analogy to a modified literature procedure, ${ }^{2}$ to a solution of (S)-2-(6-methoxynaphthalen-2-yl)propanoic acid $(1.15 \mathrm{~g}, 5 \mathrm{mmol})$ in $\mathrm{AcOH}(15 \mathrm{~mL})$ at $0{ }^{\circ} \mathrm{C}$ was added $\mathrm{Br}_{2}(0.26 \mathrm{~mL}, 5 \mathrm{mmol})$ over a period of $10 \mathrm{~min}$. The mixture was stirred at the same temperature for 1 hour and then allowed to warm to room temperature and stirred for 10 hours. The mixture was diluted with saturated $\mathrm{Na}_{2} \mathrm{~S}_{2} \mathrm{O}_{3}$ solution and stirred for $10 \mathrm{~min}$. The resulting precipitate was filtered, dried under reduced pressure and purified by column chromatography (petroleum ether and ethyl acetate) to afford (S)-2-(5-bromo-6-methoxynaphthalen-2-yl)propanoic acid (0.955 $\mathrm{g}, 62 \%$ yield) as a white solid.

To a solution of (S)-2-(5-bromo-6-methoxynaphthalen-2-yl)propanoic acid ( $0.924 \mathrm{~g}, 3 \mathrm{mmol}, 1$ equiv) in DMF $(15 \mathrm{~mL})$ was added iodomethane $\left(0.47 \mathrm{~mL}, 7.2 \mathrm{mmol}, 2.4\right.$ equiv) and $\mathrm{K}_{2} \mathrm{CO}_{3}(0.621 \mathrm{~g}, 4.5 \mathrm{mmol}, 1.5$ 
equiv) at $\mathrm{rt}$. The reaction mixture was stirred at $\mathrm{rt}$ for 8 hours. The solution was diluted with water and extracted with EtOAc. The combined organic layer was washed with water and dried over $\mathrm{Na}_{2} \mathrm{SO}_{4}$. After removal of the solvent, the crude reaction mixture was purified on column chromatography (petroleum ether and ethyl acetate) to afford the desired $\mathbf{5 i}\left(0.659 \mathrm{~g}, 68 \%\right.$ yield) as a white solid. $\mathrm{R}_{\mathrm{f}}=0.2$ (silica gel, PE:EtOAc $=20: 1)$.

${ }^{1} \mathbf{H}$ NMR $\left(500 \mathrm{MHz}, \mathbf{C D C l}_{3}\right) \delta 8.18(\mathrm{~d}, J=8.8 \mathrm{~Hz}, 1 \mathrm{H}), 7.78(\mathrm{~d}, J=9.0 \mathrm{~Hz}, 1 \mathrm{H}), 7.68(\mathrm{~d}, J=1.6 \mathrm{~Hz}, 1 \mathrm{H})$, $7.52(\mathrm{dd}, J=8.8,1.8 \mathrm{~Hz}, 1 \mathrm{H}), 7.30-7.25(\mathrm{~m}, 1 \mathrm{H}), 4.02(\mathrm{~s}, 3 \mathrm{H}), 3.89(\mathrm{q}, J=7.2 \mathrm{~Hz}, 1 \mathrm{H}), 3.67(\mathrm{~s}, 3 \mathrm{H}), 1.59$ (d, $J=7.2 \mathrm{~Hz}, 3 \mathrm{H})$. Known compound.

\section{1-Bromo-2-methoxy-3-phenylnaphthalene (5m)}

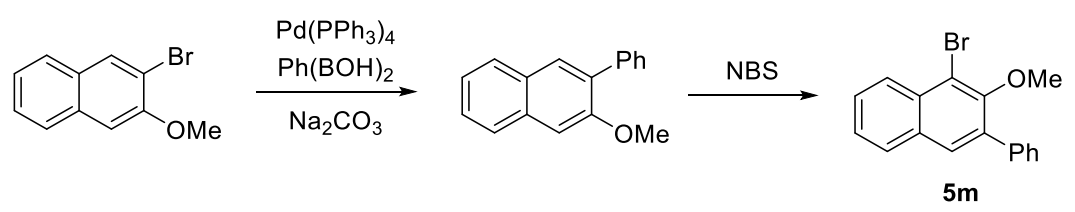

By analogy to a modified literature procedure, ${ }^{6}$ to a solution of 2-bromo-3-methoxynaphthalene $(0.236 \mathrm{~g}, 10$ $\mathrm{mmol})$, phenylboronic acid $(1.586 \mathrm{~g}, 13 \mathrm{mmol})$ and $\mathrm{Pd}\left(\mathrm{PPh}_{3}\right)_{4}(231 \mathrm{mg}, 0.2 \mathrm{mmol})$ in degassed 1,2-dimethoxyethane $(70 \mathrm{~mL})$ was added $2 \mathrm{M}$ degassed aqueous $\mathrm{Na}_{2} \mathrm{CO}_{3}(15 \mathrm{~mL}, 30 \mathrm{mmol})$. The reaction mixture was then stirred at reflux for 18 hours. After cooling to rt, the reaction was quenched with saturated aqueous ammonium chloride and the majority of 1,2-dimethoxyethane was removed under reduced pressure. EtOAc was added and the aqueous layer was separated. The organic layer was washed with saturated aqueous sodium bicarbonate and brine and then dried over $\mathrm{Na}_{2} \mathrm{SO}_{4}$. After removal of the solvent, the crude reaction mixture was purified on column chromatography (petroleum ether and ethyl acetate) to afford the desired 2-methoxy-3-phenylnaphthalene (1.92 g, $82 \%$ yield) as a colourless oil.

To a stirred solution of 2-methoxy-3-phenylnaphthalene $(1.17 \mathrm{~g}, 5 \mathrm{mmol})$ in $\mathrm{CH}_{3} \mathrm{CN}(30 \mathrm{~mL})$ was added NBS $(0.979 \mathrm{~g}, 5.5 \mathrm{mmol})$. After stirring at $\mathrm{rt}$ for 2 hours, the mixture was quenched with saturated aqueous sodium thiosulfate and the majority of the $\mathrm{CH}_{3} \mathrm{CN}$ was removed under reduced pressure. EtOAc was added and the aqueous layer separated. The organic layer was then washed with water three times, dried over $\mathrm{Na}_{2} \mathrm{SO}_{4}$. After removal of the solvent, the crude reaction mixture was purified on column chromatography (petroleum ether and ethyl acetate) to afford the desired $5 \mathrm{~m}(0.873 \mathrm{~g}, 56 \%$ yield $)$ as a white solid. $\mathrm{R}_{\mathrm{f}}=0.6$ (silica gel, PE:EtOAc $=20: 1$ ).

${ }^{1} \mathbf{H}$ NMR $\left(\mathbf{5 0 0} \mathbf{~ M H z}, \mathbf{C D C l}_{3}\right) \delta 8.26(\mathrm{dd}, J=8.5,0.8 \mathrm{~Hz}, 1 \mathrm{H}), 7.87-7.82(\mathrm{~m}, 1 \mathrm{H}), 7.81(\mathrm{~s}, 1 \mathrm{H}), 7.70-7.65$ (m, 2H), $7.62-7.57(\mathrm{~m}, 1 \mathrm{H}), 7.52-7.46(\mathrm{~m}, 3 \mathrm{H}), 7.44-7.39(\mathrm{~m}, 1 \mathrm{H}), 3.53(\mathrm{~s}, 3 \mathrm{H})$. Known compound.

Methyl 4-bromo-3-methoxy-2-naphthoate (5n)
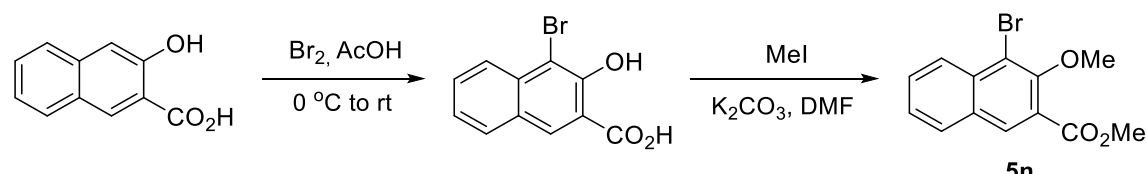

$5 n$

By analogy to a modified literature procedure, ${ }^{2}$ to a solution of 3-hydroxy-2-naphthoic acid $(0.941 \mathrm{~g}, 5$ $\mathrm{mmol})$ in $\mathrm{AcOH}(15 \mathrm{~mL})$ at $0{ }^{\circ} \mathrm{C}$ was added $\mathrm{Br}_{2}(0.26 \mathrm{~mL}, 5 \mathrm{mmol})$ over a period of $10 \mathrm{~min}$. The mixture was stirred at the same temperature for 1 hour and then allowed to warm to room temperature and stirred for 10 hours. The mixture was diluted with saturated $\mathrm{Na}_{2} \mathrm{~S}_{2} \mathrm{O}_{3}$ solution and stirred for 10 min. The resulting precipitate was filtered, dried under reduced pressure and purified by column chromatography (petroleum ether and ethyl acetate) to afford 4-bromo-3-hydroxy-2-naphthoic acid (0.904 g, 68\% yield) as a white solid. 
To a solution of 4-bromo-3-hydroxy-2-naphthoic acid (0.798 g, $3 \mathrm{mmol}, 1.0$ equiv) in DMF (15 mL) was added iodomethane ( $0.47 \mathrm{~mL}, 7.2 \mathrm{mmol}, 2.4$ equiv) and $\mathrm{K}_{2} \mathrm{CO}_{3}(1.24 \mathrm{~g}, 9 \mathrm{mmol}, 3$ equiv) at rt. The reaction mixture was stirred at $\mathrm{rt}$ for 8 hours. The solution was diluted with water and extracted with EtOAc. The combined organic layer was washed with water and dried over $\mathrm{Na}_{2} \mathrm{SO}_{4}$. After removal of the solvent, the crude reaction mixture was purified on column chromatography (petroleum ether and ethyl acetate) to afford the desired $5 \mathrm{n}\left(0.65 \mathrm{~g}, 74 \%\right.$ yield) as a white solid. $\mathrm{R}_{\mathrm{f}}=0.55$ (silica gel, PE:EtOAc $\left.=5: 1\right)$.

${ }^{1}$ H NMR (500 MHz, $\left.\mathbf{C D C l}_{3}\right) \delta 8.36(\mathrm{~s}, 1 \mathrm{H}), 8.27-8.23(\mathrm{~m}, 1 \mathrm{H}), 7.89-7.85(\mathrm{~m}, 1 \mathrm{H}), 7.69-7.63(\mathrm{~m}, 1 \mathrm{H})$, $7.54-7.49$ (m, 1H), 4.01 (s, 3H), 3.99 (s, 3H). ${ }^{13} \mathbf{C}$ NMR (126 MHz, $\left.\mathbf{C D C l}_{3}\right) \delta$ 165.7, 153.4, 134.7, 132.4, 130.2, 129.5, 129.1, 126.8, 126.3, 125.1, 117.6, 62.1, 52.5. HRMS (EI) calcd for $\mathrm{C}_{13} \mathrm{H}_{11} \mathrm{BrO}_{3}$ [M]: 293.9892 , found: 293.9893 .

Methyl 5-bromo-6-methoxy-1-naphthoate (5o)<smiles>O=C(O)c1cccc2cc(O)ccc12</smiles><smiles>O=C(O)c1cccc2c(Br)c(O)ccc12</smiles>

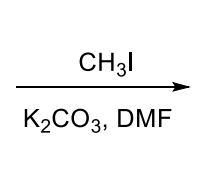<smiles>COc1ccc(OC)c2c(Br)cccc12</smiles>

50

By analogy to a modified literature procedure, ${ }^{2}$ to a solution of 6-hydroxy-1-naphthoic acid (0.94 g, $\left.5 \mathrm{mmol}\right)$ in $\mathrm{AcOH}(15 \mathrm{~mL})$ at $0{ }^{\circ} \mathrm{C}$ was added $\mathrm{Br}_{2}(0.26 \mathrm{~mL}, 15 \mathrm{mmol})$ over a period of $10 \mathrm{~min}$. The mixture was stirred at the same temperature for $1 \mathrm{~h}$ and then allowed to warm to room temperature and stirred for 10 hours. The mixture was diluted with saturated $\mathrm{Na}_{2} \mathrm{~S}_{2} \mathrm{O}_{3}$ solution and stirred for $10 \mathrm{~min}$. The resulting precipitate was filtered, dried under reduced pressure and purified by column chromatography (petroleum ether and ethyl acetate) to afford 5-bromo-6-hydroxy-1-naphthoic acid $(0.838 \mathrm{~g}, 63 \%$ yield $)$ as a white solid.

To a solution of 5-bromo-6-hydroxy-1-naphthoic acid $(0.801 \mathrm{~g}, 3 \mathrm{mmol}, 1$ equiv) in DMF (10 mL) was added iodomethane ( $0.47 \mathrm{~mL}, 7.2 \mathrm{mmol}, 2.4$ equiv) and $\mathrm{K}_{2} \mathrm{CO}_{3}$ (1.24 g, 9 mmol, 3 equiv) at rt. The reaction mixture was stirred at $\mathrm{rt}$ for 8 hours. The solution was diluted with water and extracted with EtOAc. The combined organic layer was washed with water and dried over $\mathrm{Na}_{2} \mathrm{SO}_{4}$. After removal of the solvent, the crude reaction mixture was purified on column chromatography (petroleum ether and ethyl acetate) to afford the desired $5 \mathbf{n}\left(0.335 \mathrm{~g}, 38 \%\right.$ yield) as a yellow solid. $\mathrm{R}_{\mathrm{f}}=0.5$ (silica gel, PE:EtOAc $=5: 1$ ).

${ }^{1}$ H NMR (500 MHz, CDCl $) \delta 8.94(\mathrm{dd}, J=9.5,0.7 \mathrm{~Hz}, 1 \mathrm{H}), 8.50-8.44(\mathrm{~m}, 1 \mathrm{H}), 8.07(\mathrm{dd}, J=7.2,1.2 \mathrm{~Hz}$, 1H), $7.56(\mathrm{dd}, J=8.6,7.2 \mathrm{~Hz}, 1 \mathrm{H}), 7.36$ (d, $J=9.5 \mathrm{~Hz}, 1 \mathrm{H}), 4.04$ (s, 3H), 3.99 (s, 3H). Known compound.

\section{1-bromo-2,7-dimethoxynaphthalene $(5 p)$}

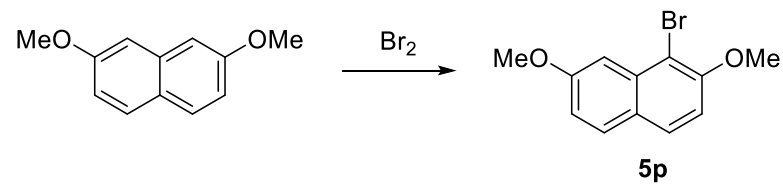

By analogy to a modified literature procedure, ${ }^{6}$ to a stirred solution of 2,7-dimethoxynaphthalene $(0.94 \mathrm{~g}$, $5 \mathrm{mmol})$ in $\mathrm{CHCl}_{3}(15 \mathrm{~mL})$ was cooled to $0{ }^{\circ} \mathrm{C}$. A solution of $\mathrm{Br}_{2}(1.54 \mathrm{~mL}, 30 \mathrm{mmol})$ in $\mathrm{CHCl}_{3}(5 \mathrm{~mL})$ was then added dropwise over a period of 1 hour. After warming to rt, the reaction was quenched with saturated aqueous sodium thiosulfate. The organic layer was separated and the aqueous layer was extracted twice with $\mathrm{CHCl}_{3}$. The combined organic extracts were dried over anhydrous $\mathrm{Na}_{2} \mathrm{SO}_{4}$. After removal of the solvent, the crude reaction mixture was purified on recrystallization from hexane/ethyl acetate afforded the desired compound 5p $(0.465 \mathrm{~g}, 35 \%)$ as a white solid. $\mathrm{R}_{\mathrm{f}}=0.4$ (silica gel, PE:EtOAc $\left.=20: 1\right)$. 
${ }^{1}$ H NMR $\left(500\right.$ MHz, $\left.\mathbf{C D C l}_{3}\right) \delta 7.73(\mathrm{~d}, J=8.9 \mathrm{~Hz}, 1 \mathrm{H}), 7.67(\mathrm{~d}, J=8.9 \mathrm{~Hz}, 1 \mathrm{H}), 7.50(\mathrm{~d}, J=2.5 \mathrm{~Hz}, 1 \mathrm{H})$, $7.11(\mathrm{~d}, J=8.9 \mathrm{~Hz}, 1 \mathrm{H}), 7.04(\mathrm{dd}, J=8.9,2.5 \mathrm{~Hz}, 1 \mathrm{H}), 4.02(\mathrm{~s}, 3 \mathrm{H}), 3.97$ (s, 3H). Known compound.

\section{4-Bromo-3-methoxyquinoline (5q)}

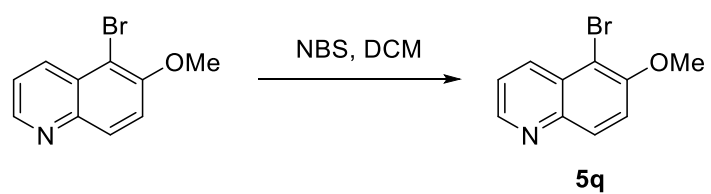

By analogy to a modified literature procedure, ${ }^{4}$ to a solution of 6-methoxyquinoline $(1.19 \mathrm{~g}, 5 \mathrm{mmol})$ in $\operatorname{DCM}(5 \mathrm{~mL})$ at $0{ }^{\circ} \mathrm{C}$ was added NBS $(1.0 \mathrm{~g}, 5.63 \mathrm{mmol})$ in one portion and the resulting mixture was stirred at $\mathrm{rt}$ for 20 hours. Upon completion, the reaction mixture was washed with $1 \mathrm{M} \mathrm{HCl}(\mathrm{aq})(2 \mathrm{~mL})$ and then brine. The organic layer was separated and dried over $\mathrm{Na}_{2} \mathrm{SO}_{4}$. After removal of the solvent, the crude reaction mixture was purified on column chromatography (petroleum ether and ethyl acetate) to afford the desired product 5q (0.948 g, 80\% yield) as a white solid. $\mathrm{R}_{\mathrm{f}}=0.1$ (silica gel, PE:EtOAc $\left.=5: 1\right)$.

${ }^{1} \mathbf{H}$ NMR $\left(\mathbf{5 0 0} \mathbf{M H z}, \mathbf{C D C l}_{3}\right) \delta 8.81(\mathrm{dd}, J=4.2,1.5 \mathrm{~Hz}, 1 \mathrm{H}), 8.57-8.51(\mathrm{~m}, 1 \mathrm{H}), 8.11(\mathrm{~d}, J=9.2 \mathrm{~Hz}, 1 \mathrm{H})$, $7.52(\mathrm{~d}, J=9.3 \mathrm{~Hz}, 1 \mathrm{H}), 7.47(\mathrm{dd}, J=8.6,4.2 \mathrm{~Hz}, 1 \mathrm{H}), 4.06(\mathrm{~s}, 3 \mathrm{H})$. Known compound.

\section{2-bromo-4-chloro-1-methoxy-3,5-dimethylbenzene (5s)}

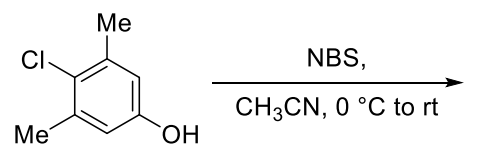<smiles>Cc1cc(O)c(Br)c([N+](=O)[O-])c1Cl</smiles><smiles>COc1cc(OC)c(Br)c([N+](=O)[O-])c1Cl</smiles>

$5 \mathrm{~s}$

By analogy to a modified literature procedure, ${ }^{5}$ to a solution of 4-chloro-3,5-dimethylphenol (1.56 g, 10.0 mmol) in ethyl acetate $(40 \mathrm{~mL})$ were added $\mathrm{HBr}(48 \%$ aq, $1.44 \mathrm{~mL}, 10 \mathrm{mmol}, 1$ equiv) and DMSO (0.71 ml, $10 \mathrm{mmol}, 1$ equiv) at $\mathrm{rt}$. The mixture were stirred at $60^{\circ} \mathrm{C}$ and monitored by TLC. After cooling down to room temperature and removal of the solvent, the crude reaction mixture was purified on column chromatography (petroleum ether and ethyl acetate) to afford the desired product 2-bromo-4-chloro-3,5-dimethylphenol (1.21 g, $52 \%$ yield) as a white solid.

To a solution of 2-bromo-4-chloro-3,5-dimethylphenol (0.424 g, $1.8 \mathrm{mmol})$ in DMF (6 mL) was added iodomethane $(0.56 \mathrm{~mL}, 9 \mathrm{mmol}, 5.0$ equiv), and $\mathrm{KOH}(0.151 \mathrm{~g}, 1.5$ equiv) at room temperature. The reaction mixture was stirred at $\mathrm{rt}$ for 8 hours. The solution was diluted with water and extracted with EtOAc. The combined organic layer was washed with water and dried over $\mathrm{Na}_{2} \mathrm{SO}_{4}$. After removal of the solvent, the crude reaction mixture was purified on column chromatography (petroleum ether and ethyl acetate) to afford the desired product $\mathbf{5 s}\left(0.42 \mathrm{~g}, 94 \%\right.$ yield) as a white solid. $\mathrm{R}_{\mathrm{f}}=0.6$ (silica gel, PE:EtOAc $\left.=20: 1\right)$.

${ }^{1}$ H NMR (500 MHz, CDCl $) \delta 6.66(\mathrm{~s}, 1 \mathrm{H}), 3.86(\mathrm{~s}, 3 \mathrm{H}), 2.55(\mathrm{~s}, 3 \mathrm{H}), 2.36$ (d, $\left.J=0.4 \mathrm{~Hz}, 3 \mathrm{H}\right)$. Known compound.

\section{5-bromo-6-methoxy-3,4-dihydronaphthalen-1(2H)-one (5u)}<smiles>COc1ccc2c(c1)CCCC2=O</smiles>

By analogy to a modified literature<smiles>COc1ccc2c(c1Br)CCCC2=O</smiles>

procedure, $^{7} \quad$ to $\quad$ a suspension of 
6-methoxy-3,4-dihydronaphthalen-1(2H)-one $(0.352 \mathrm{~g}, 2 \mathrm{mmol})$ in water $(20 \mathrm{~mL})$ was added NBS $(0.36 \mathrm{~g}, 2$ mmol ), and the reaction mixture was heated to $60{ }^{\circ} \mathrm{C}$ while stirring. $\mathrm{H}_{2} \mathrm{SO}_{4}(40 \%$ aq solution, 4 mmol ) was then added and stirring continued for 5 hours. The reaction solution was cooled to $\mathrm{rt}$ and extracted with tert-butyl methyl ether, dried over $\mathrm{Na}_{2} \mathrm{SO}_{4}$. After removal of the solvent, the crude reaction mixture was purified on column chromatography (petroleum ether and ethyl acetate) to afford the desired product 5u $(0.31 \mathrm{~g}, 61 \%$ yield $)$, which was additionally purified by crystallisation from ethanol to give pure $\mathbf{5 u}$ as a white solid. $\mathrm{R}_{\mathrm{f}}=0.35$ (silica gel, PE:EtOAc $=5: 1$ ).

${ }^{1} \mathbf{H}$ NMR $\left(500 \mathbf{M H z} \mathbf{C D C l}_{3}\right) \delta 8.05(\mathrm{~d}, J=8.7 \mathrm{~Hz}, 1 \mathrm{H}), 6.87(\mathrm{~d}, J=8.7 \mathrm{~Hz}, 1 \mathrm{H}), 3.96(\mathrm{~s}, 3 \mathrm{H}), 3.02(\mathrm{t}, J=6.2$ $\mathrm{Hz}, 2 \mathrm{H}), 2.64-2.56(\mathrm{~m}, 2 \mathrm{H}), 2.19-2.07(\mathrm{~m}, 2 \mathrm{H})$.

\section{General Procedure for the Atroposelective Miyaura Borylation Reaction}

In air, a $10 \mathrm{~mL}$ schlenk tube was charged with 5 (0.1 mmol, 1 equiv), 4 (0.15 mmol, 1.5 equiv), $\mathrm{Pd}(\mathrm{OAc})_{2}(4$ mol\%), $\mathbf{L 4}(6 \mathrm{~mol} \%)$ and $\mathrm{K}_{3} \mathrm{PO}_{4}(0.25 \mathrm{mmol}, 2.5$ equiv). The tube was evacuated and filled with argon for three cycles. Then, $1 \mathrm{~mL}$ of 1,4-dioxane was added under argon. The reaction was allowed to stir at $30{ }^{\circ} \mathrm{C}$ for 24 hours. Upon completion, proper amount of silica gel was added to the reaction mixture. After removal of the solvent, the crude reaction mixture was purified on silica gel (petroleum ether and ethyl acetate) to afford the desired products.

(S)-1-ethyl-2-(2-methoxynaphthalen-1-yl)-2,3-dihydro-1H-naphtho[1,8-de][1,3,2]diazaborinine (6a)

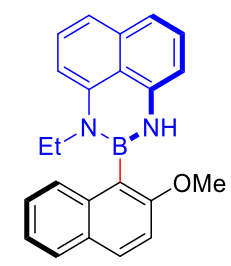

White solid, mp: $88-89^{\circ} \mathrm{C}$. Yield: $82 \% . \mathrm{R}_{\mathrm{f}}=0.25$ (silica gel, PE:EtOAc $=20: 1$ ).

${ }^{1} \mathbf{H}$ NMR $\left(400 \mathbf{M H z} \mathbf{C D C l}_{3}\right) \delta 7.90(\mathrm{~d}, J=9.0 \mathrm{~Hz}, 1 \mathrm{H}), 7.81(\mathrm{~d}, J=7.8 \mathrm{~Hz}, 1 \mathrm{H}), 7.78(\mathrm{~d}, J=8.2 \mathrm{~Hz}, 1 \mathrm{H})$, $7.42-7.23(\mathrm{~m}, 4 \mathrm{H}), 7.17-7.07(\mathrm{~m}, 3 \mathrm{H}), 6.53(\mathrm{~d}, J=7.7 \mathrm{~Hz}, 1 \mathrm{H}), 6.30(\mathrm{dd}, J=6.9,1.2 \mathrm{~Hz}, 1 \mathrm{H}), 5.94(\mathrm{~s}, 1 \mathrm{H})$, $3.90(\mathrm{~s}, 3 \mathrm{H}), 3.51-3.26(\mathrm{~m}, 2 \mathrm{H}), 1.07$ (t, $J=7.0 \mathrm{~Hz}, 3 \mathrm{H}) .{ }^{\mathbf{1 3}} \mathbf{C} \mathbf{N M R}\left(\mathbf{1 2 6} \mathbf{M H z}, \mathbf{C D C l}_{\mathbf{3}}\right) \delta 159.0,142.4,140.8$, 136.6, 136.5, 130.5, 128.9, 128.2, 127.6, 127.2, 127.2, 126.4, 123.5, 120.9, 117.7, 117.5, 112.6, 105.6, 103.7, 56.0, 41.1, 12.9. ${ }^{11} \mathbf{B}$ NMR (128 $\left.\mathbf{M H z}, \mathbf{C D C l}_{3}\right) \delta 31.8$. HRMS (ESI) calcd for $\mathrm{C}_{23} \mathrm{H}_{22} \mathrm{BN}_{2} \mathrm{O}[\mathrm{M}+\mathrm{H}]^{+}: 353.1820$, found: 353.1816. HPLC analysis: DAICEL CHIRALCEL IA-3, hexane/isopropanol $=95 / 5,1 \mathrm{~mL} / \mathrm{min}, \lambda=$ $254 \mathrm{~nm}, \mathrm{t}_{\mathrm{R}}($ minor $)=7.24 \mathrm{~min}, \mathrm{t}_{\mathrm{R}}($ major $)=8.25 \mathrm{~min}, 94 \%$ ee. $[\boldsymbol{\alpha}]^{25}{ }_{\mathrm{D}}:+89.2\left(c 0.5, \mathrm{CHCl}_{3}\right)$.

(S)-1-ethyl-2-(2-isopropoxynaphthalen-1-yl)-2,3-dihydro-1H-naphtho[1,8-de][1,3,2]diazaborinine (6b)

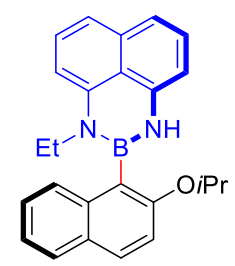

White solid, mp: $68-70{ }^{\circ} \mathrm{C}$. Yield: $32 \% . \mathrm{R}_{\mathrm{f}}=0.45$ (silica gel, PE:EtOAc $=20: 1$ ).

${ }^{1} \mathbf{H}$ NMR $\left(\mathbf{5 0 0} \mathbf{M H z}, \mathbf{C D C l}_{3}\right) \delta 7.87(\mathrm{~d}, J=9.0 \mathrm{~Hz}), 7.82(\mathrm{~d}, J=7.9 \mathrm{~Hz}, 1 \mathrm{H}), 7.79(\mathrm{~d}, J=8.2 \mathrm{~Hz}, 1 \mathrm{H})$, 7.39-7.32 (m, 2H), $7.31-7.27(\mathrm{~m}, 2 \mathrm{H}), 7.18-7.10(\mathrm{~m}, 3 \mathrm{H}), 6.56(\mathrm{~d}, J=7.6 \mathrm{~Hz}, 1 \mathrm{H}), 6.32(\mathrm{~d}, J=6.9 \mathrm{~Hz}, 1 \mathrm{H})$, $5.93(\mathrm{~s}, 1 \mathrm{H}), 4.71(\mathrm{dt}, J=12.0,6.0 \mathrm{~Hz}, 1 \mathrm{H}), 3.57-3.34(\mathrm{~m}, 2 \mathrm{H}), 1.34(\mathrm{~d}, J=6.0 \mathrm{~Hz}, 3 \mathrm{H}), 1.31(\mathrm{~d}, J=5.9 \mathrm{~Hz}$, 3H), $1.10(\mathrm{t}, J=7.0 \mathrm{~Hz}, 3 \mathrm{H}) .{ }^{\mathbf{1 3}} \mathbf{C} \mathbf{N M R}\left(\mathbf{1 2 6} \mathbf{~ M H z}, \mathbf{C D C l}_{3}\right) \delta 157.6,142.5,141.0,136.7,136.6,130.3,128.9$, 
128.1, 127.6, 127.3, 127.2, 126.3, 123.5, 120.9, 117.5, 117.4, 115.4, 105.4, 103.7, 70.8, 41.2, 22.6, 22.5, 12.9.

${ }^{11}$ B NMR (128 $\mathbf{M H z}, \mathbf{C D C l}_{3}$ ) $\delta$ 32.6. HRMS (ESI) calcd for $\mathrm{C}_{25} \mathrm{H}_{26} \mathrm{BN}_{2} \mathrm{O}[\mathrm{M}+\mathrm{H}]^{+}:$381.2133, found: 381.2126. HPLC analysis: DAICEL CHIRALCEL IA-3, hexane/isopropanol $=98 / 2,1 \mathrm{~mL} / \mathrm{min}, \lambda=254 \mathrm{~nm}$, $\mathrm{t}_{\mathrm{R}}($ minor $)=6.54 \mathrm{~min}, \mathrm{t}_{\mathrm{R}}($ major $)=8.63 \mathrm{~min}, 93 \%$ ee. $[\boldsymbol{\alpha}]^{25}{ }_{\mathrm{D}}:-413.0\left(c 0.5, \mathrm{CHCl}_{3}\right)$.

(S)-2-(2-(benzyloxy)naphthalen-1-yl)-1-ethyl-2,3-dihydro-1H-naphtho[1,8-de][1,3,2]diazaborinine (6c)

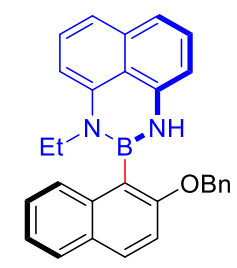

White solid, mp: $54-55^{\circ} \mathrm{C}$. Yield: $64 \% . \mathrm{R}_{\mathrm{f}}=0.3$ (silica gel, PE:EtOAc $=20: 1$ ).

${ }^{1} \mathbf{H}$ NMR $\left(500 \mathbf{M H z} \mathbf{C D C l}_{3}\right) \delta 7.86(\mathrm{~d}, J=9.1 \mathrm{~Hz}, 1 \mathrm{H}), 7.80(\mathrm{t}, J=8.9 \mathrm{~Hz}, 2 \mathrm{H}), 7.43-7.32(\mathrm{~m}, 5 \mathrm{H}), 7.30-$ $7.22(\mathrm{~m}, 4 \mathrm{H}), 7.19-7.10(\mathrm{~m}, 3 \mathrm{H}), 6.55$ (d, $J=7.7 \mathrm{~Hz}, 1 \mathrm{H}), 6.29(\mathrm{dd}, J=7.1,1.2 \mathrm{~Hz}, 1 \mathrm{H}), 5.83(\mathrm{~s}, 1 \mathrm{H}), 5.26$ $(\mathrm{q}, J=12.7 \mathrm{~Hz}, 2 \mathrm{H}), 3.52-3.36(\mathrm{~m}, 2 \mathrm{H}), 1.08(\mathrm{t}, J=7.0 \mathrm{~Hz}, 3 \mathrm{H}) .{ }^{\mathbf{1 3}} \mathbf{C} \mathbf{N M R}\left(\mathbf{1 2 6} \mathbf{M H z}, \mathbf{C D C l}_{\mathbf{3}}\right) \delta 158.0$, $142.4,140.8,137.3,136.6,136.5,130.5,129.2$, 128.5, 128.2, 127.7, 127.6, 127.4, 127.2, 126.4, 123.8, 120.9, 117.7, 117.5, 114.8, 105.6, 103.8, 70.9, 41.2, 13.1. ${ }^{11} \mathbf{B}$ NMR (128 MHz, CDCl $) \delta 31.7$. HRMS (ESI) calcd for $\mathrm{C}_{29} \mathrm{H}_{26} \mathrm{BN}_{2} \mathrm{O}[\mathrm{M}+\mathrm{H}]^{+}$: 429.2133, found: 429.2137. HPLC analysis: DAICEL CHIRALCEL IA-3, hexane/isopropanol $=98 / 2,1 \mathrm{~mL} / \mathrm{min}, \lambda=254 \mathrm{~nm}, \mathrm{t}_{\mathrm{R}}$ (major) $=20.0 \mathrm{~min}, \mathrm{t}_{\mathrm{R}}($ minor $)=51.41 \mathrm{~min}, 96 \%$ ee. $[\alpha]^{25}$ : $+30.1\left(c 0.5, \mathrm{CHCl}_{3}\right)$.

(S)-1-ethyl-2-(2-(methoxymethoxy)naphthalen-1-yl)-2,3-dihydro-1H-naphtho[1,8-de][1,3,2]diazaborinin e (6d)

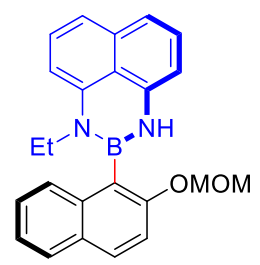

White solid, mp: $49-50^{\circ} \mathrm{C}$. Yield: $40 \% . \mathrm{R}_{\mathrm{f}}=0.3$ (silica gel, PE:EtOAc $=20: 1$ ).

${ }^{1} \mathbf{H}$ NMR (500 MHz, CDCl $) \delta 7.89(\mathrm{~d}, J=9.0 \mathrm{~Hz}, 1 \mathrm{H}), 7.86-7.79(\mathrm{~m}, 2 \mathrm{H}), 7.47(\mathrm{~d}, J=9.0 \mathrm{~Hz}, 1 \mathrm{H}), 7.44-$ $7.36(\mathrm{~m}, 2 \mathrm{H}), 7.29(\mathrm{t}, J=8.0 \mathrm{~Hz}, 1 \mathrm{H}), 7.17-7.11(\mathrm{~m}, 3 \mathrm{H}), 6.56(\mathrm{~d}, J=7.6 \mathrm{~Hz}, 1 \mathrm{H}), 6.32(\mathrm{dd}, J=7.0,1.3 \mathrm{~Hz}$, $1 \mathrm{H}), 5.98(\mathrm{~s}, 1 \mathrm{H}), 5.26(\mathrm{dd}, J=23.1,7.0 \mathrm{~Hz}, 2 \mathrm{H}), 3.48(\mathrm{~s}, 3 \mathrm{H}) 3.53-3.33(\mathrm{~m}, 2 \mathrm{H}), 1.10(\mathrm{t}, J=7.0 \mathrm{~Hz}, 3 \mathrm{H})$. ${ }^{13}$ C NMR (126 MHz, $\left.\mathbf{C D C l}_{3}\right) \delta 157.0,142.5,140.9,136.7,136.5,130.7,129.7,128.3,127.7,127.6,127.3$, 126.5, 124.1, 121.0, 117.9, 117.7, 115.8, 105.7, 103.9, 95.0, 56.3, 41.3, 13.1. ${ }^{11} \mathbf{B}$ NMR (128 $\left.\mathbf{M H z}_{\mathbf{1}} \mathbf{C D C l}_{\mathbf{3}}\right) \delta$ 31.8. HRMS (ESI) calcd for $\mathrm{C}_{24} \mathrm{H}_{24} \mathrm{BN}_{2} \mathrm{O}_{2}[\mathrm{M}+\mathrm{H}]^{+}: 383.1925$, found: 383.1920. HPLC analysis: DAICEL CHIRALCEL IA-3, hexane/isopropanol $=98 / 2,1 \mathrm{~mL} / \mathrm{min}, \lambda=254 \mathrm{~nm}, \mathrm{t}_{\mathrm{R}}($ minor $)=10.97 \mathrm{~min}, \mathrm{t}_{\mathrm{R}}($ major $)=$ $13.93 \mathrm{~min}, 97 \%$ ee. $[\boldsymbol{\alpha}]^{25}$ : $-595.8\left(c 0.5, \mathrm{CHCl}_{3}\right)$.

(S)-5-(1-ethyl-1H-naphtho[1,8-de][1,3,2] diazaborinin-2(3H)-yl)-6-methoxy-2-naphthaldehyde (6e)<smiles></smiles> 
Yellow solid, mp: $92-93{ }^{\circ} \mathrm{C}$. Yield: $96 \% . \mathrm{R}_{\mathrm{f}}=0.33$ (silica gel, PE: EtOAc $=5: 1$ ).

${ }^{1} \mathbf{H}$ NMR (500 MHz, $\left.\mathbf{C D C l}_{3}\right) \delta 10.07(\mathrm{~s}, 1 \mathrm{H}), 8.30(\mathrm{~s}, 1 \mathrm{H}), 8.07(\mathrm{~d}, J=9.0 \mathrm{~Hz}, 1 \mathrm{H}), 7.87-7.81(\mathrm{~m}, 1 \mathrm{H}), 7.38$ $(\mathrm{d}, J=9.1 \mathrm{~Hz}, 1 \mathrm{H}), 7.29-7.25(\mathrm{~m}, 1 \mathrm{H}), 7.17-7.14(\mathrm{~m}, 1 \mathrm{H}), 7.14-7.10(\mathrm{~m}, 1 \mathrm{H}), 6.54(\mathrm{~d}, J=7.6 \mathrm{~Hz}, 1 \mathrm{H})$, $6.33(\mathrm{dd}, J=6.9,1.4 \mathrm{~Hz}, 1 \mathrm{H}), 5.95(\mathrm{~s}, 1 \mathrm{H}), 3.94(\mathrm{~s}, 3 \mathrm{H}), 3.44-3.29(\mathrm{~m}, 2 \mathrm{H}), 1.06(\mathrm{t}, J=7.0 \mathrm{~Hz}, 3 \mathrm{H}) .{ }^{13} \mathbf{C}$ NMR (126 MHz, $\left.\mathbf{C D C l}_{3}\right) \delta 192.0,161.6,142.1,140.5,139.9,136.5,135.1,132.6,132.1,128.2,127.8,127.6$,

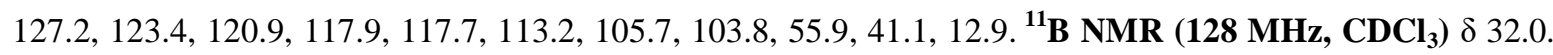
HRMS (ESI) calcd for $\mathrm{C}_{24} \mathrm{H}_{22} \mathrm{BN}_{2} \mathrm{O}_{2}[\mathrm{M}+\mathrm{H}]^{+}: 381.1769$, found: 381.1771. HPLC analysis: DAICEL CHIRALCEL IA-3, hexane/isopropanol $=90 / 10,1 \mathrm{~mL} / \mathrm{min}, \lambda=254 \mathrm{~nm}, \mathrm{t}_{\mathrm{R}}($ minor $)=15.82 \mathrm{~min}, \mathrm{t}_{\mathrm{R}}$ (major) $=$ $17.13 \min , 92 \%$ ee. $[\boldsymbol{\alpha}]^{25}{ }_{\mathbf{D}}:+129.5\left(c 0.5, \mathrm{CHCl}_{3}\right)$.

(S)-5-(1-ethyl-1H-naphtho[1,8-de][1,3,2]diazaborinin-2(3H)-yl)-6-methoxy-2-naphthonitrile (6f)<smiles></smiles>

White solid, mp: $68-69^{\circ} \mathrm{C}$. Yield: $90 \% . \mathrm{R}_{\mathrm{f}}=0.37$ (silica gel, PE:EtOAc $=5: 1$ ).

${ }^{1} \mathbf{H}$ NMR (500 MHz, CDCl $\left.\mathbf{3}\right) \delta 8.20(\mathrm{~d}, J=1.0 \mathrm{~Hz}, 1 \mathrm{H}), 7.97(\mathrm{~d}, J=9.1 \mathrm{~Hz}, 1 \mathrm{H}), 7.85(\mathrm{~d}, J=8.7 \mathrm{~Hz}, 1 \mathrm{H})$, $7.50(\mathrm{dd}, J=8.8,1.5 \mathrm{~Hz}, 1 \mathrm{H}), 7.41(\mathrm{~d}, J=9.1 \mathrm{~Hz}, 1 \mathrm{H}), 7.32-7.26(\mathrm{~m}, 1 \mathrm{H}), 7.18-7.09(\mathrm{~m}, 3 \mathrm{H}), 6.54(\mathrm{~d}, J=$ $7.6 \mathrm{~Hz}, 1 \mathrm{H}), 6.34(\mathrm{dd}, J=6.9,1.1 \mathrm{~Hz}, 1 \mathrm{H}), 5.91(\mathrm{~s}, 1 \mathrm{H}), 3.95(\mathrm{~s}, 3 \mathrm{H}), 3.48-3.25(\mathrm{~m}, 2 \mathrm{H}), 1.06(\mathrm{t}, J=7.0 \mathrm{~Hz}$, 3H). ${ }^{13} \mathbf{C}$ NMR (126 MHz, $\left.\mathbf{C D C l}_{3}\right) \delta 161.4,142.0,140.4,138.1,136.5,134.5,131.4,128.3,127.6,127.6$, 127.2, 126.8, 120.9, 119.5, 118.0, 117.8, 113.9, 106.6, 105.7, 103.9, 55.9, 41.1, 12.9. ${ }^{11}$ B NMR (128 MHz, $\left.\mathbf{C D C l}_{3}\right) \delta$ 31.8. HRMS (ESI) calcd for $\mathrm{C}_{24} \mathrm{H}_{21} \mathrm{BN}_{3} \mathrm{O}[\mathrm{M}+\mathrm{H}]^{+}: 378.1772$, found: 378.1767. HPLC analysis: DAICEL CHIRALCEL A-SH, hexane/isopropanol $=80 / 20,1 \mathrm{~mL} / \mathrm{min}, \lambda=254 \mathrm{~nm}, \mathrm{t}_{\mathrm{R}}($ minor $)=15.47 \mathrm{~min}, \mathrm{t}_{\mathrm{R}}$ $($ major $)=23.11 \mathrm{~min}, 91 \%$ ee. $[\boldsymbol{\alpha}]^{25}{ }_{\mathbf{D}}:+134.0\left(c 0.5, \mathrm{CHCl}_{3}\right)$.

(S)-1-(5-(1-ethyl-1H-naphtho[1,8-de][1,3,2]diazaborinin-2(3H)-yl)-6-methoxynaphthalen-2-yl)ethan-1-o ne $(6 \mathrm{~g})$

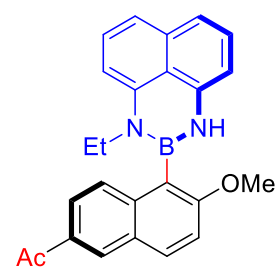

Yellow solid, mp: $205-208^{\circ} \mathrm{C}$. Yield: $80 \% . \mathrm{R}_{\mathrm{f}}=0.2$ (silica gel, PE:EtOAc $=5: 1$ ).

${ }^{1} \mathbf{H}$ NMR $\left(\mathbf{5 0 0} \mathbf{M H z}, \mathbf{C D C l}_{3}\right) \delta 8.46(\mathrm{~d}, J=1.6 \mathrm{~Hz}, 1 \mathrm{H}), 8.04(\mathrm{~d}, J=9.0 \mathrm{~Hz}, 1 \mathrm{H}), 7.93(\mathrm{dd}, J=8.9,1.8 \mathrm{~Hz}$, $1 \mathrm{H}), 7.82(\mathrm{~d}, J=8.9 \mathrm{~Hz}, 1 \mathrm{H}), 7.37(\mathrm{~d}, J=9.1 \mathrm{~Hz}, 1 \mathrm{H}), 7.30-7.26(\mathrm{~m}, 1 \mathrm{H}), 7.20-7.08(\mathrm{~m}, 3 \mathrm{H}), 6.54(\mathrm{~d}, J=$ $7.6 \mathrm{~Hz}, 1 \mathrm{H}), 6.33(\mathrm{dd}, J=7.0,1.2 \mathrm{~Hz}, 1 \mathrm{H}), 5.94(\mathrm{~s}, 1 \mathrm{H}), 3.94(\mathrm{~s}, 3 \mathrm{H}), 3.48-3.27(\mathrm{~m}, 2 \mathrm{H}), 2.70(\mathrm{~s}, 3 \mathrm{H}), 1.06(\mathrm{t}$, $J=7.0 \mathrm{~Hz}, 3 \mathrm{H}) .{ }^{13} \mathbf{C}$ NMR (126 MHz, $\left.\mathbf{C D C l}_{3}\right) \delta 197.9,161.2,142.2,140.6,139.0,136.6,132.6,132.4,130.7$, 127.7, 127.6, 127.6, 127.2, 124.6, 120.9, 117.9, 117.7, 113.2, 105.7, 103.8, 55.9, 41.1, 26.6, 12.9. ${ }^{11} \mathbf{B}$ NMR (128 MHz, $\mathbf{C D C l}_{3}$ ) $\delta$ 32.1. HRMS (ESI) calcd for $\mathrm{C}_{25} \mathrm{H}_{24} \mathrm{BN}_{2} \mathrm{O}_{2}[\mathrm{M}+\mathrm{H}]^{+}: 395.1925$, found: 395.1921. HPLC analysis: DAICEL CHIRALCEL IA-3, hexane/isopropanol $=90 / 10,1 \mathrm{~mL} / \mathrm{min}, \lambda=254 \mathrm{~nm}, \mathrm{t}_{\mathrm{R}}($ minor) $=16.46$ $\min , \mathrm{t}_{\mathrm{R}}($ major $)=20.62 \mathrm{~min}, 94 \%$ ee. $[\boldsymbol{\alpha}]_{\mathbf{D}}^{25}:+125.0\left(c 0.5, \mathrm{CHCl}_{3}\right)$. 


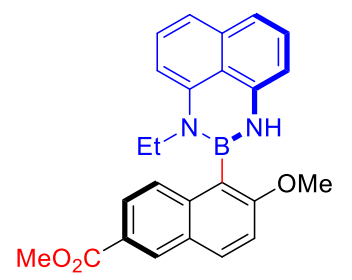

Light Yellow solid, mp: $224-226{ }^{\circ} \mathrm{C}$. Yield: $76 \% . \mathrm{R}_{\mathrm{f}}=0.2$ (silica gel, PE:EtOAc $=10: 1$ ).

${ }^{1}$ H NMR $\left(500 ~ M H z, \mathbf{C D C l}_{3}\right) \delta 8.59(\mathrm{~d}, J=1.3 \mathrm{~Hz}, 1 \mathrm{H}), 8.02(\mathrm{~d}, J=9.0 \mathrm{~Hz}, 1 \mathrm{H}), 7.95(\mathrm{dd}, J=8.7,1.6 \mathrm{~Hz}$, $1 \mathrm{H}), 7.80(\mathrm{~d}, J=8.8 \mathrm{~Hz}, 1 \mathrm{H}), 7.36(\mathrm{~d}, J=9.0 \mathrm{~Hz}, 1 \mathrm{H}), 7.32-7.26(\mathrm{~m}, 1 \mathrm{H}), 7.20-7.07(\mathrm{~m}, 3 \mathrm{H}), 6.55(\mathrm{~d}, J=$ $7.7 \mathrm{~Hz}, 1 \mathrm{H}), 6.38-6.30(\mathrm{~m}, 1 \mathrm{H}), 5.97(\mathrm{~s}, 1 \mathrm{H}), 3.96(\mathrm{~s}, 3 \mathrm{H}), 3.94(\mathrm{~s}, 3 \mathrm{H}), 3.50-3.28(\mathrm{~m}, 3 \mathrm{H}), 1.07(\mathrm{t}, J=7.0$ $\mathrm{Hz}, 3 \mathrm{H}) .{ }^{13} \mathbf{C} \mathbf{N M R}\left(\mathbf{1 2 6} \mathbf{~ M H z}, \mathbf{C D C l}_{3}\right) \delta 167.3,160.9,142.2,140.6,138.9,136.6,132.3,131.5,127.8,127.6$,

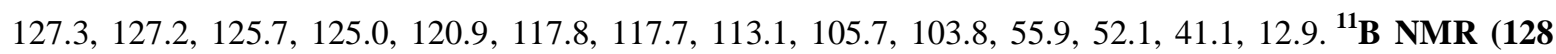
MHz, $\left.\mathbf{C D C l}_{3}\right) \delta$ 33.2. HRMS (ESI) calcd for $\mathrm{C}_{25} \mathrm{H}_{24} \mathrm{BN}_{2} \mathrm{O}_{3}[\mathrm{M}+\mathrm{H}]^{+}:$411.1874, found: 411.1876. HPLC analysis: DAICEL CHIRALCEL IA-3, hexane/isopropanol $=90 / 10,1 \mathrm{~mL} / \mathrm{min}, \lambda=254 \mathrm{~nm}, \mathrm{t}_{\mathrm{R}}(\operatorname{minor})=16.31$ $\min , \mathrm{t}_{\mathrm{R}}($ major $)=17.77 \min , 84 \%$ ee $[\boldsymbol{\alpha}]^{25}{ }_{\mathbf{D}}:+122.4\left(c 0.5, \mathrm{CHCl}_{3}\right)$.

(S)-1-ethyl-2-(2-methoxy-6-phenylnaphthalen-1-yl)-2,3-dihydro-1H-naphtho[1,8-de][1,3,2]diazaborinine (6i)

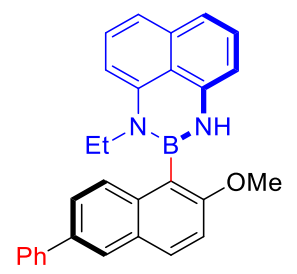

White solid, mp: $111-113{ }^{\circ} \mathrm{C}$. Yield: $95 \% . \mathrm{R}_{\mathrm{f}}=0.15$ (silica gel, PE:EtOAc $=20: 1$ ).

${ }^{1} \mathbf{H}$ NMR $(500$ MHz, CDCl $) \delta 8.04(\mathrm{~d}, J=1.9 \mathrm{~Hz}, 1 \mathrm{H}), 7.97(\mathrm{~d}, J=8.9 \mathrm{~Hz}, 1 \mathrm{H}), 7.86(\mathrm{~d}, J=8.7 \mathrm{~Hz}, 1 \mathrm{H})$, 7.73-7.70 (m, 2H), $7.67(\mathrm{dd}, J=8.7,2.0 \mathrm{~Hz}, 1 \mathrm{H}), 7.51-7.46(\mathrm{~m}, 2 \mathrm{H}), 7.41-7.33(\mathrm{~m}, 2 \mathrm{H}), 7.31-7.27(\mathrm{~m}$, $1 \mathrm{H}), 7.21-7.09(\mathrm{~m}, 3 \mathrm{H}), 6.56(\mathrm{~d}, J=7.6 \mathrm{~Hz}, 1 \mathrm{H}), 6.33(\mathrm{dd}, J=7.1,1.3 \mathrm{~Hz}, 1 \mathrm{H}), 5.98(\mathrm{~s}, 1 \mathrm{H}), 3.93(\mathrm{~s}, 3 \mathrm{H})$, 3.50-3.37 (m, 2H, 2H), $1.11(\mathrm{t}, J=7.0 \mathrm{~Hz}, 3 \mathrm{H}) .{ }^{13} \mathbf{C}$ NMR (126 MHz, $\left.\mathbf{C D C l}_{3}\right) \delta$ 159.2, 142.4, 141.0, 140.8, 136.6, 136.2, 135.6, 130.9, 129.1, 128.8, 127.7, 127.6, 127.2, 127.2, 127.1, 126.1, 126.0, 120.9, 117.7, 117.5, 113.0, 105.6, 103.8, 56.0, 41.2, 12.9. ${ }^{11} \mathbf{B}$ NMR (128 $\left.\mathbf{~ M H z , ~} \mathbf{C D C l}_{3}\right) \delta$ 32.0. HRMS (ESI) calcd for $\mathrm{C}_{29} \mathrm{H}_{26} \mathrm{BN}_{2} \mathrm{O}[\mathrm{M}+\mathrm{H}]^{+}:$429.2133, found: 429.2126. HPLC analysis: DAICEL CHIRALCEL IA-3, hexane/isopropanol $=98 / 2,1 \mathrm{~mL} / \mathrm{min}, \lambda=254 \mathrm{~nm}, \mathrm{t}_{\mathrm{R}}($ minor $)=19.27 \mathrm{~min}, \mathrm{t}_{\mathrm{R}}$ (major) $=23.64 \mathrm{~min}, 91 \%$ ee. $[\boldsymbol{\alpha}]^{25}:+132.8\left(c 0.5, \mathrm{CHCl}_{3}\right)$.

(S)-1-ethyl-2-(2-methoxy-6-methylnaphthalen-1-yl)-2,3-dihydro-1H-naphtho[1,8-de][1,3,2]diazaborinine (6j)

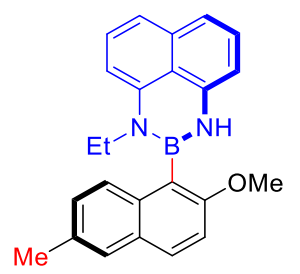

White solid, mp: $88-89^{\circ} \mathrm{C}$. Yield: $95 \% . \mathrm{R}_{\mathrm{f}}=0.37$ (silica gel, PE:EtOAc $=20: 1$ ).

${ }^{1} \mathbf{H}$ NMR $\left(\mathbf{5 0 0} \mathbf{M H z}_{\mathbf{C}} \mathbf{C D C l}_{\mathbf{3}}\right) \delta 7.83(\mathrm{~d}, J=8.8 \mathrm{~Hz}, 1 \mathrm{H}), 7.71(\mathrm{~d}, J=8.3 \mathrm{~Hz}, 1 \mathrm{H}), 7.61(\mathrm{~s}, 1 \mathrm{H}), 7.32-7.23$ (dd, $J=16.9,9.5 \mathrm{~Hz}, 3 \mathrm{H}), 7.20-7.08(\mathrm{~m}, 3 \mathrm{H}), 6.56(\mathrm{~d}, J=7.1 \mathrm{~Hz}, 1 \mathrm{H}), 6.32(\mathrm{~d}, J=4.5 \mathrm{~Hz}, 1 \mathrm{H}), 5.96(\mathrm{~s}, 1 \mathrm{H})$, 
$3.91(\mathrm{~s}, 3 \mathrm{H}), 3.51-3.33(\mathrm{~m}, 2 \mathrm{H}), 2.50(\mathrm{~s}, 3 \mathrm{H}), 1.10(\mathrm{~s}, 3 \mathrm{H}) .{ }^{13} \mathbf{C} \mathbf{N M R}\left(\mathbf{1 2 6} \mathbf{~ M H z}, \mathbf{C D C l}_{\mathbf{3}}\right) \delta$ 158.5, 142.4, 140.8, 136.6, 134.6, 133.0, 129.8, 129.1, 128.7, 127.6, 127.2, 127.1, 120.9, 117.6, 117.5, 112.7, 105.5, 103.7, 56.0, 41.1, 21.4, 12.9. ${ }^{11} \mathbf{B}$ NMR (128 MHz, $\left.\mathbf{C D C l}_{3}\right) \delta 31.9$. HRMS (ESI) calcd for $\mathrm{C}_{24} \mathrm{H}_{24} \mathrm{BN}_{2} \mathrm{O}[\mathrm{M}+\mathrm{H}]^{+}$: 367.1976, found: 367.1971. HPLC analysis: DAICEL CHIRALCEL IA-3, hexane/isopropanol $=98 / 2$, $1 \mathrm{~mL} / \min , \lambda=254 \mathrm{~nm}, \mathrm{t}_{\mathrm{R}}($ minor $)=10.0 \mathrm{~min}, \mathrm{t}_{\mathrm{R}}($ major $)=13.49 \mathrm{~min}, 95 \%$ ee. $[\boldsymbol{\alpha}]^{25}{ }_{\mathbf{D}}:+83.8\left(c 0.5, \mathrm{CHCl}_{3}\right)$.

(S)-2-(2,6-dimethoxynaphthalen-1-yl)-1-ethyl-2,3-dihydro-1H-naphtho[1,8-de][1,3,2]diazaborinine (6k)

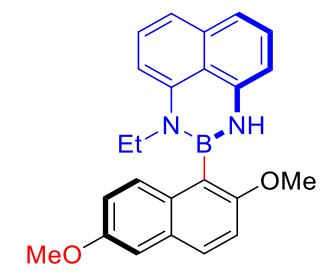

White solid, mp: $173-174{ }^{\circ} \mathrm{C}$. Yield: $95 \% . \mathrm{R}_{\mathrm{f}}=0.2$ (silica gel, PE:EtOAc $=20: 1$ ).

${ }^{1} \mathbf{H}$ NMR $\left(\mathbf{5 0 0 ~ M H z}, \mathbf{C D C l}_{\mathbf{3}}\right) \delta 7.80(\mathrm{~d}, J=8.9 \mathrm{~Hz}, 1 \mathrm{H}), 7.70(\mathrm{~d}, J=9.0 \mathrm{~Hz}, 1 \mathrm{H}), 7.30-7.24(\mathrm{~m}, 2 \mathrm{H}), 7.17$ $7.05(\mathrm{~m}, 5 \mathrm{H}), 6.54(\mathrm{~d}, J=7.5 \mathrm{~Hz}, 1 \mathrm{H}), 6.32(\mathrm{dd}, J=7.1,1.2 \mathrm{~Hz}, 1 \mathrm{H}), 5.95(\mathrm{~s}, 1 \mathrm{H}), 3.90(\mathrm{~s}, 3 \mathrm{H}), 3.87(\mathrm{~s}, 3 \mathrm{H})$, $3.48-3.30(\mathrm{~m}, 2 \mathrm{H}), 1.07(\mathrm{t}, J=6.6 \mathrm{~Hz}, 3 \mathrm{H}) .{ }^{13} \mathbf{C} \mathbf{~ N M R}\left(\mathbf{1 2 6} \mathbf{~ M H z}, \mathbf{C D C l}_{\mathbf{3}}\right) \delta 157.6,155.9,142.4,140.7$, 136.6, 131.7, 129.7, 129.1, 128.7, 127.6, 127.1, 120.9, 119.3, 117.6, 117.5, 113.2, 106.0, 105.6, 103.7, 56.1, 55.3, 41.1, 12.9. ${ }^{11} \mathbf{B}$ NMR (128 MHz, $\left.\mathbf{C D C l}_{3}\right) \delta$ 32.4. HRMS (ESI) calcd for $\mathrm{C}_{24} \mathrm{H}_{24} \mathrm{BN}_{2} \mathrm{O}_{2}[\mathrm{M}+\mathrm{H}]^{+}$: 383.1925, found: 383.1921. HPLC analysis: DAICEL CHIRALCEL IA-3, hexane/isopropanol $=98 / 2$, $1 \mathrm{~mL} / \min , \lambda=254 \mathrm{~nm}, \mathrm{t}_{\mathrm{R}}($ major $)=24.47 \mathrm{~min}, \mathrm{t}_{\mathrm{R}}($ minor $)=37.73 \mathrm{~min}, 96 \%$ ee. $[\boldsymbol{\alpha}]^{25}{ }_{\mathbf{D}}:+98.5\left(c 0.5, \mathrm{CHCl}_{3}\right)$.

(S,S)-methyl-2-(5-(1-ethyl-1H-naphtho[1,8-de][1,3,2]diazaborinin-2(3H)-yl)-6-methoxynaphthalen-2-yl) propanoate (61)

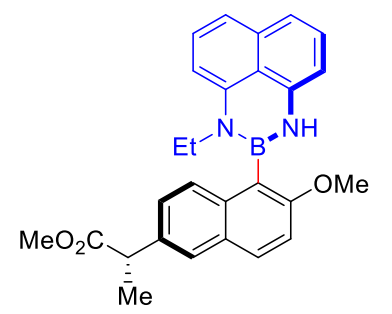

White solid, mp: $79-80^{\circ} \mathrm{C}$. Yield: $74 \% . \mathrm{R}_{\mathrm{f}}=0.4$ (silica gel, PE:EtOAc $=5: 1$ ).

${ }^{1}$ H NMR (500 MHz, $\left.\mathbf{C D C l}_{\mathbf{3}}\right) \delta 7.88(\mathrm{~d}, J=8.9 \mathrm{~Hz}, 1 \mathrm{H}), 7.74(\mathrm{dd}, J=14.1,5.2 \mathrm{~Hz}, 2 \mathrm{H}), 7.34(\mathrm{dd}, J=8.7,1.9$ $\mathrm{Hz}, 1 \mathrm{H}), 7.32-7.25(\mathrm{~m}, 2 \mathrm{H}), 7.18-7.09(\mathrm{~m}, 3 \mathrm{H}), 6.55(\mathrm{~d}, J=7.6 \mathrm{~Hz}, 1 \mathrm{H}), 6.30(\mathrm{dd}, J=7.0,1.3 \mathrm{~Hz}, 1 \mathrm{H}), 5.93$ (s, 1H), $3.90(\mathrm{~s}, 3 \mathrm{H}), 3.89-3.81(\mathrm{~m}, 1 \mathrm{H}), 3.68(\mathrm{~s}, 3 \mathrm{H}), 3.50-3.27(\mathrm{~m}, 2 \mathrm{H}), 1.58(\mathrm{~d}, J=7.2 \mathrm{~Hz}, 3 \mathrm{H}), 1.09(\mathrm{t}, J$ $=7.0 \mathrm{~Hz}, 3 \mathrm{H}) .{ }^{13} \mathbf{C}$ NMR (126 MHz, $\left.\mathbf{C D C l}_{3}\right) \delta 175.1,159.0,142.4,140.7,136.6,135.6,135.5,130.4,128.8$, 127.7, 127.6, 127.2, 126.3, 120.9, 117.7, 117.5, 112.9, 105.6, 103.7, 55.9, 52.1, 45.3, 41.1, 18.6, 12.9. ${ }^{11} \mathbf{B}$ NMR (128 MHz, $\mathbf{C D C l}_{3}$ ) $\delta$ 32.9. HRMS (ESI) calcd for $\mathrm{C}_{27} \mathrm{H}_{28} \mathrm{BN}_{2} \mathrm{O}_{3}[\mathrm{M}+\mathrm{H}]^{+}:$439.2187, found: 439.2183. HPLC analysis: DAICEL CHIRALCEL OD-H, hexane/isopropanol $=90 / 10,1 \mathrm{~mL} / \mathrm{min}, \lambda=254 \mathrm{~nm}, \mathrm{t}_{\mathrm{R}}$ (minor) $=10.76 \mathrm{~min}, \mathrm{t}_{\mathrm{R}}($ major $)=22.31 \mathrm{~min}, 94 \%$ de. $[\boldsymbol{\alpha}]^{25}{ }_{\mathbf{D}}:+146.0\left(c 0.5, \mathrm{CHCl}_{3}\right)$. 

(6m)

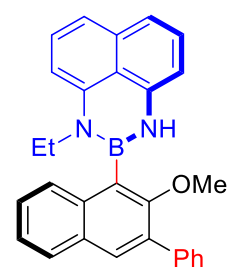

White solid, mp: 83-85 ${ }^{\circ} \mathrm{C}$. Yield: $94 \% . \mathrm{R}_{\mathrm{f}}=0.15$ (silica gel, PE:EtOAc $=20: 1$ ).

${ }^{1} \mathbf{H}$ NMR (500 MHz, $\left.\mathbf{C D C l}_{3}\right) \delta 7.94-7.85(\mathrm{~m}, 3 \mathrm{H}), 7.73(\mathrm{~d}, J=7.8 \mathrm{~Hz}, 2 \mathrm{H}), 7.55-7.39(\mathrm{~m}, 5 \mathrm{H}), 7.31(\mathrm{t}, J=$ $8.0 \mathrm{~Hz}, 1 \mathrm{H}), 7.20-7.13(\mathrm{~m}, 3 \mathrm{H}), 6.60(\mathrm{~d}, J=7.7 \mathrm{~Hz}, 1 \mathrm{H}), 6.35(\mathrm{~d}, J=6.9 \mathrm{~Hz}, 1 \mathrm{H}), 6.06(\mathrm{~s}, 1 \mathrm{H}), 3.65-3.44$ $(\mathrm{m}, 5 \mathrm{H}), 1.18(\mathrm{t}, J=6.9 \mathrm{~Hz}, 3 \mathrm{H}) .{ }^{13} \mathbf{C} \mathbf{N M R}\left(\mathbf{1 2 6} \mathbf{M H z}, \mathbf{C D C l}_{3}\right) \delta 157.9,142.2,140.5,138.7,136.5,135.8$, 134.4, 131.5, 130.8, 129.1, 128.4, 128.2, 127.6, 127.5, 127.3, 127.2, 126.3, 125.0, 120.9, 117.9, 117.7, 105.6, 104.0, 61.0, 41.5, 12.9. ${ }^{11} \mathbf{B}$ NMR (128 MHz, $\left.\mathbf{C D C l}_{3}\right) \delta 31.80$. HRMS (ESI) calcd for $\mathrm{C}_{29} \mathrm{H}_{26} \mathrm{BN}_{2} \mathrm{O}_{2}[\mathrm{M}+\mathrm{H}]^{+}$: 429.2133, found: 429.2128. HPLC analysis: DAICEL CHIRALCEL IA-3, hexane/isopropanol = 98/2, $1 \mathrm{~mL} / \mathrm{min}, \lambda=254 \mathrm{~nm}, \mathrm{t}_{\mathrm{R}}($ minor $)=7.76 \mathrm{~min}, \mathrm{t}_{\mathrm{R}}($ major $)=12.27 \mathrm{~min}, 80 \%$ ee $[\boldsymbol{\alpha}]^{25}{ }_{\mathrm{D}}:+133.0\left(c 0.5, \mathrm{CHCl}_{3}\right)$.

(S)-methyl 4-(1-ethyl-1H-naphtho[1,8-de][1,3,2]diazaborinin-2(3H)-yl)-3-methoxy-2-naphthoate (6n)

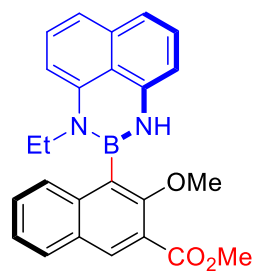

Light Green solid, mp: $56-57{ }^{\circ} \mathrm{C}$. Yield: $88 \% . \mathrm{R}_{\mathrm{f}}=0.1$ (silica gel, PE:EtOAc $=5: 1$ ).

${ }^{1}$ H NMR $\left(500\right.$ MHz, $\left.\mathbf{C D C l}_{3}\right) \delta 8.43(\mathrm{~s}, 1 \mathrm{H}), 7.94-7.89(\mathrm{~m}, 1 \mathrm{H}), 7.89-7.85(\mathrm{~m}, 1 \mathrm{H}), 7.53-7.44(\mathrm{~m}, 2 \mathrm{H})$, $7.29(\mathrm{t}, J=8.1 \mathrm{~Hz}, 1 \mathrm{H}), 7.19-7.12(\mathrm{~m}, 3 \mathrm{H}), 6.57(\mathrm{~d}, J=7.6 \mathrm{~Hz}, 1 \mathrm{H}), 6.35(\mathrm{dd}, J=6.8,1.6 \mathrm{~Hz}, 1 \mathrm{H}), 6.04(\mathrm{~s}$, 1H), 4.00 (s, 3H), $3.94(\mathrm{~s}, 3 \mathrm{H}), 3.53-3.35(\mathrm{~m}, 2 \mathrm{H}), 1.08(\mathrm{t}, J=7.0 \mathrm{~Hz}, 3 \mathrm{H}) .{ }^{\mathbf{1 3}} \mathbf{C} \mathbf{N M R}\left(\mathbf{1 2 6} \mathbf{M H z}, \mathbf{C D C l}_{3}\right) \delta$ 166.9, 158.1, 142.0, 140.3, 138.1, 136.5, 134.2, 129.5, 129.3, 128.3, 127.6, 127.5, 127.2, 125.5, 123.9, 120.9, 118.1, 117.9, 105.7, 104.2, 62.5, 52.5, 41.4, 12.9. ${ }^{\mathbf{1 1}} \mathbf{B}$ NMR (128 MHz, $\left.\mathbf{C D C l}_{3}\right) \delta 32.7 . \mathbf{H R M S}(\mathbf{E S I})$ calcd for $\mathrm{C}_{25} \mathrm{H}_{24} \mathrm{BN}_{2} \mathrm{O}_{3}[\mathrm{M}+\mathrm{H}]^{+}$: 411.1874, found: 411.1868. HPLC analysis: DAICEL CHIRALCEL IA-3, hexane/isopropanol $=90 / 10,1 \mathrm{~mL} / \mathrm{min}, \lambda=254 \mathrm{~nm}, \mathrm{t}_{\mathrm{R}}\left(\right.$ minor) $=9.21 \mathrm{~min}, \mathrm{t}_{\mathrm{R}}$ (major) $=10.19 \mathrm{~min}, 81 \%$ ee. $[\alpha]^{25}:+1.2\left(c 0.5, \mathrm{CHCl}_{3}\right)$.

(S)-methyl 5-(1-ethyl-1H-naphtho[1,8-de][1,3,2]diazaborinin-2(3H)-yl)-6-methoxy-1-naphthoate (6o)<smiles>CCN1BNc2cccc3cccc(c23)N1c1c(OC)ccc2c(C(C)=O)cccc12</smiles>

Yellow solid, mp: $101-102{ }^{\circ} \mathrm{C}$. Yield: $80 \% . \mathrm{R}_{\mathrm{f}}=0.2$ (silica gel, PE:EtOAc $=10: 1$ ).

${ }^{1}$ H NMR $\left(500 ~ M H z, \mathbf{C D C l}_{3}\right) \delta 9.00(\mathrm{~d}, J=9.4 \mathrm{~Hz}, 1 \mathrm{H}), 8.03(\mathrm{~d}, J=7.0 \mathrm{~Hz}, 1 \mathrm{H}), 7.99(\mathrm{~d}, J=8.2 \mathrm{~Hz}, 1 \mathrm{H})$, $7.46-7.37(\mathrm{~m}, 2 \mathrm{H}), 7.31-7.26(\mathrm{~m}, 1 \mathrm{H}), 7.20-7.09(\mathrm{~m}, 3 \mathrm{H}), 6.54(\mathrm{~d}, J=7.7 \mathrm{~Hz}, 1 \mathrm{H}), 6.33(\mathrm{~d}, J=6.9 \mathrm{~Hz}$, 1H), $5.96(\mathrm{~s}, 1 \mathrm{H}), 4.02(\mathrm{~s}, 3 \mathrm{H}), 3.93(\mathrm{~s}, 3 \mathrm{H}), 3.47-3.28(\mathrm{~m}, 2 \mathrm{H}), 1.06(\mathrm{t}, J=6.9 \mathrm{~Hz}, 3 \mathrm{H}) .{ }^{\mathbf{1 3}} \mathbf{C} \mathbf{N M R}(\mathbf{1 2 6}$ MHz, $\left.\mathbf{C D C l}_{3}\right) \delta 168.2,159.0,142.3,140.6,137.1,136.5,132.5,128.5,127.8,127.6,127.4,127.2,126.5$, 
125.1, 120.9, 117.8, 117.6, 113.9, 105.6, 103.8, 55.8, 52.2, 41.1, 12.8. ${ }^{11} \mathbf{B}$ NMR (128 MHz, $\left.\mathbf{C D C l}_{\mathbf{3}}\right) \delta 30.6$. HRMS (ESI) calcd for $\mathrm{C}_{25} \mathrm{H}_{24} \mathrm{BN}_{2} \mathrm{O}_{3}[\mathrm{M}+\mathrm{H}]^{+}$: 411.1874, found: 411.1869. HPLC analysis: DAICEL CHIRALCEL IA-3, hexane/isopropanol $=90 / 10,1 \mathrm{~mL} / \mathrm{min}, \lambda=254 \mathrm{~nm}, \mathrm{t}_{\mathrm{R}}($ minor $)=10.08 \mathrm{~min}, \mathrm{t}_{\mathrm{R}}$ (major) $=$ $16.04 \min , 95 \%$ ee. $[\alpha]^{25}$ : $+66.5\left(c 0.5, \mathrm{CHCl}_{3}\right)$.

(S)-2-(2,7-dimethoxynaphthalen-1-yl)-1-ethyl-2,3-dihydro-1H-naphtho[1,8-de][1,3,2]diazaborinine (6p)

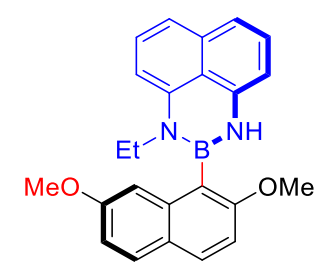

White solid, mp: $80-83{ }^{\circ} \mathrm{C}$. Yield: $40 \% . \mathrm{R}_{\mathrm{f}}=0.55$ (silica gel, PE:EtOAc $=5: 1$ ).

${ }^{1} \mathbf{H}$ NMR $\left(\mathbf{5 0 0} \mathbf{~ M H z}, \mathbf{C D C l}_{3}\right) \delta 7.83(\mathrm{~d}, J=8.9 \mathrm{~Hz}, 1 \mathrm{H}), 7.72(\mathrm{~d}, J=9.6 \mathrm{~Hz}, 1 \mathrm{H}), 7.29(\mathrm{t}, J=8.0 \mathrm{~Hz}, 1 \mathrm{H})$, $7.20-7.10(\mathrm{~m}, 4 \mathrm{H}), 7.02(\mathrm{dt}, J=4.4,2.5 \mathrm{~Hz}, 2 \mathrm{H}), 6.56(\mathrm{~d}, J=7.6 \mathrm{~Hz}, 1 \mathrm{H}), 6.34(\mathrm{dd}, J=7.1,1.3 \mathrm{~Hz}, 1 \mathrm{H})$, 5.98 (s, 1H), 3.89 (s, 3H), 3.79 (s, 3H), $3.53-3.33$ (m, 2H), $1.11(\mathrm{t}, J=7.0 \mathrm{~Hz}, 3 \mathrm{H}) .{ }^{13} \mathbf{C}$ NMR (126 MHz, $\left.\mathbf{C D C l}_{\mathbf{3}}\right) \delta 159.7,158.2,142.5,140.9,137.8,136.6,130.3,129.7,127.6,127.2,124.4,121.0,117.6,117.4$,

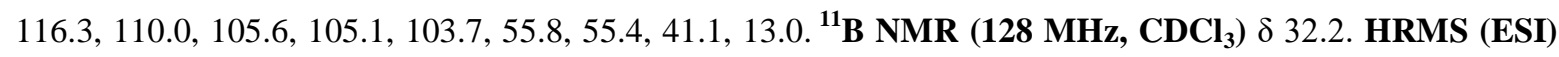
calcd for $\mathrm{C}_{24} \mathrm{H}_{24} \mathrm{BN}_{2} \mathrm{O}_{2}[\mathrm{M}+\mathrm{H}]^{+}: 383.1925$, found: 383.1923 . HPLC analysis: DAICEL CHIRALCEL IA-3, hexane/isopropanol $=98 / 2,1 \mathrm{~mL} / \mathrm{min}, \lambda=254 \mathrm{~nm}, \mathrm{t}_{\mathrm{R}}($ minor $)=17.68 \mathrm{~min}, \mathrm{t}_{\mathrm{R}}$ (major) $=24.57 \mathrm{~min}, 77 \%$ ee . $[\alpha]^{25}$ D $+79.6\left(c 0.5, \mathrm{CHCl}_{3}\right)$.

(S)-1-ethyl-2-(6-methoxyquinolin-5-yl)-2,3-dihydro-1H-naphtho[1,8-de][1,3,2]diazaborinine (6q)

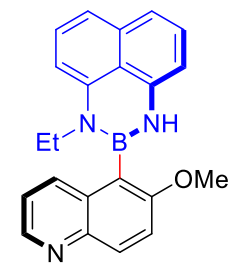

Light Green solid, mp: $262-263{ }^{\circ} \mathrm{C}$. Yield: $92 \% . \mathrm{R}_{\mathrm{f}}=0.2$ (silica gel, PE:EtOAc $=2: 1$ ).

${ }^{1} \mathbf{H}$ NMR $\left(\mathbf{5 0 0 ~ M H z}, \mathbf{C D C l}_{\mathbf{3}}\right) \delta 8.79(\mathrm{dd}, J=4.1,1.5 \mathrm{~Hz}, 1 \mathrm{H}), 8.17(\mathrm{~d}, J=9.3 \mathrm{~Hz}, 1 \mathrm{H}), 8.12(\mathrm{~d}, J=7.9 \mathrm{~Hz}$, $1 \mathrm{H}), 7.53(\mathrm{~d}, J=9.3 \mathrm{~Hz}, 1 \mathrm{H}), 7.34-7.26(\mathrm{~m}, 2 \mathrm{H}), 7.18-7.10(\mathrm{~m}, 3 \mathrm{H}), 6.54(\mathrm{~d}, J=7.7 \mathrm{~Hz}, 1 \mathrm{H}), 6.34(\mathrm{dd}, J=$ 7.0, $1.1 \mathrm{~Hz}, 1 \mathrm{H}), 5.97$ (s, 1H), $3.94(\mathrm{~s}, 3 \mathrm{H}), 3.46-3.29(\mathrm{~m}, 2 \mathrm{H}), 1.06$ (t, $J=7.0 \mathrm{~Hz}, 3 \mathrm{H}) .{ }^{13} \mathbf{C}$ NMR (101 MHz, $\left.\mathbf{C D C l}_{3}\right) \delta 159.0,148.1,144.0,142.1,140.5,136.5,135.4,131.8,131.3,127.6,127.2,121.3,120.9,117.9$, 117.8, 115.9, 105.7, 103.9, 56.0, 41.1, 12.9. ${ }^{11} \mathbf{B}$ NMR (128 $\left.\mathbf{~ M H z , ~} \mathbf{C D C l}_{\mathbf{3}}\right) \delta 31.6$. HRMS (ESI) calcd for $\mathrm{C}_{22} \mathrm{H}_{21} \mathrm{BN}_{3} \mathrm{O}[\mathrm{M}+\mathrm{H}]^{+}:$354.1772, found: 354.1771. HPLC analysis: DAICEL CHIRALCEL IA-3, hexane/isopropanol $=90 / 10,1 \mathrm{~mL} / \mathrm{min}, \lambda=254 \mathrm{~nm}, \mathrm{t}_{\mathrm{R}}($ major $)=14.03 \mathrm{~min}, \mathrm{t}_{\mathrm{R}}($ minor $)=17.55 \mathrm{~min}, 88 \%$ ee . $[\alpha]^{25}$ : $+83.7\left(c 0.5, \mathrm{CHCl}_{3}\right)$.

(S)-1-ethyl-2-(2-methoxy-6-methylphenyl)-2,3-dihydro-1H-naphtho[1,8-de][1,3,2]diazaborinine (6r)

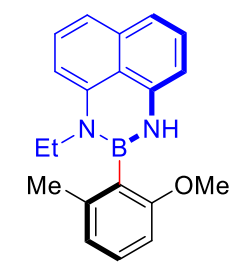

White solid, mp: $115-116^{\circ} \mathrm{C}$. Yield: $88 \% . \mathrm{R}_{\mathrm{f}}=0.3$ (silica gel, PE:EtOAc $=20: 1$ ). 
${ }^{1} \mathbf{H}$ NMR $\left(\mathbf{5 0 0} \mathbf{~ M H z}, \mathbf{C D C l}_{3}\right) \delta 7.27(\mathrm{dt}, J=12.6,7.6 \mathrm{~Hz}, 2 \mathrm{H}), 7.18-7.07(\mathrm{~m}, 3 \mathrm{H}), 6.86(\mathrm{~d}, J=7.6 \mathrm{~Hz}, 1 \mathrm{H})$, $6.74(\mathrm{~d}, J=8.3 \mathrm{~Hz}, 1 \mathrm{H}), 6.52(\mathrm{~d}, J=7.7 \mathrm{~Hz}, 1 \mathrm{H}), 6.32(\mathrm{dd}, J=7.2,1.1 \mathrm{~Hz}, 1 \mathrm{H}), 5.85(\mathrm{~s}, 1 \mathrm{H}), 3.77(\mathrm{~s}, 3 \mathrm{H})$, 3.50 - 3.34 (m, 2H), 2.34 (s, 3H), 1.14 (t, $J=7.0 \mathrm{~Hz}, 3 \mathrm{H}) .{ }^{\mathbf{1 3}} \mathbf{C}$ NMR (126 MHz, $\left.\mathbf{C D C l}_{\mathbf{3}}\right) \delta$ 161.5, 142.4, 141.6, 140.7, 136.5, 129.6, 127.5, 127.1, 122.0, 120.8, 117.5, 117.4, 106.8, 105.5, 103.6, 55.1, 40.8, 21.9, 12.7. ${ }^{11} \mathbf{B}$ NMR (128 MHz, $\mathbf{C D C l}_{3}$ ) $\delta 30.8$. HRMS (ESI) calcd for $\mathrm{C}_{20} \mathrm{H}_{22} \mathrm{BN}_{2} \mathrm{O}[\mathrm{M}+\mathrm{H}]^{+}: 317.1820$, found: 317.1816 . HPLC analysis: DAICEL CHIRALCEL IA-3, hexane/isopropanol $=98 / 2,1 \mathrm{~mL} / \mathrm{min}, \lambda=254 \mathrm{~nm}, \mathrm{t}_{\mathrm{R}}$ (major) $=$ $7.42 \mathrm{~min}, \mathrm{t}_{\mathrm{R}}($ minor $)=8.63 \mathrm{~min}, 92 \%$ ee. $[\boldsymbol{\alpha}]^{25}{ }_{\mathbf{D}}:+58.8\left(c 0.5, \mathrm{CHCl}_{3}\right)$.

(S)-2-(3-chloro-6-methoxy-2,4-dimethylphenyl)-1-ethyl-2,3-dihydro-1H-naphtho[1,8-de][1,3,2]diazabori nine (6s)

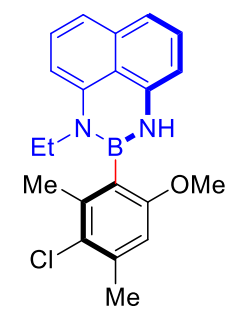

White solid, mp: $57-59^{\circ} \mathrm{C}$. Yield: $60 \%$. $\mathrm{R}_{\mathrm{f}}=0.5$ (silica gel, PE: $\mathrm{DCM}=2: 1$ ).

${ }^{1} \mathbf{H}$ NMR $\left(\mathbf{5 0 0 ~ M H z}, \mathbf{C D C l}_{\mathbf{3}}\right) \delta 7.26(\mathrm{t}, J=8.0 \mathrm{~Hz}, 1 \mathrm{H}), 7.18-7.08(\mathrm{~m}, 3 \mathrm{H}), 6.66(\mathrm{~s}, 1 \mathrm{H}), 6.52(\mathrm{~d}, J=7.7 \mathrm{~Hz}$, $1 \mathrm{H}), 6.32(\mathrm{dd}, J=7.2,1.2 \mathrm{~Hz}, 1 \mathrm{H}), 5.80(\mathrm{~s}, 1 \mathrm{H}), 3.75(\mathrm{~s}, 3 \mathrm{H}), 3.52-3.28(\mathrm{~m}, 2 \mathrm{H}), 2.45(\mathrm{~s}, 3 \mathrm{H}), 2.38(\mathrm{~s}, 3 \mathrm{H})$, $1.13(\mathrm{t}, J=7.0 \mathrm{~Hz}, 3 \mathrm{H}) .{ }^{\mathbf{1 3}} \mathbf{C}$ NMR (126 MHz, $\left.\mathbf{C D C l}_{\mathbf{3}}\right) \delta 159.3,142.2,140.6,138.9,137.4,136.5,127.5$, 127.1, 126.8, 120.7, 117.6, 117.5, 110.2, 105.6, 103.7, 55.3, 40.9, 21.4, 20.8, 12.7. ${ }^{11} \mathbf{B}$ NMR (128 MHz, $\mathbf{C D C l}_{3}$ ) $\delta$ 31.3. HRMS (ESI) calcd for $\mathrm{C}_{21} \mathrm{H}_{23} \mathrm{ClBN}_{2} \mathrm{O}[\mathrm{M}+\mathrm{H}]^{+}: 365.1586$, found: 365.1579 . HPLC analysis: DAICEL CHIRALCEL IA-3, hexane/isopropanol $=95 / 5,1 \mathrm{~mL} / \mathrm{min}, \lambda=254 \mathrm{~nm}, \mathrm{t}_{\mathrm{R}}$ (major) $=6.50 \mathrm{~min}, \mathrm{t}_{\mathrm{R}}$ $($ minor $)=8.14 \min , 98 \%$ ee. $[\alpha]^{25} \mathbf{D}:+69.0\left(c 0.5, \mathrm{CHCl}_{3}\right)$.

(S)-1-ethyl-2-(2-methoxy-5,6,7,8-tetrahydronaphthalen-1-yl)-2,3-dihydro-1H-naphtho[1,8-de][1,3,2]diaz aborinine (6t)

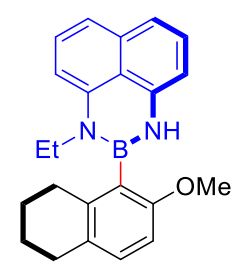

White solid, mp: $75-76{ }^{\circ} \mathrm{C}$. Yield: $92 \% . \mathrm{R}_{\mathrm{f}}=0.5$ (silica gel, PE:EtOAc $=20: 1$ ).

${ }^{1} \mathbf{H}$ NMR (400 MHz, $\left.\mathbf{C D C l}_{3}\right) \delta 7.30-7.21(\mathrm{~m}, 1 \mathrm{H}), 7.16-7.03(\mathrm{~m}, 4 \mathrm{H}), 6.70(\mathrm{~d}, J=8.4 \mathrm{~Hz}, 1 \mathrm{H}), 6.50(\mathrm{~d}, J=$ $7.7 \mathrm{~Hz}, 1 \mathrm{H}), 6.31(\mathrm{~d}, J=7.0 \mathrm{~Hz}, 1 \mathrm{H}), 5.83(\mathrm{~s}, 1 \mathrm{H}), 3.74(\mathrm{~s}, 3 \mathrm{H}), 3.50-3.30(\mathrm{~m}, 2 \mathrm{H}), 2.85-2.59(\mathrm{~m}, 4 \mathrm{H})$, $1.90-1.67(\mathrm{~m}, 4 \mathrm{H}), 1.14(\mathrm{t}, J=7.0 \mathrm{~Hz}, 3 \mathrm{H}) .{ }^{13} \mathbf{C}$ NMR (126 MHz, $\left.\mathbf{C D C l}_{3}\right) \delta 159.3,142.4,140.8,140.5$, 136.5, 130.3, 129.2, 127.5, 127.1, 120.7, 117.4, 117.3, 107.4, 105.4, 103.6, 55.1, 40.9, 29.9, 29.1, 23.3, 23.3, 12.7. ${ }^{11} \mathbf{B}$ NMR (128 MHz, $\left.\mathbf{C D C l}_{3}\right) \delta$ 30.7. HRMS (ESI) calcd for $\mathrm{C}_{23} \mathrm{H}_{26} \mathrm{BN}_{2} \mathrm{O}[\mathrm{M}+\mathrm{H}]^{+}: 357.2133$, found: 357.2129. HPLC analysis: DAICEL CHIRALCEL OD-H, hexane/isopropanol $=98 / 2,0.5 \mathrm{~mL} / \mathrm{min}, \lambda=254$ $\mathrm{nm}, \mathrm{t}_{\mathrm{R}}($ minor $)=13.80 \mathrm{~min}, \mathrm{t}_{\mathrm{R}}($ major $)=15.04 \mathrm{~min}, 85 \%$ ee. $[\boldsymbol{\alpha}]^{25}{ }_{\mathrm{D}}:+129.5\left(c 0.5, \mathrm{CHCl}_{3}\right)$. 


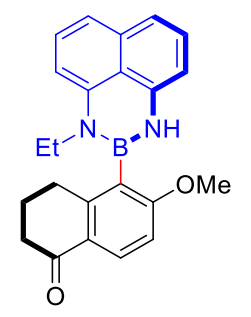

Light Yellow solid, mp: $84-88{ }^{\circ} \mathrm{C}$. Yield: $30 \% . \mathrm{R}_{\mathrm{f}}=0.25$ (silica gel, PE:EtOAc $=5: 1$ ).

${ }^{1} \mathbf{H}$ NMR $\left(\mathbf{5 0 0} \mathbf{M H z}_{\mathbf{C}} \mathbf{C D C l}_{3}\right) \delta 8.15(\mathrm{~d}, J=8.8 \mathrm{~Hz}, 1 \mathrm{H}), 7.26(\mathrm{t}, J=7.9 \mathrm{~Hz}, 1 \mathrm{H}), 7.19-7.07(\mathrm{~m}, 3 \mathrm{H}), 6.87(\mathrm{~d}$, $J=8.8 \mathrm{~Hz}, 1 \mathrm{H}), 6.52(\mathrm{~d}, J=7.7 \mathrm{~Hz}, 1 \mathrm{H}), 6.35(\mathrm{dd}, J=7.2,0.8 \mathrm{~Hz}, 1 \mathrm{H}), 5.88(\mathrm{~s}, 1 \mathrm{H}), 3.84(\mathrm{~s}, 3 \mathrm{H}), 3.44-3.32$ $(\mathrm{m}, 2 \mathrm{H}), 3.01-2.95(\mathrm{~m}, 1 \mathrm{H}), 2.90-2.78(\mathrm{~m}, 1 \mathrm{H}), 2.72-2.56(\mathrm{~m}, 2 \mathrm{H}), 2.16-2.00(\mathrm{~m}, 2 \mathrm{H}), 1.13(\mathrm{t}, J=7.0$ $\mathrm{Hz}, 3 \mathrm{H}) .{ }^{13} \mathbf{C}$ NMR (126 MHz, $\left.\mathbf{C D C l}_{3}\right) \delta$ 197.6, 165.2, 148.7, 142.0, 140.4, 136.5, 130.7, 127.6, 127.1, 126.5, 120.7, 117.8, 117.7, 108.4, 105.6, 103.8, 55.3, 40.9, 39.0, 30.2, 23.4, 12.8. ${ }^{11} \mathbf{B}$ NMR (128 $\left.\mathbf{M H z}, \mathbf{C D C l}_{3}\right) \delta$ 31.3. HRMS (ESI) calcd for $\mathrm{C}_{23} \mathrm{H}_{24} \mathrm{BN}_{2} \mathrm{O}_{2}[\mathrm{M}+\mathrm{H}]^{+}: 371.1925$, found: 371.1921. HPLC analysis: DAICEL CHIRALCEL OD-H, hexane/isopropanol $=90 / 10,0.5 \mathrm{~mL} / \mathrm{min}, \lambda=254 \mathrm{~nm}, \mathrm{t}_{\mathrm{R}}$ (minor) $=36.59$ min, $\mathrm{t}_{\mathrm{R}}$ (major) $=39.04 \min , 88 \%$ ee $[\boldsymbol{\alpha}]^{\mathbf{2 5}}{ }_{\mathbf{D}}:+61.2\left(c 0.5, \mathrm{CHCl}_{3}\right)$.

(S)-1-(cyclopropylmethyl)-2-(2-methoxynaphthalen-1-yl)-2,3-dihydro-1H-naphtho[1,8-de][1,3,2]diazabo rinine $(6 \mathbf{v})$

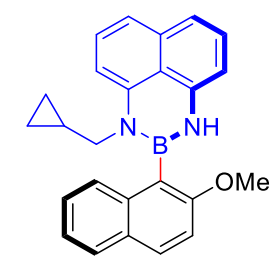

Yellow solid, mp: $146-148{ }^{\circ} \mathrm{C}$. Yield: $86 \% . \mathrm{R}_{\mathrm{f}}=0.3$ (silica gel, PE:EtOAc $=20: 1$ ).

${ }^{1} \mathbf{H}$ NMR (400 MHz, $\left.\mathbf{C D C l}_{3}\right) \delta 7.91(\mathrm{~d}, J=9.0 \mathrm{~Hz}, 1 \mathrm{H}), 7.87-7.72(\mathrm{~m}, 2 \mathrm{H}), 7.54-7.27$ (m, 4H), $7.23-7.07$ $(\mathrm{m}, 3 \mathrm{H}), 6.74(\mathrm{~d}, J=7.6 \mathrm{~Hz}, 1 \mathrm{H}), 6.33(\mathrm{~d}, J=6.6 \mathrm{~Hz}, 1 \mathrm{H}), 5.97(\mathrm{~s}, 1 \mathrm{H}), 3.92(\mathrm{~s}, 3 \mathrm{H}), 3.39-3.16(\mathrm{~m}, 2 \mathrm{H})$, $1.35-1.18(\mathrm{~m}, 1 \mathrm{H}), 0.40-0.27(\mathrm{~m}, 1 \mathrm{H}), 0.26-0.14(\mathrm{~m}, 1 \mathrm{H}),-0.05--0.21(\mathrm{~m}, 1 \mathrm{H}),-0.30--0.47(\mathrm{~m}, 1 \mathrm{H})$. ${ }^{13}$ C NMR (101 MHz, $\left.\mathbf{C D C l}_{3}\right) \delta$ 159.0, 143.0, 140.8, 136.5, 136.5, 130.7, 128.8, 128.1, 127.5, 127.3, 127.2, 126.5, 123.5, 120.8, 117.8, 117.5, 112.5, 105.6, 104.4, 55.9, 50.7, 9.1, 4.2, 3.8. ${ }^{11} \mathbf{B}$ NMR (128 $\left.\mathbf{M H z}, \mathbf{C D C l}_{3}\right)$ $\delta$ 31.8. HRMS (ESI) calcd for $\mathrm{C}_{25} \mathrm{H}_{24} \mathrm{BN}_{2} \mathrm{O}[\mathrm{M}+\mathrm{H}]^{+}: 379.1976$, found: 379.1974. IA-3, hexane/isopropanol = $98 / 2,1 \mathrm{~mL} / \mathrm{min}, \lambda=254 \mathrm{~nm}, \mathrm{t}_{\mathrm{R}}($ minor $)=10.34 \mathrm{~min}, \mathrm{t}_{\mathrm{R}}$ (major) $=11.97 \mathrm{~min}, 91 \%$ ee. $[\boldsymbol{\alpha}]^{25}{ }_{\mathrm{D}}:+59.4(c 0.5$, $\left.\mathrm{CHCl}_{3}\right)$.

(S)-1-(cyclopentylmethyl)-2-(2,6-dimethoxynaphthalen-1-yl)-2,3-dihydro-1H-naphtho[1,8-de][1,3,2]diaz aborinine (6w)

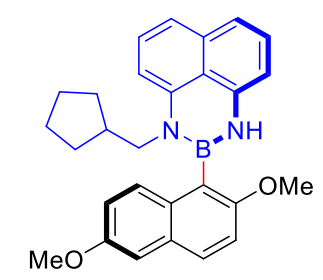

White solid, mp: $54-55^{\circ} \mathrm{C}$. Yield: $62 \% . \mathrm{R}_{\mathrm{f}}=0.3$ (silica gel, PE:EtOAc $=10: 1$ ). 
${ }^{1}$ H NMR (500 MHz, $\left.\mathbf{C D C l}_{3}\right) \delta 7.80(\mathrm{~d}, J=9.0 \mathrm{~Hz}, 1 \mathrm{H}), 7.71(\mathrm{~d}, J=9.1 \mathrm{~Hz}, 1 \mathrm{H}), 7.31-7.23(\mathrm{~m}, 2 \mathrm{H}), 7.19$ $7.08(\mathrm{~m}, 5 \mathrm{H}), 6.56(\mathrm{~d}, J=7.7 \mathrm{~Hz}, 1 \mathrm{H}), 6.33(\mathrm{dd}, J=7.1,1.2 \mathrm{~Hz}, 1 \mathrm{H}), 5.97(\mathrm{~s}, 1 \mathrm{H}), 3.92(\mathrm{~s}, 3 \mathrm{H}), 3.89(\mathrm{~s}, 3 \mathrm{H})$, $3.37(\mathrm{dd}, J=14.2,6.3 \mathrm{~Hz}, 1 \mathrm{H}), 3.25(\mathrm{dd}, J=14.2,7.4 \mathrm{~Hz}, 1 \mathrm{H}), 2.50-2.33(\mathrm{~m}, 1 \mathrm{H}), 1.72-1.61(\mathrm{~m}, 1 \mathrm{H}), 1.61$ - $1.52(\mathrm{~m}, 1 \mathrm{H}), 1.36-1.14(\mathrm{~m}, 1 \mathrm{H}), 1.10-0.97(\mathrm{~m}, 1 \mathrm{H}), 0.82-0.70(\mathrm{~m}, 1 \mathrm{H}) .{ }^{13} \mathbf{C}$ NMR $\left(\mathbf{1 2 6} \mathbf{~ M H z}, \mathbf{C D C l}_{\mathbf{3}}\right)$ $\delta 157.7,155.8,142.8,140.8,136.5,131.7,129.7,129.3,128.7,127.4,127.1,120.9,119.3,117.7,117.4,112.9$, 106.1, 105.5, 104.5, 55.9, 55.2, 51.3, 36.1, 30.4, 30.1, 24.6, 24.5. $\left.{ }^{11} \mathbf{B} \mathbf{~ N M R ~ ( 1 2 8 ~} \mathbf{~ M H z ,} \mathbf{C D C l}_{3}\right) \delta 32.4$. HRMS (ESI) calcd for $\mathrm{C}_{28} \mathrm{H}_{30} \mathrm{BN}_{2} \mathrm{O}_{2}[\mathrm{M}+\mathrm{H}]^{+}$: 437.2395, found: 437.2386. HPLC analysis: DAICEL CHIRALCEL IA-3, hexane/isopropanol $=90 / 10,1 \mathrm{~mL} / \mathrm{min}, \lambda=254 \mathrm{~nm}, \mathrm{t}_{\mathrm{R}}$ (major) $=7.85 \mathrm{~min}, \mathrm{t}_{\mathrm{R}}($ minor $)=$ $13.92 \mathrm{~min}, 86 \%$ ee. $[\boldsymbol{\alpha}]^{25}{ }_{\mathbf{D}}:+50.0\left(c 0.5, \mathrm{CHCl}_{3}\right)$.

(S)-5-(1-benzyl-1H-naphtho[1,8-de][1,3,2]diazaborinin-2(3H)-yl)-6-methoxy-2-naphthaldehyde (6x)

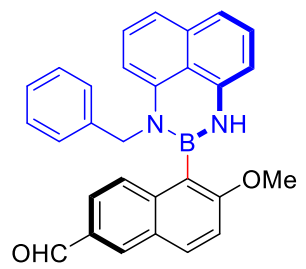

Light Yellow solid, mp: $37-38^{\circ} \mathrm{C}$. Yield: $34 \% . \mathrm{R}_{\mathrm{f}}=0.1$ (silica gel, PE:EtOAc $=5: 1$ ).

${ }^{1} \mathbf{H}$ NMR $\left(\mathbf{5 0 0} \mathbf{~ M H z}, \mathbf{C D C l}_{3}\right) \delta 10.08(\mathrm{~s}, 1 \mathrm{H}), 8.28(\mathrm{~d}, J=1.3 \mathrm{~Hz}, 1 \mathrm{H}), 8.02(\mathrm{~d}, J=9.0 \mathrm{~Hz}, 1 \mathrm{H}), 7.94(\mathrm{~d}, J=$ $8.7 \mathrm{~Hz}, 1 \mathrm{H}), 7.87$ (dd, $J=8.7,1.6 \mathrm{~Hz}), 7.33-7.27(\mathrm{~m}, 3 \mathrm{H}), 7.25-7.18(\mathrm{~m}, 3 \mathrm{H}), 7.17-7.06(\mathrm{~m}, 4 \mathrm{H}), 6.43$ (dd, $J=7.1,0.9 \mathrm{~Hz}, 1 \mathrm{H}), 6.35-6.27(\mathrm{~m}, 1 \mathrm{H}), 6.10(\mathrm{~s}, 1 \mathrm{H}), 4.66-4.49(\mathrm{~m}, 2 \mathrm{H}), 3.80(\mathrm{~s}, 3 \mathrm{H}) .{ }^{13} \mathbf{C}$ NMR $(\mathbf{1 2 6} \mathbf{~ M H z}$, $\left.\mathbf{C D C l}_{3}\right) \delta 191.9,162.0,142.1,140.3,139.9,138.1,136.2,135.1,132.8,132.1,128.3,128.0,127.8,127.5$, 127.2, 126.5, 126.3, 123.6, 120.7, 118.4, 118.0, 113.2, 106.2, 106.0, 55.6, 50.8. ${ }^{11} \mathbf{B}_{\mathbf{~ N M R}}\left(\mathbf{1 2 8} \mathbf{~ M H z}, \mathbf{C D C l}_{\mathbf{3}}\right)$ $\delta$ 33.4. HRMS (ESI) calcd for $\mathrm{C}_{29} \mathrm{H}_{24} \mathrm{BN}_{2} \mathrm{O}_{2}[\mathrm{M}+\mathrm{H}]^{+}: 443.1925$, found: 443.1920. HPLC analysis: DAICEL CHIRALCEL IG, hexane/isopropanol $=80 / 20,1 \mathrm{~mL} / \mathrm{min}, \lambda=254 \mathrm{~nm}, \mathrm{t}_{\mathrm{R}}($ minor $)=19.79 \mathrm{~min}, \mathrm{t}_{\mathrm{R}}$ (major) $=$ $21.91 \mathrm{~min}, 94 \%$ ee. $[\boldsymbol{\alpha}]^{25}{ }_{\mathbf{D}}$ : $-57.6\left(\right.$ c $\left.0.5, \mathrm{CHCl}_{3}\right)$.

(S)-2-(2,6-dimethoxynaphthalen-1-yl)-1-(2-methylbenzyl)-2,3-dihydro-1H-naphtho[1,8-de][1,3,2]diazabo rinine $(6 \mathbf{6 y})$

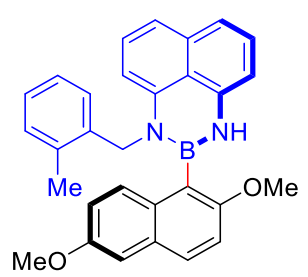

White solid, mp: $66-67{ }^{\circ} \mathrm{C}$. Yield: $90 \% . \mathrm{R}_{\mathrm{f}}=0.1$ (silica gel, PE:EtOAc $=20: 1$ ).

${ }^{1}$ H NMR $\left(\mathbf{5 0 0 ~ M H z}, \mathbf{C D C l}_{\mathbf{3}}\right) \quad \delta 7.80(\mathrm{~d}, J=10.0 \mathrm{~Hz}, 1 \mathrm{H}), 7.74(\mathrm{~d}, J=9.0 \mathrm{~Hz}, 1 \mathrm{H}), 7.49(\mathrm{dd}, J=6.1,3.0 \mathrm{~Hz}$, 1H), $7.24-7.02(\mathrm{~m}, 10 \mathrm{H}), 6.42(\mathrm{dd}, J=7.2,1.1 \mathrm{~Hz}, 1 \mathrm{H}), 6.18-6.11(\mathrm{~m}, 2 \mathrm{H}), 4.52(\mathrm{~d}, J=3.6 \mathrm{~Hz}, 2 \mathrm{H}), 3.91$ (s, 3H), 3.66 (s, 3H), 2.13 (s, 3H). ${ }^{13} \mathbf{C}$ NMR (126 MHz, $\mathbf{C D C l}_{3}$ ) $\delta$ 162.5, 160.5, 157.8, 155.9, 142.1, 140.4, 136.3, 133.9, 133.8, 131.7, 129.7, 129.3, 128.4, 127.9, 127.8, 127.4, 127.2, 120.7, 119.5, 118.3, 117.8, 115.1, 114.9, 112.9, 106.2, 106.0, 105.9, 55.7, 55.3, 50.1. ${ }^{11} \mathbf{B}$ NMR (128 $\left.\mathbf{~ M H z , ~} \mathbf{C D C l}_{\mathbf{3}}\right) \delta 33.2$. HRMS (ESI) calcd for $\mathrm{C}_{30} \mathrm{H}_{28} \mathrm{BN}_{2} \mathrm{O}_{2}[\mathrm{M}+\mathrm{H}]^{+}:$459.2238, found: 459.2230. HPLC analysis: DAICEL CHIRALCEL IA-3, hexane $/$ isopropanol $=90 / 10,1 \mathrm{~mL} / \mathrm{min}, \lambda=254 \mathrm{~nm}, \mathrm{t}_{\mathrm{R}}($ major $)=12.60 \mathrm{~min}, \mathrm{t}_{\mathrm{R}}($ minor $)=17.98 \mathrm{~min}, 93 \%$ ee. $[\alpha]^{25}$ : $-52.0\left(c 0.5, \mathrm{CHCl}_{3}\right)$. 
(S)-2-(2,6-dimethoxynaphthalen-1-yl)-1-(3-methylbenzyl)-2,3-dihydro-1H-naphtho[1,8-de][1,3,2]diazabo rinine $(6 z)$

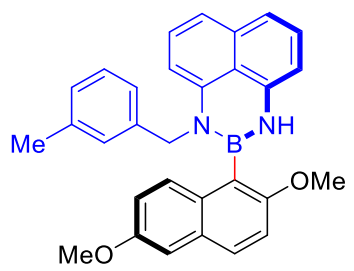

White solid, mp: $63-65^{\circ} \mathrm{C}$. Yield: $95 \% . \mathrm{R}_{\mathrm{f}}=0.23$ (silica gel, PE:EtOAc $=10: 1$ ).

${ }^{1} \mathbf{H}$ NMR $\left(\mathbf{5 0 0}\right.$ MHz, $\left.\mathbf{C D C l}_{3}\right) \delta 7.82(\mathrm{~d}, J=8.8 \mathrm{~Hz}, 1 \mathrm{H}), 7.76(\mathrm{~d}, J=9.0 \mathrm{~Hz}, 1 \mathrm{H}), 7.24-7.08(\mathrm{~m}, 10 \mathrm{H}), 6.96$ $(\mathrm{d}, J=6.0 \mathrm{~Hz}, 1 \mathrm{H}), 6.42(\mathrm{~d}, J=7.1 \mathrm{~Hz}, 1 \mathrm{H}), 6.34(\mathrm{~d}, J=5.8 \mathrm{~Hz}, 1 \mathrm{H}), 6.14(\mathrm{~s}, 1 \mathrm{H}), 4.62(\mathrm{~d}, J=16.5 \mathrm{~Hz}, 1 \mathrm{H})$, $4.52(\mathrm{~d}, J=16.5 \mathrm{~Hz}, 1 \mathrm{H}), 3.91(\mathrm{~s}, 3 \mathrm{H}), 3.79$ (s, 3H), 2.27 (s, 3H). ${ }^{13} \mathbf{C}$ NMR (126 MHz, CDCl$) \delta 157.8$, 155.9, 142.4, 140.5, 138.4, 137.8, 136.2, 131.7, 129.7, 129.2, 128.5, 128.1, 127.5, 127.1, 127.1, 127.0, 123.5, 120.7, 119.4, 118.1, 117.7, 112.9, 106.2, 106.1, 105.7, 55.7, 55.3, 50.9, 21.4. ${ }^{11} \mathbf{B}$ NMR (128 $\left.\mathbf{M H z} \mathbf{C D C l}_{3}\right) \delta$ 33.4. HRMS (ESI) calcd for $\mathrm{C}_{30} \mathrm{H}_{28} \mathrm{BN}_{2} \mathrm{O}_{2}[\mathrm{M}+\mathrm{H}]^{+}: 459.2238$, found: 459.2231.HPLC analysis: DAICEL CHIRALCEL IA-3, hexane/isopropanol $=90 / 10,1 \mathrm{~mL} / \mathrm{min}, \lambda=254 \mathrm{~nm}, \mathrm{t}_{\mathrm{R}}($ major $)=11.99 \mathrm{~min}, \mathrm{t}_{\mathrm{R}}($ minor $)=$ $15.20 \min , 93 \%$ ee. $[\boldsymbol{\alpha}]^{25}$ : $-74.8\left(c 0.5, \mathrm{CHCl}_{3}\right)$.

(S)-2-(2,6-dimethoxynaphthalen-1-yl)-1-(4-methylbenzyl)-2,3-dihydro-1H-naphtho[1,8-de][1,3,2]diazabo rinine (6aa)

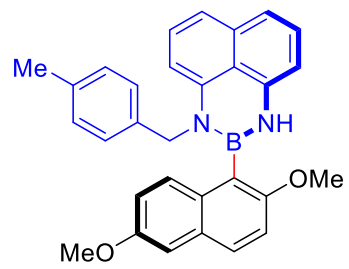

White solid, mp: $70-72{ }^{\circ} \mathrm{C}$. Yield: $80 \% . \mathrm{R}_{\mathrm{f}}=0.3$ (silica gel, PE:EtOAc $=10: 1$ ).

${ }^{1} \mathbf{H}$ NMR $\left(\mathbf{5 0 0} \mathbf{M H z}, \mathbf{C D C l}_{3}\right) \quad \delta 7.81(\mathrm{~d}, J=9.8 \mathrm{~Hz}, 1 \mathrm{H}), 7.76(\mathrm{~d}, J=9.0 \mathrm{~Hz}, 1 \mathrm{H}), 7.26-7.17(\mathrm{~m}, 4 \mathrm{H}), 7.17$ $-7.08(\mathrm{~m}, 5 \mathrm{H}), 7.04(\mathrm{~d}, J=7.7 \mathrm{~Hz}, 2 \mathrm{H}), 6.44-6.37(\mathrm{~m}, 1 \mathrm{H}), 6.33(\mathrm{~d}, J=6.4 \mathrm{~Hz}, 1 \mathrm{H}), 6.13(\mathrm{~s}, 1 \mathrm{H}), 4.62(\mathrm{~d}, J$ $=16.5 \mathrm{~Hz}, 1 \mathrm{H}), 4.53(\mathrm{~d}, J=16.4 \mathrm{~Hz}, 1 \mathrm{H}), 3.91(\mathrm{~s}, 3 \mathrm{H}), 3.78(\mathrm{~s}, 3 \mathrm{H}), 2.28(\mathrm{~s}, 3 \mathrm{H}) .{ }^{13} \mathbf{C}$ NMR (126 MHz, $\left.\mathbf{C D C l}_{3}\right) \delta 157.8,155.9,142.3,140.5,136.2,135.8,135.3,131.7,129.7,129.2,128.9,128.5,127.5,127.1$,

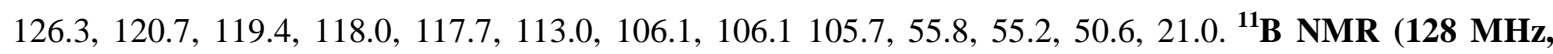
$\left.\mathbf{C D C l}_{3}\right) \delta$ 33.4. HRMS (ESI) calcd for $\mathrm{C}_{30} \mathrm{H}_{28} \mathrm{BN}_{2} \mathrm{O}_{2}[\mathrm{M}+\mathrm{H}]^{+}: 459.2238$, found: 459.2232. HPLC analysis: DAICEL CHIRALCEL IA-3, hexane/isopropanol $=90 / 10,1 \mathrm{~mL} / \mathrm{min}, \lambda=254 \mathrm{~nm}, \mathrm{t}_{\mathrm{R}}($ major$)=11.56 \mathrm{~min}, \mathrm{t}_{\mathrm{R}}$ $($ minor $)=16.98 \mathrm{~min}, 82 \%$ ee. $[\alpha]^{25}$ : $-92.5\left(c 0.5, \mathrm{CHCl}_{3}\right)$.

(S)-2-(2,6-dimethoxynaphthalen-1-yl)-1-(4-fluorobenzyl)-2,3-dihydro-1H-naphtho[1,8-de][1,3,2]diazabo rinine (6ab)

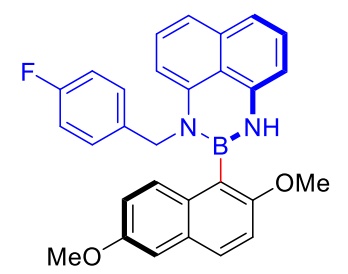

White solid, mp: 66 - $69^{\circ} \mathrm{C}$. Yield: $99 \% . \mathrm{R}_{\mathrm{f}}=0.1$ (silica gel, PE:EtOAc $\left.=20: 1\right) .{ }^{\mathbf{1}} \mathbf{H}$ NMR (500 MHz, CDCl $)$ $(500 \mathrm{MHz},) \delta 7.77(\mathrm{~d}, J=9.1 \mathrm{~Hz}, 2 \mathrm{H}), 7.31-7.25(\mathrm{~m}, 2 \mathrm{H}), 7.22-7.18(\mathrm{~m}, 2 \mathrm{H}), 7.17-7.08(\mathrm{~m}, 5 \mathrm{H}), 6.91(\mathrm{t}$, $J=8.7 \mathrm{~Hz}, 2 \mathrm{H}), 6.40(\mathrm{dd}, J=7.2,1.2 \mathrm{~Hz}, 1 \mathrm{H}), 6.26(\mathrm{dd}, J=7.2,1.4 \mathrm{~Hz}, 1 \mathrm{H}), 6.13(\mathrm{~s}, 1 \mathrm{H}), 4.60(\mathrm{~d}, J=16.6$ 
Hz, 1H), $4.51(\mathrm{~d}, J=16.5 \mathrm{~Hz}, 1 \mathrm{H}), 3.91(\mathrm{~s}, 3 \mathrm{H}), 3.75(\mathrm{~s}, 3 \mathrm{H}) .{ }^{13} \mathbf{C}$ NMR (126 MHz, $\left.\mathbf{C D C l}_{\mathbf{3}}\right) \delta 161.5(\mathrm{~d}, J=$ $244.4 \mathrm{~Hz}), 157.8,155.9,142.0,140.4,136.2,133.9(\mathrm{~d}, J=3.2 \mathrm{~Hz}), 131.7,129.7,129.3,128.3,127.9$ (d, $J=$ $7.9 \mathrm{~Hz}), 127.4,127.2,120.6,119.5,118.3,117.8,115.1,114.9,112.9,106.2,106.0$ (d, $J=7.1 \mathrm{~Hz}), 55.7,55.3$, 50.1. ${ }^{19}$ F NMR (471 MHz, $\left.\mathbf{C D C l}_{3}\right) \delta$-116.7. ${ }^{11}$ B NMR (128 MHz, $\left.\mathbf{C D C l}_{3}\right) \delta 33.6$. HRMS (ESI) calcd for $\mathrm{C}_{29} \mathrm{H}_{25} \mathrm{FBN}_{2} \mathrm{O}_{2}[\mathrm{M}+\mathrm{H}]^{+}:$463.1988, found: 463.1981. HPLC analysis: DAICEL CHIRALCEL IA-3, hexane/isopropanol $=90 / 10,1 \mathrm{~mL} / \mathrm{min}, \lambda=254 \mathrm{~nm}, \mathrm{t}_{\mathrm{R}}$ (major) $=14.24 \mathrm{~min}, \mathrm{t}_{\mathrm{R}}($ minor $)=17.37 \mathrm{~min}, 92 \%$ ee. $[\alpha]^{25}$ : $-78.8\left(c 0.5, \mathrm{CHCl}_{3}\right)$.

(S)-2-(2,6-dimethoxynaphthalen-1-yl)-1-(thiophen-3-ylmethyl)-2,3-dihydro-1H-naphtho[1,8-de][1,3,2]di azaborinine (6ac)

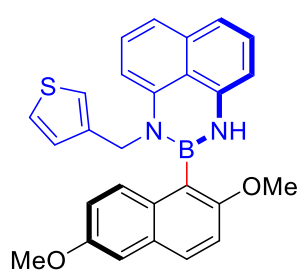

White solid, mp: $74-76{ }^{\circ} \mathrm{C}$. Yield: $73 \% . \mathrm{R}_{\mathrm{f}}=0.25$ (silica gel, PE:EtOAc = 10:1). ${ }^{1} \mathbf{H}$ NMR (500 MHz, $\left.\mathbf{C D C l}_{3}\right) \quad \delta 7.78(\mathrm{~d}, J=9.1 \mathrm{~Hz}, 1 \mathrm{H}), 7.75(\mathrm{~d}, J=9.2 \mathrm{~Hz}, 1 \mathrm{H}), 7.25-7.05(\mathrm{~m}, 9 \mathrm{H}), 6.95(\mathrm{~d}, J=4.4 \mathrm{~Hz}, 1 \mathrm{H})$, $6.43(\mathrm{~d}, J=6.4 \mathrm{~Hz}, 1 \mathrm{H}), 6.37(\mathrm{~d}, J=6.9 \mathrm{~Hz}, 1 \mathrm{H}), 6.10(\mathrm{~s}, 1 \mathrm{H}), 4.57(\mathrm{~s}, 2 \mathrm{H}), 3.91(\mathrm{~s}, 3 \mathrm{H}), 3.78(\mathrm{~s}, 3 \mathrm{H}) .{ }^{13} \mathbf{C}$ NMR (126 MHz, CDCl $) \delta 157.8,155.9,142.3,140.5,139.7,136.3,131.7,129.7,129.3,128.4,127.5,127.1$, $126.7,125.2,120.7,120.7,119.4,118.1,117.7,113.0,106.2,105.8,105.5,55.8,55.3,46.9 .{ }^{11} \mathbf{B}$ NMR (128 MHz, $\mathbf{C D C l}_{3}$ ) $\delta 32.8$. HRMS (ESI) calcd for $\mathrm{C}_{27} \mathrm{H}_{24} \mathrm{BSN}_{2} \mathrm{O}_{2}[\mathrm{M}+\mathrm{H}]^{+}: 451.1646$, found: 451.1641. HPLC analysis: DAICEL CHIRALCEL IA-3, hexane/isopropanol $=90 / 10,1 \mathrm{~mL} / \mathrm{min}, \lambda=254 \mathrm{~nm}, \mathrm{t}_{\mathrm{R}}$ (major) $=14.55$ $\min , \mathrm{t}_{\mathrm{R}}(\operatorname{minor})=18.54 \mathrm{~min}, 92 \%$ ee. $[\boldsymbol{\alpha}]^{25}$ : $-27.6\left(c 0.5, \mathrm{CHCl}_{3}\right)$.

(S)-2-(2,6-dimethoxynaphthalen-1-yl)-1-(naphthalen-2-ylmethyl)-2,3-dihydro-1H-naphtho[1,8-de][1,3,2] diazaborinine (6ad)

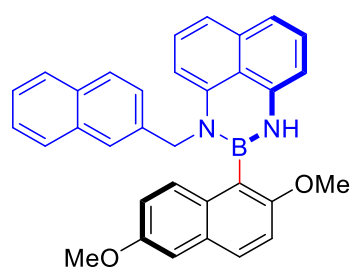

White solid, mp: $78-80{ }^{\circ} \mathrm{C}$. Yield: $44 \% . \mathrm{R}_{\mathrm{f}}=0.2$ (silica gel, PE:EtOAc $=10: 1$ ).

${ }^{1} \mathbf{H}$ NMR (500 MHz, CDCl $) \delta 7.84(\mathrm{~d}, J=9.0 \mathrm{~Hz}, 1 \mathrm{H}), 7.79(\mathrm{~s}, 1 \mathrm{H}), 7.76-7.68(\mathrm{~m}, 4 \mathrm{H}), 7.44-7.35(\mathrm{~m}$, 3H), $7.21(\mathrm{dd}, J=8.1,7.4 \mathrm{~Hz}, 1 \mathrm{H}), 7.17-7.08(\mathrm{~m}, 5 \mathrm{H}), 7.03(\mathrm{t}, J=7.9 \mathrm{~Hz}, 1 \mathrm{H}), 6.44(\mathrm{dd}, J=7.3,1.1 \mathrm{~Hz}$, 1H), $6.32(\mathrm{~d}, J=7.3 \mathrm{~Hz}, 1 \mathrm{H}), 6.17(\mathrm{~s}, 1 \mathrm{H}), 4.79(\mathrm{~d}, J=16.8 \mathrm{~Hz}, 1 \mathrm{H}), 4.68(\mathrm{~d}, J=16.8 \mathrm{~Hz}, 1 \mathrm{H}), 3.89(\mathrm{~s}, 3 \mathrm{H})$, 3.71 (s, 3H). ${ }^{13} \mathbf{C}$ NMR (126 MHz, $\left.\mathbf{C D C l}_{3}\right) \delta 157.9,155.9,142.3,140.6,136.3,135.9,133.4,132.4,131.7$, 129.7, 129.3, 128.4, 127.8, 127.6, 127.5, 127.1, 125.8, 125.2, 124.9, 124.8, 120.7, 119.5, 118.2, 117.8, 112.9, 106.2, 106.2, 105.9, 55.8, 55.3, 51.1. ${ }^{11} \mathbf{B}$ NMR (128 $\left.\mathbf{~ M H z , ~} \mathbf{C D C l}_{3}\right) \delta$ 33.6. HRMS (ESI) calcd for $\mathrm{C}_{33} \mathrm{H}_{28} \mathrm{BN}_{2} \mathrm{O}_{2} \quad[\mathrm{M}+\mathrm{H}]^{+}:$495.2238, found: 495.2232. HPLC analysis: DAICEL CHIRALCEL IA-3, hexane/isopropanol $=90 / 10,1 \mathrm{~mL} / \mathrm{min}, \lambda=254 \mathrm{~nm}, \mathrm{t}_{\mathrm{R}}$ (major) $=15.83 \mathrm{~min}, \mathrm{t}_{\mathrm{R}}($ minor $)=21.71 \mathrm{~min}, 86 \%$ ee. $[\alpha]^{25}$ : $-187.5\left(c 0.5, \mathrm{CHCl}_{3}\right)$. 


\section{Synthetic Transformations}

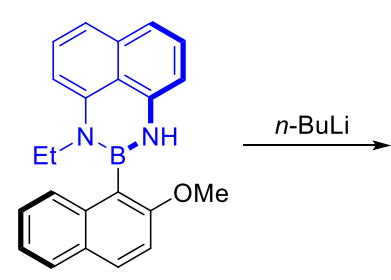

6a, $94 \%$ ee

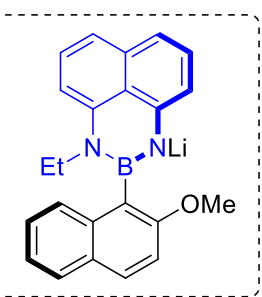

$\mathrm{El}$

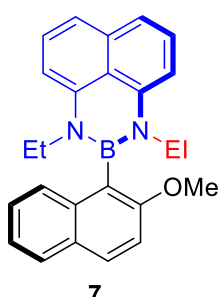

7

In air, a $10 \mathrm{~mL}$ schlenk tube was charged with $\mathbf{6 a}(0.1 \mathrm{mmol}, 1$ equiv). The tube was evacuated and filled with argon for three cycles. $1 \mathrm{~mL}$ of dry THF was added under argon. The reaction was allowed to stir at -78 ${ }^{\circ} \mathrm{C}$ for $20 \mathrm{~min}$. Then, $n \mathrm{BuLi}(0.075 \mathrm{~mL}, 0.12 \mathrm{mmol}, 1.2$ equiv, $1.6 \mathrm{M}$ in hexane) was added to the cooled tube. The reaction mixture was allowed to stir at $-30{ }^{\circ} \mathrm{C}$ for 1 hour. Next, the electrophile (3 equiv of $\mathrm{CH}_{3} \mathrm{I}$ or $\mathrm{C}_{3} \mathrm{H}_{5} \mathrm{Br}$, 1.2 equiv of $\mathrm{Ph}_{2} \mathrm{PCl}$ or $\left.(S)-\mathrm{C}_{20} \mathrm{H}_{12} \mathrm{ClO}_{2} \mathrm{P}\right)$ was added to the reaction mixture, and then slowly raised to rt and stirred overnight. Upon completion, Saturated $\mathrm{NH}_{4} \mathrm{Cl}$ solution was added, and the mixture was extracted with EtOAc and dried over $\mathrm{Na}_{2} \mathrm{SO}_{4}$. After removal of the solvent, the crude reaction mixture was purified on column chromatography (petroleum ether and ethyl acetate) to afford the desired product 7 .

(S)-1-ethyl-2-(2-methoxynaphthalen-1-yl)-3-methyl-2,3-dihydro-1H-naphtho[1,8-de][1,3,2]diazaborinine (7a)

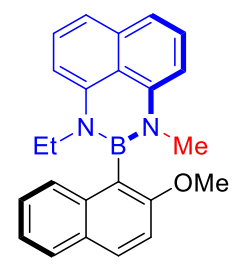

White solid, mp: $47-49^{\circ} \mathrm{C}$. Yield: $96 \% . \mathrm{R}_{\mathrm{f}}=0.3$ (silica gel, PE:EtOAc $=20: 1$ ).

${ }^{1} \mathbf{H}$ NMR $\left(\mathbf{5 0 0 ~ M H z}, \mathbf{C D C l}_{3}\right) \delta 7.94(\mathrm{~d}, J=9.0 \mathrm{~Hz}, 1 \mathrm{H}), 7.86(\mathrm{dd}, J=6.8,2.6 \mathrm{~Hz}, 1 \mathrm{H}), 7.68-7.63(\mathrm{~m}, 1 \mathrm{H})$, $7.42-7.30(\mathrm{~m}, 5 \mathrm{H}), 7.25-7.20(\mathrm{~m}, 2 \mathrm{H}), 6.61(\mathrm{~d}, J=7.7 \mathrm{~Hz}, 1 \mathrm{H}), 6.51(\mathrm{~d}, J=7.5 \mathrm{~Hz}, 1 \mathrm{H}), 3.91(\mathrm{~s}, 3 \mathrm{H}), 3.48$ $-3.26(\mathrm{~m}, 2 \mathrm{H}), 2.80$ (s, 3H), $1.03(\mathrm{t}, J=7.0 \mathrm{~Hz}, 3 \mathrm{H}) .{ }^{\mathbf{1 3}} \mathbf{C} \mathbf{~ N M R}\left(\mathbf{1 2 6} \mathbf{~ M H z}, \mathbf{C D C l}_{\mathbf{3}}\right) \delta 158.6,143.3,141.7$, $136.3,135.9,130.3,129.0,128.2,127.2,127.2,126.9,126.5,123.6,121.1,118.0,117.6,112.8,103.8,103.1$, 55.9, 41.7, 34.8, 12.8. ${ }^{11} \mathrm{~B}$ NMR $\left(128 \mathrm{MHz}, \mathrm{CDCl}_{3}\right) \delta 32.90$ (s). HRMS (ESI) calcd for $\mathrm{C}_{24} \mathrm{H}_{24} \mathrm{BN}_{2} \mathrm{O}[\mathrm{M}+\mathrm{H}]^{+}$: 367.1976, found: 367.1979. HPLC analysis: DAICEL CHIRALCEL OD-H, hexane/isopropanol = 99/1, $1 \mathrm{~mL} / \mathrm{min}, \lambda=254 \mathrm{~nm}, \mathrm{t}_{\mathrm{R}}($ major $)=7.15 \mathrm{~min}, \mathrm{t}_{\mathrm{R}}($ minor $)=8.32 \mathrm{~min}, 95 \%$ ee. $[\boldsymbol{\alpha}]^{25}{ }_{\mathrm{D}}:+17.1\left(c 0.5, \mathrm{CHCl}_{3}\right)$.

\section{(R)-1-allyl-3-ethyl-2-(2-methoxynaphthalen-1-yl)-2,3-dihydro-1H-naphtho[1,8-de][1,3,2]diazaborinine}

(7b)

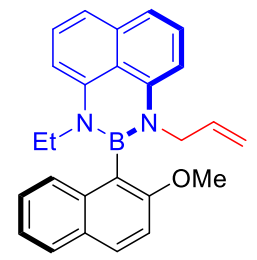

White solid, mp: $172-174{ }^{\circ} \mathrm{C}$. Yield: $51 \% . \mathrm{R}_{\mathrm{f}}=0.3$ (silica gel, PE:EtOAc $=10: 1$ ).

${ }^{1} \mathbf{H}$ NMR $\left(\mathbf{5 0 0} \mathbf{M H z}, \mathbf{C D C l}_{\mathbf{3}}\right) \delta 7.94(\mathrm{~d}, J=9.0 \mathrm{~Hz}, 1 \mathrm{H}), 7.86(\mathrm{~d}, J=7.3 \mathrm{~Hz}, 1 \mathrm{H}), 7.70(\mathrm{~d}, J=7.8 \mathrm{~Hz}, 1 \mathrm{H})$, $7.42-7.27(\mathrm{~m}, 5 \mathrm{H}), 7.22(\mathrm{~d}, J=7.7 \mathrm{~Hz}, 1 \mathrm{H}), 6.61(\mathrm{~d}, J=7.7 \mathrm{~Hz}, 1 \mathrm{H}), 6.55(\mathrm{~d}, J=7.6 \mathrm{~Hz}, 1 \mathrm{H}), 5.75-5.65$ (m, 1H), $5.07(\mathrm{dd}, J=17.4,1.0 \mathrm{~Hz}, 1 \mathrm{H}), 5.04-4.96(\mathrm{~m}, 1 \mathrm{H}), 3.96-3.81(\mathrm{~m}, 5 \mathrm{H}), 3.50-3.29(\mathrm{~m}, 2 \mathrm{H}), 1.05(\mathrm{t}$, $J=7.0 \mathrm{~Hz}, 3 \mathrm{H}) .{ }^{13} \mathbf{C} \mathbf{N M R}\left(\mathbf{1 2 6} \mathbf{~ M H z}, \mathbf{C D C l}_{3}\right) \delta 158.5,142.1,141.7,136.5,135.9,134.8,130.3,128.9,128.2$, 127.1, 127.1, 127.0, 126.4, 123.5, 121.4, 117.9, 117.7, 115.8, 112.4, 104.9, 103.7, 55.5, 49.9, 41.8, 12.7. ${ }^{11} \mathbf{B}$ 
NMR (128 MHz, $\left.\mathbf{C D C l}_{3}\right) \delta$ 33.1. HRMS (ESI) calcd for $\mathrm{C}_{26} \mathrm{H}_{26} \mathrm{BN}_{2} \mathrm{O}[\mathrm{M}+\mathrm{H}]^{+}:$393.2133, found: 393.2131 . HPLC analysis: DAICEL CHIRALCEL OD-3, $n$-hexane/isopropanol $=99 / 1,0.5 \mathrm{~mL} / \mathrm{min}, \lambda=230 \mathrm{~nm}, \mathrm{t}_{\mathrm{R}}$ $($ major $)=13.9 \mathrm{~min}, \mathrm{t}_{\mathrm{R}}(\operatorname{minor})=15.7 \mathrm{~min}, \mathrm{ee}=98 \% .[\boldsymbol{\alpha}]^{25}{ }_{\mathbf{D}}:+18.4\left(c 0.5, \mathrm{CHCl}_{3}\right)$.

(R)-1-(diphenylphosphanyl)-3-ethyl-2-(2-methoxynaphthalen-1-yl)-2,3-dihydro-1H-naphtho[1,8-de][1,3, 2]diazaborinine (7c)

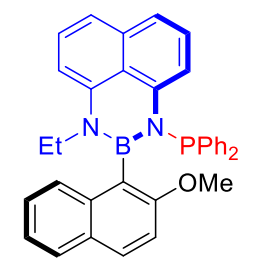

White solid, mp: $150-153^{\circ} \mathrm{C}$. Yield: $42 \%$ by recrystallization. $\mathrm{R}_{\mathrm{f}}=0.3$ (silica gel, PE:EtOAc $=20: 1$ ).

${ }^{1} \mathbf{H}$ NMR $\left(400 \mathrm{MHz}, \mathbf{C D C l}_{3}\right) \delta 7.84(\mathrm{t}, J=8.8 \mathrm{~Hz}, 2 \mathrm{H}), 7.78(\mathrm{~d}, J=8.0 \mathrm{~Hz}, 1 \mathrm{H}), 7.61(\mathrm{t}, J=6.6 \mathrm{~Hz}, 2 \mathrm{H}), 7.48$ (t, $J=6.4 \mathrm{~Hz}, 2 \mathrm{H}), 7.39-7.34(\mathrm{~m}, 1 \mathrm{H}), 7.33-7.24(\mathrm{~m}, 5 \mathrm{H}), 7.24-7.15(\mathrm{~m}, 5 \mathrm{H}), 7.12(\mathrm{~d}, J=8.1 \mathrm{~Hz}, 1 \mathrm{H})$, $6.78(\mathrm{t}, J=8.0 \mathrm{~Hz}, 1 \mathrm{H}), 6.69(\mathrm{~d}, J=7.6 \mathrm{~Hz}, 1 \mathrm{H}), 6.59(\mathrm{~d}, J=7.9 \mathrm{~Hz}, 1 \mathrm{H}), 3.70(\mathrm{~s}, 3 \mathrm{H}), 3.55-3.35(\mathrm{~m}, 2 \mathrm{H})$, $1.08(\mathrm{t}, J=7.0 \mathrm{~Hz}, 3 \mathrm{H}) .{ }^{13} \mathbf{C}$ NMR $\left(\mathbf{1 2 6} \mathbf{~ M H z}, \mathbf{C D C l}_{3}\right) \delta 158.1(\mathrm{~d}, J=3.1 \mathrm{~Hz}), 140.8,138.2(\mathrm{~d}, J=9.9 \mathrm{~Hz})$, 136.1, $135.7(\mathrm{~d}, J=6.3 \mathrm{~Hz}), 135.6(\mathrm{~d}, J=3.8 \mathrm{~Hz}), 135.5(\mathrm{~d}, J=5.0 \mathrm{~Hz}), 130.6(\mathrm{~d}, J=10.1 \mathrm{~Hz}), 130.4(\mathrm{~d}, J=$ $11.3 \mathrm{~Hz}), 129.9,128.9,128.4,128.0(\mathrm{~d}, J=5.0 \mathrm{~Hz}), 127.9(\mathrm{~d}, J=3.8 \mathrm{~Hz}), 127.8(\mathrm{~d}, J=1.3 \mathrm{~Hz}), 127.7(\mathrm{~d}, J=$ $1.5 \mathrm{~Hz}$ ), 127.1, 126.6, 126.3, 125.7, 123.4, 119.8, 118.9, 114.8 (d, $J=1.8 \mathrm{~Hz}), 112.1,104.6,55.2,42.1,12.8$. ${ }^{11}$ B NMR (128 MHz, $\left.\mathbf{C D C l}_{3}\right) \delta 37.0 .{ }^{31} \mathbf{P}$ NMR (162 $\left.\mathbf{~ M H z ,} \mathbf{C D C l}_{3}\right) \delta$ 49.3. HRMS (ESI) calcd for $\mathrm{C}_{35} \mathrm{H}_{31} \mathrm{BN}_{2} \mathrm{OP}[\mathrm{M}+\mathrm{H}]^{+}:$537.2262, found: 537.2266. HPLC analysis: DAICEL CHIRALCEL AD-H, hexane/isopropanol $=98 / 2,1 \mathrm{~mL} / \mathrm{min}, \lambda=254 \mathrm{~nm}, \mathrm{t}_{\mathrm{R}}($ major $)=4.41 \mathrm{~min}, \mathrm{t}_{\mathrm{R}}($ minor $)=5.81 \mathrm{~min}, 99 \%$ ee . $[\alpha]^{25}:+54.0\left(c 0.5, \mathrm{CHCl}_{3}\right)$.

(R)-1-((11bR)-dinaphtho[2,1-d:1',2'-f][1,3,2]dioxaphosphepin-4-yl)-3-ethyl-2-(2-methoxynaphthalen-1-yl )-2,3-dihydro-1H-naphtho[1,8-de][1,3,2]diazaborinine (7d)

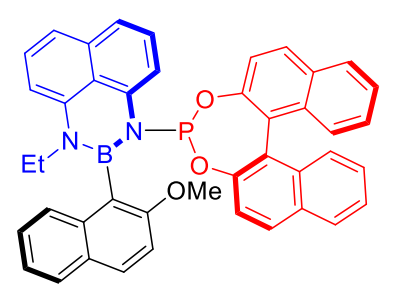

White solid, Yield: $25 \% . \mathrm{R}_{\mathrm{f}}=0.3$ (silica gel, PE:EtOAc $=20: 1$ ).

${ }^{1} \mathbf{H}$ NMR $\left(\mathbf{5 0 0} \mathbf{~ M H z}, \mathbf{C D C l}_{3}\right) \delta 7.92-7.83(\mathrm{~m}, 4 \mathrm{H}), 7.81(\mathrm{~d}, J=8.2 \mathrm{~Hz}, 1 \mathrm{H}), 7.75(\mathrm{~d}, J=8.8 \mathrm{~Hz}, 1 \mathrm{H}), 7.70(\mathrm{~d}$, $J=8.8 \mathrm{~Hz}, 1 \mathrm{H}), 7.54-7.48(\mathrm{~m}, 1 \mathrm{H}), 7.47-7.39(\mathrm{~m}, 2 \mathrm{H}), 7.39-7.25(\mathrm{~m}, 6 \mathrm{H}), 7.23-7.17(\mathrm{~m}, 3 \mathrm{H}), 7.12-$ $7.08(\mathrm{~m}, 1 \mathrm{H}), 6.96(\mathrm{~d}, J=8.8 \mathrm{~Hz}, 1 \mathrm{H}), 6.88(\mathrm{~d}, J=7.9 \mathrm{~Hz}, 1 \mathrm{H}), 6.72(\mathrm{~d}, J=7.6 \mathrm{~Hz}, 1 \mathrm{H}), 6.18(\mathrm{t}, J=8.0 \mathrm{~Hz}$, 1H), $4.02(\mathrm{~s}, 3 \mathrm{H}), 3.51-3.35(\mathrm{~m}, 2 \mathrm{H}), 1.08(\mathrm{t}, J=7.0 \mathrm{~Hz}, 3 \mathrm{H}) .{ }^{13} \mathbf{C} \mathbf{~ N M R}\left(\mathbf{1 2 6} \mathbf{~ M H z}, \mathbf{C D C l}_{3}\right) \delta 158.5(\mathrm{~d}, J=$ $5.5 \mathrm{~Hz}), 153.2(\mathrm{~d}, J=9.2 \mathrm{~Hz}), 149.5,139.9,137.0(\mathrm{~d}, J=7.4 \mathrm{~Hz}), 135.8(\mathrm{~d}, J=4.8 \mathrm{~Hz}), 135.6,132.9$ (d, $J=$ $2.0 \mathrm{~Hz}), 132.6(\mathrm{~d}, J=1.7 \mathrm{~Hz}), 131.4(\mathrm{~d}, J=1.3 \mathrm{~Hz}), 130.7(\mathrm{~d}, J=2.2 \mathrm{~Hz}), 130.6,130.4(\mathrm{~d}, J=1.1 \mathrm{~Hz}), 130.2$ (d, $J=1.2 \mathrm{~Hz}), 129.0(\mathrm{~d}, J=1.8 \mathrm{~Hz}), 128.3,128.2,127.3,127.0,126.9,126.6(\mathrm{~d}, J=19.1 \mathrm{~Hz}), 126.1,126.0$, 126.0, 124.9, 124.7 (d, $J=7.0 \mathrm{~Hz}), 124.5,123.5,122.5,122.1$ (d, $J=2.7 \mathrm{~Hz}), 121.7$ (d, $J=1.5 \mathrm{~Hz}), 121.0$, $120.9(\mathrm{~d}, J=2.8 \mathrm{~Hz}), 119.4,115.5(\mathrm{~d}, J=3.2 \mathrm{~Hz}), 112.4(\mathrm{~d}, J=1.7 \mathrm{~Hz}), 105.4,55.8,41.3,12.9 .{ }^{11} \mathbf{B}$ NMR $\left(128 \mathrm{MHz}, \mathbf{C D C l}_{3}\right) \delta$ 37.1. ${ }^{31} \mathbf{P}$ NMR (202 $\left.\mathbf{~ M H z}, \mathbf{C D C l}_{3}\right) \delta$ 146.0. HRMS (ESI) calcd for $\mathrm{C}_{43} \mathrm{H}_{33} \mathrm{BN}_{2} \mathrm{O}_{3} \mathrm{P}$ $[\mathrm{M}+\mathrm{H}]^{+}:$667.2316, found: 667.2313 . 


\section{Application of atropisomeric mono-phosphine 7c or 7d as ligand.}

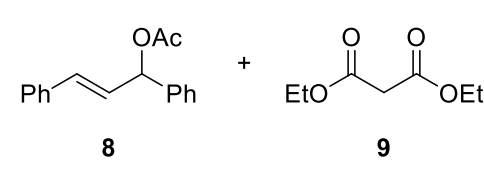

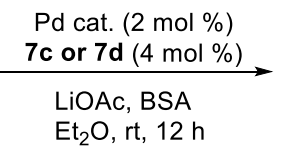

with 7 d, $87 \%$ yield, $42 \%$ ee

To a mixture of chiral ligand $7 \mathbf{c}$ or $\mathbf{7 d}(0.004 \mathrm{mmol}),\left[\mathrm{Pd}\left(\eta-\mathrm{C}_{3} \mathrm{H}_{5}\right) \mathrm{Cl}\right]_{2}(0.0025 \mathrm{mmol}, 0.56 \mathrm{mg})$ and LiOAc $(0.008 \mathrm{mmol}, 0.53 \mathrm{mg})$ in diethyl ether $(1 \mathrm{~mL})$ were added BSA $(0.3 \mathrm{mmol}, 60.8 \mathrm{mg})$ and $8(0.1 \mathrm{mmol}, 25.2$ $\mathrm{mg}$ ) at $\mathrm{rt}$ under argon atmosphere. After $30 \mathrm{~min}, 9(0.3 \mathrm{mmol}, 48 \mathrm{mg})$ was added. After $12 \mathrm{~h}$, the reaction mixture was diluted with diethyl ether and water. The organic layer was washed with brine and dried over $\mathrm{Na}_{2} \mathrm{SO}_{4}$. The filtrate was concentrated and purified by column chromatography to afford $\mathbf{1 0}$.

Colorless oil. Yield: $87 \%$ with $\mathbf{7 d}$ as ligand. $\mathrm{R}_{\mathrm{f}}=0.25$ (silica gel, PE:EtOAc $\left.=15: 1\right) .{ }^{\mathbf{1}} \mathbf{H}$ NMR $(\mathbf{5 0 0} \mathbf{~ M H z}$, $\left.\mathbf{C D C l}_{3}\right) \delta 7.31-7.19(\mathrm{~m}, 10 \mathrm{H}), 6.47(\mathrm{~d}, J=15.8 \mathrm{~Hz}, 1 \mathrm{H}), 6.40-6.29(\mathrm{~m}, 2 \mathrm{H}), 4.30-4.22(\mathrm{~m}, 1 \mathrm{H}), 4.22-$ $4.13(\mathrm{~m}, 2 \mathrm{H}), 4.02-3.86(\mathrm{~m}, 3 \mathrm{H}), 1.21(\mathrm{t}, J=7.1 \mathrm{~Hz}, 3 \mathrm{H}), 1.01(\mathrm{t}, J=7.1 \mathrm{~Hz}, 3 \mathrm{H})$. HPLC analysis: DAICEL CHIRALCEL OJ-H, hexane/isopropanol $=87 / 13,0.5 \mathrm{~mL} / \mathrm{min}, \lambda=254 \mathrm{~nm}, \mathrm{t}_{\mathrm{R}}$ (major) $=18.06 \mathrm{~min}, \mathrm{t}_{\mathrm{R}}$ (minor) $=22.74 \mathrm{~min}, 42 \%$ ee. Known compound (the absolute configuration was determined according to the previous study). ${ }^{8}$

\section{Rotational Barriers of Axially Chiral Arylborons}

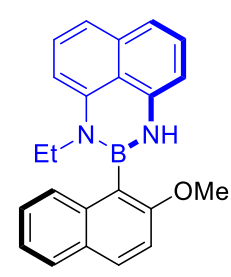

$6 a$

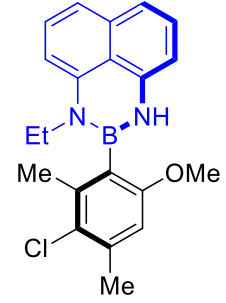

$6 s$

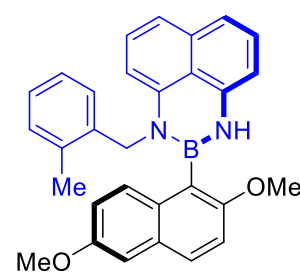

$6 y$

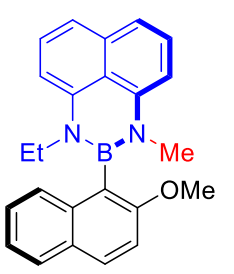

$7 \mathbf{a}$

A solution of $6(5.0 \mathrm{mg})$ in $i \mathrm{PrOH}(1 \mathrm{~mL})$ was heated at the specific temperatures. A solution of $7 \mathbf{a}(5.0 \mathrm{mg}) \mathrm{in}$ $p$-xylene $(1 \mathrm{~mL})$ was heated at the specific temperatures. The ee value was determined by chiral HPLC analysis at different intervals.

Table S1. Rotational Barrier of $6 a$ at $80^{\circ} \mathrm{C}$

\begin{tabular}{ccc}
\hline $\mathrm{t}(\mathrm{s})$ & ee $(\%)$ & $\ln \left(\mathrm{ee}_{0} / \mathrm{ee}_{\mathrm{t}}\right)$ \\
\hline 0 & 94.3 & 0 \\
1200 & 83.1 & 0.126436 \\
2400 & 76.3 & 0.211808 \\
3600 & 62.7 & 0.40812 \\
4800 & 54.7 & 0.544617 \\
6000 & 47.4 & 0.687859 \\
7200 & 40.9 & 0.835351 \\
9000 & 33.8 & 1.02602 \\
10800 & 27.8 & 1.221445 \\
\hline
\end{tabular}




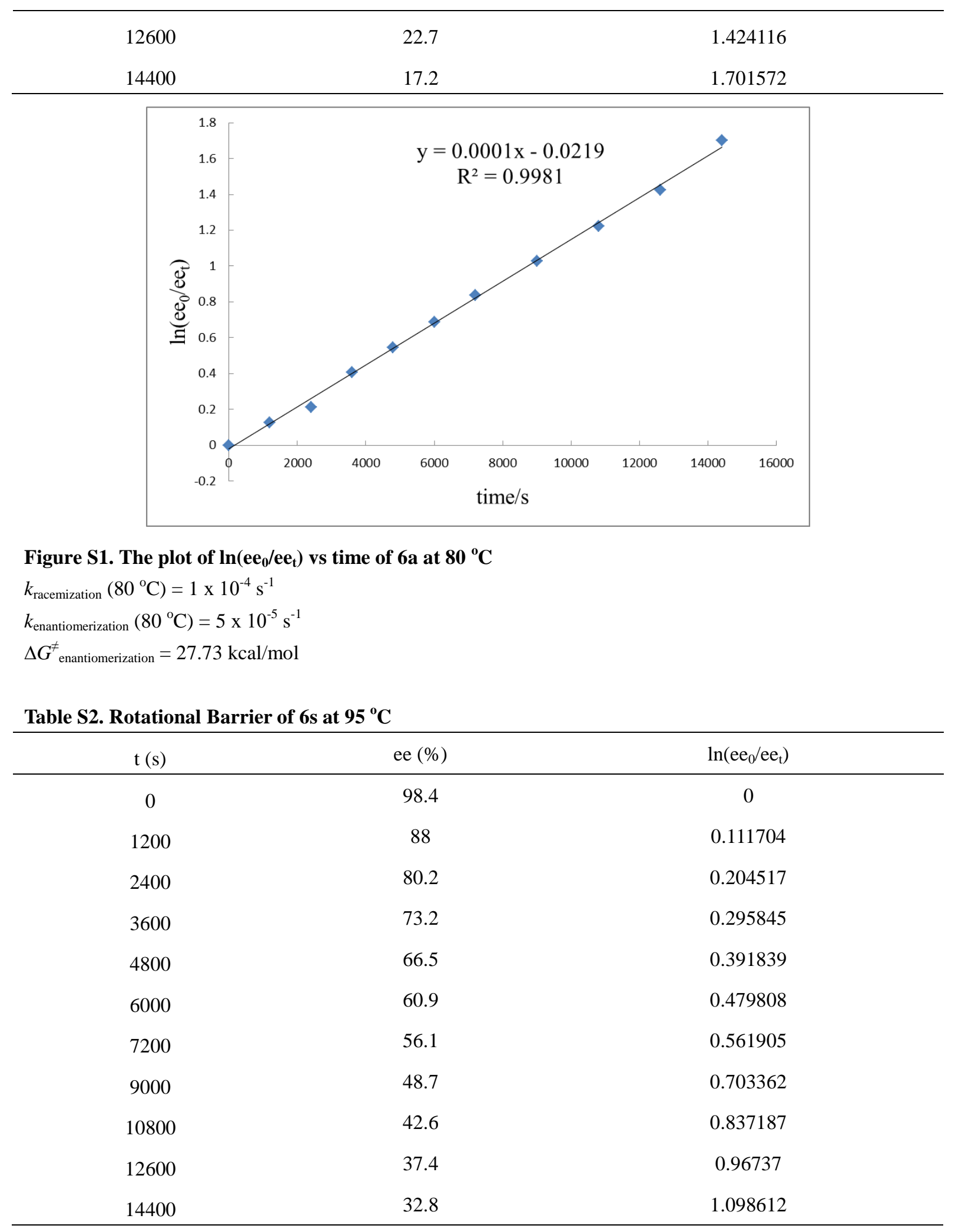




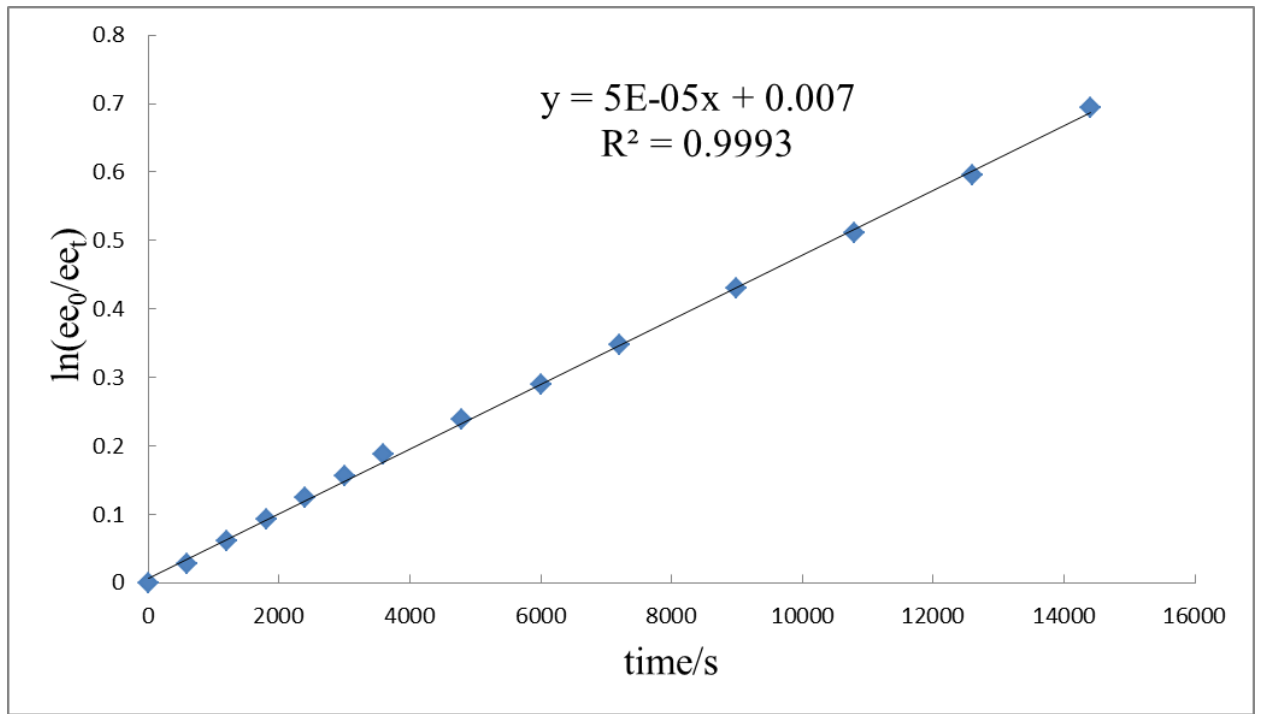

Figure S2. The plot of $\ln \left(\mathrm{ee}_{0} / \mathrm{ee}_{\mathrm{t}}\right)$ vs time of $6 \mathrm{~s}$ at $95^{\circ} \mathrm{C}$

$k_{\text {racemization }}\left(95^{\circ} \mathrm{C}\right)=5 \times 10^{-5} \mathrm{~s}^{-1}$

$k_{\text {enantiomerization }}\left(95^{\circ} \mathrm{C}\right)=2.5 \times 10^{-5} \mathrm{~s}^{-1}$

$\Delta G_{\text {enantiomerization }}^{\neq}=29.10 \mathrm{kcal} / \mathrm{mol}$

Table S3. Rotational Barrier of $6 y$ at $50{ }^{\circ} \mathrm{C}$

\begin{tabular}{ccl}
\hline $\mathrm{t}(\mathrm{s})$ & ee $(\%)$ & $\ln \left(\mathrm{ee}_{0} / \mathrm{ee}_{\mathrm{t}}\right)$ \\
\hline 0 & 93.6 & 0 \\
600 & 91.1 & 0.027073 \\
1200 & 88.1 & 0.060558 \\
1800 & 85.3 & 0.092856 \\
2400 & 82.6 & 0.125021 \\
3000 & 80.1 & 0.155755 \\
3600 & 77.6 & 0.187463 \\
4800 & 73.7 & 0.239028 \\
6000 & 70.1 & 0.289108 \\
7200 & 66.1 & 0.347862 \\
9000 & 60.9 & 0.429797 \\
10800 & 56.1 & 0.511895 \\
12600 & 51.6 & 0.595509 \\
14400 & 46.8 & 0.693147 \\
\hline
\end{tabular}




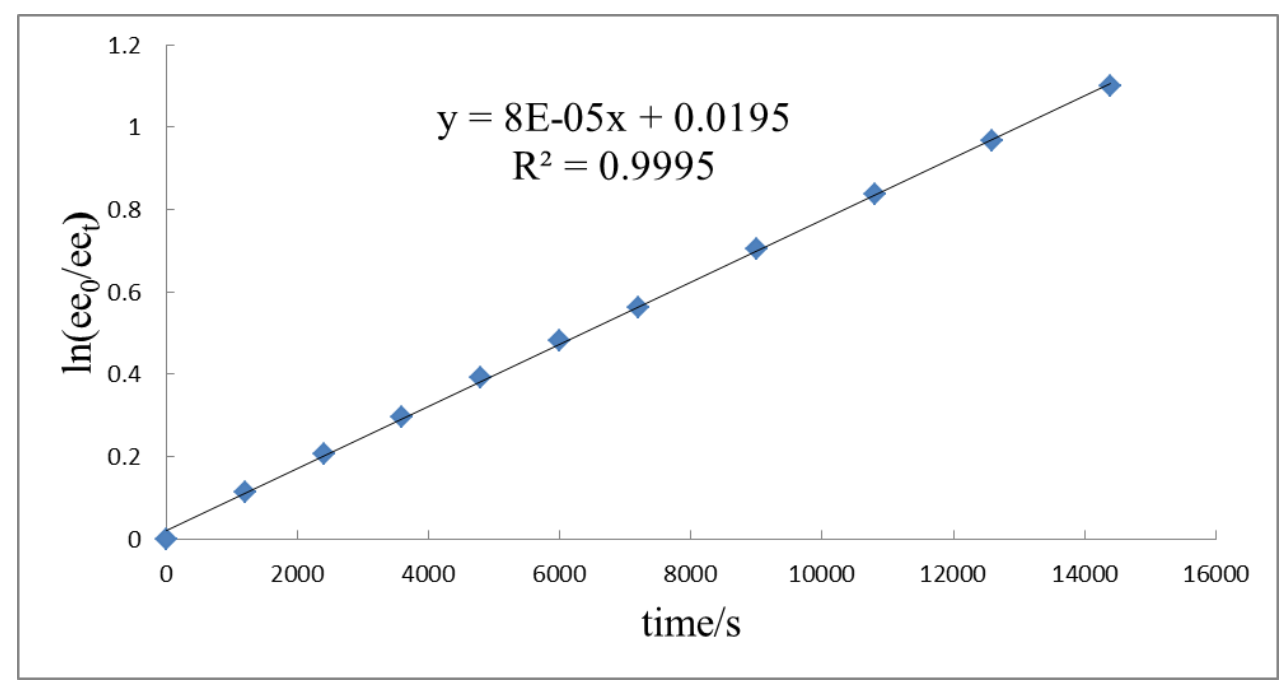

Figure S3. The plot of $\ln \left(\mathrm{ee}_{0} / \mathrm{ee}_{\mathrm{t}}\right)$ vs time of $6 \mathrm{y}$ at $50^{\circ} \mathrm{C}$

$k_{\text {racemization }}\left(50^{\circ} \mathrm{C}\right)=8 \times 10^{-5} \mathrm{~s}^{-1}$

$k_{\text {enantiomerization }}\left(50{ }^{\circ} \mathrm{C}\right)=4 \times 10^{-5} \mathrm{~s}^{-1}$

$\Delta G_{\text {enantiomerization }}^{\neq}=25.76 \mathrm{kcal} / \mathrm{mol}$

Table S4. Thermal Racemization of 7a

\begin{tabular}{|c|c|c|c|c|c|c|}
\hline time (h) & 1 & 2 & 4 & 6 & 8 & 24 \\
\hline temp $\left({ }^{\circ} \mathrm{C}\right)$ ee (\%) & 95.1 & 95.1 & 95.1 & 95.1 & 95.1 & 94.1 \\
\hline 130 & 95.1 & 95.1 & 95.1 & -- & -- & 93.2 \\
\hline 140 & 95.1 & 95.1 & 94.5 & -- & -- & 93.8 \\
\hline 150 & & & & & \\
\hline
\end{tabular}




\section{Crystal Structure of Compound 4a and 7c}

For 4a: the data was collected by using molybdenum (Mo) irradiation source at room temperature.

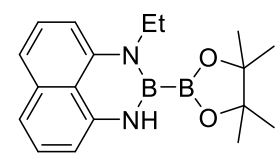

$4 a$

CCDC 2079780

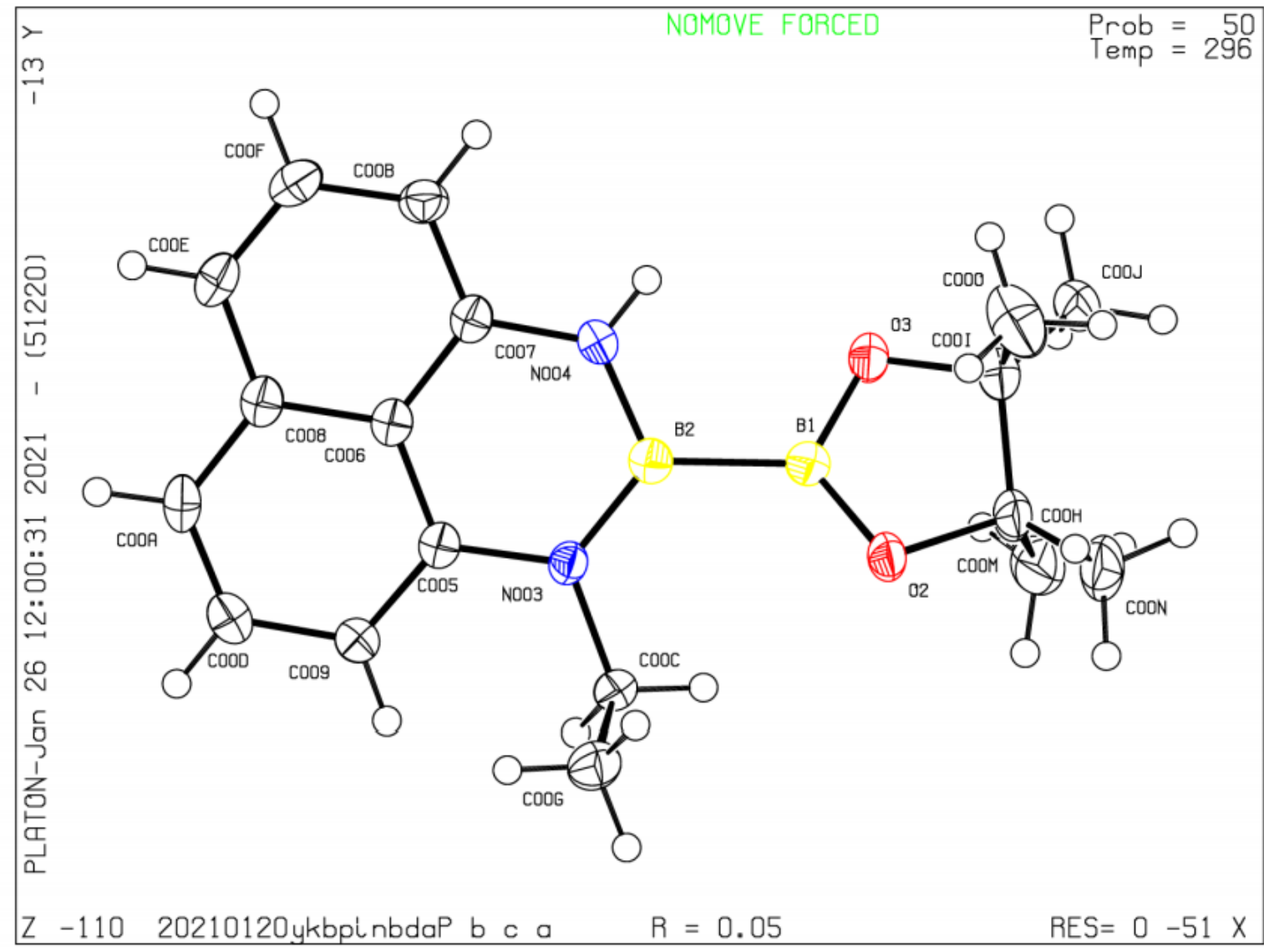

Bond precision:

$\mathrm{C}-\mathrm{C}=0.0030 \mathrm{~A}$

Wavelength $=0.71073$

Cell:

$$
\begin{array}{cc}
\mathrm{a}=11.4527(8) & \mathrm{b}=17.1932(13) \\
\text { alpha }=90 & \text { beta }=90
\end{array}
$$

$$
\begin{gathered}
\mathrm{c}=18.0614(10) \\
\text { gamma }=90
\end{gathered}
$$

Temperature: 296 K

Volume

Space group

Hall group

Moiety formula

Sum formula

$\mathrm{Mr}$

Dx, g cm-3

$\mathrm{Z}$

$\mathrm{Mu}(\mathrm{mm}-1)$

\section{Calculated}

3556.4(4)

$\mathrm{Pb} \mathrm{c} \mathrm{a}$

-P $2 \mathrm{ac} 2 \mathrm{ab}$

C18 H24 B2 N2 O2

C18 H24 B2 N2 O2

322.01

1.203

8

0.076
Reported

3556.4(4)

$\mathrm{P} \mathrm{b} \mathrm{c} \mathrm{a}$

$-\mathrm{P} 2 \mathrm{ac} 2 \mathrm{ab}$

C18 H24 B2 N2 O2

C18 H24 B2 N2 O2

322.01

1.203

8

0.076 
F000

F000'

h,k, Imax

Nref

Tmin,Tmax

Tmin'

Correction method $=$ Not given

Data completeness $=0.996$

$\mathrm{R}($ reflections $)=0.0486(2358)$

$\mathrm{S}=1.044$
1376.0

1376.52

$13,20,21$

$13,20,21$

3136

$\operatorname{Theta}(\max )=24.999$

$\mathrm{wR} 2($ reflections $)=0.1268(3125)$

Npar $=222$

For 7c: the data was collected by using molybdenum (Mo) irradiation source at room temperature.
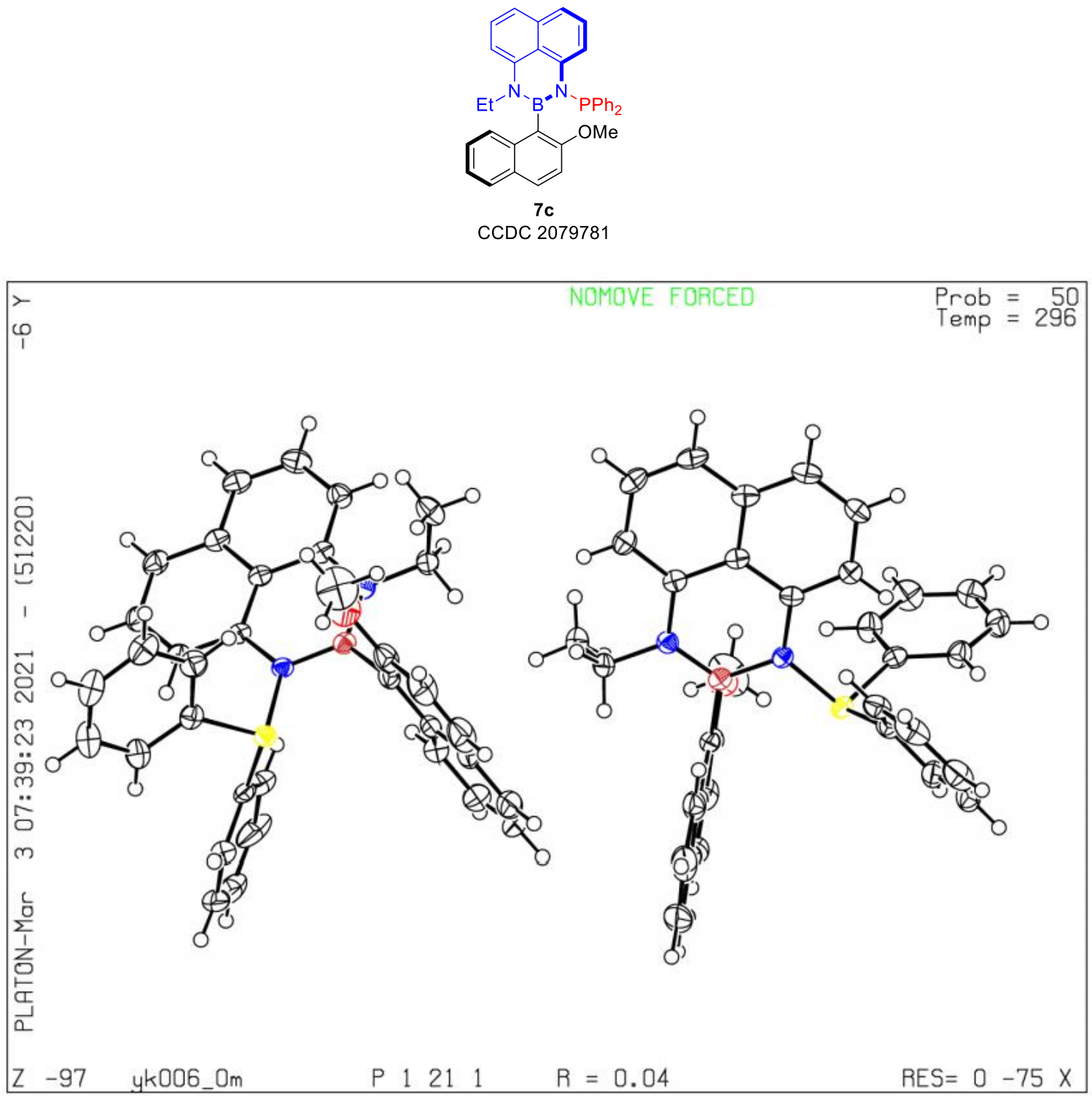

Bond precision:

$\mathrm{C}-\mathrm{C}=0.0041 \mathrm{~A}$

Wavelength $=0.71073$ 
Cell:

$$
a=17.0299(10)
$$$$
\text { alpha }=90
$$

$\mathrm{b}=8.3709(4)$

beta $=98.129(2)$ $c=19.8873(12)$

gamma $=90$

Temperature: $296 \mathrm{~K}$

Volume

Calculated

Reported

Space group

2806.6(3)

2806.6(3)

Hall group

P 21

P 1211

$\mathrm{P} 2 \mathrm{yb}$

$\mathrm{P} 2 \mathrm{yb}$

Moiety formula

C35 H30 B N2 O P

C35 H30 B N2 O P

Sum formula

C35 H30 B N2 O P

C35 H30 B N2 O P

$\mathrm{Mr}$

536.39

Dx, g cm-3

1.269

536.39

1.269

4

4

0.130

0.130

$\mathrm{Mu}(\mathrm{mm}-1)$

1128.0

1128.0

F000'

1128.80

$\mathrm{h}, \mathrm{k}, \operatorname{lmax}$

$22,11,26$

$22,11,26$

$13763[7348]$

12877

Tmin,Tmax

$0.954,0.964$

$0.549,0.758$

Tmin'

0.948

Correction method $=$ \# Reported $\mathrm{T}$ Limits: $\mathrm{Tmin}=0.549 \mathrm{Tmax}=0.758$

AbsCorr $=$ MULTI-SCAN

Data completeness $=1.75 / 0.94$

$\operatorname{Theta}(\max )=28.150$

$\mathrm{R}$ (reflections) $=0.0404(10424)$

$\mathrm{wR} 2$ (reflections) $=0.0846(12877)$

$\mathrm{S}=1.042$

Npar $=726$

Flack parameter

$-0.05(3)$

\section{References}

(1) Iwadate, N.; Suginome, M. Differentially Protected Diboron for Regioselective Diboration of Alkynes: Internal-Selective Cross-Coupling of 1-Alkene-1,2-diboronic Acid Derivatives. J. Am. Chem. Soc. 2010, 132, 2548-2549.

(2) Nguyen, Q.-H.; Guo, S.-M.; Royal, T.; Baudoin, O.; Cramer, N. Intermolecular Palladium(0)-Catalyzed Atropo-enantioselective C-H Arylation of Heteroarenes. J. Am. Chem. Soc. 2020, 142, 2161-2167.

(3) Heid, B.; Plietker, B. Iron-Catalyzed C-Allylating Partial Dearomatization of Naphthols. Synthesis 2016, 48, 340-350.

(4) Yang, H.; Sun, J.; Gu, W.; Tang, W. Enantioselective Cross-Coupling for Axially Chiral Tetra-ortho-Substituted Biaryls and Asymmetric Synthesis of Gossypol. J. Am. Chem. Soc. 2020, 142, 8036-8043.

(5) Pan, C.; Zhu, Z.; Zhang, M.; Gu, Z. Palladium-Catalyzed Enantioselective Synthesis of 2-Aryl Cyclohex-2-enone Atropisomers: Platform Molecules for the Divergent Synthesis of Axially Chiral Biaryl Compounds. Angew. Chem. Int. Ed. 2017, 56, 4777-4781.

(6) Jolliffe, J. D.; Armstrong, R. J.; Smith, M. D. Catalytic enantioselective synthesis of atropisomeric biaryls by a cation-directed O-alkylation. Nat. Chem. 2017, 9, 558-562.

(7) Pravst, I.; Zupan, M.; Stavber, S. Directed regioselectivity of bromination of ketones with NBS: solvent-free conditions versus water. Tetrahedron Lett. 2006, 47, 4707-4710 
(8) Yao, L.; Nie, H.; Zhang, D.; Zhang, S. Chiral Ferrocenyl N,N Ligands with Intramolecular Hydrogen Bonds for Highly Enantioselective Allylic Alkylations. ChemCatChem. 2018, 10, 804-809. 


\section{NMR Spectra}

1-ethyl-2-(4,4,5,5-tetramethyl-1,3,2-dioxaborolan-2-yl)-2,3-dihydro-1H-naphtho[1,8-de][1,3,2] diazabori nine (4a)

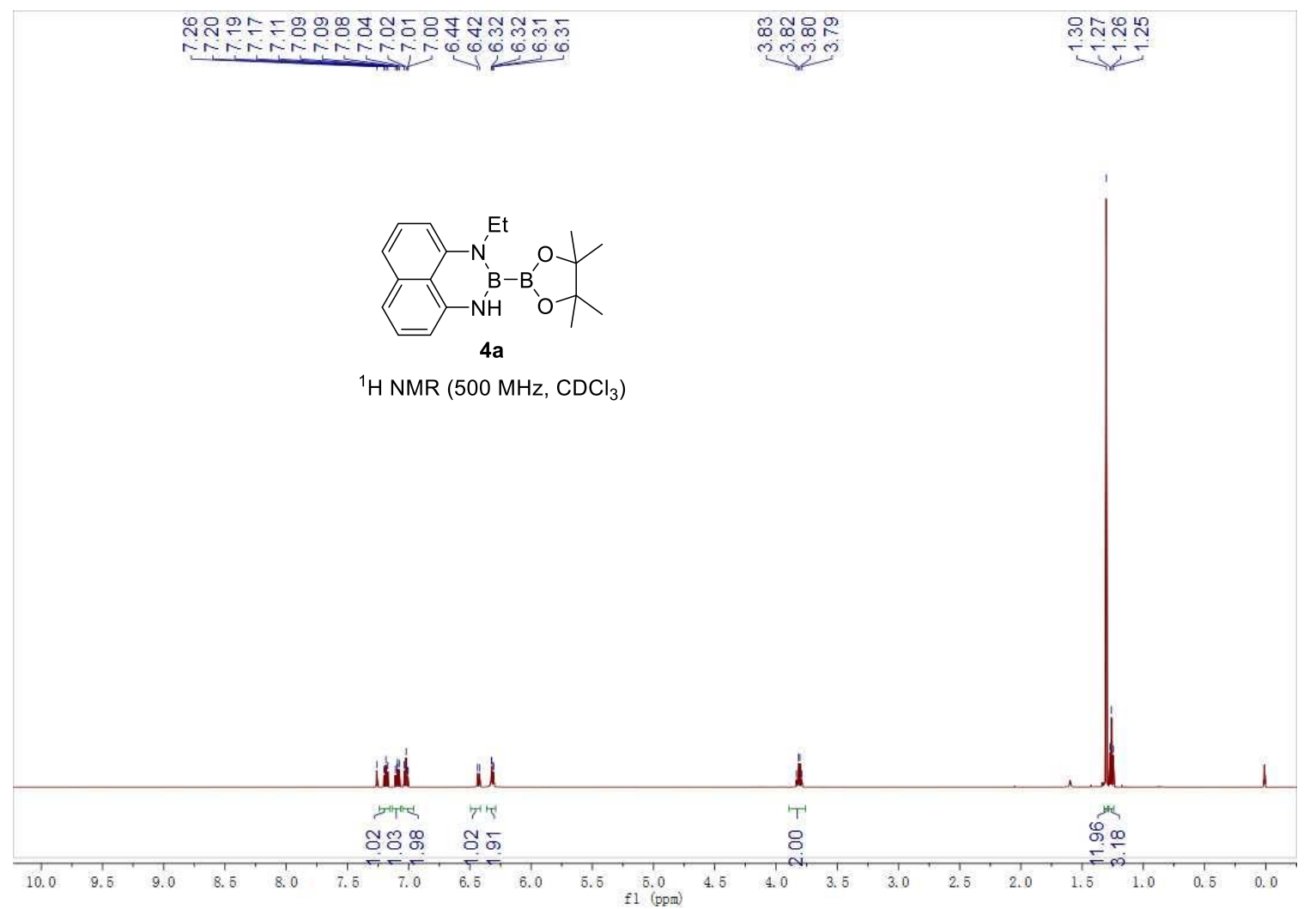

1-ethyl-2-(4,4,5,5-tetramethyl-1,3,2-dioxaborolan-2-yl)-2,3-dihydro-1H-naphtho[1,8-de][1,3,2]diazabori nine (4a)

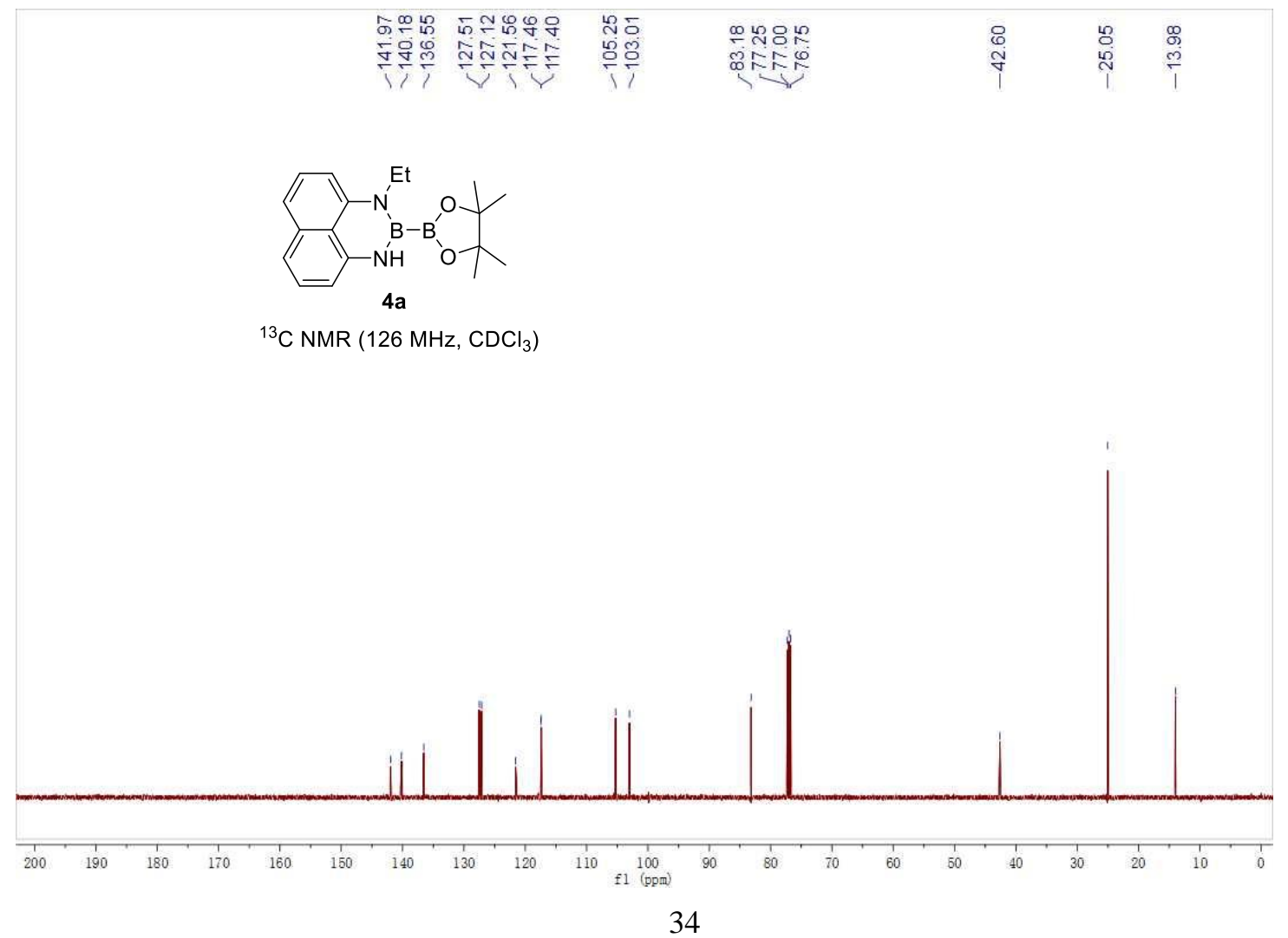


1-ethyl-2-(4,4,5,5-tetramethyl-1,3,2-dioxaborolan-2-yl)-2,3-dihydro-1H-naphtho[1,8-de][1,3,2]diazabori nine (4a)

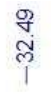

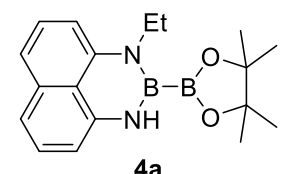

${ }^{11} \mathrm{~B}$ NMR $\left(128 \mathrm{MHz}, \mathrm{CDCl}_{3}\right)$

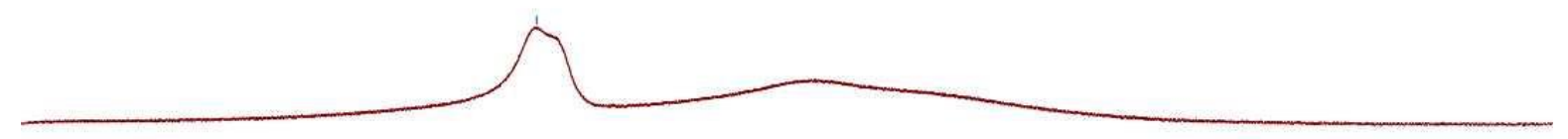

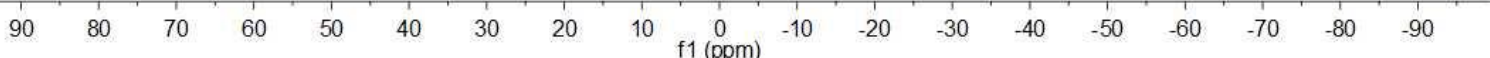

1-(cyclopropylmethyl)-2-(4,4,5,5-tetramethyl-1,3,2-dioxaborolan-2-yl)-2,3-dihydro-1H-naphtho[1,8-de][ $1,3,2]$ diazaborinine (4b)

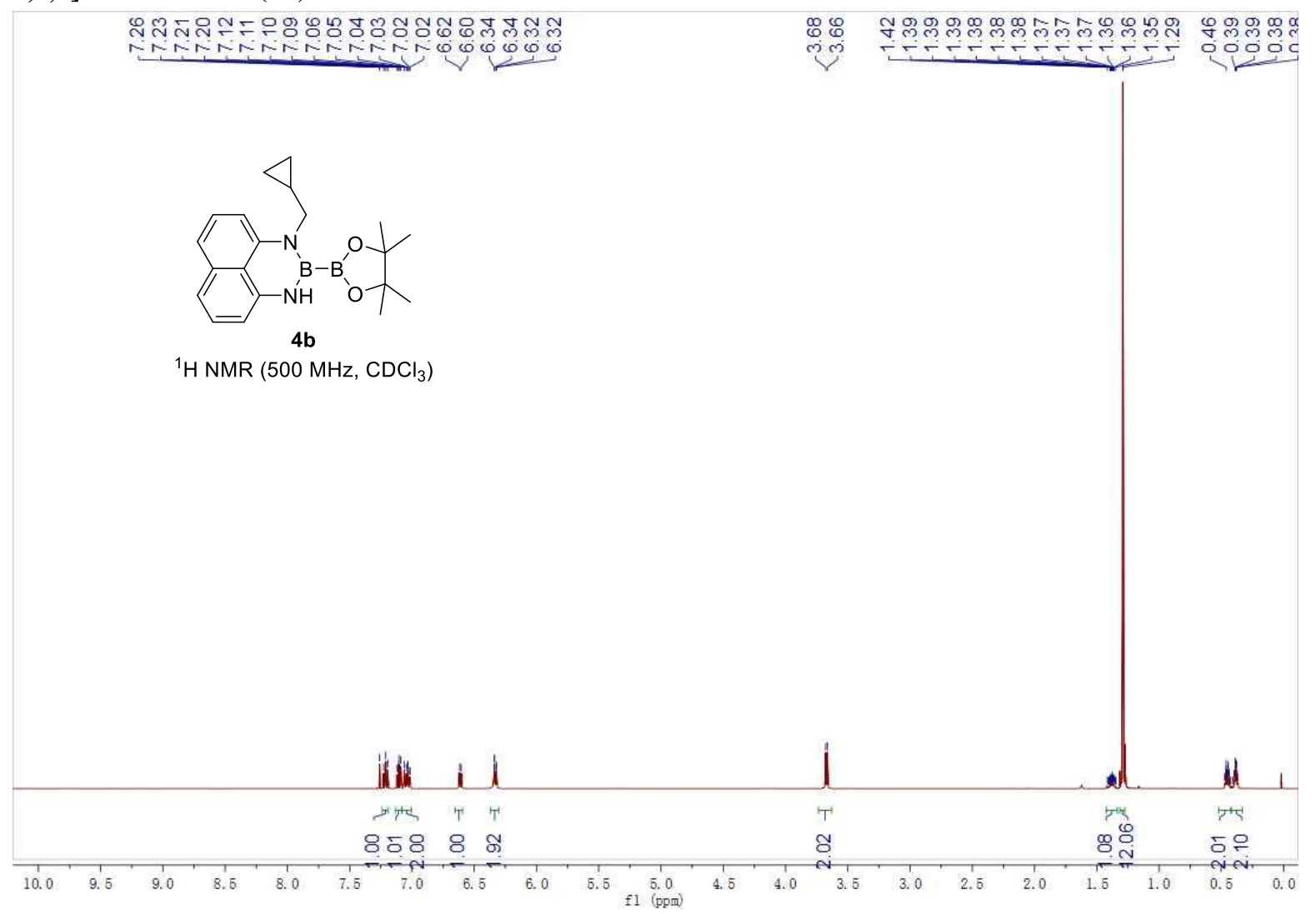


1-(cyclopropylmethyl)-2-(4,4,5,5-tetramethyl-1,3,2-dioxaborolan-2-yl)-2,3-dihydro-1H-naphtho[1,8-de][ $1,3,2]$ diazaborinine (4b)

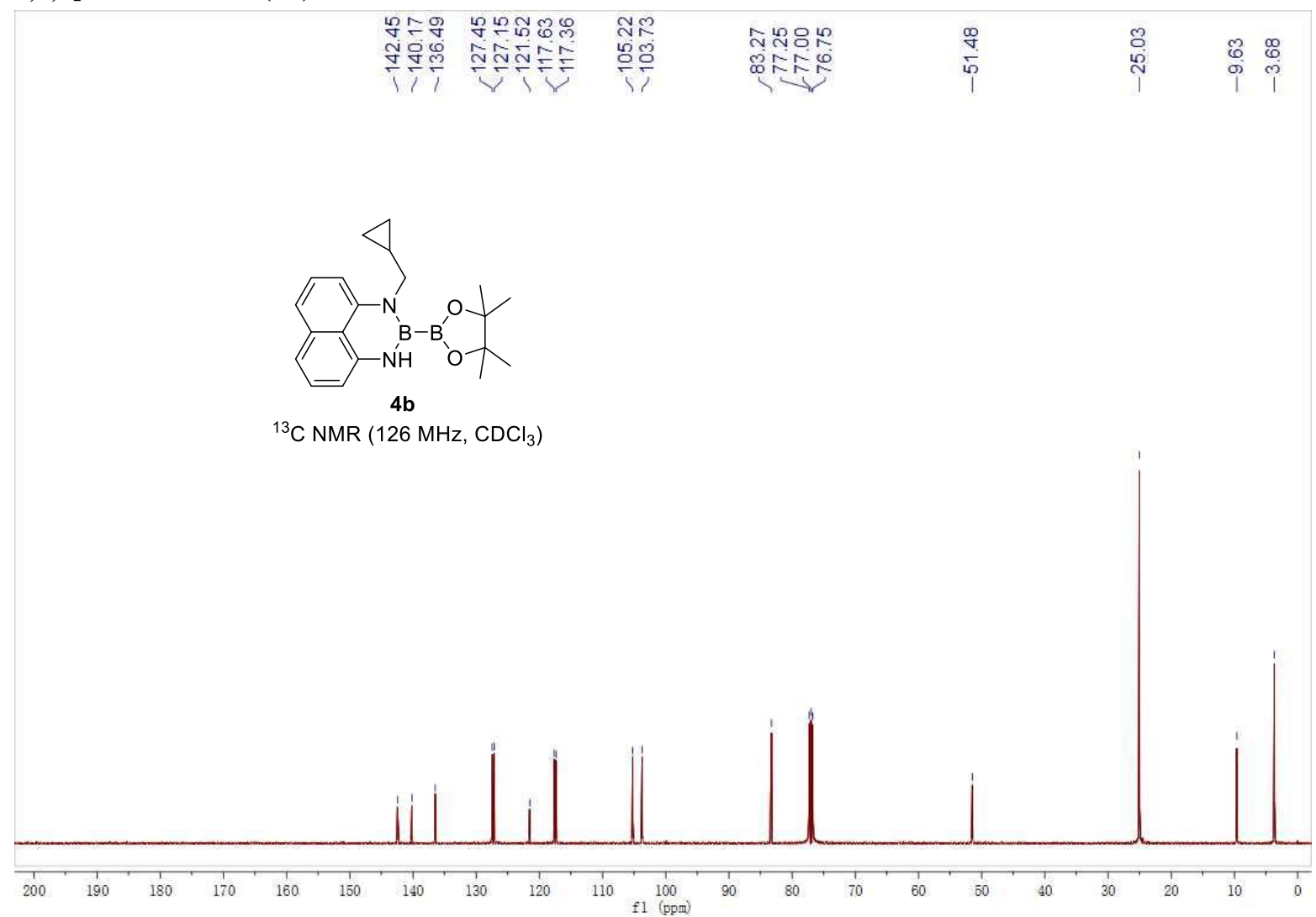

1-(cyclopropylmethyl)-2-(4,4,5,5-tetramethyl-1,3,2-dioxaborolan-2-yl)-2,3-dihydro-1H-naphtho[1,8-de][ $1,3,2]$ diazaborinine (4b)

मू
ले

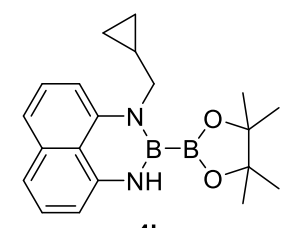

4b

${ }^{11} \mathrm{~B}$ NMR (128 $\left.\mathrm{MHz}, \mathrm{CDCl}_{3}\right)$

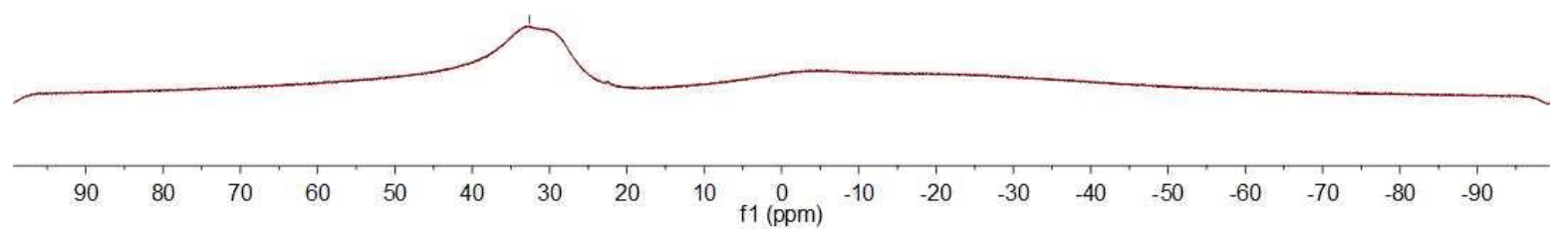


1-(cyclopentylmethyl)-2-(4,4,5,5-tetramethyl-1,3,2-dioxaborolan-2-yl)-2,3-dihydro-1H-naphtho[1,8-de][1 ,3,2]diazaborinine (4c)

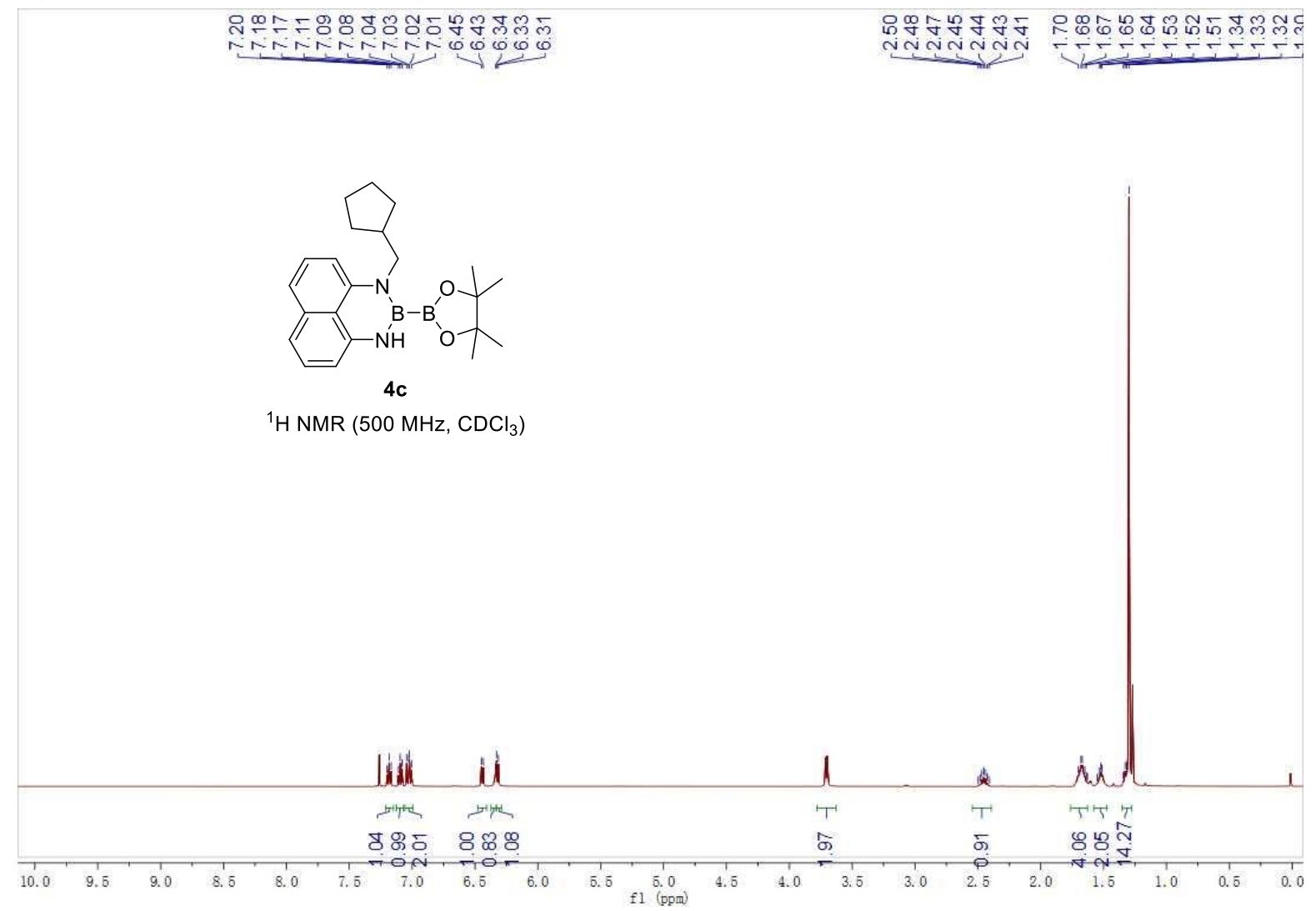

1-(cyclopentylmethyl)-2-(4,4,5,5-tetramethyl-1,3,2-dioxaborolan-2-yl)-2,3-dihydro-1H-naphtho[1,8-de][1 ,3,2]diazaborinine (4c)

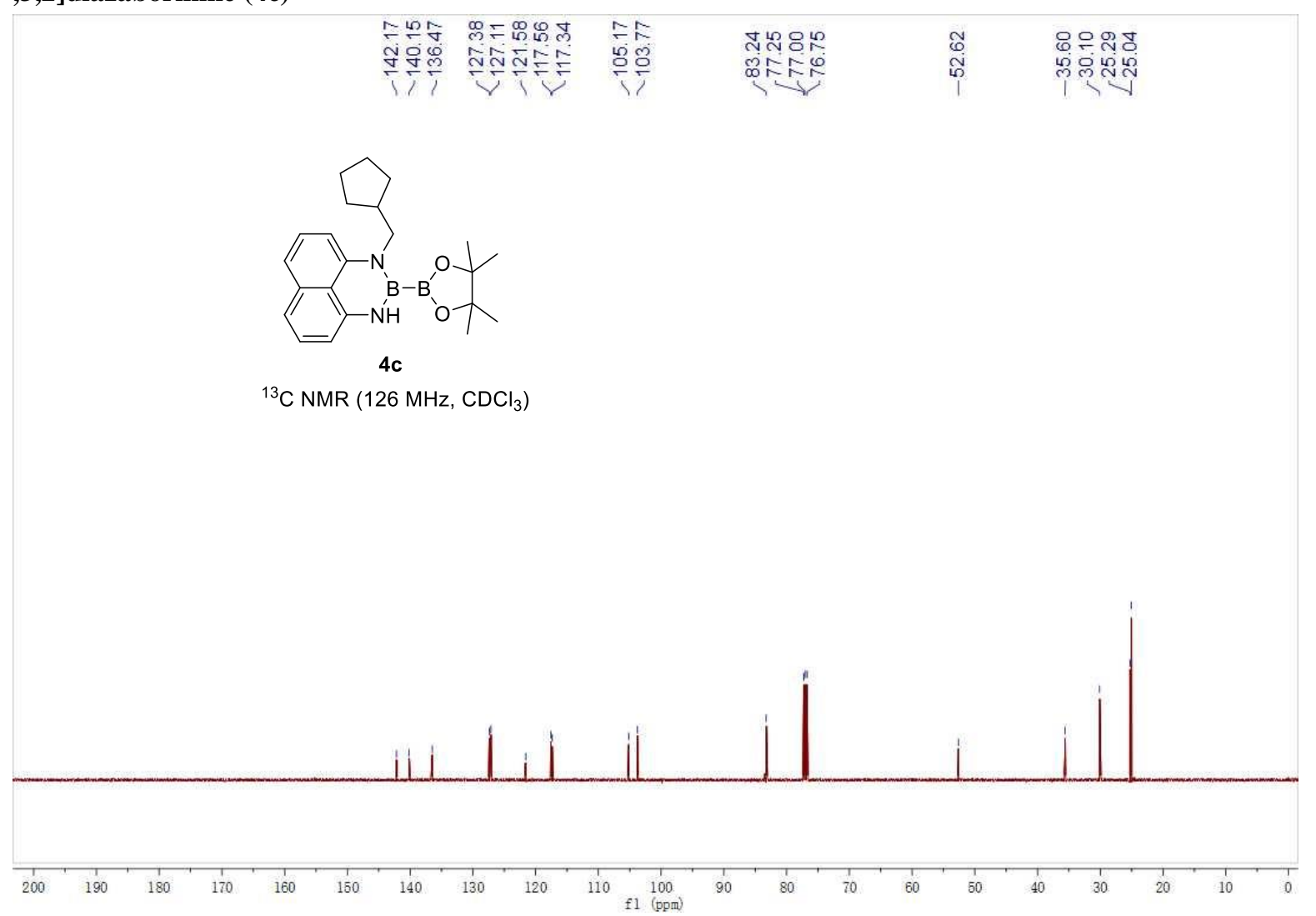


1-(cyclopentylmethyl)-2-(4,4,5,5-tetramethyl-1,3,2-dioxaborolan-2-yl)-2,3-dihydro-1H-naphtho[1,8-de][1 ,3,2]diazaborinine (4c)

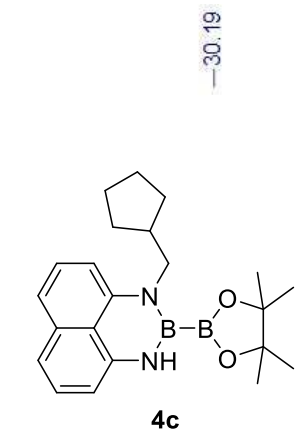

${ }^{11} \mathrm{~B}$ NMR (128 MHz, $\mathrm{CDCl}_{3}$ )

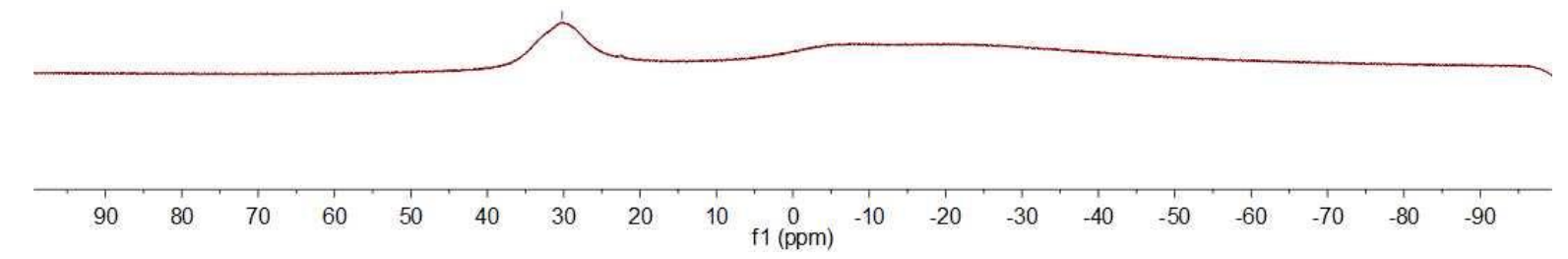

1-benzyl-2-(4,4,5,5-tetramethyl-1,3,2-dioxaborolan-2-yl)-2,3-dihydro-1H-naphtho[1,8-de][1,3,2]diazabor inine (4d)

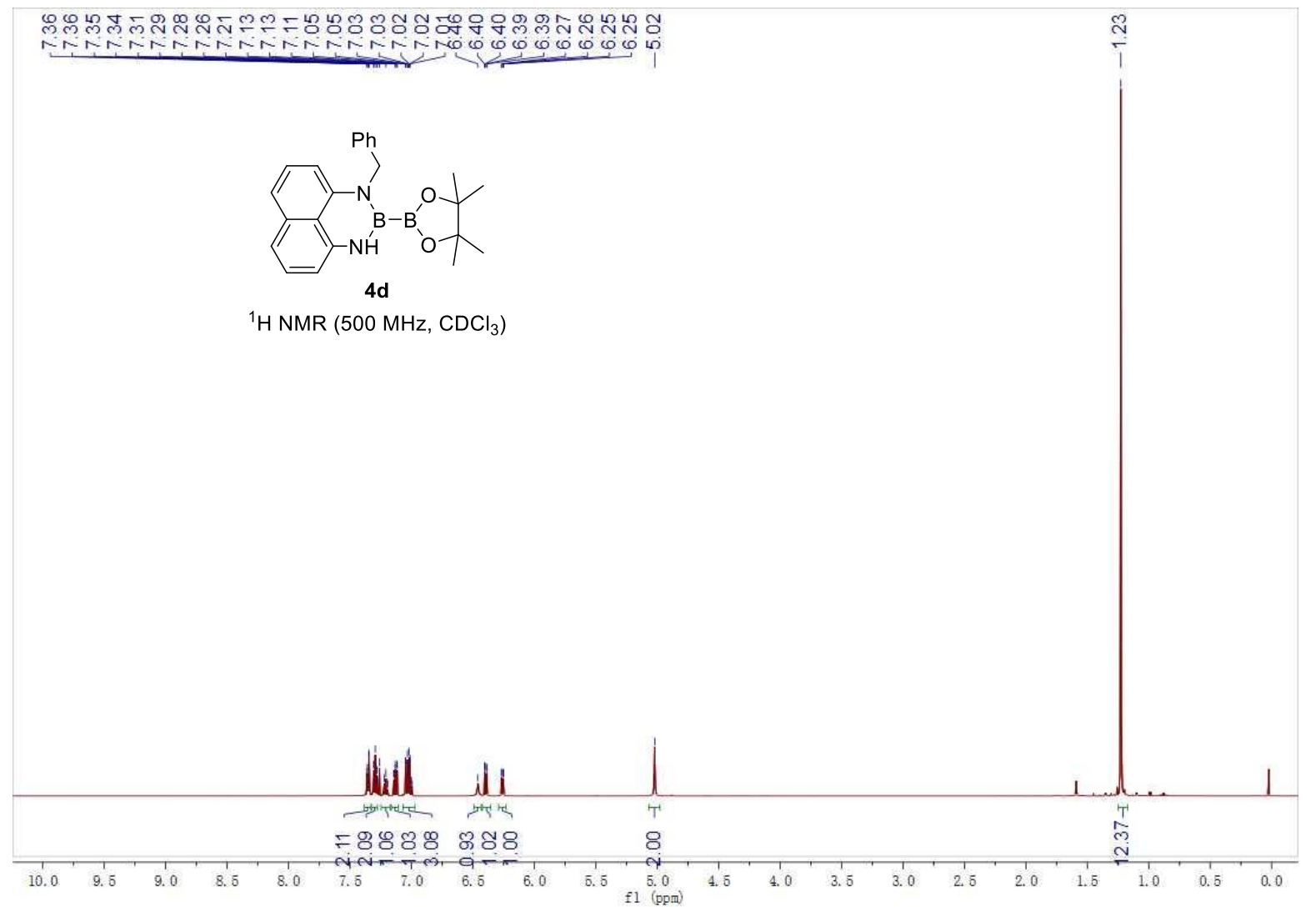


1-benzyl-2-(4,4,5,5-tetramethyl-1,3,2-dioxaborolan-2-yl)-2,3-dihydro-1H-naphtho[1,8-de][1,3,2]diazabor inine (4d)

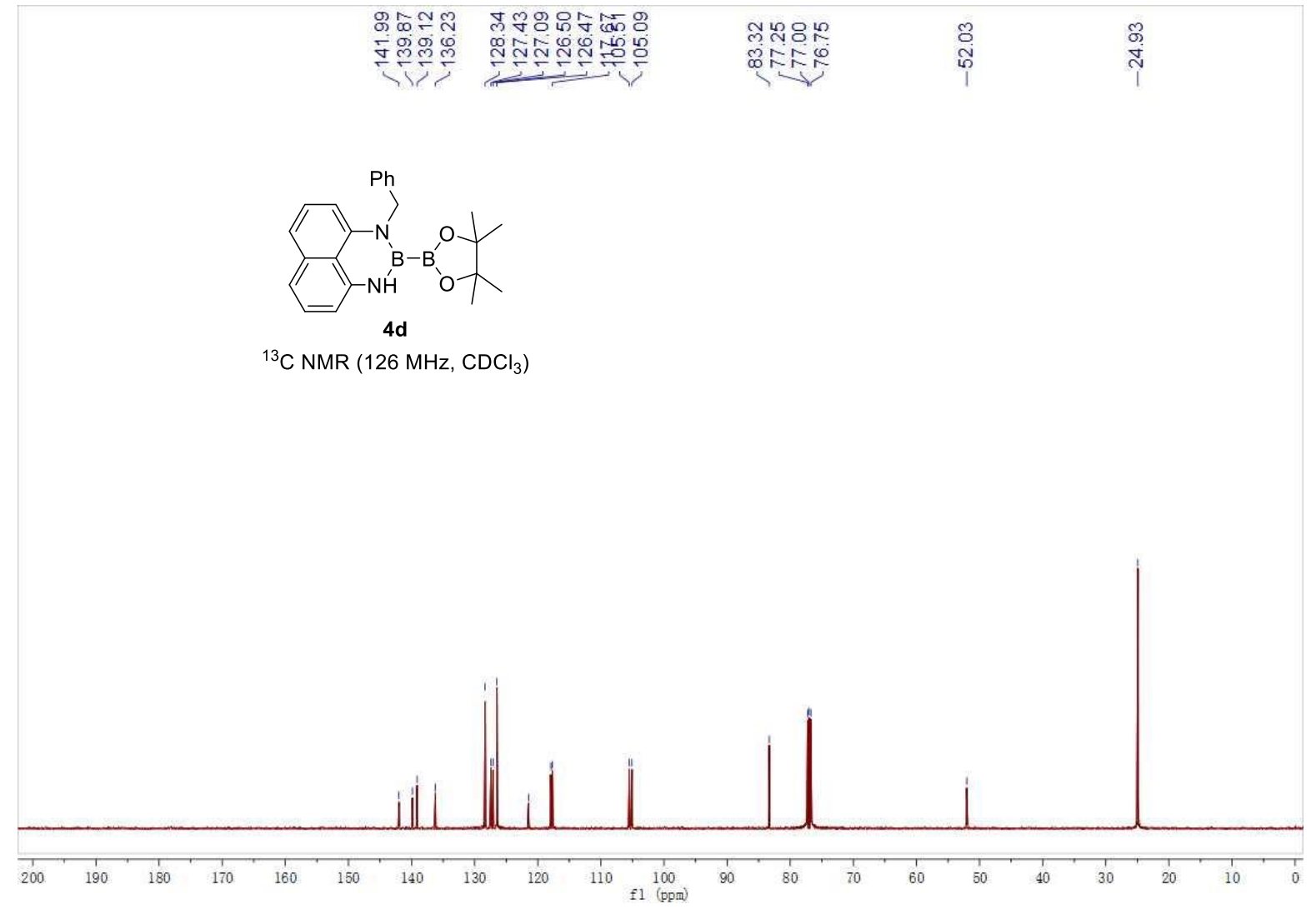

1-benzyl-2-(4,4,5,5-tetramethyl-1,3,2-dioxaborolan-2-yl)-2,3-dihydro-1H-naphtho[1,8-de][1,3,2]diazabor inine (4d)

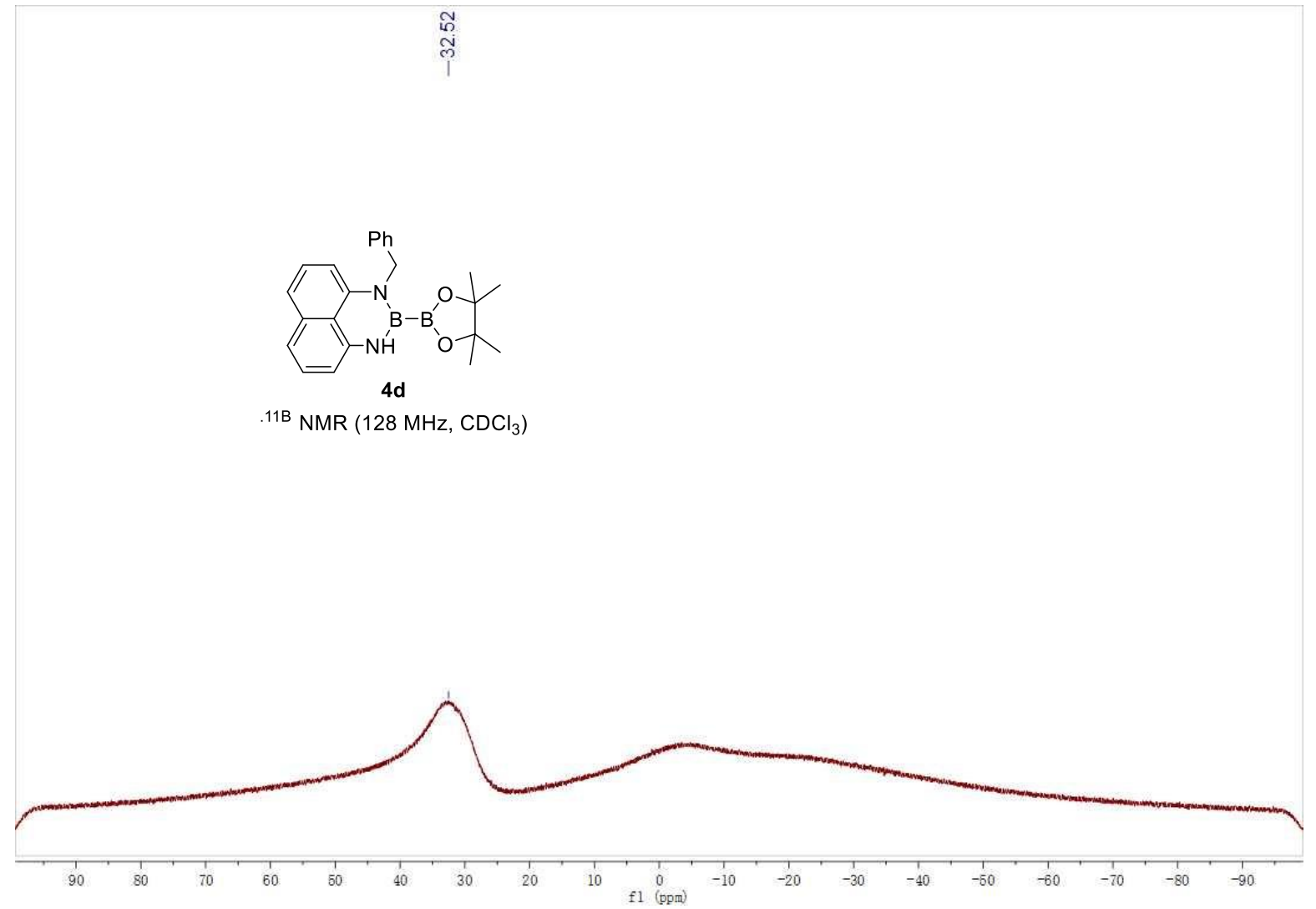


1-(2-methylbenzyl)-2-(4,4,5,5-tetramethyl-1,3,2-dioxaborolan-2-yl)-2,3-dihydro-1H-naphtho[1,8-de][1,3, 2]diazaborinine (4e)

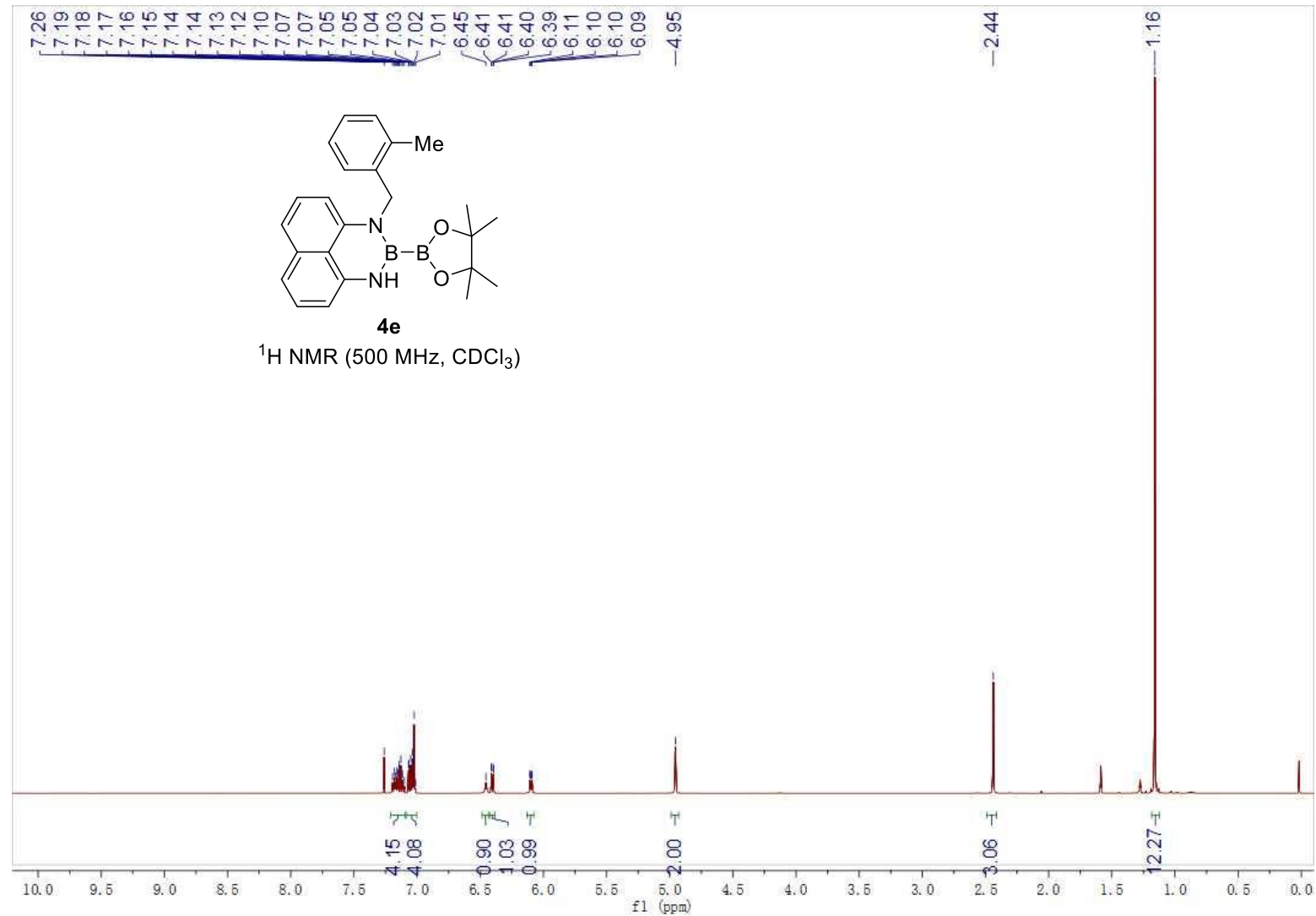

1-(2-methylbenzyl)-2-(4,4,5,5-tetramethyl-1,3,2-dioxaborolan-2-yl)-2,3-dihydro-1H-naphtho[1,8-de][1,3, 2]diazaborinine (4e)

\begin{tabular}{|c|c|c|}
\hline 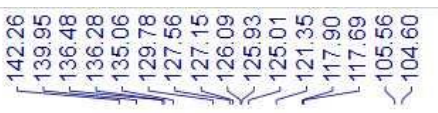 & 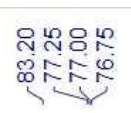 & 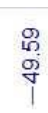 \\
\hline
\end{tabular}

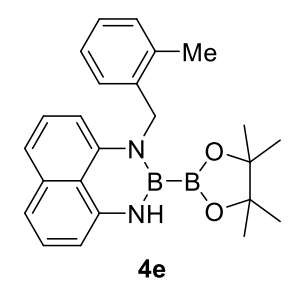

${ }^{13} \mathrm{C} \mathrm{NMR} \mathrm{(126} \mathrm{MHz,} \mathrm{CDCl}_{3}$ )

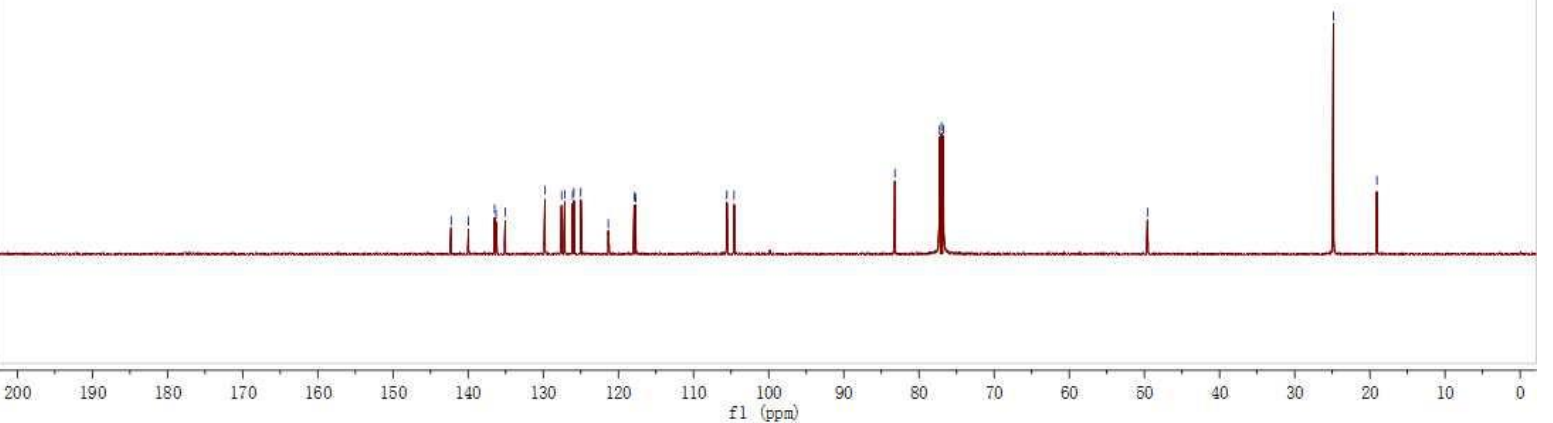


1-(2-methylbenzyl)-2-(4,4,5,5-tetramethyl-1,3,2-dioxaborolan-2-yl)-2,3-dihydro-1H-naphtho[1,8-de][1,3, 2]diazaborinine (4e)
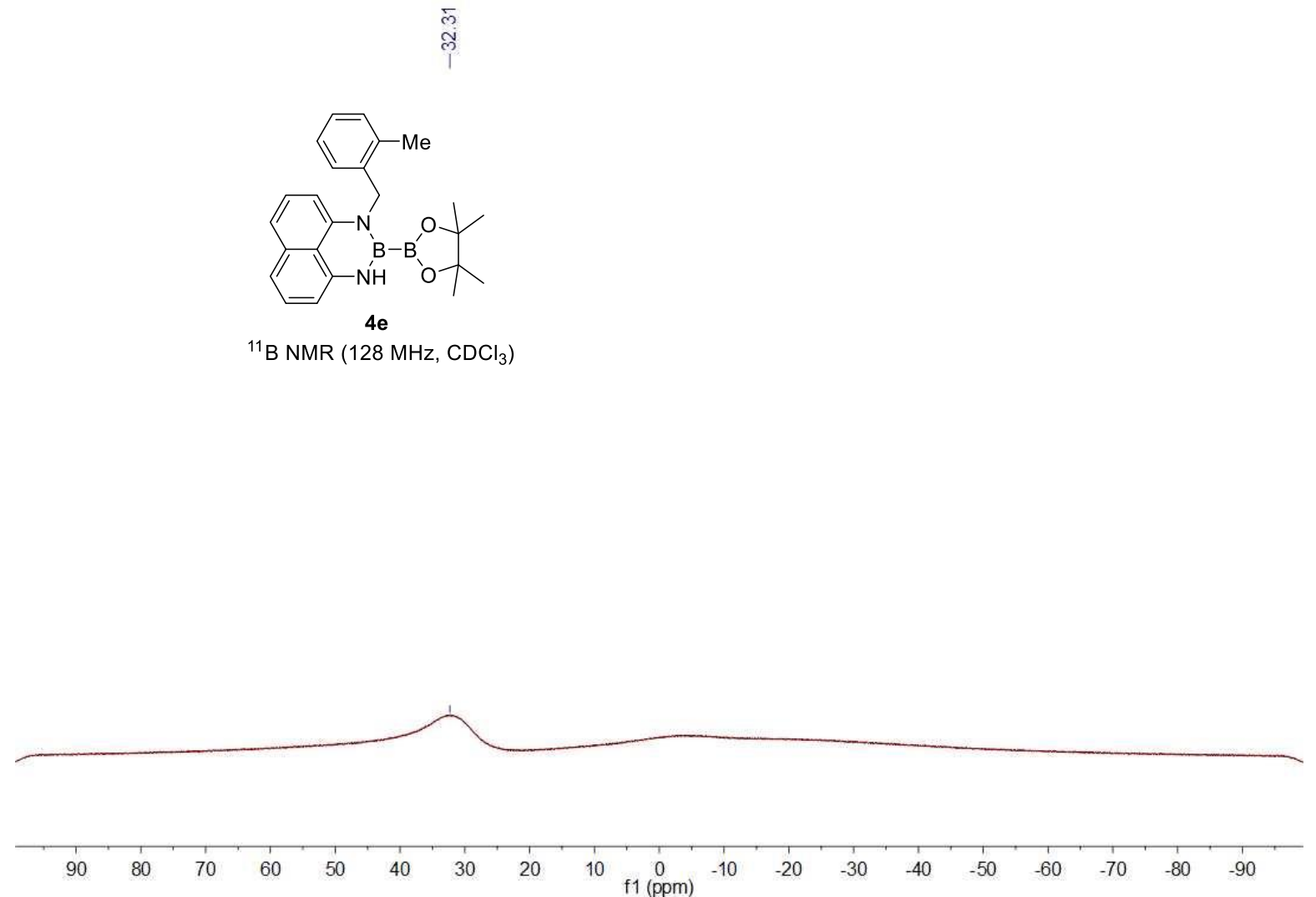

1-(3-methylbenzyl)-2-(4,4,5,5-tetramethyl-1,3,2-dioxaborolan-2-yl)-2,3-dihydro-1H-naphtho[1,8-de][1,3, 2]diazaborinine (4f)

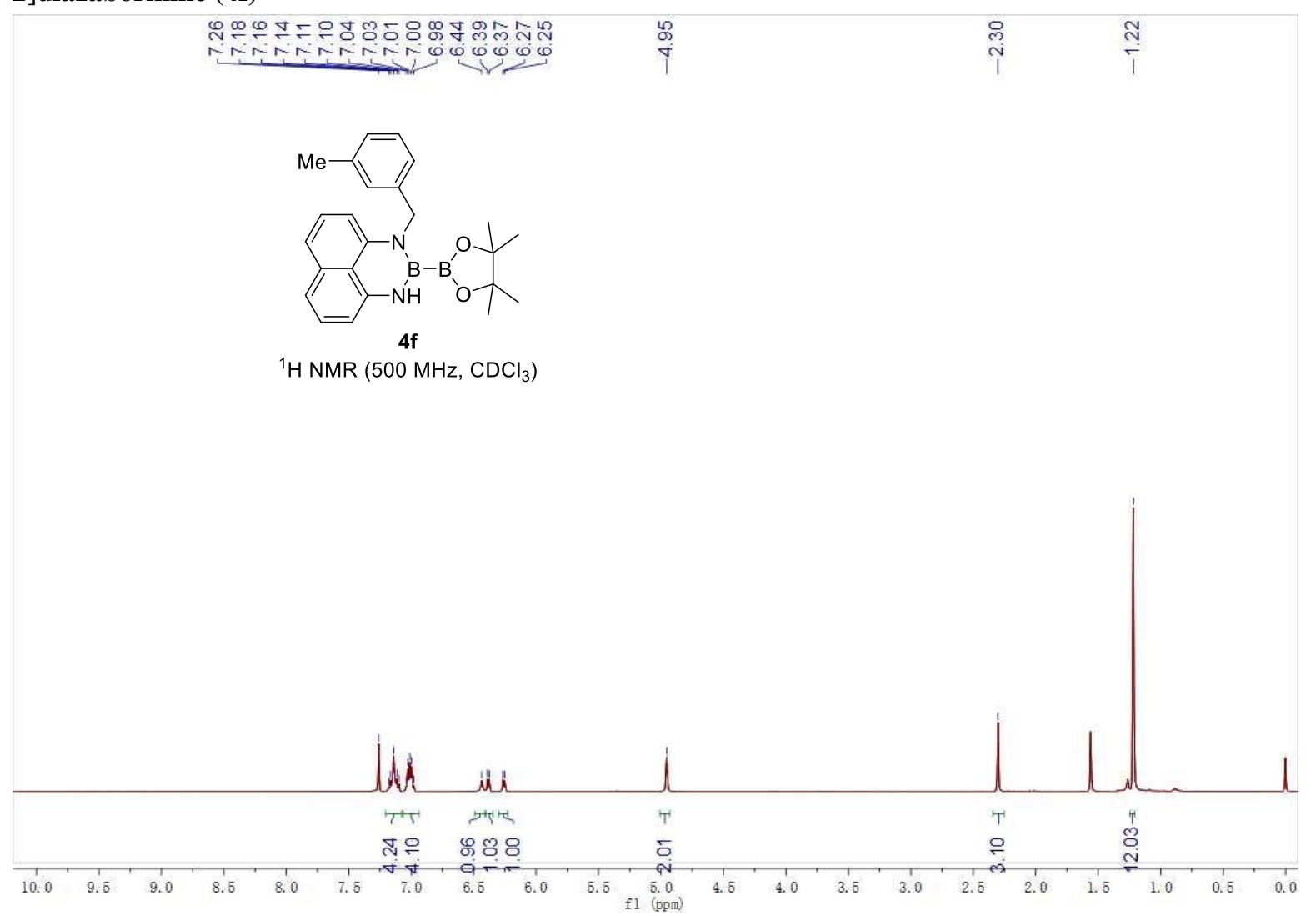


1-(3-methylbenzyl)-2-(4,4,5,5-tetramethyl-1,3,2-dioxaborolan-2-yl)-2,3-dihydro-1H-naphtho[1,8-de][1,3, 2]diazaborinine (4f)

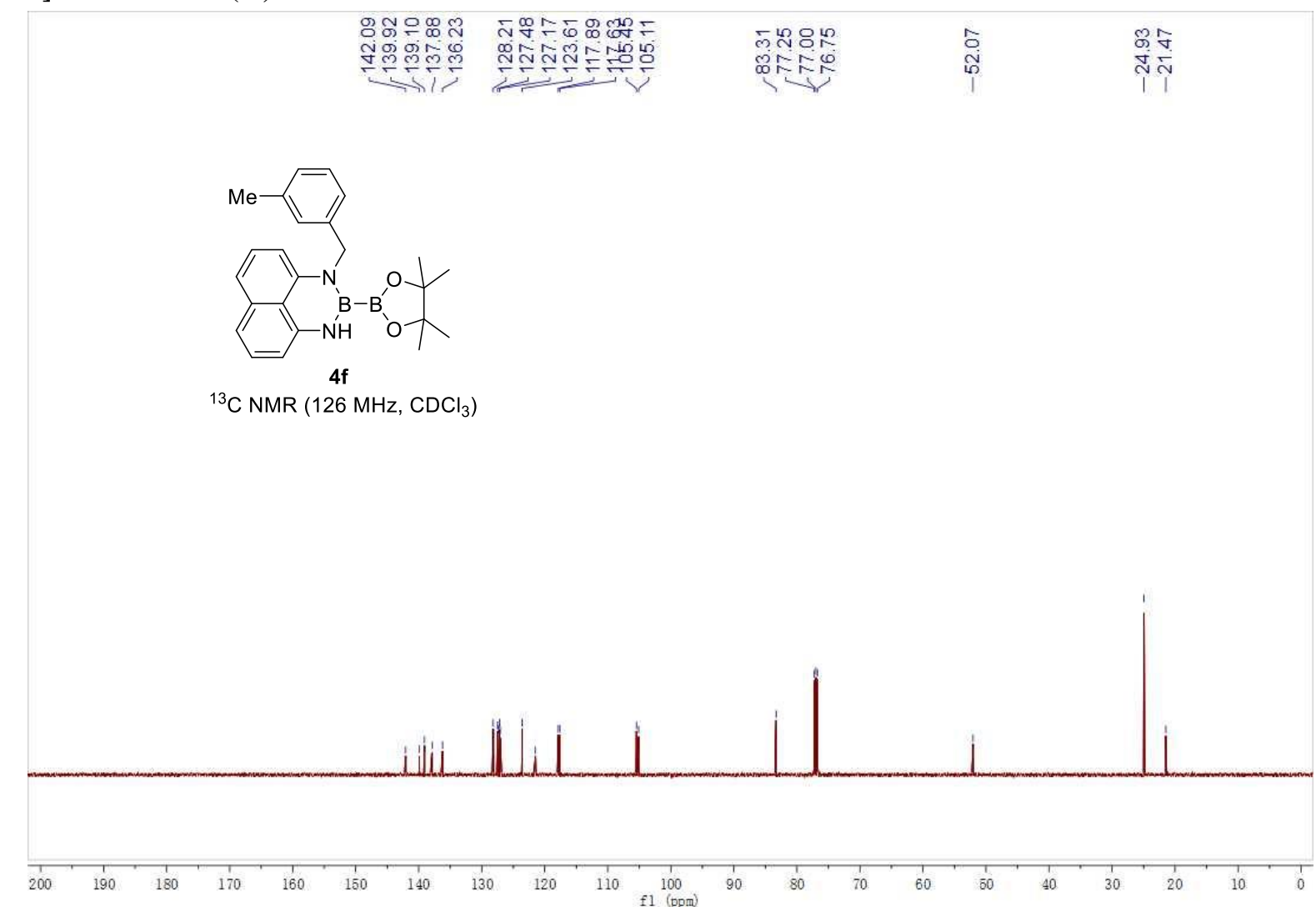

1-(3-methylbenzyl)-2-(4,4,5,5-tetramethyl-1,3,2-dioxaborolan-2-yl)-2,3-dihydro-1H-naphtho[1,8-de][1,3, 2]diazaborinine (4f)
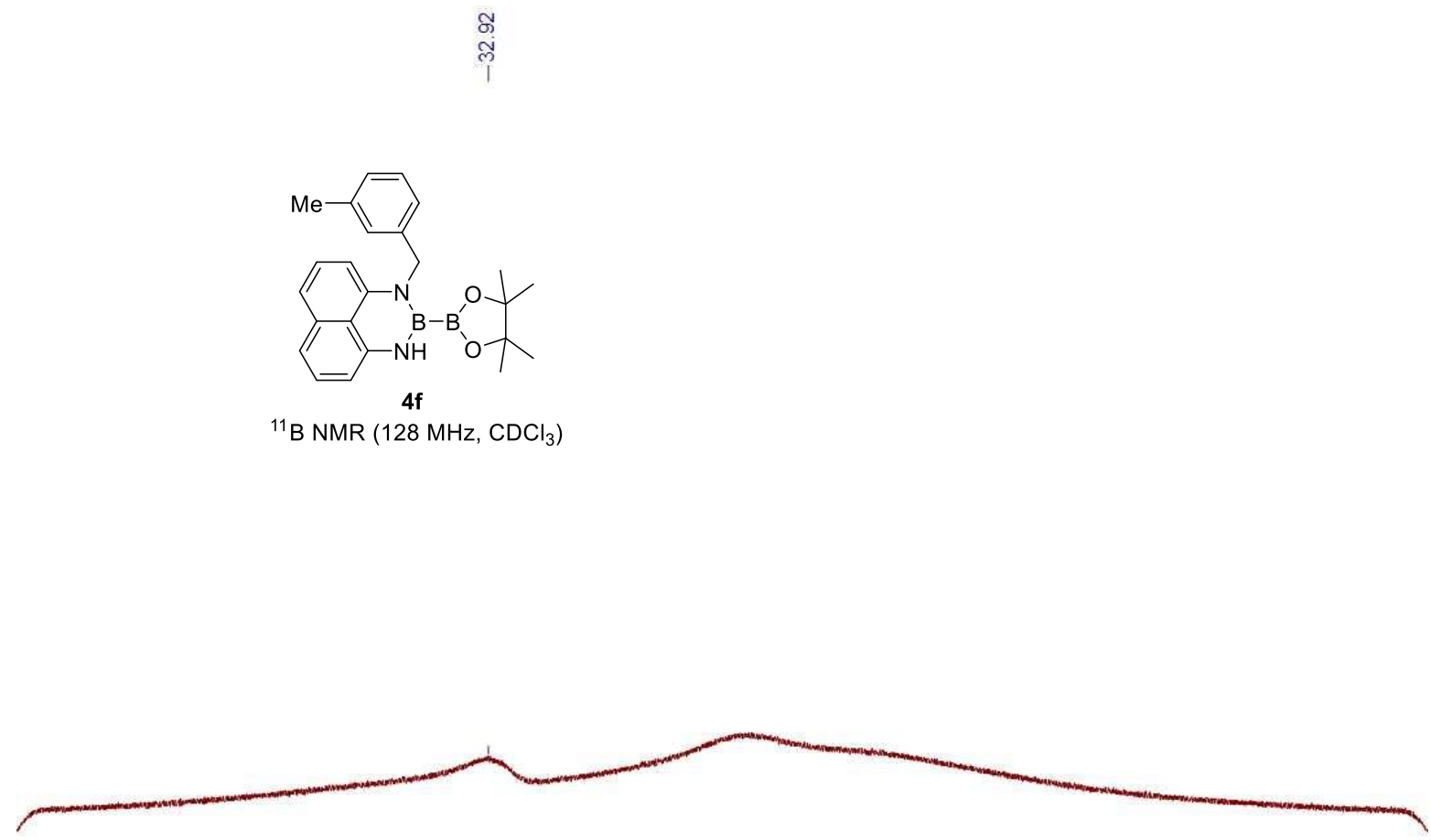

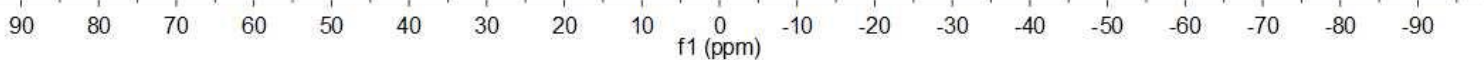


1-(4-methylbenzyl)-2-(4,4,5,5-tetramethyl-1,3,2-dioxaborolan-2-yl)-2,3-dihydro-1H-naphtho[1,8-de][1,3, 2]diazaborinine $(4 \mathrm{~g})$

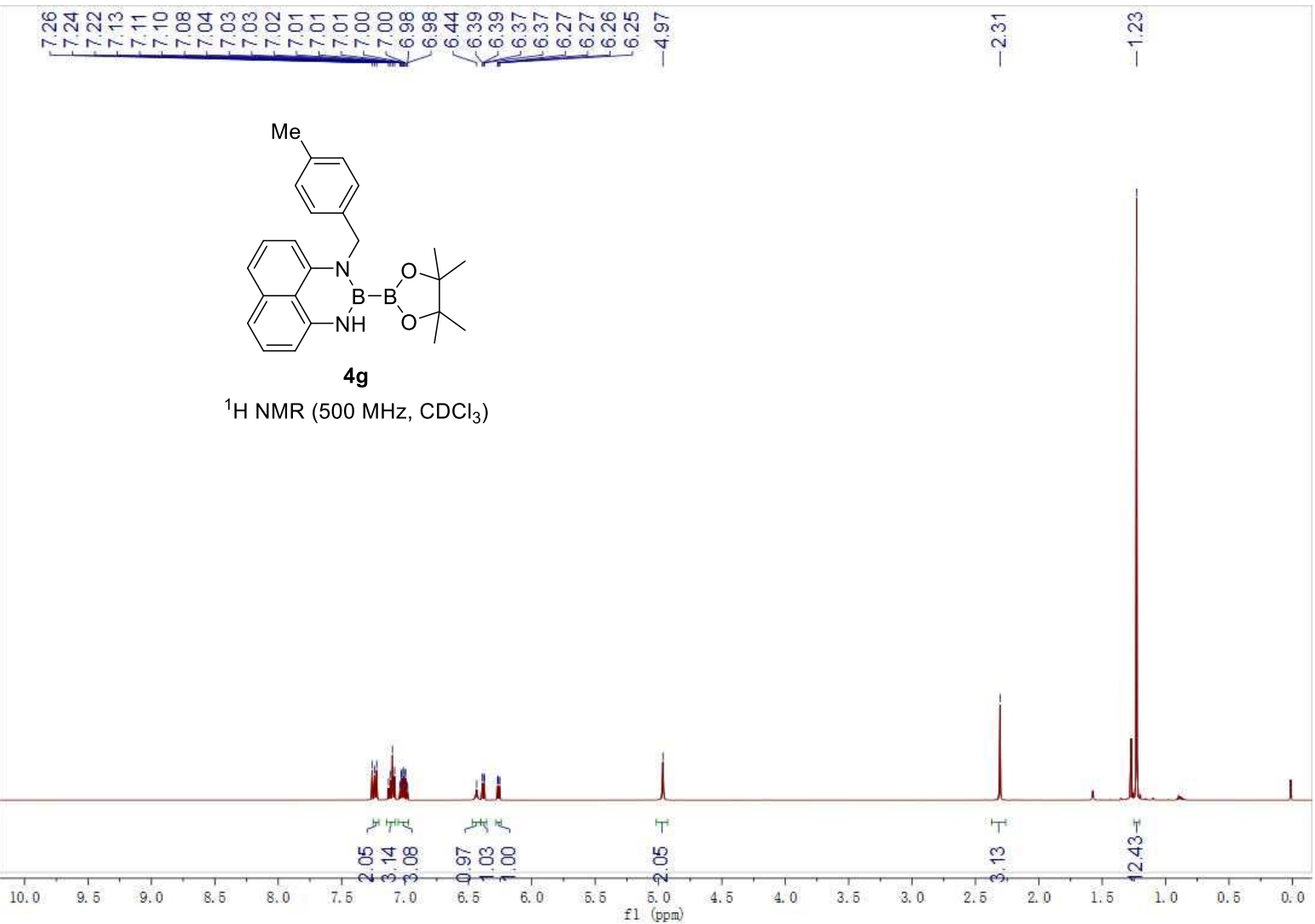

1-(4-methylbenzyl)-2-(4,4,5,5-tetramethyl-1,3,2-dioxaborolan-2-yl)-2,3-dihydro-1H-naphtho[1,8-de][1,3, 2]diazaborinine (4g)

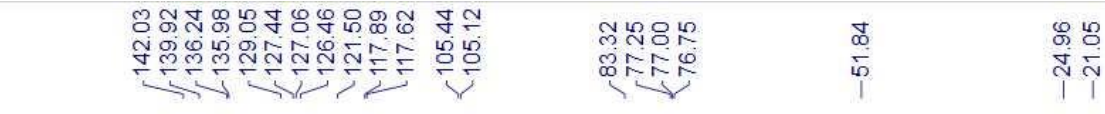

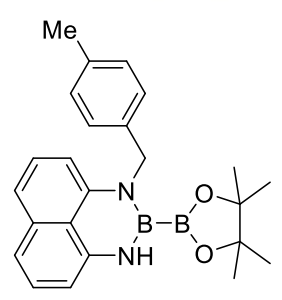

$4 \mathrm{~g}$

${ }^{13} \mathrm{C}$ NMR $\left(126 \mathrm{MHz}, \mathrm{CDCl}_{3}\right)$

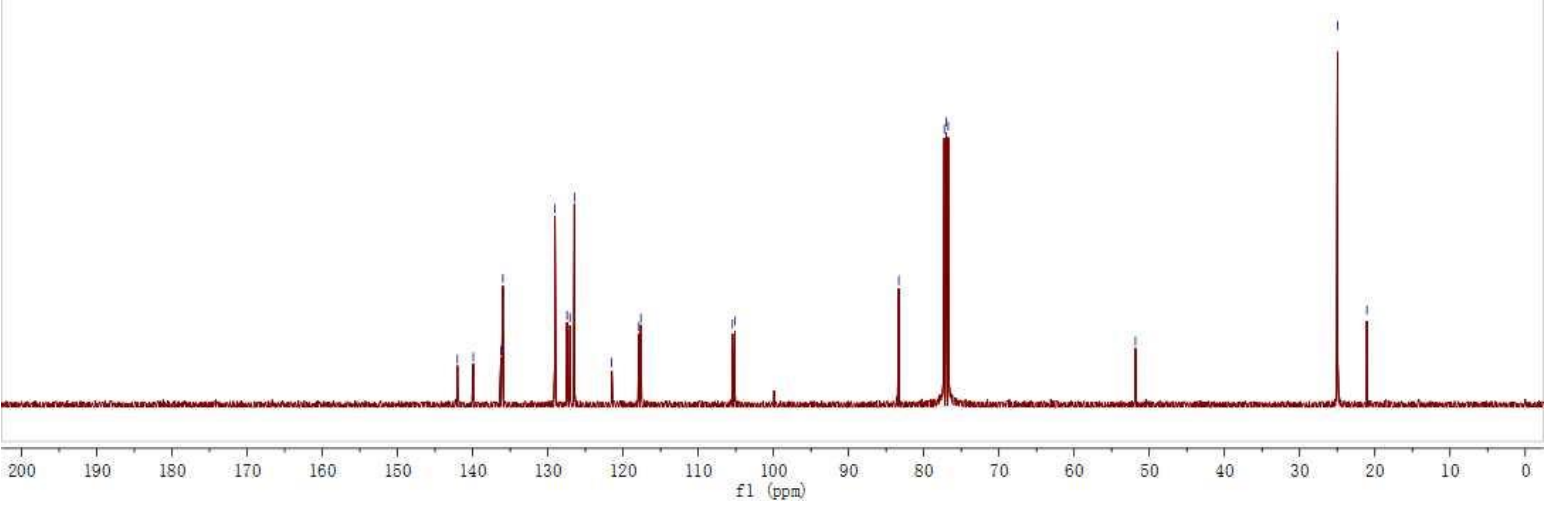


1-(4-methylbenzyl)-2-(4,4,5,5-tetramethyl-1,3,2-dioxaborolan-2-yl)-2,3-dihydro-1H-naphtho[1,8-de][1,3, 2]diazaborinine (4g)

ल

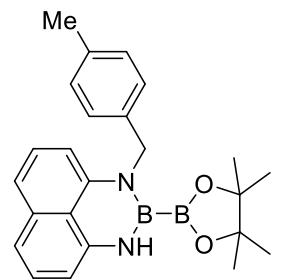

$\mathbf{4 g}$

${ }^{1} \mathrm{H}$ NMR $\left(500 \mathrm{MHz}, \mathrm{CDCl}_{3}\right)$

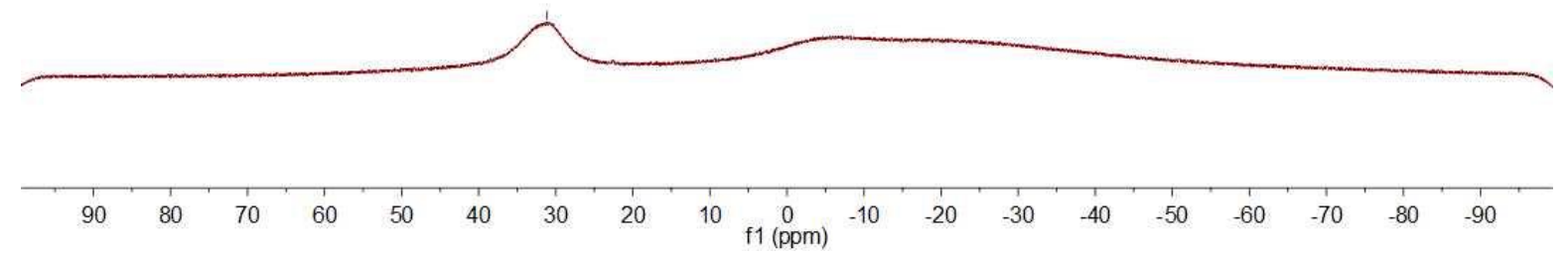

1-(4-fluorobenzyl)-2-(4,4,5,5-tetramethyl-1,3,2-dioxaborolan-2-yl)-2,3-dihydro-1H-naphtho[1,8-de][1,3,2 ]diazaborinine (4h)

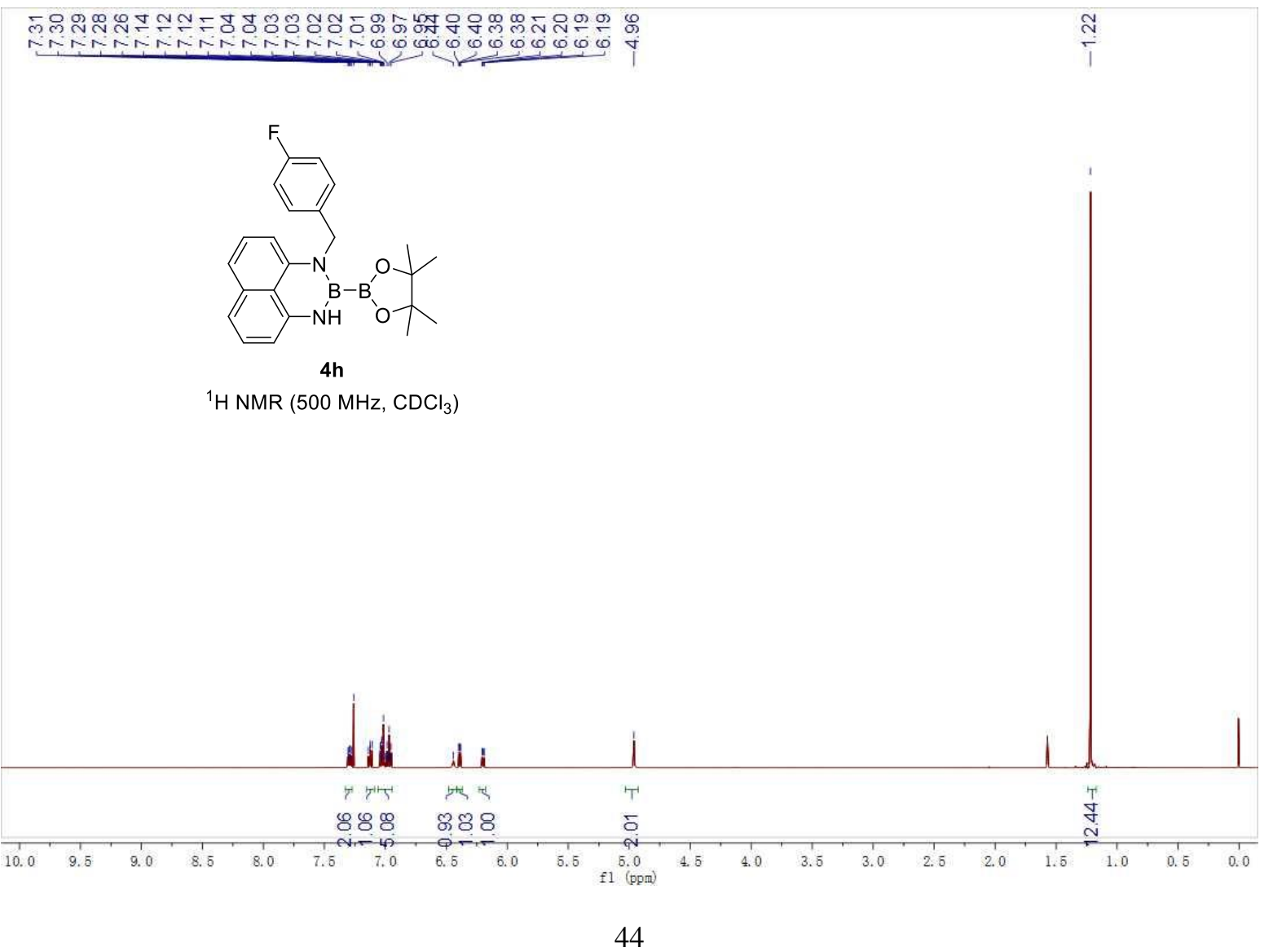


1-(4-fluorobenzyl)-2-(4,4,5,5-tetramethyl-1,3,2-dioxaborolan-2-yl)-2,3-dihydro-1H-naphtho[1,8-de][1,3,2 ddiazaborinine (4h)

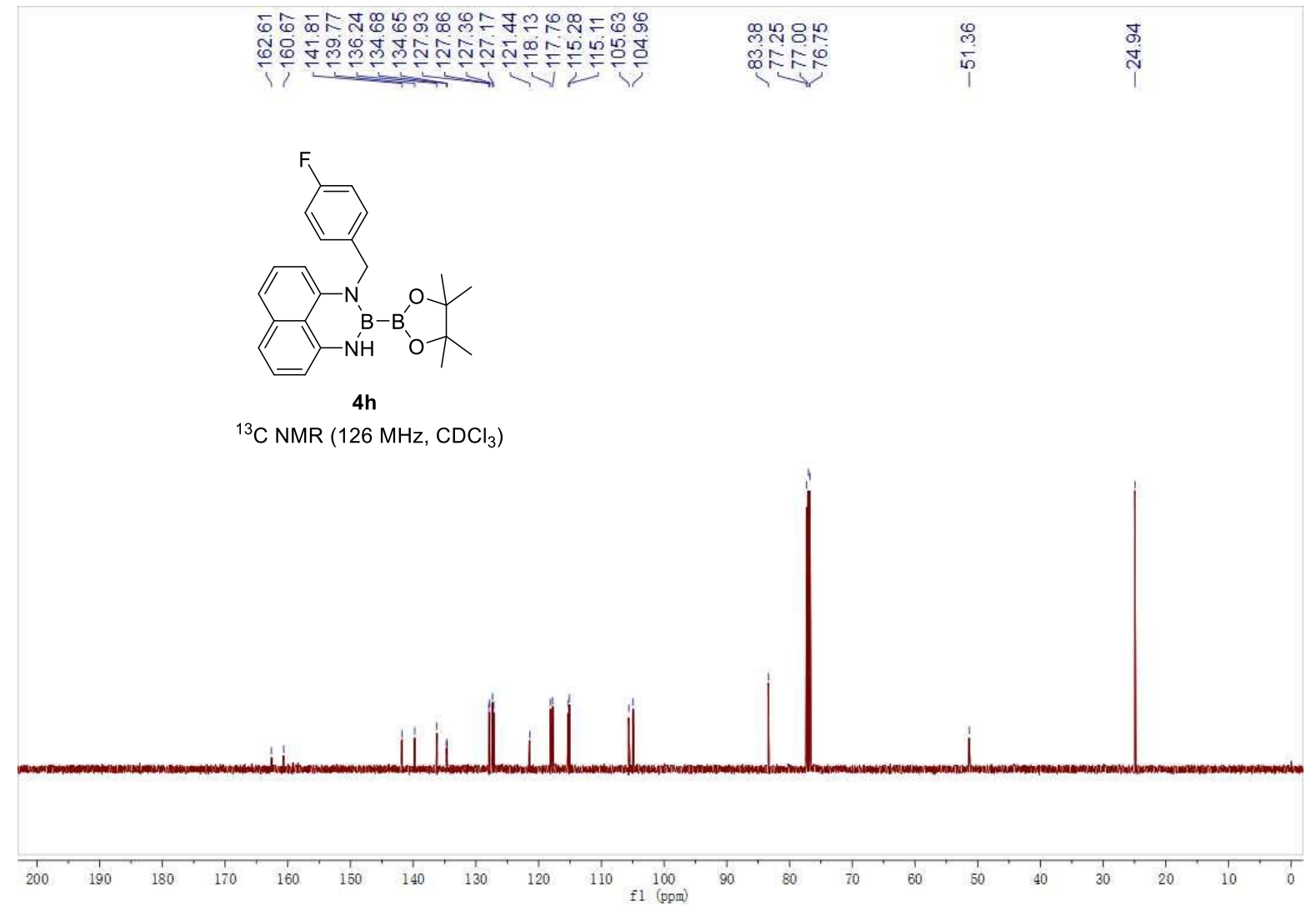

1-(4-fluorobenzyl)-2-(4,4,5,5-tetramethyl-1,3,2-dioxaborolan-2-yl)-2,3-dihydro-1H-naphtho[1,8-de][1,3,2 ]diazaborinine $(4 \mathrm{~h})$

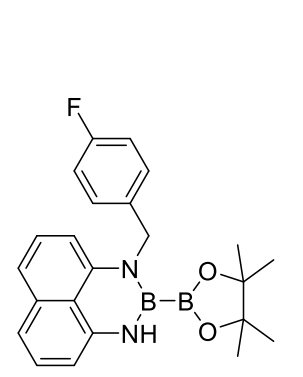

4h

${ }^{11} \mathrm{~B}$ NMR $\left(128 \mathrm{MHz}, \mathrm{CDCl}_{3}\right)$

$\begin{array}{lllllllllllllllllll}90 & 80 & 70 & 60 & 50 & 40 & 30 & 20 & 10 & 0 & -10 & -20 & -30 & -40 & -50 & -60 & -70 & -80 & -90\end{array}$


1-(4-fluorobenzyl)-2-(4,4,5,5-tetramethyl-1,3,2-dioxaborolan-2-yl)-2,3-dihydro-1H-naphtho[1,8-de][1,3,2 ]diazaborinine $(4 \mathrm{~h})$

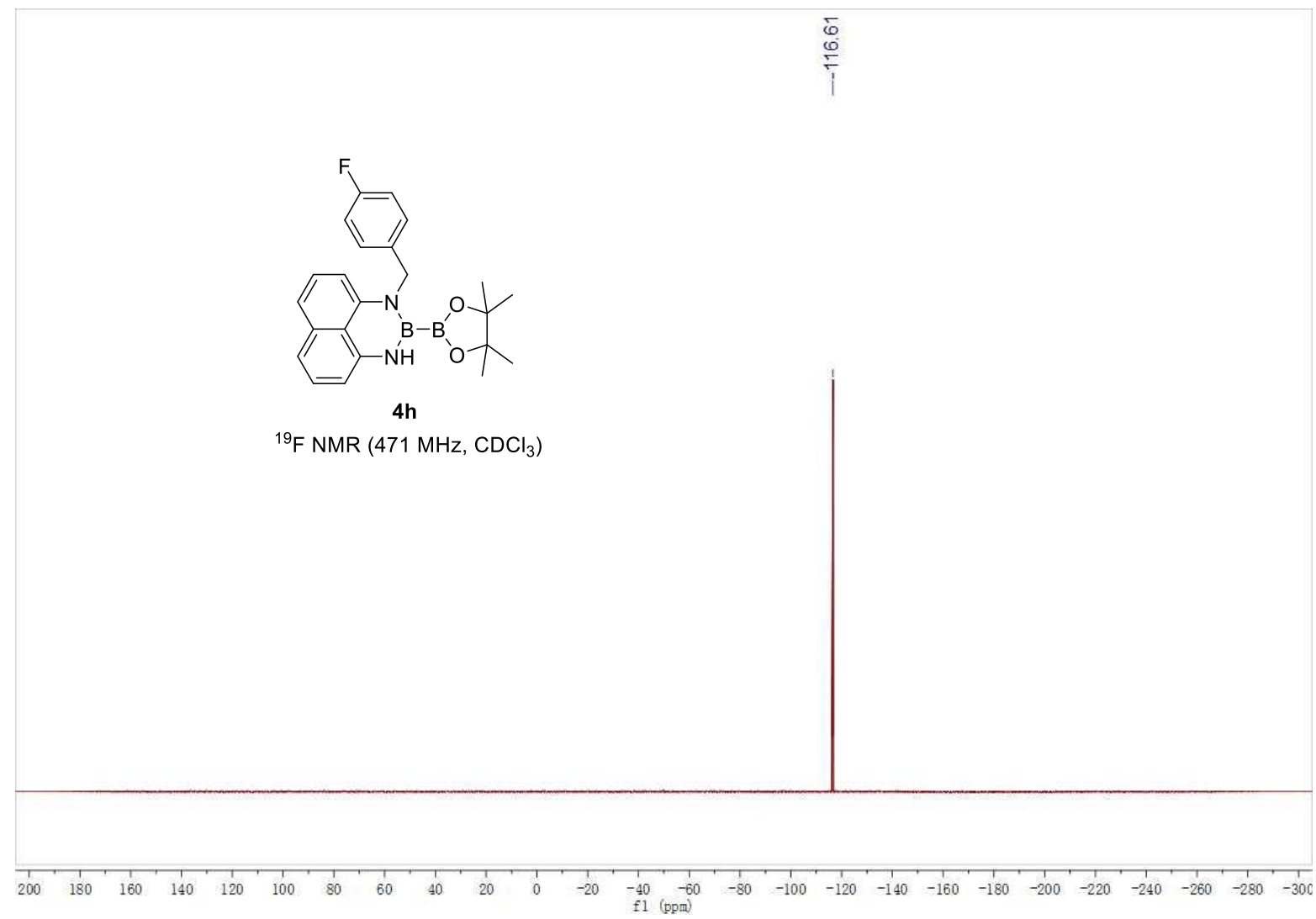

2-(4,4,5,5-tetramethyl-1,3,2-dioxaborolan-2-yl)-1-(thiophen-3-ylmethyl)-2,3-dihydro-1H-naphtho[1,8-de ][1,3,2] diazaborinine (4i)

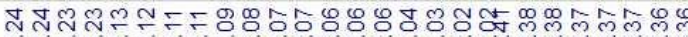

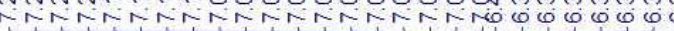

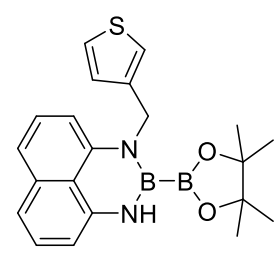

$4 i$

${ }^{1} \mathrm{H}$ NMR $\left(500 \mathrm{MHz}, \mathrm{CDCl}_{3}\right)$

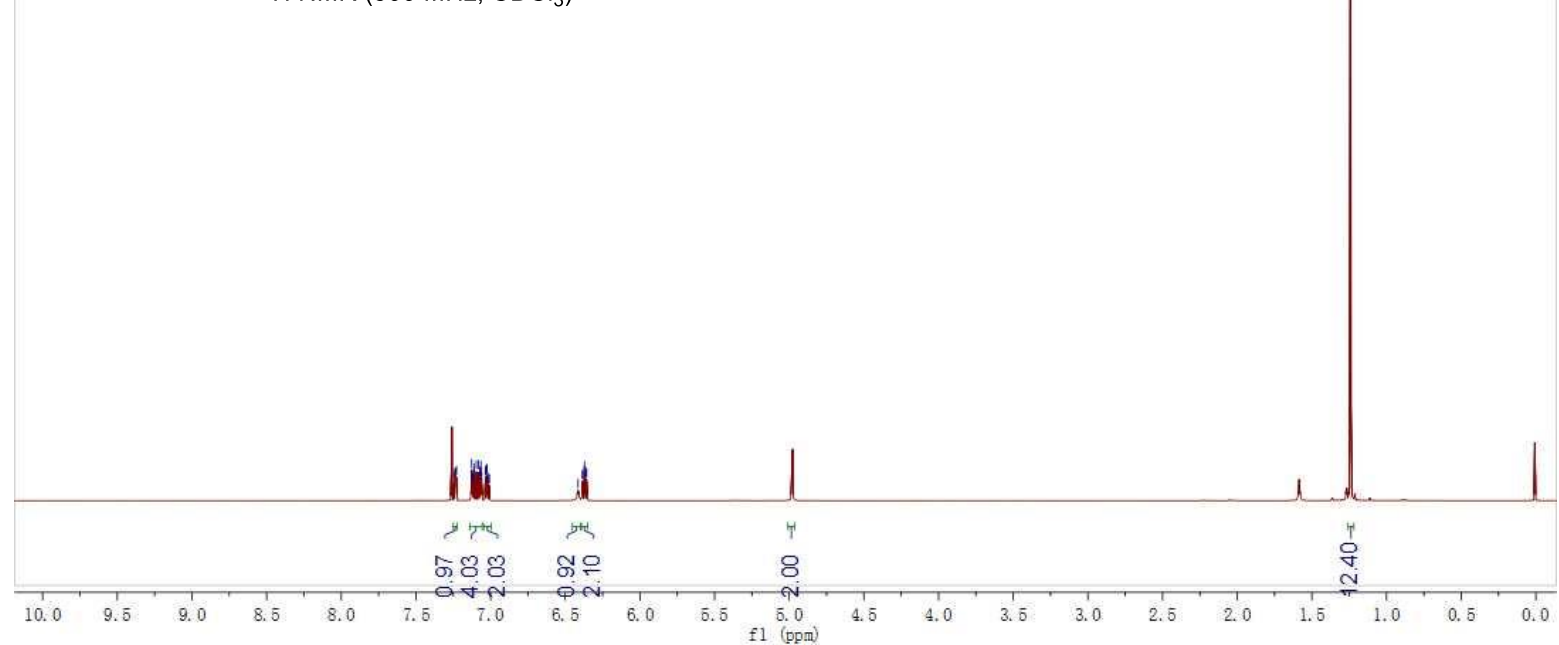


2-(4,4,5,5-tetramethyl-1,3,2-dioxaborolan-2-yl)-1-(thiophen-3-ylmethyl)-2,3-dihydro-1H-naphtho[1,8-de ]$[1,3,2]$ diazaborinine (4i)

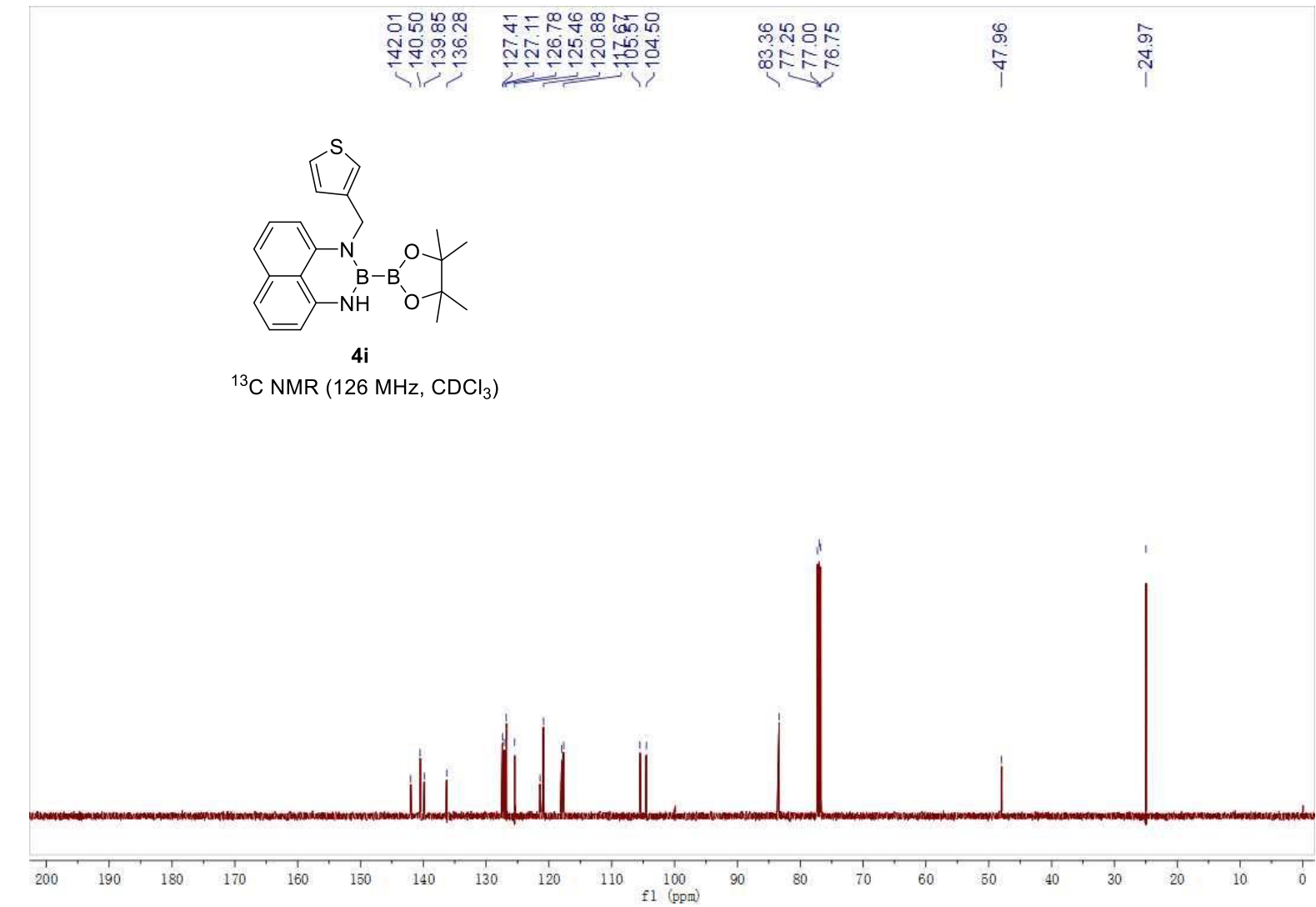

2-(4,4,5,5-tetramethyl-1,3,2-dioxaborolan-2-yl)-1-(thiophen-3-ylmethyl)-2,3-dihydro-1H-naphtho[1,8-de ]$[1,3,2]$ diazaborinine $(4 \mathbf{i})$

$\stackrel{8}{\stackrel{8}{\bar{m}}}$

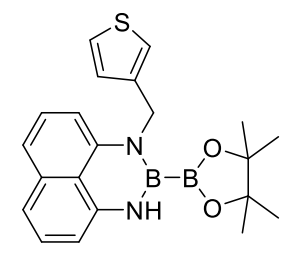

4i

${ }^{11} \mathrm{~B} \mathrm{NMR}\left(128 \mathrm{MHz}, \mathrm{CDCl}_{3}\right)$

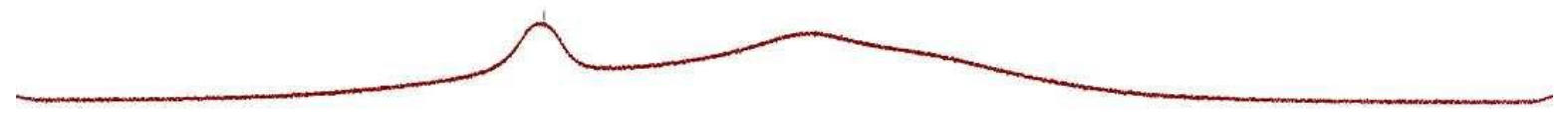

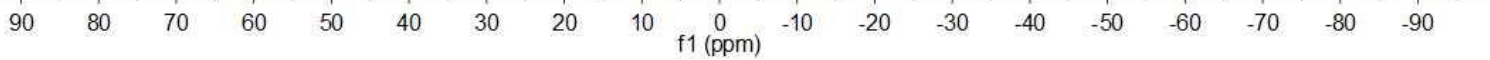


1-(naphthalen-2-ylmethyl)-2-(4,4,5,5-tetramethyl-1,3,2-dioxaborolan-2-yl)-2,3-dihydro-1H-naphtho[1,8de][1,3,2] diazaborinine $(4 \mathbf{j})$

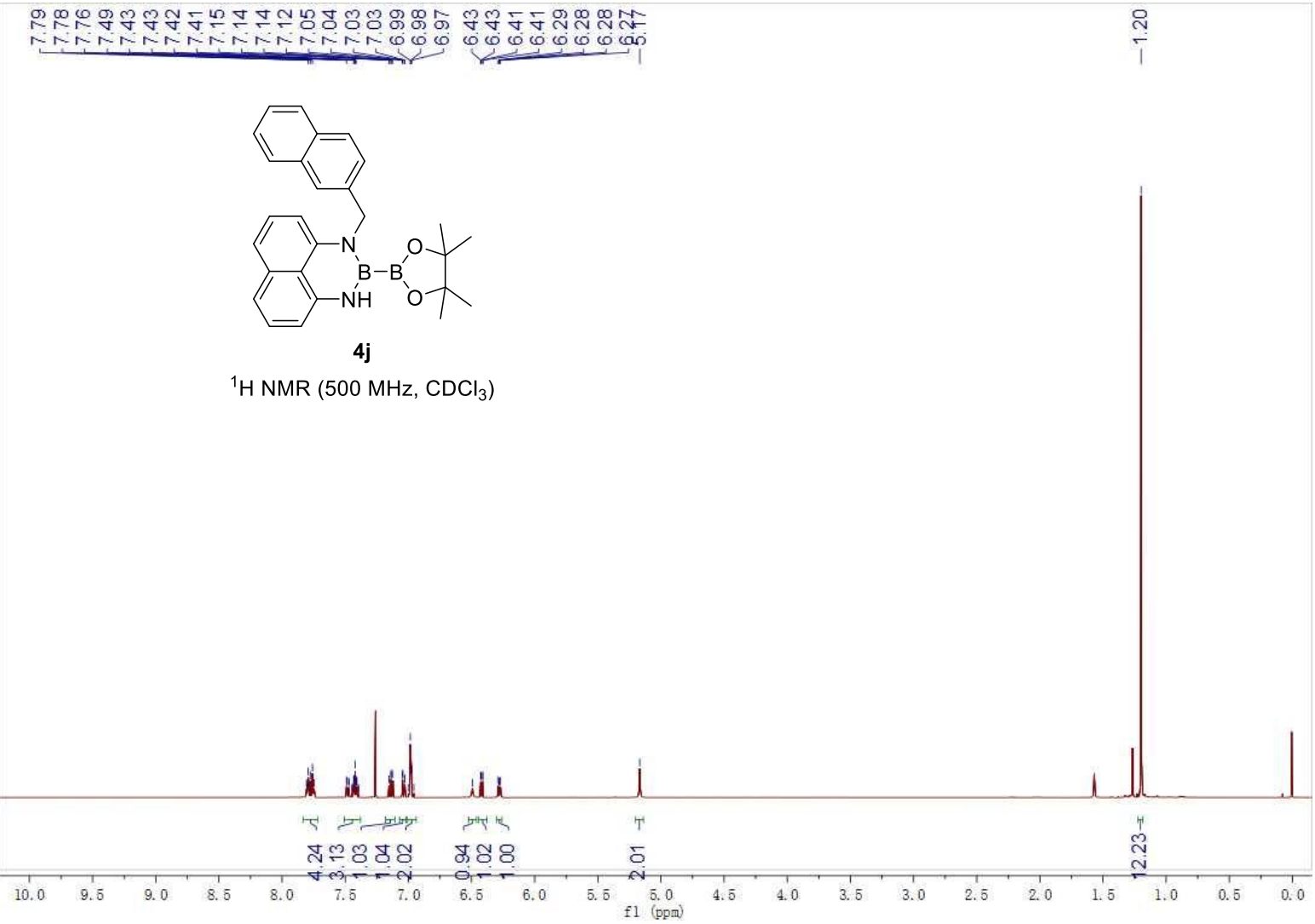

1-(naphthalen-2-ylmethyl)-2-(4,4,5,5-tetramethyl-1,3,2-dioxaborolan-2-yl)-2,3-dihydro-1H-naphtho[1,8de][1,3,2]diazaborinine $(4 \mathrm{j})$

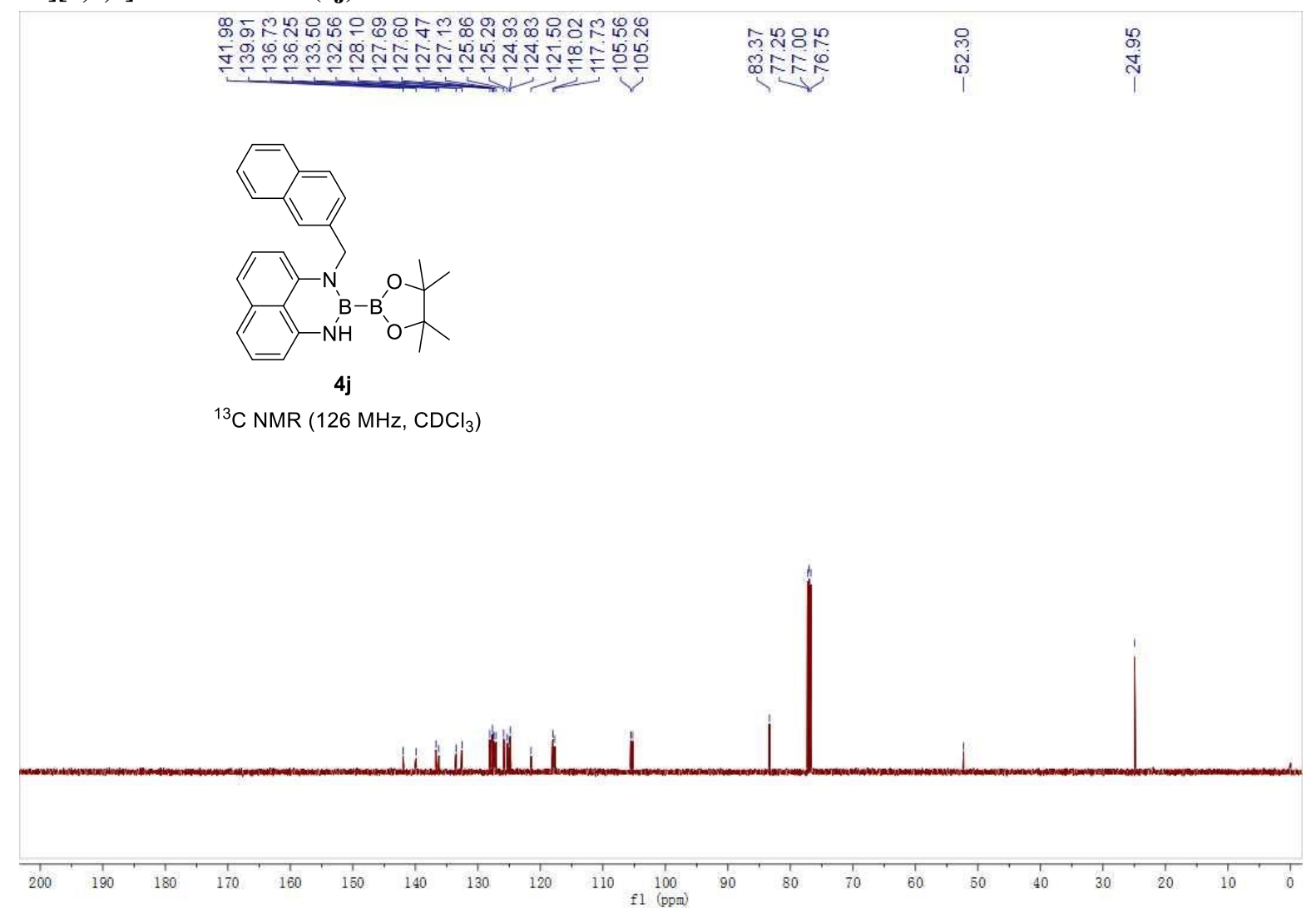


1-(naphthalen-2-ylmethyl)-2-(4,4,5,5-tetramethyl-1,3,2-dioxaborolan-2-yl)-2,3-dihydro-1H-naphtho[1,8de][1,3,2]diazaborinine $(4 \mathbf{j})$

लै

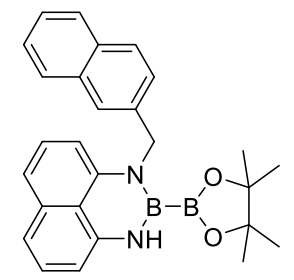

$4 \mathrm{j}$

${ }^{11} \mathrm{~B} N M R\left(128 \mathrm{MHz}, \mathrm{CDCl}_{3}\right)$

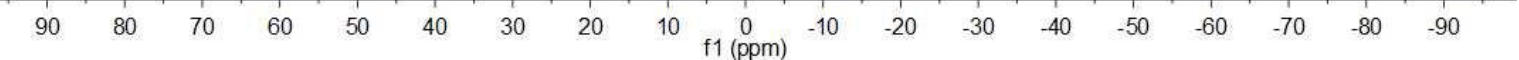

1-Bromo-2-isopropoxynaphthalene (5b)

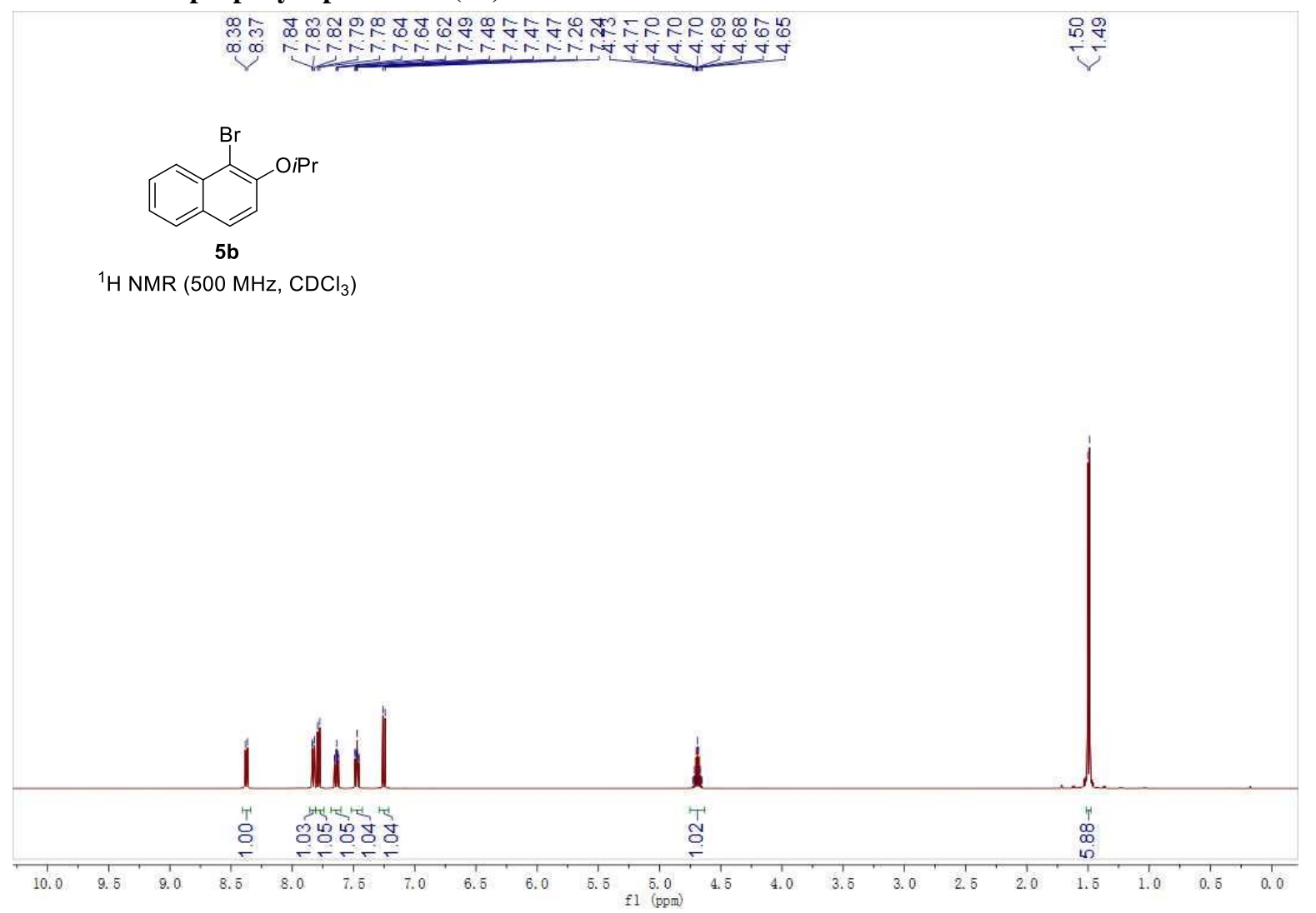


2-(Benzyloxy)-1-bromonaphthalene (5c)

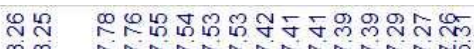

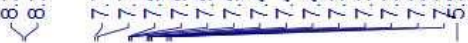

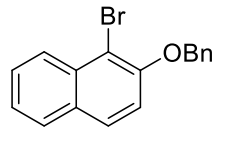

$5 \mathrm{c}$

${ }^{1} \mathrm{H}$ NMR $\left(500 \mathrm{MHz}, \mathrm{CDCl}_{3}\right)$

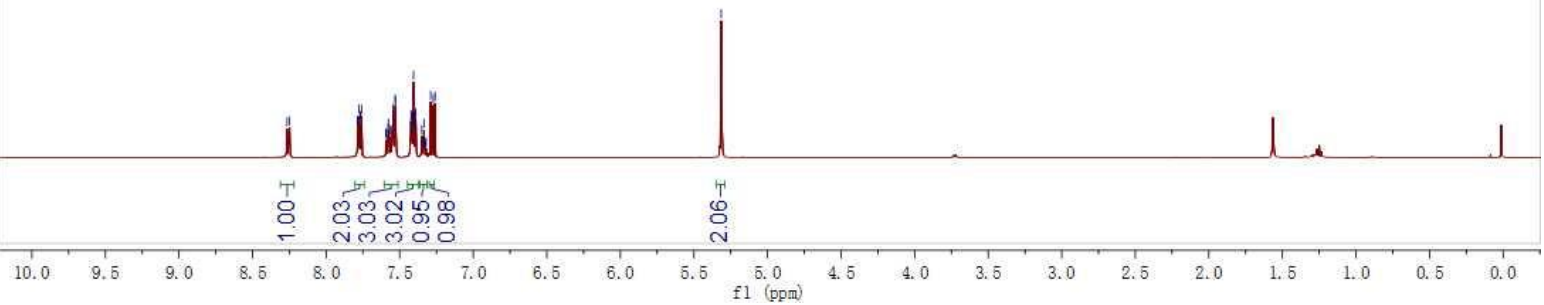

1-bromo-2-(methoxymethoxy)naphthalene (5d)

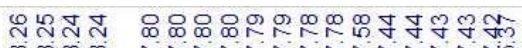

0

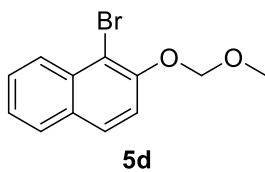

${ }^{1} \mathrm{H}$ NMR $\left(500 \mathrm{MHz}, \mathrm{CDCl}_{3}\right)$

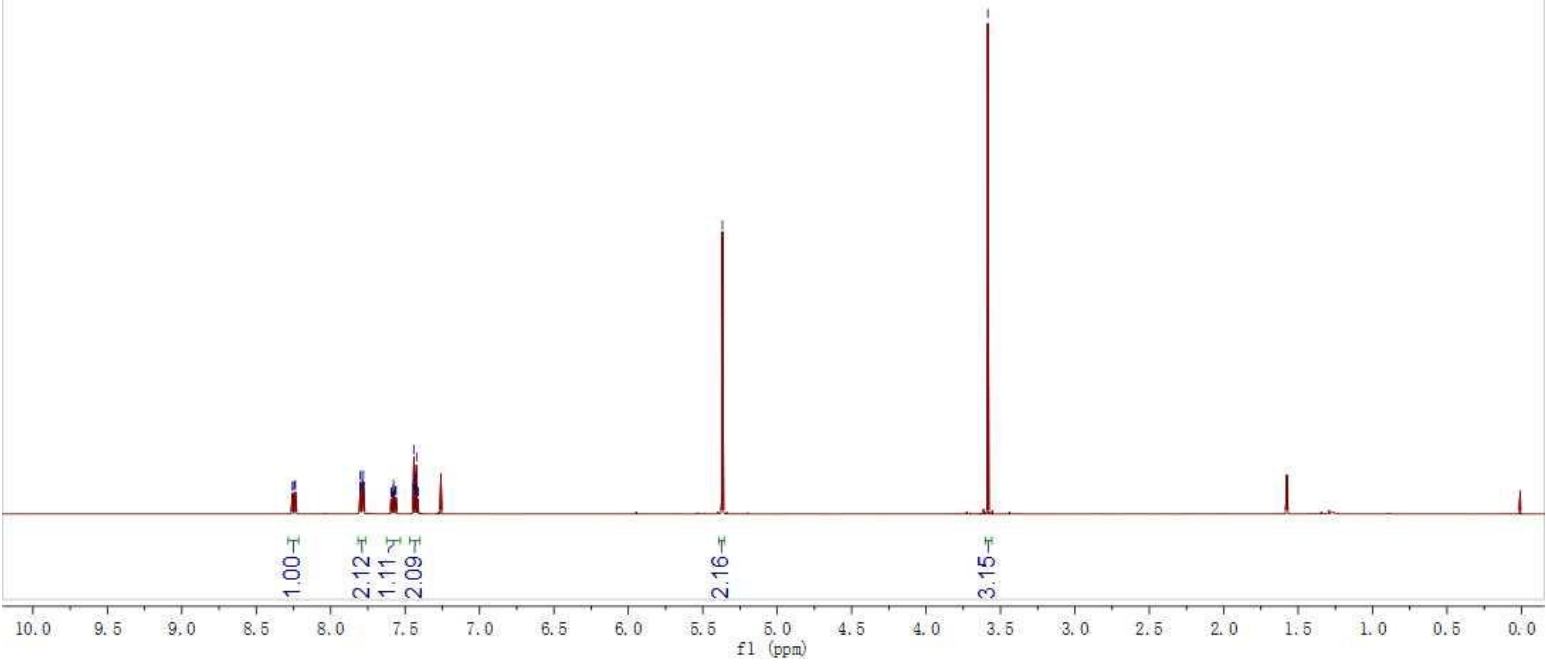


5-bromo-6-methoxy-2-naphthaldehyde (5e)

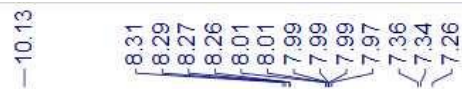

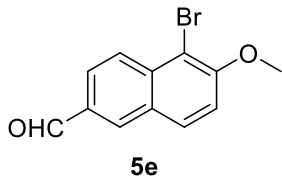

${ }^{1} \mathrm{H} \mathrm{NMR}\left(500 \mathrm{MHz}, \mathrm{CDCl}_{3}\right)$

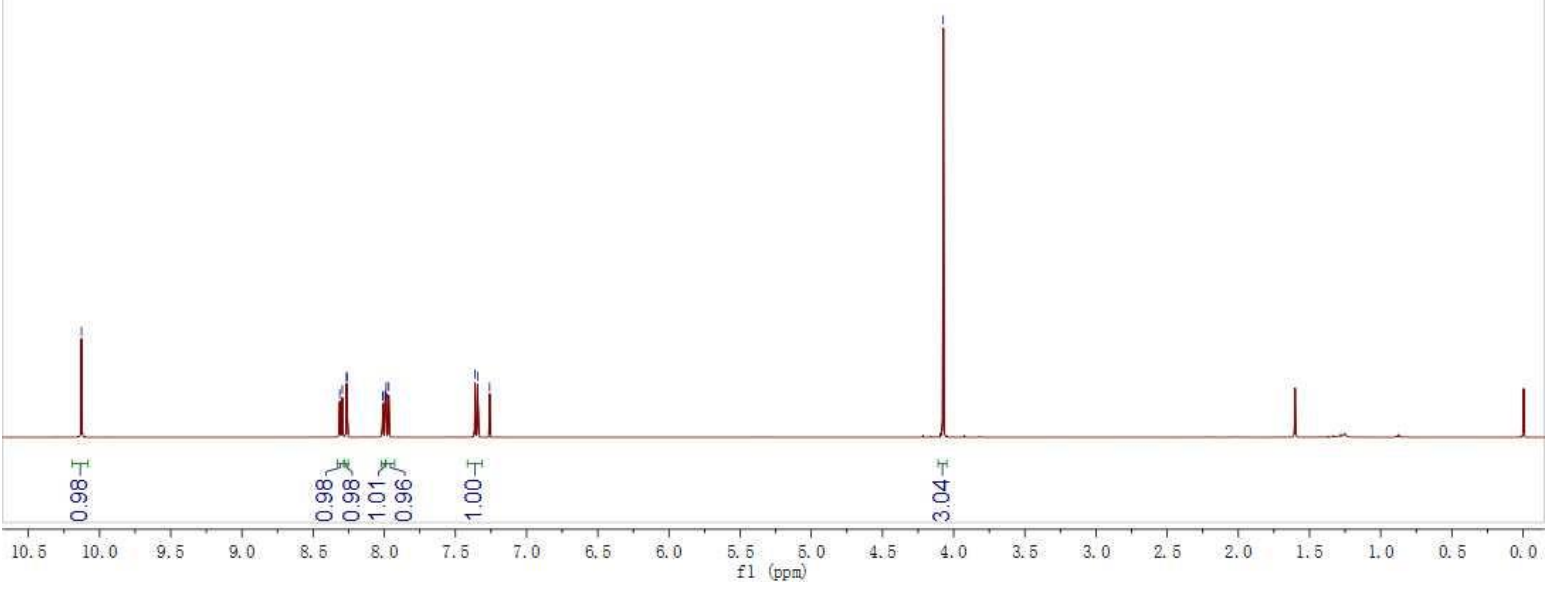

5-Bromo-6-methoxy-2-naphthonitrile (5f)

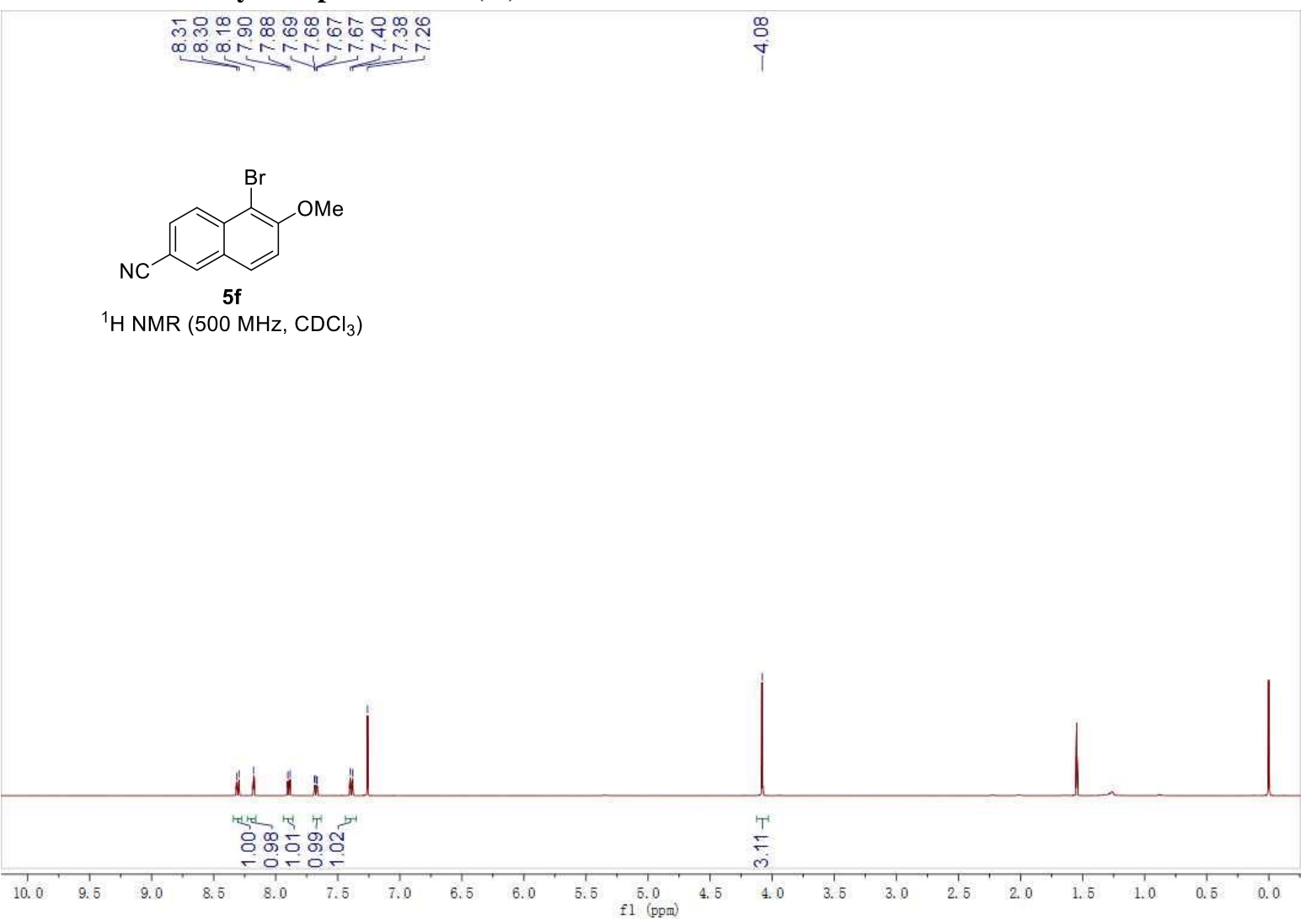


1-(5-Bromo-6-methoxynaphthalen-2-yl)ethan-1-one (5g)

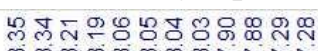

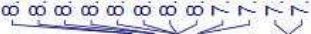

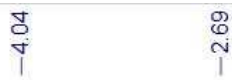

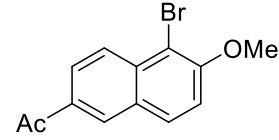

$5 \mathrm{~g}$

${ }^{1} \mathrm{H}$ NMR $\left(500 \mathrm{MHz}, \mathrm{CDCl}_{3}\right)$

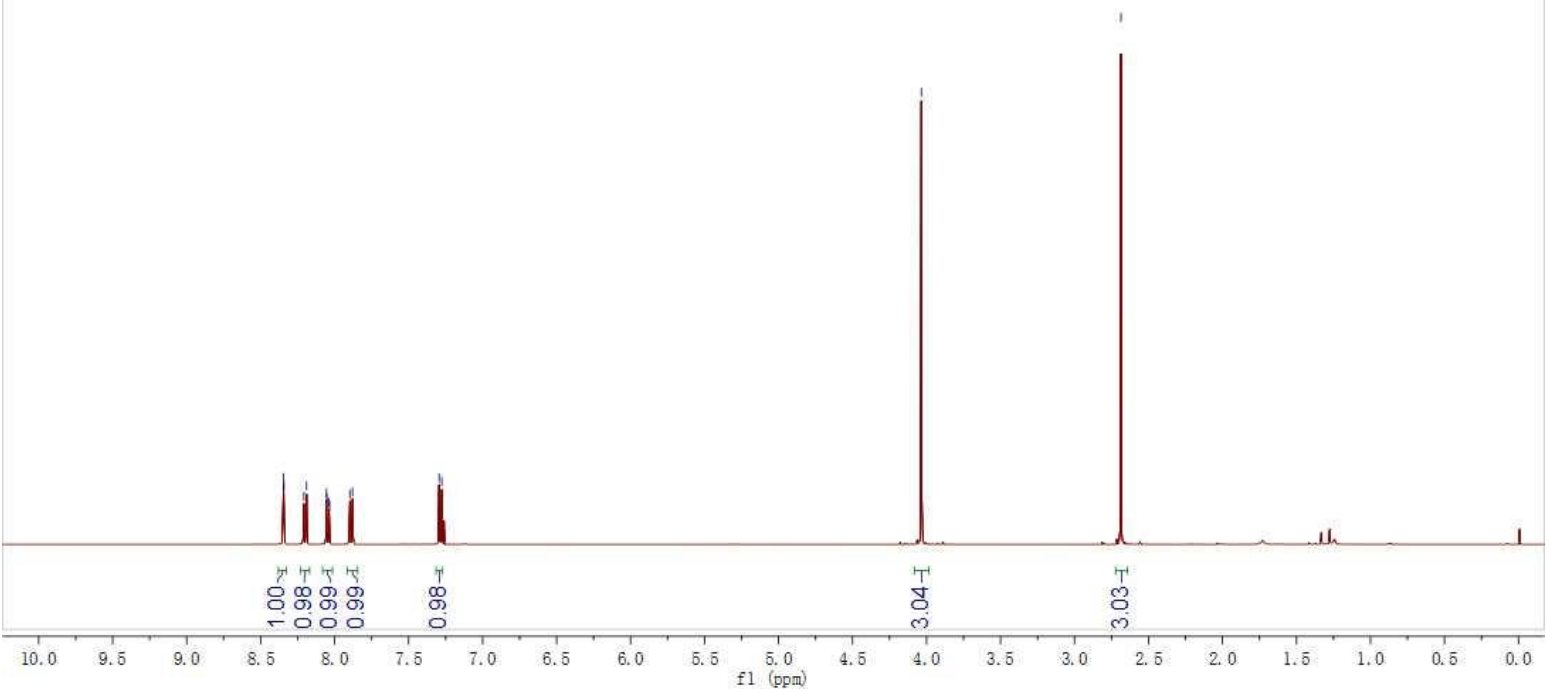

Methyl 5-bromo-6-methoxy-2-naphthoate (5h)

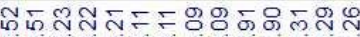

$000000000 N-N N$

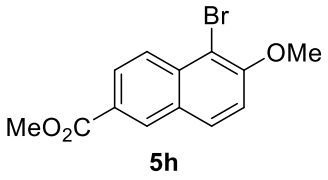

${ }^{1} \mathrm{H}$ NMR $\left(500 \mathrm{MHz}, \mathrm{CDCl}_{3}\right)$

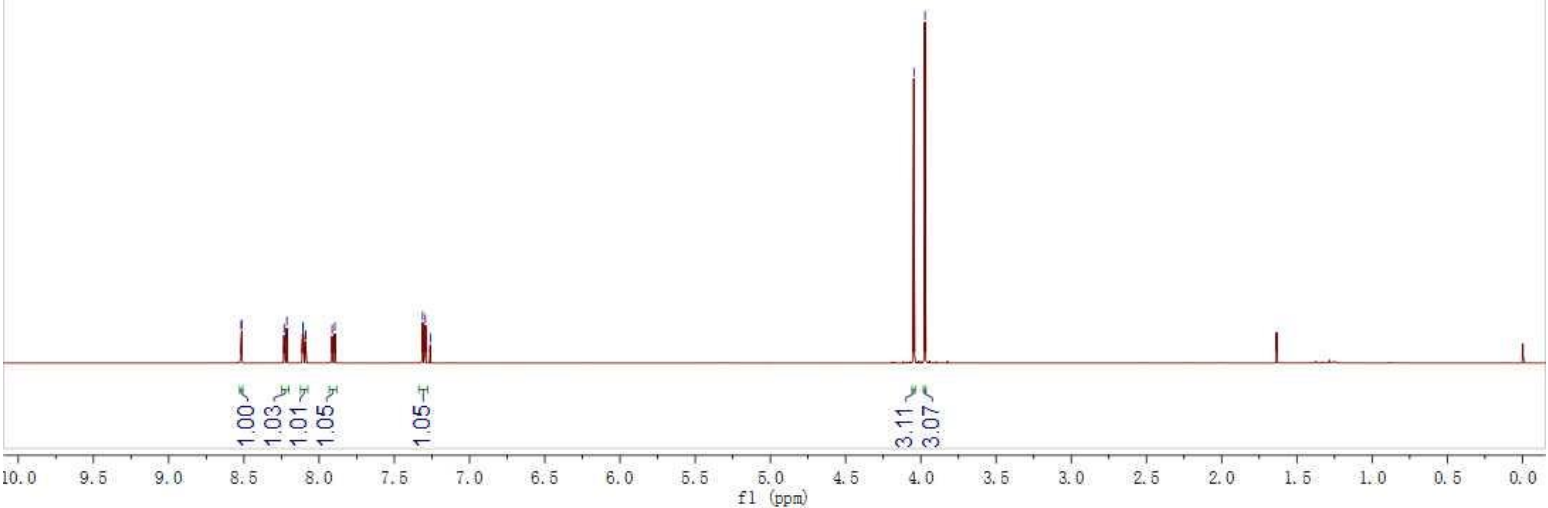


1-Bromo-2-methoxy-6-phenylnaphthalene (5i)

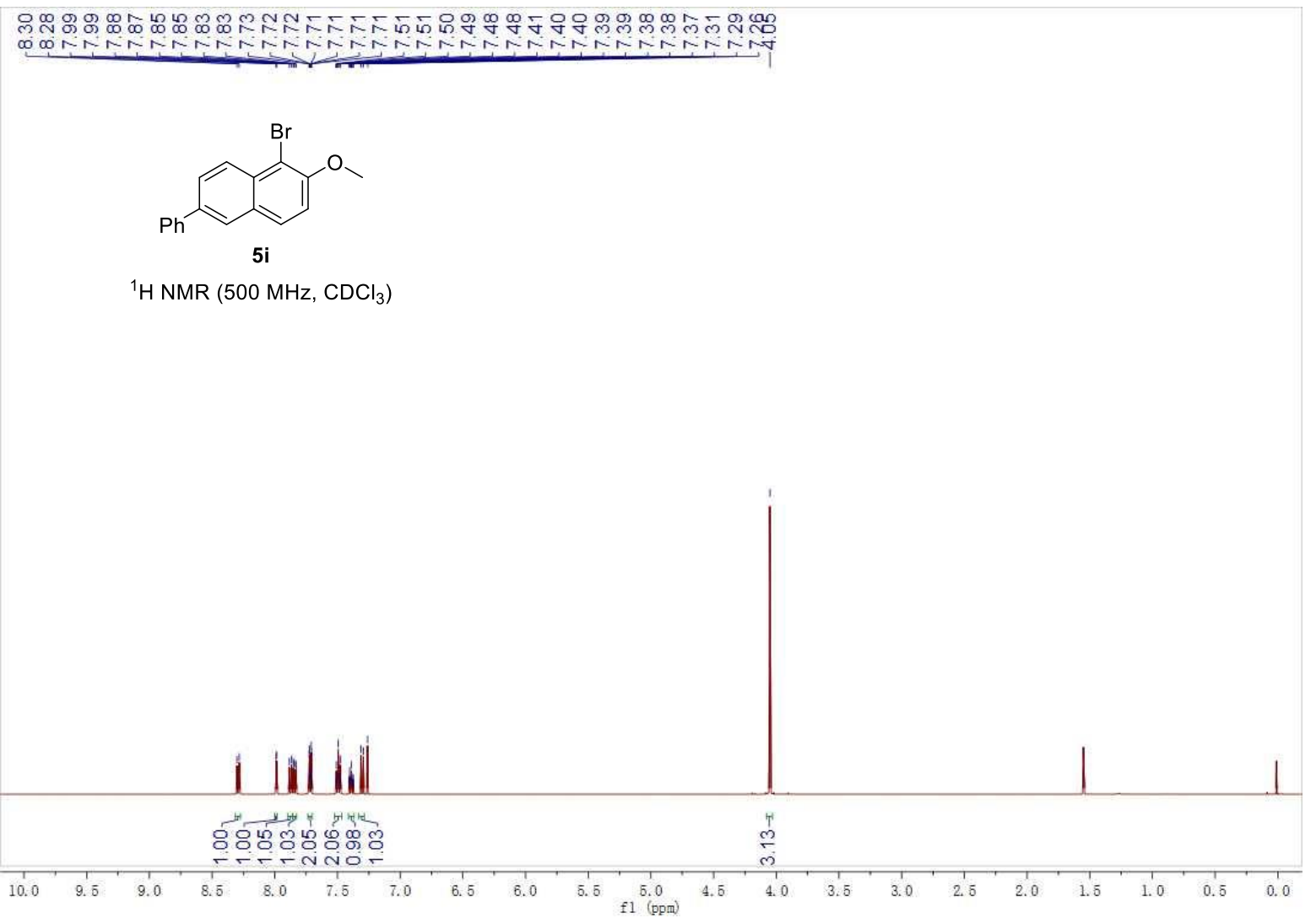

1-Bromo-2-methoxy-6-methylnaphthalene $(5 \mathbf{j})$

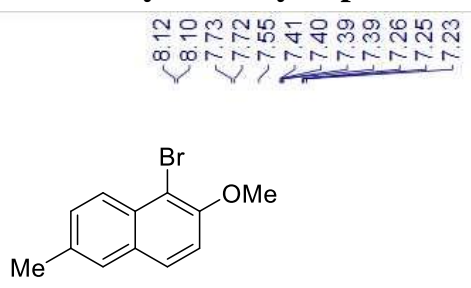

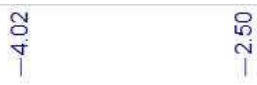

$5 j$

${ }^{1} \mathrm{H}$ NMR $\left(500 \mathrm{MHz}, \mathrm{CDCl}_{3}\right)$

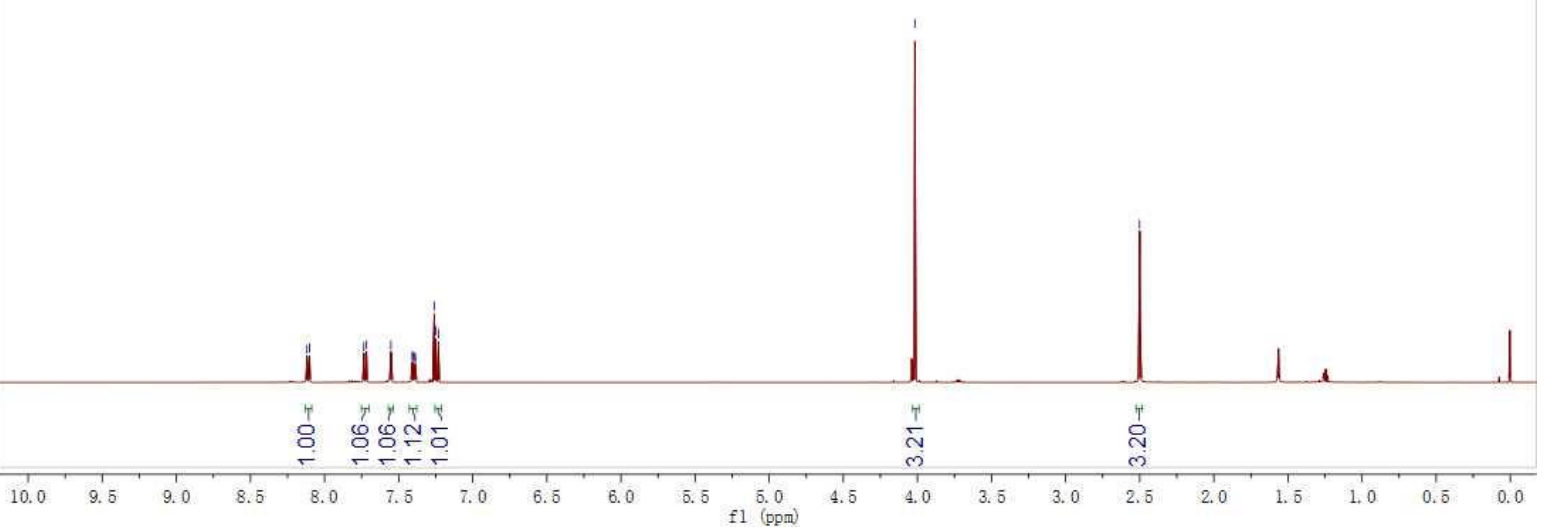


1-Bromo-2,6-dimethoxynaphthalene (5k)

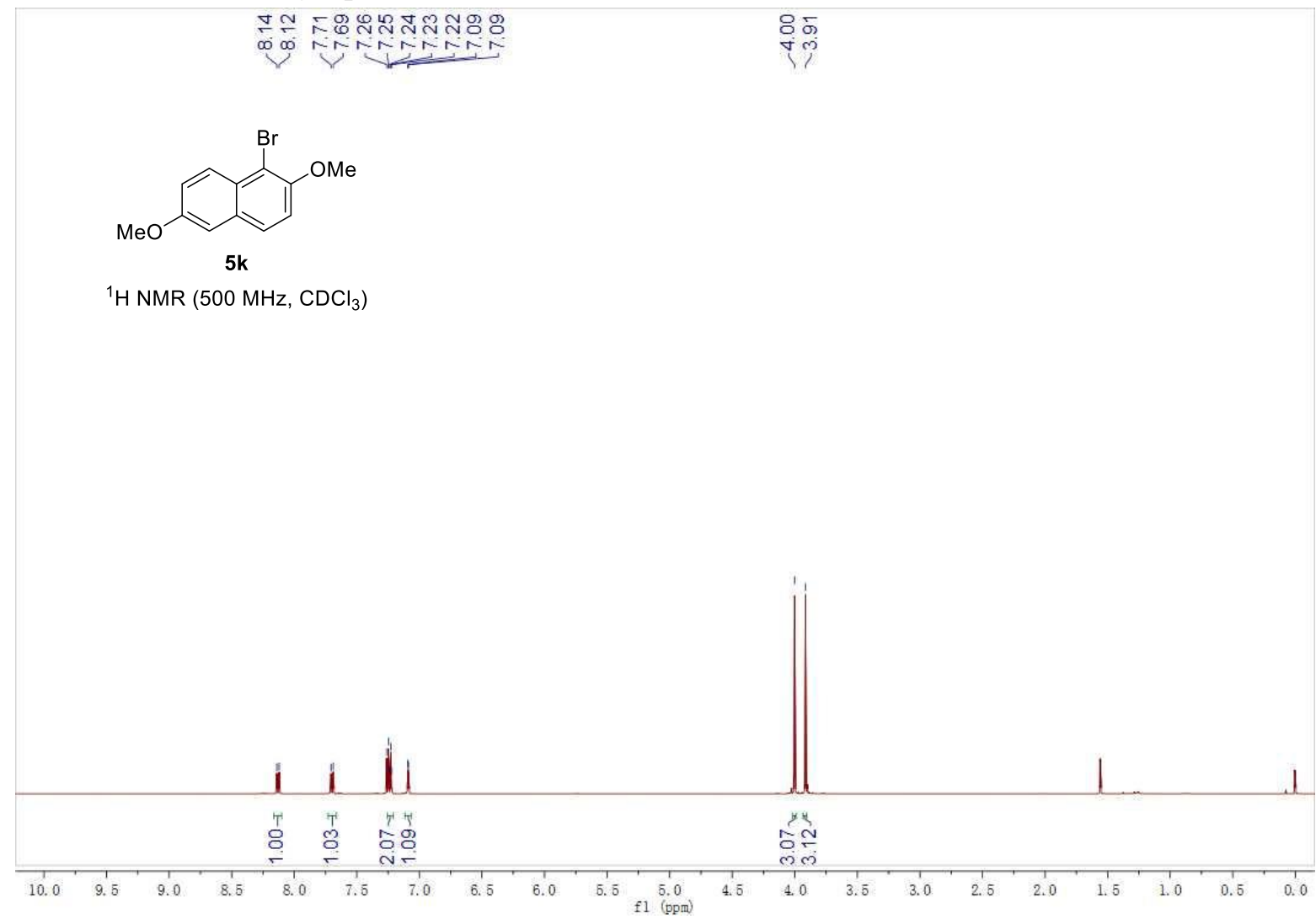

Methyl (S)-2-(5-bromo-6-methoxynaphthalen-2-yl)propanoate (5l)

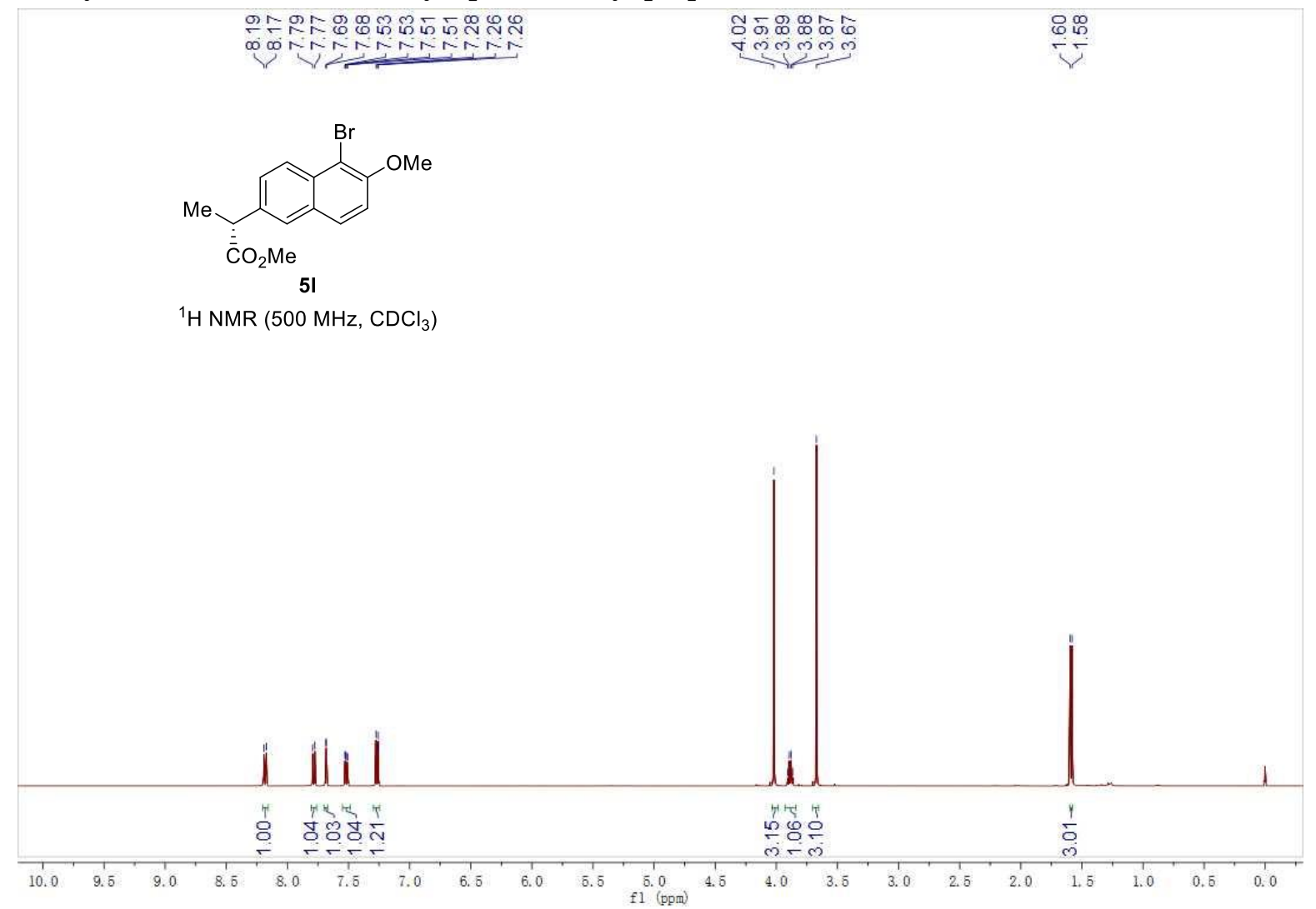


1-Bromo-2-methoxy-3-phenylnaphthalene (5m)

ㅊำน

皮每

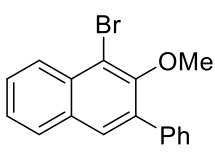

$5 \mathrm{~m}$

${ }^{1} \mathrm{H}$ NMR $\left(500 \mathrm{MHz}, \mathrm{CDCl}_{3}\right)$

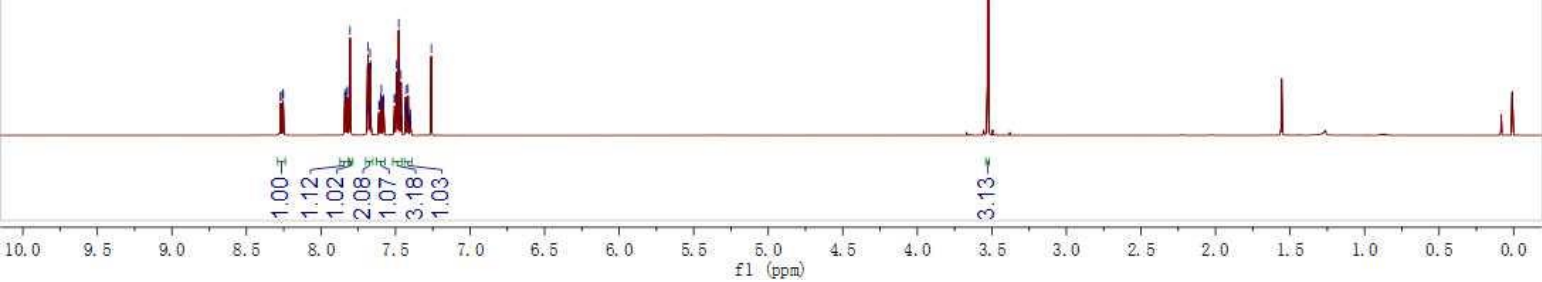

Methyl 4-bromo-3-methoxy-2-naphthoate (5n)

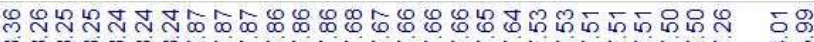

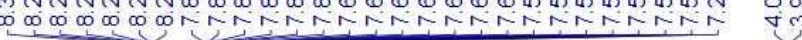

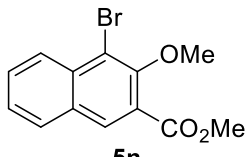

${ }^{1} \mathrm{H} \mathrm{NMR}\left(500 \mathrm{MHz}, \mathrm{CDCl}_{3}\right)$

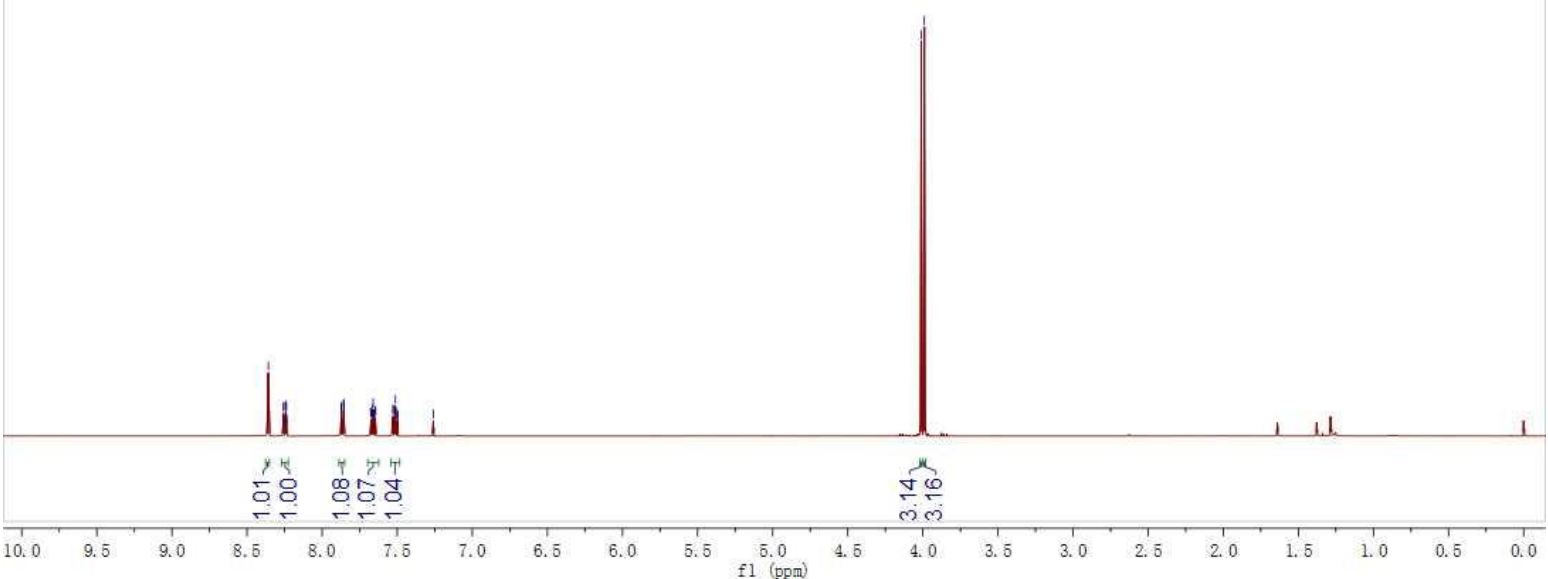


Methyl 4-bromo-3-methoxy-2-naphthoate (5n)

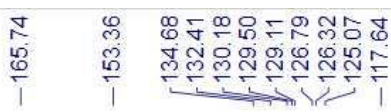

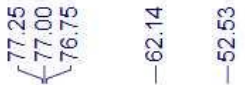

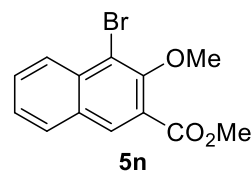

${ }^{13} \mathrm{C}$ NMR (126 MHz, $\mathrm{CDCl}_{3}$ )
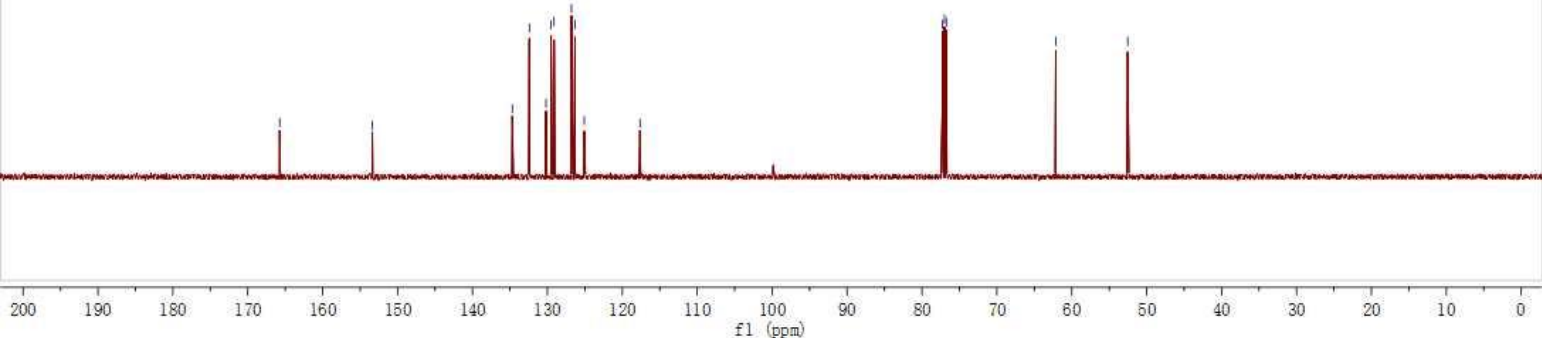

Methyl 5-bromo-6-methoxy-1-naphthoate (5o)

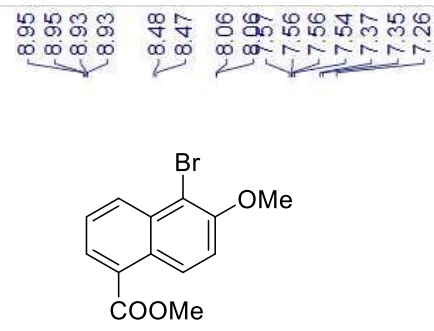

50

${ }^{1} \mathrm{H}$ NMR (500 MHz, CDCl ${ }_{3}$ )

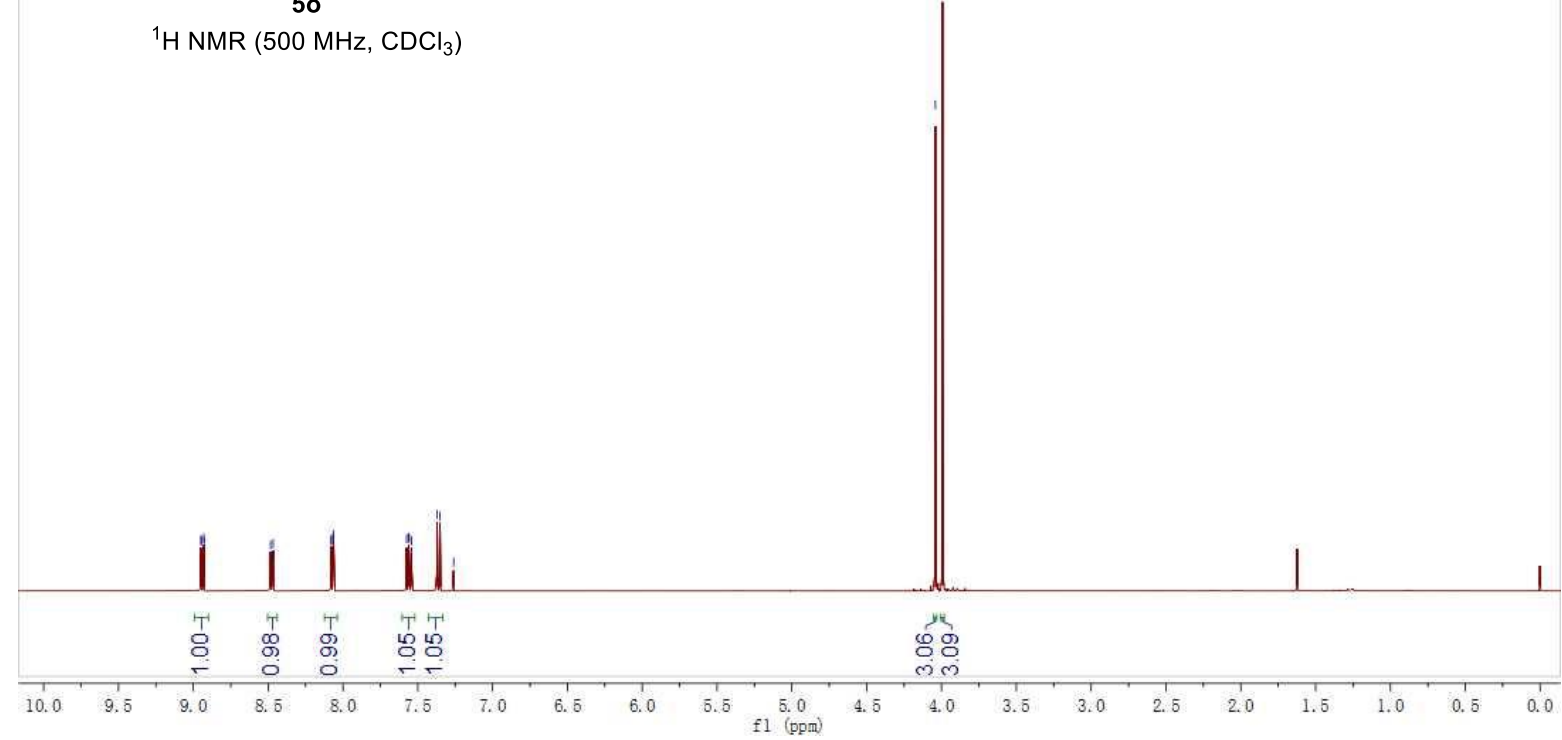

1-bromo-2,7-dimethoxynaphthalene (5p) 


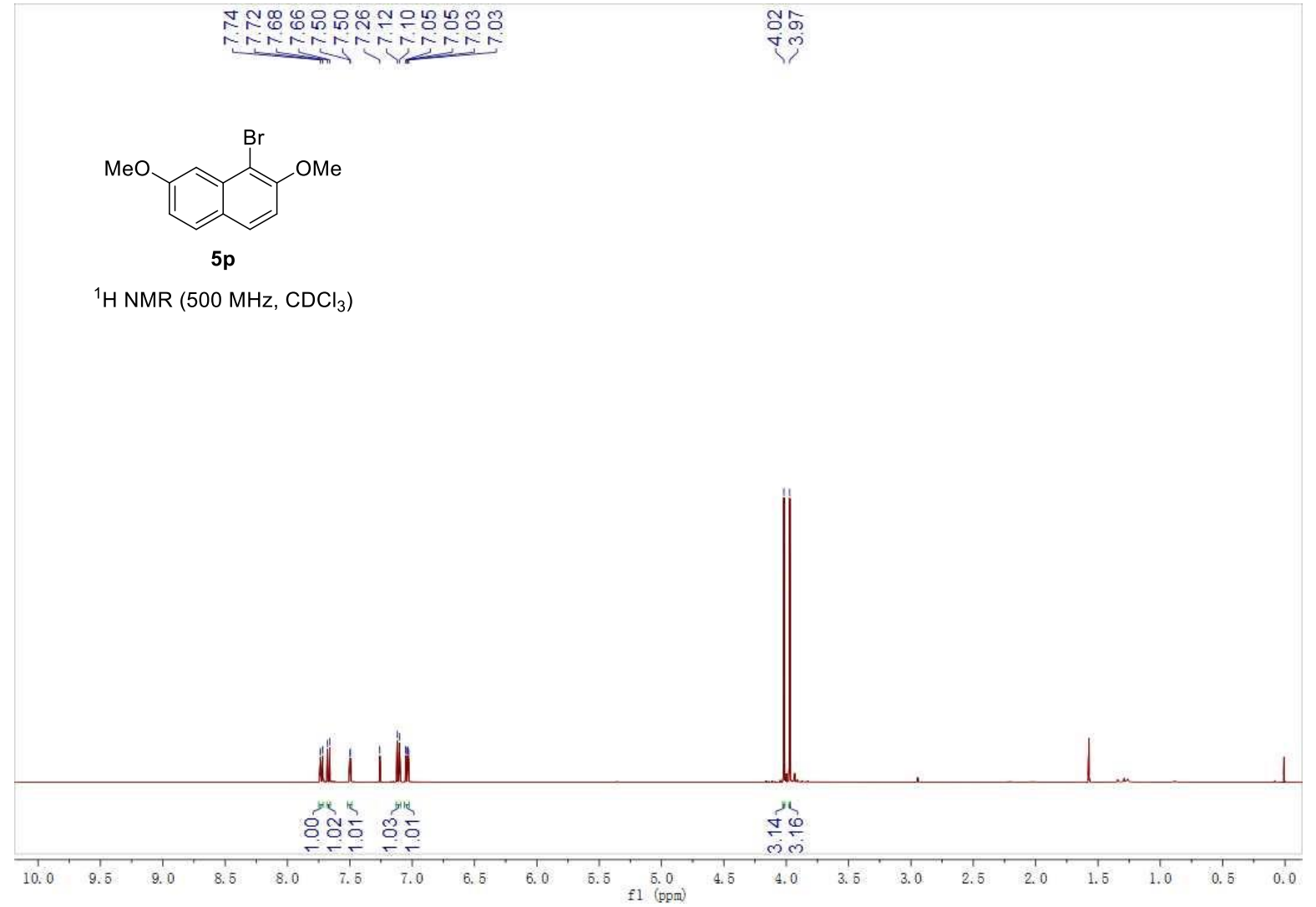

\section{4-Bromo-3-methoxyquinoline (5q)}

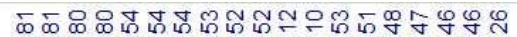

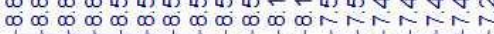

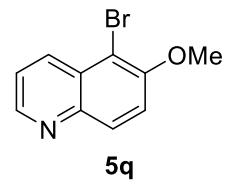

${ }^{1} \mathrm{H}$ NMR $\left(500 \mathrm{MHz}, \mathrm{CDCl}_{3}\right)$

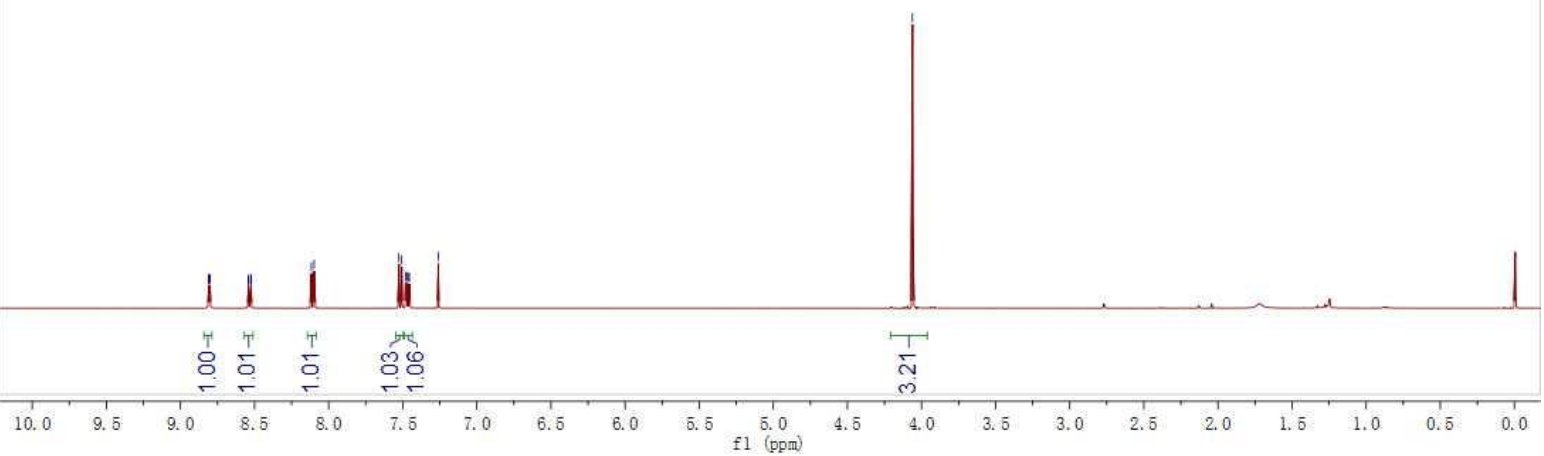


2-bromo-4-chloro-1-methoxy-3,5-dimethylbenzene (5s)

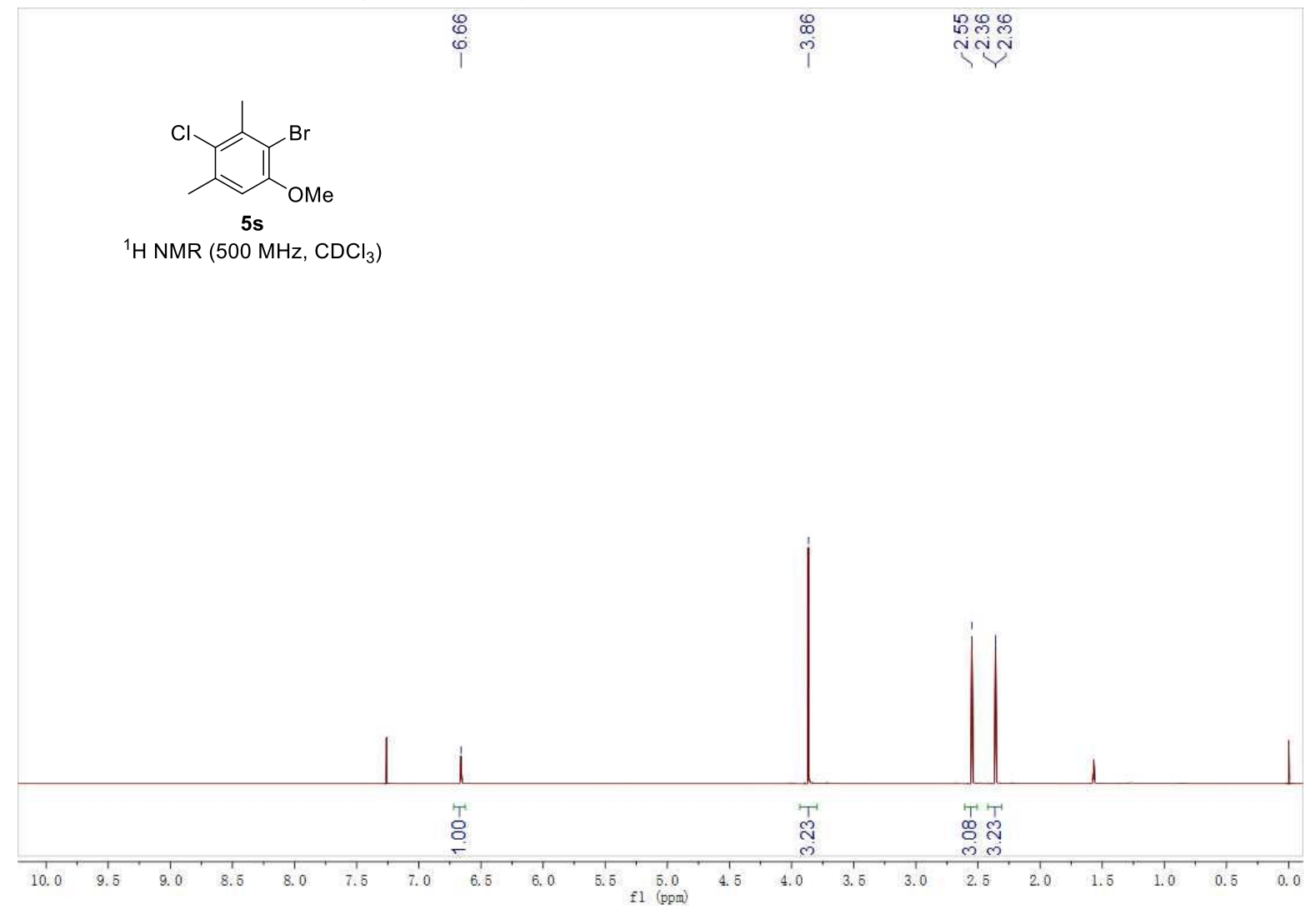

5-bromo-6-methoxy-3,4-dihydronaphthalen-1(2H)-one (5u)

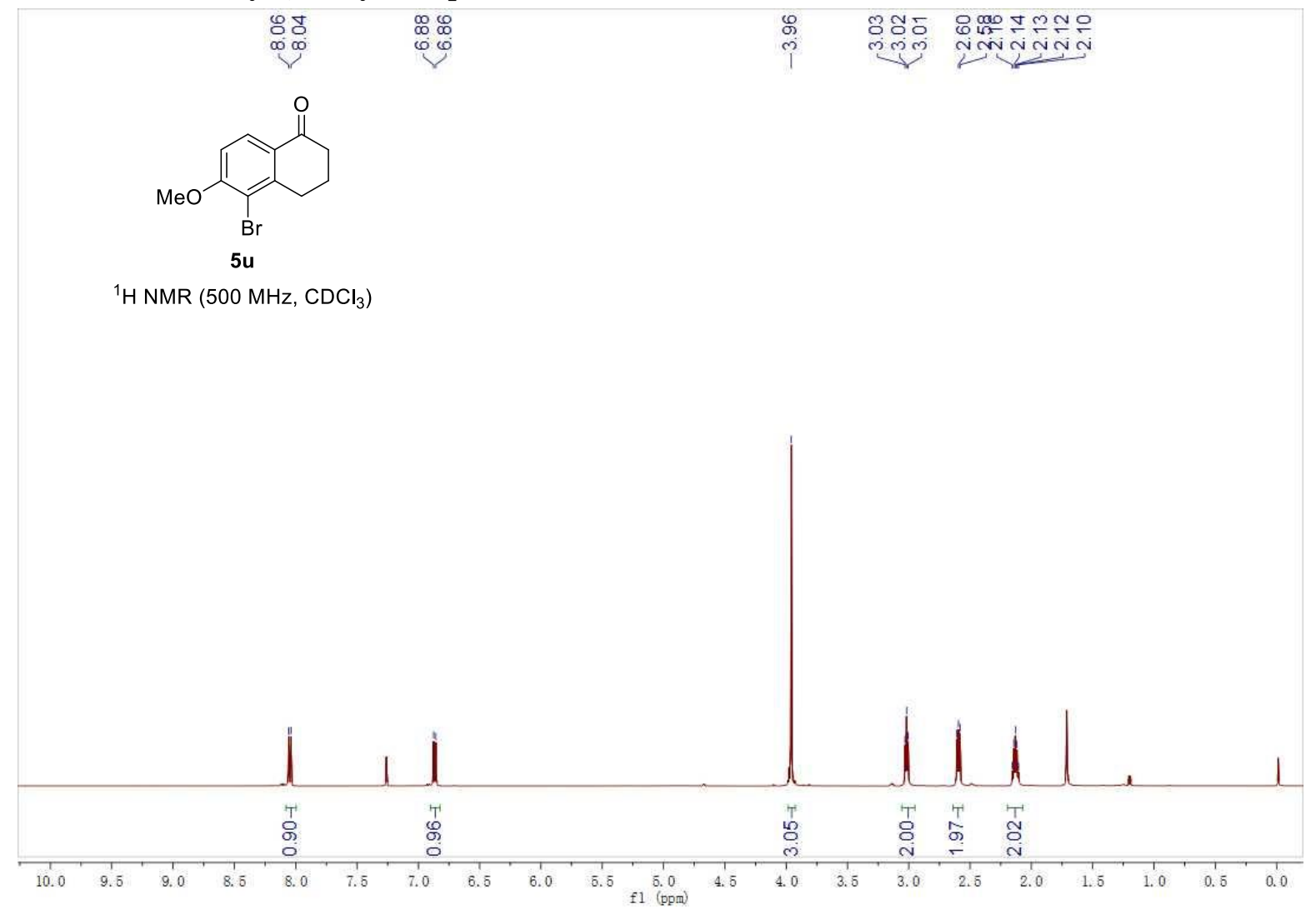


(S)-1-ethyl-2-(2-methoxynaphthalen-1-yl)-2,3-dihydro-1H-naphtho[1,8-de][1,3,2]diazaborinine (6a)

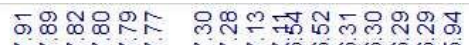

Ninin TiN

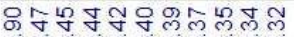

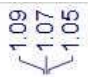

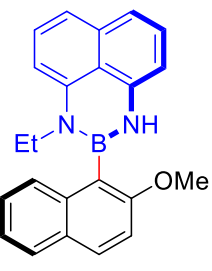

$6 a$

${ }^{1} \mathrm{H}$ NMR $\left(400 \mathrm{MHz}, \mathrm{CDCl}_{3}\right)$

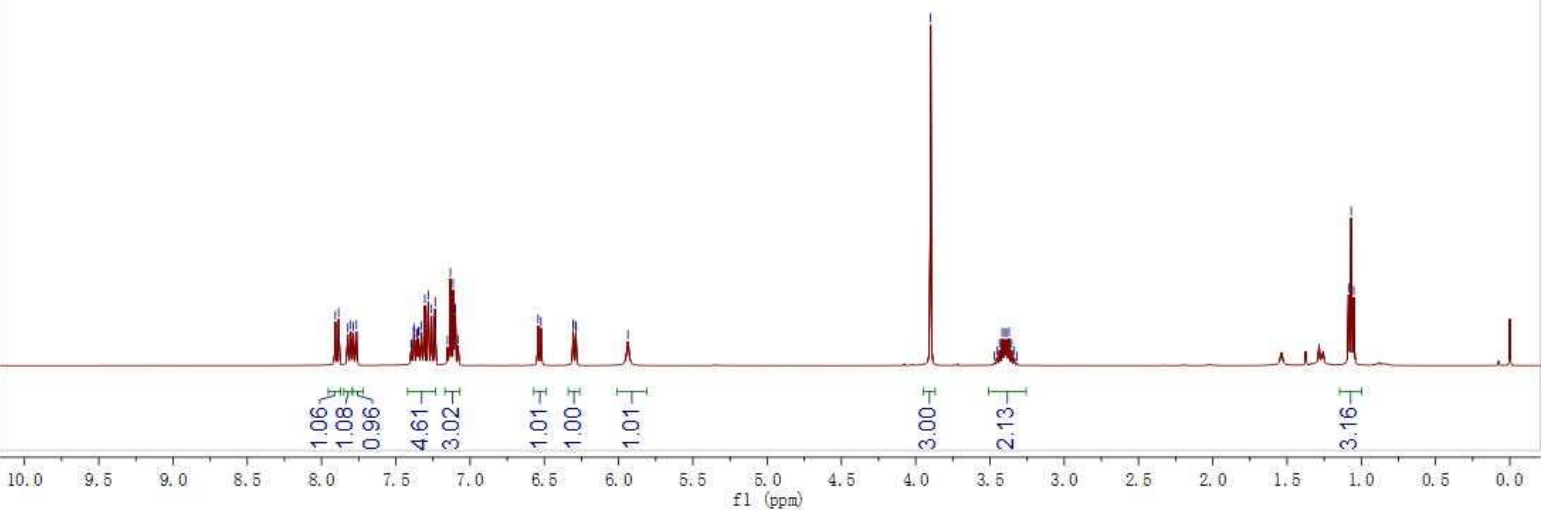

(S)-1-ethyl-2-(2-methoxynaphthalen-1-yl)-2,3-dihydro-1H-naphtho[1,8-de][1,3,2]diazaborinine (6a)

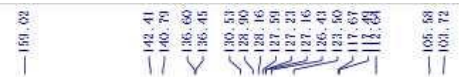

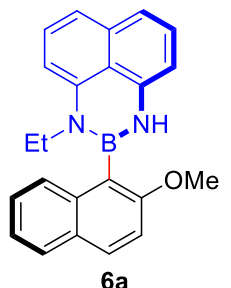

${ }^{13} \mathrm{C}$ NMR (126 MHz, $\mathrm{CDCl}_{3}$ )

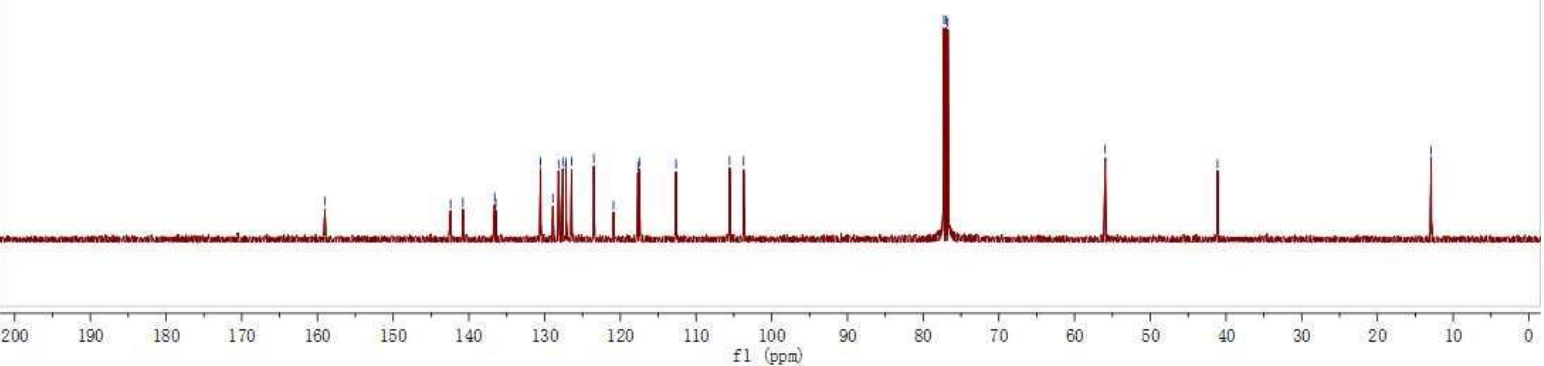


(S)-1-ethyl-2-(2-methoxynaphthalen-1-yl)-2,3-dihydro-1H-naphtho[1,8-de][1,3,2]diazaborinine (6a)

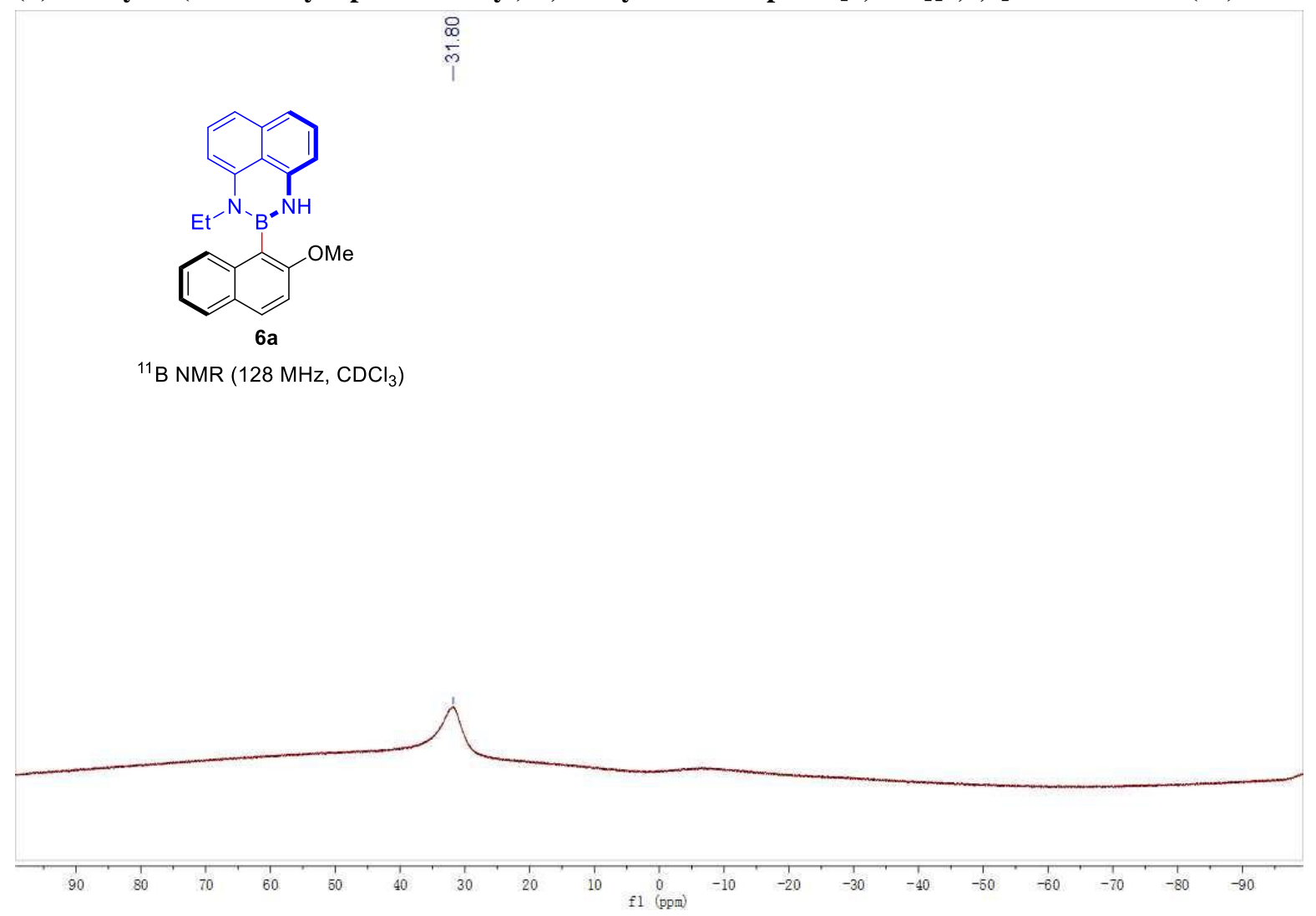

(S)-1-ethyl-2-(2-isopropoxynaphthalen-1-yl)-2,3-dihydro-1H-naphtho[1,8-de][1,3,2]diazaborinine (6b)

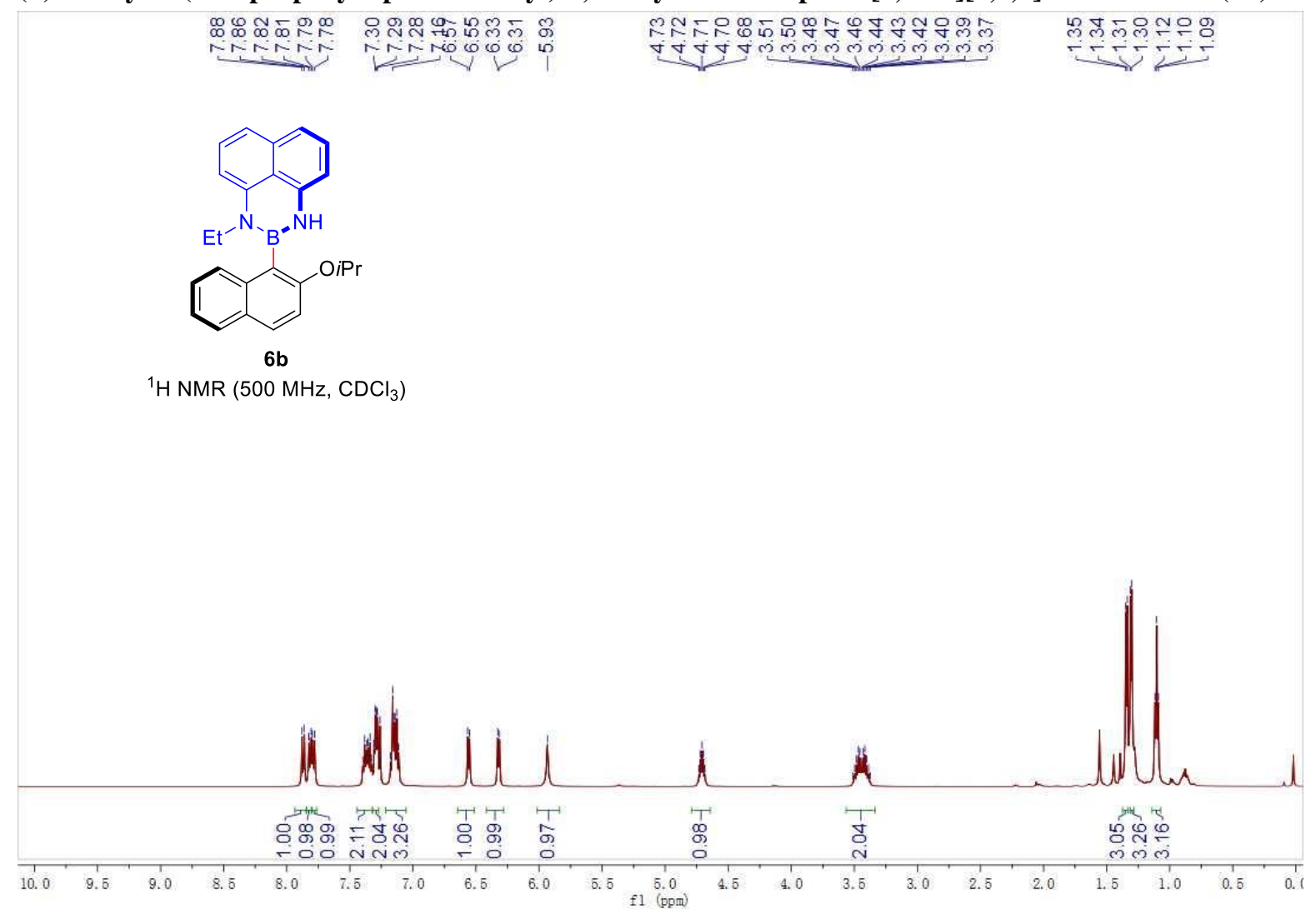


(S)-1-ethyl-2-(2-isopropoxynaphthalen-1-yl)-2,3-dihydro-1H-naphtho[1,8-de][1,3,2]diazaborinine (6b)

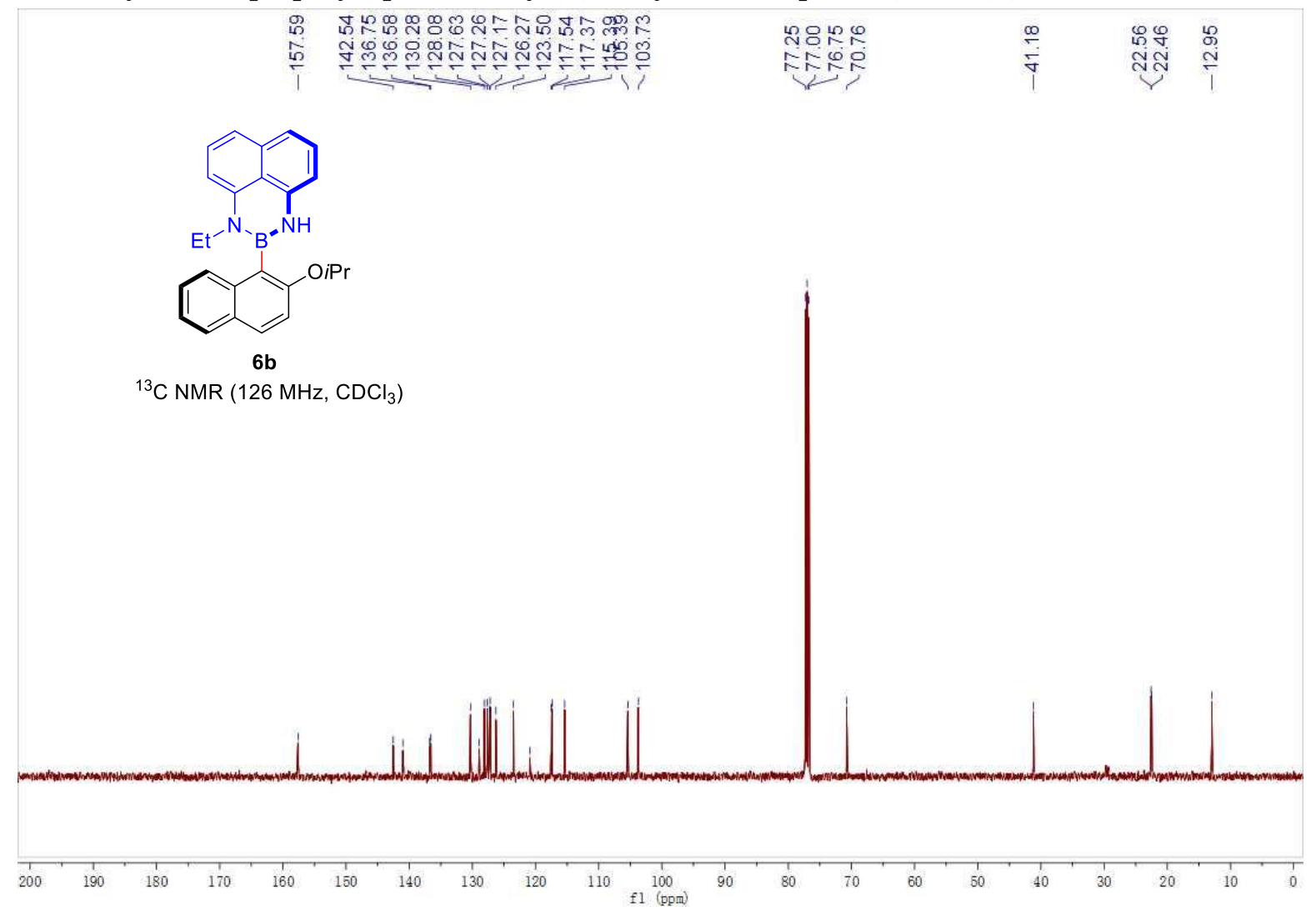

(S)-1-ethyl-2-(2-isopropoxynaphthalen-1-yl)-2,3-dihydro-1H-naphtho[1,8-de][1,3,2]diazaborinine (6b)

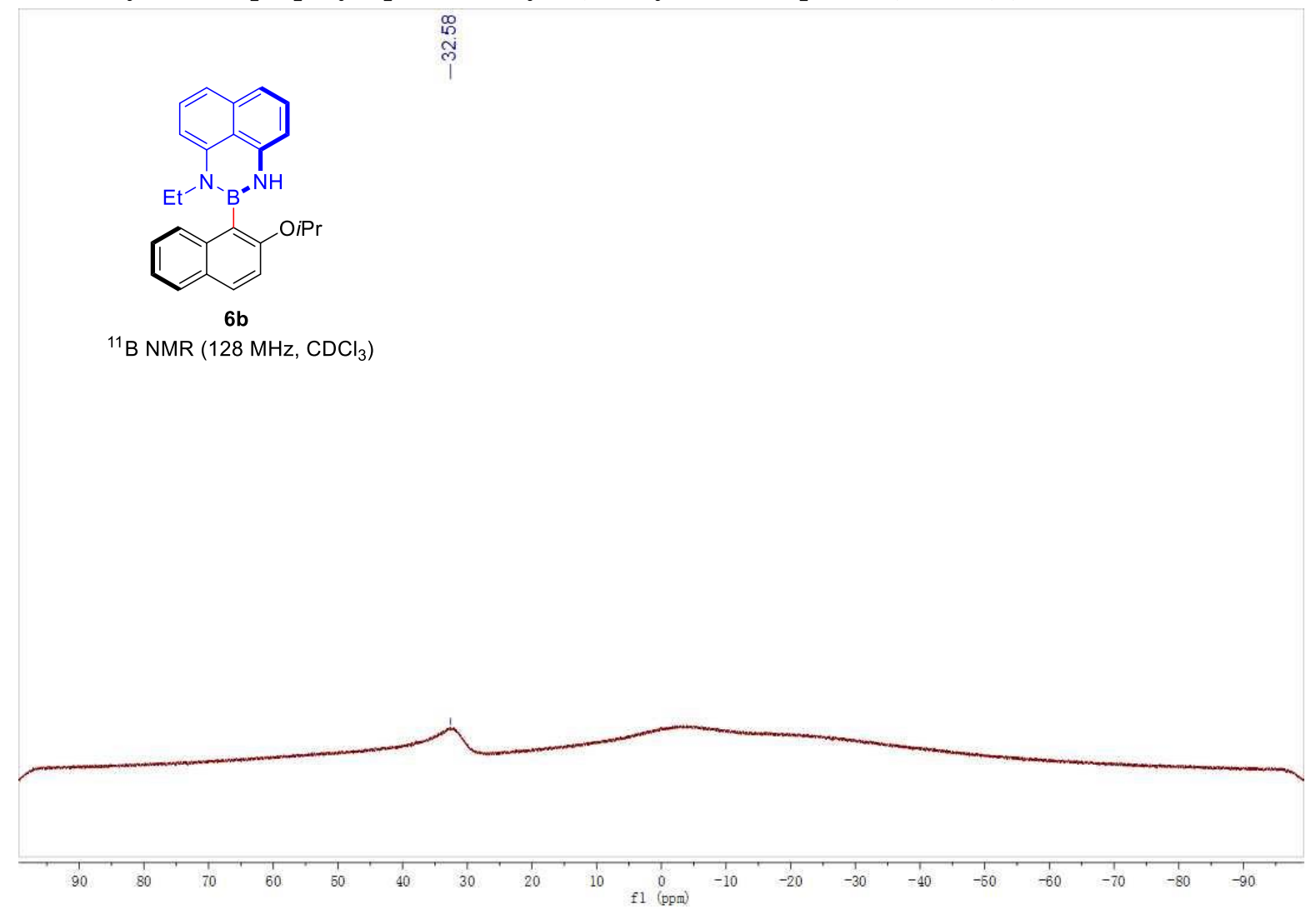




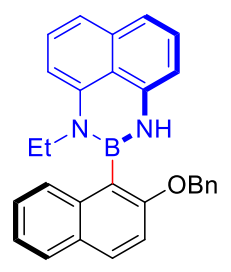

6c

${ }^{1} \mathrm{H}$ NMR $\left(500 \mathrm{MHz}, \mathrm{CDCl}_{3}\right)$

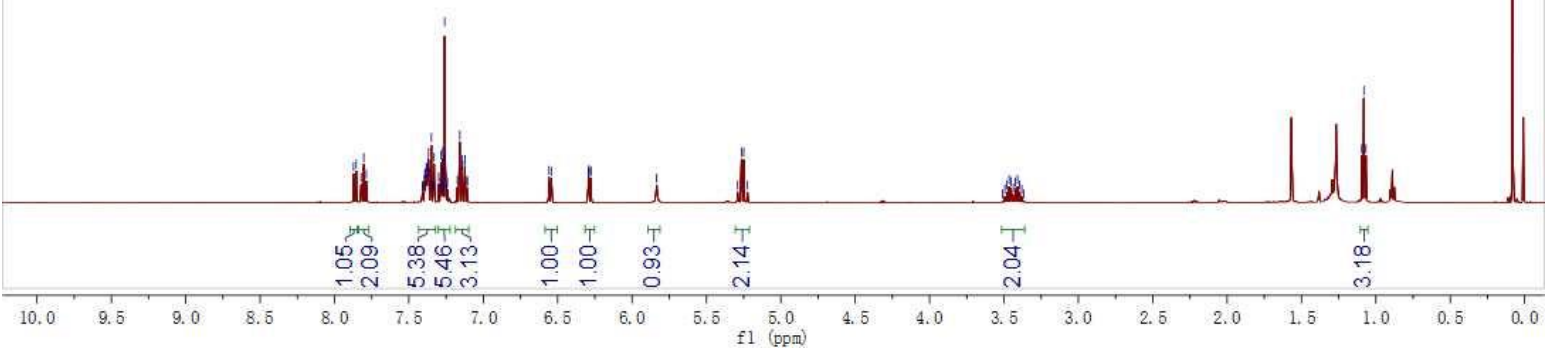

(S)-2-(2-(benzyloxy)naphthalen-1-yl)-1-ethyl-2,3-dihydro-1H-naphtho[1,8-de][1,3,2]diazaborinine (6c)

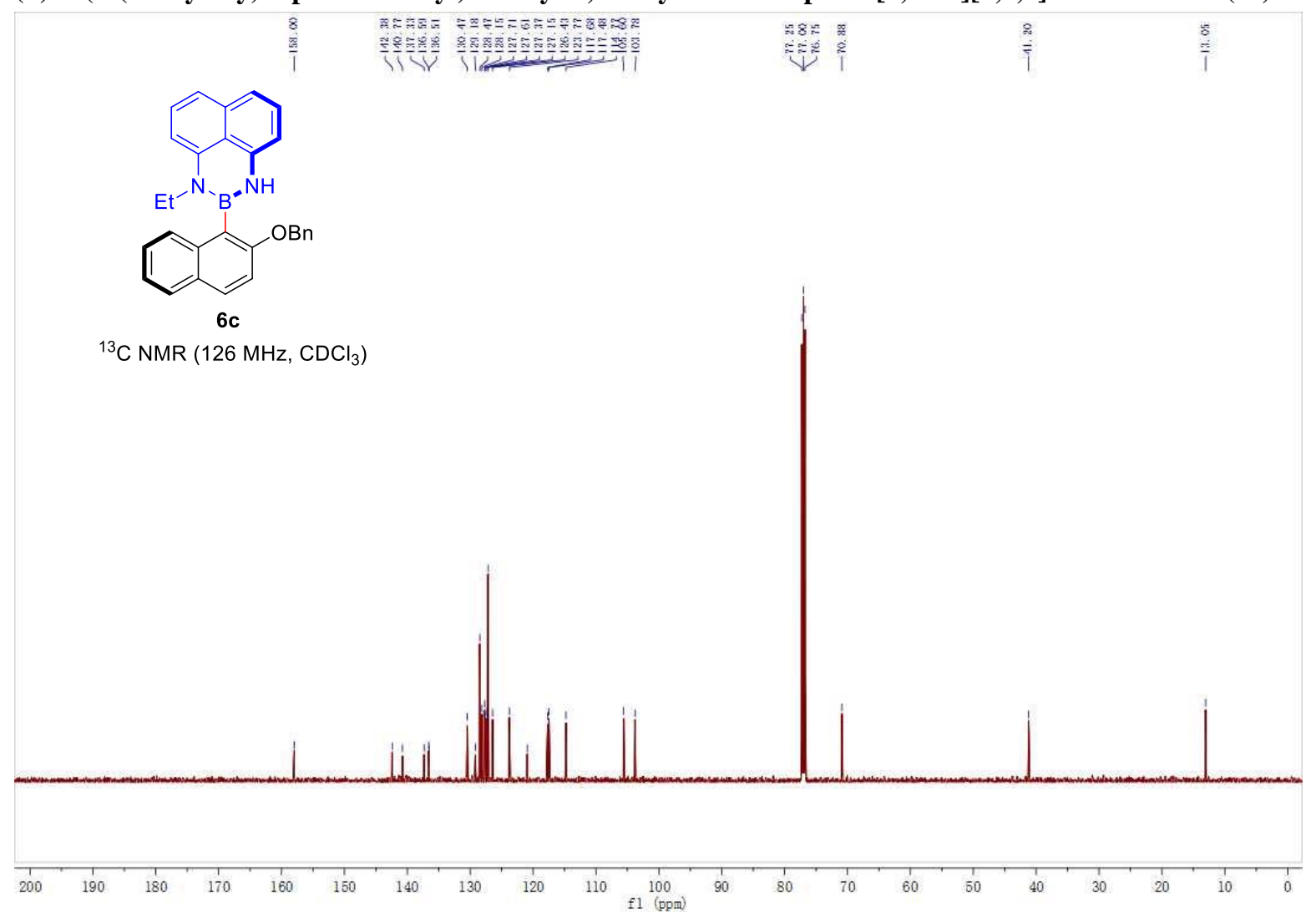


(S)-2-(2-(benzyloxy)naphthalen-1-yl)-1-ethyl-2,3-dihydro-1H-naphtho[1,8-de][1,3,2]diazaborinine (6c)

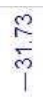

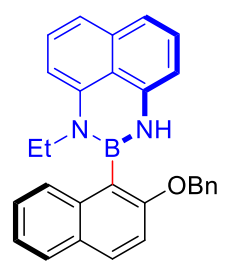

6c

${ }^{11} \mathrm{~B}$ NMR $\left(128 \mathrm{MHz}, \mathrm{CDCl}_{3}\right)$

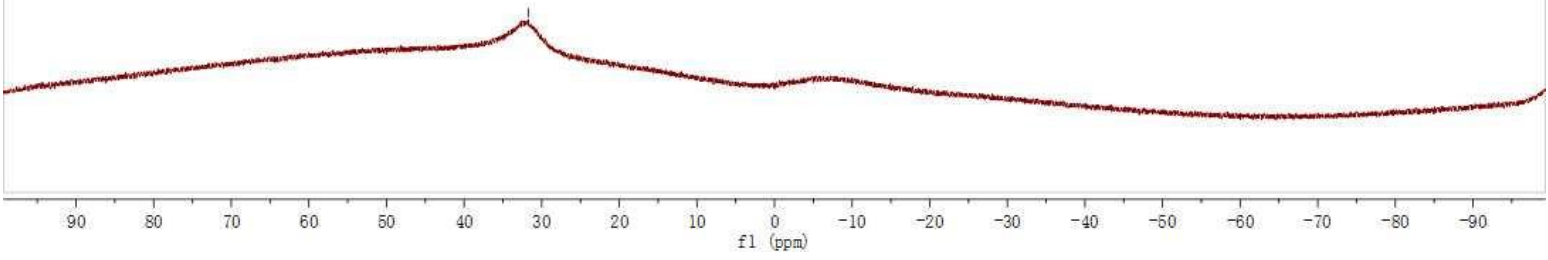

(S)-1-ethyl-2-(2-(methoxymethoxy)naphthalen-1-yl)-2,3-dihydro-1H-naphtho[1,8-de][1,3,2]diazaborinin e (6d)

ద쌔

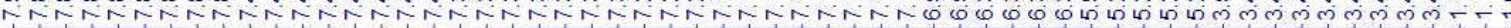

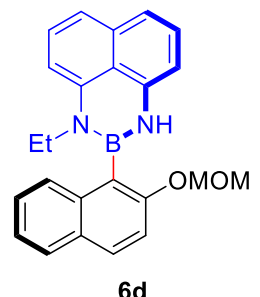

${ }^{1} \mathrm{H}$ NMR $\left(500 \mathrm{MHz}, \mathrm{CDCl}_{3}\right)$

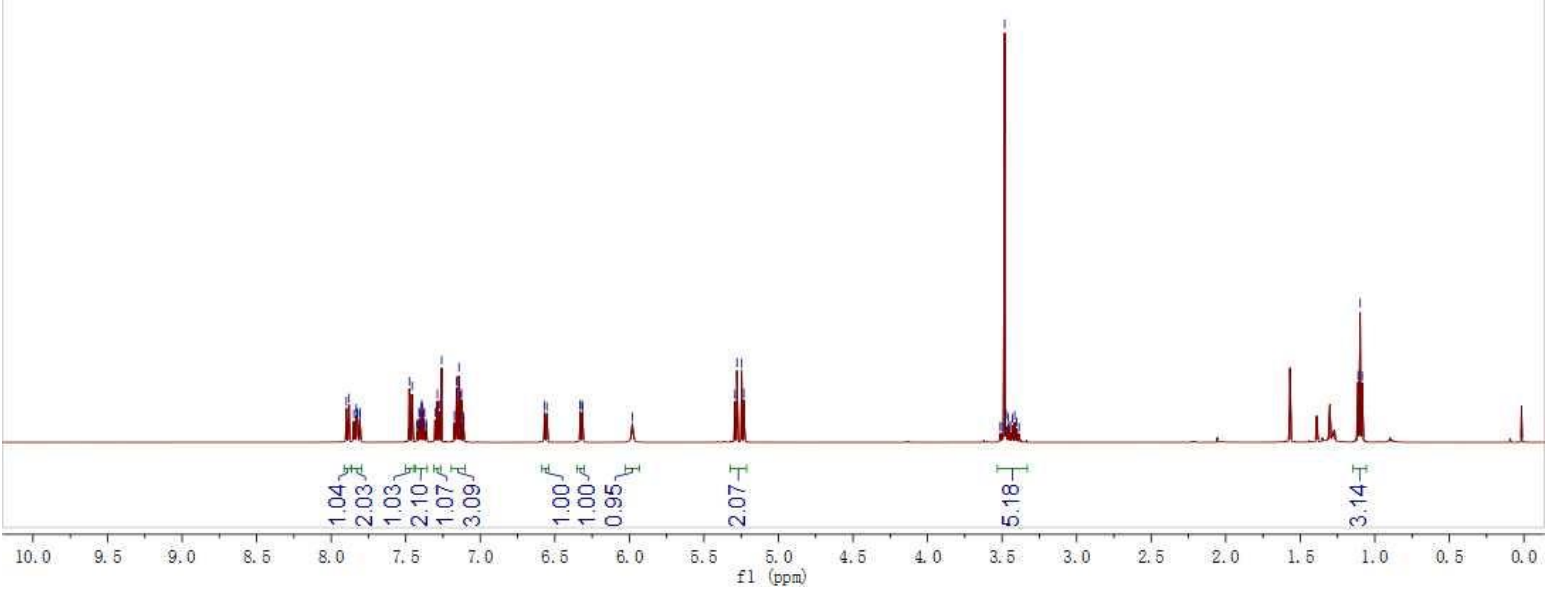


(S)-1-ethyl-2-(2-(methoxymethoxy)naphthalen-1-yl)-2,3-dihydro-1H-naphtho[1,8-de][1,3,2]diazaborinin e (6d)

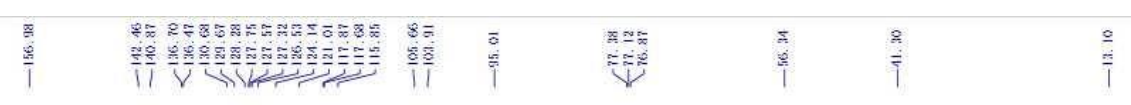<smiles>CCN1B2C(=CC=Cc3ccccc32)Nc2cccc3cccc1c23</smiles>

6d

${ }^{13} \mathrm{C}$ NMR (126 $\left.\mathrm{MHz}, \mathrm{CDCl}_{3}\right)$
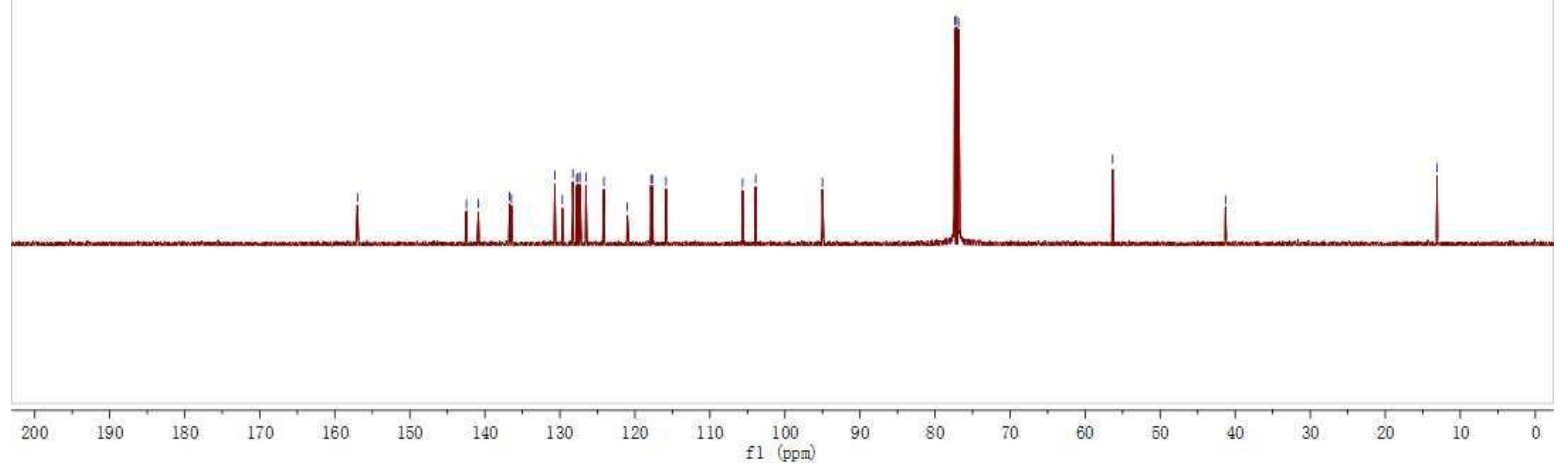

(S)-1-ethyl-2-(2-(methoxymethoxy)naphthalen-1-yl)-2,3-dihydro-1H-naphtho[1,8-de][1,3,2]diazaborinin e (6d)

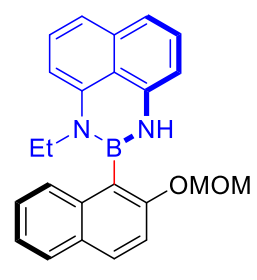

6d

${ }^{11} \mathrm{~B}$ NMR (128 MHz, $\left.\mathrm{CDCl}_{3}\right)$

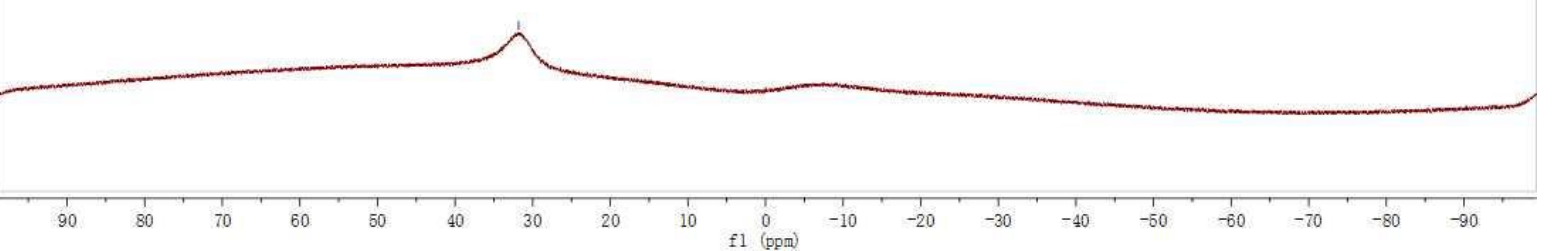


(S)-5-(1-ethyl-1H-naphtho[1,8-de][1,3,2]diazaborinin-2(3H)-yl)-6-methoxy-2-naphthaldehyde (6e)

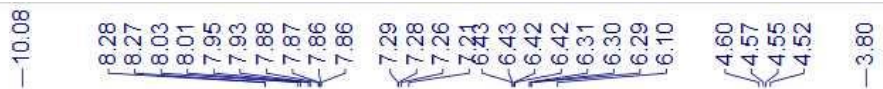<smiles>CCN1BNc2cccc3cccc(c23)B1c1c(OC)ccc2ccc(C=O)cc12</smiles>

${ }^{1} \mathrm{H}$ NMR $\left(500 \mathrm{MHz}, \mathrm{CDCl}_{3}\right)$

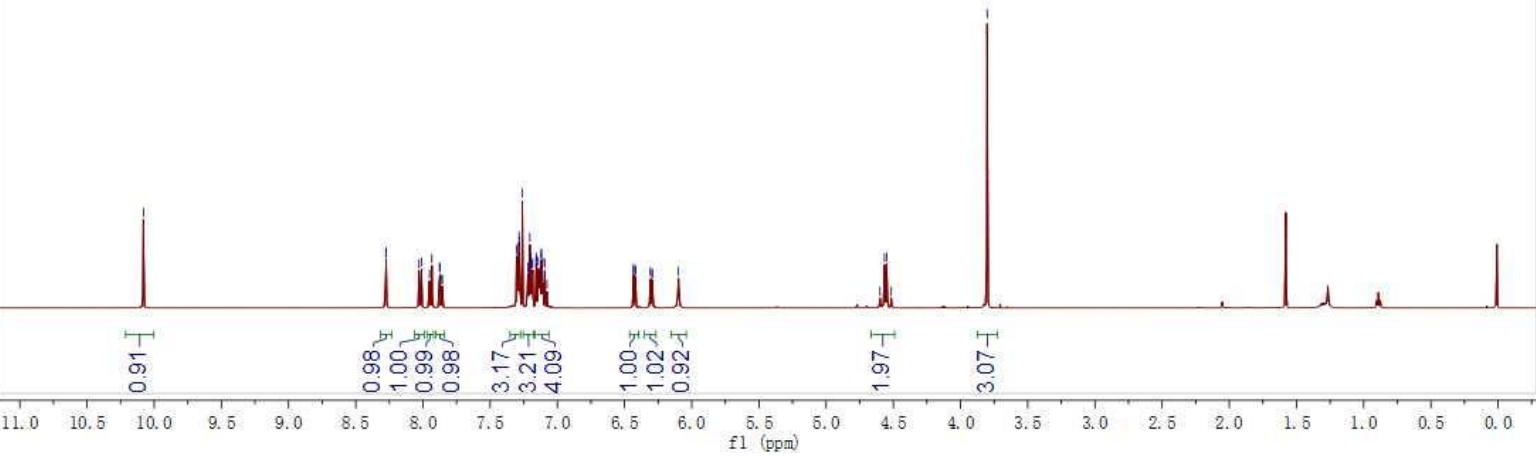

(S)-5-(1-ethyl-1H-naphtho[1,8-de][1,3,2]diazaborinin-2(3H)-yl)-6-methoxy-2-naphthaldehyde (6e)

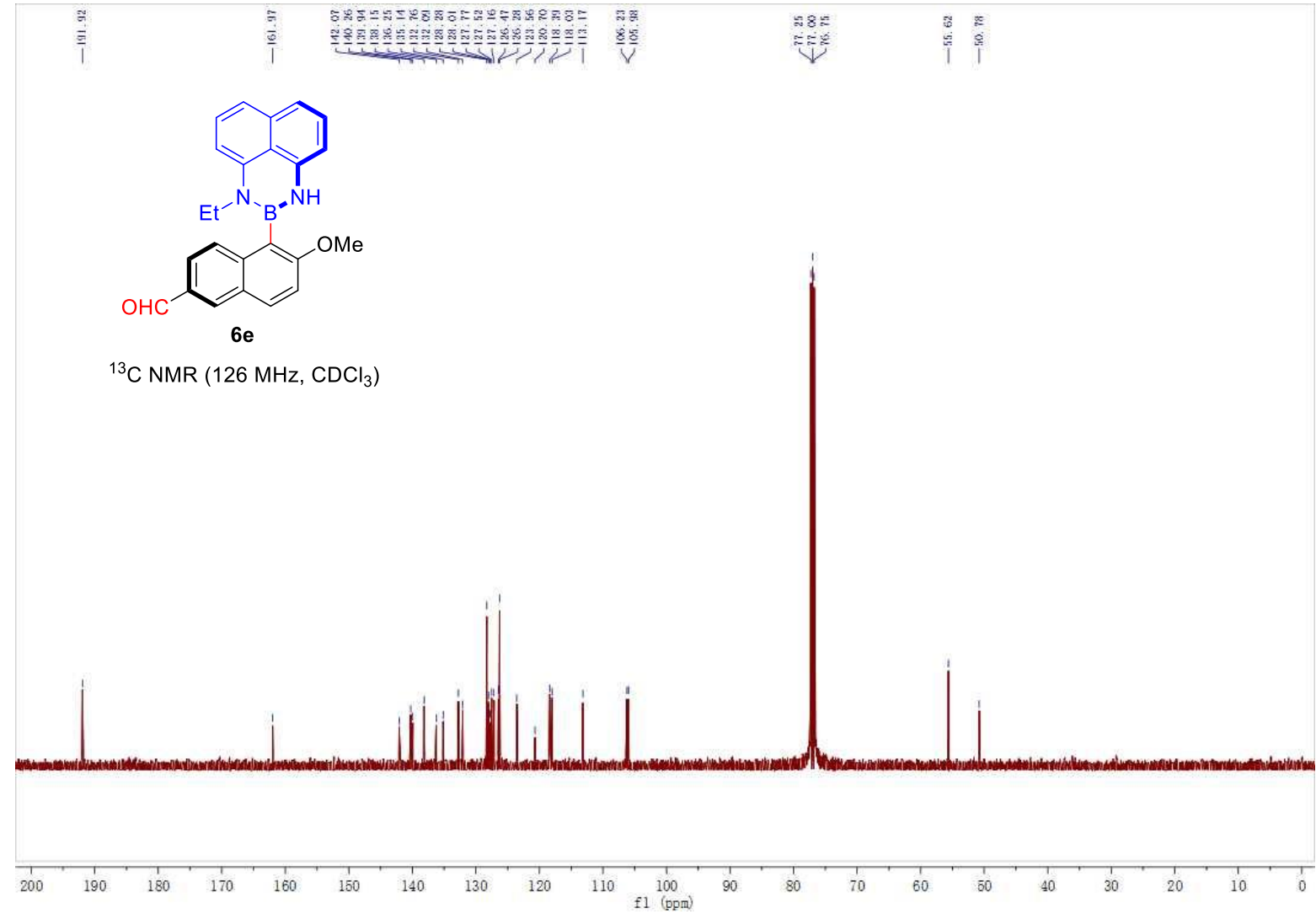


(S)-5-(1-ethyl-1H-naphtho[1,8-de][1,3,2] diazaborinin-2(3H)-yl)-6-methoxy-2-naphthaldehyde (6e) m<smiles>CCN1c2cccc3cccc(c23)N1c1c(OC)ccc2cccc(OC)c12</smiles>

${ }^{11} \mathrm{~B} \mathrm{NMR}\left(128 \mathrm{MHz}, \mathrm{CDCl}_{3}\right)$

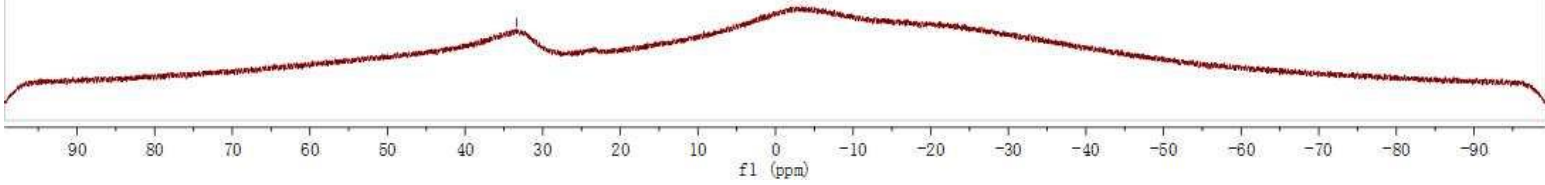

(S)-5-(1-ethyl-1H-naphtho[1,8-de][1,3,2]diazaborinin-2(3H)-yl)-6-methoxy-2-naphthonitrile (6f)

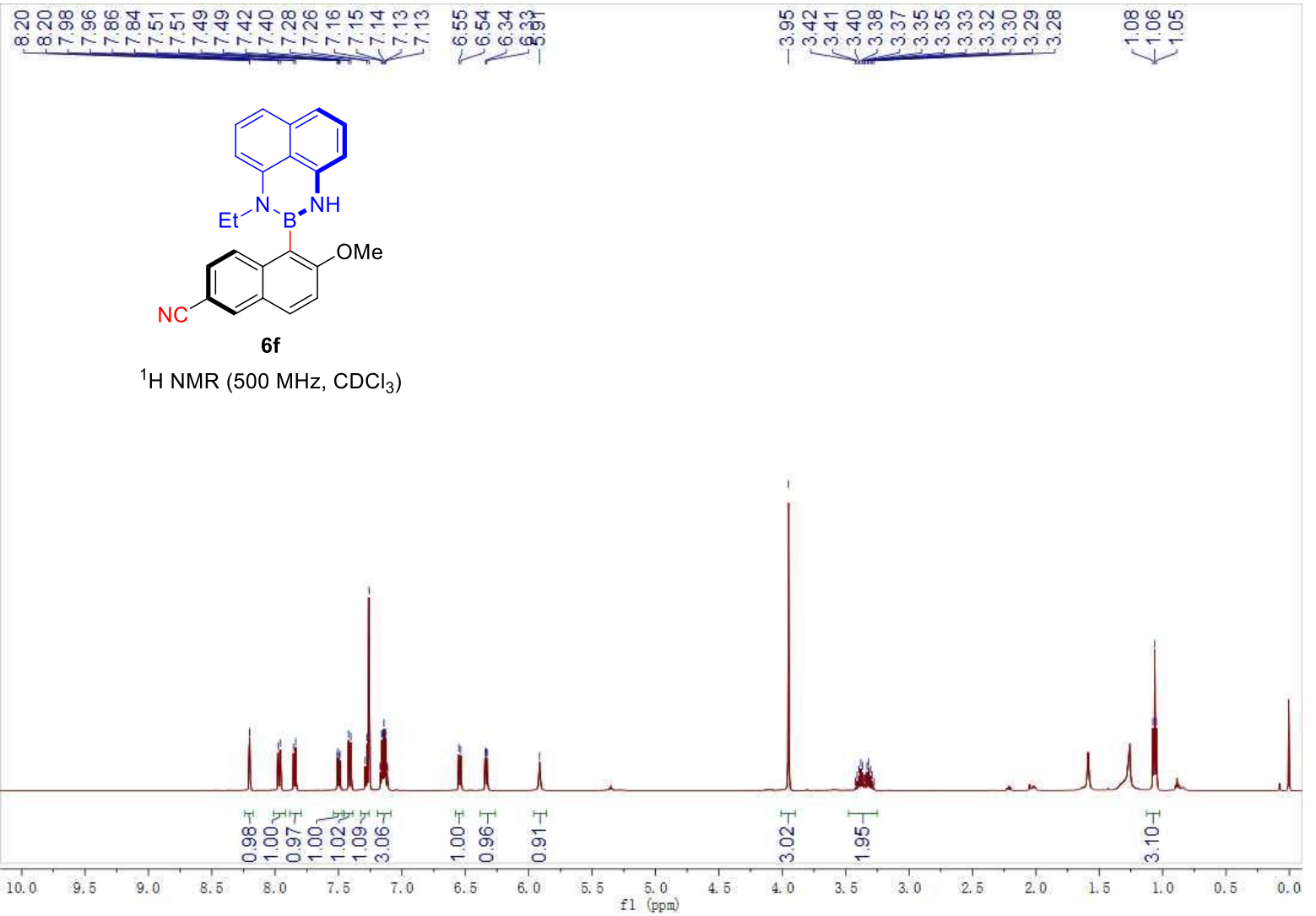


(S)-5-(1-ethyl-1H-naphtho[1,8-de][1,3,2] diazaborinin-2(3H)-yl)-6-methoxy-2-naphthonitrile (6f)

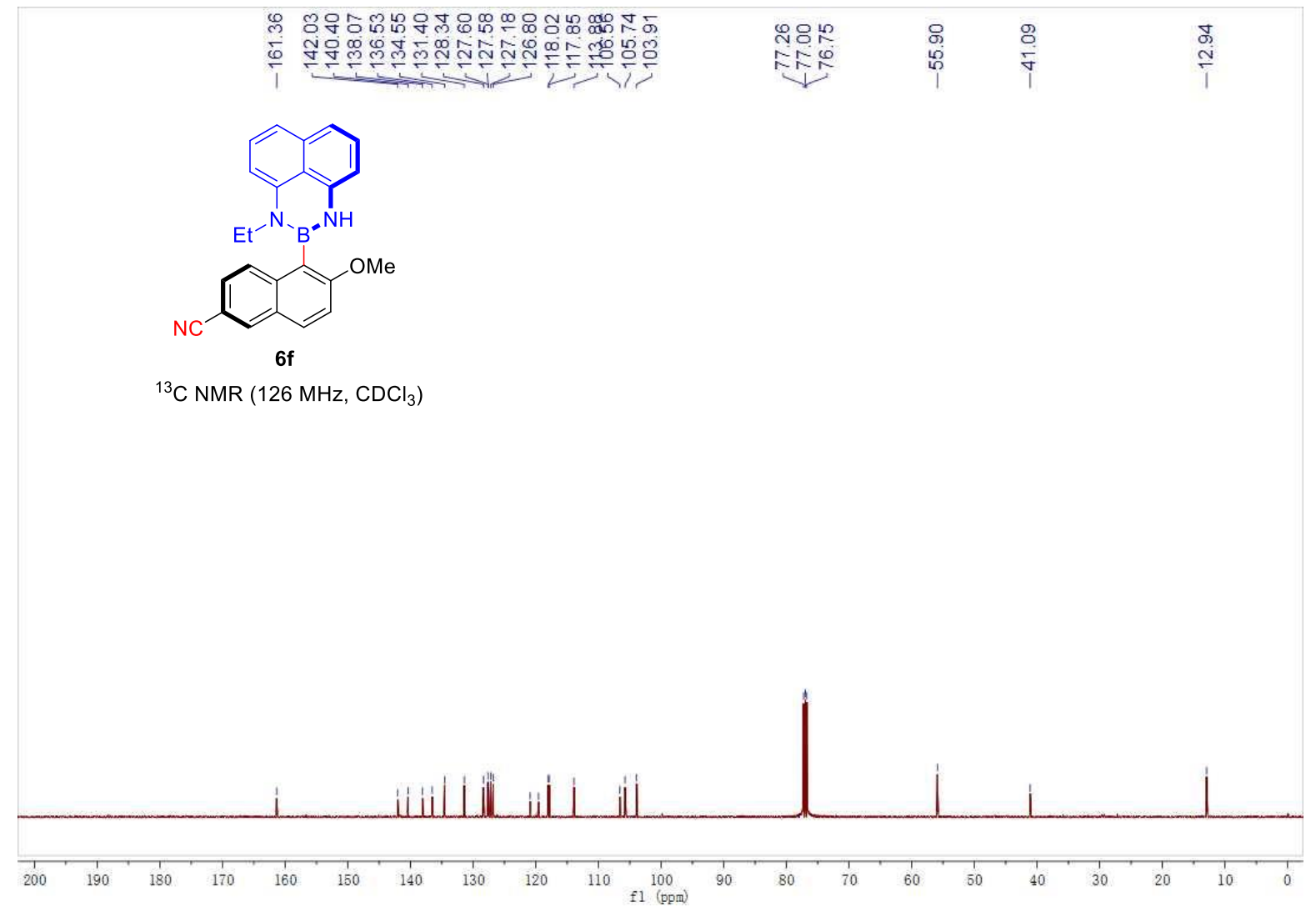

(S)-5-(1-ethyl-1H-naphtho[1,8-de][1,3,2] diazaborinin-2(3H)-yl)-6-methoxy-2-naphthonitrile (6f)

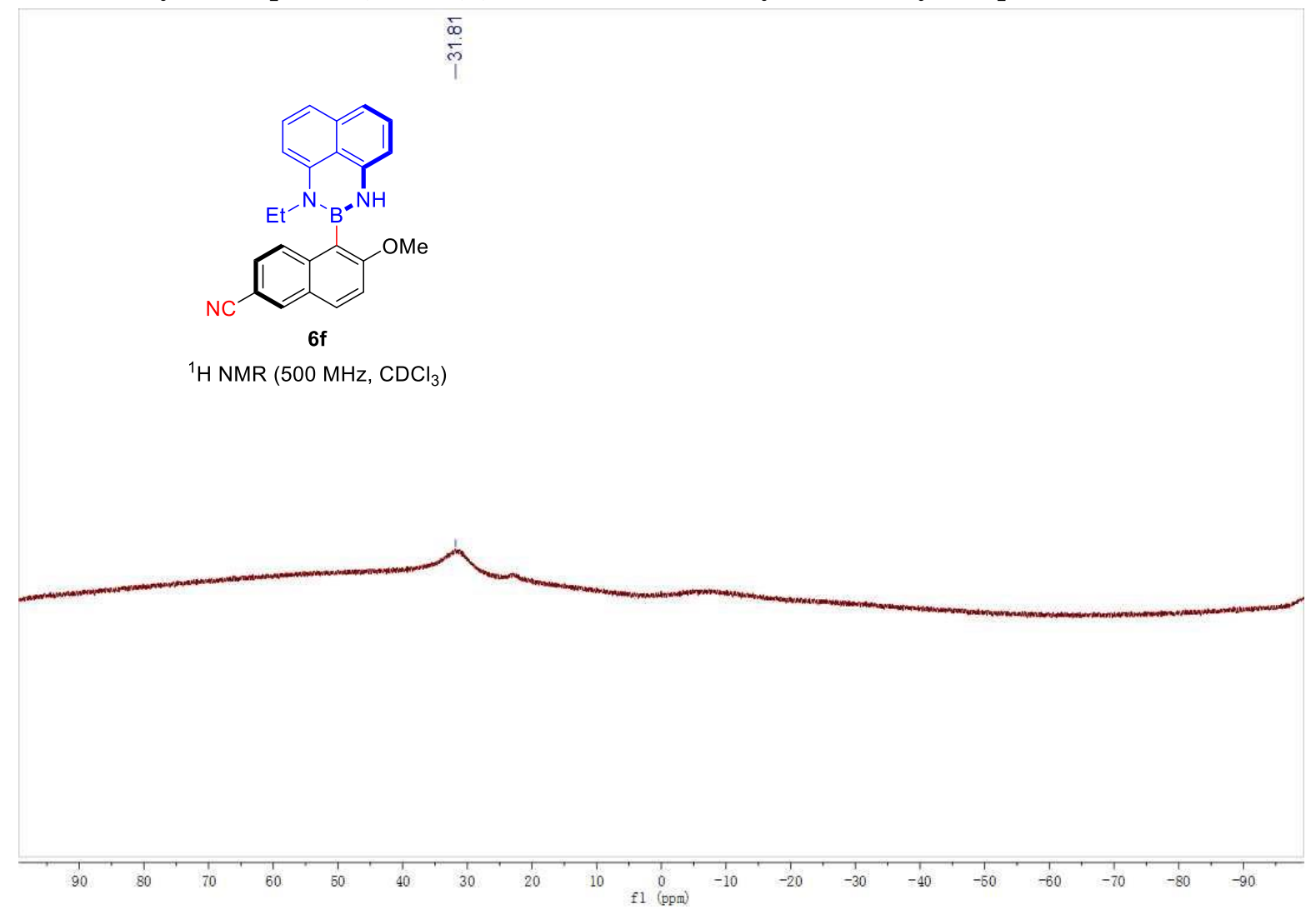


(S)-1-(5-(1-ethyl-1H-naphtho[1,8-de][1,3,2]diazaborinin-2(3H)-yl)-6-methoxynaphthalen-2-yl)ethan-1-o ne $(6 g)$

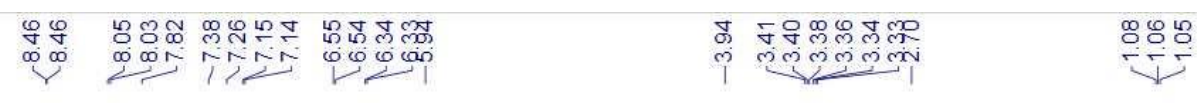

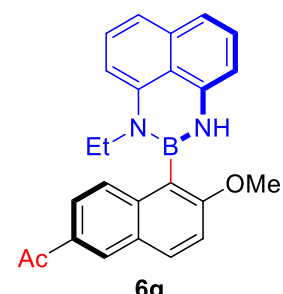

${ }^{1} \mathrm{H}$ NMR $\left(500 \mathrm{MHz}, \mathrm{CDCl}_{3}\right)$

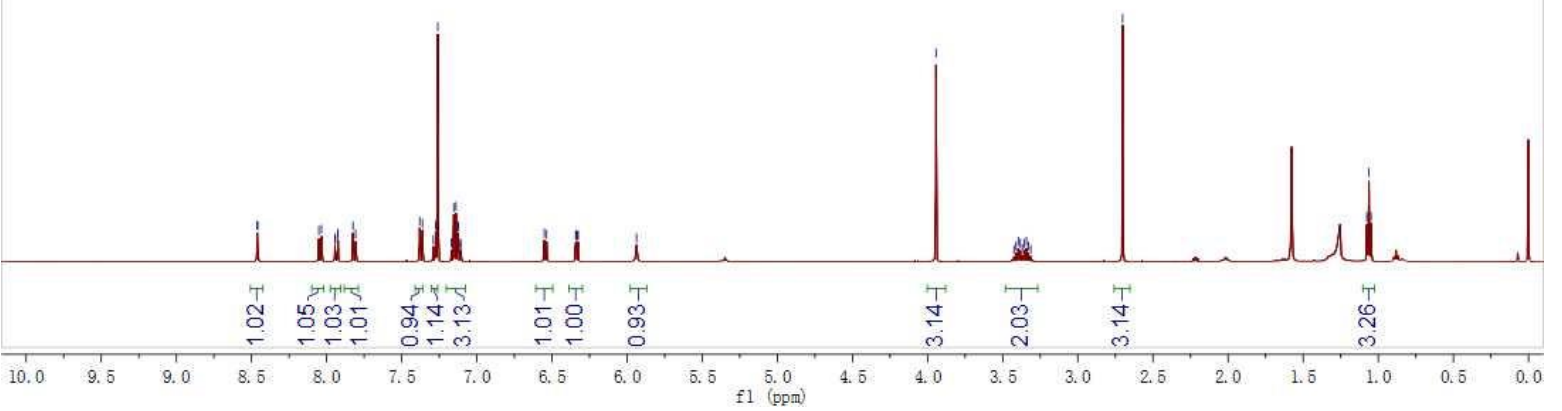

(S)-1-(5-(1-ethyl-1H-naphtho[1,8-de][1,3,2] diazaborinin-2(3H)-yl)-6-methoxynaphthalen-2-yl)ethan-1-o ne (6g)

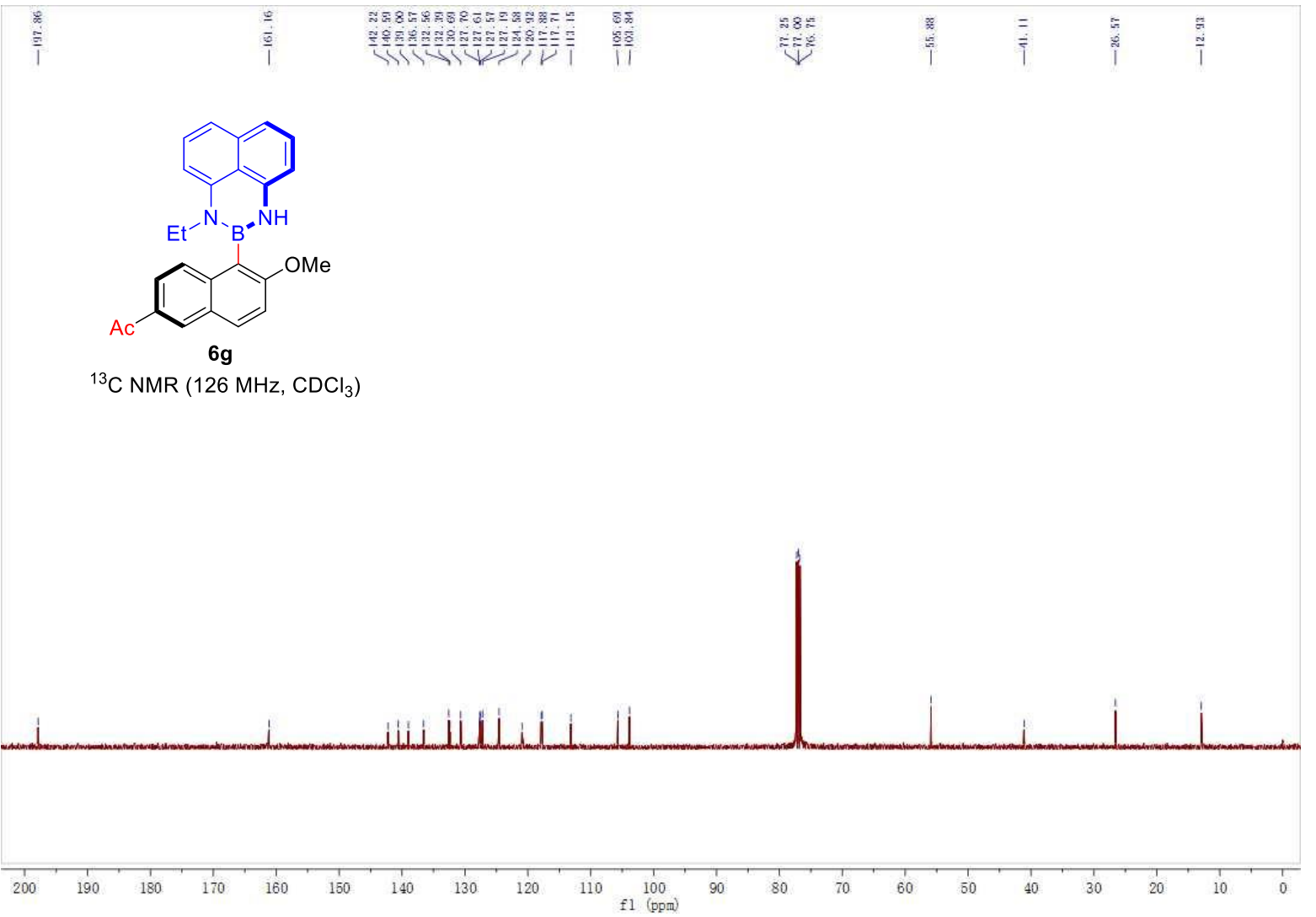


(S)-1-(5-(1-ethyl-1H-naphtho[1,8-de][1,3,2]diazaborinin-2(3H)-yl)-6-methoxynaphthalen-2-yl)ethan-1-o ne $(6 \mathrm{~g})$

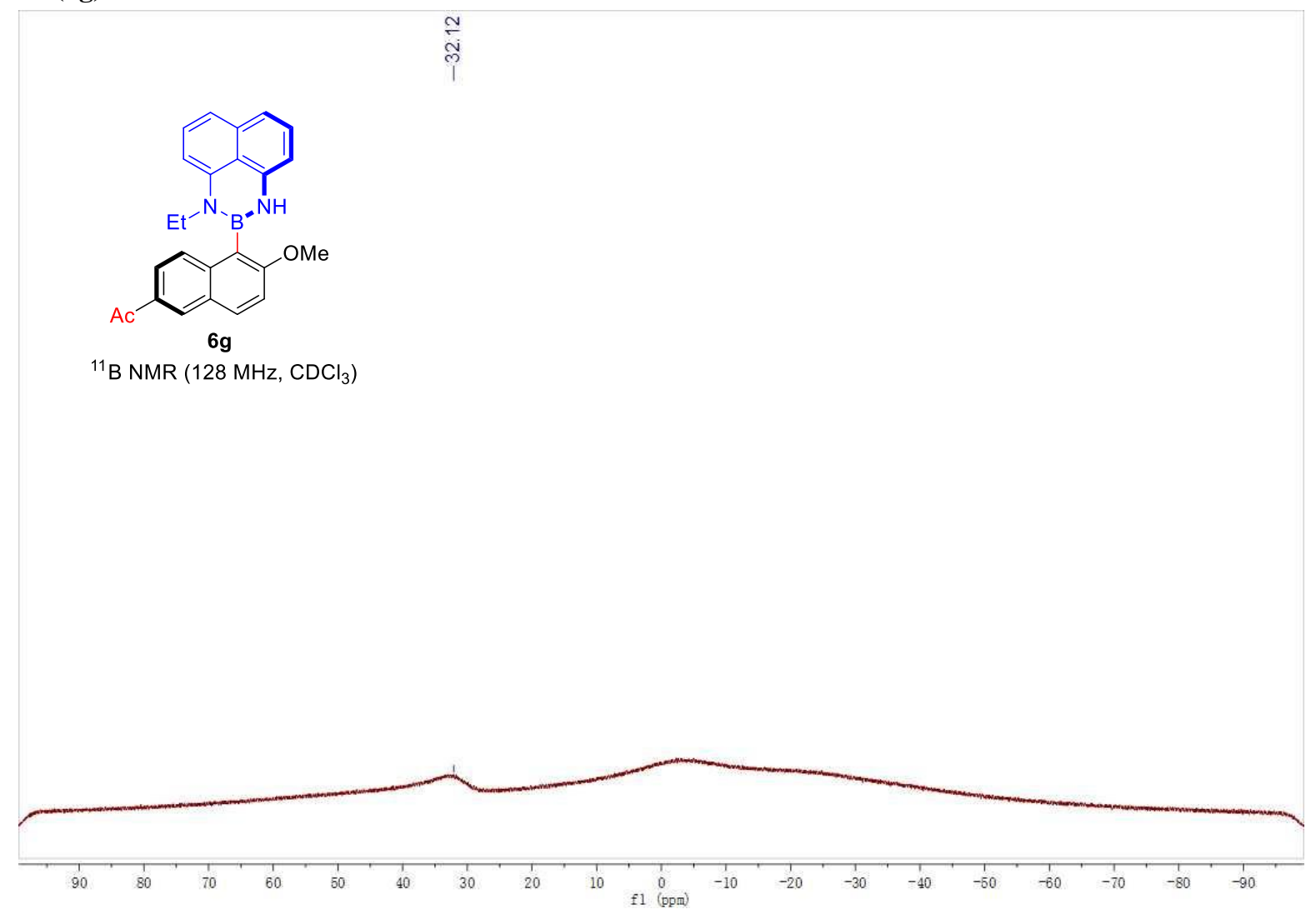

(S)-methyl 5-(1-ethyl-1H-naphtho[1,8-de][1,3,2]diazaborinin-2(3H)-yl)-6-methoxy-2-naphthoate (6h)

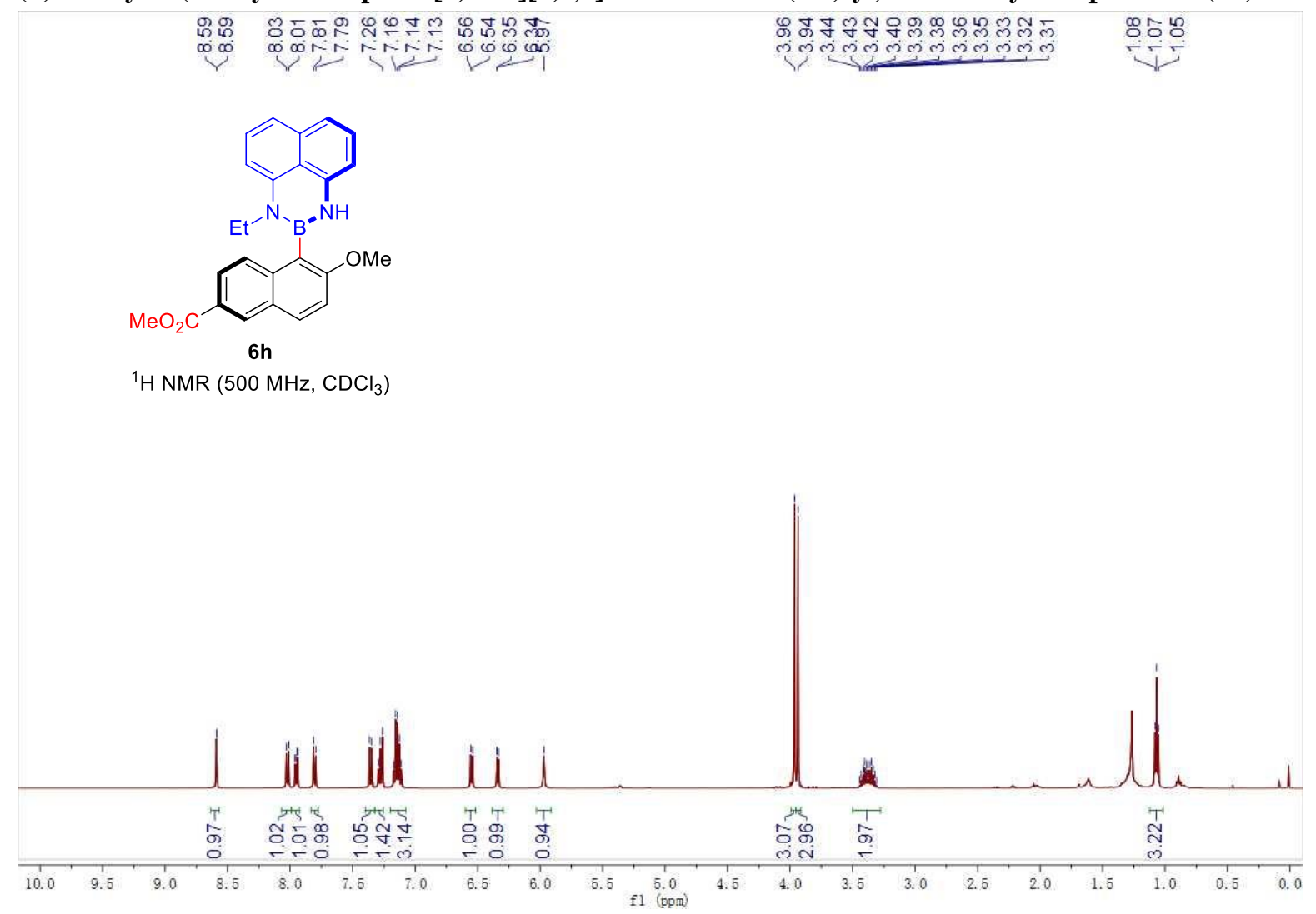


(S)-methyl 5-(1-ethyl-1H-naphtho[1,8-de][1,3,2]diazaborinin-2(3H)-yl)-6-methoxy-2-naphthoate (6h)

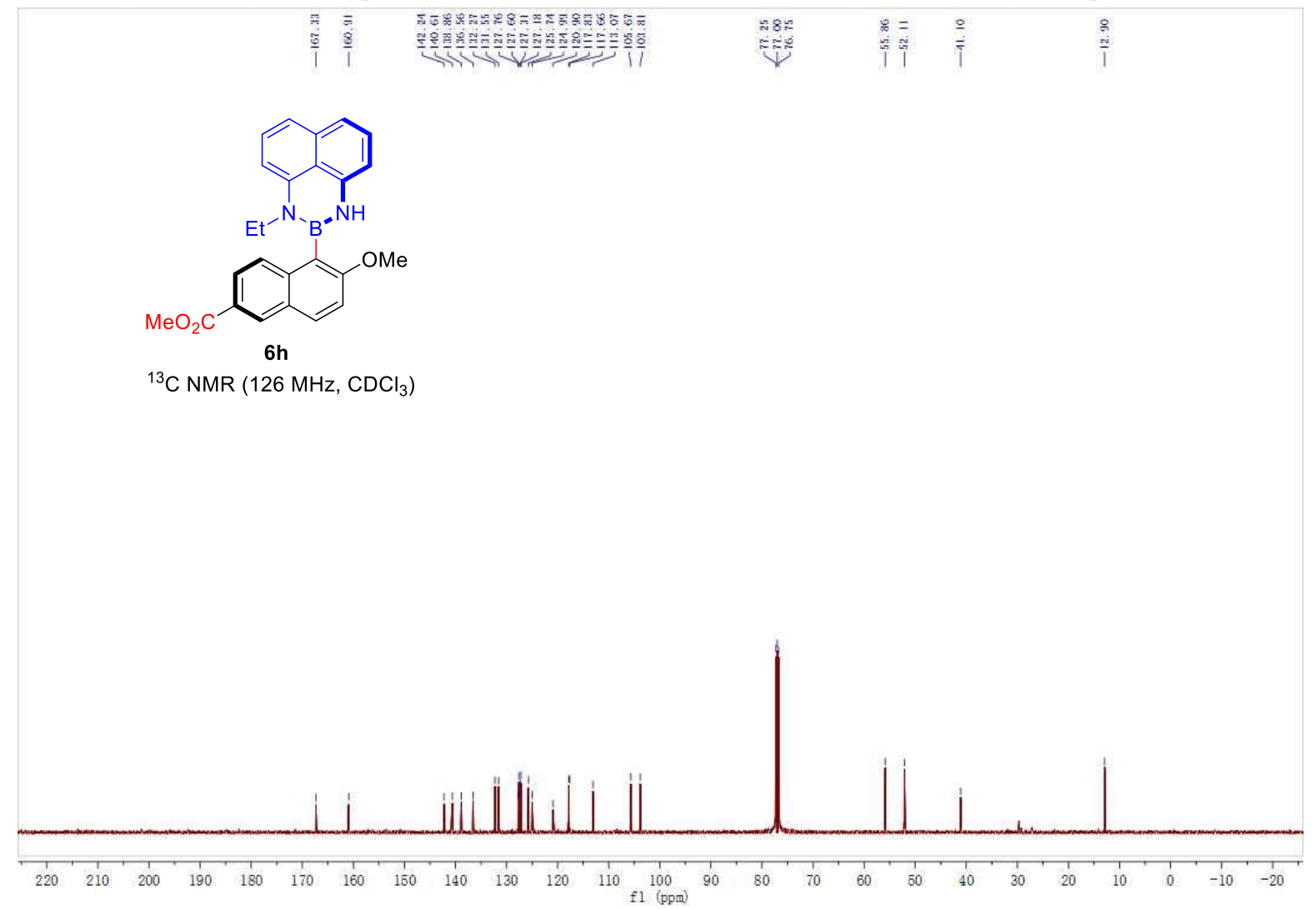

(S)-methyl 5-(1-ethyl-1H-naphtho[1,8-de][1,3,2]diazaborinin-2(3H)-yl)-6-methoxy-2-naphthoate (6h)

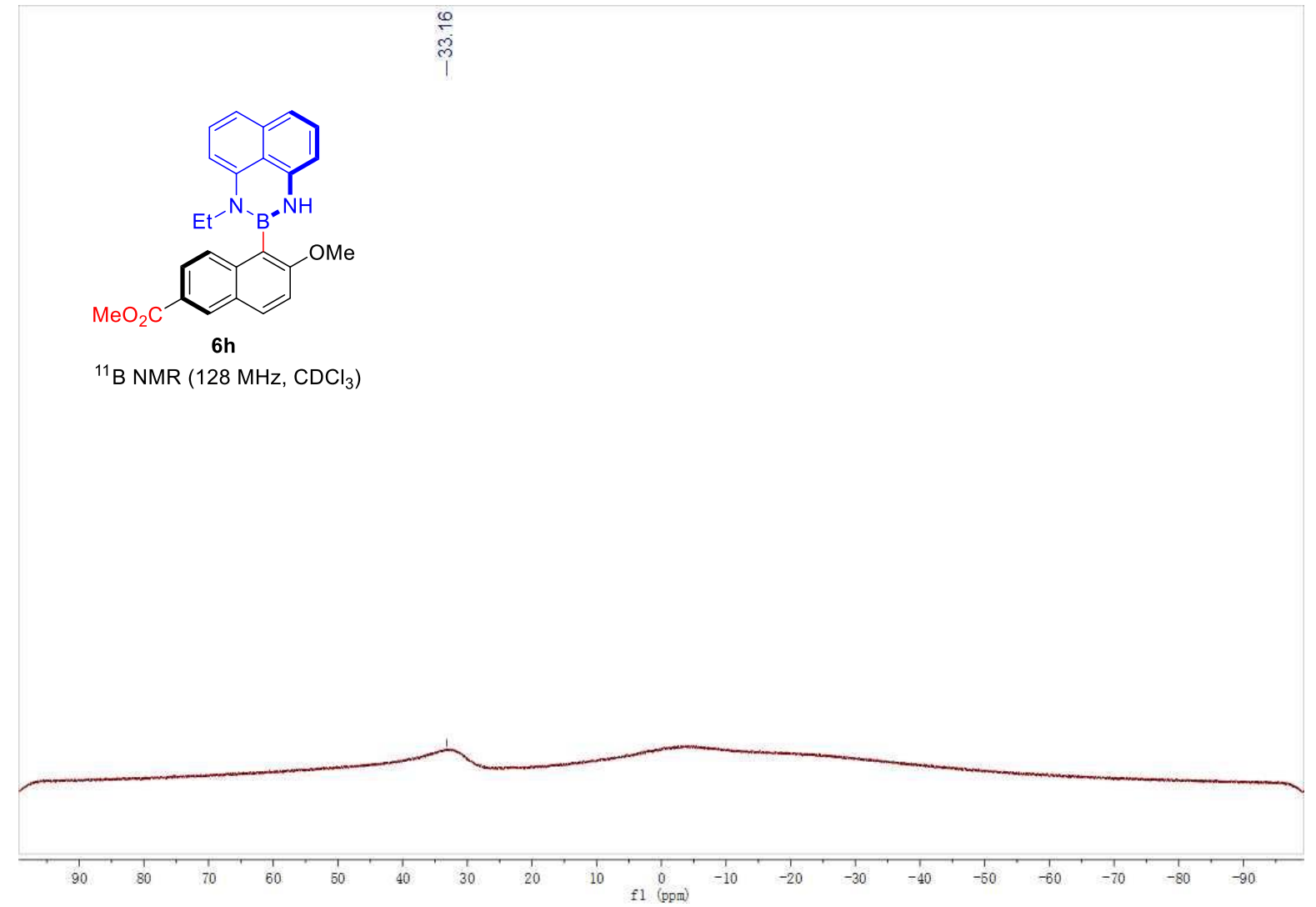


(S)-1-ethyl-2-(2-methoxy-6-phenylnaphthalen-1-yl)-2,3-dihydro-1H-naphtho[1,8-de][1,3,2]diazaborinine (6i)

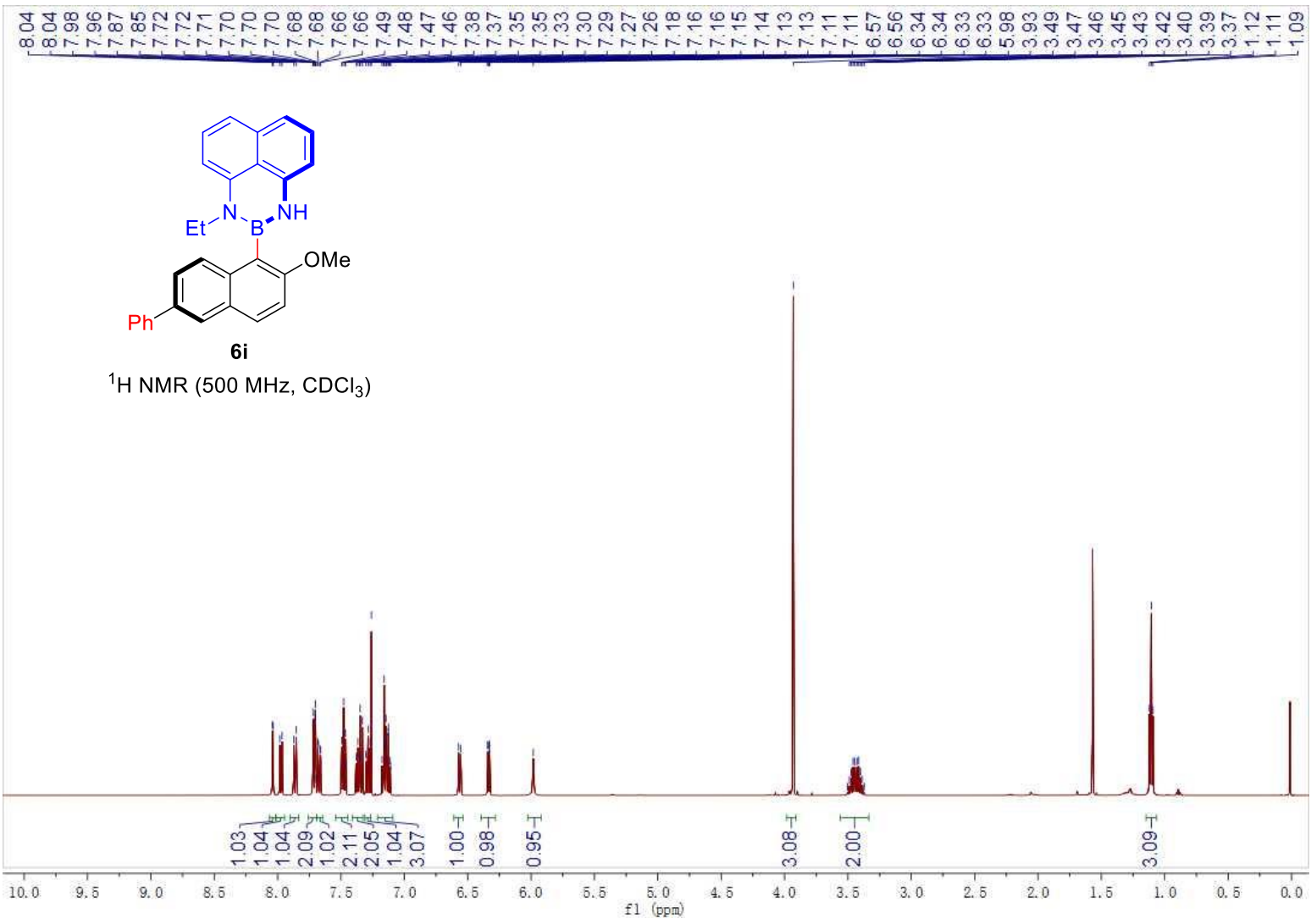

(S)-1-ethyl-2-(2-methoxy-6-phenylnaphthalen-1-yl)-2,3-dihydro-1H-naphtho[1,8-de][1,3,2]diazaborinine (6i)

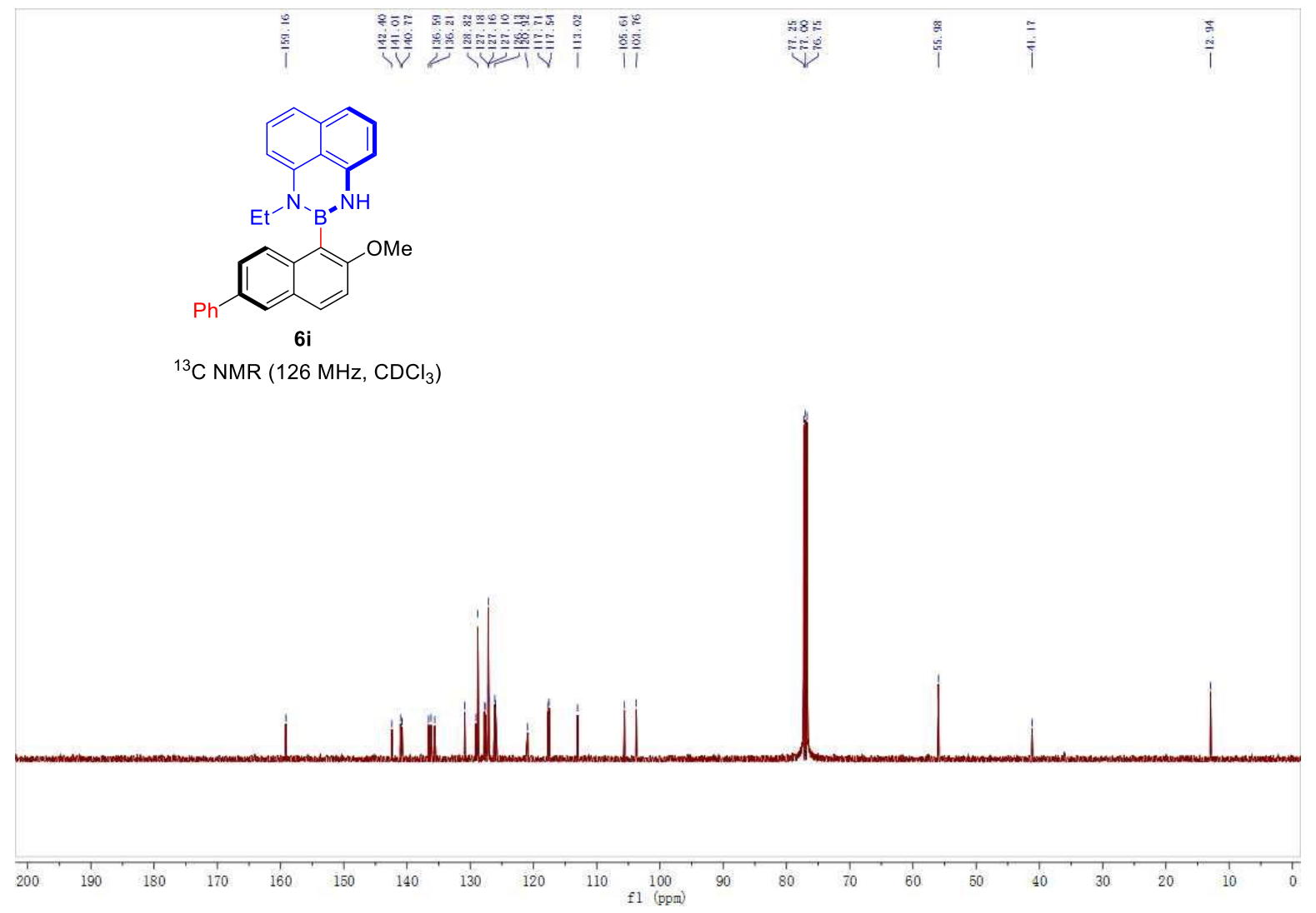


(S)-1-ethyl-2-(2-methoxy-6-phenylnaphthalen-1-yl)-2,3-dihydro-1H-naphtho[1,8-de][1,3,2]diazaborinine (6i)

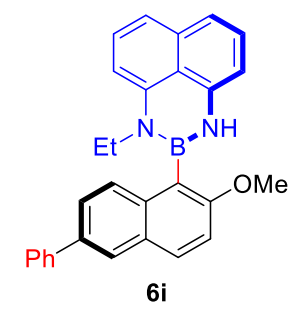

$$
\text { ণ্ণ }
$$

${ }^{11} \mathrm{~B}$ NMR $\left(128 \mathrm{MHz}, \mathrm{CDCl}_{3}\right)$

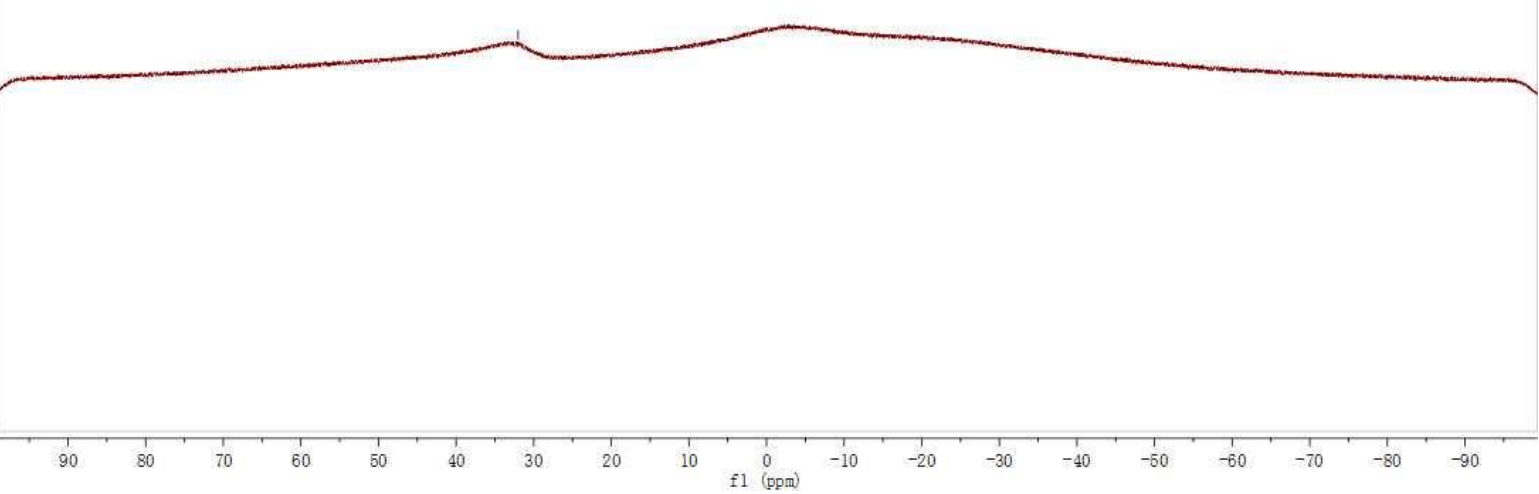

(S)-1-ethyl-2-(2-methoxy-6-methylnaphthalen-1-yl)-2,3-dihydro-1H-naphtho[1,8-de][1,3,2]diazaborinine (6j)

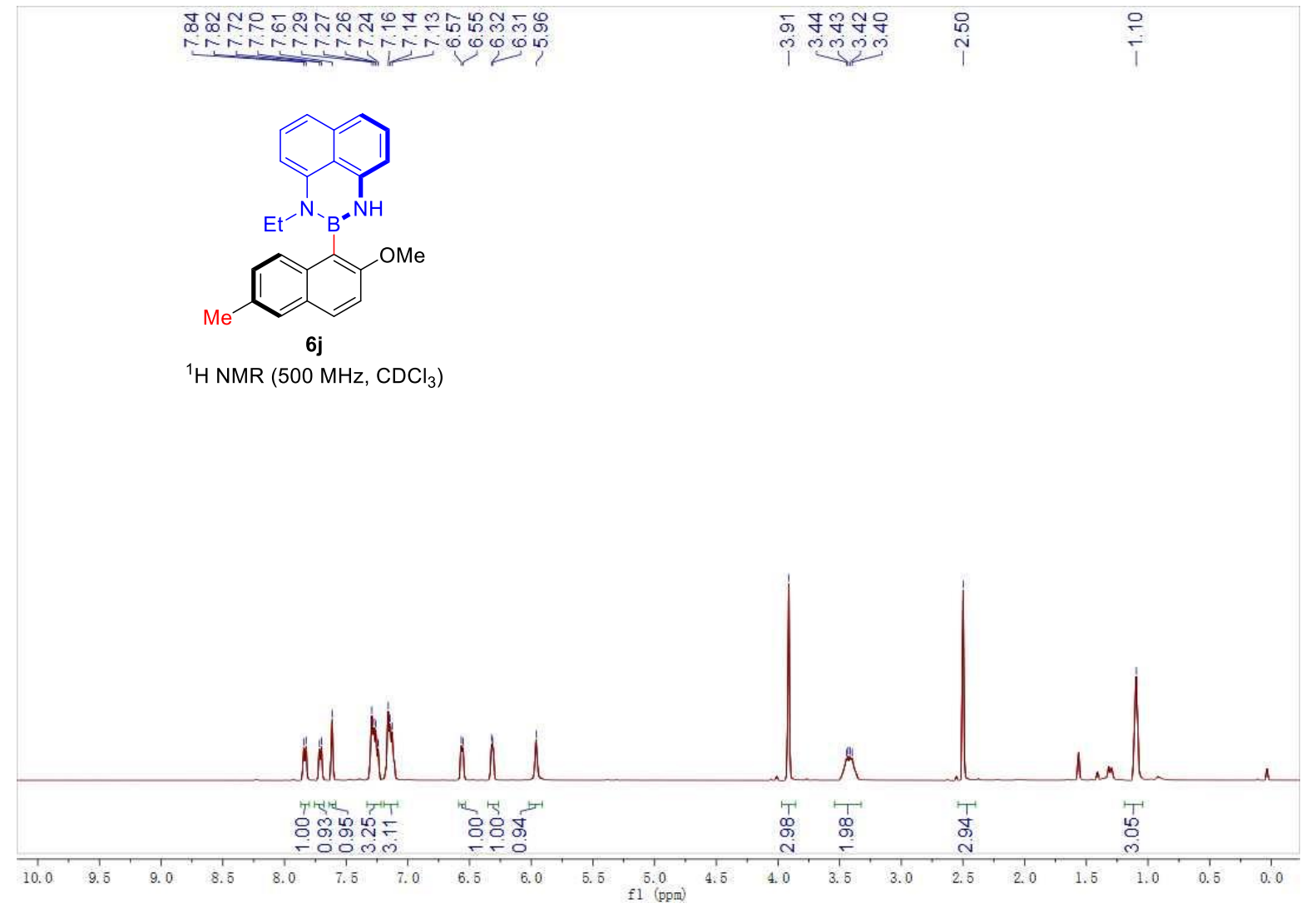


(S)-1-ethyl-2-(2-methoxy-6-methylnaphthalen-1-yl)-2,3-dihydro-1H-naphtho[1,8-de][1,3,2]diazaborinine (6j)

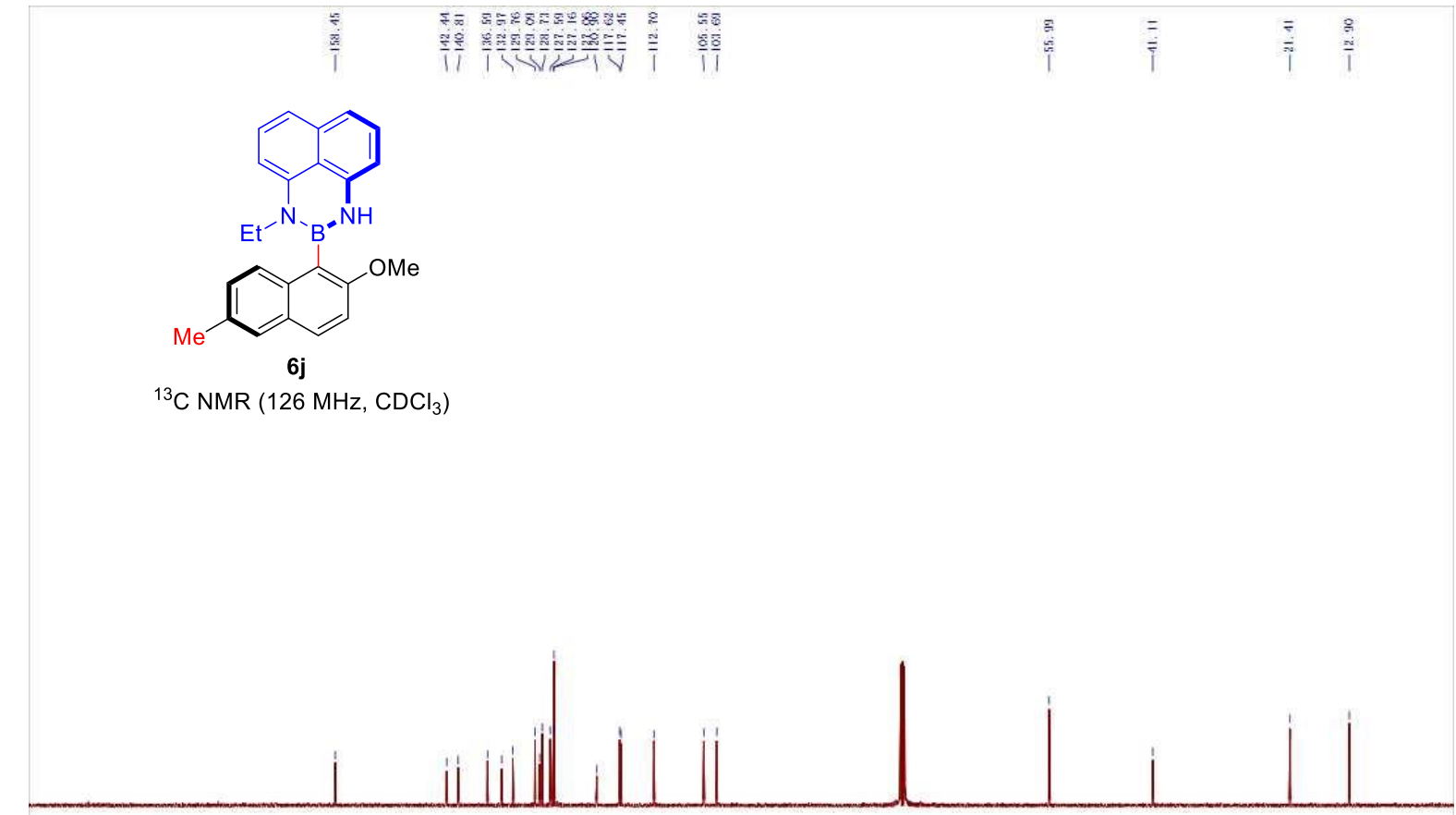

$\begin{array}{llll}= & = & 8 \\ i & i & i\end{array}$

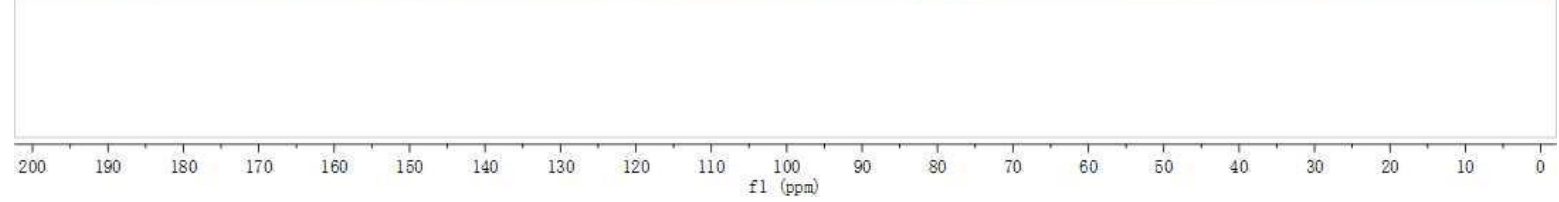

(S)-1-ethyl-2-(2-methoxy-6-methylnaphthalen-1-yl)-2,3-dihydro-1H-naphtho[1,8-de][1,3,2]diazaborinine (6j)<smiles>CCN1B(c2c(OC)ccc3cc(C)ccc23)Nc2cccc3cccc1c23</smiles>

\section{$\stackrel{\substack{\infty \\ \stackrel{\infty}{i}}}{\stackrel{\infty}{i}}$}

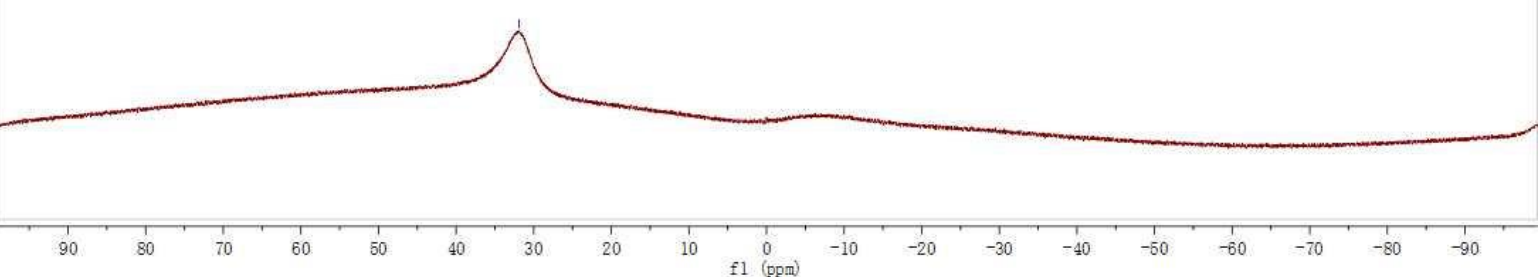


(S)-2-(2,6-dimethoxynaphthalen-1-yl)-1-ethyl-2,3-dihydro-1H-naphtho[1,8-de][1,3,2]diazaborinine (6k)

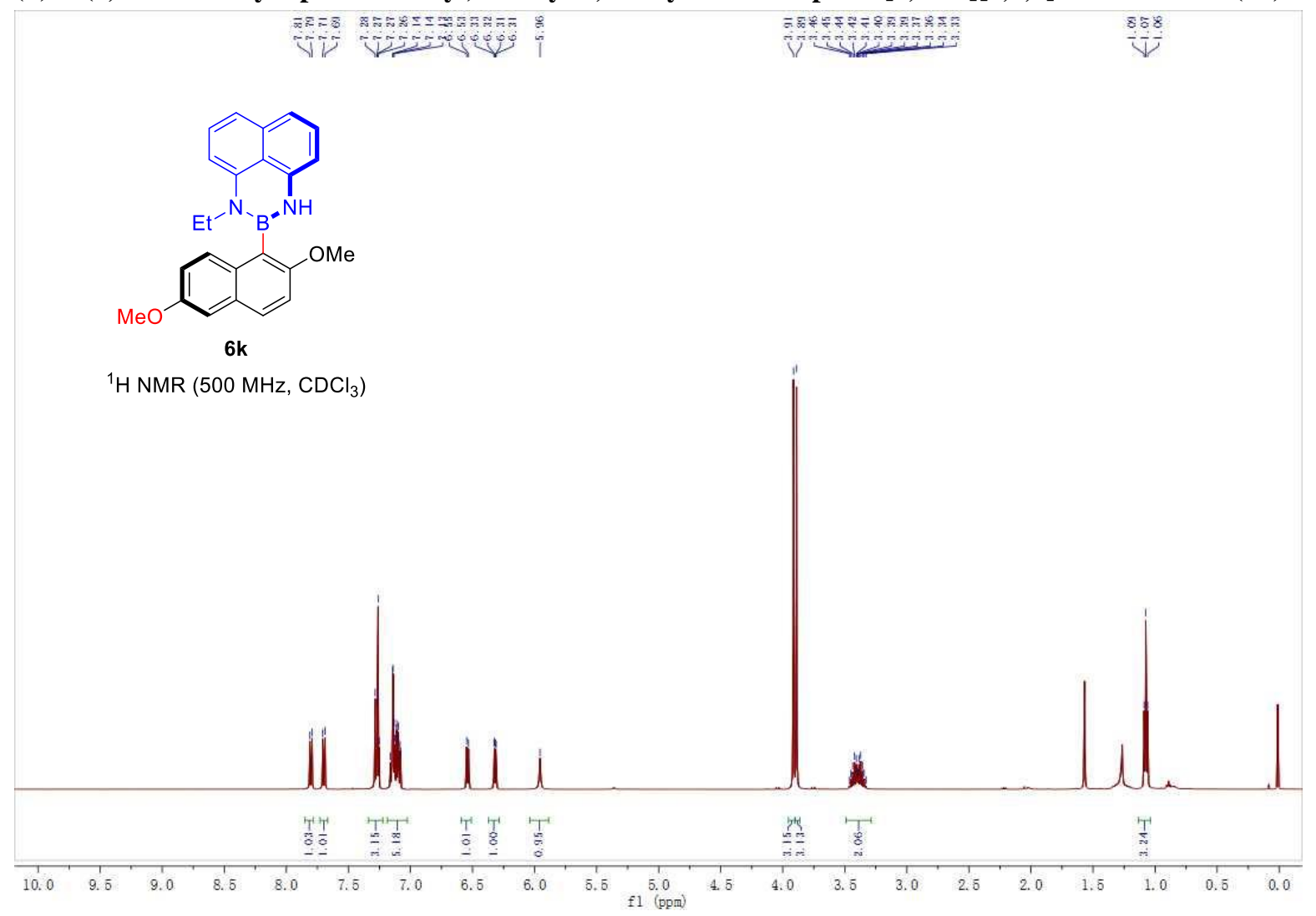

(S)-2-(2,6-dimethoxynaphthalen-1-yl)-1-ethyl-2,3-dihydro-1H-naphtho[1,8-de][1,3,2]diazaborinine (6k)

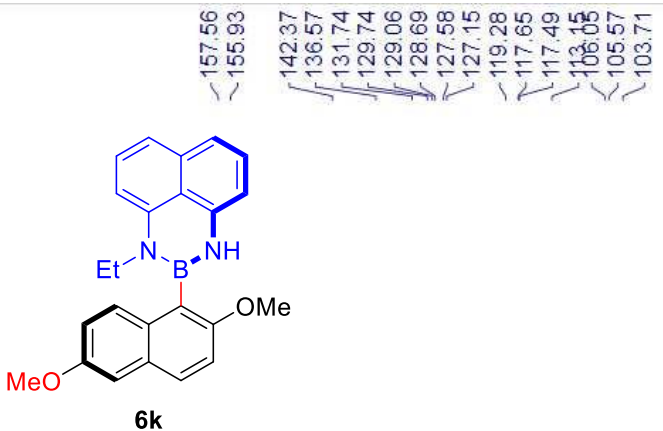

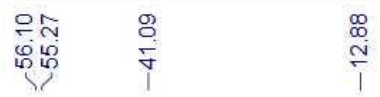

${ }^{13} \mathrm{C} \mathrm{NMR}\left(126 \mathrm{MHz}, \mathrm{CDCl}_{3}\right)$

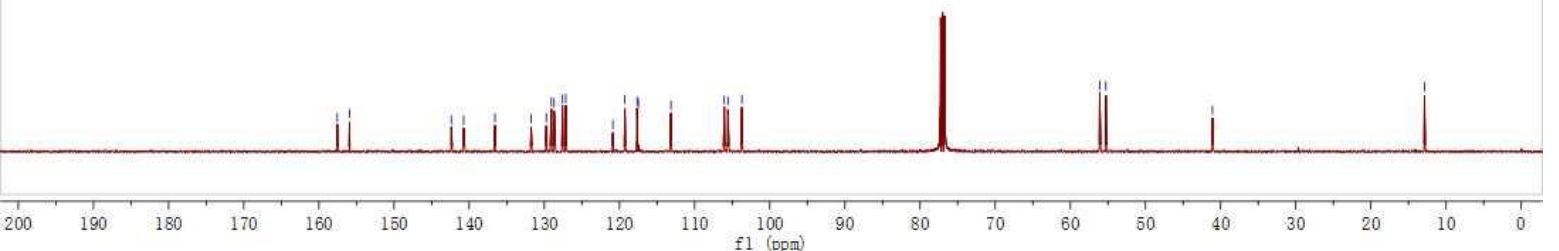


(S)-2-(2,6-dimethoxynaphthalen-1-yl)-1-ethyl-2,3-dihydro-1H-naphtho[1,8-de][1,3,2]diazaborinine (6k)

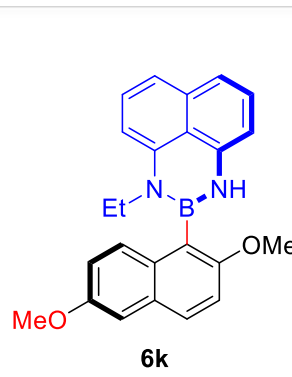

$\stackrel{\substack{m\\}}{i}$

${ }^{11}$ B NMR $\left(128 \mathrm{MHz}, \mathrm{CDCl}_{3}\right)$

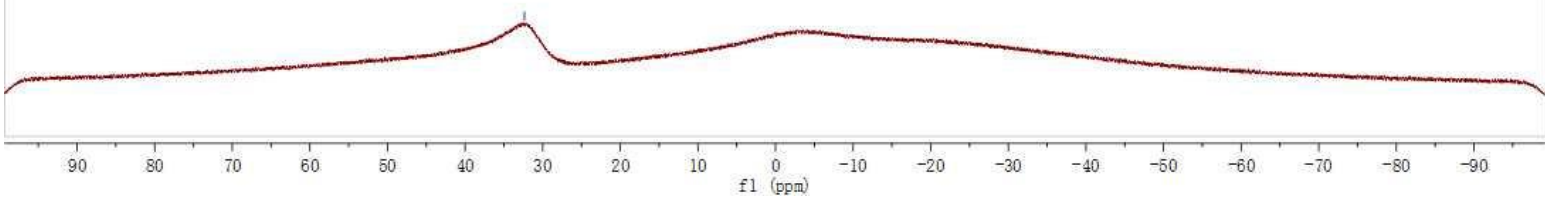

(S,S)-methyl-2-(5-(1-ethyl-1H-naphtho[1,8-de][1,3,2]diazaborinin-2(3H)-yl)-6-methoxynaphthalen-2-yl) propanoate (61)

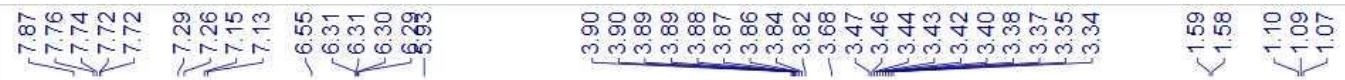

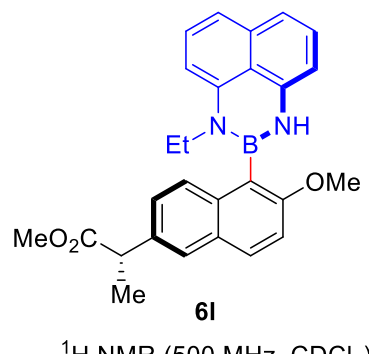

${ }^{1} \mathrm{H}$ NMR $\left(500 \mathrm{MHz}, \mathrm{CDCl}_{3}\right)$

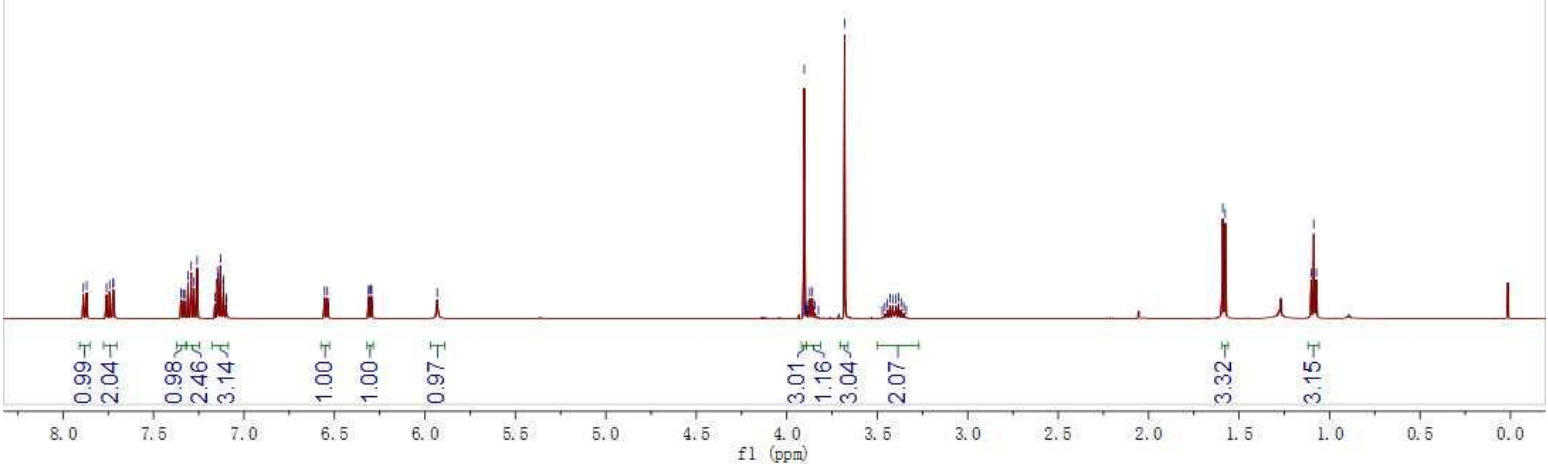


(S,S)-methyl-2-(5-(1-ethyl-1H-naphtho[1,8-de][1,3,2]diazaborinin-2(3H)-yl)-6-methoxynaphthalen-2-yl) propanoate (61)

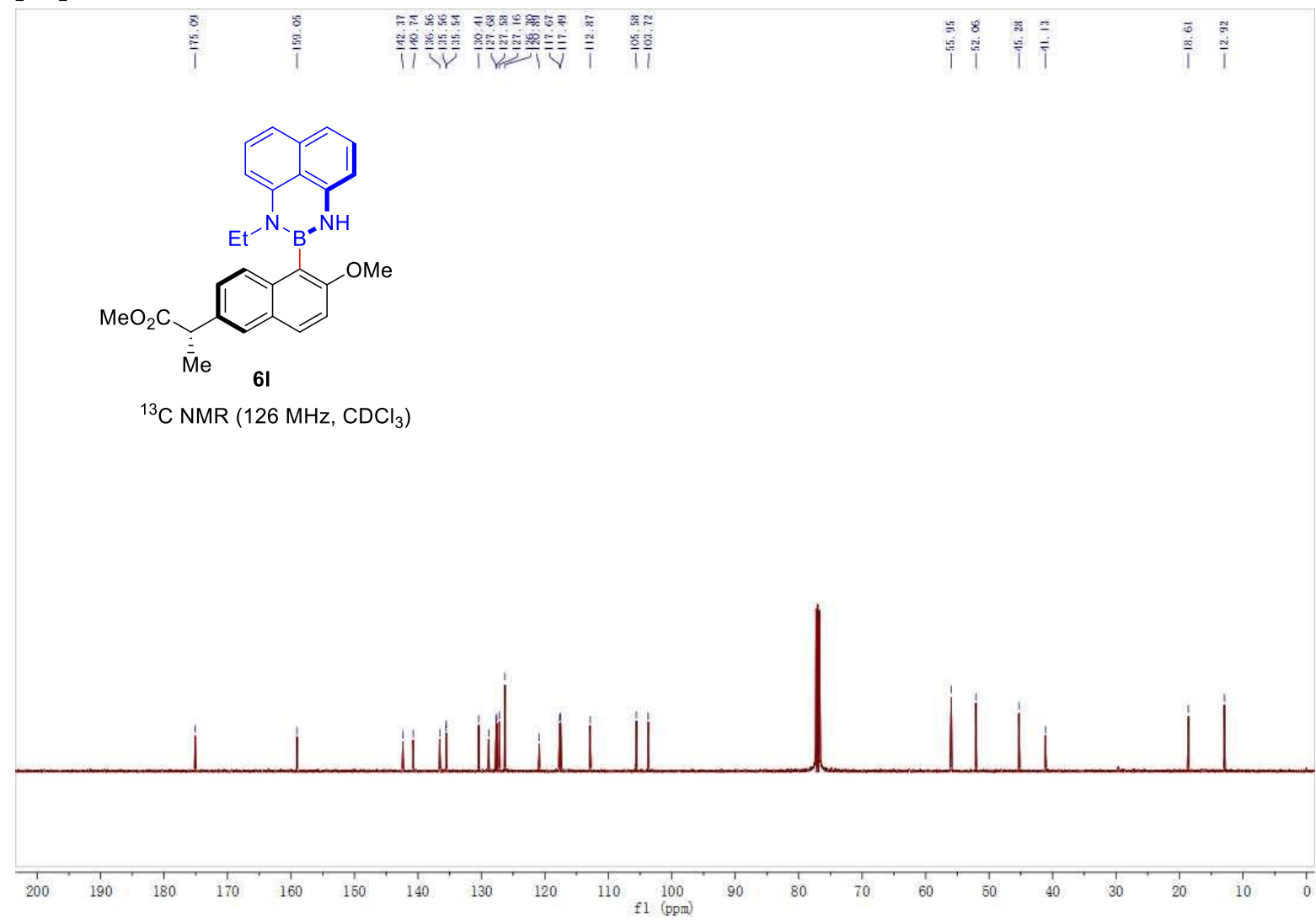

(S,S)-methyl-2-(5-(1-ethyl-1H-naphtho[1,8-de][1,3,2]diazaborinin-2(3H)-yl)-6-methoxynaphthalen-2-yl) propanoate (6l)

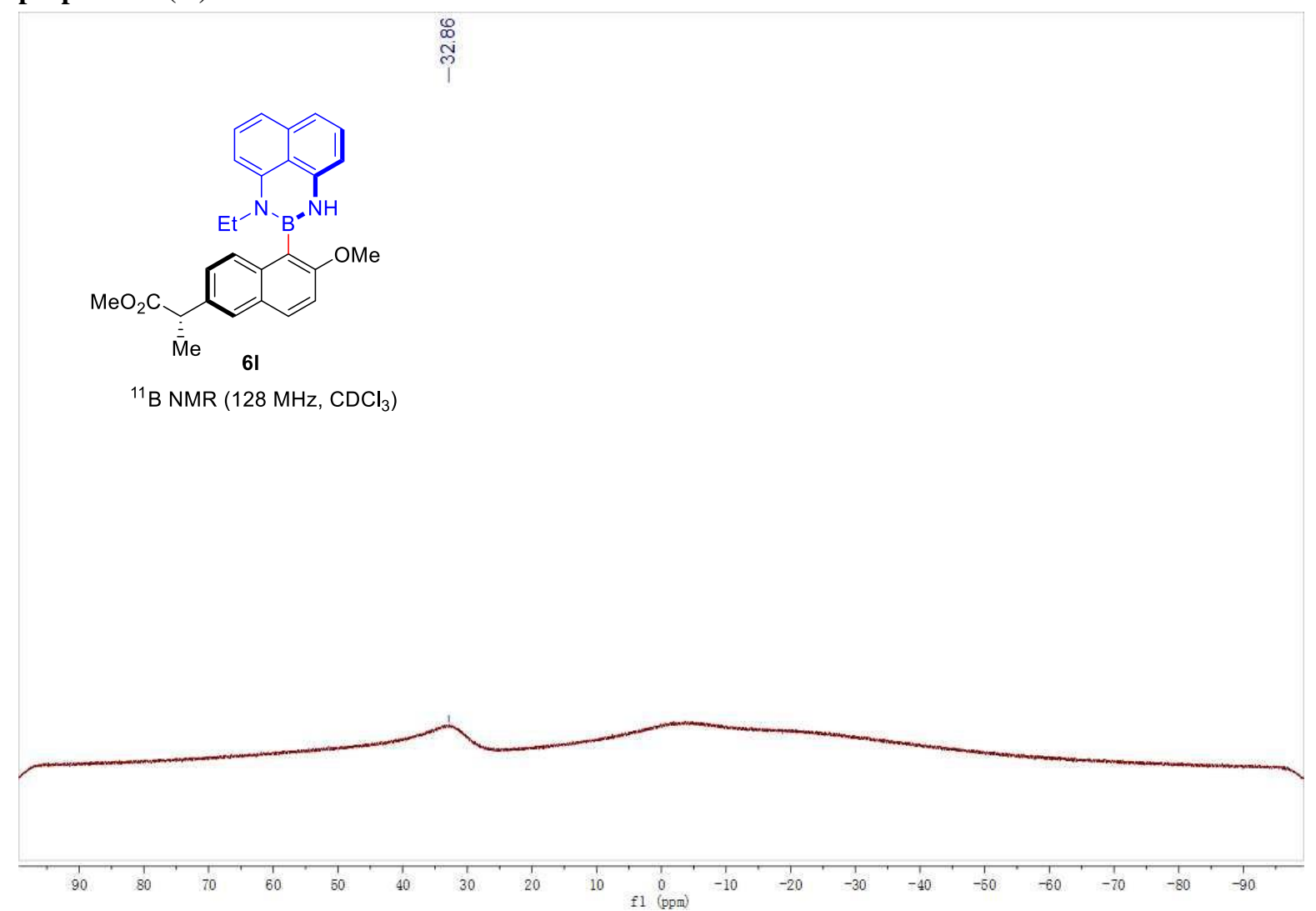


(S)-1-ethyl-2-(2-methoxy-3-phenylnaphthalen-1-yl)-2,3-dihydro-1H-naphtho[1,8-de][1,3,2]diazaborinine $(6 \mathrm{~m})$

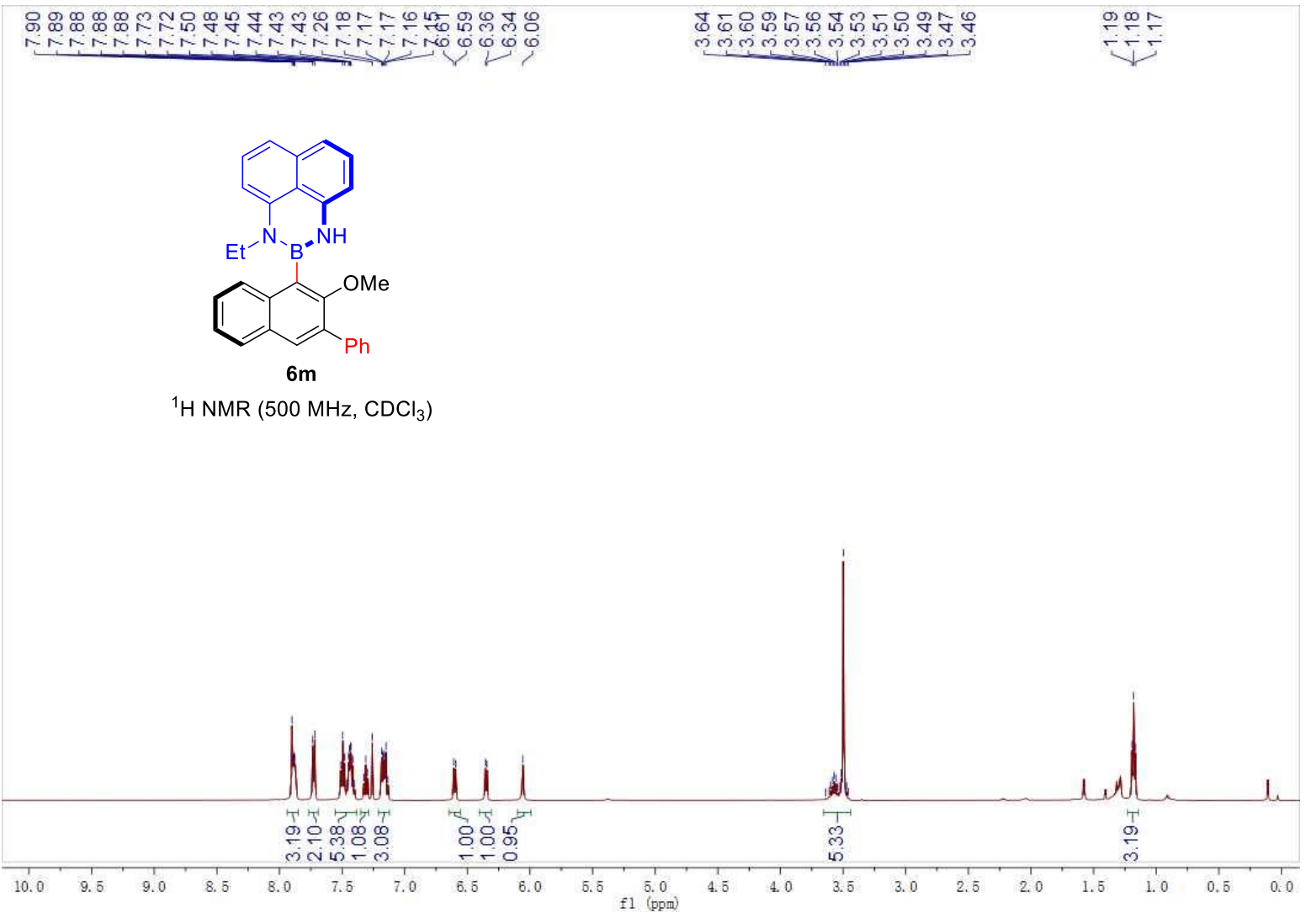

(S)-1-ethyl-2-(2-methoxy-3-phenylnaphthalen-1-yl)-2,3-dihydro-1H-naphtho[1,8-de][1,3,2]diazaborinine $(6 \mathrm{~m})$

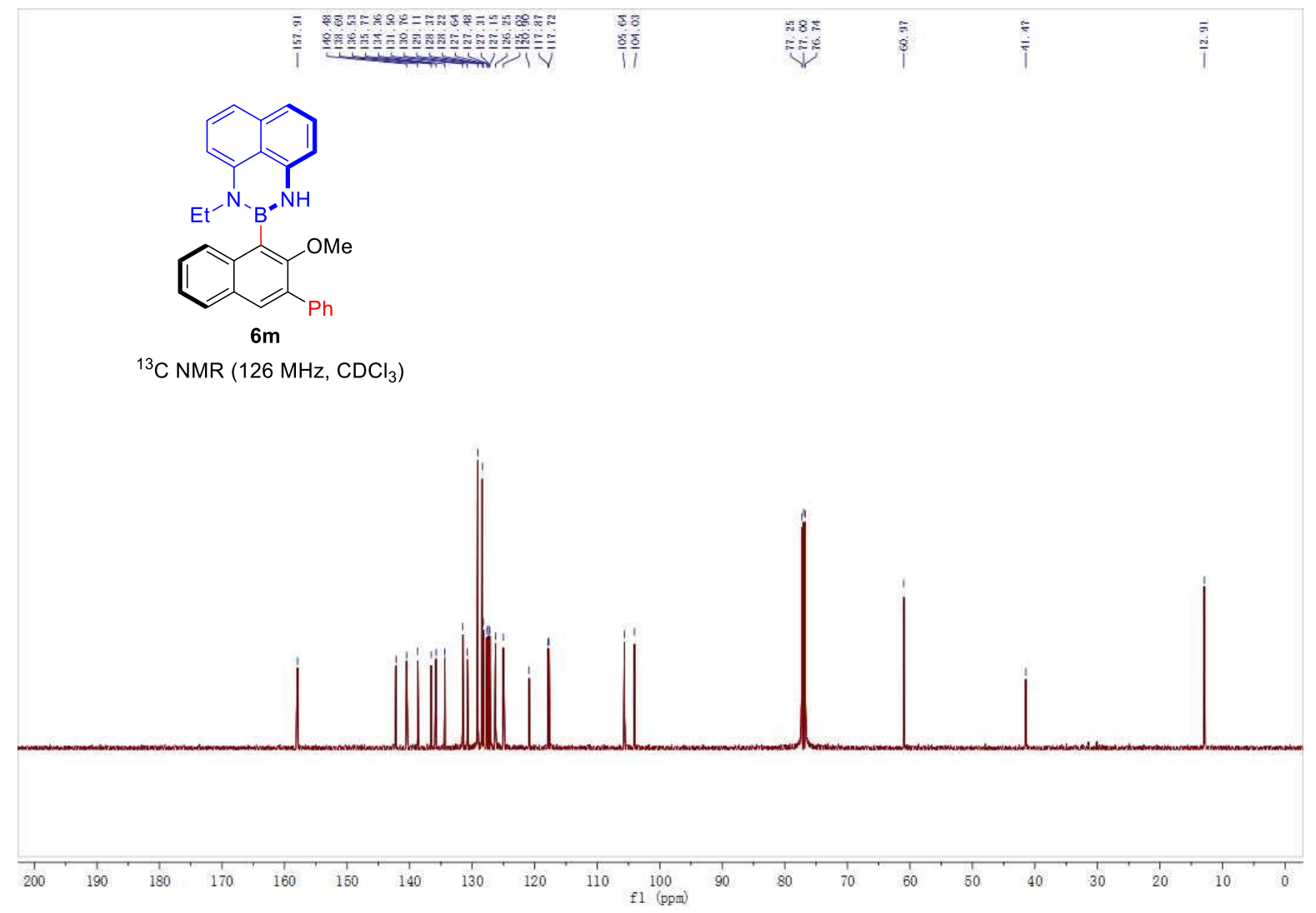


(S)-1-ethyl-2-(2-methoxy-3-phenylnaphthalen-1-yl)-2,3-dihydro-1H-naphtho[1,8-de][1,3,2] diazaborinine $(6 \mathrm{~m})$

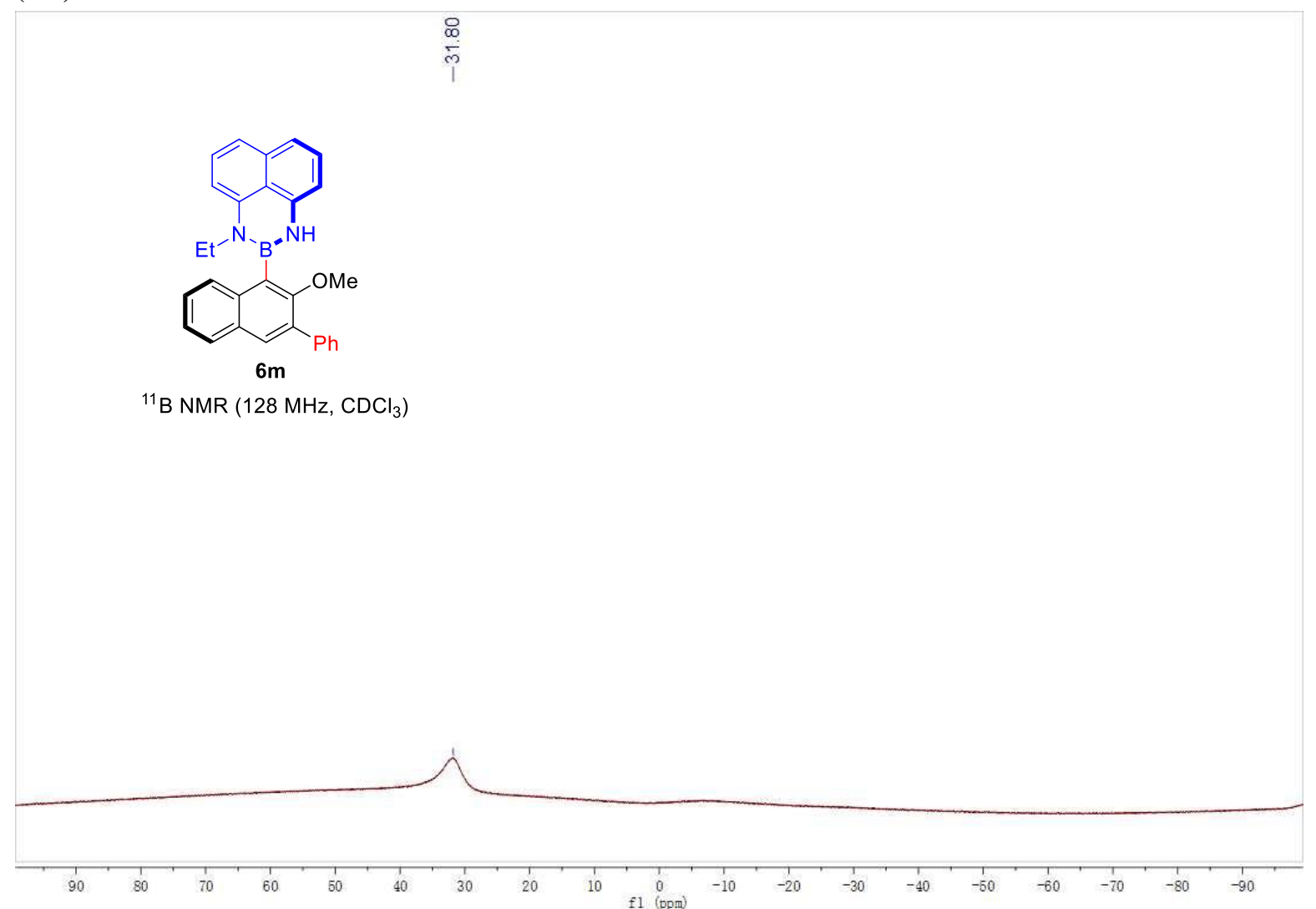

(S)-methyl 4-(1-ethyl-1H-naphtho[1,8-de][1,3,2]diazaborinin-2(3H)-yl)-3-methoxy-2-naphthoate (6n)

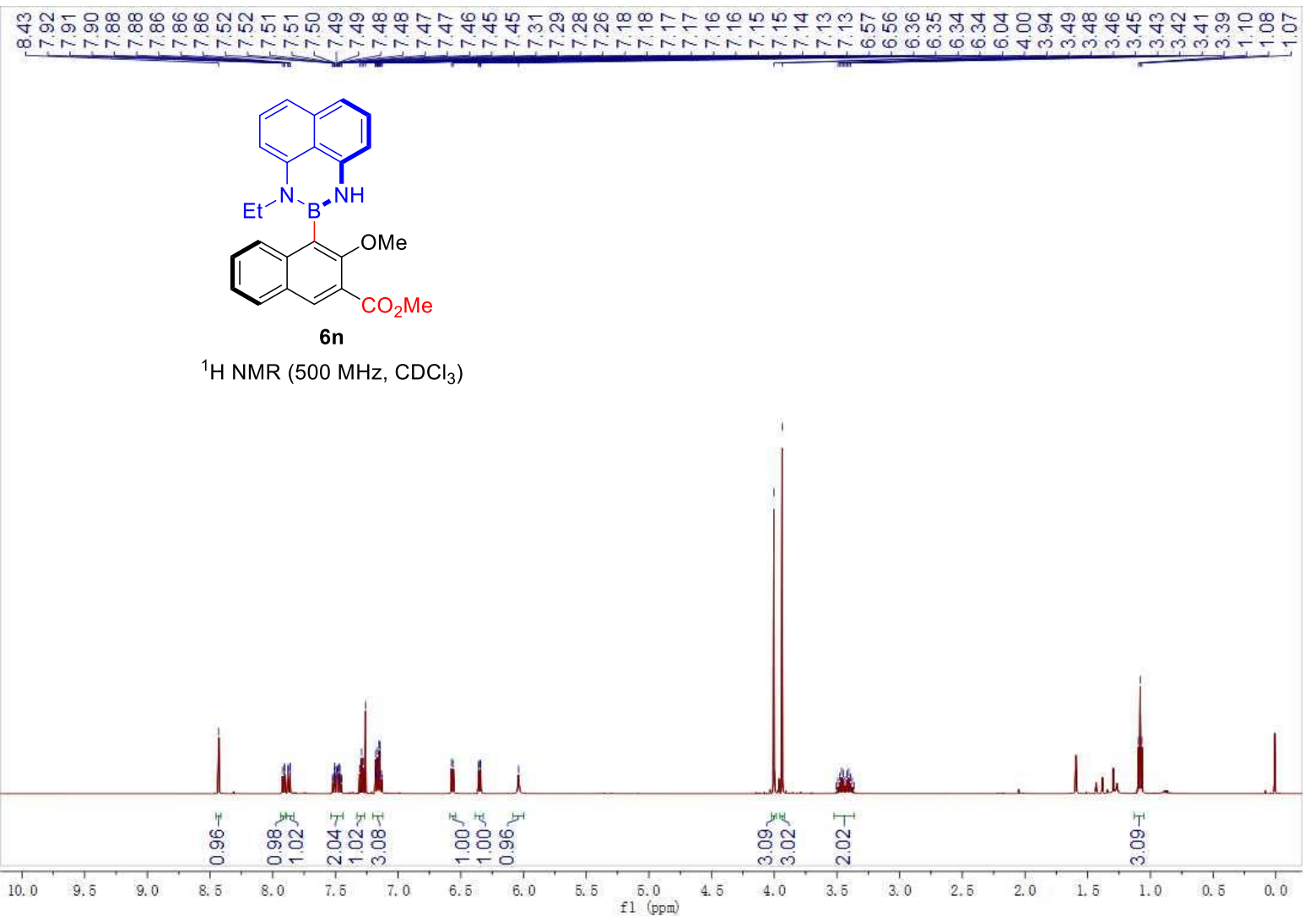


(S)-methyl 4-(1-ethyl-1H-naphtho[1,8-de][1,3,2]diazaborinin-2(3H)-yl)-3-methoxy-2-naphthoate (6n)

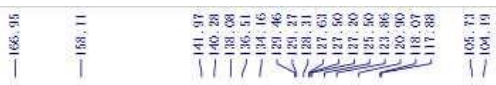

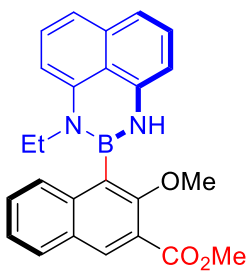

6n

${ }^{13} \mathrm{C}$ NMR $\left(126 \mathrm{MHz}, \mathrm{CDCl}_{3}\right)$
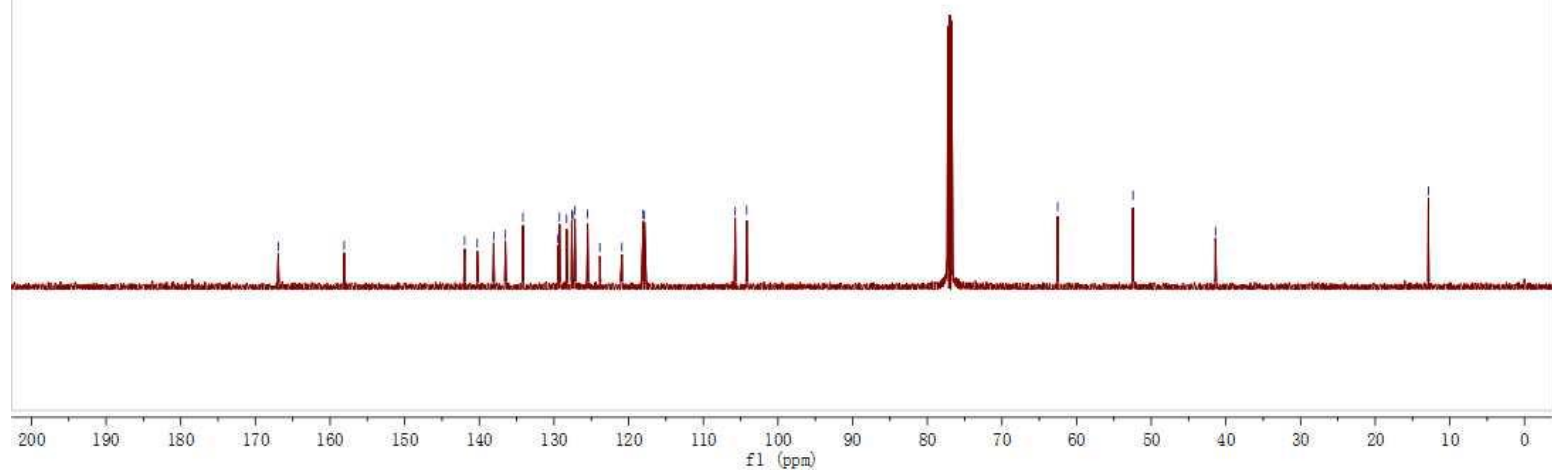

(S)-methyl 4-(1-ethyl-1H-naphtho[1,8-de][1,3,2]diazaborinin-2(3H)-yl)-3-methoxy-2-naphthoate (6n)

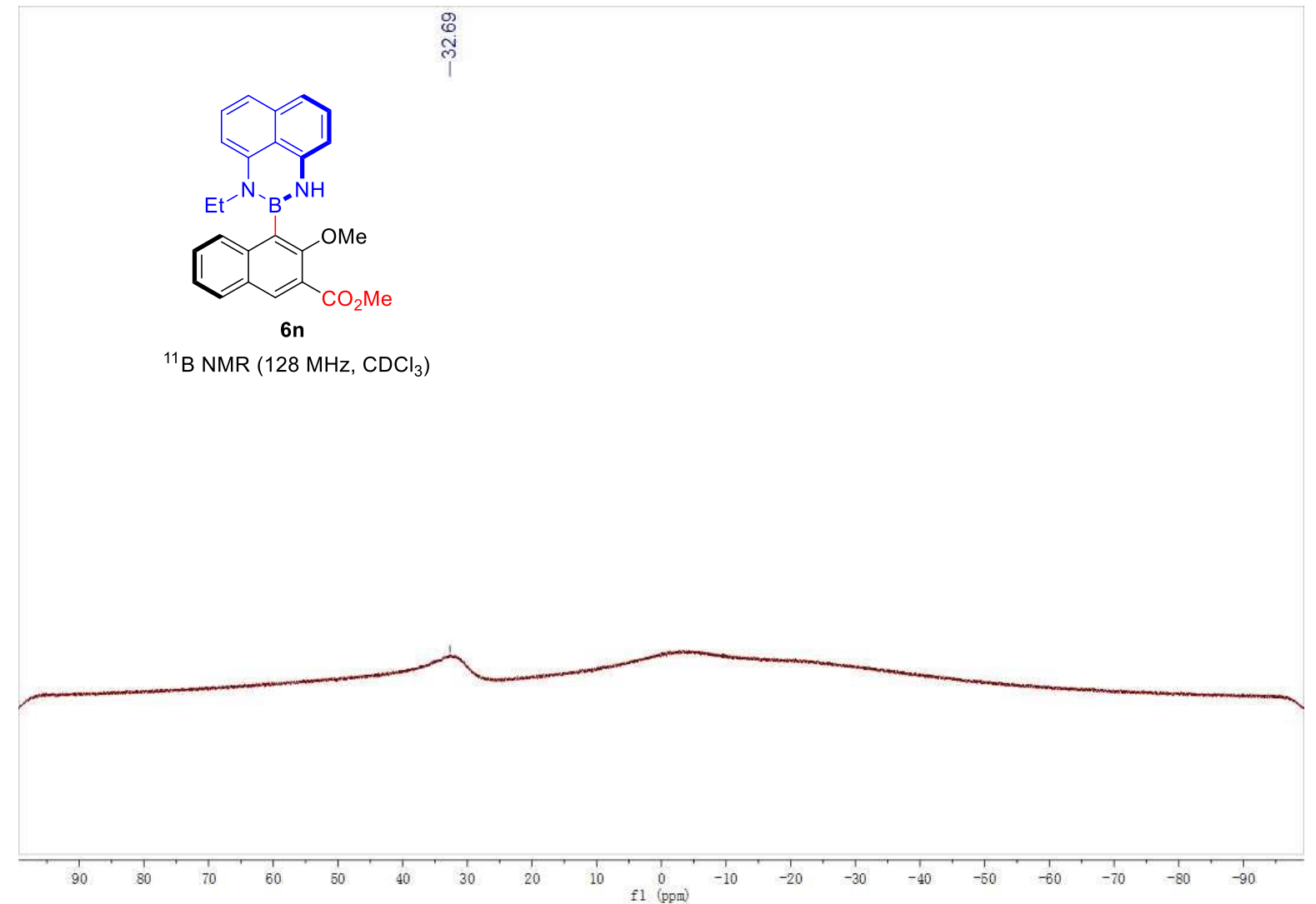


(S)-methyl 5-(1-ethyl-1H-naphtho[1,8-de][1,3,2]diazaborinin-2(3H)-yl)-6-methoxy-1-naphthoate (6o)

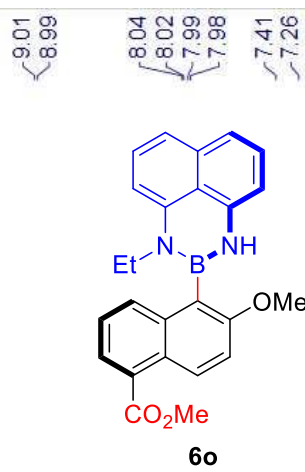

${ }^{1} \mathrm{H}$ NMR $\left(500 \mathrm{MHz}, \mathrm{CDCl}_{3}\right)$

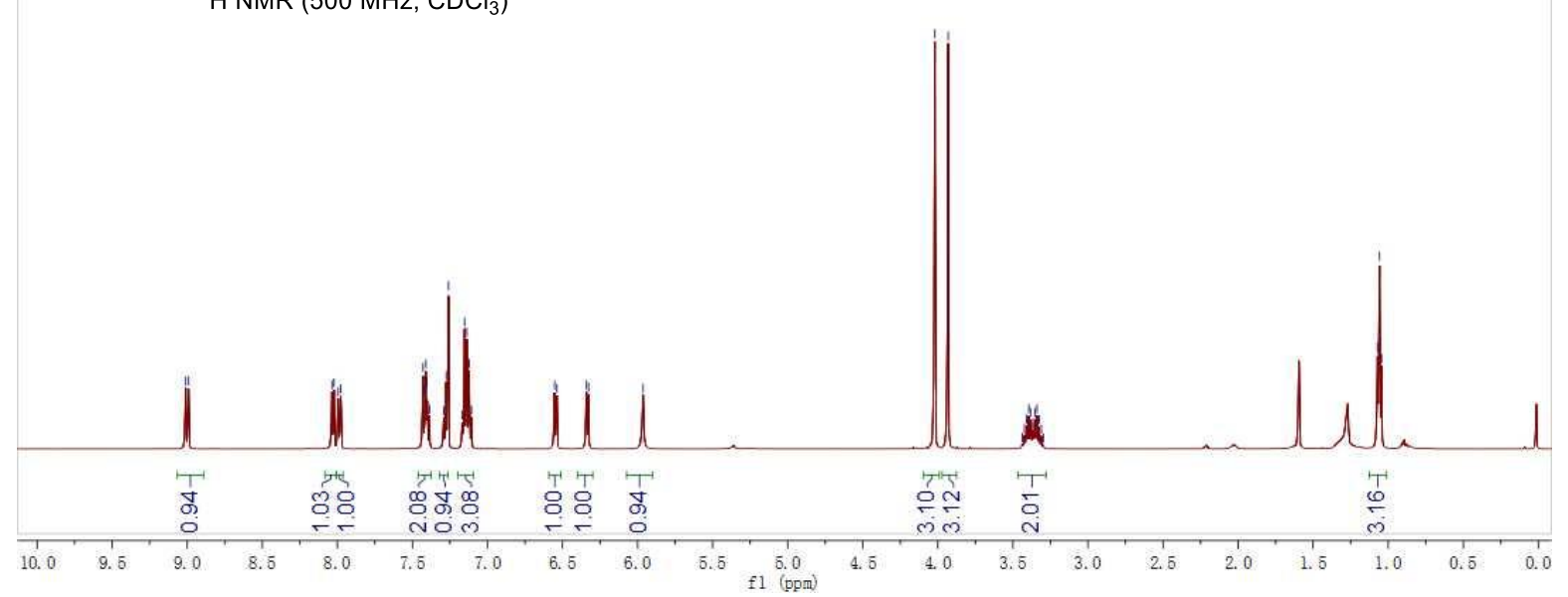

(S)-methyl 5-(1-ethyl-1H-naphtho[1,8-de][1,3,2]diazaborinin-2(3H)-yl)-6-methoxy-1-naphthoate (6o)

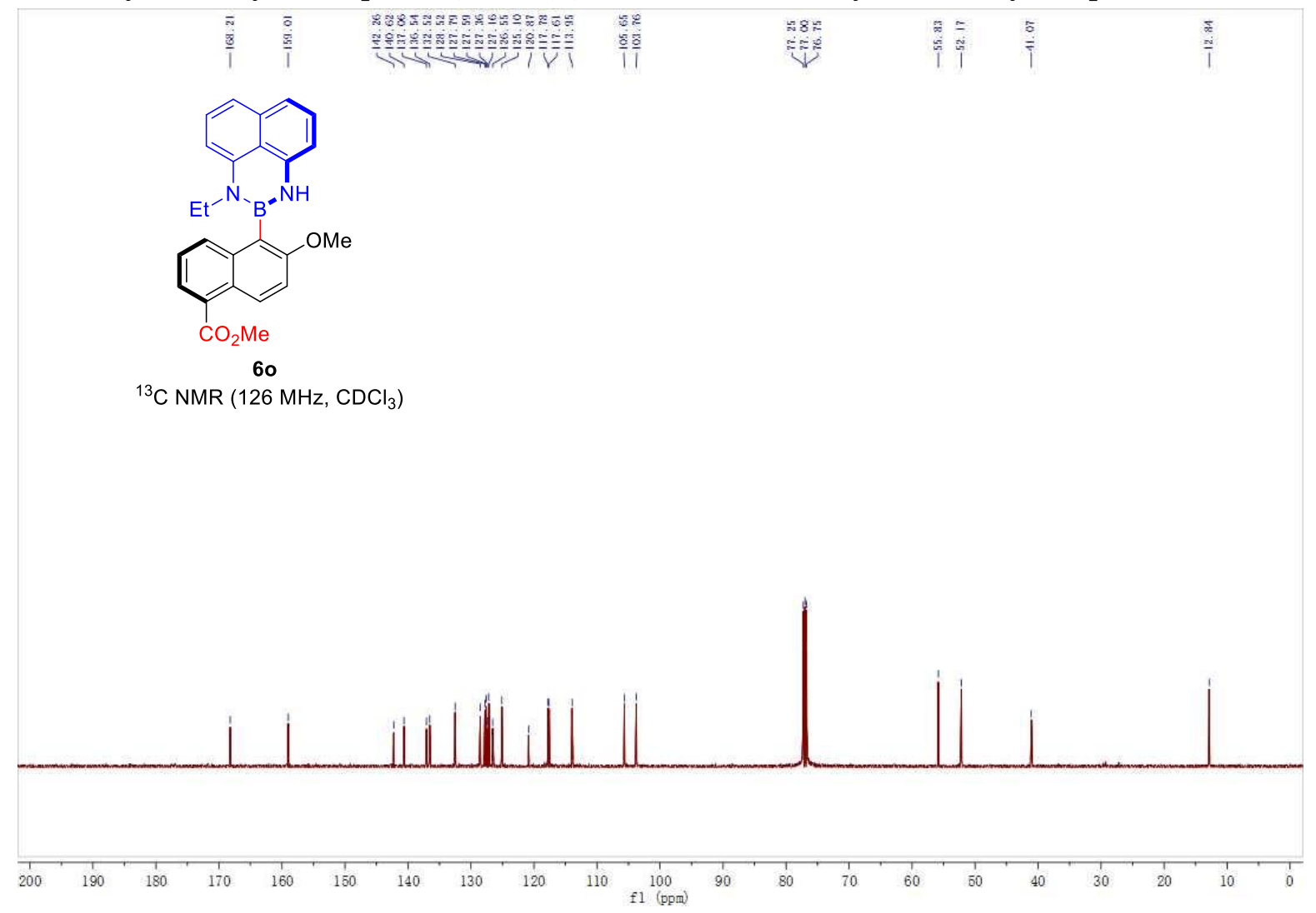


(S)-methyl 5-(1-ethyl-1H-naphtho[1,8-de][1,3,2]diazaborinin-2(3H)-yl)-6-methoxy-1-naphthoate (6o)

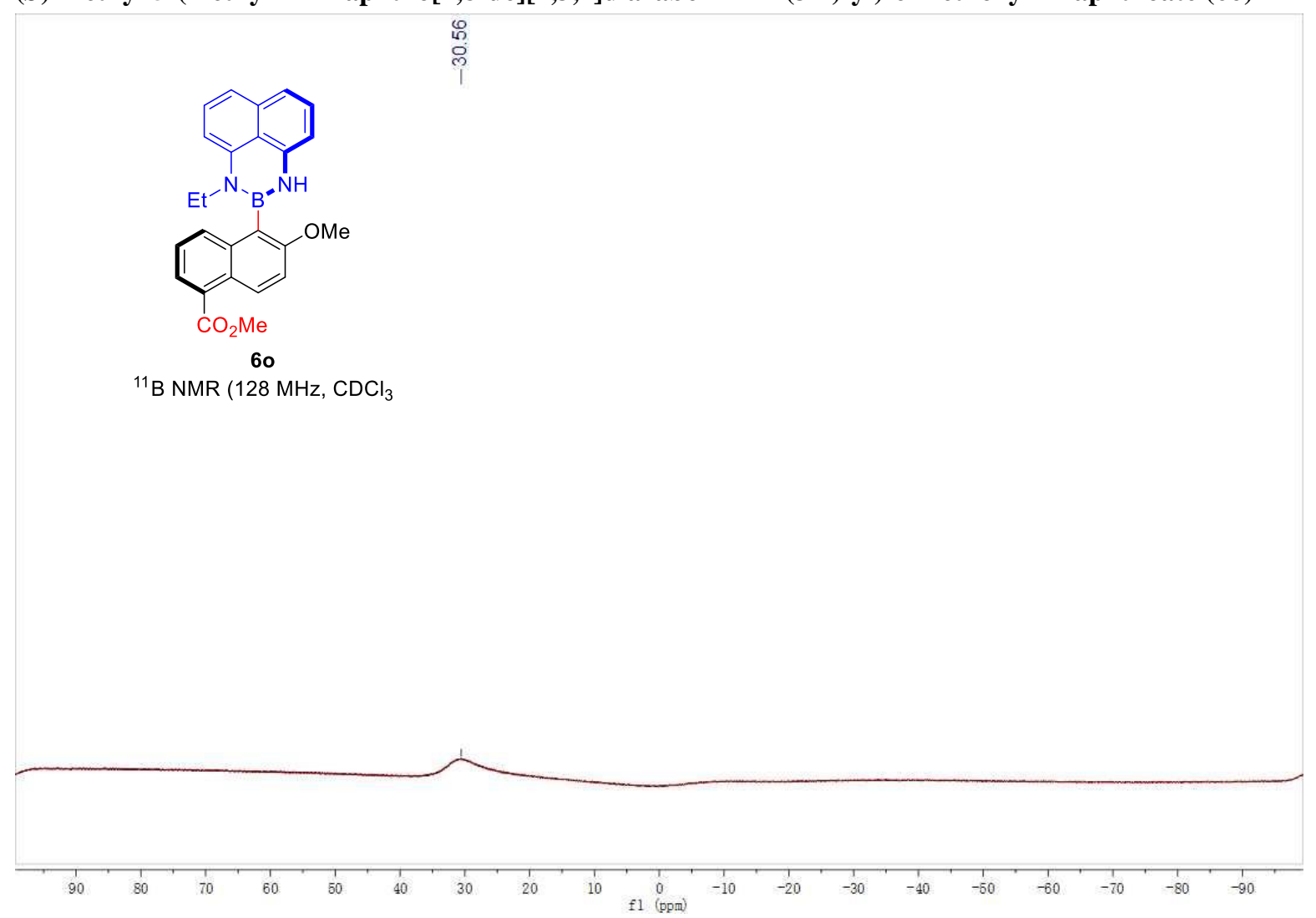

(S)-2-(2,7-dimethoxynaphthalen-1-yl)-1-ethyl-2,3-dihydro-1H-naphtho[1,8-de][1,3,2]diazaborinine (6p)

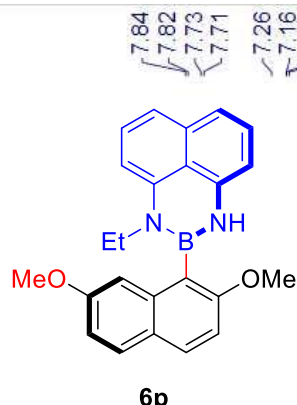

${ }^{1} \mathrm{H}$ NMR $\left(500 \mathrm{MHz}, \mathrm{CDCl}_{3}\right)$

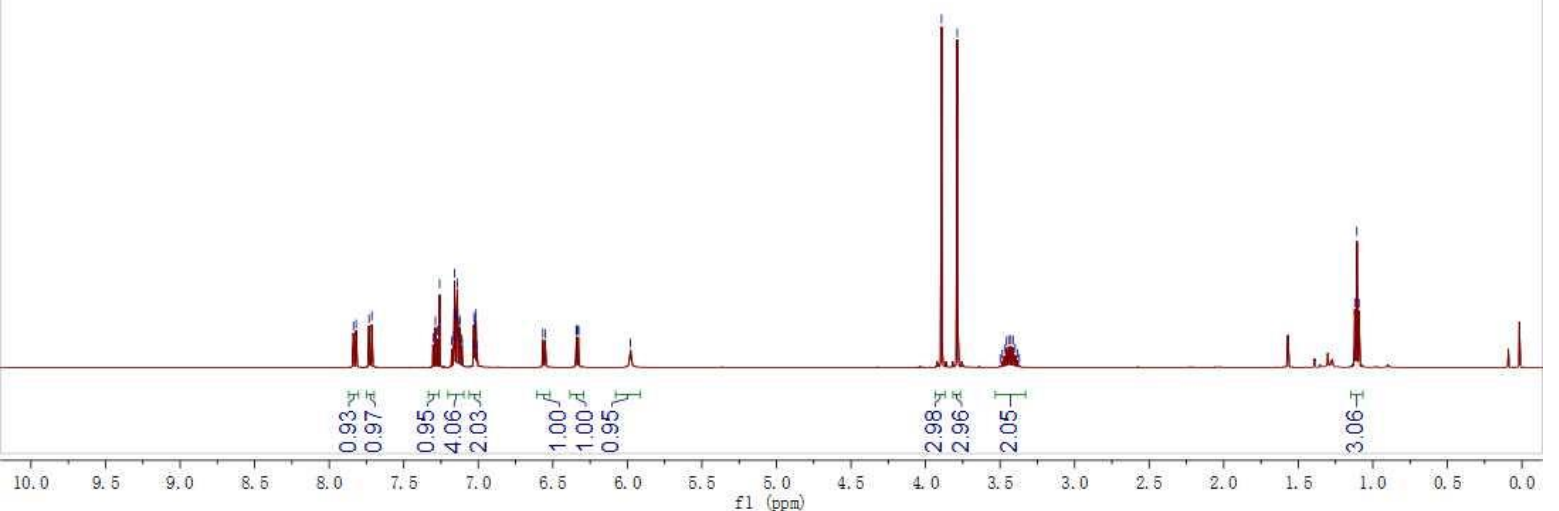


(S)-2-(2,7-dimethoxynaphthalen-1-yl)-1-ethyl-2,3-dihydro-1H-naphtho[1,8-de][1,3,2]diazaborinine (6p)

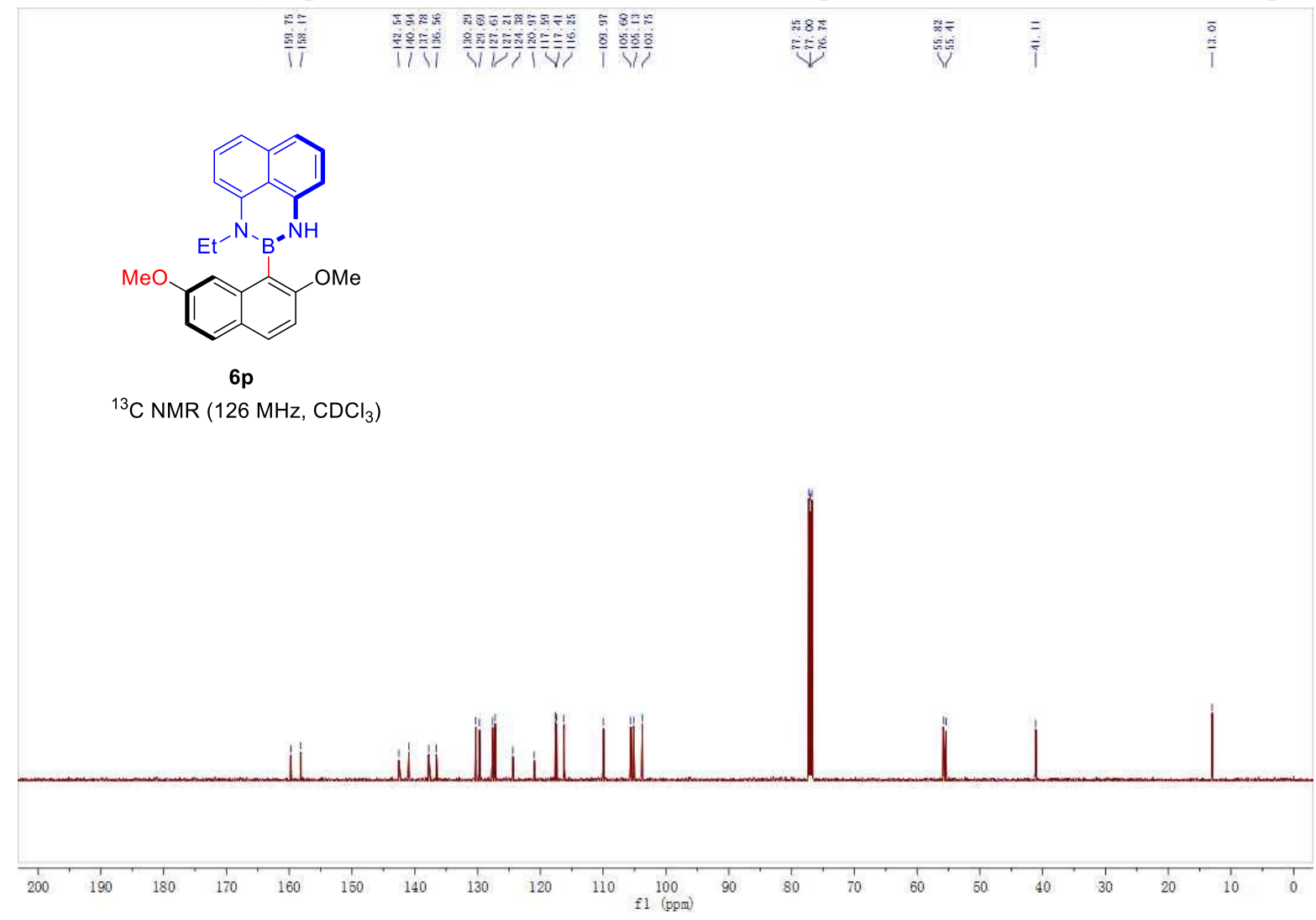

(S)-2-(2,7-dimethoxynaphthalen-1-yl)-1-ethyl-2,3-dihydro-1H-naphtho[1,8-de][1,3,2]diazaborinine (6p)

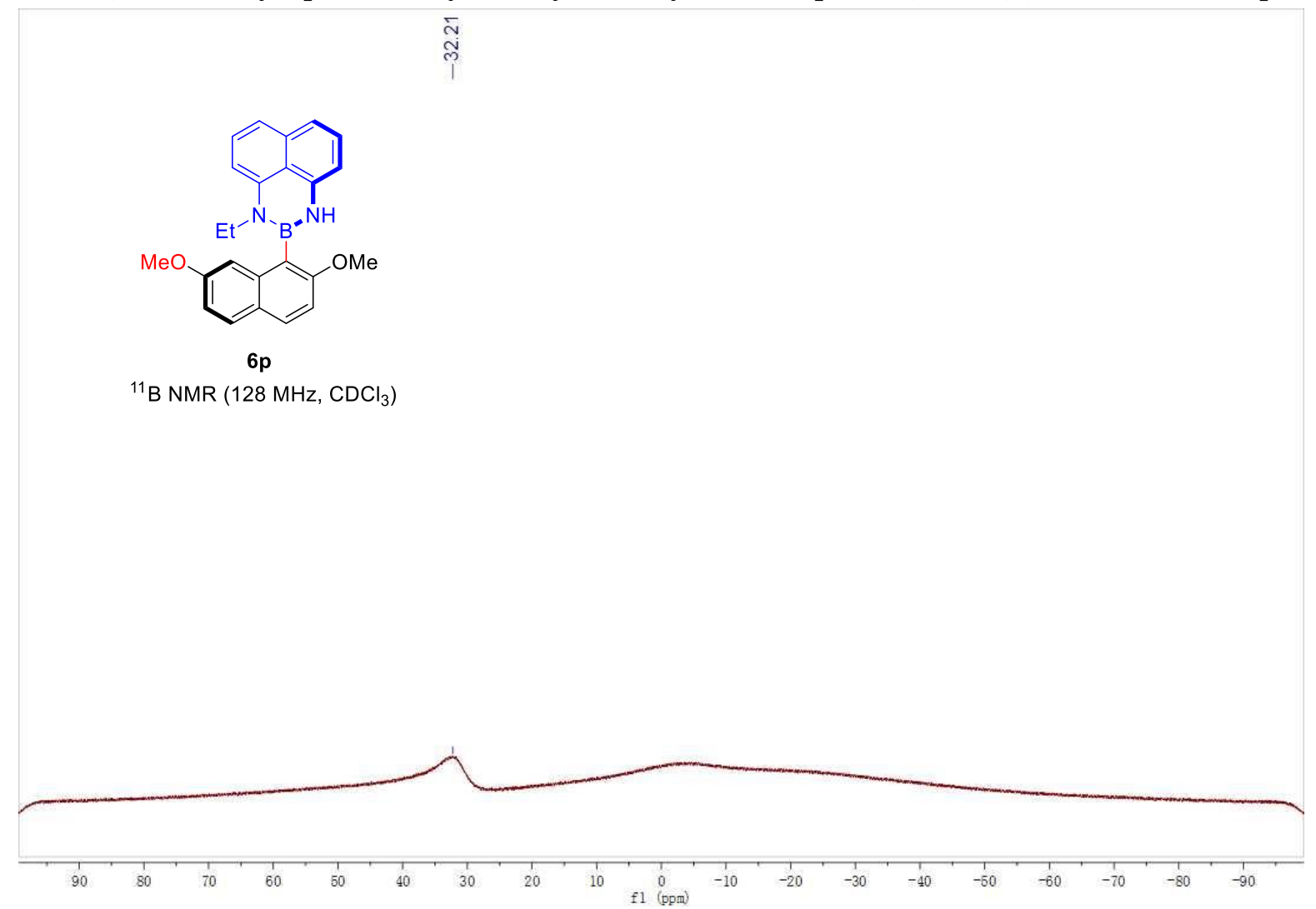


(S)-1-ethyl-2-(6-methoxyquinolin-5-yl)-2,3-dihydro-1H-naphtho[1,8-de][1,3,2]diazaborinine (6q)

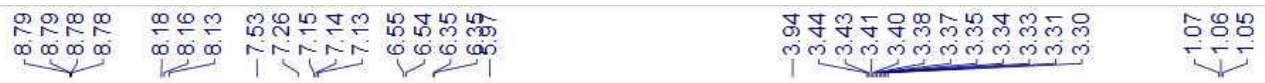
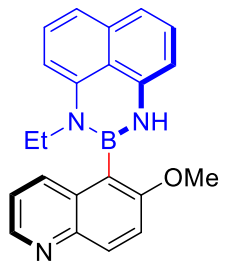

$6 q$

${ }^{1} \mathrm{H} \mathrm{NMR}\left(500 \mathrm{MHz}, \mathrm{CDCl}_{3}\right)$

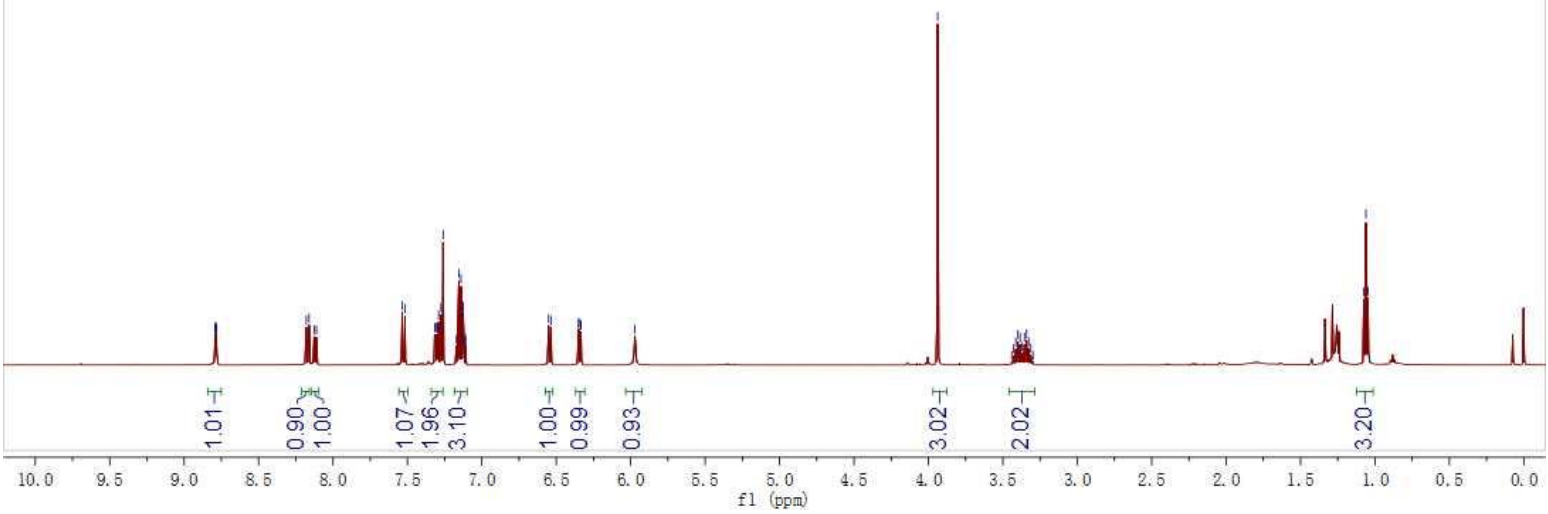

(S)-1-ethyl-2-(6-methoxyquinolin-5-yl)-2,3-dihydro-1H-naphtho[1,8-de][1,3,2]diazaborinine (6q)

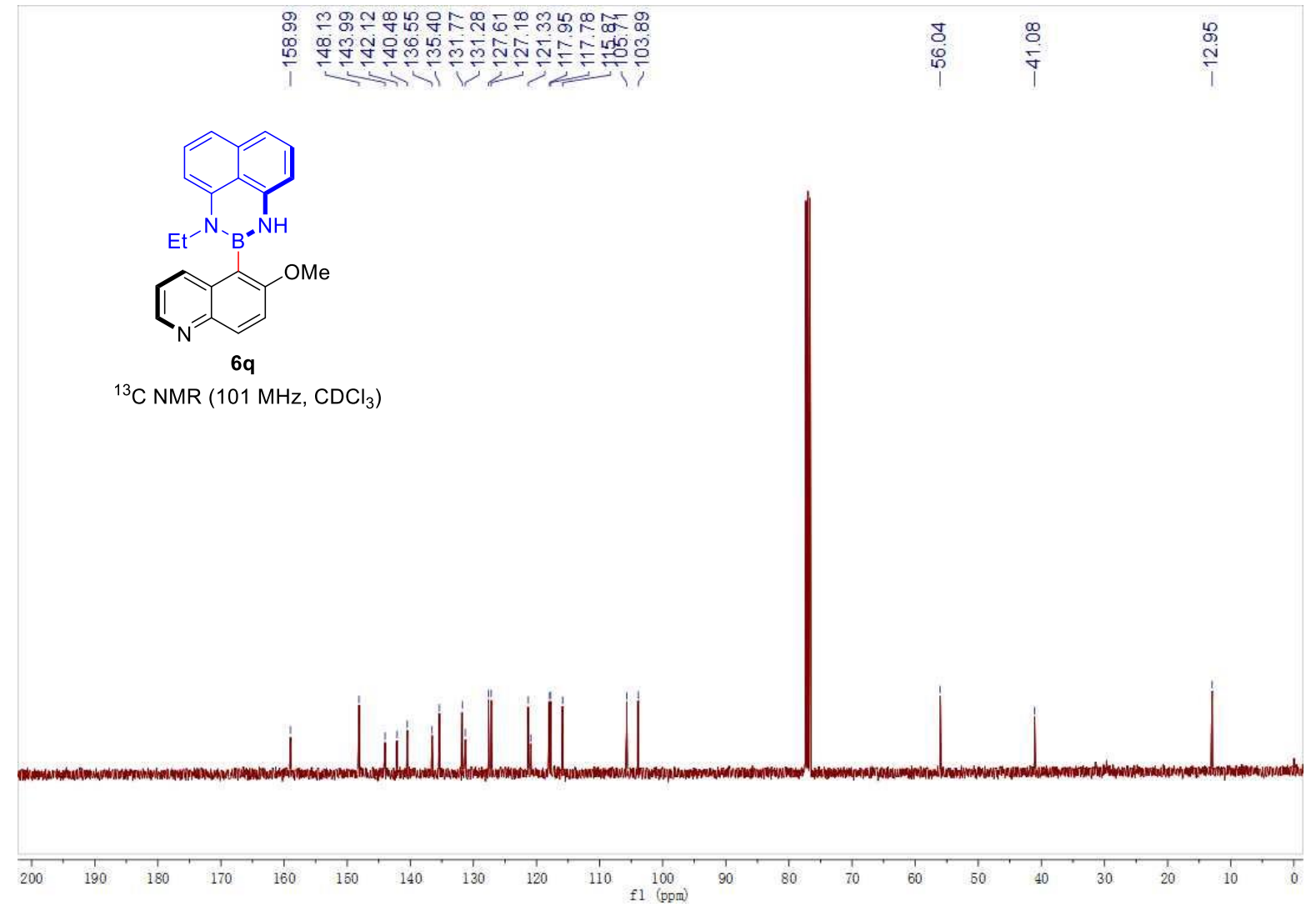


(S)-1-ethyl-2-(6-methoxyquinolin-5-yl)-2,3-dihydro-1H-naphtho[1,8-de][1,3,2]diazaborinine (6q)

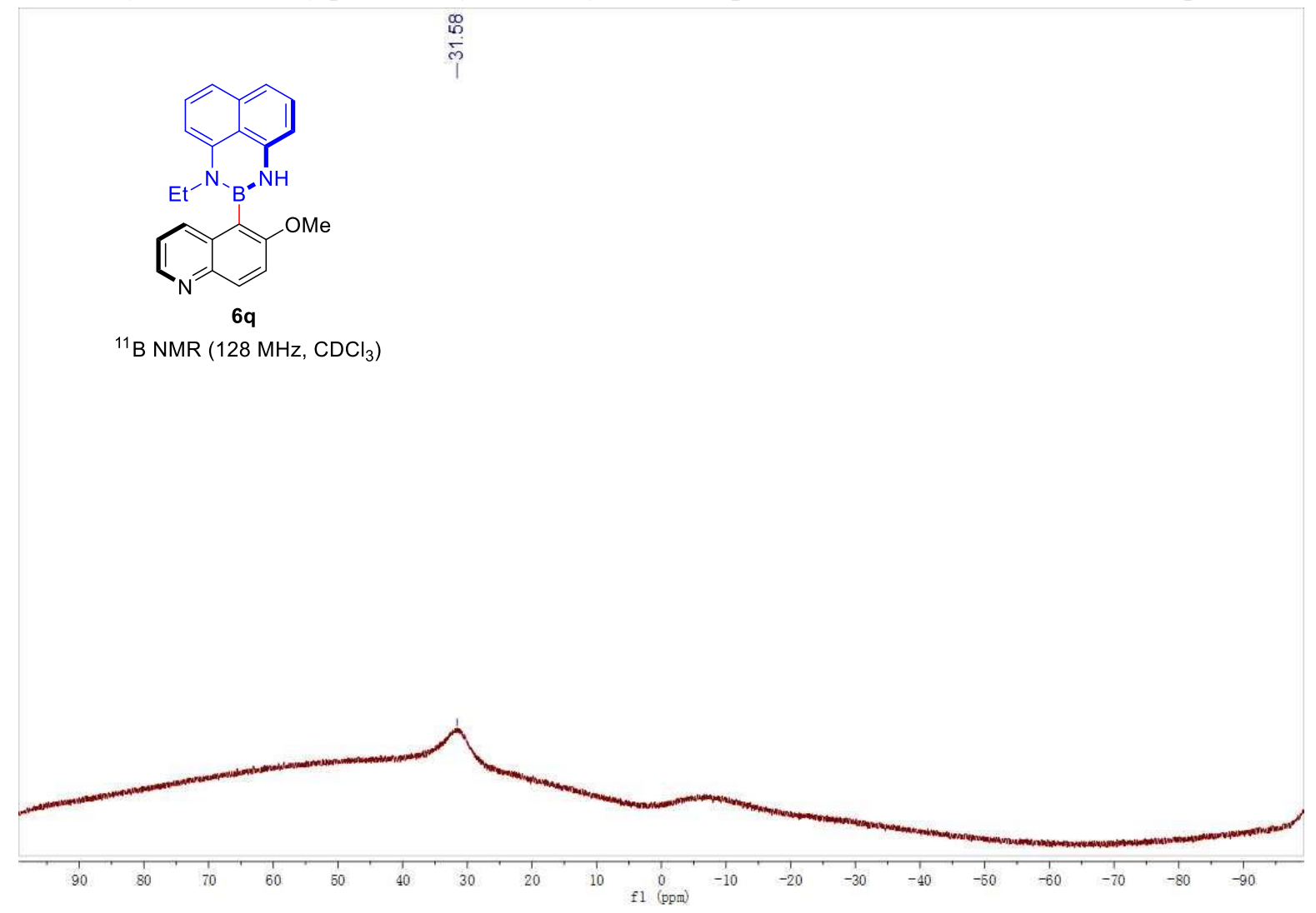

(S)-1-ethyl-2-(2-methoxy-6-methylphenyl)-2,3-dihydro-1H-naphtho[1,8-de][1,3,2]diazaborinine (6r)

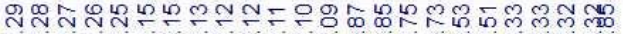

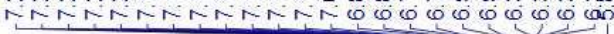

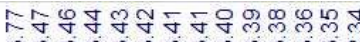

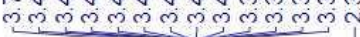
$\stackrel{\text { in }}{\frac{\pi}{5}}$

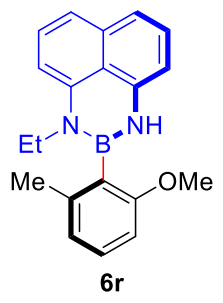

${ }^{1} \mathrm{H} \mathrm{NMR}\left(500 \mathrm{MHz}, \mathrm{CDCl}_{3}\right)$

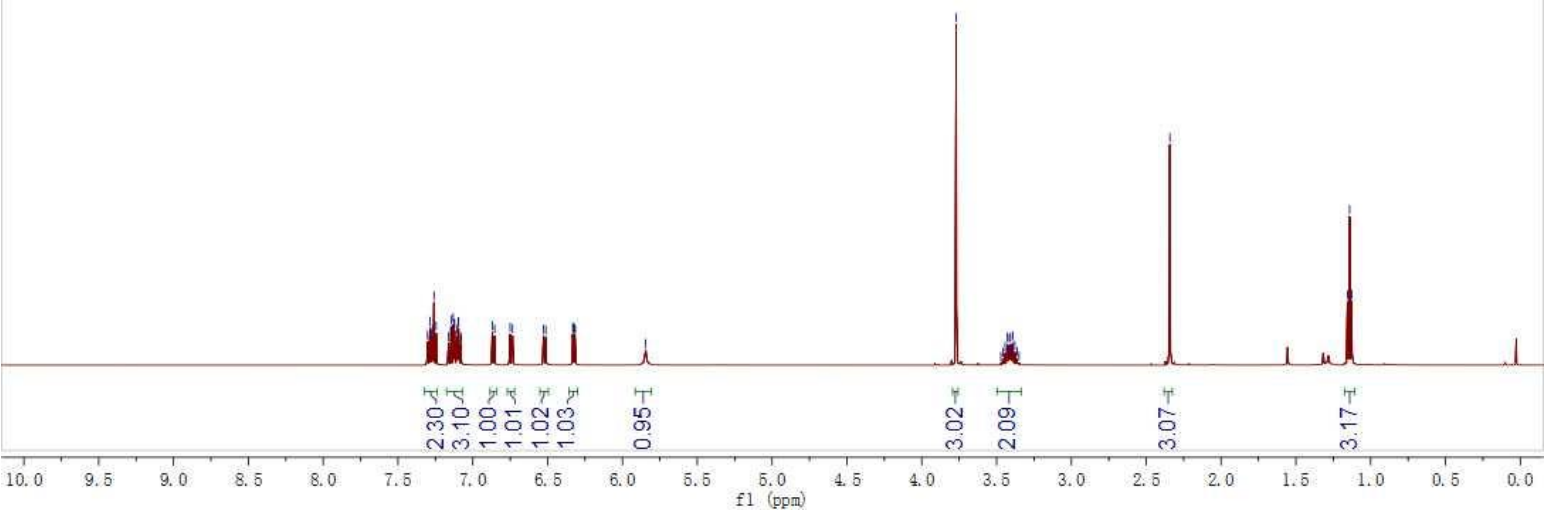


(S)-1-ethyl-2-(2-methoxy-6-methylphenyl)-2,3-dihydro-1H-naphtho[1,8-de][1,3,2]diazaborinine (6r)

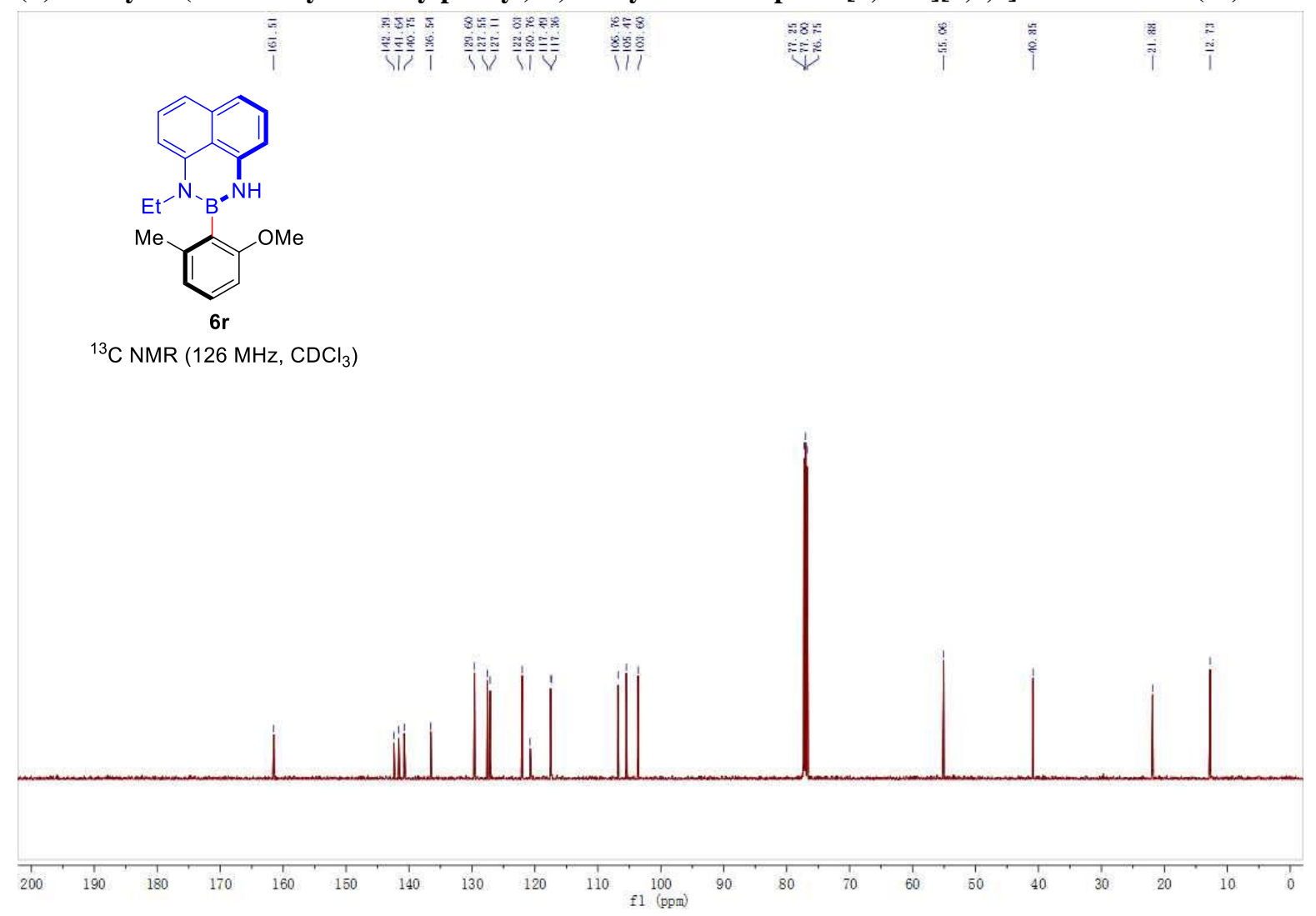

(S)-1-ethyl-2-(2-methoxy-6-methylphenyl)-2,3-dihydro-1H-naphtho[1,8-de][1,3,2]diazaborinine (6r)

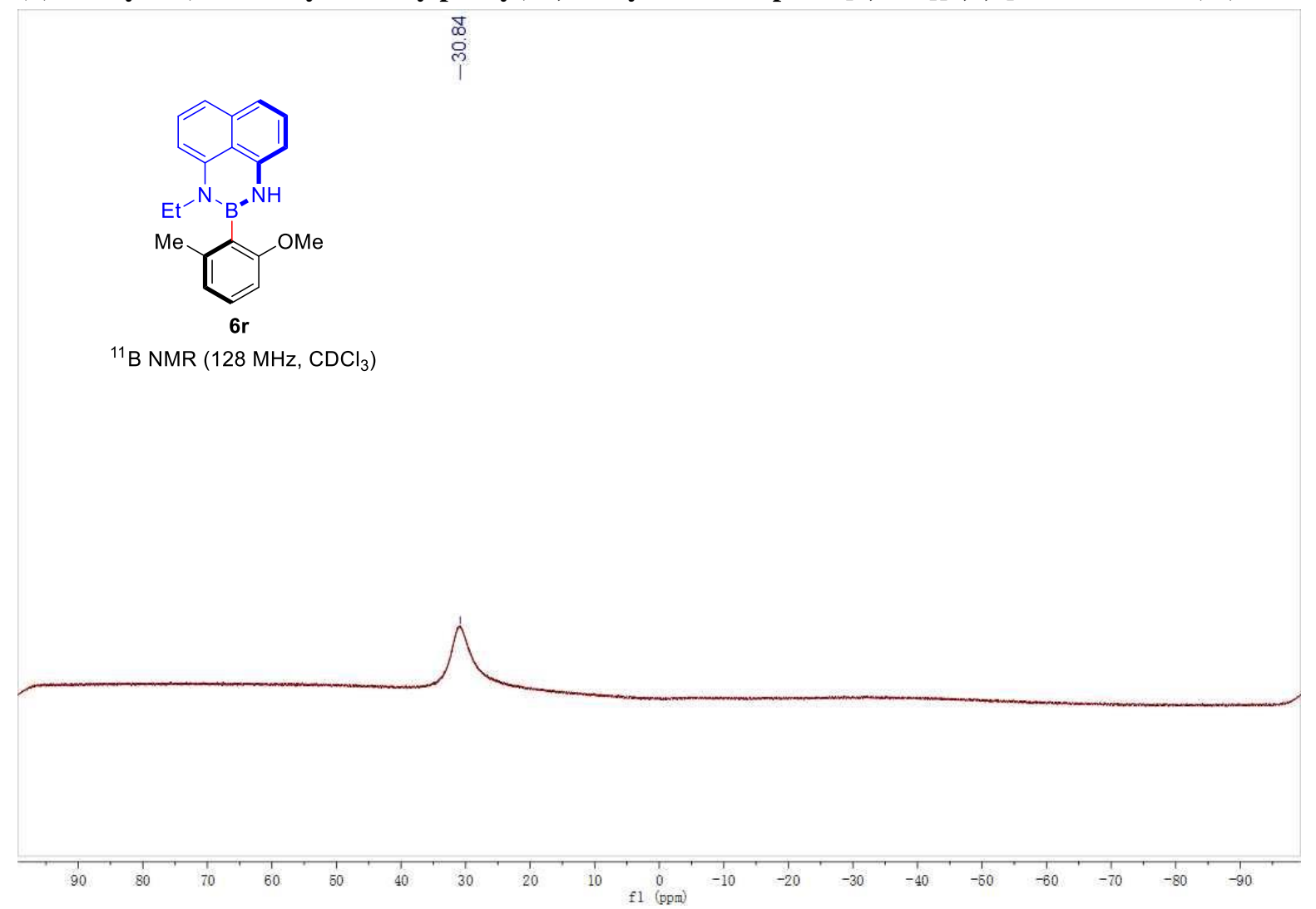


(S)-2-(3-chloro-6-methoxy-2,4-dimethylphenyl)-1-ethyl-2,3-dihydro-1H-naphtho[1,8-de][1,3,2]diazabori nine $(6 s)$

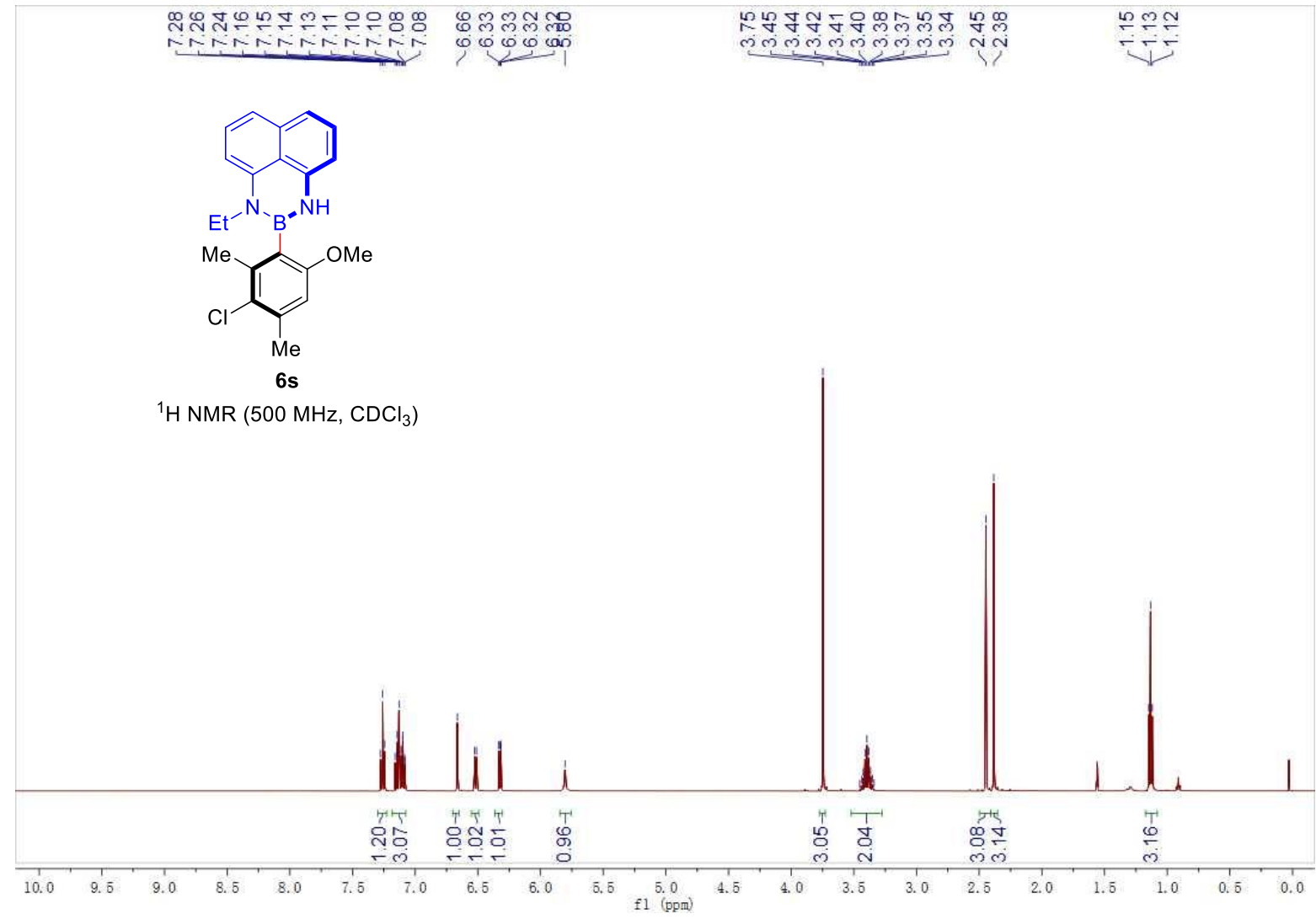

(S)-2-(3-chloro-6-methoxy-2,4-dimethylphenyl)-1-ethyl-2,3-dihydro-1H-naphtho[1,8-de][1,3,2]diazabori nine $(6 s)$

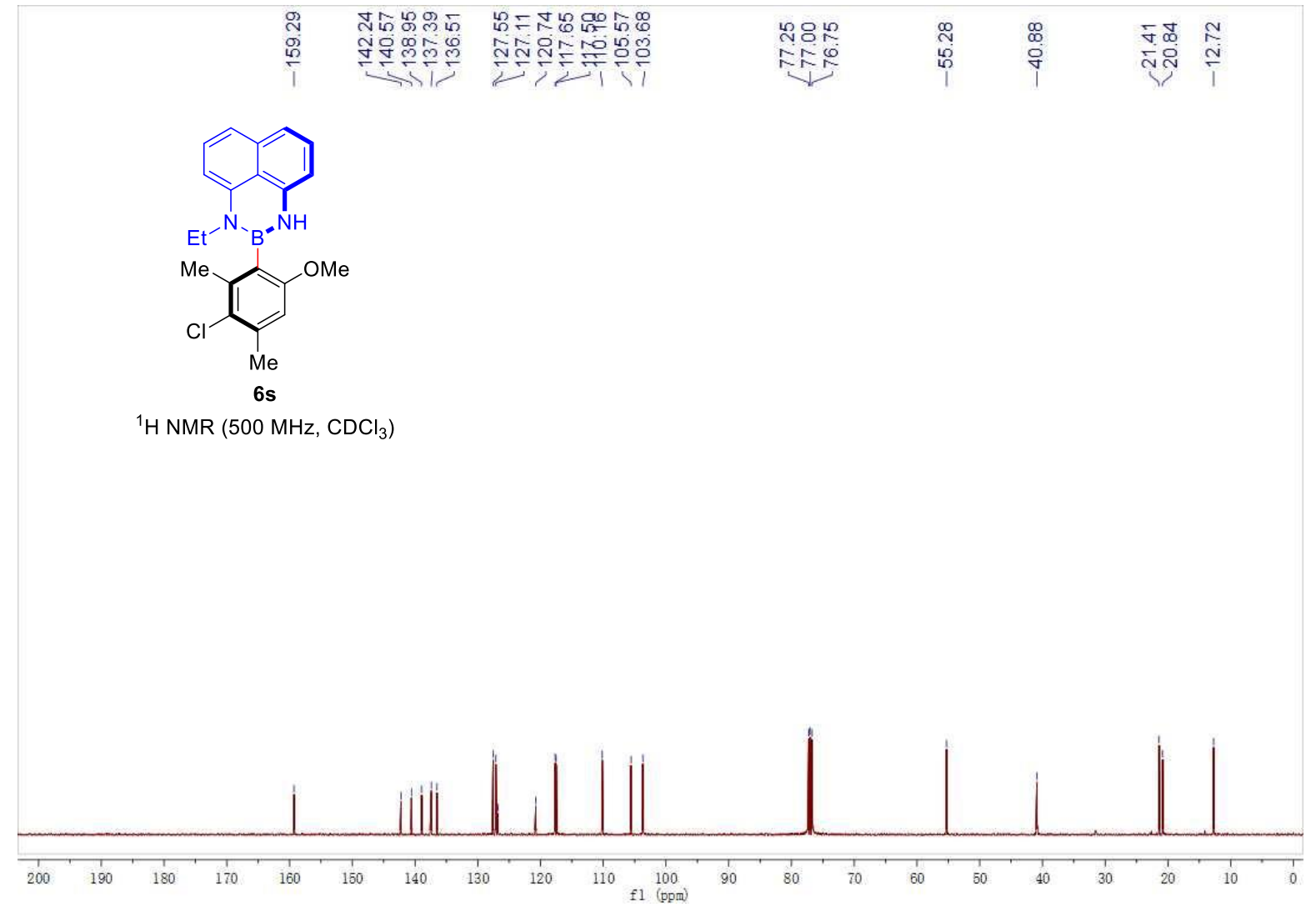


(S)-2-(3-chloro-6-methoxy-2,4-dimethylphenyl)-1-ethyl-2,3-dihydro-1H-naphtho[1,8-de][1,3,2]diazabori nine $(6 s)$

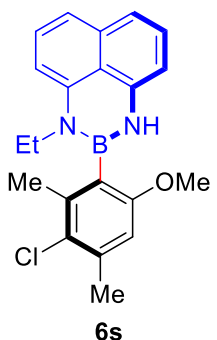

${ }^{1} \mathrm{H}$ NMR $\left(500 \mathrm{MHz}, \mathrm{CDCl}_{3}\right)$

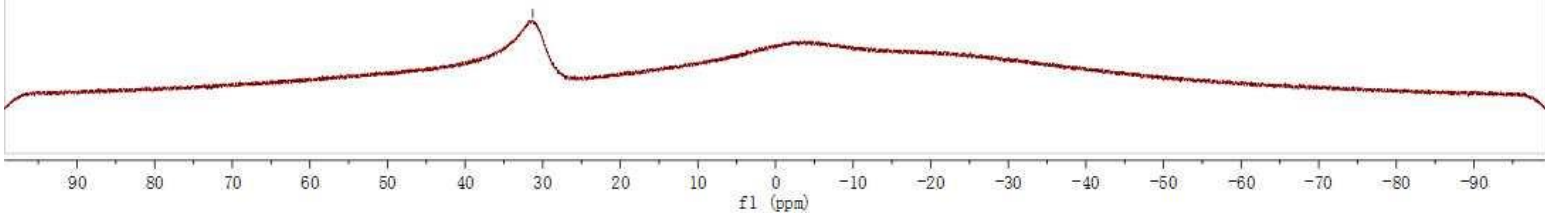

(S)-1-ethyl-2-(2-methoxy-5,6,7,8-tetrahydronaphthalen-1-yl)-2,3-dihydro-1H-naphtho[1,8-de][1,3,2]diaz aborinine (6t)

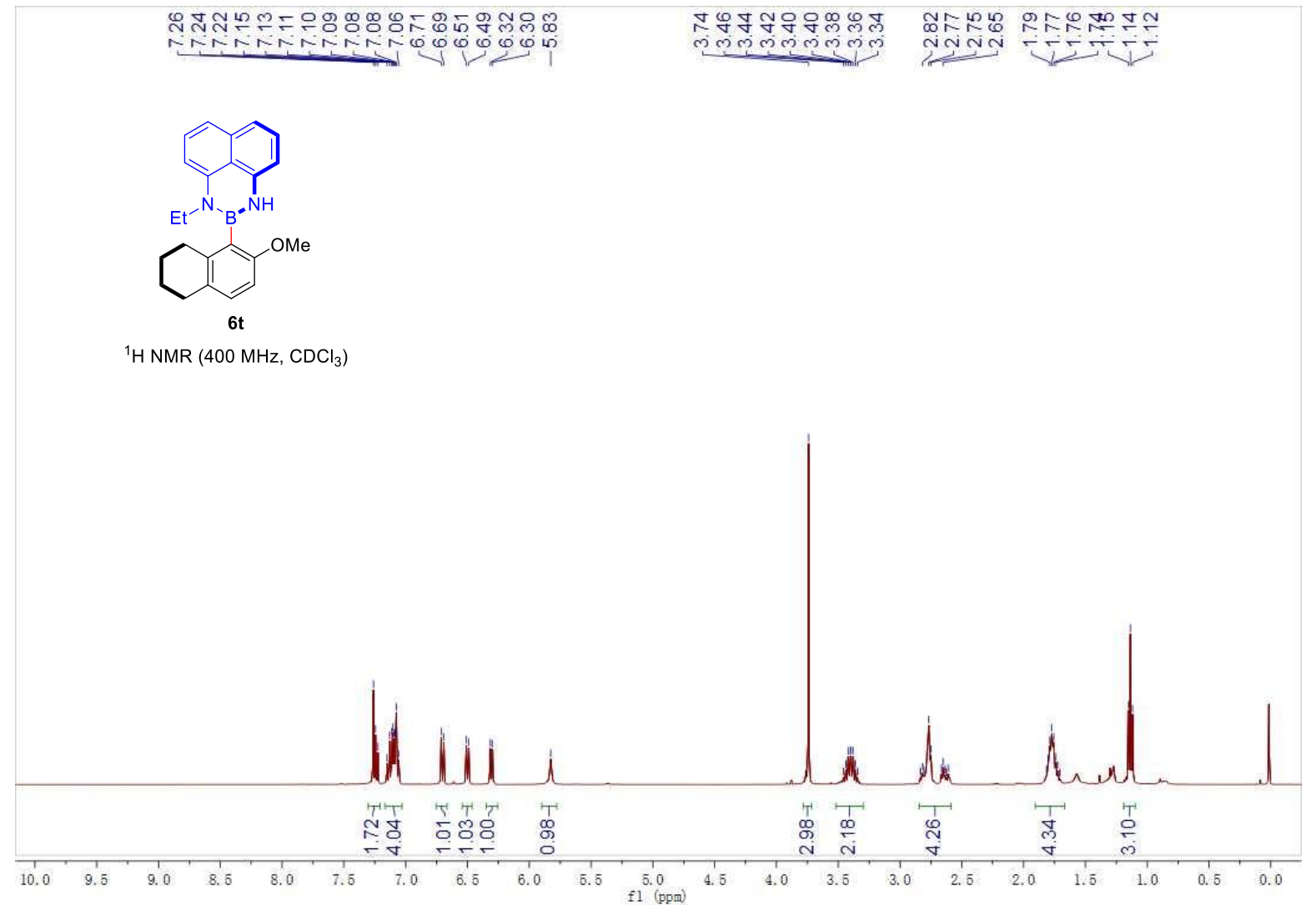


(S)-1-ethyl-2-(2-methoxy-5,6,7,8-tetrahydronaphthalen-1-yl)-2,3-dihydro-1H-naphtho[1,8-de][1,3,2]diaz aborinine (6t)

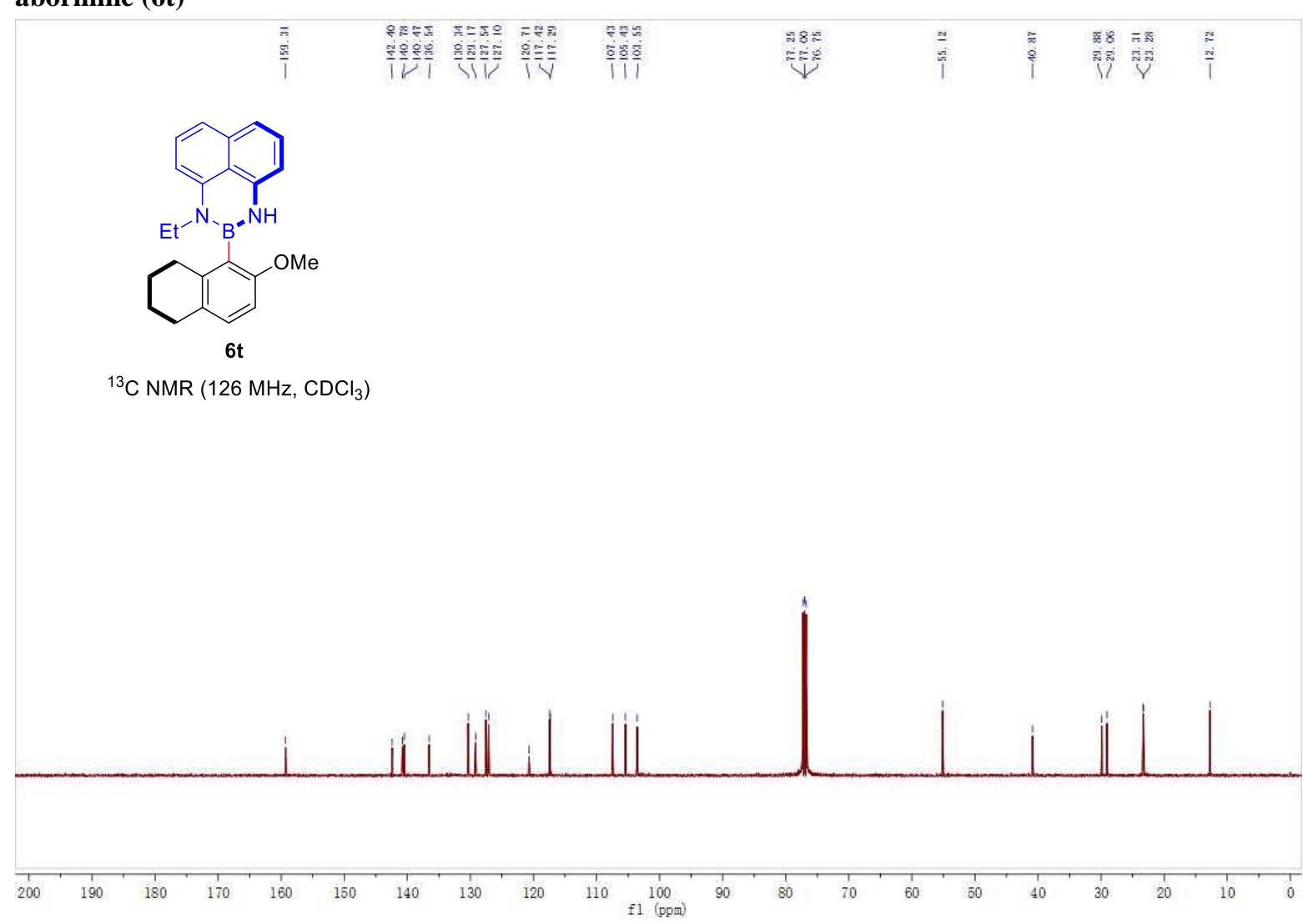

(S)-1-ethyl-2-(2-methoxy-5,6,7,8-tetrahydronaphthalen-1-yl)-2,3-dihydro-1H-naphtho[1,8-de][1,3,2]diaz aborinine (6t)

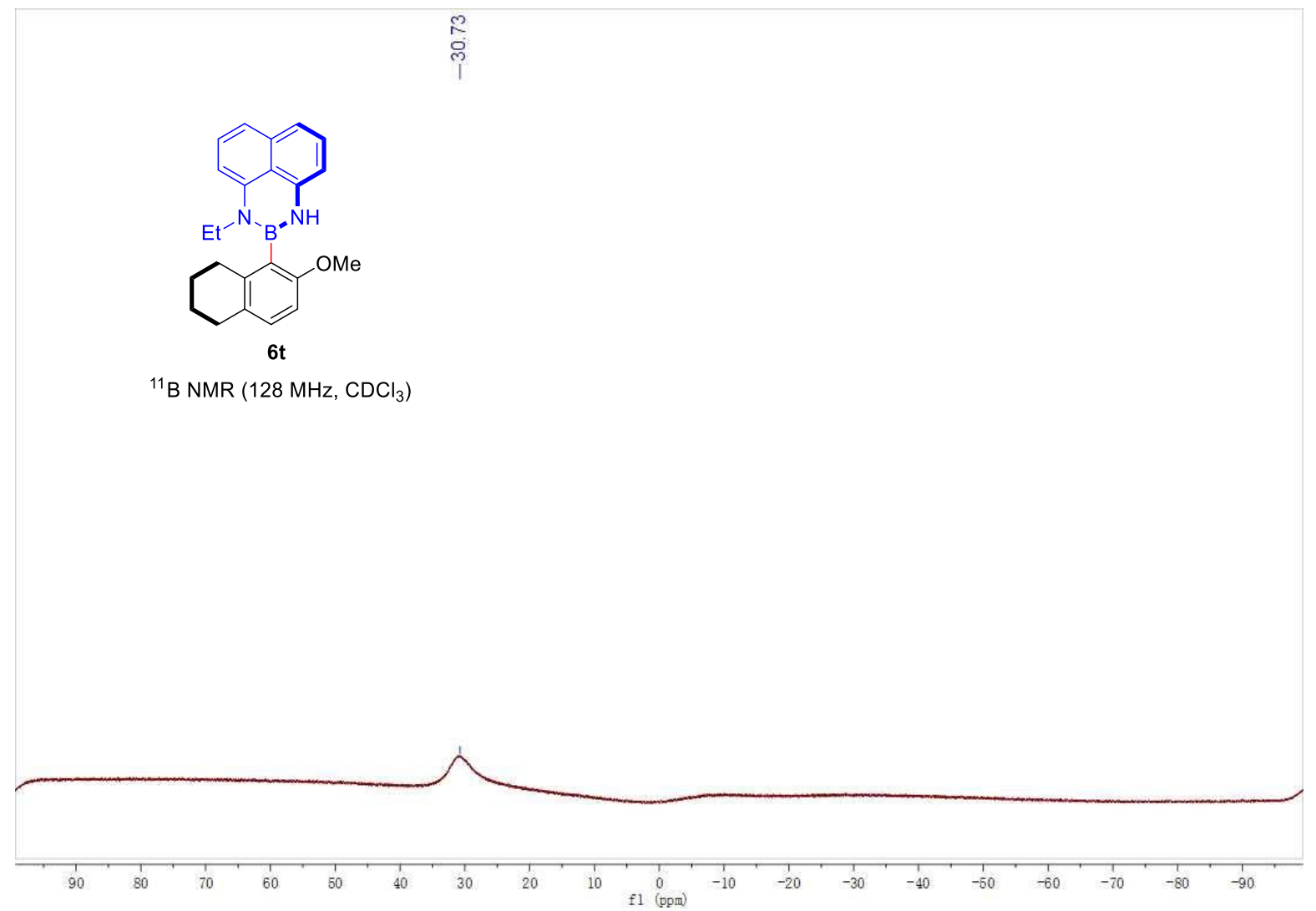


(S)-5-(1-ethyl-1H-naphtho[1,8-de][1,3,2]diazaborinin-2(3H)-yl)-6-methoxy-3,4-dihydronaphthalen-1(2H )-one (6u)

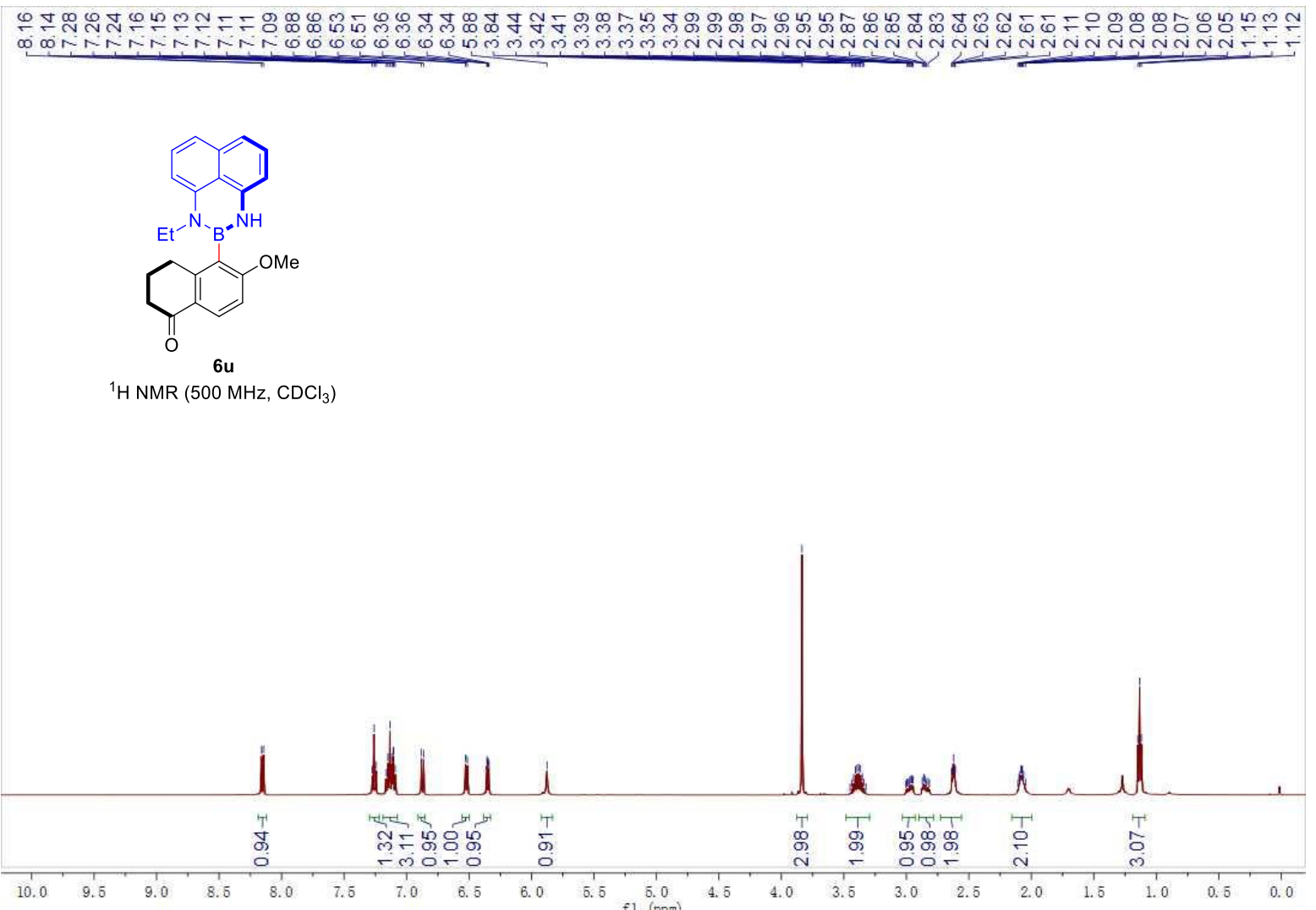

(S)-5-(1-ethyl-1H-naphtho[1,8-de][1,3,2]diazaborinin-2(3H)-yl)-6-methoxy-3,4-dihydronaphthalen-1(2H )-one (6u)

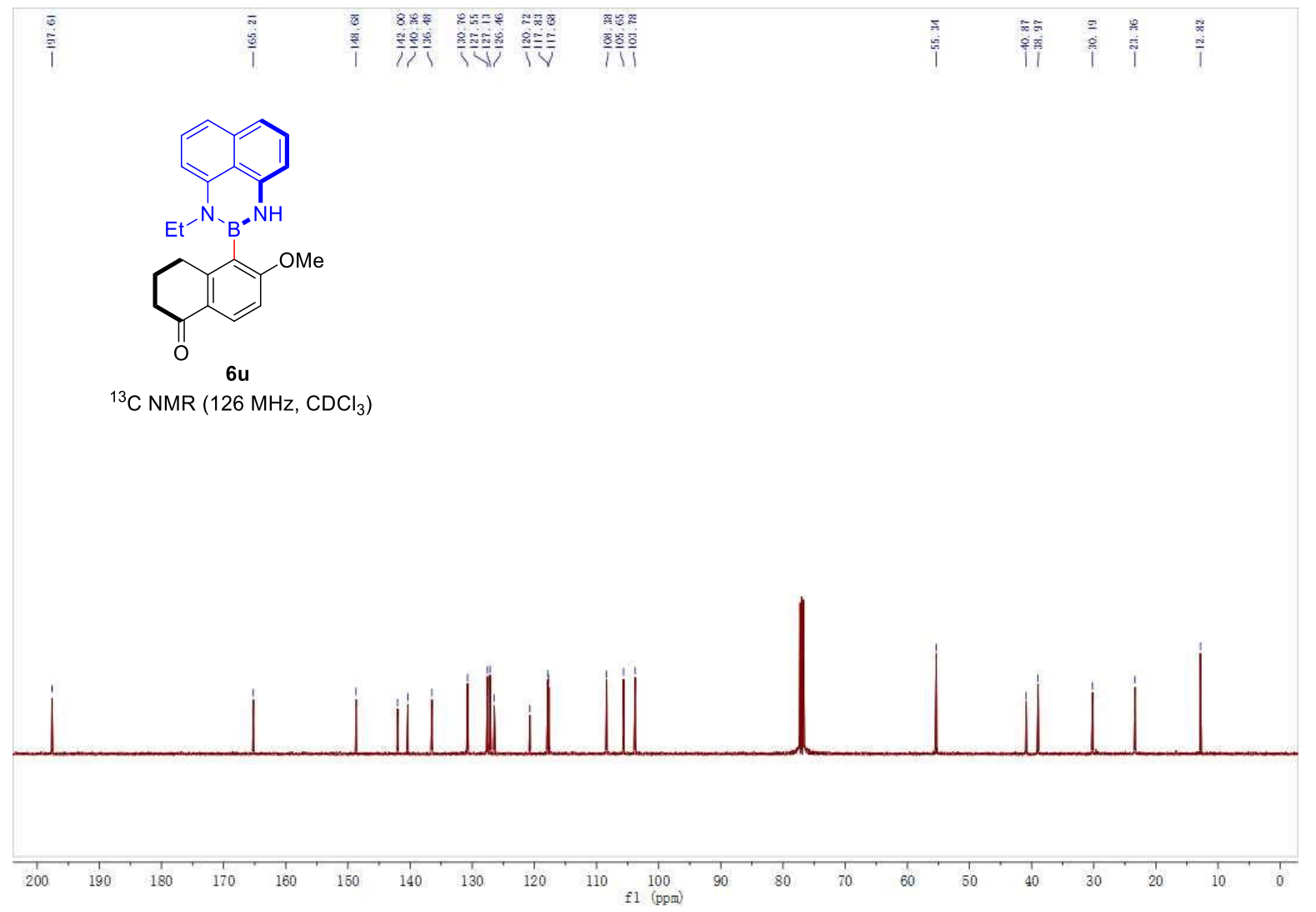


(S)-5-(1-ethyl-1H-naphtho[1,8-de][1,3,2]diazaborinin-2(3H)-yl)-6-methoxy-3,4-dihydronaphthalen-1(2H )-one (6u)

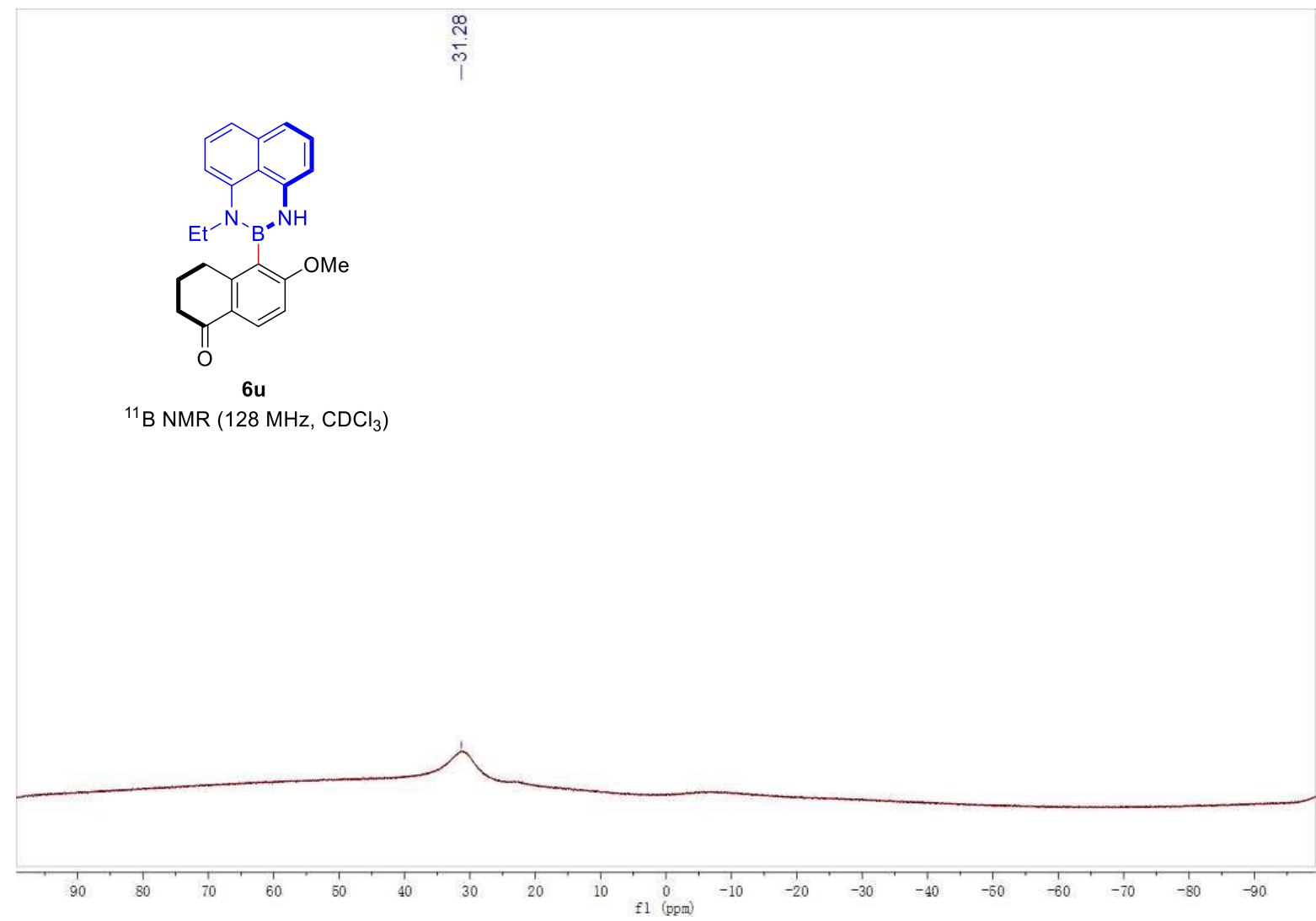

(S)-1-(cyclopropylmethyl)-2-(2-methoxynaphthalen-1-yl)-2,3-dihydro-1H-naphtho[1,8-de][1,3,2] diazabo rinine $(6 v)$

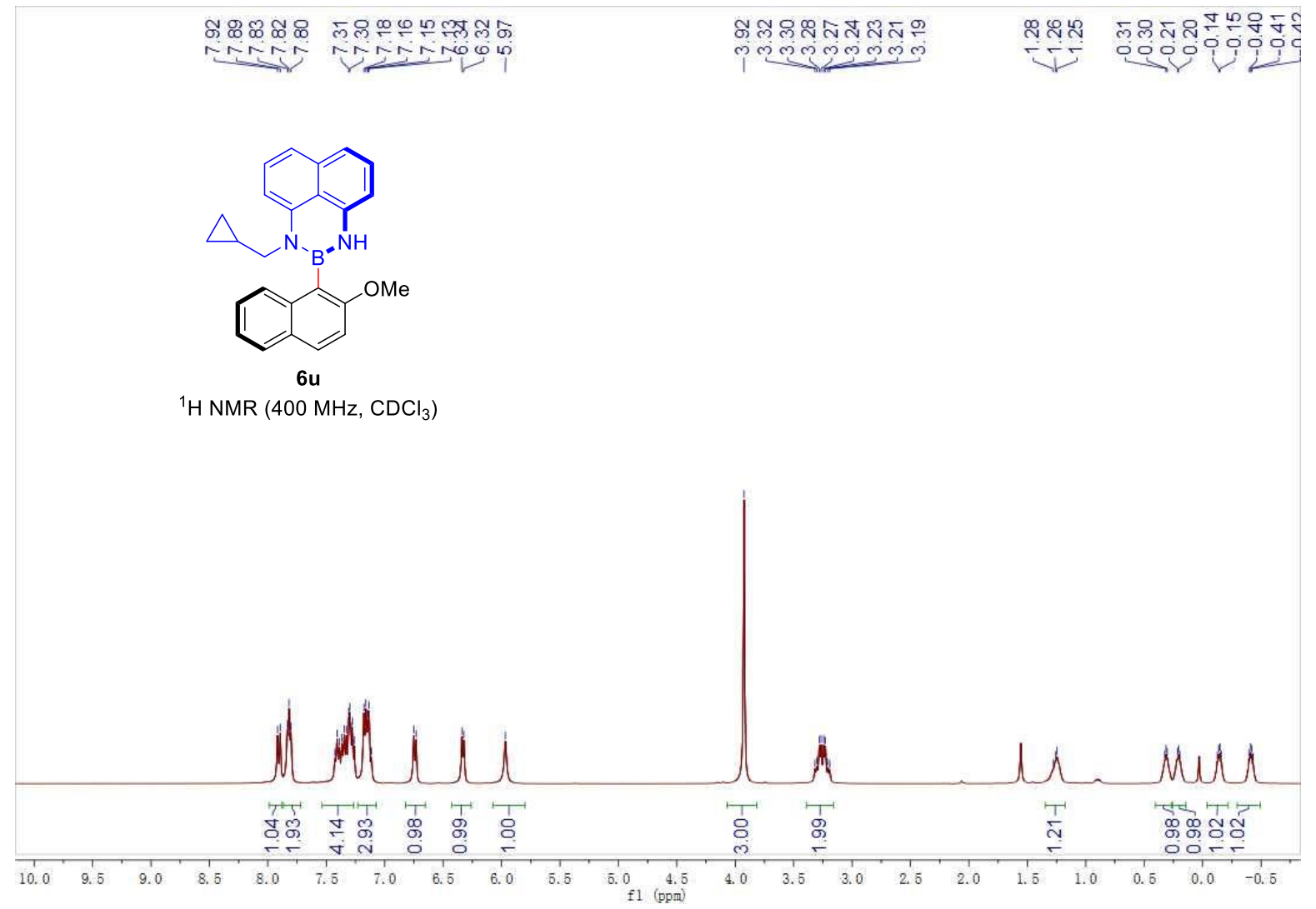


(S)-1-(cyclopropylmethyl)-2-(2-methoxynaphthalen-1-yl)-2,3-dihydro-1H-naphtho[1,8-de][1,3,2]diazabo rinine (6v)

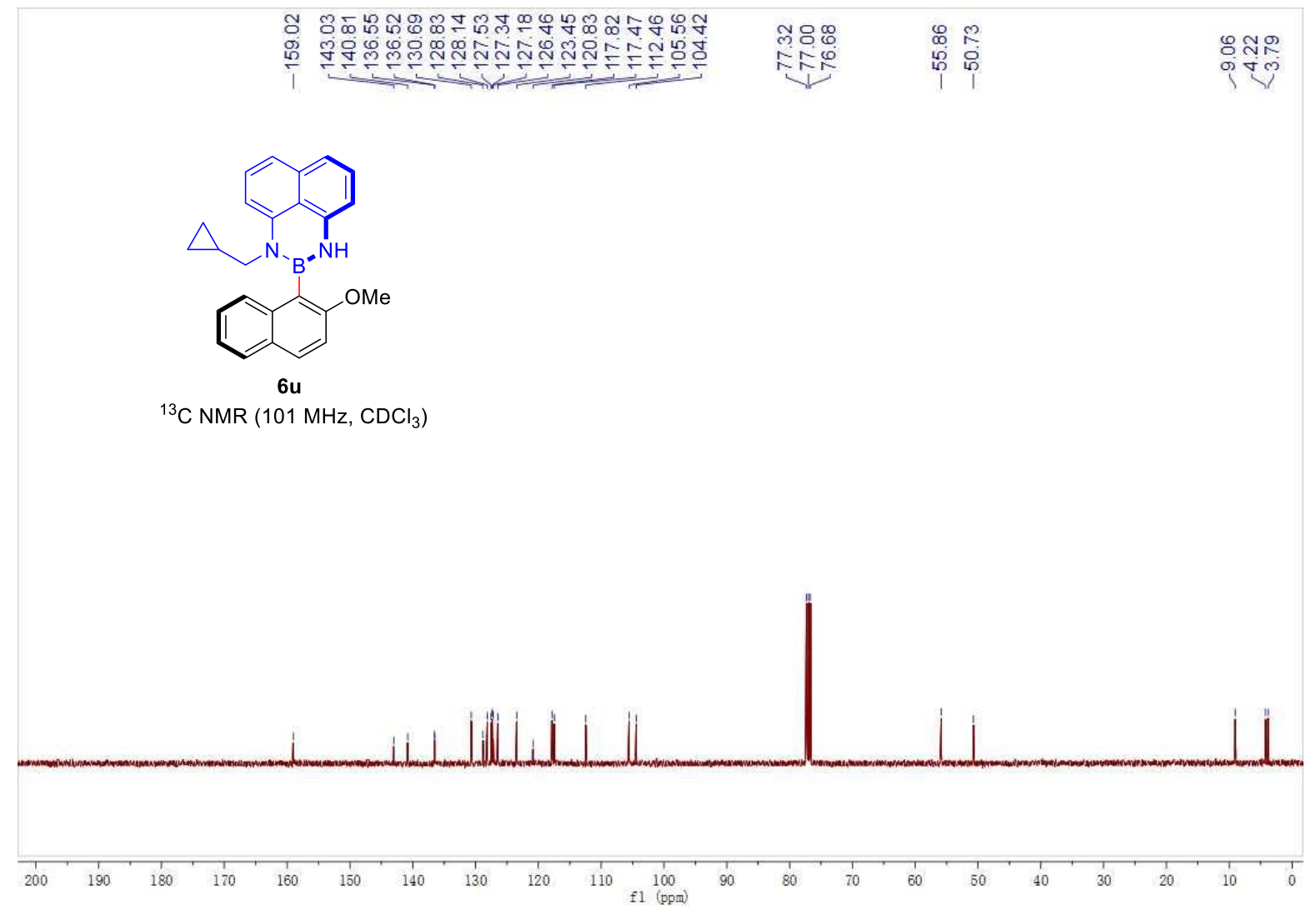

(S)-1-(cyclopropylmethyl)-2-(2-methoxynaphthalen-1-yl)-2,3-dihydro-1H-naphtho[1,8-de][1,3,2]diazabo rinine (6v)

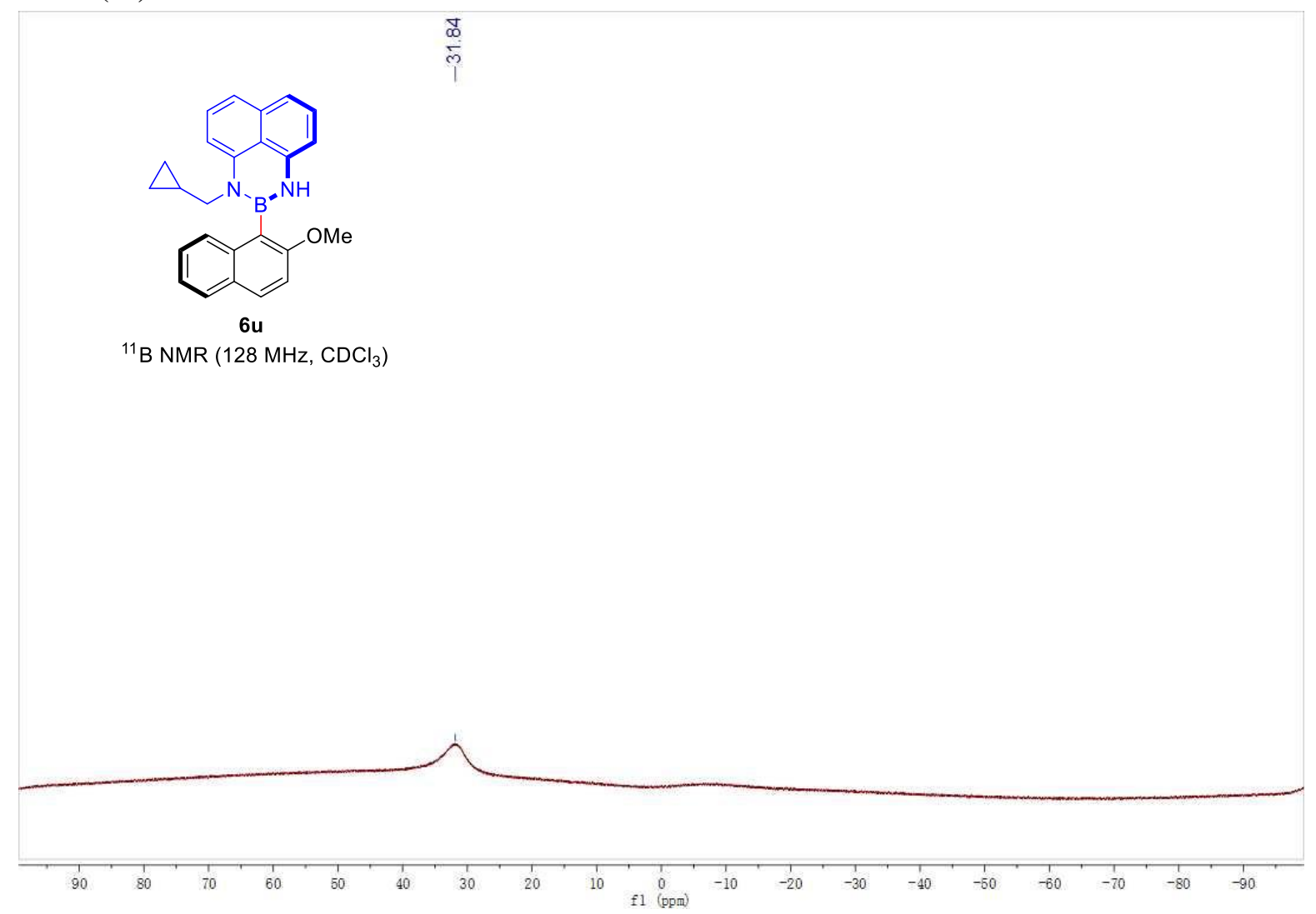


(S)-1-(cyclopentylmethyl)-2-(2,6-dimethoxynaphthalen-1-yl)-2,3-dihydro-1H-naphtho[1,8-de][1,3,2]diaz aborinine (6w)

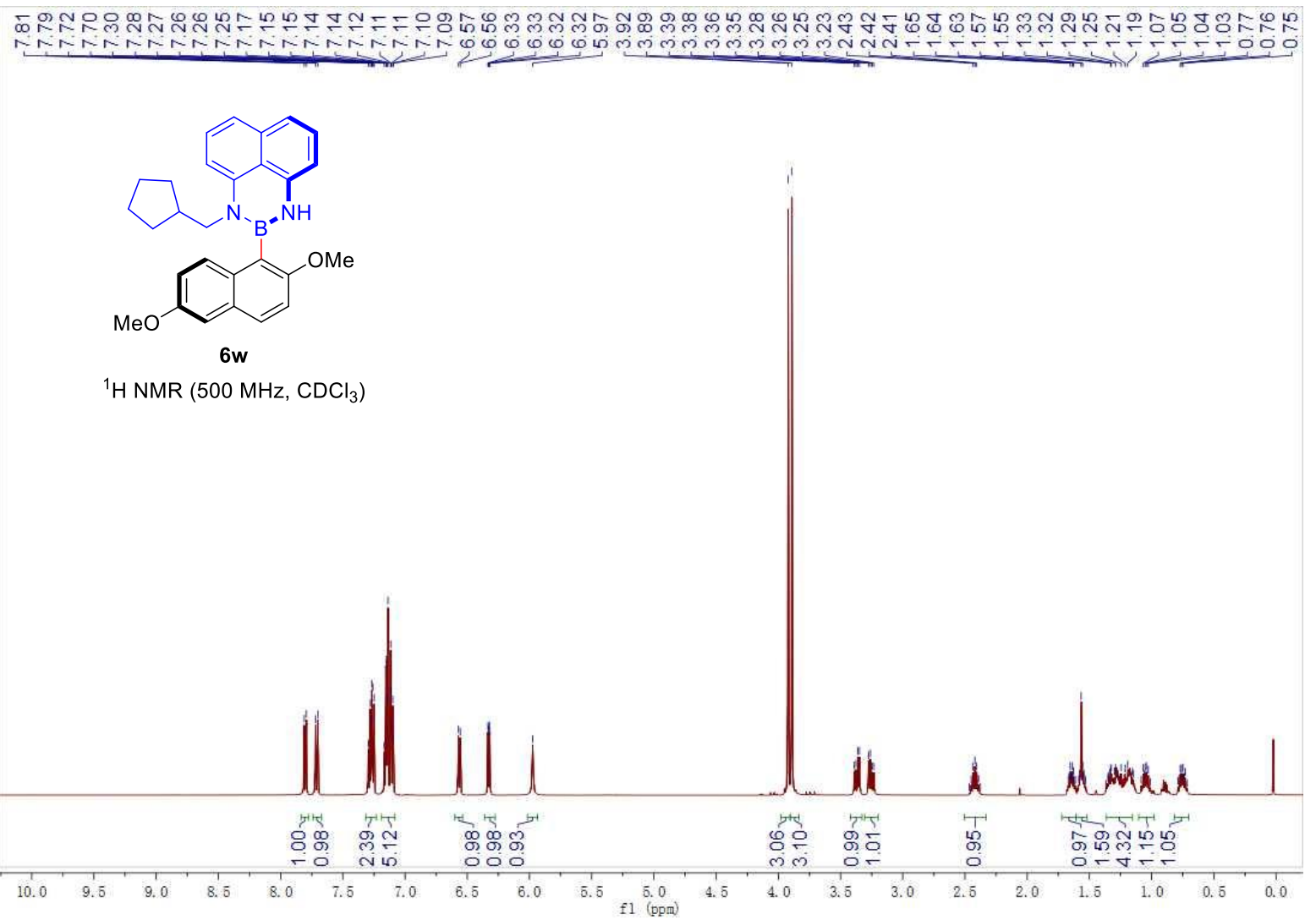

(S)-1-(cyclopentylmethyl)-2-(2,6-dimethoxynaphthalen-1-yl)-2,3-dihydro-1H-naphtho[1,8-de][1,3,2]diaz aborinine (6w)

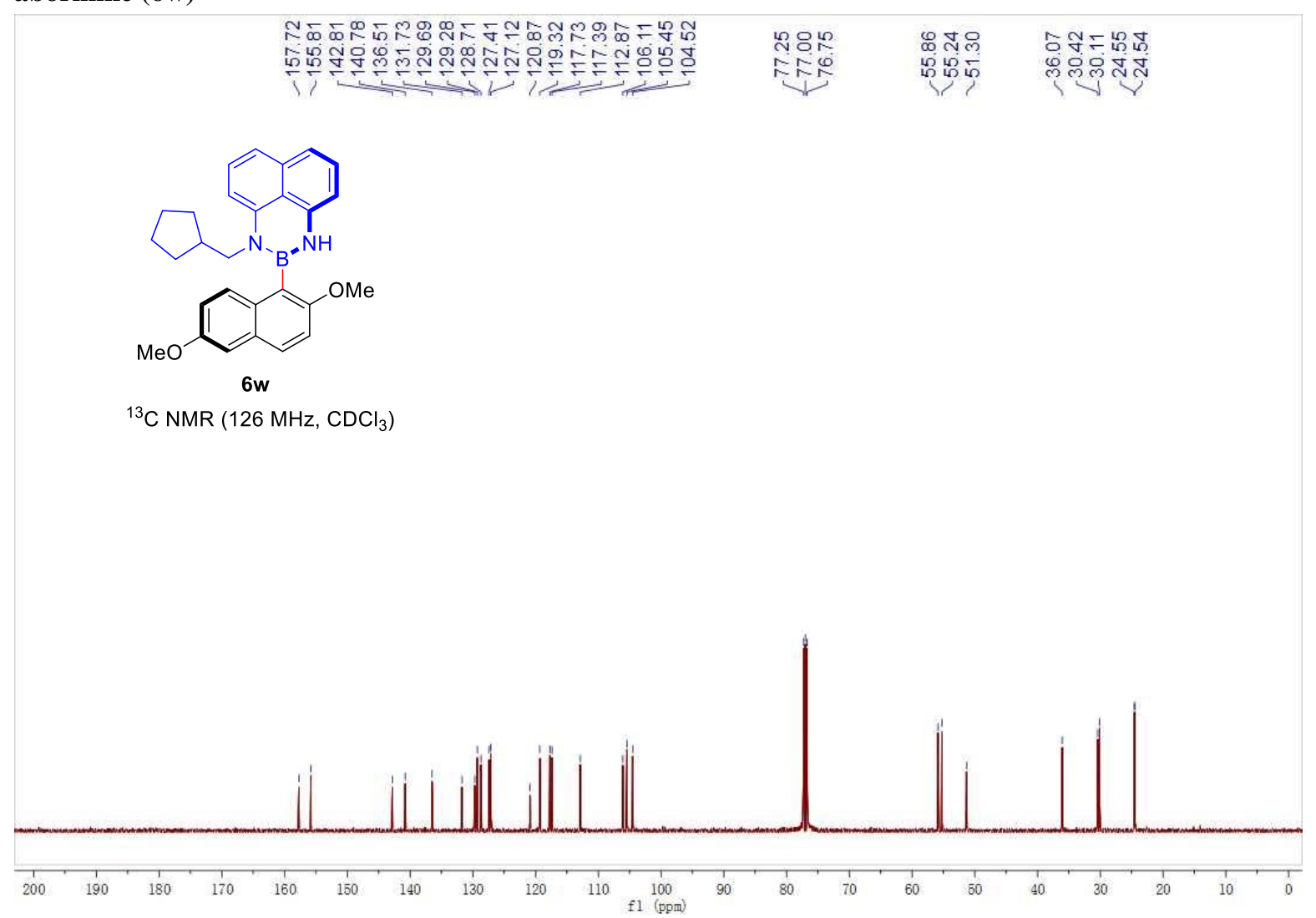


(S)-1-(cyclopentylmethyl)-2-(2,6-dimethoxynaphthalen-1-yl)-2,3-dihydro-1H-naphtho[1,8-de][1,3,2]diaz aborinine (6w)

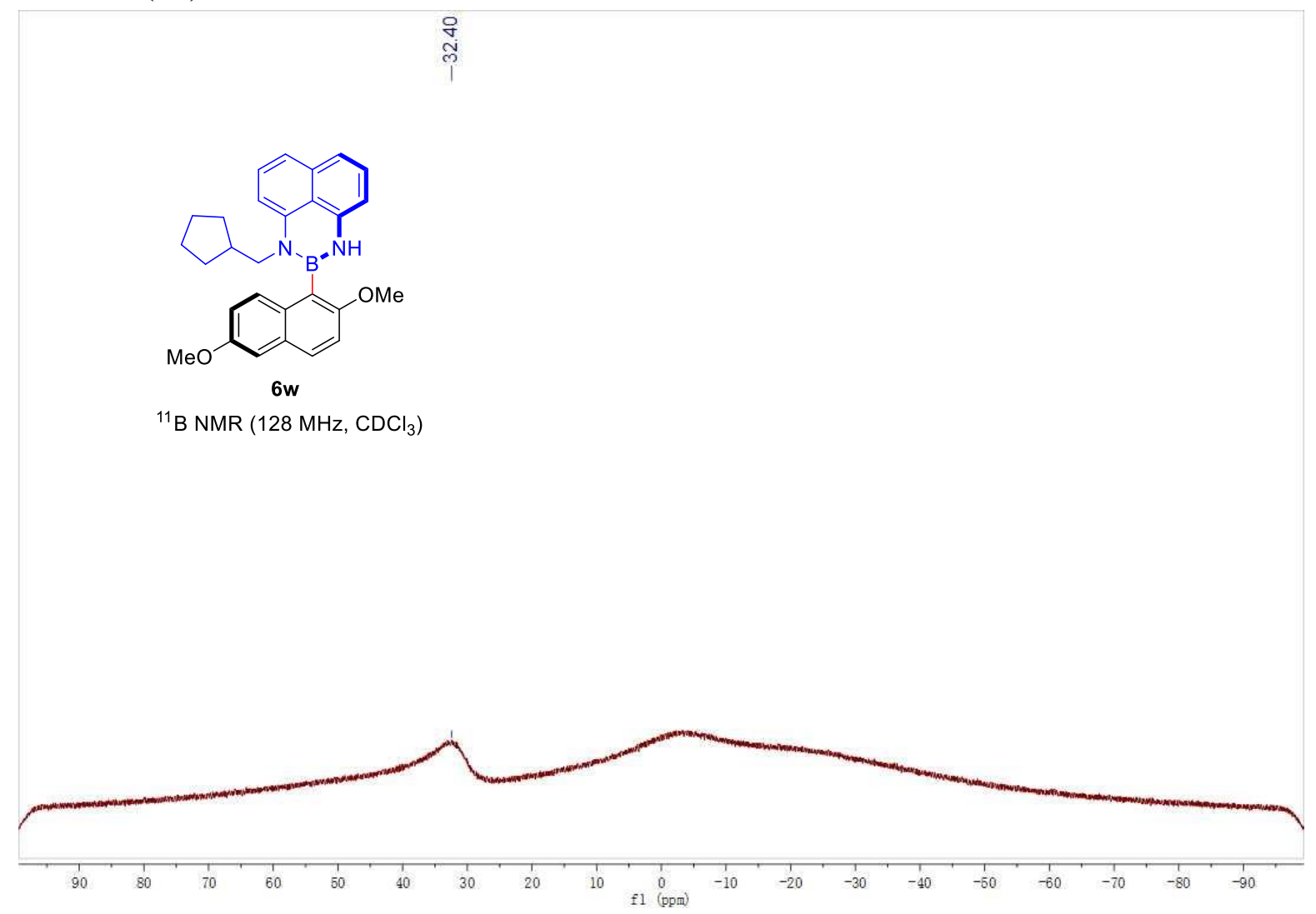

(S)-5-(1-benzyl-1H-naphtho[1,8-de][1,3,2]diazaborinin-2(3H)-yl)-6-methoxy-2-naphthaldehyde (6x)

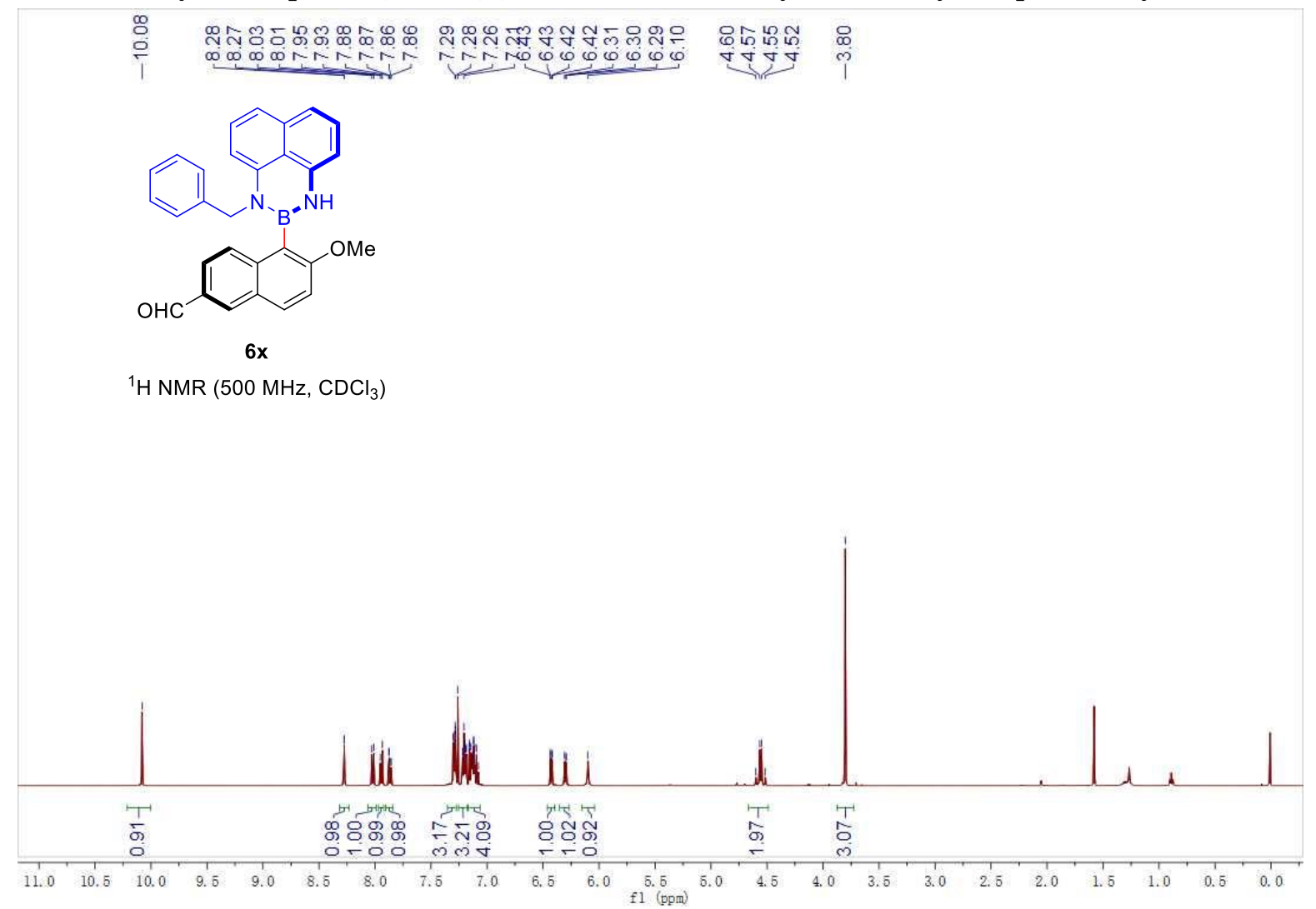


(S)-5-(1-benzyl-1H-naphtho[1,8-de][1,3,2] diazaborinin-2(3H)-yl)-6-methoxy-2-naphthaldehyde (6x)

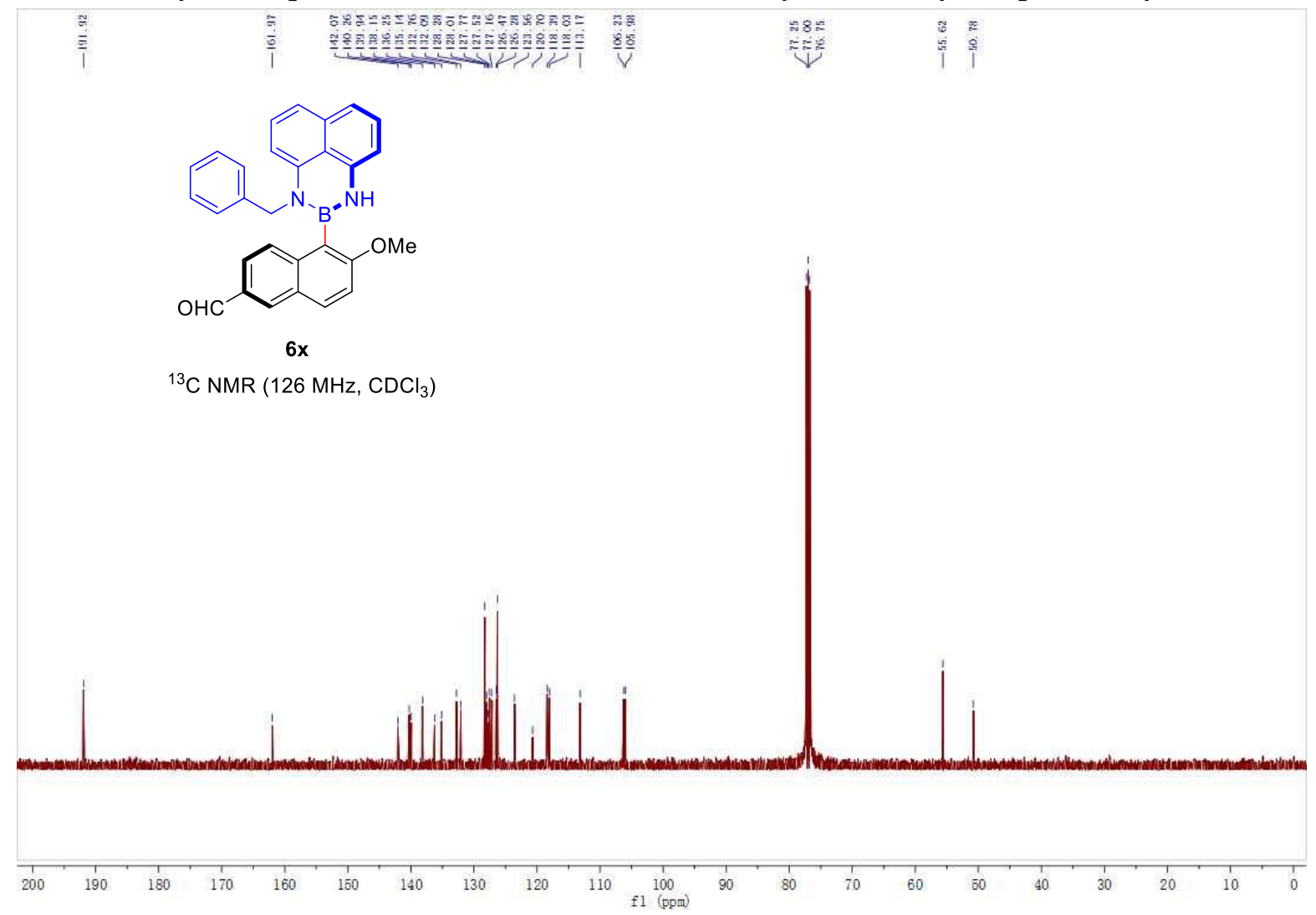

(S)-5-(1-benzyl-1H-naphtho[1,8-de][1,3,2]diazaborinin-2(3H)-yl)-6-methoxy-2-naphthaldehyde (6x)

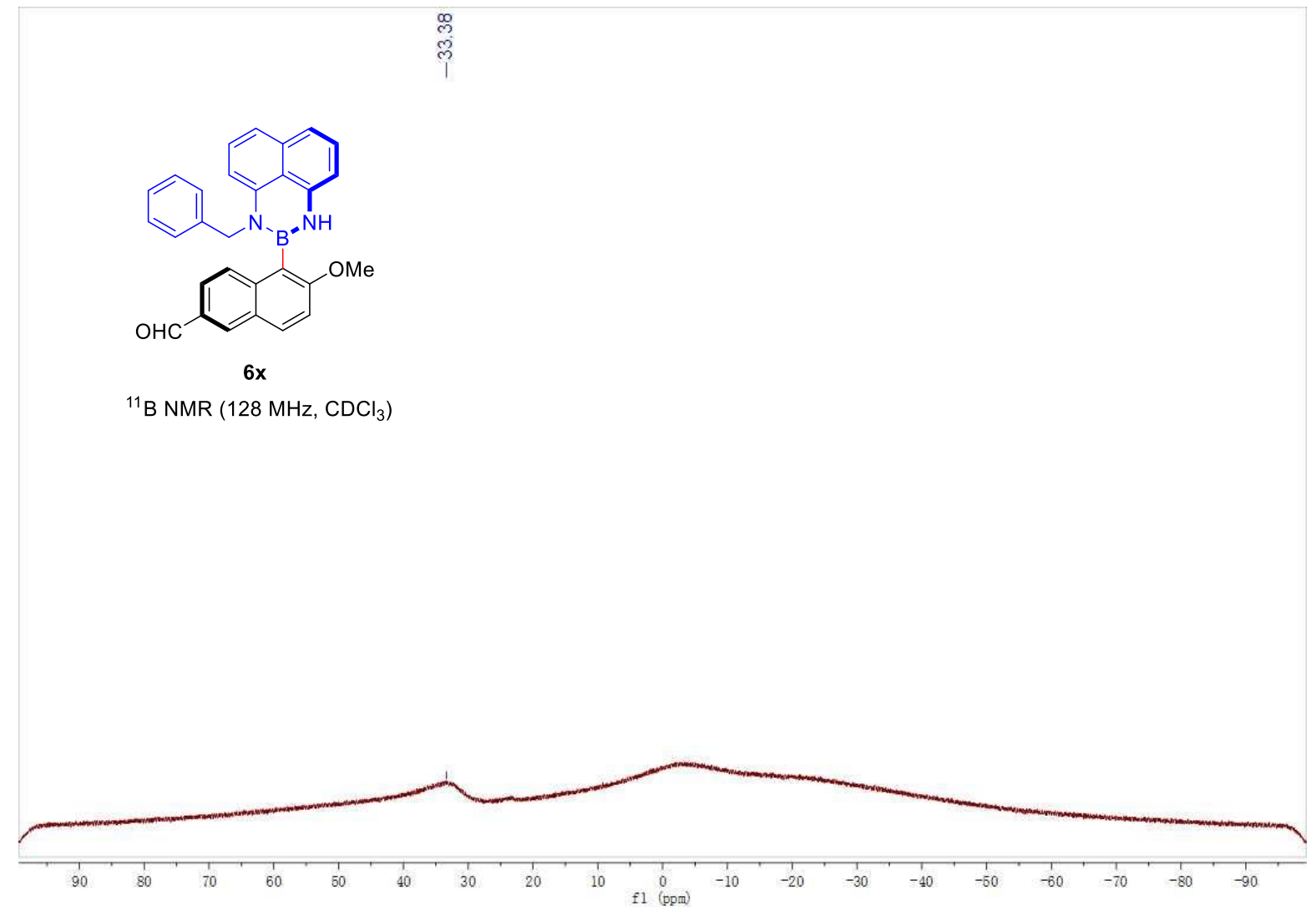

94 
(S)-2-(2,6-dimethoxynaphthalen-1-yl)-1-(2-methylbenzyl)-2,3-dihydro-1H-naphtho[1,8-de][1,3,2]diazabo rinine (6y)

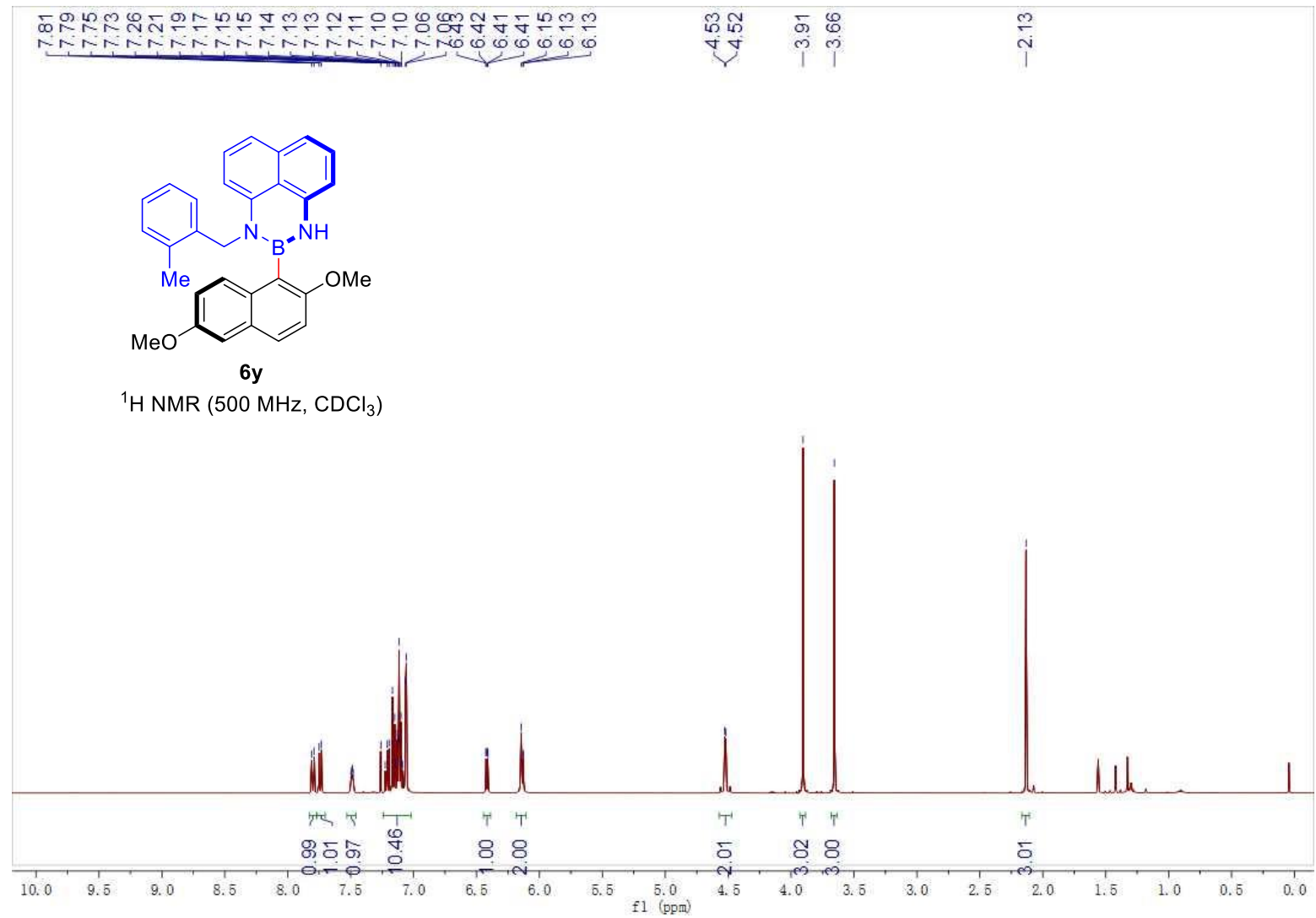

(S)-2-(2,6-dimethoxynaphthalen-1-yl)-1-(2-methylbenzyl)-2,3-dihydro-1H-naphtho[1,8-de][1,3,2]diazabo rinine (6y)

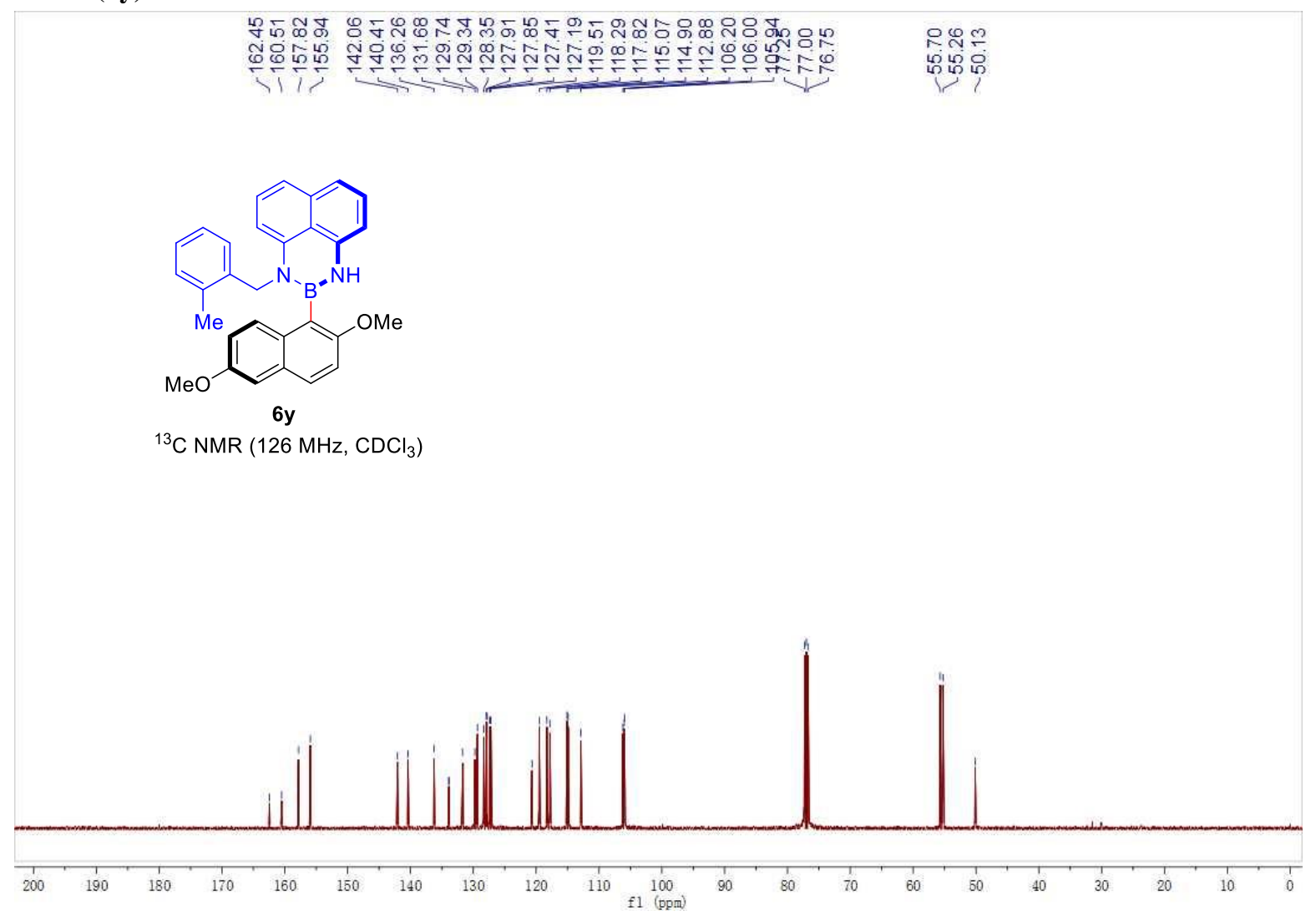


(S)-2-(2,6-dimethoxynaphthalen-1-yl)-1-(2-methylbenzyl)-2,3-dihydro-1H-naphtho[1,8-de][1,3,2]diazabo rinine $(6 y)$

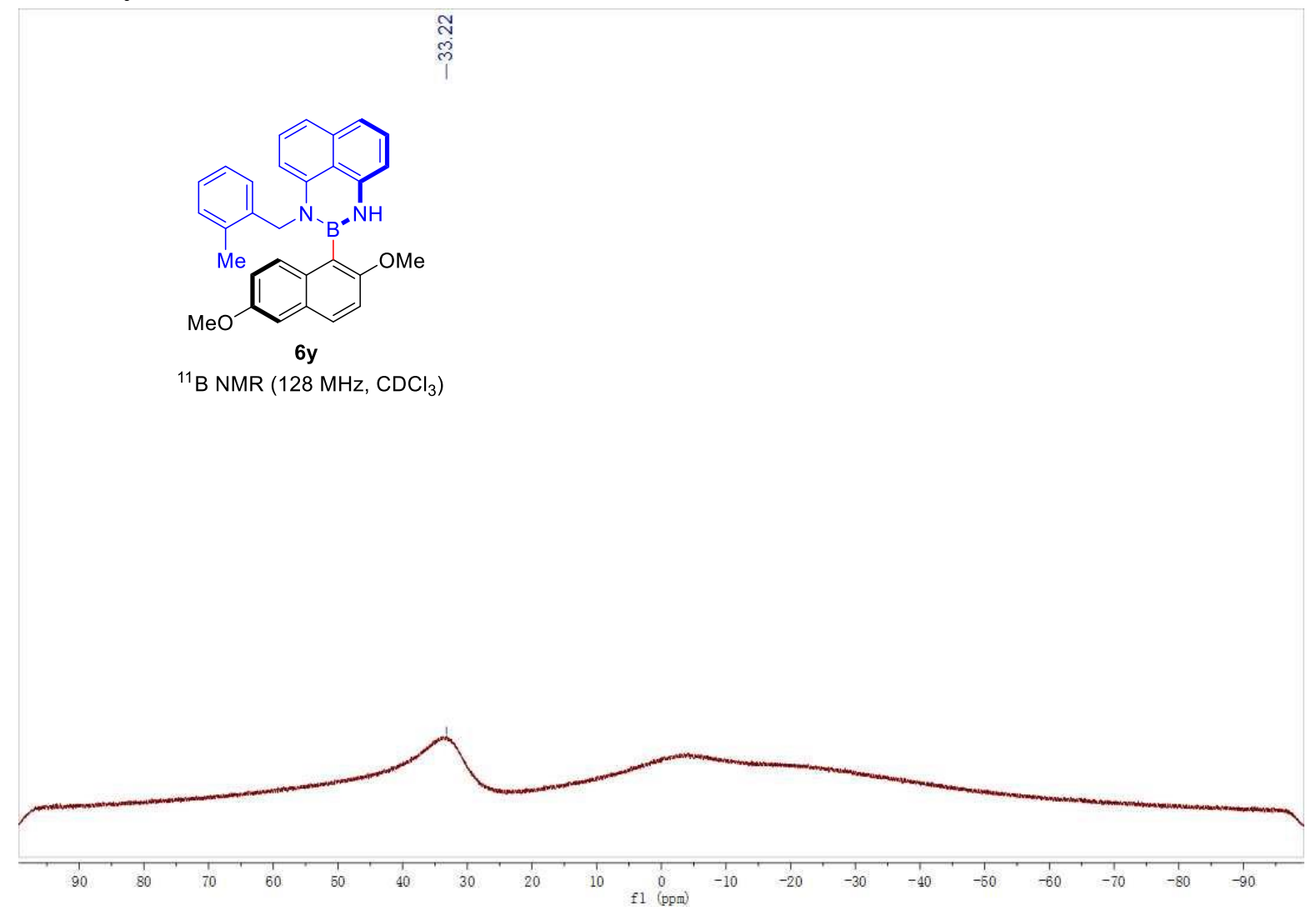

(S)-2-(2,6-dimethoxynaphthalen-1-yl)-1-(3-methylbenzyl)-2,3-dihydro-1H-naphtho[1,8-de][1,3,2]diazabo rinine $(6 z)$

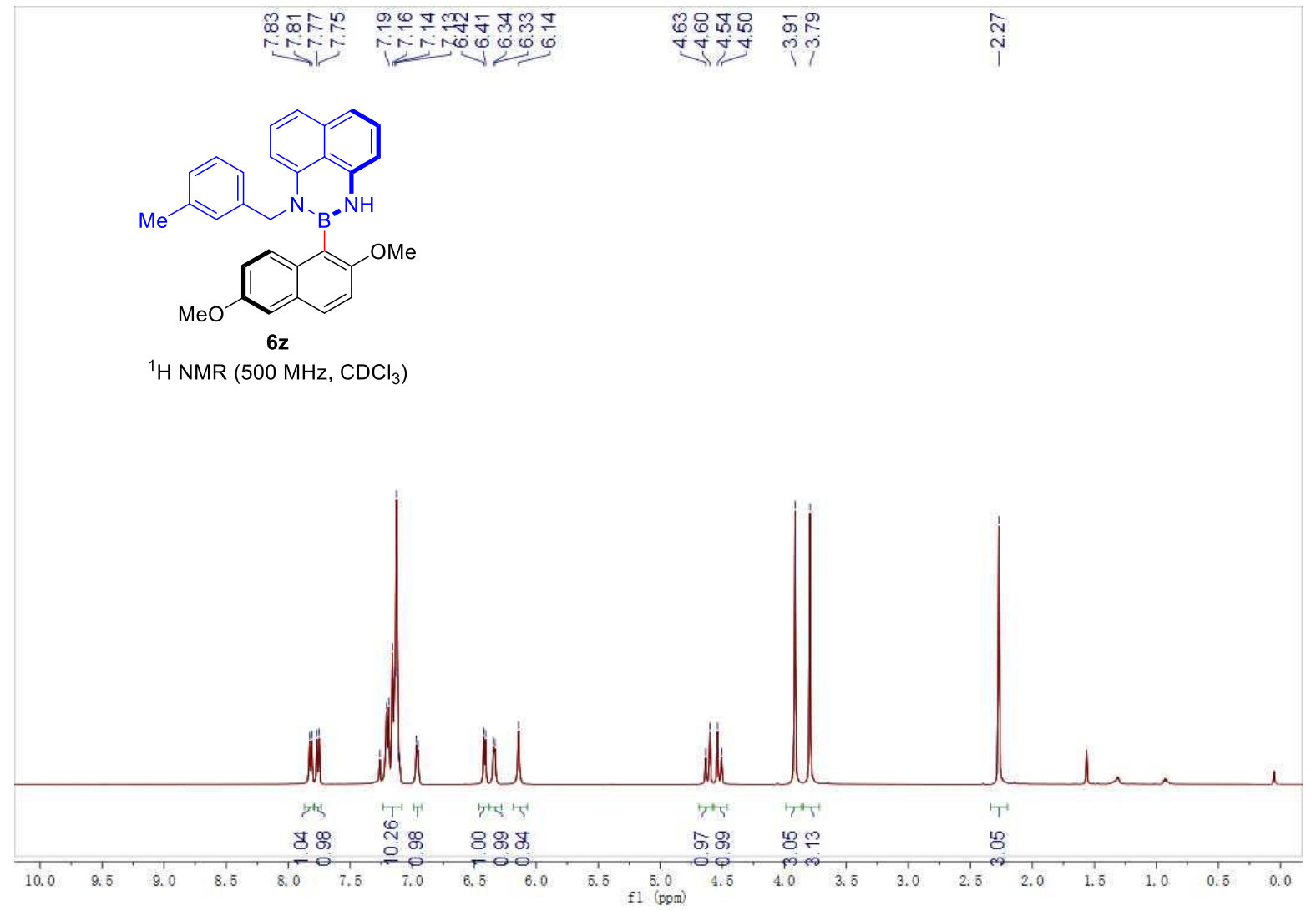


(S)-2-(2,6-dimethoxynaphthalen-1-yl)-1-(3-methylbenzyl)-2,3-dihydro-1H-naphtho[1,8-de][1,3,2]diazabo rinine $(6 z)$
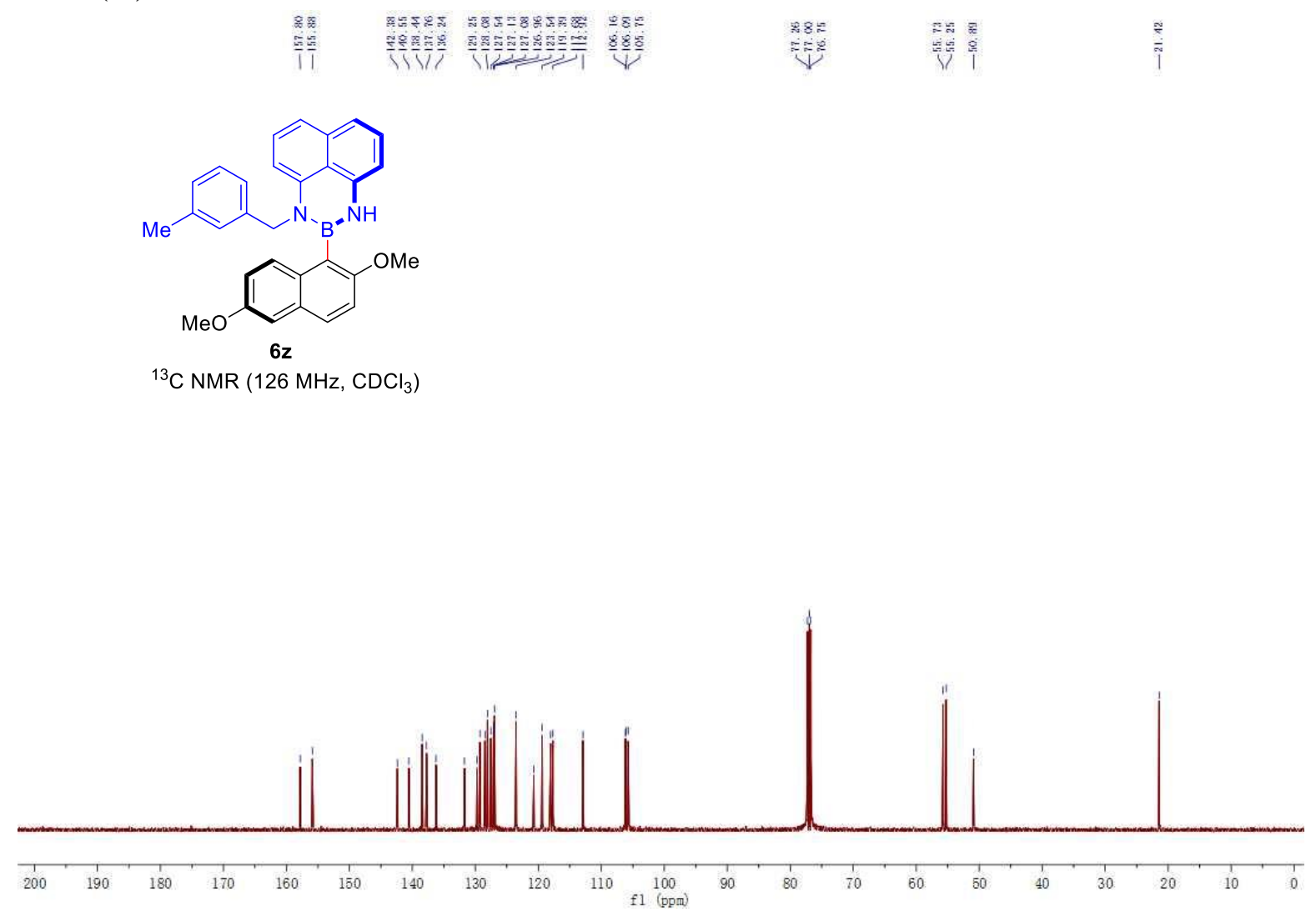

(S)-2-(2,6-dimethoxynaphthalen-1-yl)-1-(3-methylbenzyl)-2,3-dihydro-1H-naphtho[1,8-de][1,3,2]diazabo rinine $(6 z)$

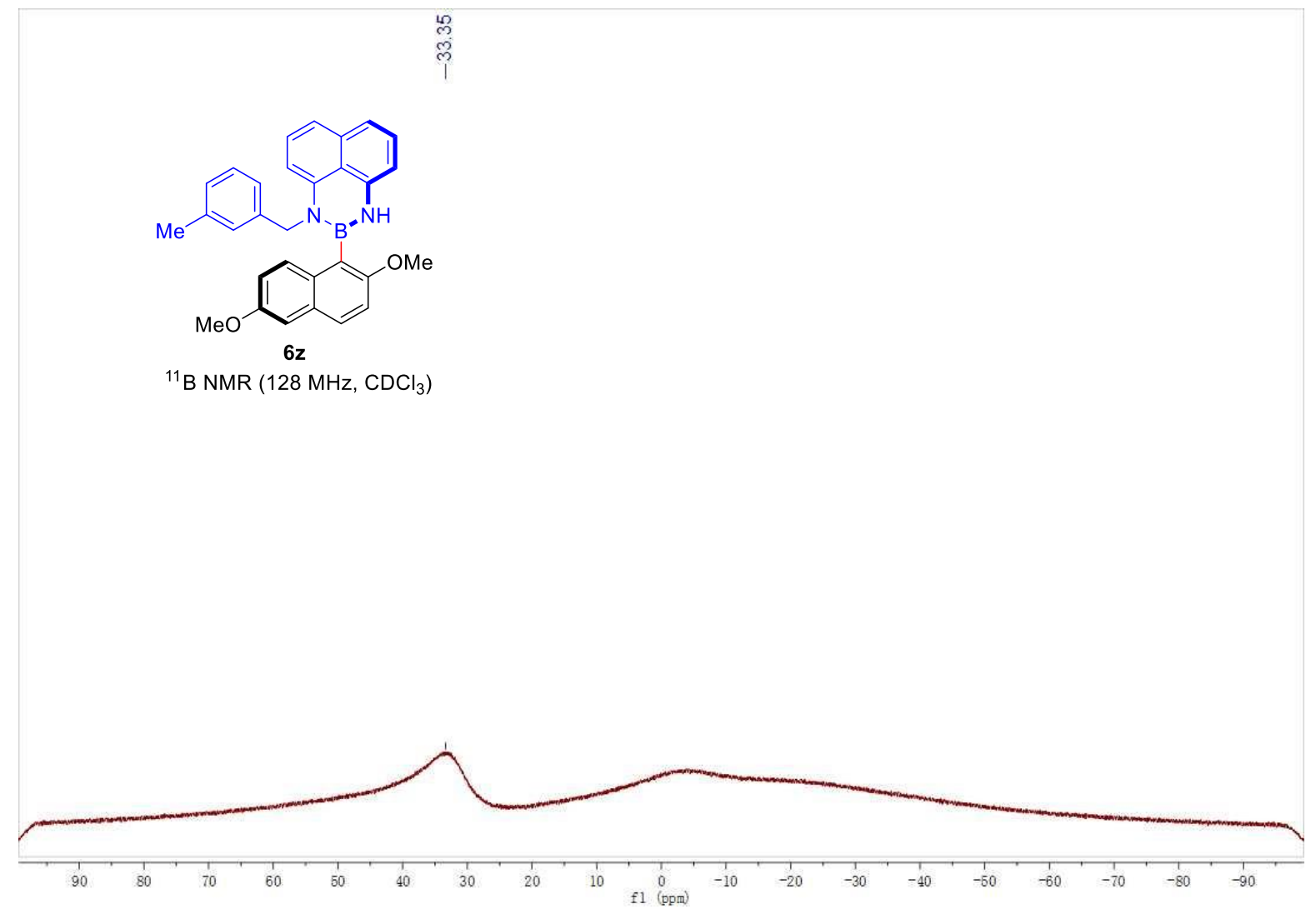


(S)-2-(2,6-dimethoxynaphthalen-1-yl)-1-(4-methylbenzyl)-2,3-dihydro-1H-naphtho[1,8-de][1,3,2]diazabo rinine (6aa)

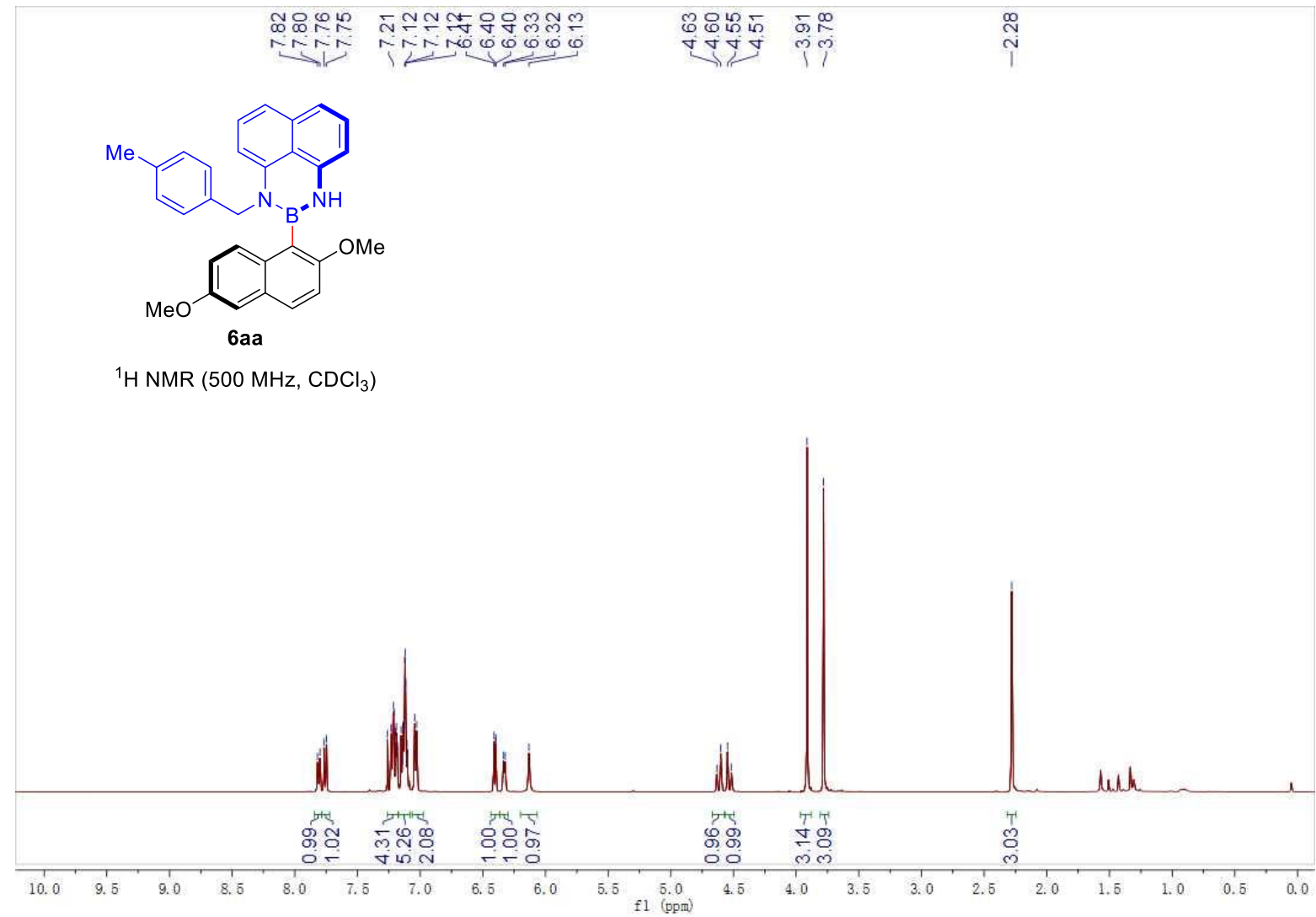

(S)-2-(2,6-dimethoxynaphthalen-1-yl)-1-(4-methylbenzyl)-2,3-dihydro-1H-naphtho[1,8-de][1,3,2]diazabo rinine (6aa)

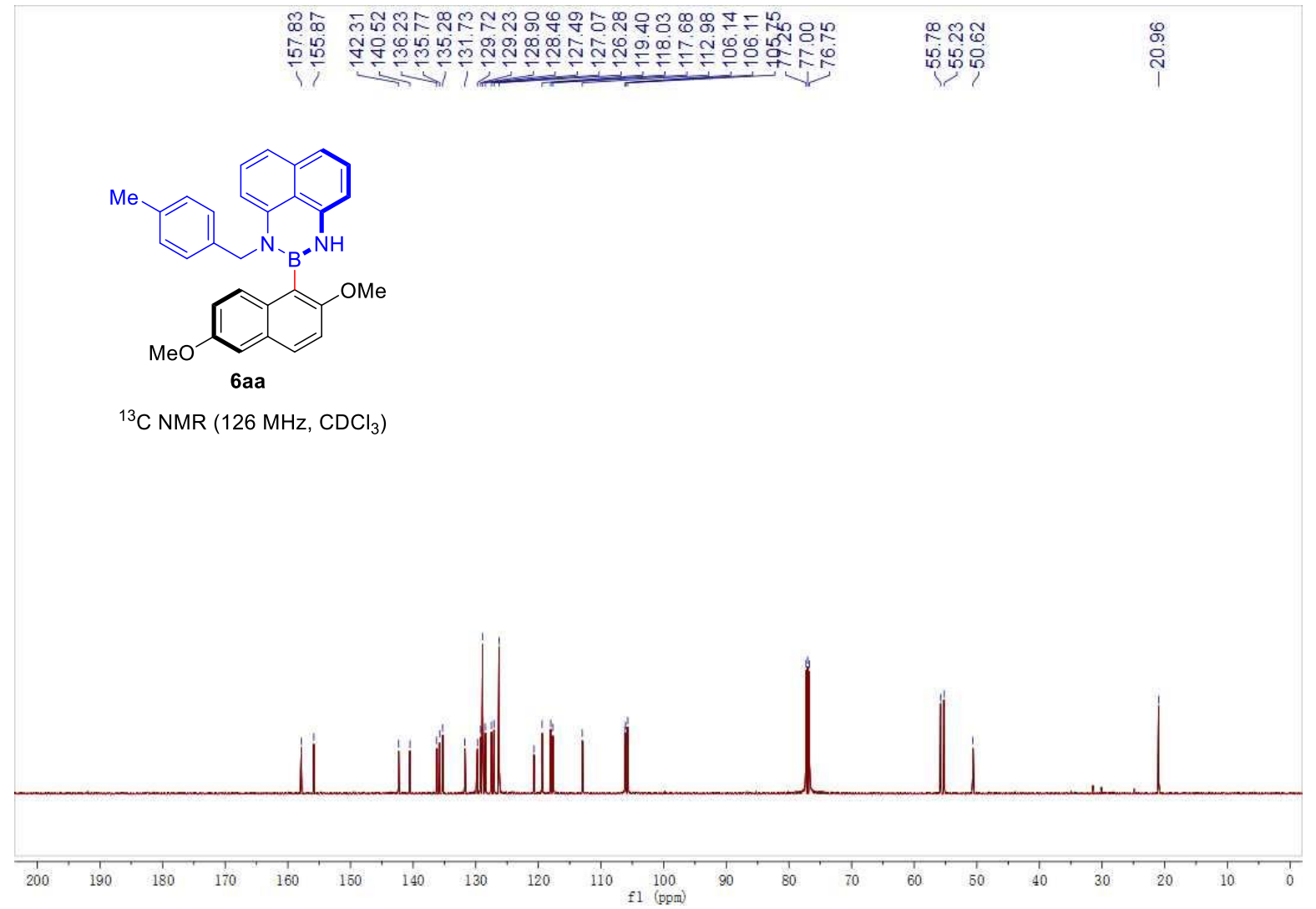


(S)-2-(2,6-dimethoxynaphthalen-1-yl)-1-(4-methylbenzyl)-2,3-dihydro-1H-naphtho[1,8-de][1,3,2]diazabo rinine (6aa)

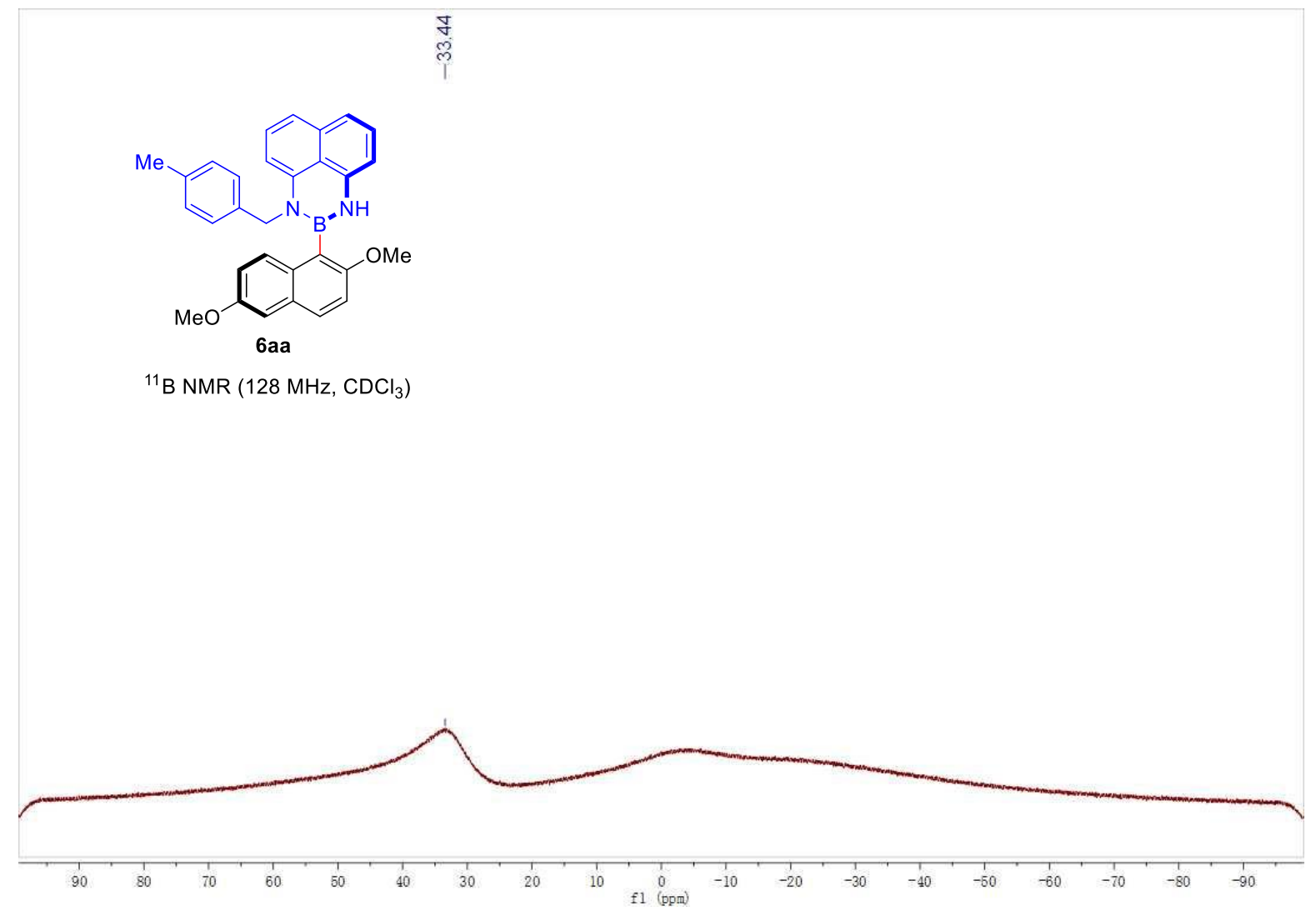

(S)-2-(2,6-dimethoxynaphthalen-1-yl)-1-(4-fluorobenzyl)-2,3-dihydro-1H-naphtho[1,8-de][1,3,2]diazabo rinine (6ab)

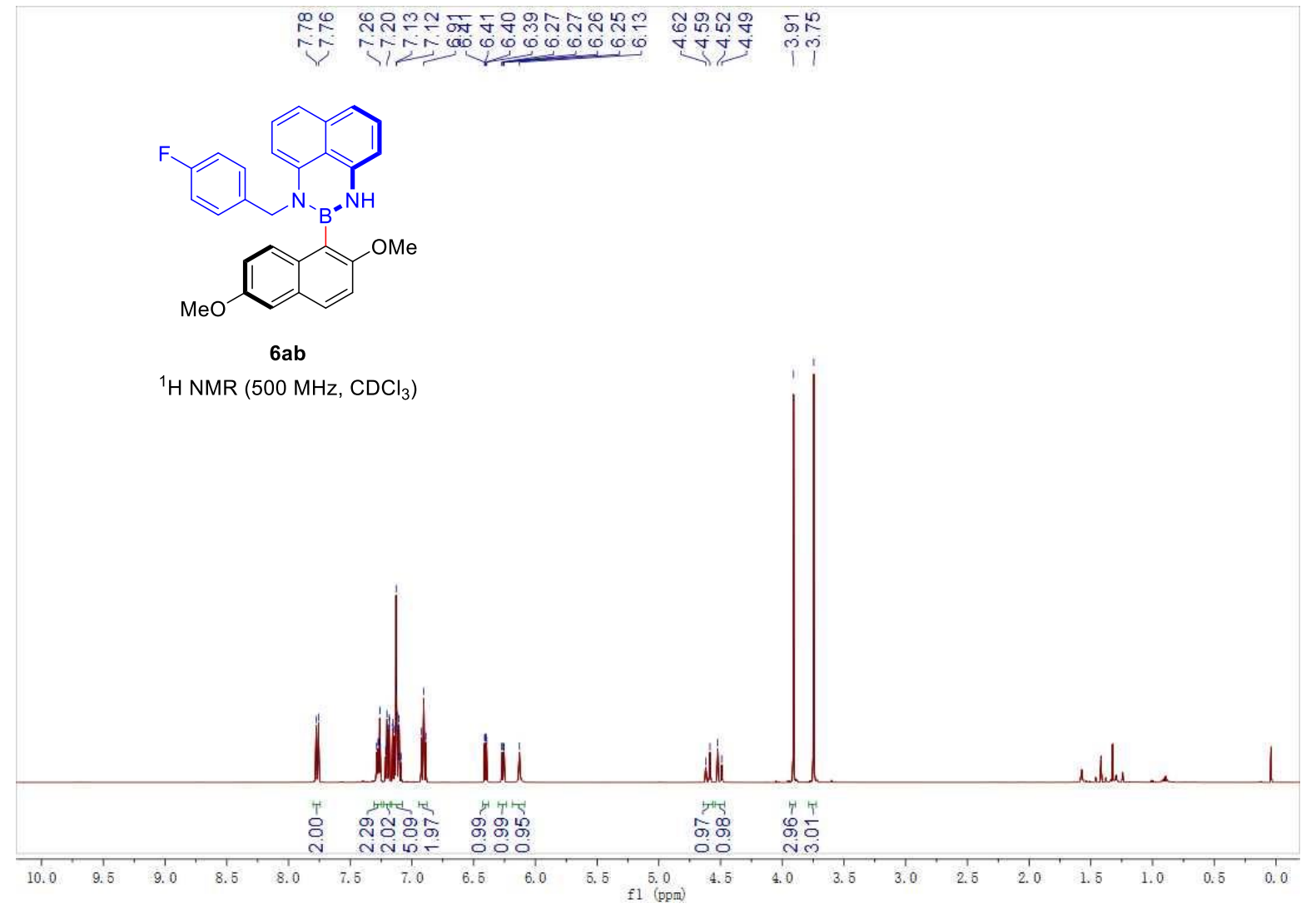


(S)-2-(2,6-dimethoxynaphthalen-1-yl)-1-(4-fluorobenzyl)-2,3-dihydro-1H-naphtho[1,8-de][1,3,2]diazabo rinine (6ab)

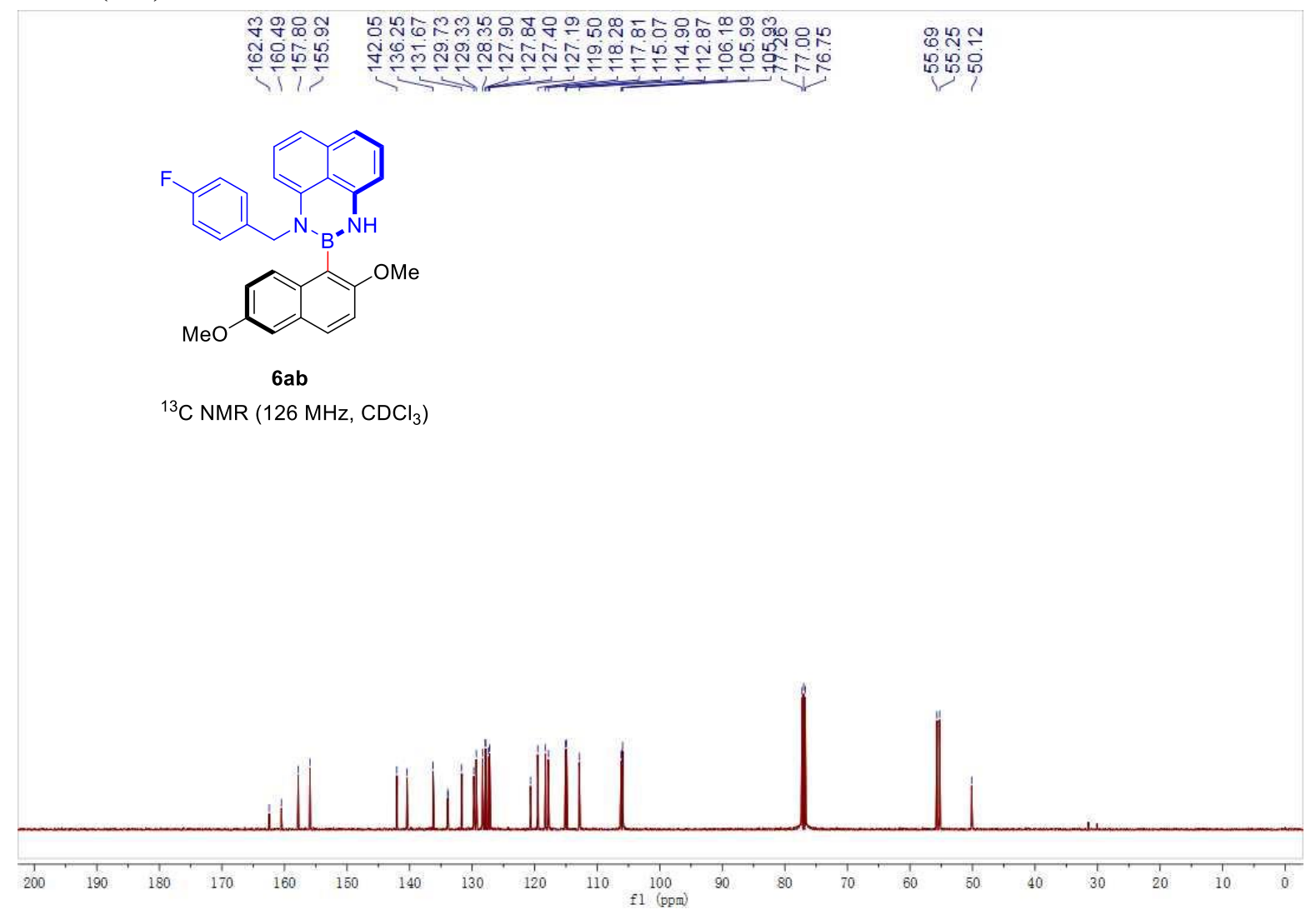

(S)-2-(2,6-dimethoxynaphthalen-1-yl)-1-(4-fluorobenzyl)-2,3-dihydro-1H-naphtho[1,8-de][1,3,2]diazabo rinine (6ab)

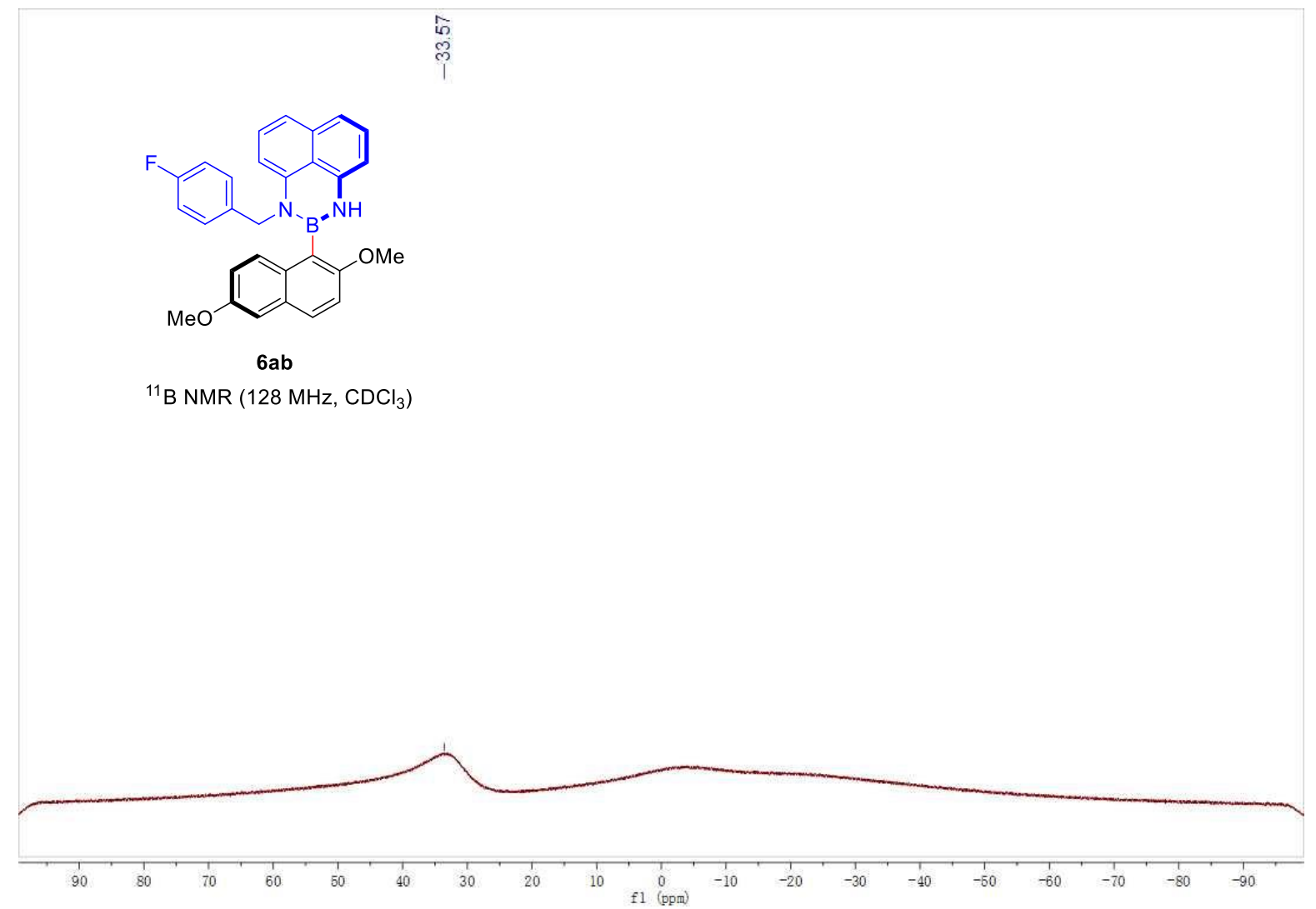


(S)-2-(2,6-dimethoxynaphthalen-1-yl)-1-(thiophen-3-ylmethyl)-2,3-dihydro-1H-naphtho[1,8-de][1,3,2]di azaborinine (6ac)

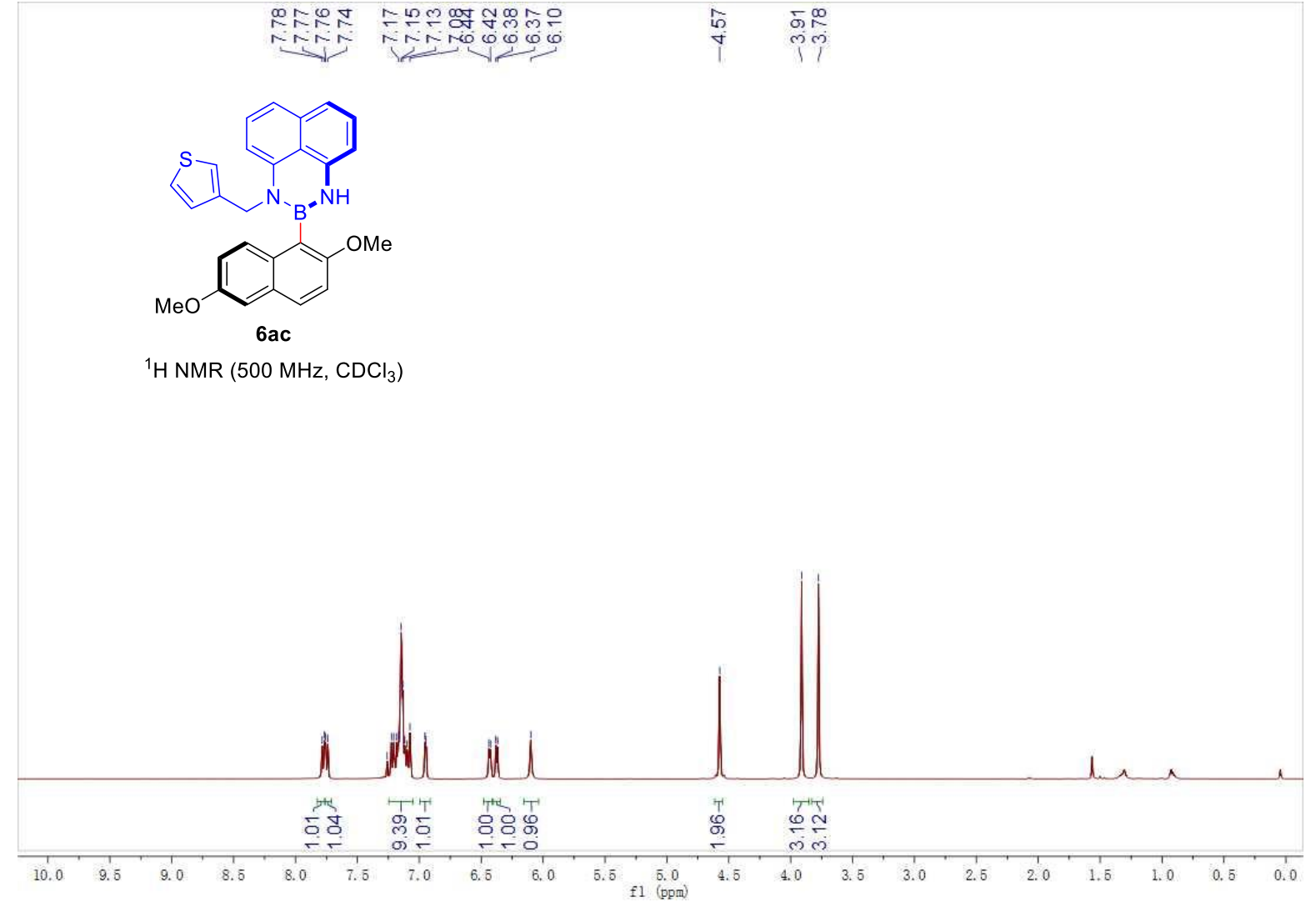

(S)-2-(2,6-dimethoxynaphthalen-1-yl)-1-(thiophen-3-ylmethyl)-2,3-dihydro-1H-naphtho[1,8-de][1,3,2]di azaborinine (6ac)

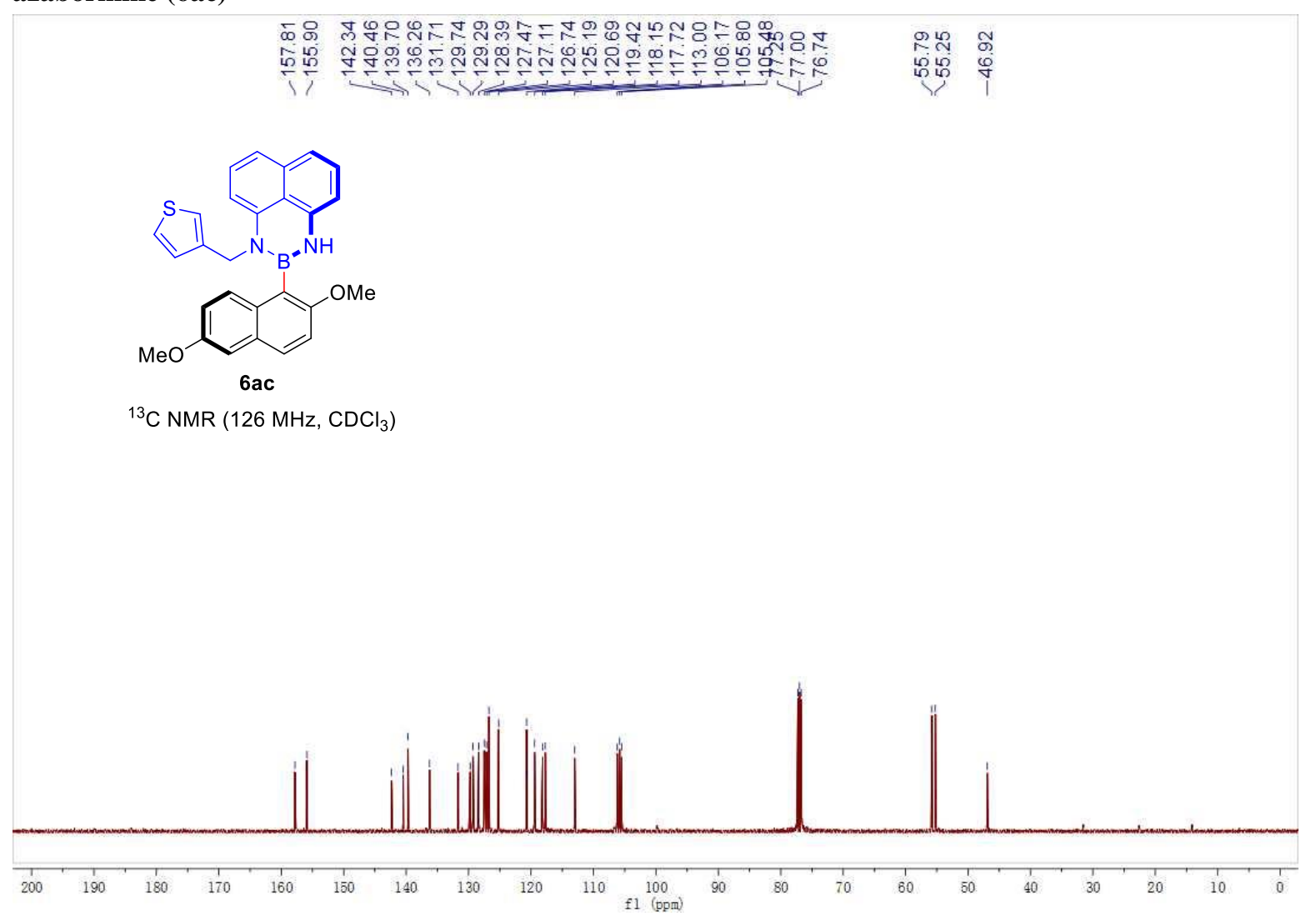


(S)-2-(2,6-dimethoxynaphthalen-1-yl)-1-(thiophen-3-ylmethyl)-2,3-dihydro-1H-naphtho[1,8-de][1,3,2]di azaborinine (6ac)

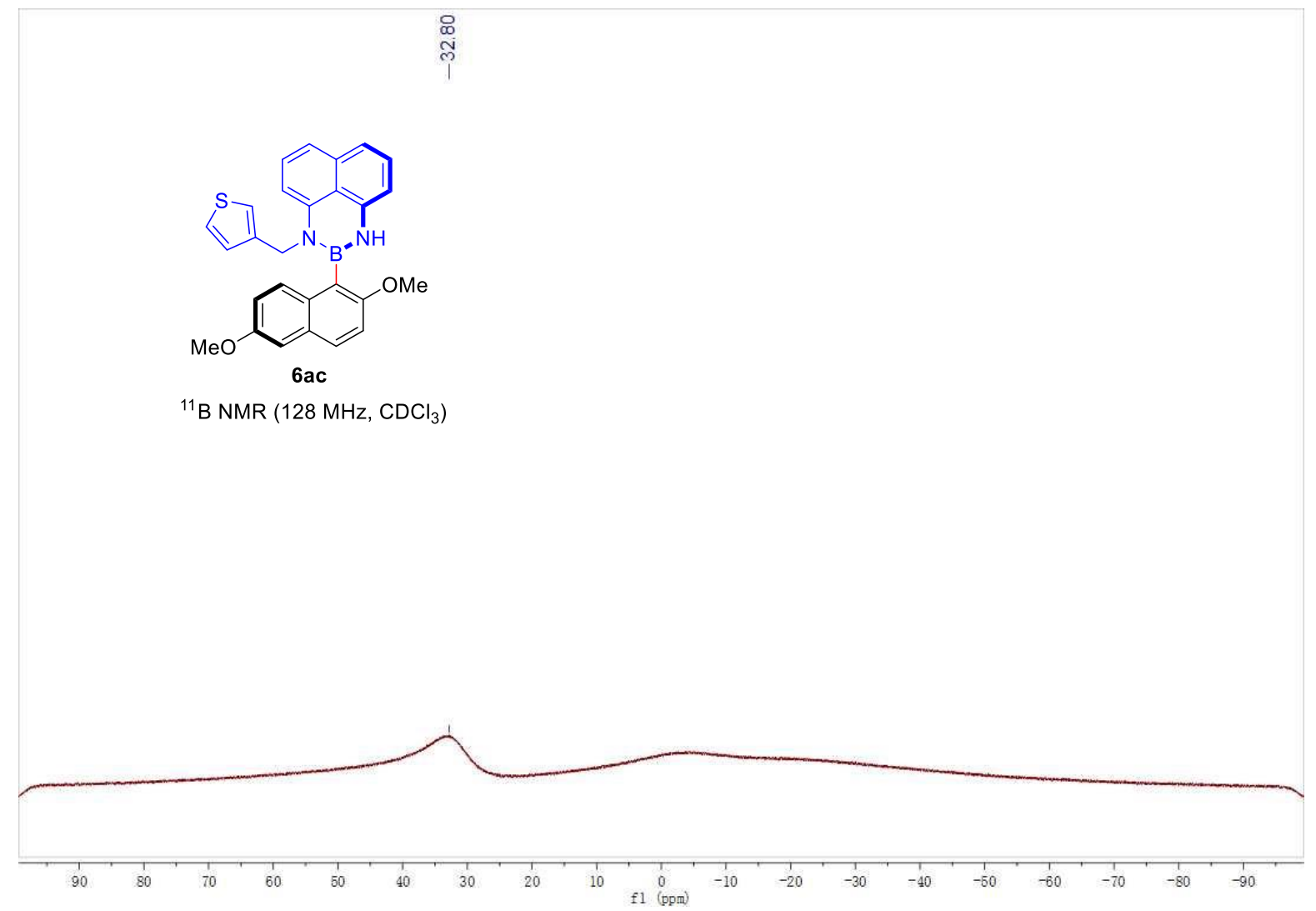

(S)-2-(2,6-dimethoxynaphthalen-1-yl)-1-(naphthalen-2-ylmethyl)-2,3-dihydro-1H-naphtho[1,8-de][1,3,2] diazaborinine (6ad)

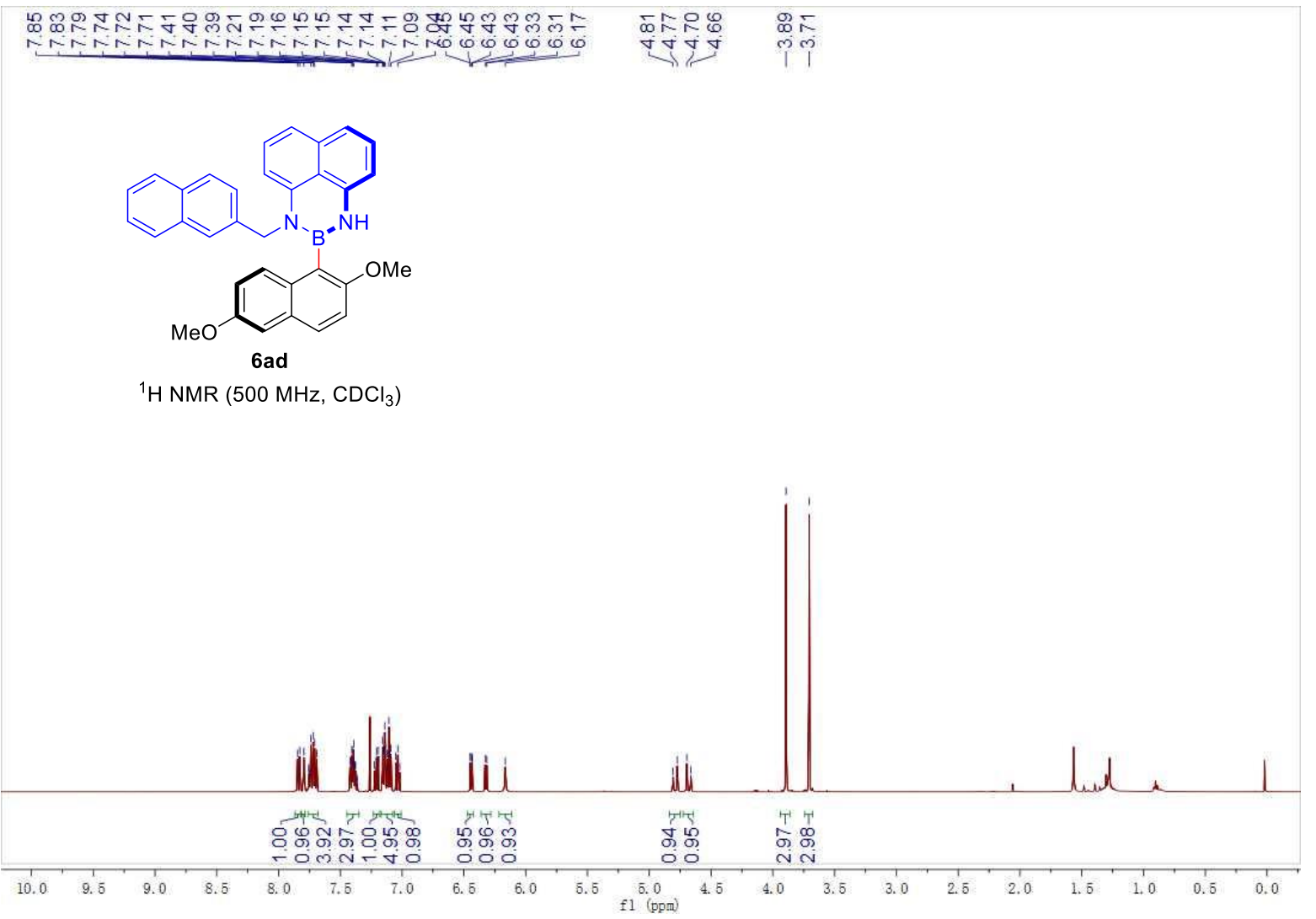


(S)-2-(2,6-dimethoxynaphthalen-1-yl)-1-(naphthalen-2-ylmethyl)-2,3-dihydro-1H-naphtho[1,8-de][1,3,2] diazaborinine (6ad)

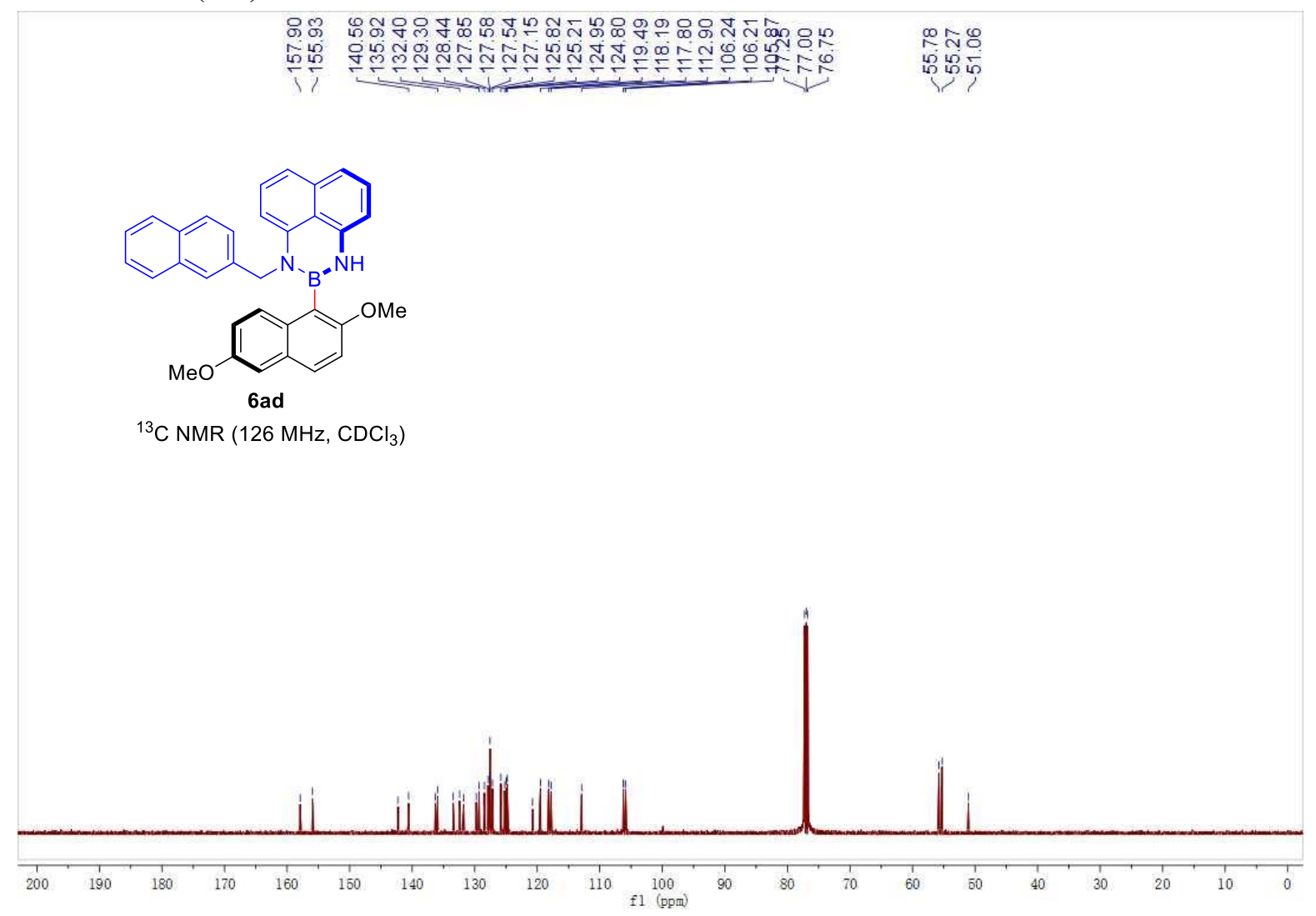

(S)-2-(2,6-dimethoxynaphthalen-1-yl)-1-(naphthalen-2-ylmethyl)-2,3-dihydro-1H-naphtho[1,8-de] [1,3,2] diazaborinine (6ad)

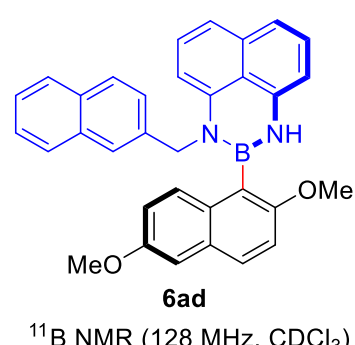

$\underset{\widetilde{1}}{\stackrel{1}{1}}$

${ }^{11} \mathrm{~B}$ NMR $\left(128 \mathrm{MHz}, \mathrm{CDCl}_{3}\right)$

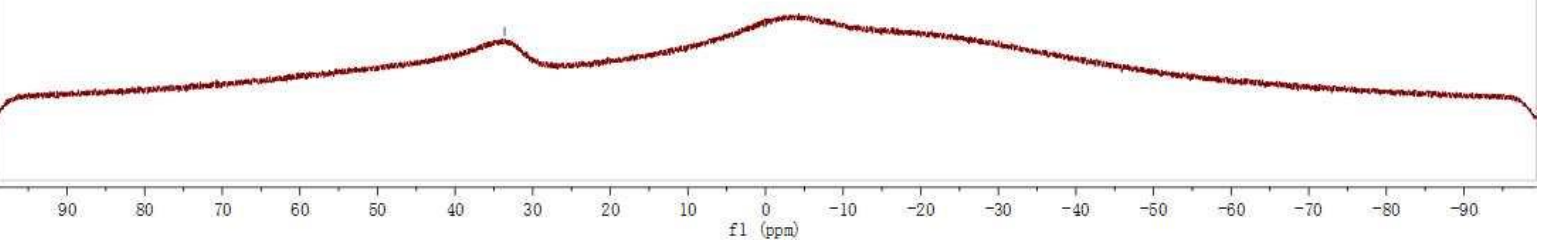


(S)-1-ethyl-2-(2-methoxynaphthalen-1-yl)-3-methyl-2,3-dihydro-1H-naphtho[1,8-de][1,3,2]diazaborinine (7a)

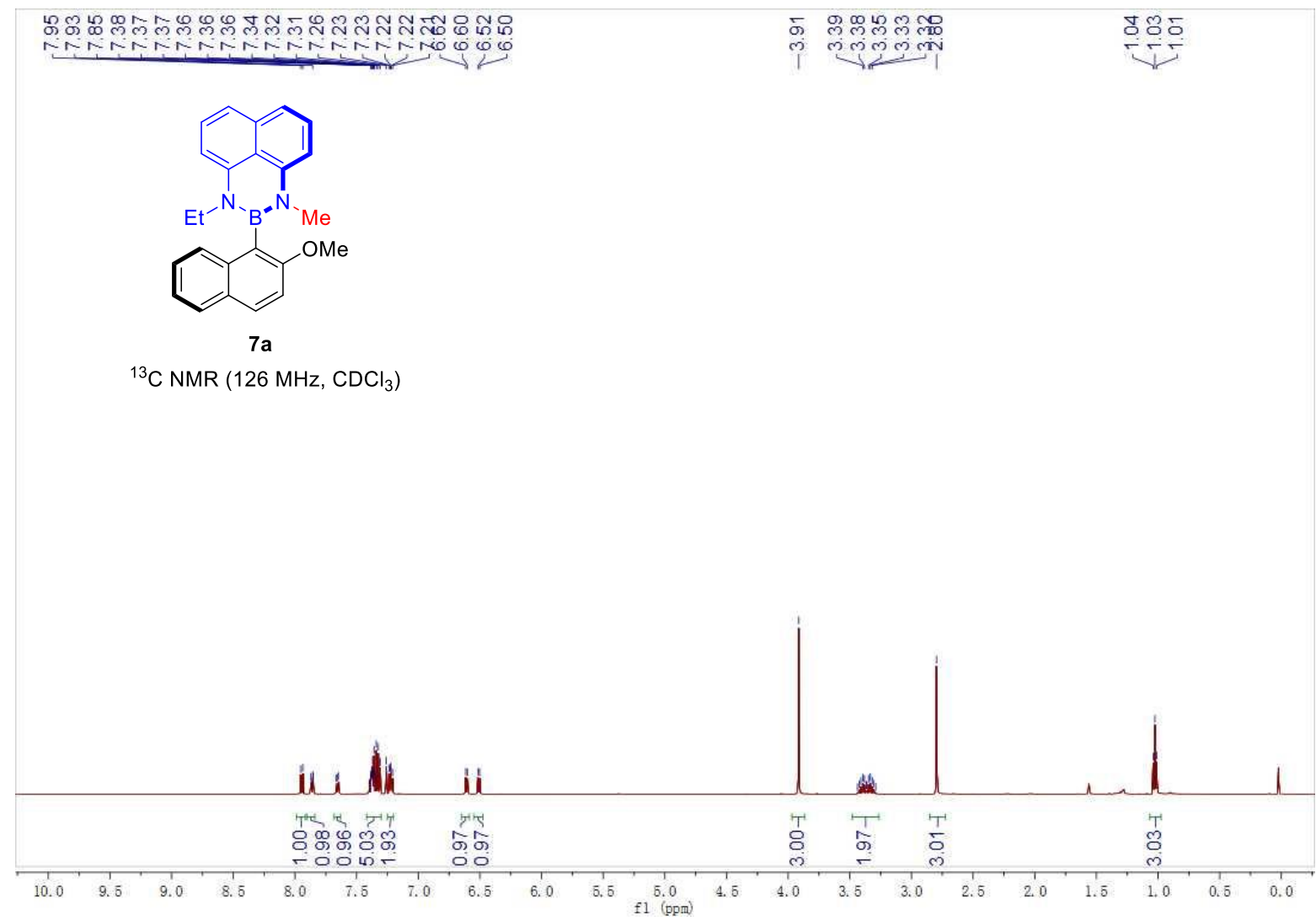

(S)-1-ethyl-2-(2-methoxynaphthalen-1-yl)-3-methyl-2,3-dihydro-1H-naphtho[1,8-de][1,3,2]diazaborinine (7a)

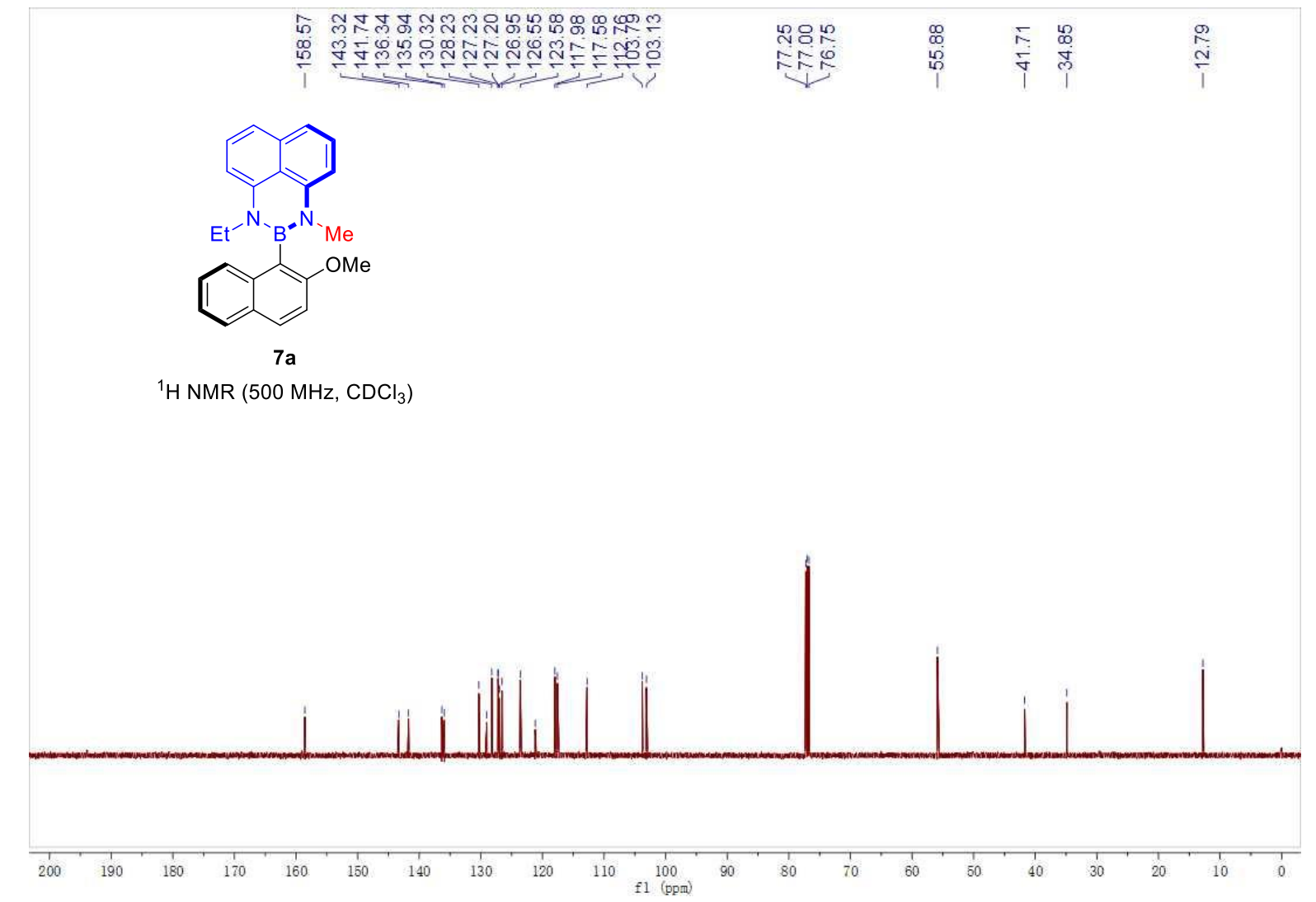


(S)-1-ethyl-2-(2-methoxynaphthalen-1-yl)-3-methyl-2,3-dihydro-1H-naphtho[1,8-de][1,3,2]diazaborinine (7a)

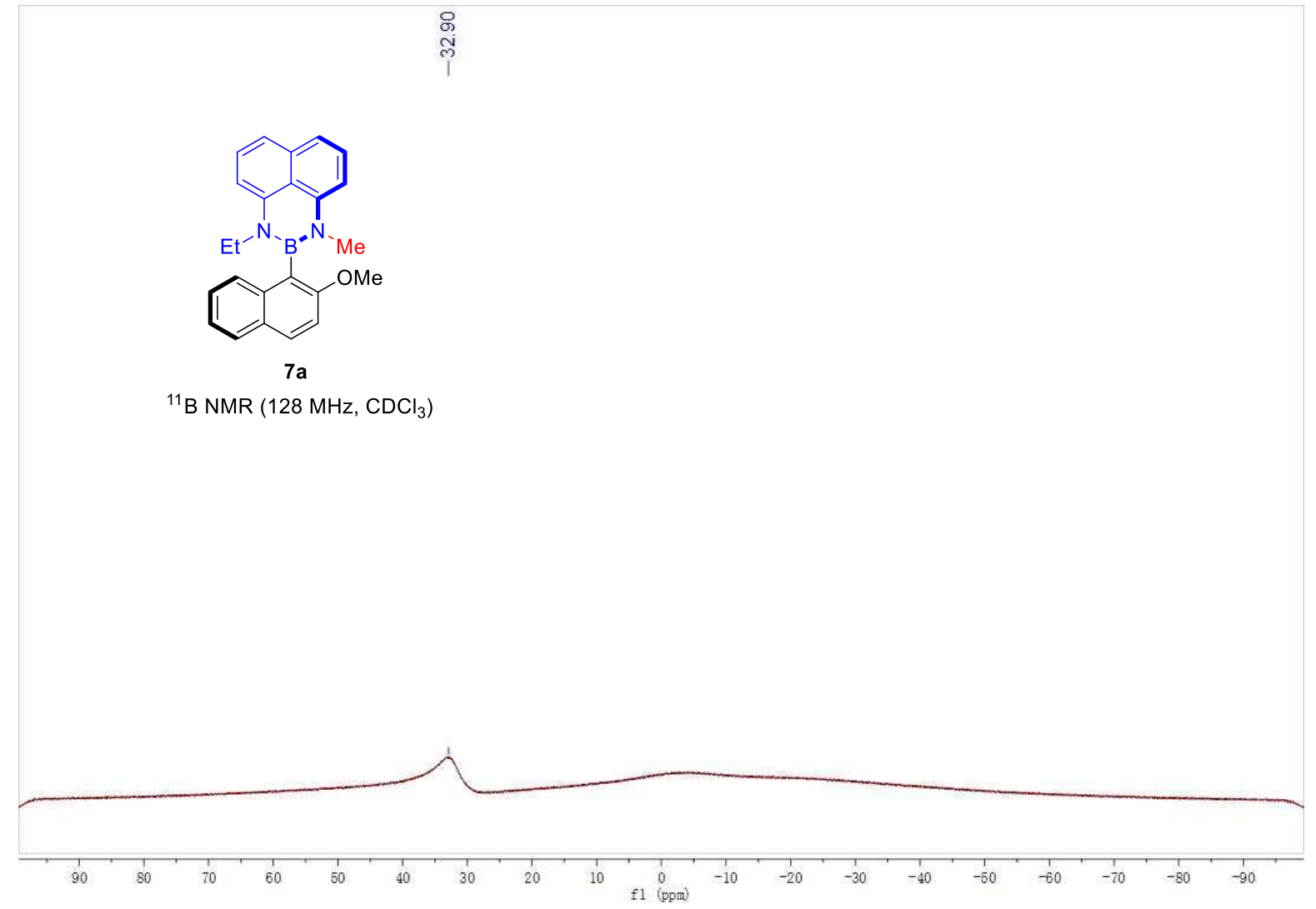

(R)-1-allyl-3-ethyl-2-(2-methoxynaphthalen-1-yl)-2,3-dihydro-1H-naphtho[1,8-de][1,3,2]diazaborinine

(7b)

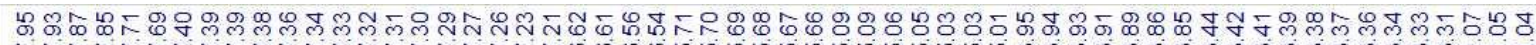

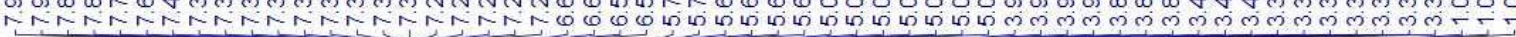

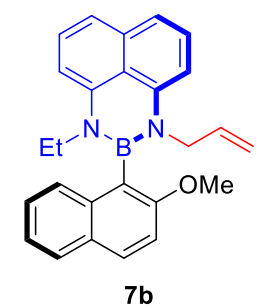

${ }^{1} \mathrm{H} \mathrm{NMR}\left(500 \mathrm{MHz}, \mathrm{CDCl}_{3}\right)$

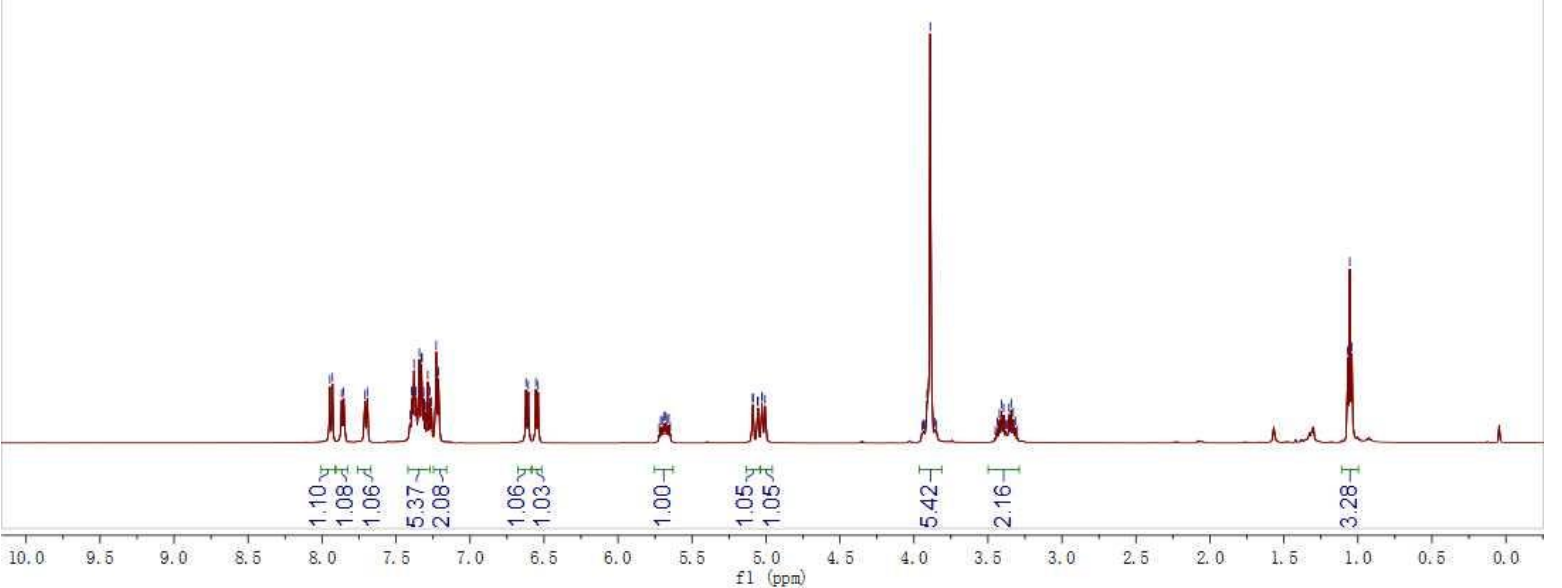


(R)-1-allyl-3-ethyl-2-(2-methoxynaphthalen-1-yl)-2,3-dihydro-1H-naphtho[1,8-de][1,3,2]diazaborinine (7b)

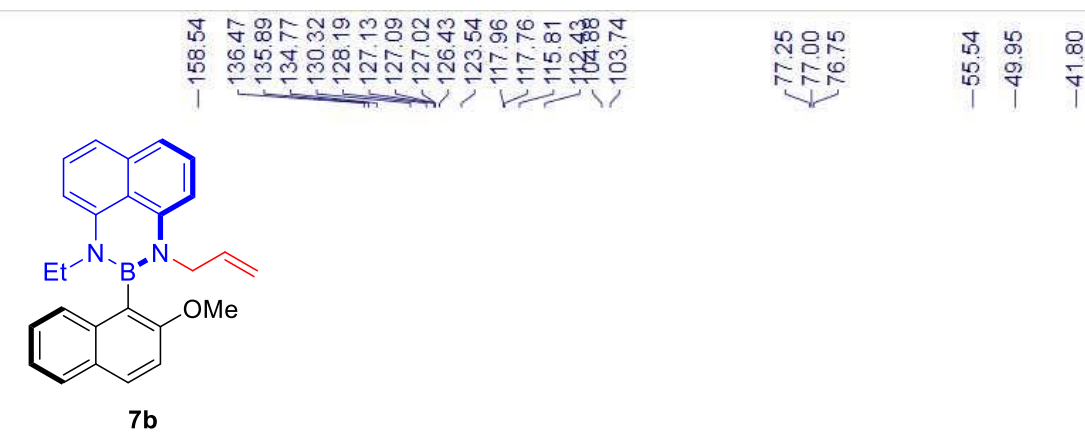

${ }^{13} \mathrm{C} \mathrm{NMR}\left(126 \mathrm{MHz}, \mathrm{CDCl}_{3}\right)$
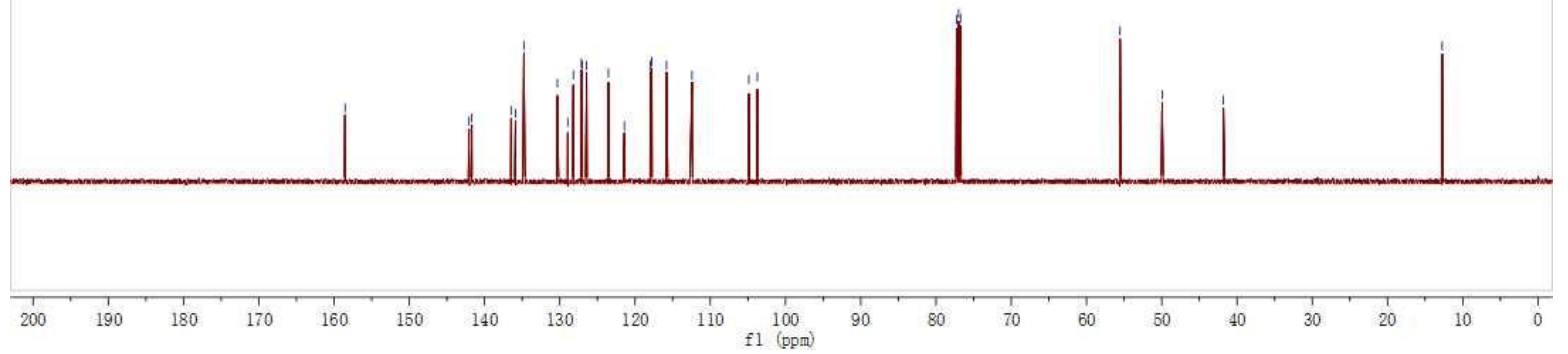

(R)-1-allyl-3-ethyl-2-(2-methoxynaphthalen-1-yl)-2,3-dihydro-1H-naphtho[1,8-de][1,3,2]diazaborinine (7b)

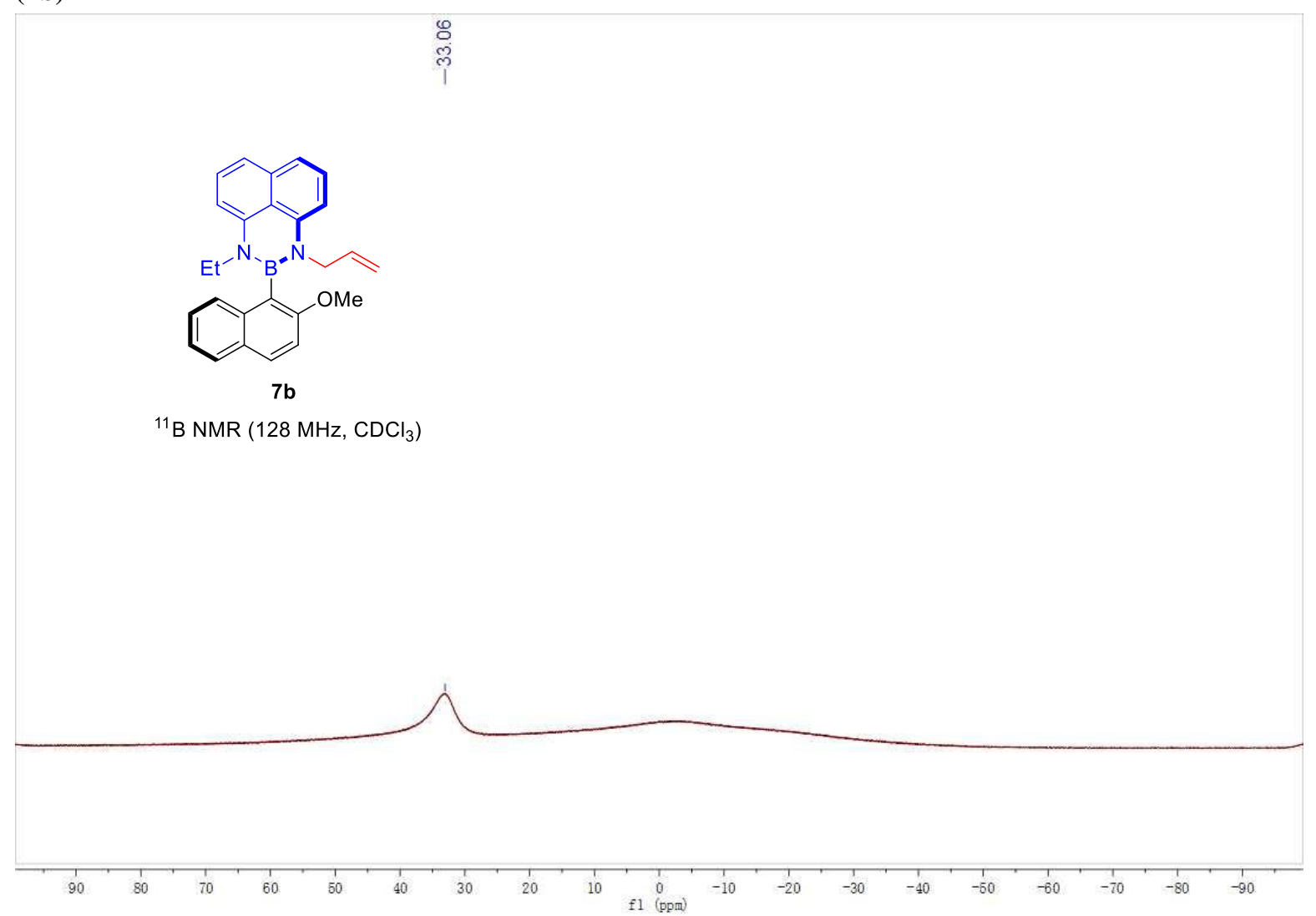


(R)-1-(diphenylphosphanyl)-3-ethyl-2-(2-methoxynaphthalen-1-yl)-2,3-dihydro-1H-naphtho[1,8-de][1,3, 2]diazaborinine (7c)

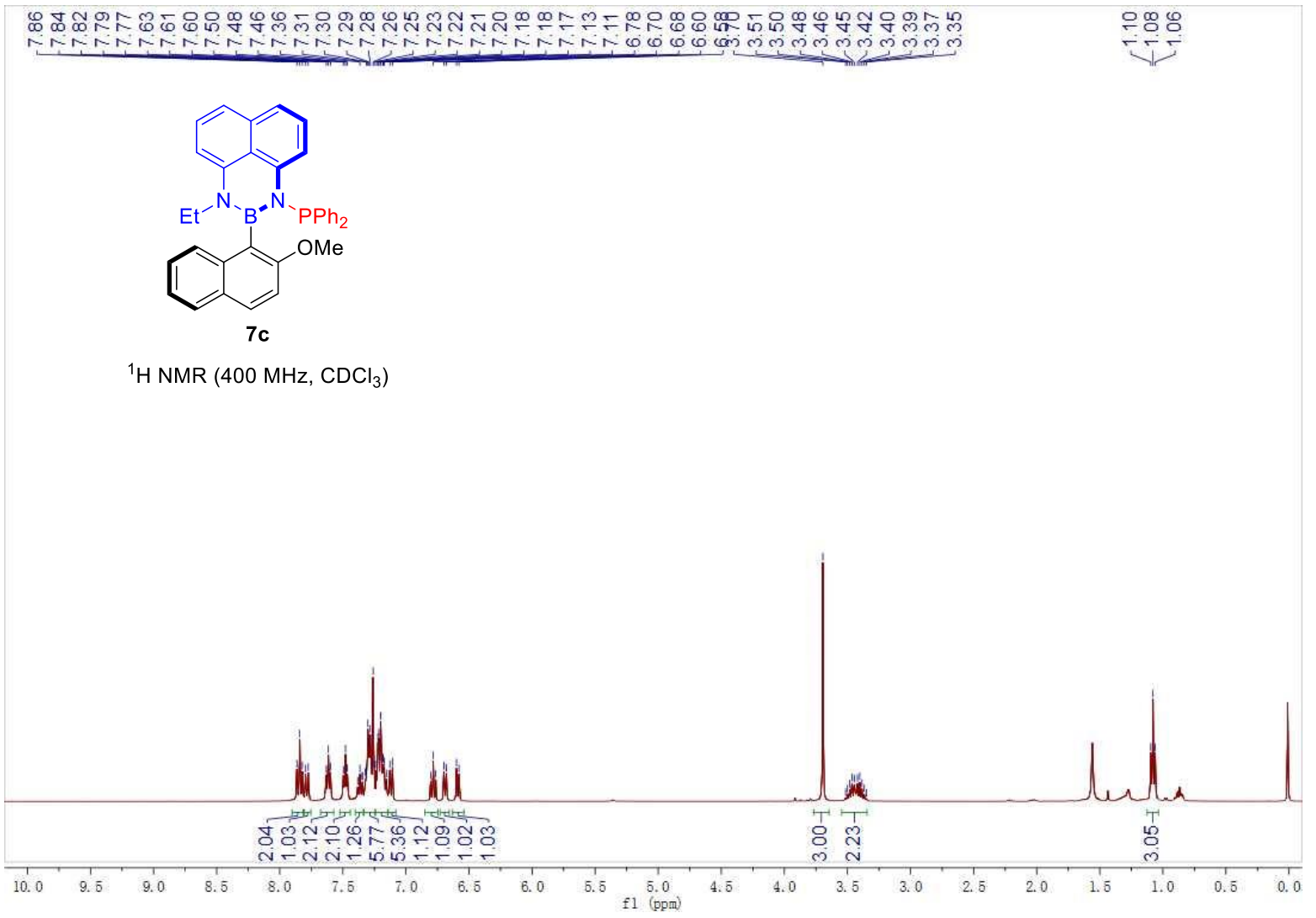

(R)-1-(diphenylphosphanyl)-3-ethyl-2-(2-methoxynaphthalen-1-yl)-2,3-dihydro-1H-naphtho[1,8-de][1,3, 2]diazaborinine (7c)

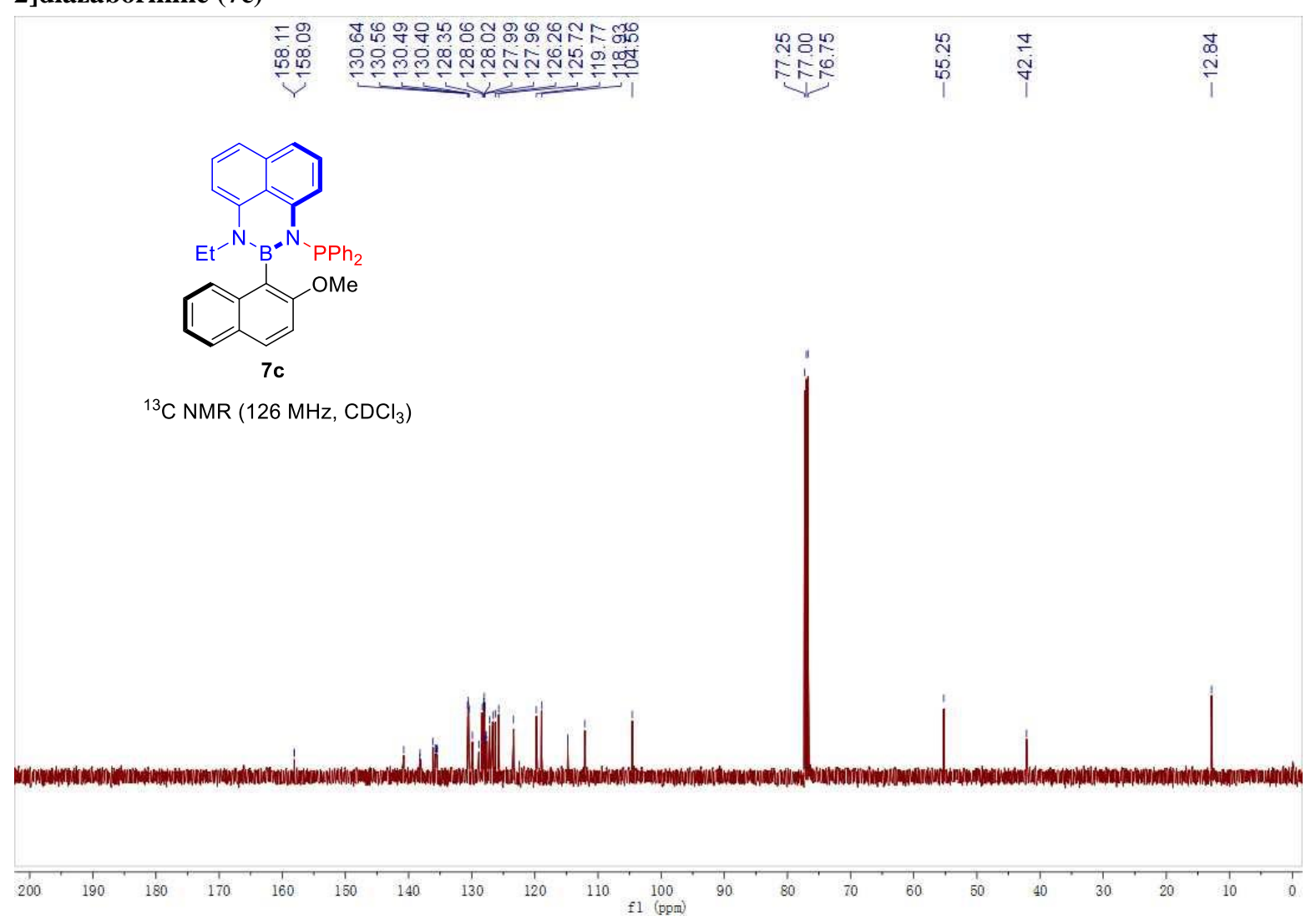


(R)-1-(diphenylphosphanyl)-3-ethyl-2-(2-methoxynaphthalen-1-yl)-2,3-dihydro-1H-naphtho[1,8-de][1,3, 2]diazaborinine (7c)

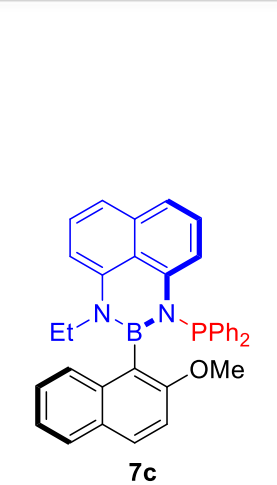

${ }^{11} \mathrm{~B}$ NMR $\left(128 \mathrm{MHz}, \mathrm{CDCl}_{3}\right)$

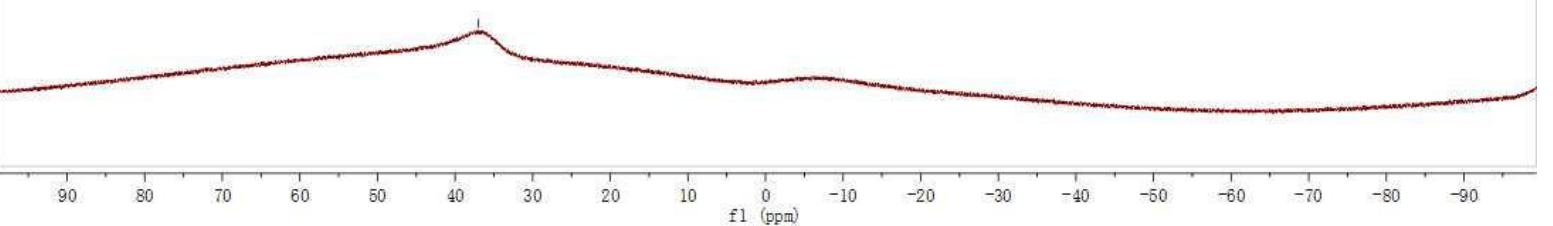

(R)-1-(diphenylphosphanyl)-3-ethyl-2-(2-methoxynaphthalen-1-yl)-2,3-dihydro-1H-naphtho[1,8-de][1,3, 2]diazaborinine (7c)

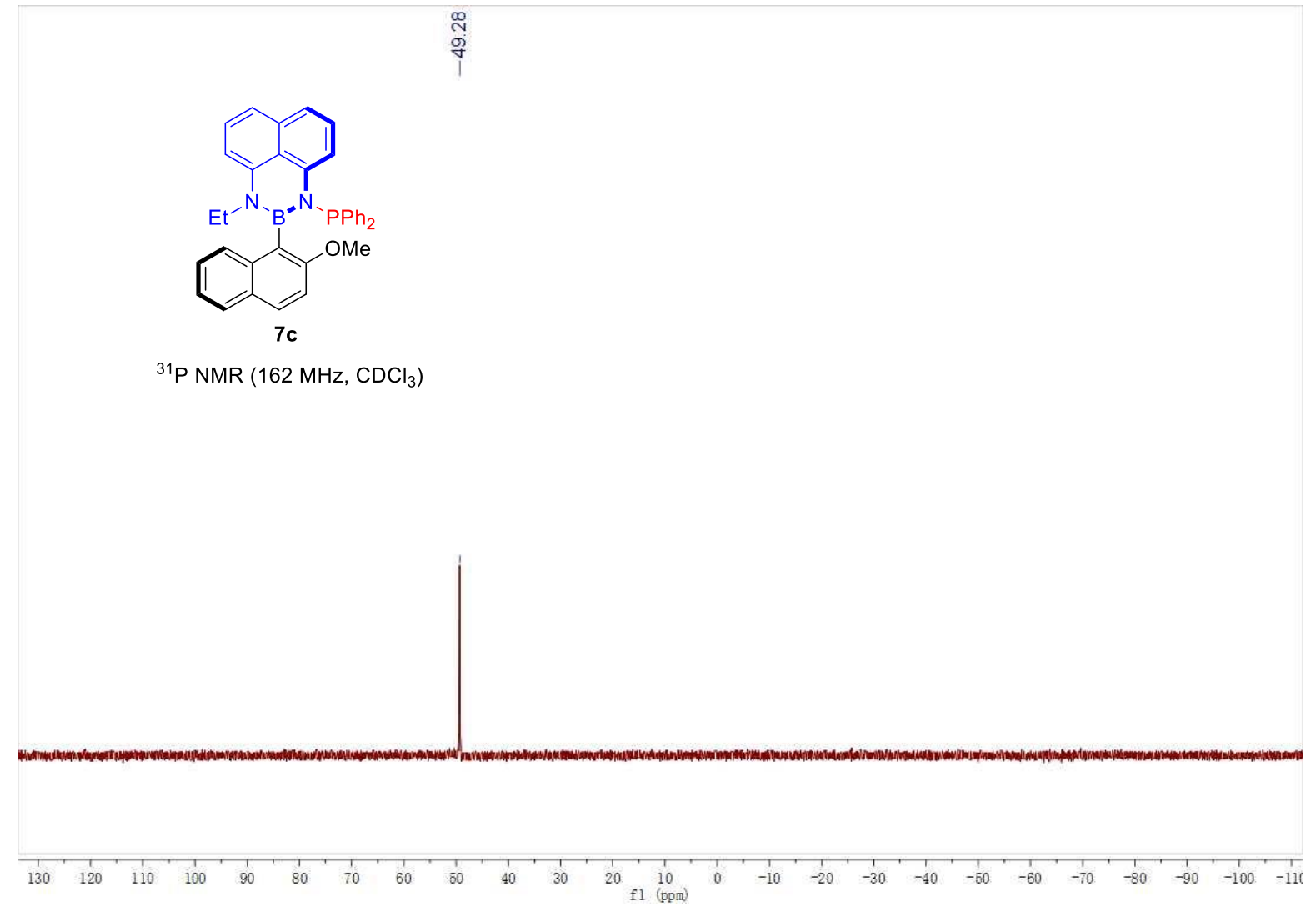


(R)-1-((11bR)-dinaphtho[2,1-d:1',2'-f][1,3,2]dioxaphosphepin-4-yl)-3-ethyl-2-(2-methoxynaphthalen-1-yl )-2,3-dihydro-1H-naphtho[1,8-de][1,3,2]diazaborinine (7d)

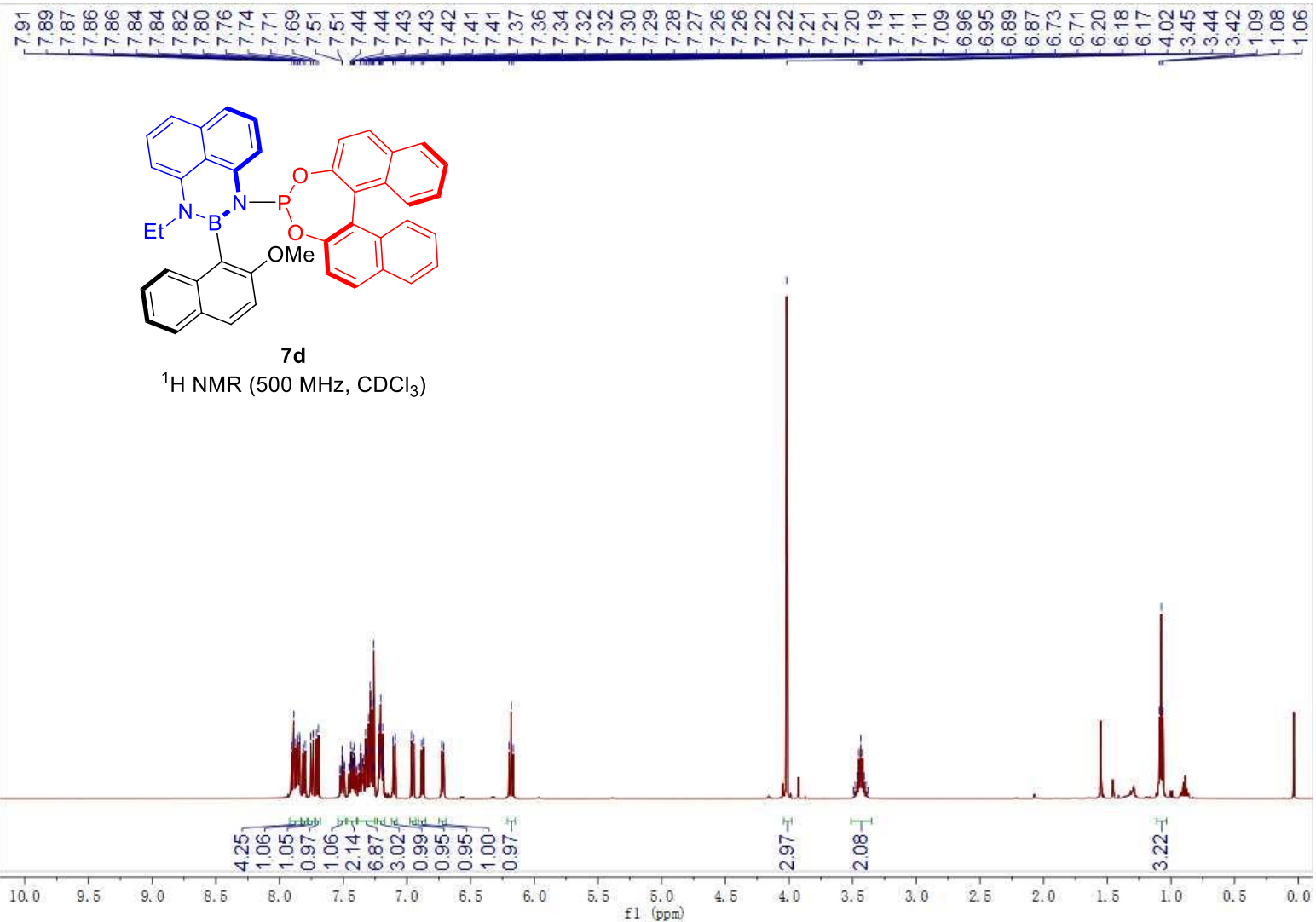

(R)-1-((11bR)-dinaphtho[2,1-d:1',2'-f][1,3,2]dioxaphosphepin-4-yl)-3-ethyl-2-(2-methoxynaphthalen-1-yl )-2,3-dihydro-1H-naphtho[1,8-de][1,3,2]diazaborinine (7d)

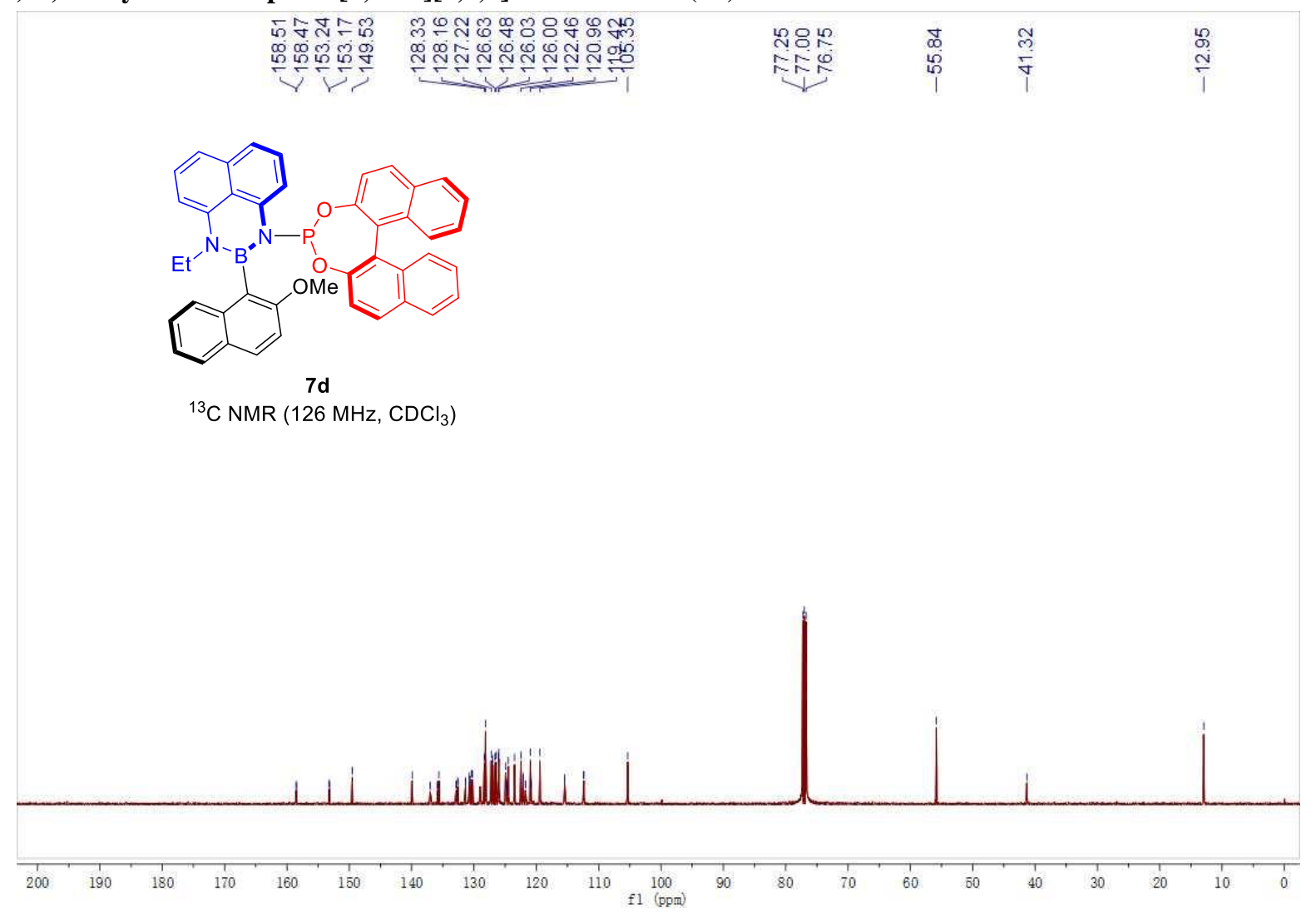


(R)-1-((11bR)-dinaphtho[2,1-d:1',2'-f][1,3,2] dioxaphosphepin-4-yl)-3-ethyl-2-(2-methoxynaphthalen-1-yl )-2,3-dihydro-1H-naphtho[1,8-de][1,3,2]diazaborinine (7d)

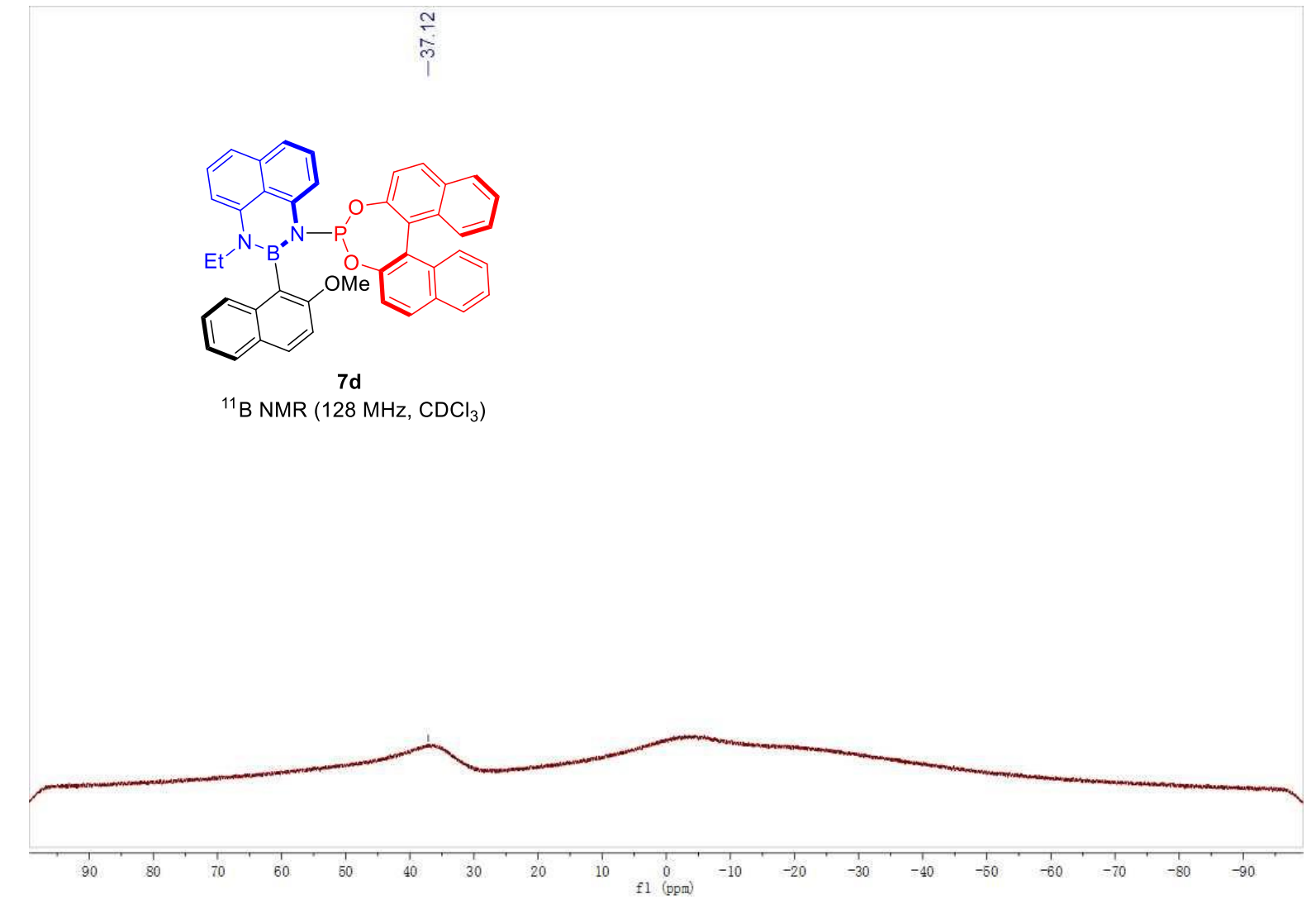

(R)-1-((11bR)-dinaphtho[2,1-d:1',2'-f][1,3,2] dioxaphosphepin-4-yl)-3-ethyl-2-(2-methoxynaphthalen-1-yl )-2,3-dihydro-1H-naphtho[1,8-de][1,3,2]diazaborinine (7d)
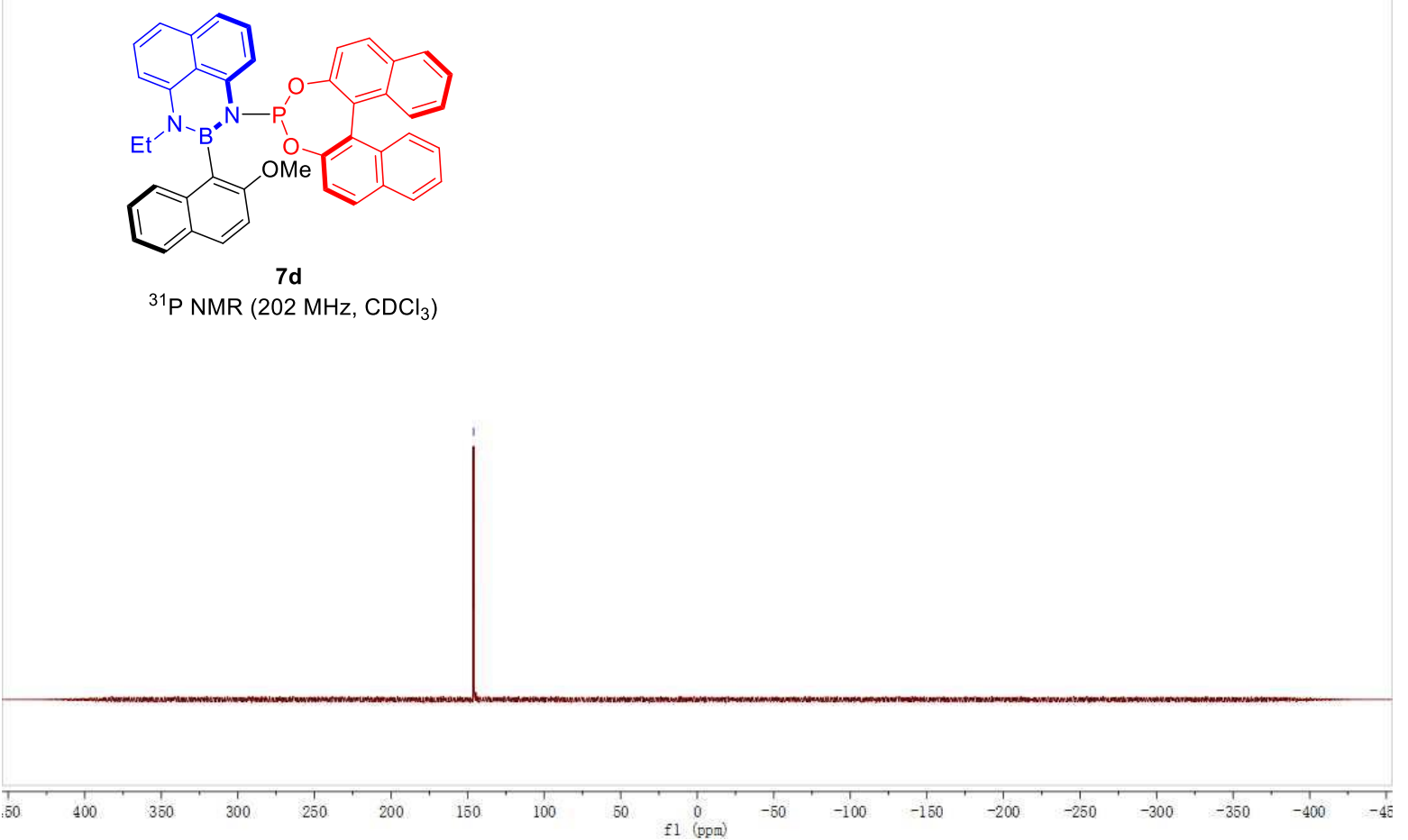
Diethyl $(R, E)$-2-(1,3-diphenylallyl)malonate (10)

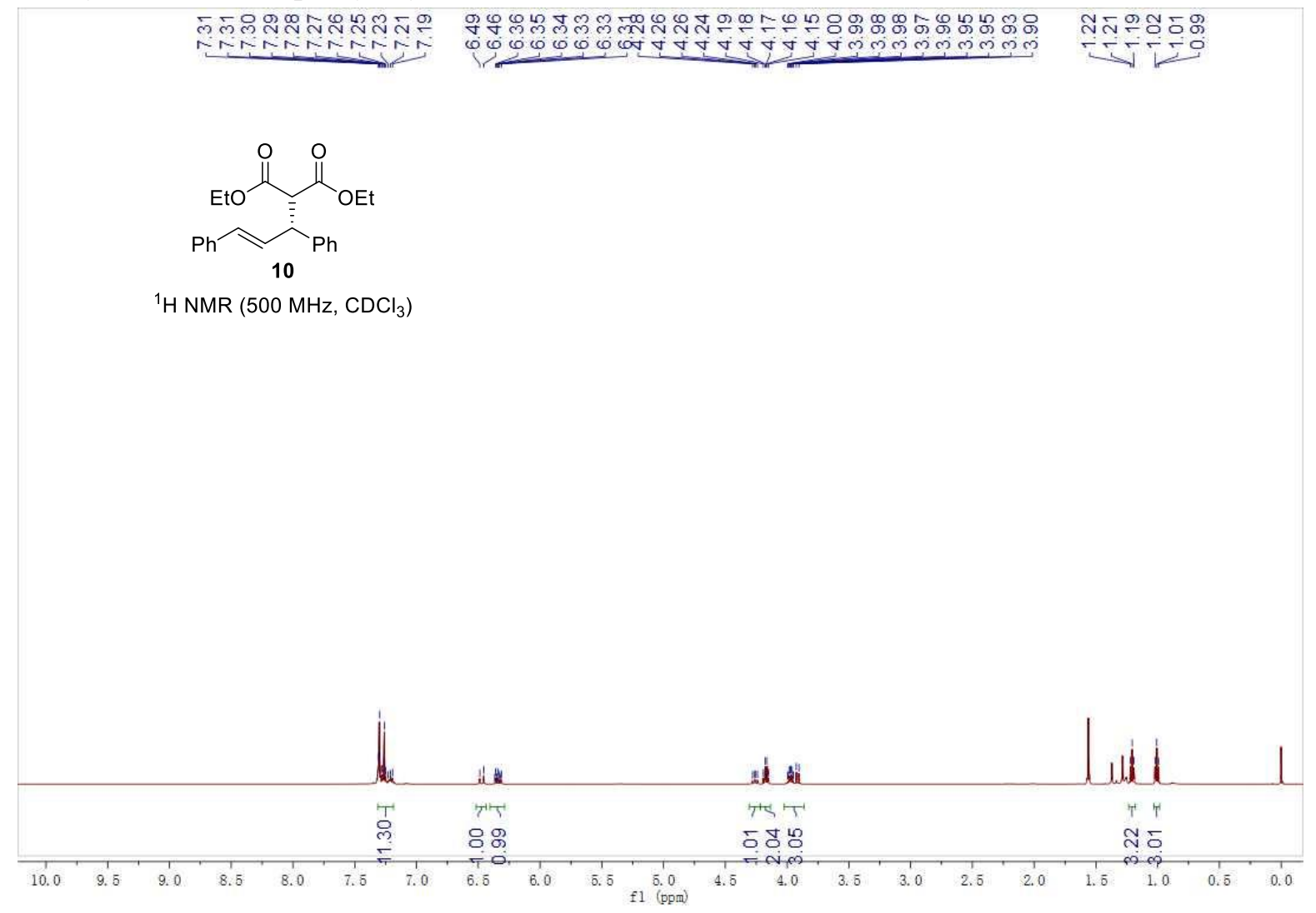




\section{Chiral HPLC Charts}

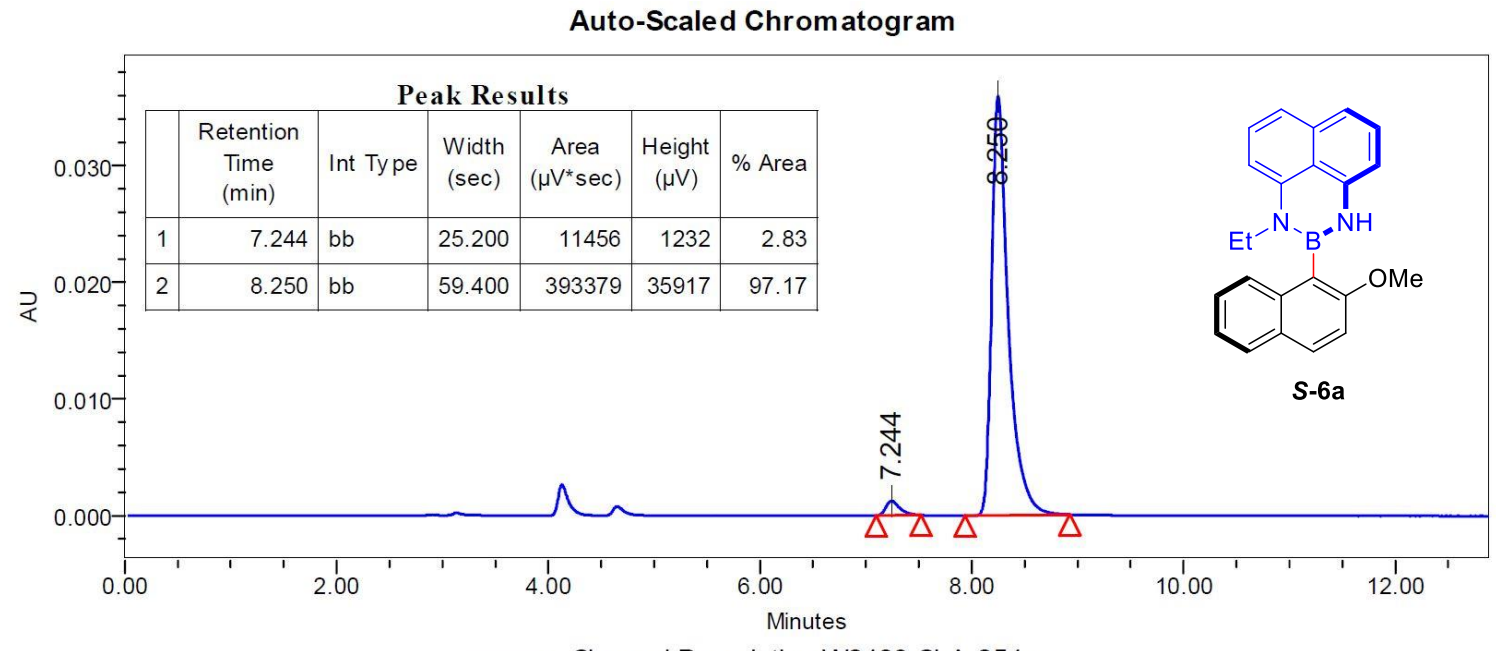

Channel Description W2489 ChA 254nm

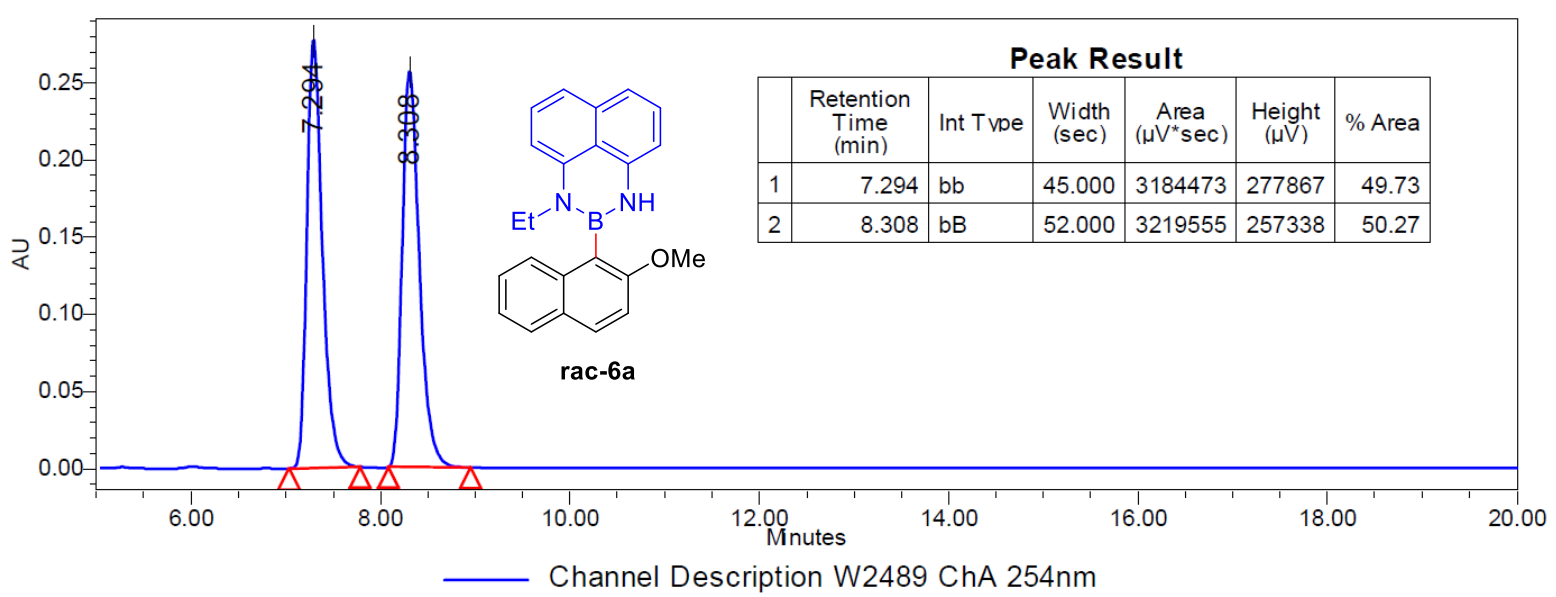



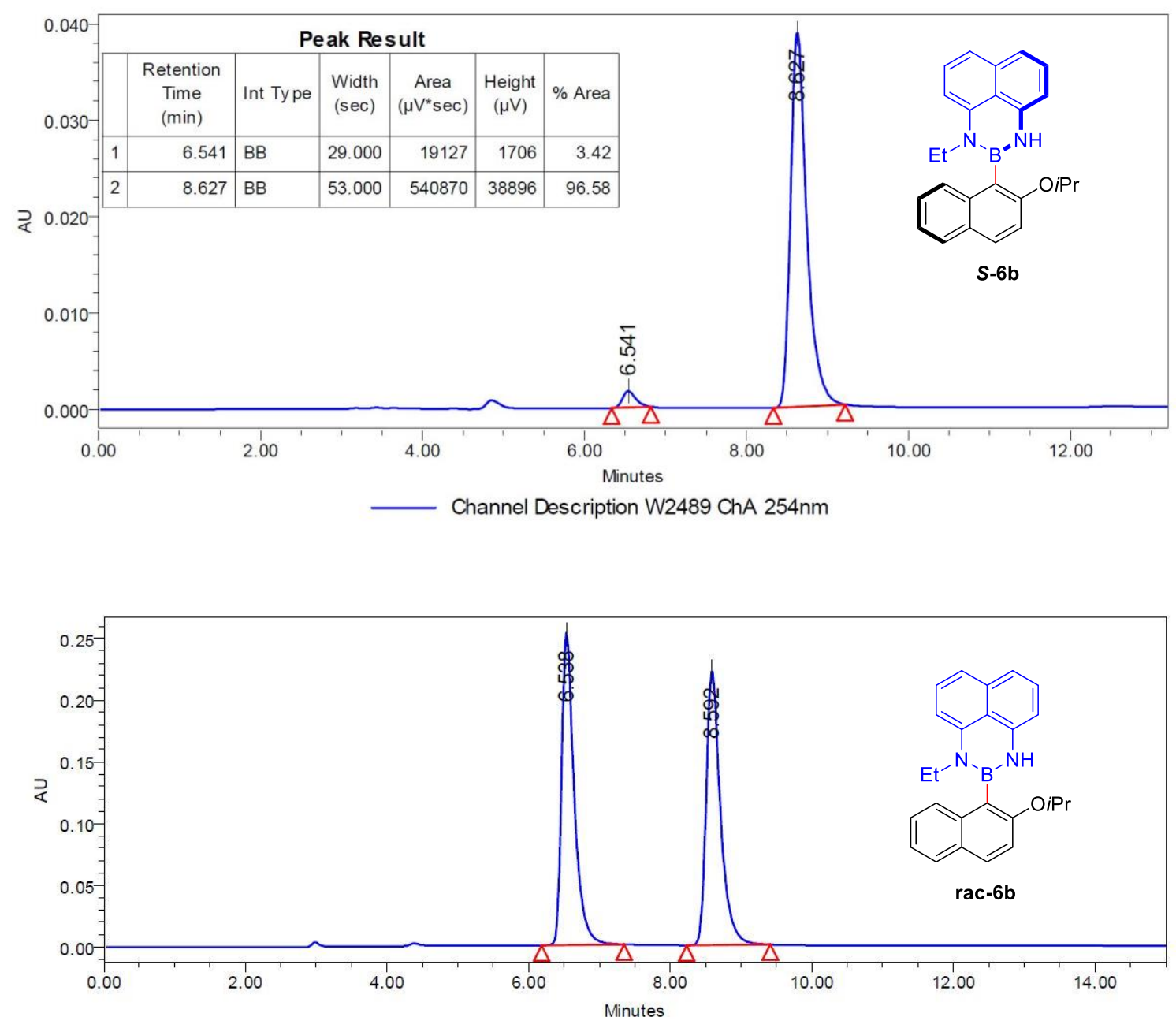

Channel Description W2489 ChA 254nm

Peak Result

\begin{tabular}{|c|c|l|l|l|l|l|}
\hline & $\begin{array}{c}\text { Retention } \\
\text { Time } \\
(\mathrm{min})\end{array}$ & Int Type & $\begin{array}{c}\text { Width } \\
(\mathrm{sec})\end{array}$ & $\begin{array}{c}\text { Area } \\
\left(\mu \mathrm{V}^{*} \mathrm{sec}\right)\end{array}$ & $\begin{array}{c}\text { Height } \\
(\mu \mathrm{V})\end{array}$ & $\%$ Area \\
\hline 1 & 6.538 & BB & 70.000 & 3155078 & 253538 & 50.18 \\
\hline 2 & 8.592 & BB & 71.000 & 3132600 & 222709 & 49.82 \\
\hline
\end{tabular}




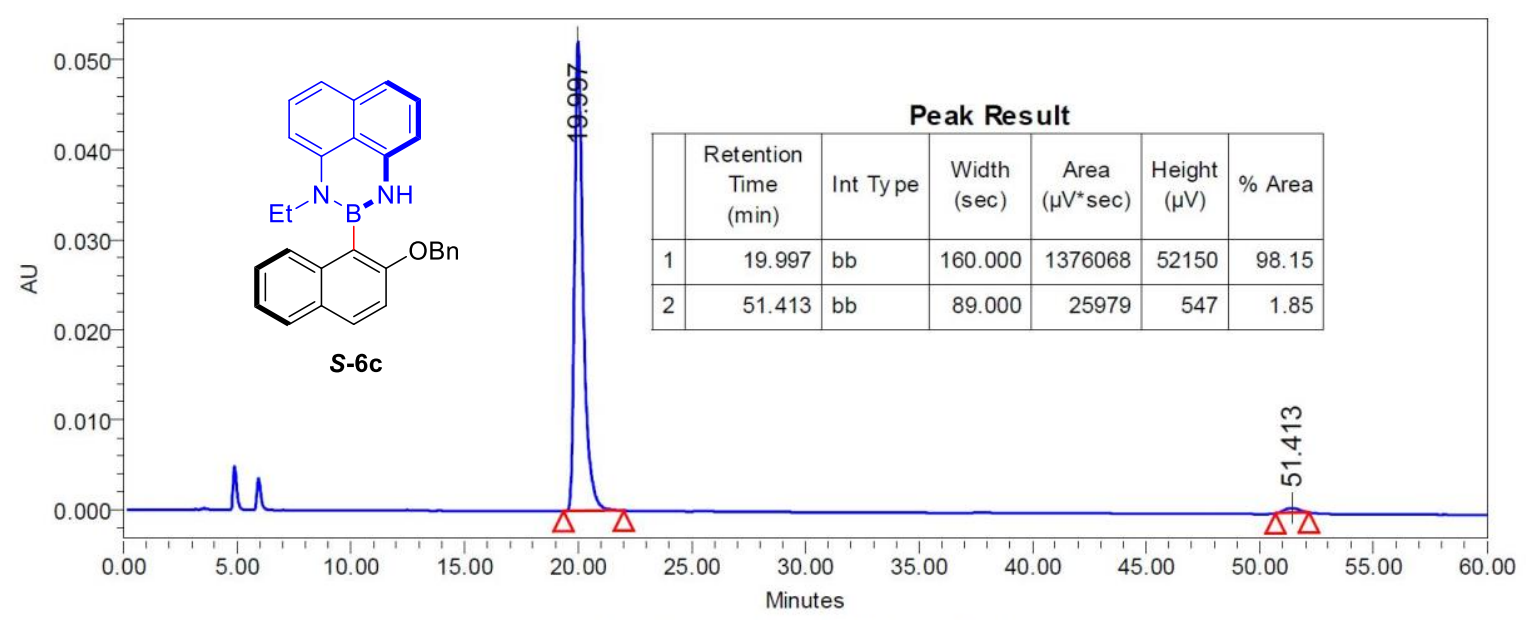

Channel Description W2489 ChA 254nm

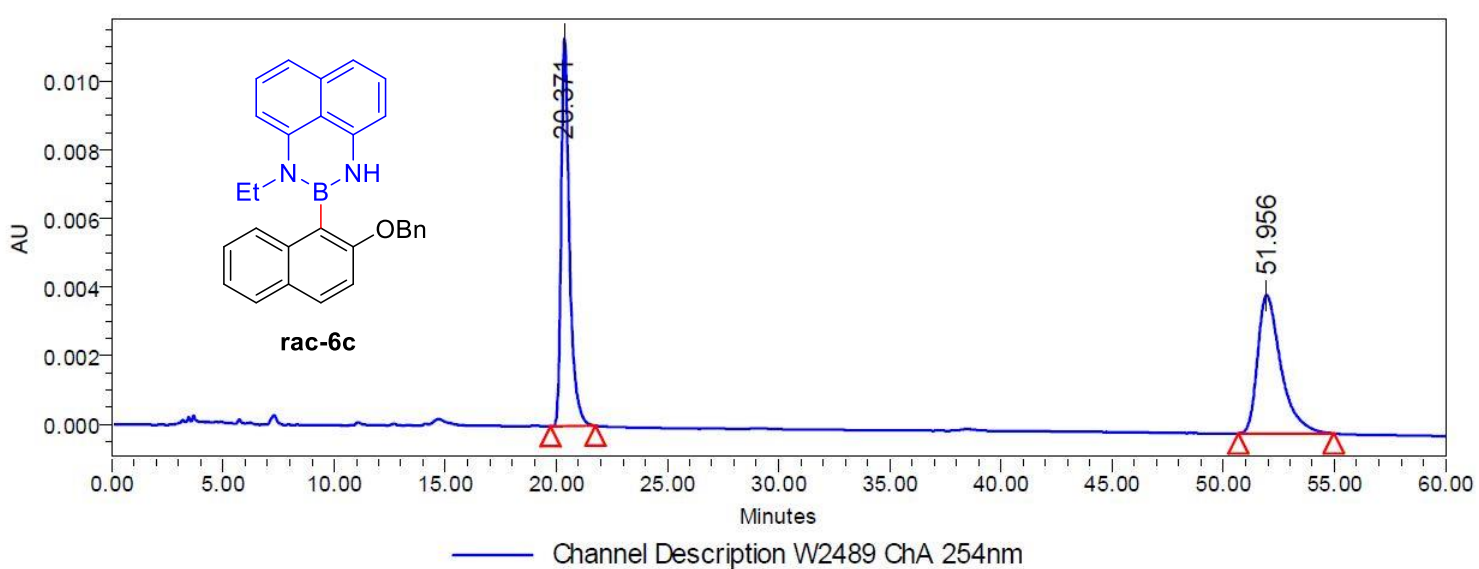

Peak Result

\begin{tabular}{|c|c|l|r|r|r|r|}
\hline & $\begin{array}{c}\text { Retention } \\
\text { Time } \\
(\mathrm{min})\end{array}$ & Int Ty pe & $\begin{array}{c}\text { Width } \\
(\mathrm{sec})\end{array}$ & $\begin{array}{c}\text { Area } \\
\left(\mu \mathrm{V}^{*} \mathrm{sec}\right)\end{array}$ & $\begin{array}{c}\text { Height } \\
(\mu \mathrm{V})\end{array}$ & \% Area \\
\hline 1 & 20.371 & bb & 121.000 & 295979 & 11288 & 50.08 \\
\hline 2 & 51.956 & bb & 257.000 & 295046 & 4029 & 49.92 \\
\hline
\end{tabular}



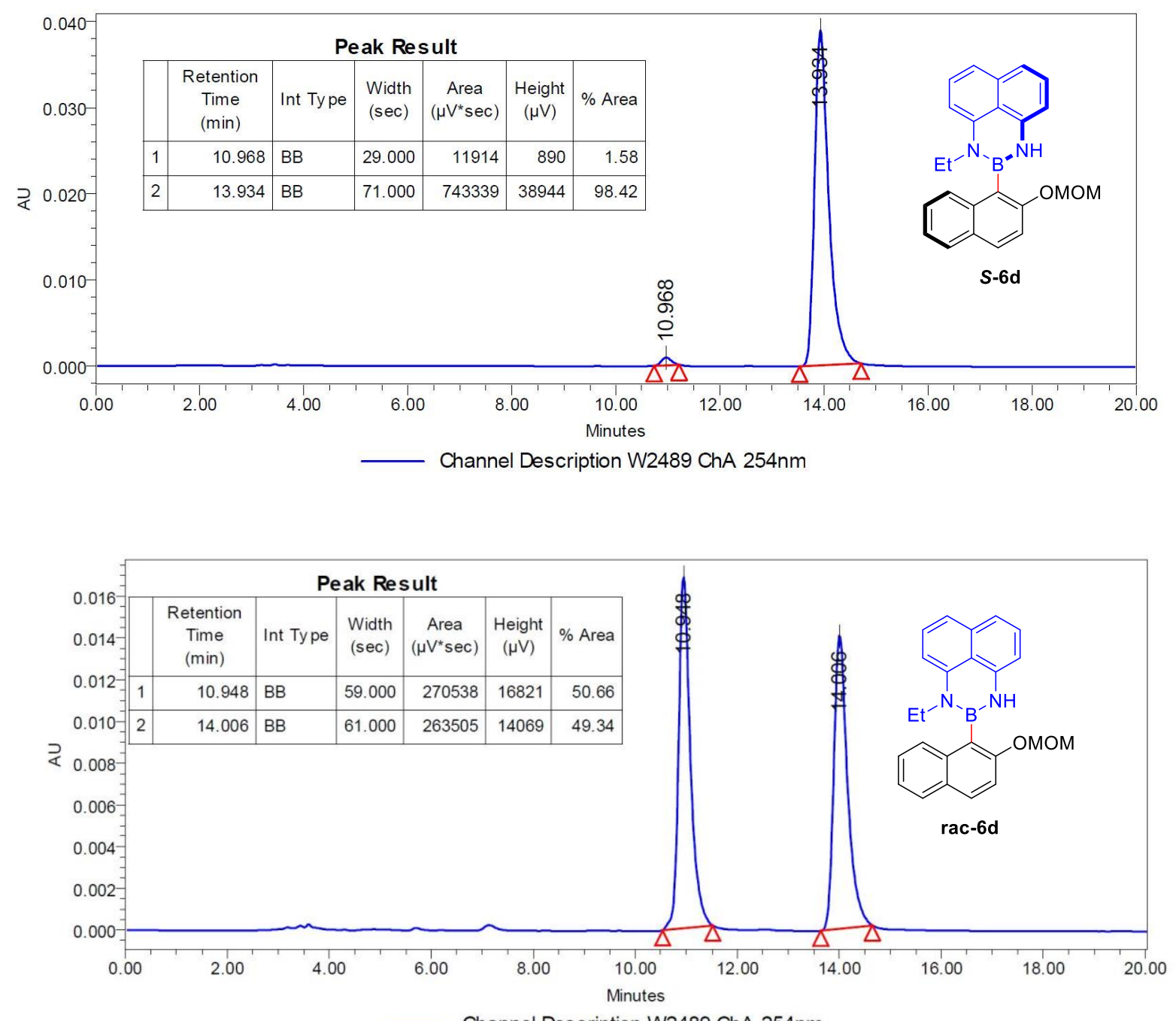

Channel Description W2489 ChA 254nm 
Auto-Scaled Chromatogram

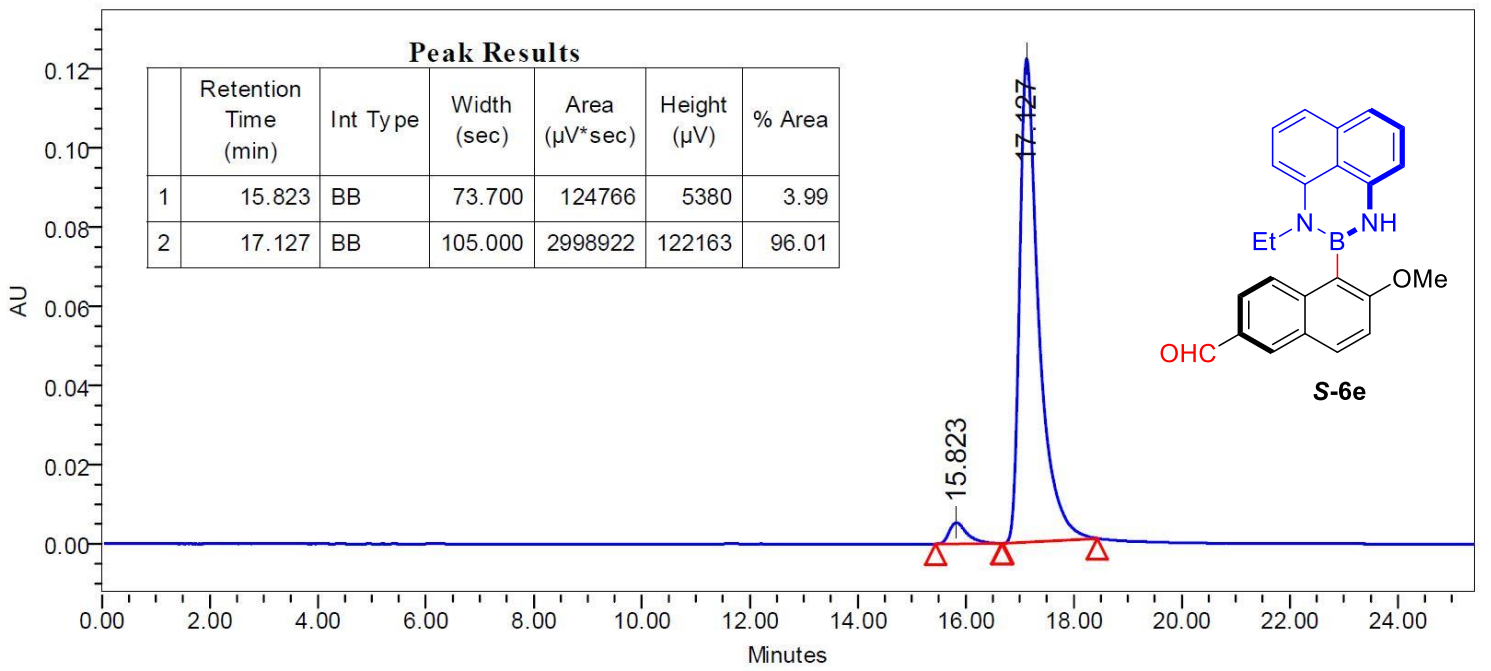

Channel Description W2489 ChA 254nm

Auto-Scaled Chromatogram

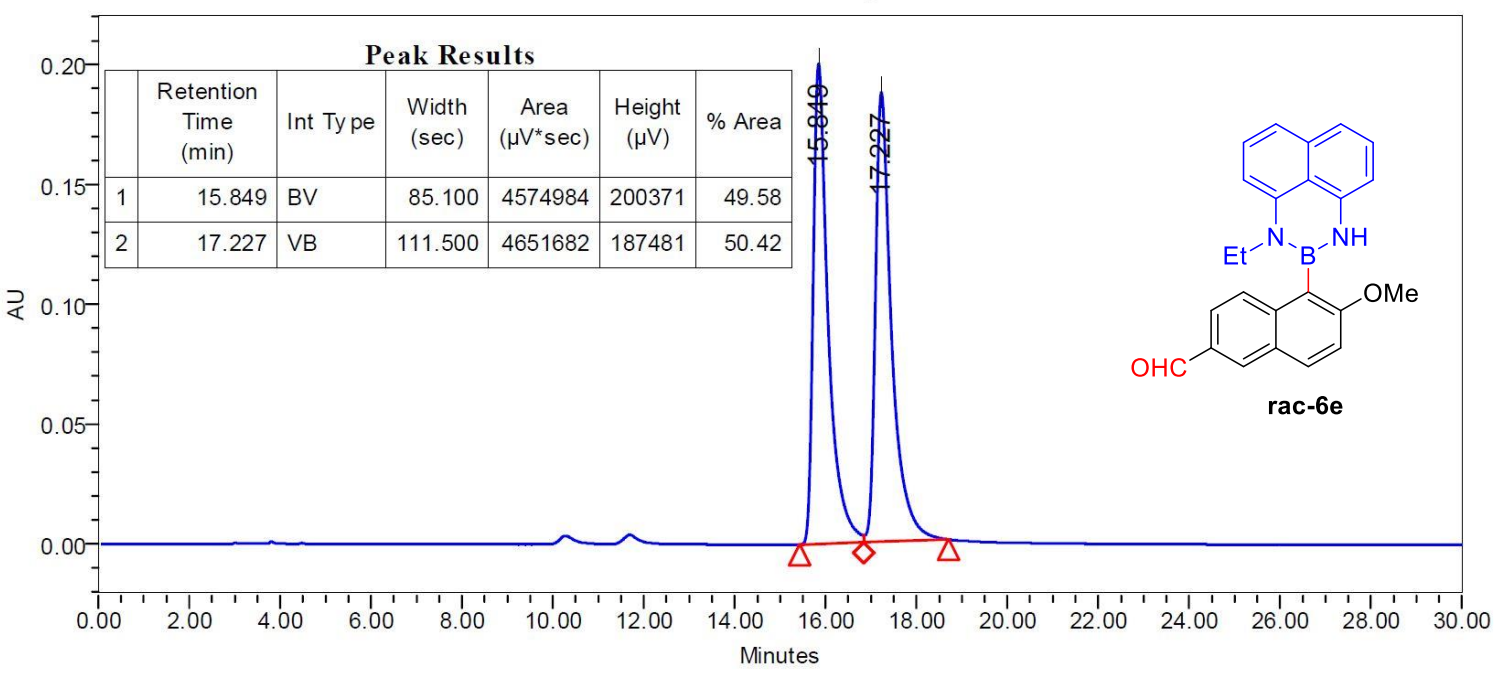

Channel Description W2489 ChA 254nm 
Auto-Scaled Chromatogram

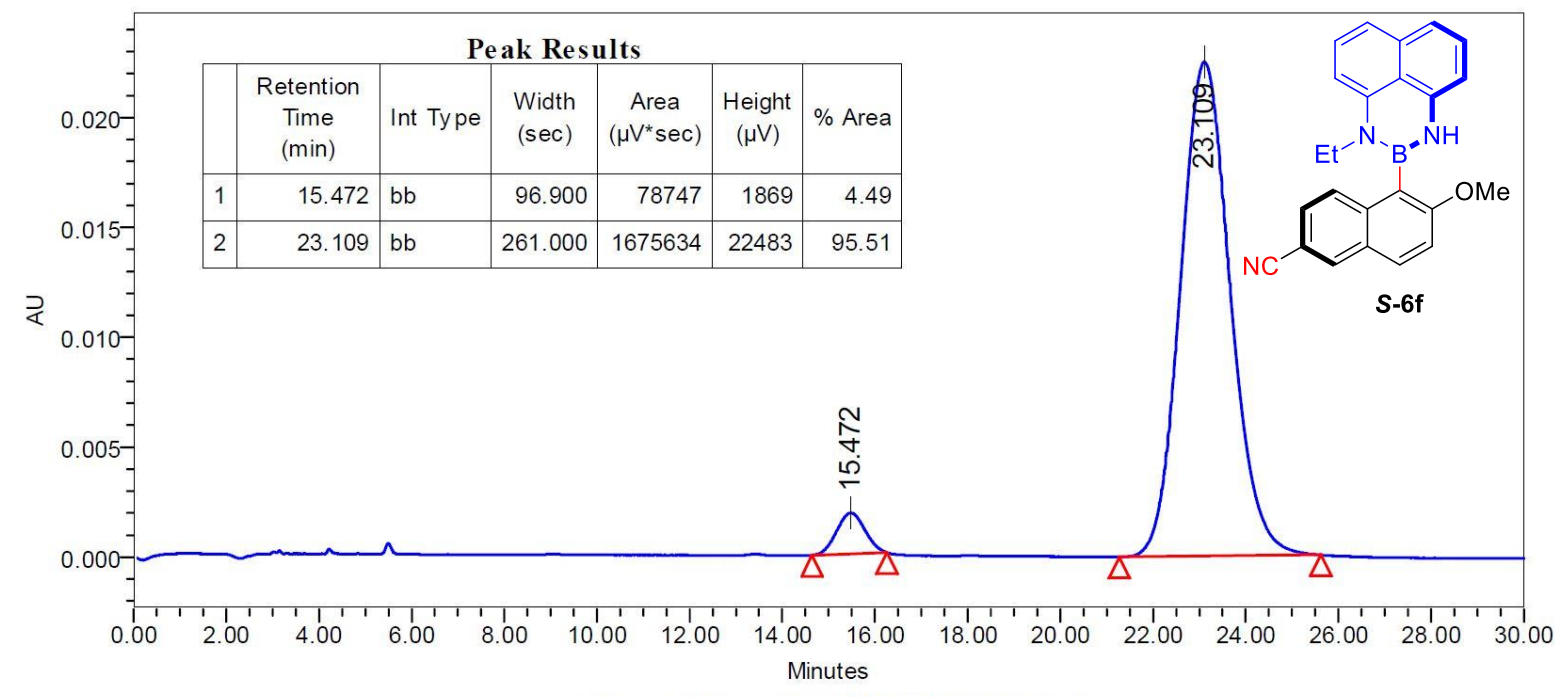

Channel Description W2489 ChA 254nm

Auto-Scaled Chromatogram

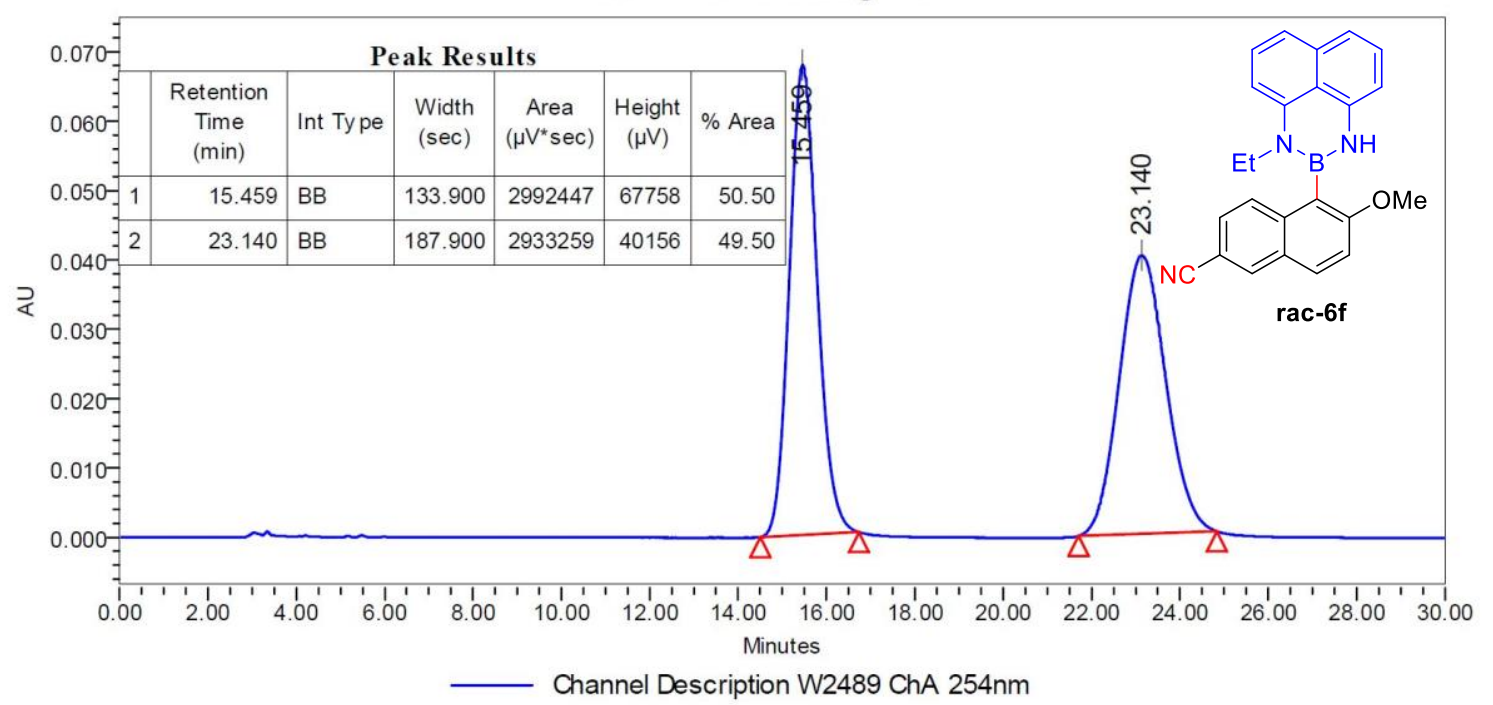


Auto-Scaled Chromatogram

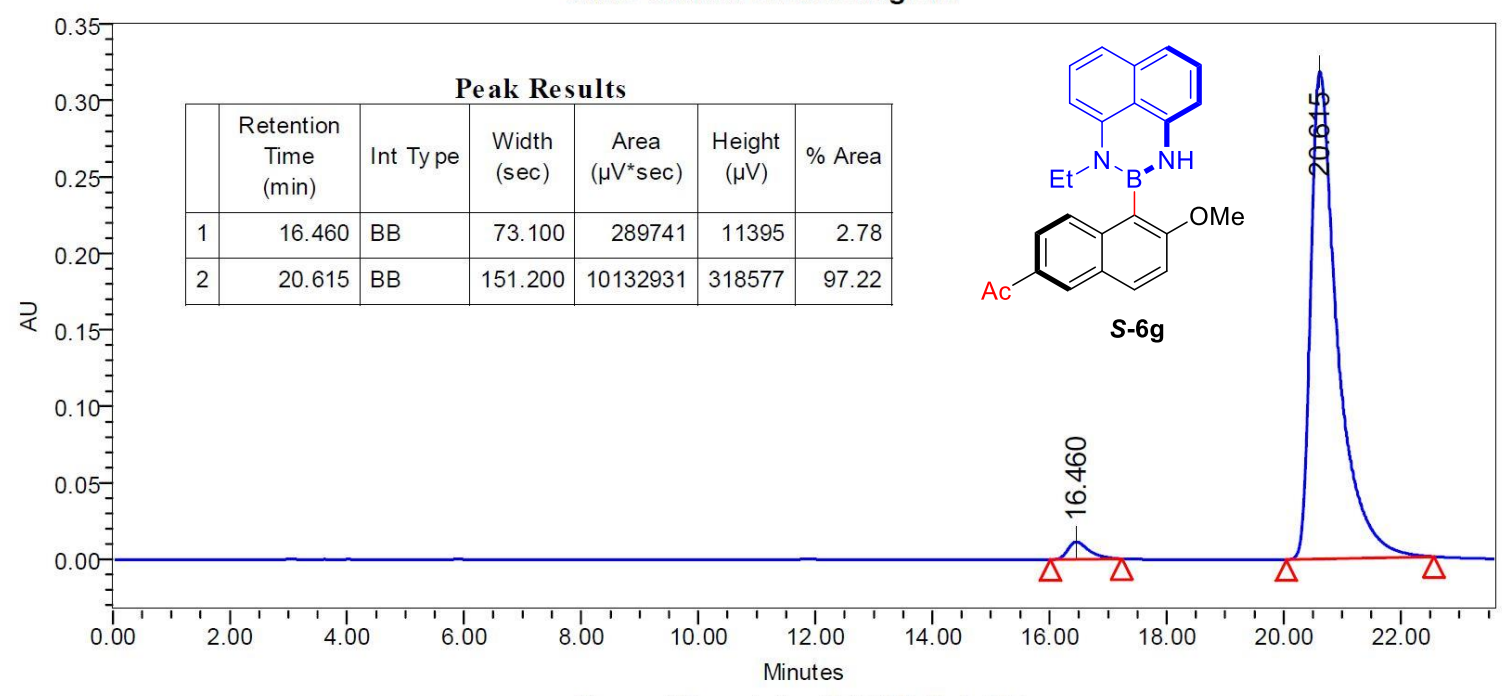

Channel Description W2489 ChA 254nm

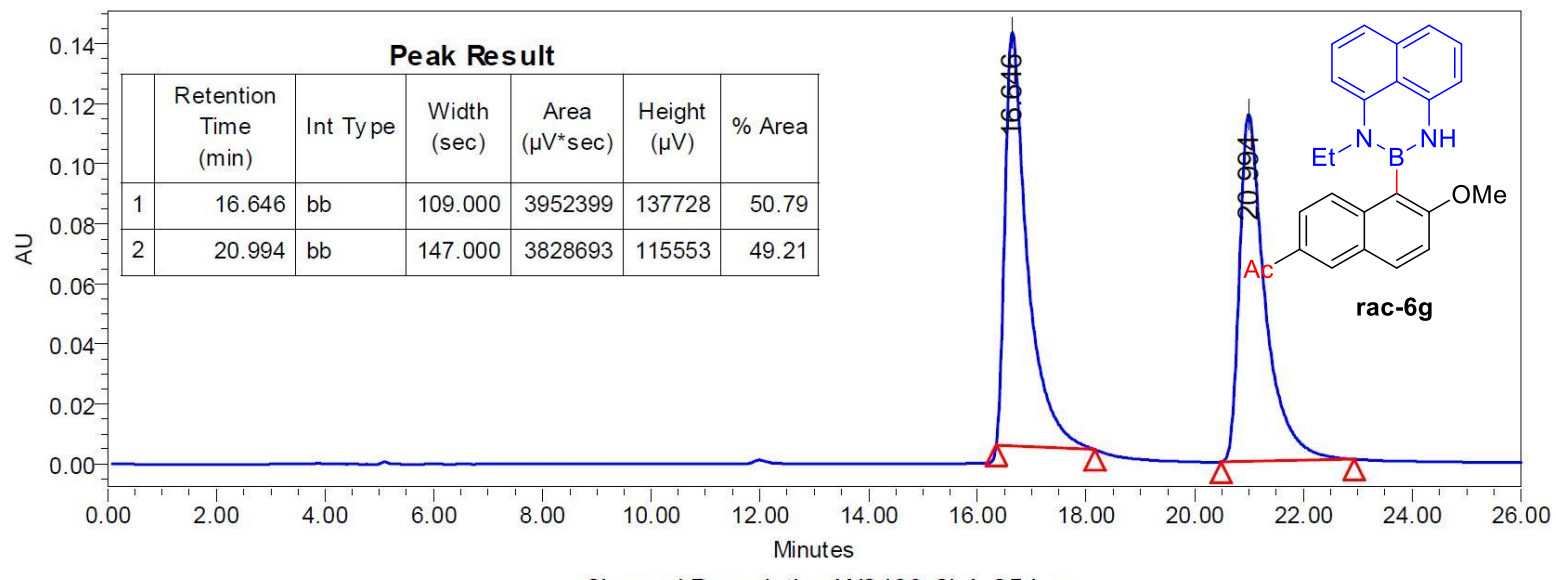

Channel Description W2489 ChA 254nm 


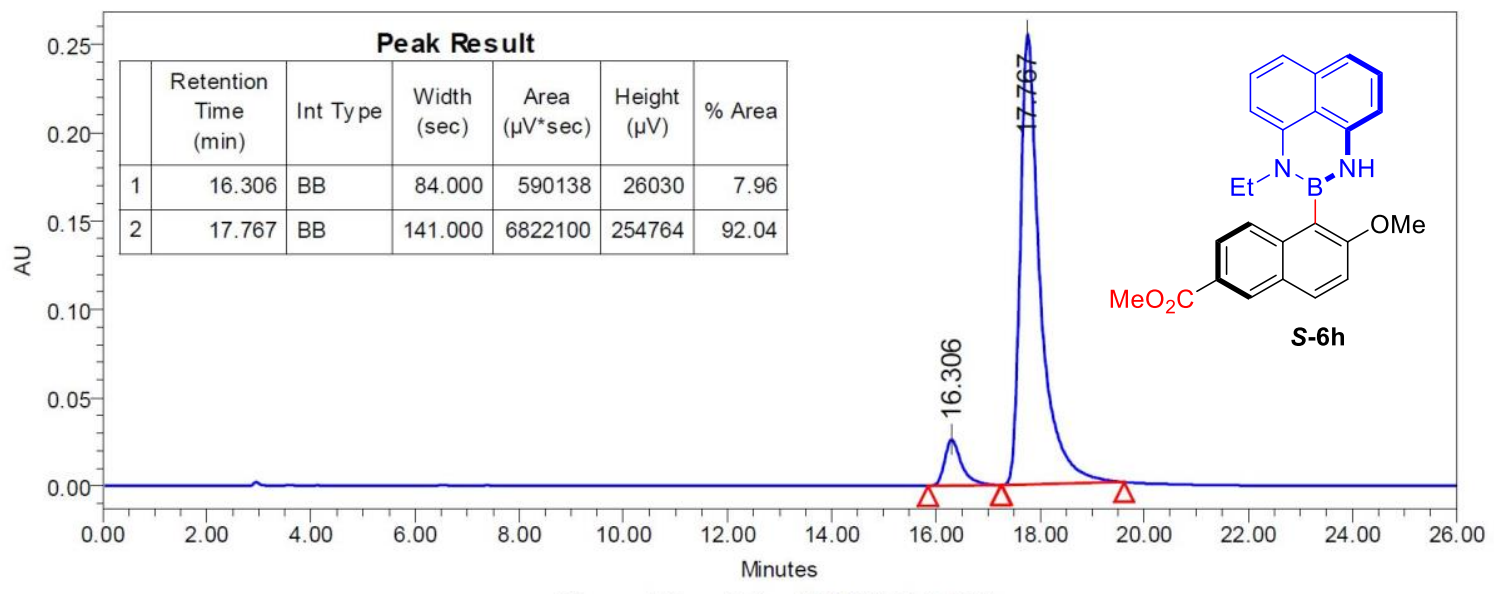

Channel Description W2489 ChA 254nm

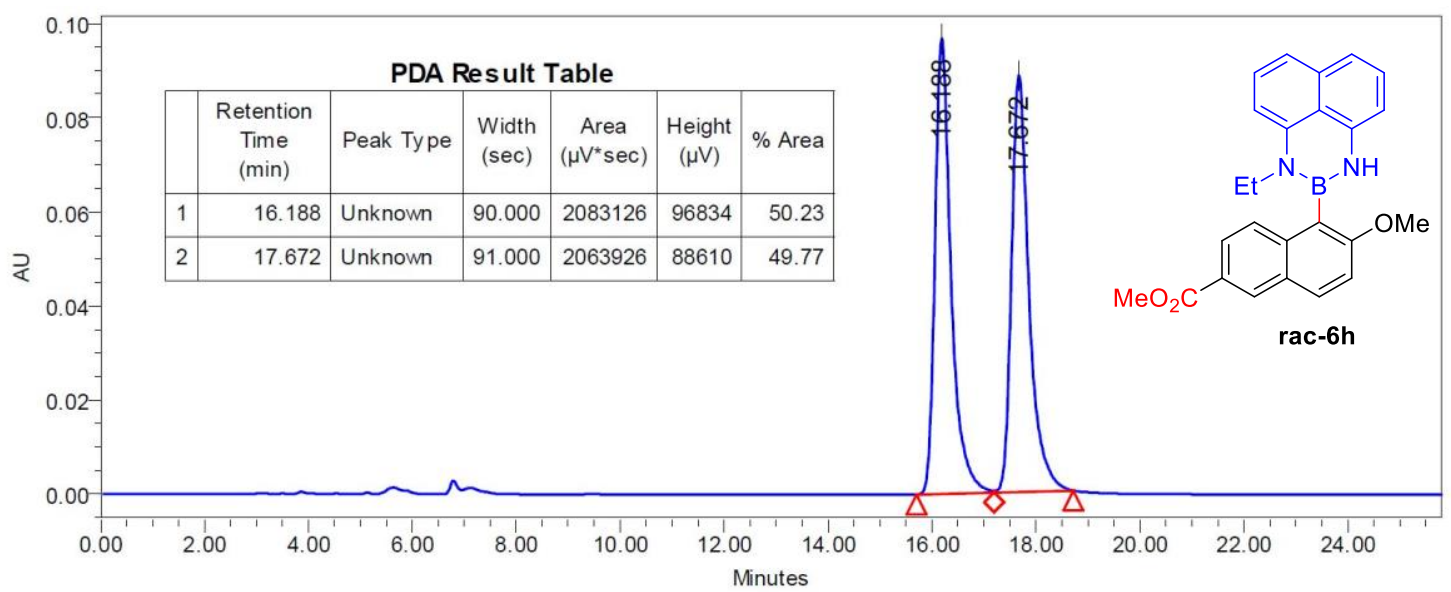

Channel Description W2489 ChA 254nm 
Auto-Scaled Chromatogram

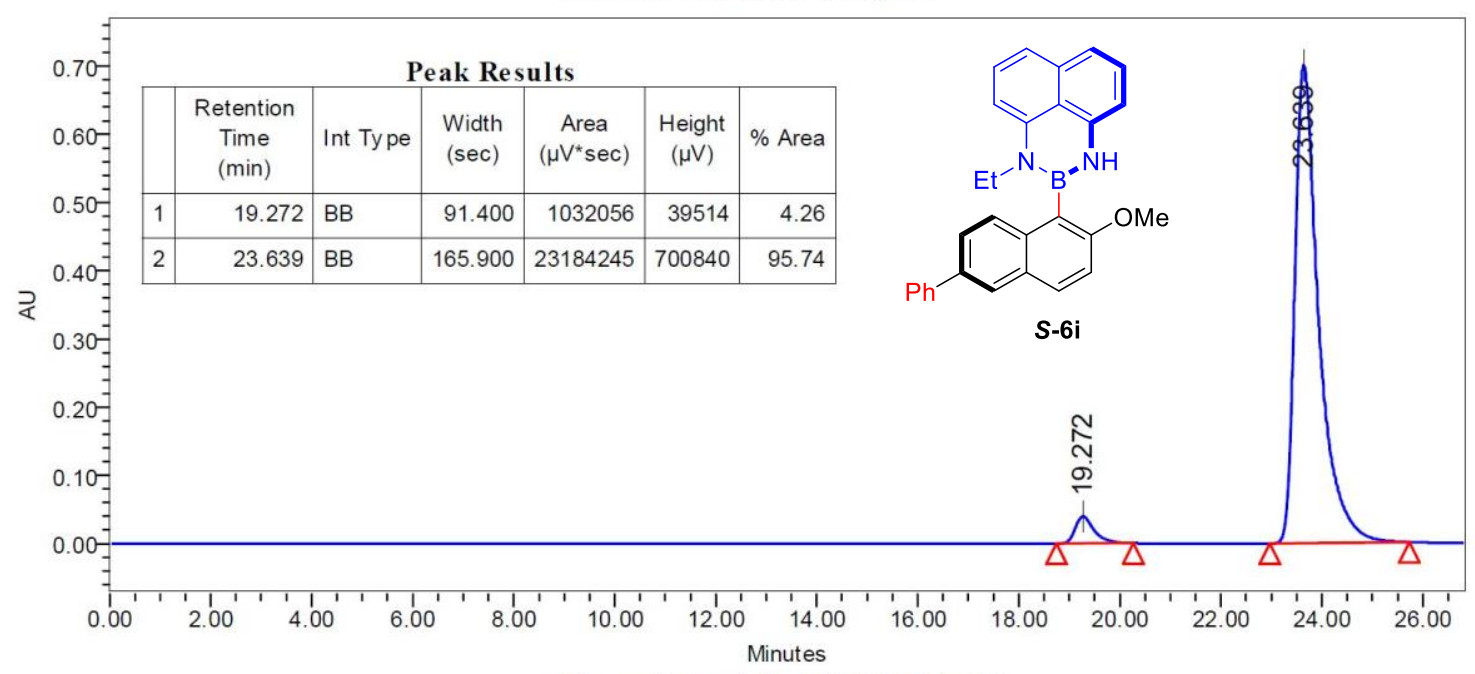

Channel Description W2489 ChA 254nm

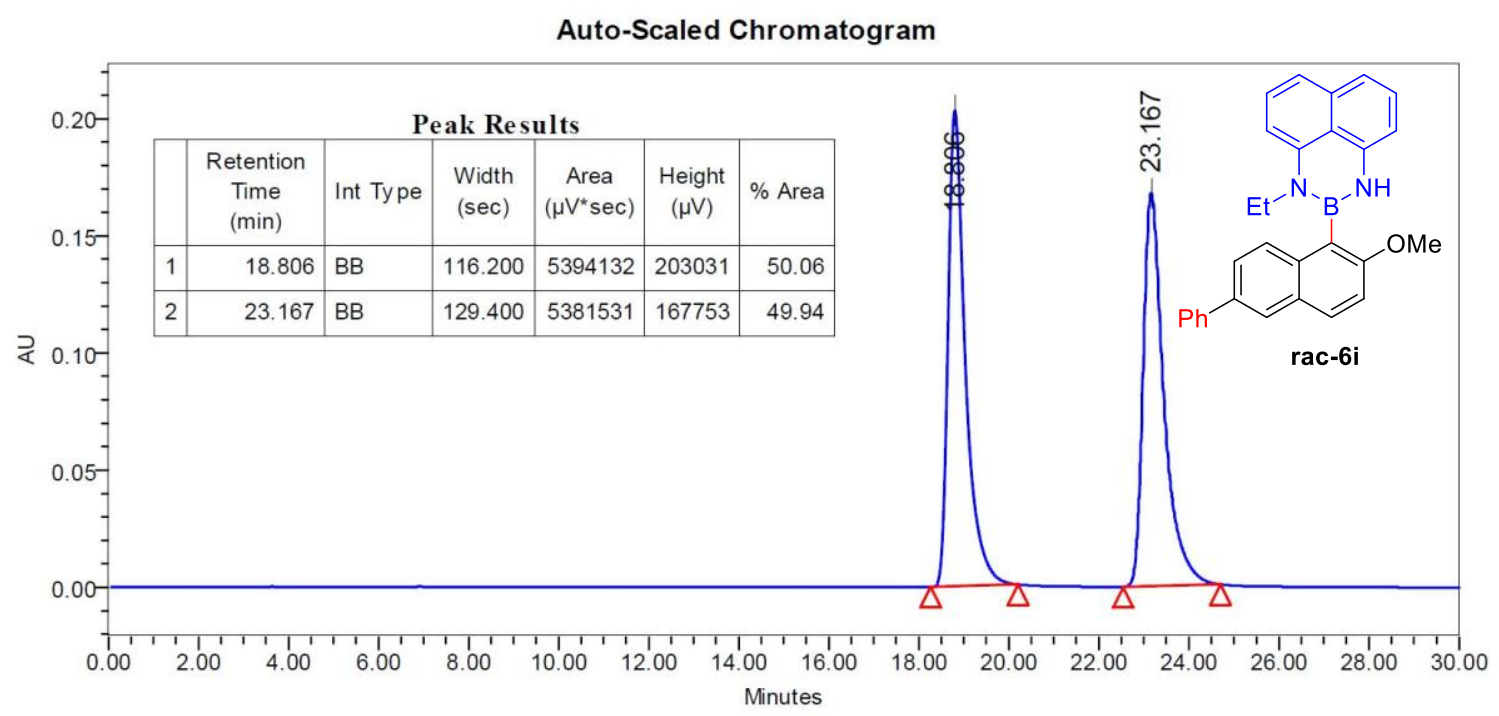

Channel Description W2489 ChA 254nm 


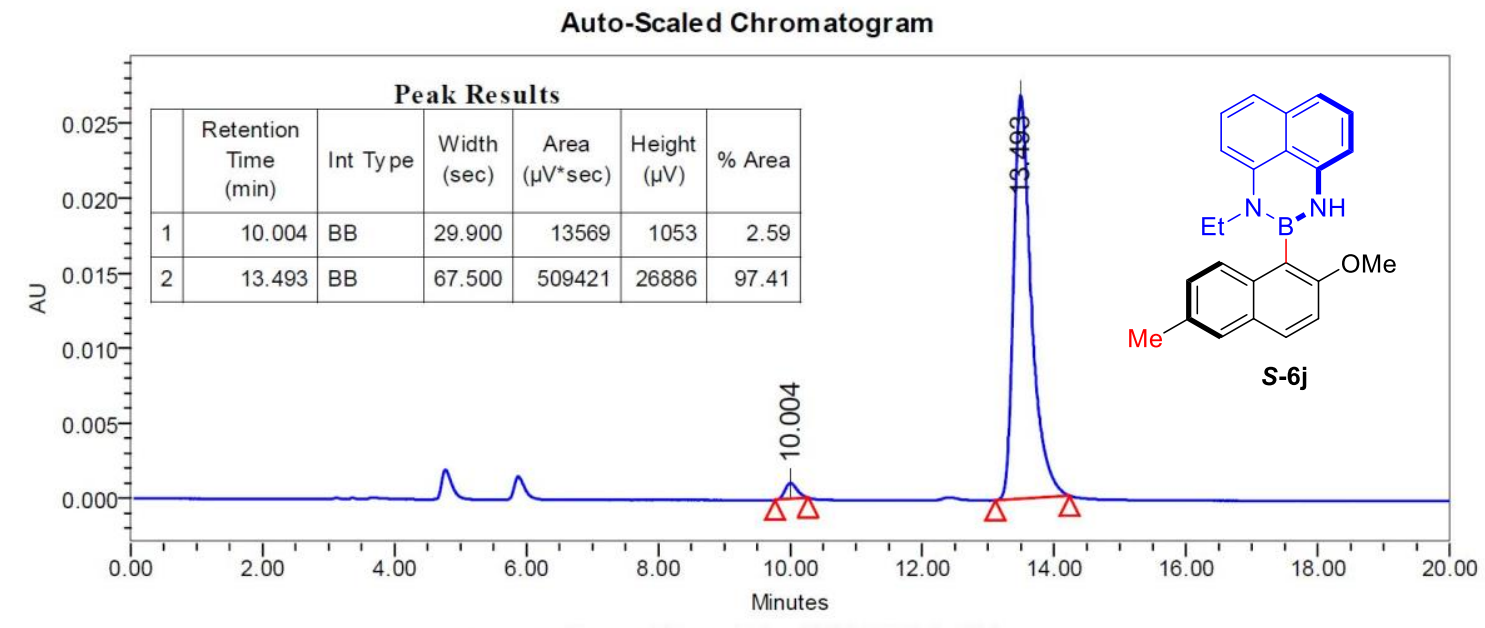

Channel Description W2489 ChA 254nm

Auto-Scaled Chromatogram

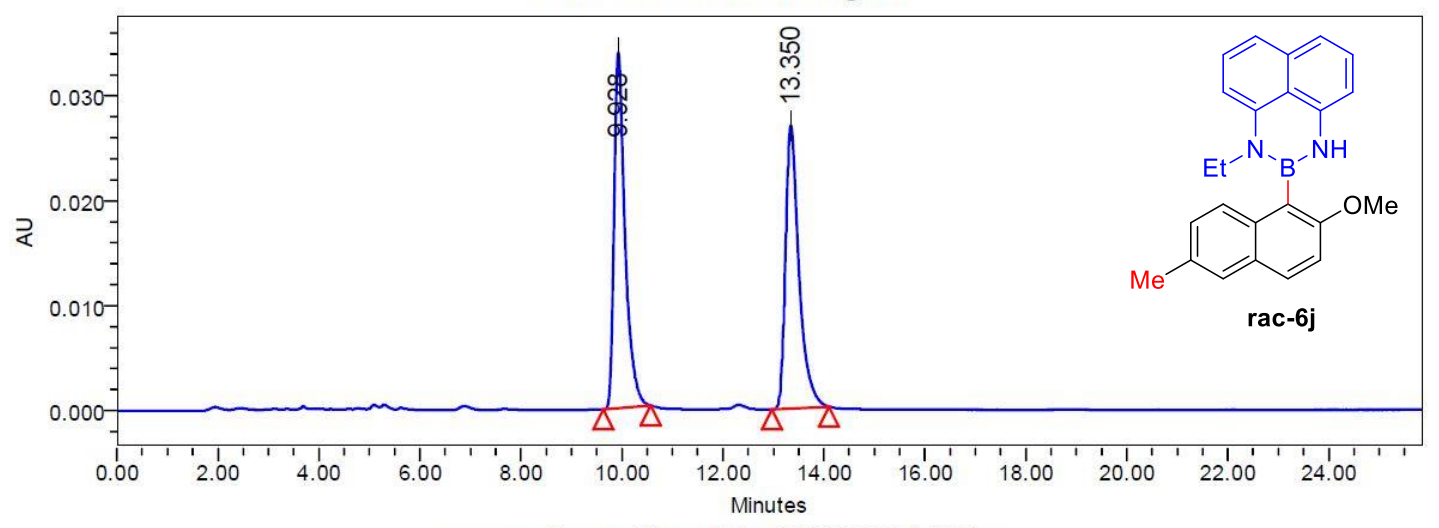

Channel Description W2489 ChA 254nm

Peak Results

\begin{tabular}{|c|r|l|l|l|l|l|}
\hline & $\begin{array}{c}\text { Retention } \\
\text { Time } \\
(\mathrm{min})\end{array}$ & Int Type & $\begin{array}{c}\text { Width } \\
(\mathrm{sec})\end{array}$ & $\begin{array}{c}\text { Area } \\
\left(\mu \mathrm{V}^{*} \mathrm{sec}\right)\end{array}$ & $\begin{array}{c}\text { Height } \\
(\mu \mathrm{V})\end{array}$ & $\%$ Area \\
\hline 1 & 9.928 & BB & 56.200 & 507228 & 33894 & 50.32 \\
\hline 2 & 13.350 & BB & 67.900 & 500794 & 26984 & 49.68 \\
\hline
\end{tabular}




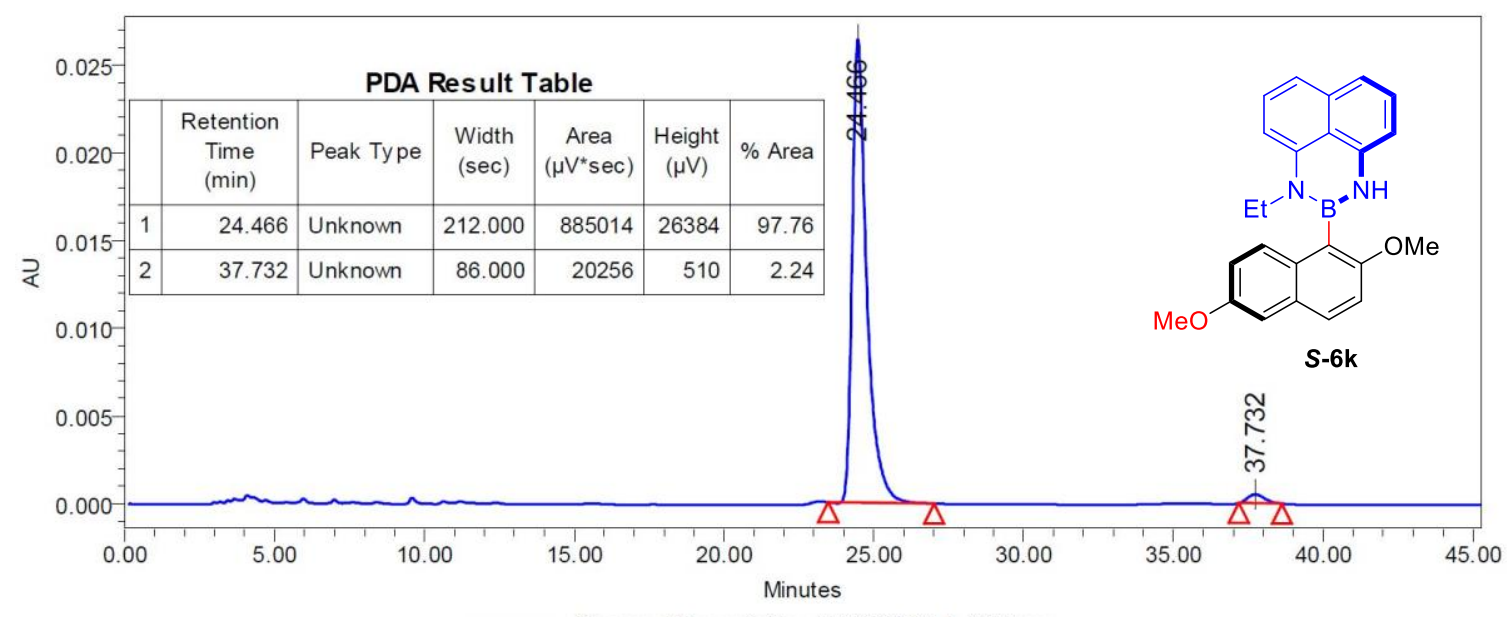

Channel Description W2489 ChA 254nm

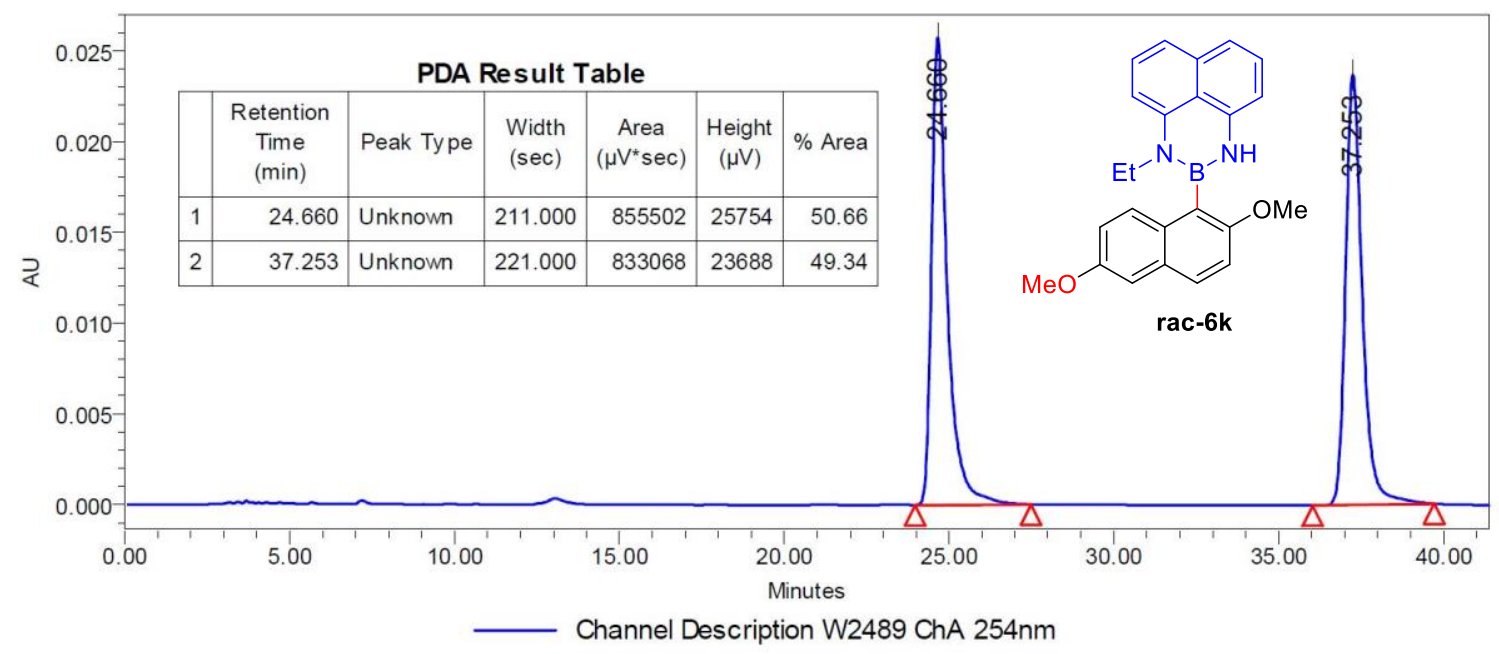




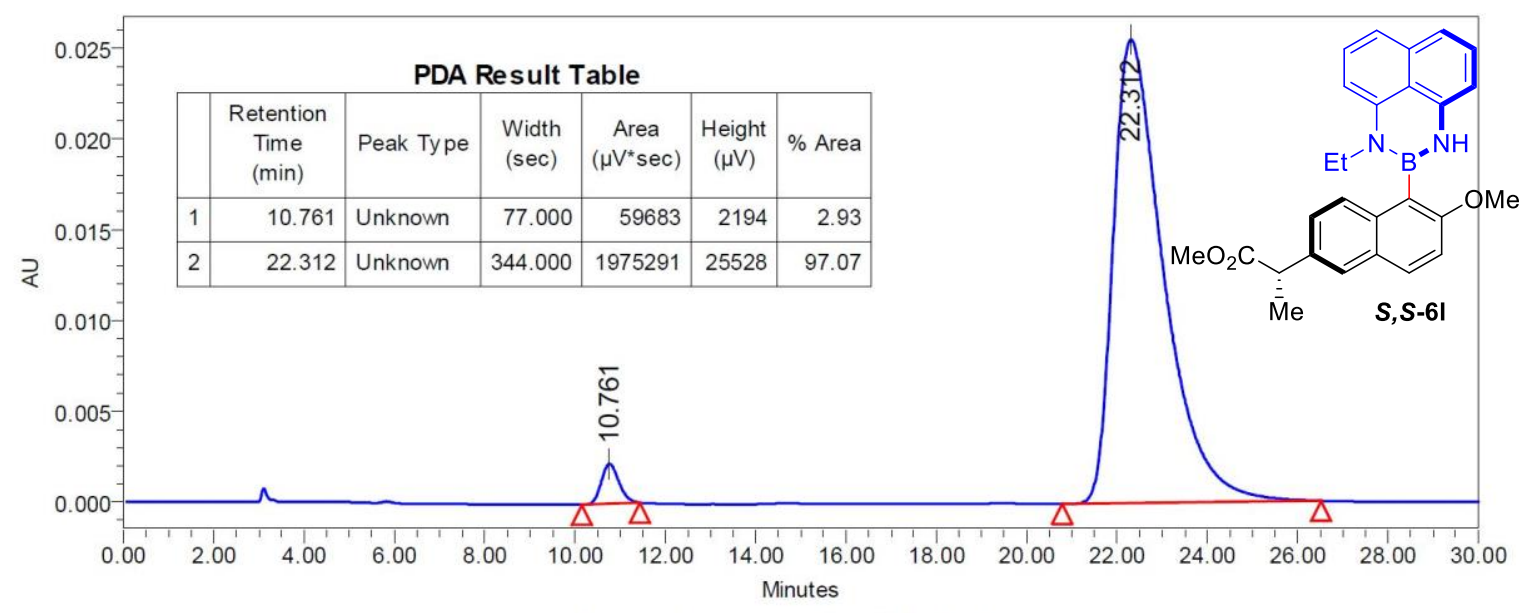

Channel Description W2489 ChA 254nm

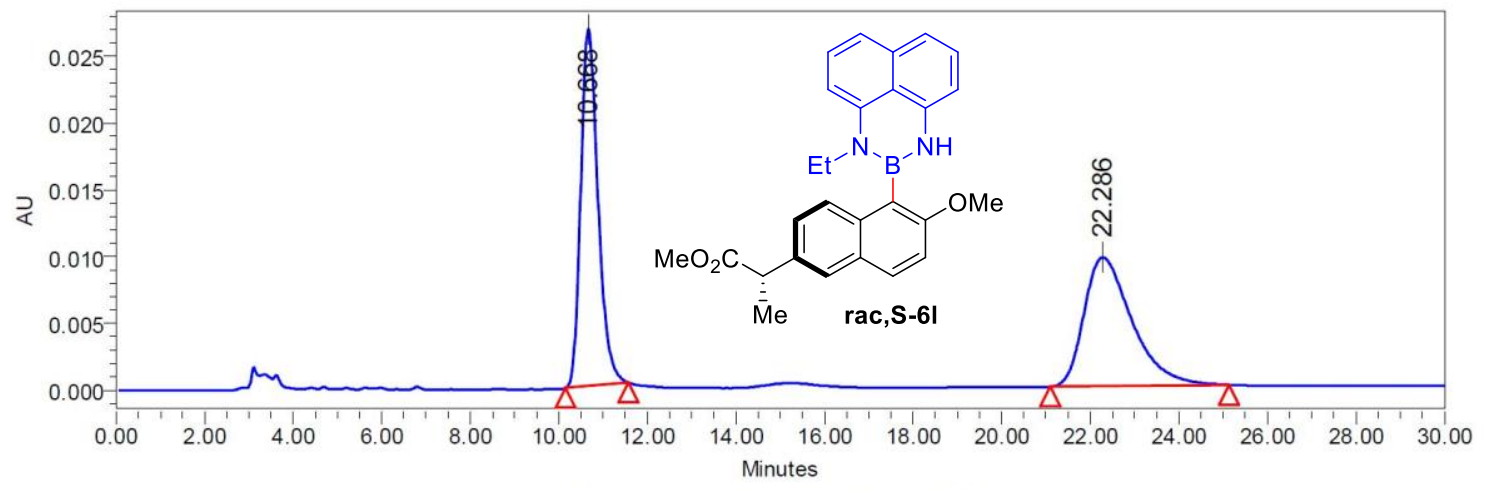

Channel Description W2489 ChA 254nm

PDA Result Table
\begin{tabular}{|c|c|l|r|r|r|r|}
\hline & $\begin{array}{c}\text { Retention } \\
\text { Time } \\
(\mathrm{min})\end{array}$ & Peak Type & $\begin{array}{c}\text { Width } \\
(\mathrm{sec})\end{array}$ & $\begin{array}{c}\text { Area } \\
\left(\mu \mathrm{V}^{*} \mathrm{sec}\right)\end{array}$ & $\begin{array}{c}\text { Height } \\
(\mu \mathrm{V})\end{array}$ & $\%$ Area \\
\hline 1 & 10.668 & Unknown & 85.000 & 723255 & 26706 & 49.99 \\
\hline 2 & 22.286 & Unknown & 242.000 & 723467 & 9642 & 50.01 \\
\hline
\end{tabular}


Auto-Scaled Chromatogram

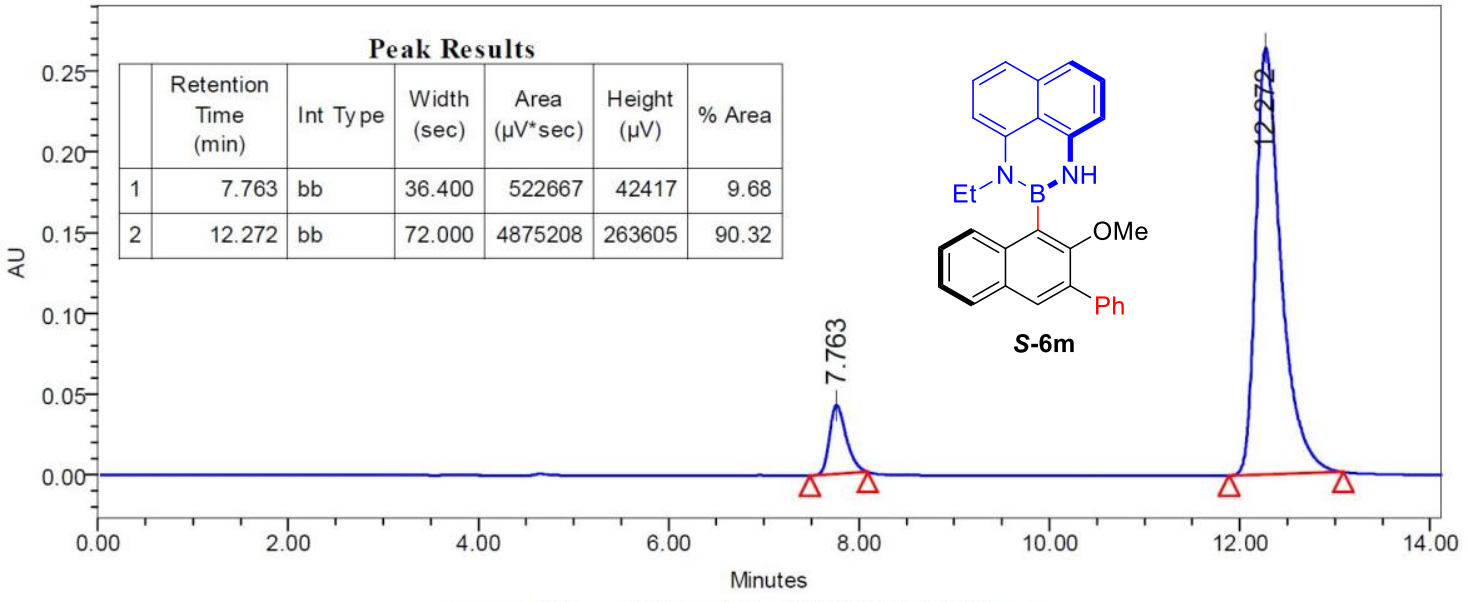

Channel Description W2489 ChA 254nm

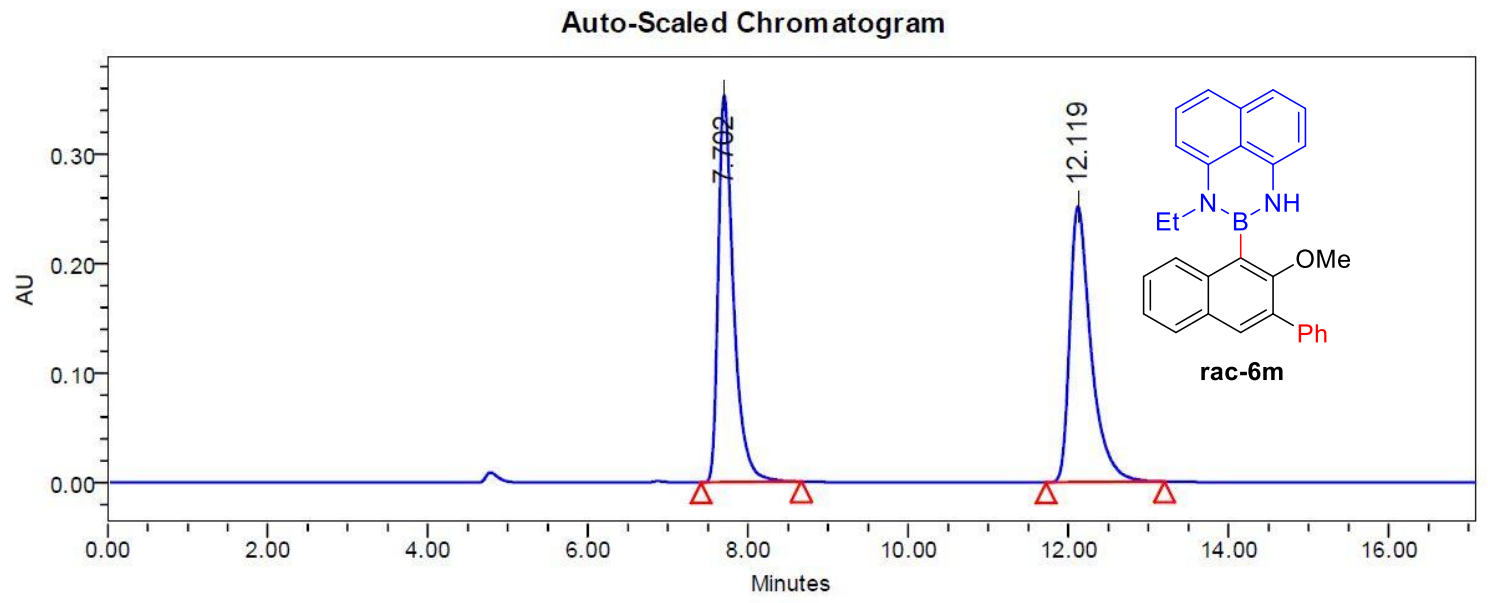

Channel Description W2489 ChA 254nm

Peak Results

\begin{tabular}{|c|r|l|l|l|l|r|}
\hline & $\begin{array}{c}\text { Retention } \\
\text { Time } \\
(\mathrm{min})\end{array}$ & Int Ty pe & $\begin{array}{c}\text { Width } \\
(\mathrm{sec})\end{array}$ & $\begin{array}{c}\text { Area } \\
\left(\mu \mathrm{V}^{*} \mathrm{sec}\right)\end{array}$ & $\begin{array}{c}\text { Height } \\
(\mu \mathrm{V})\end{array}$ & $\%$ Area \\
\hline 1 & 7.702 & BB & 75.300 & 4698920 & 353313 & 50.13 \\
\hline 2 & 12.119 & BB & 88.600 & 4675333 & 251961 & 49.87 \\
\hline
\end{tabular}




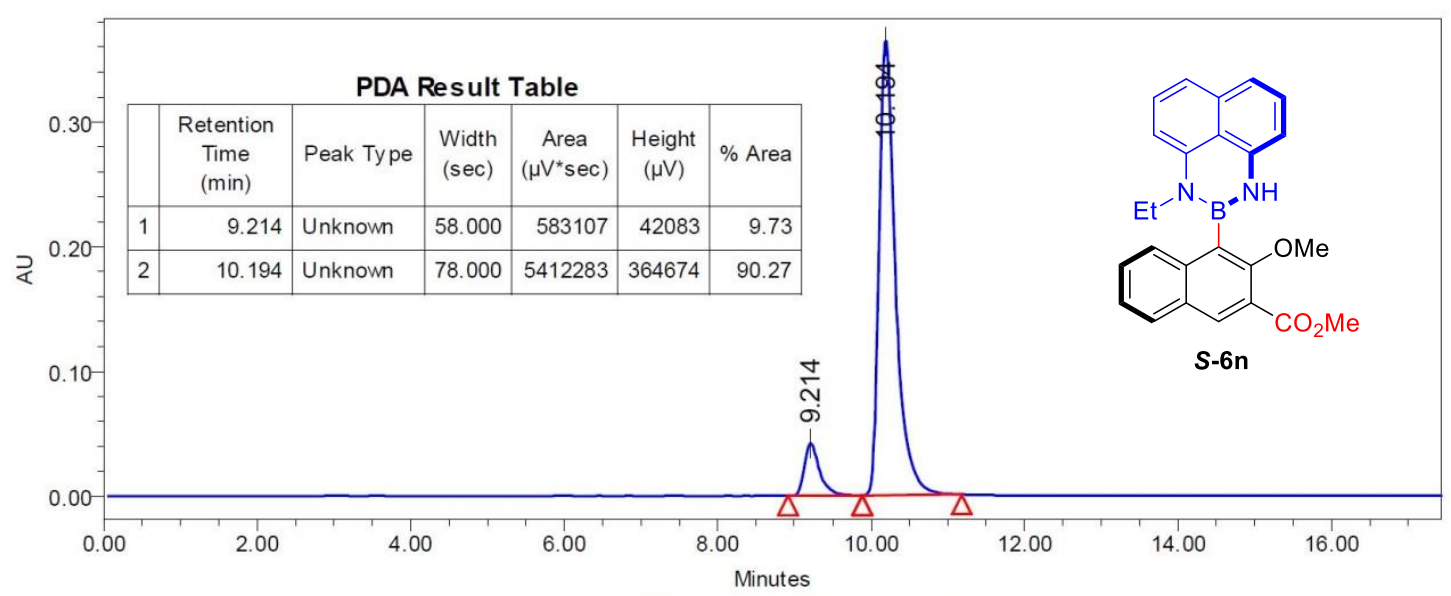

Channel Description W2489 ChA 254nm

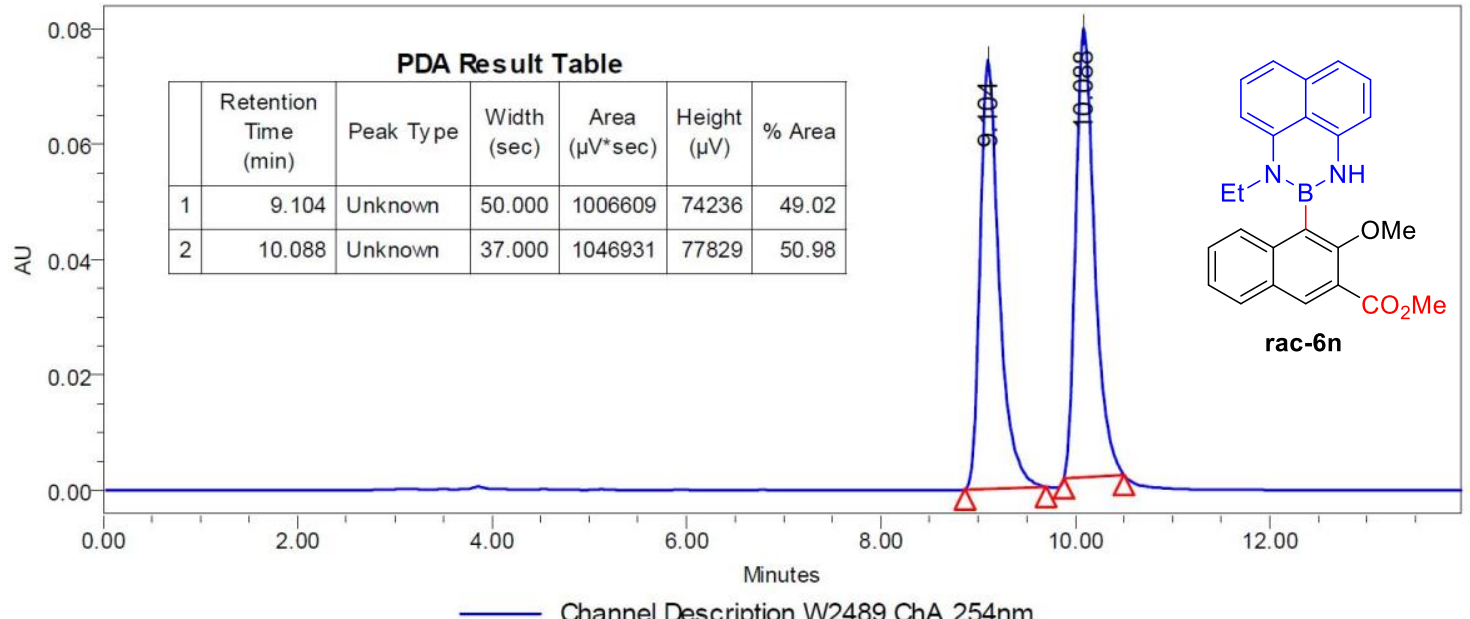




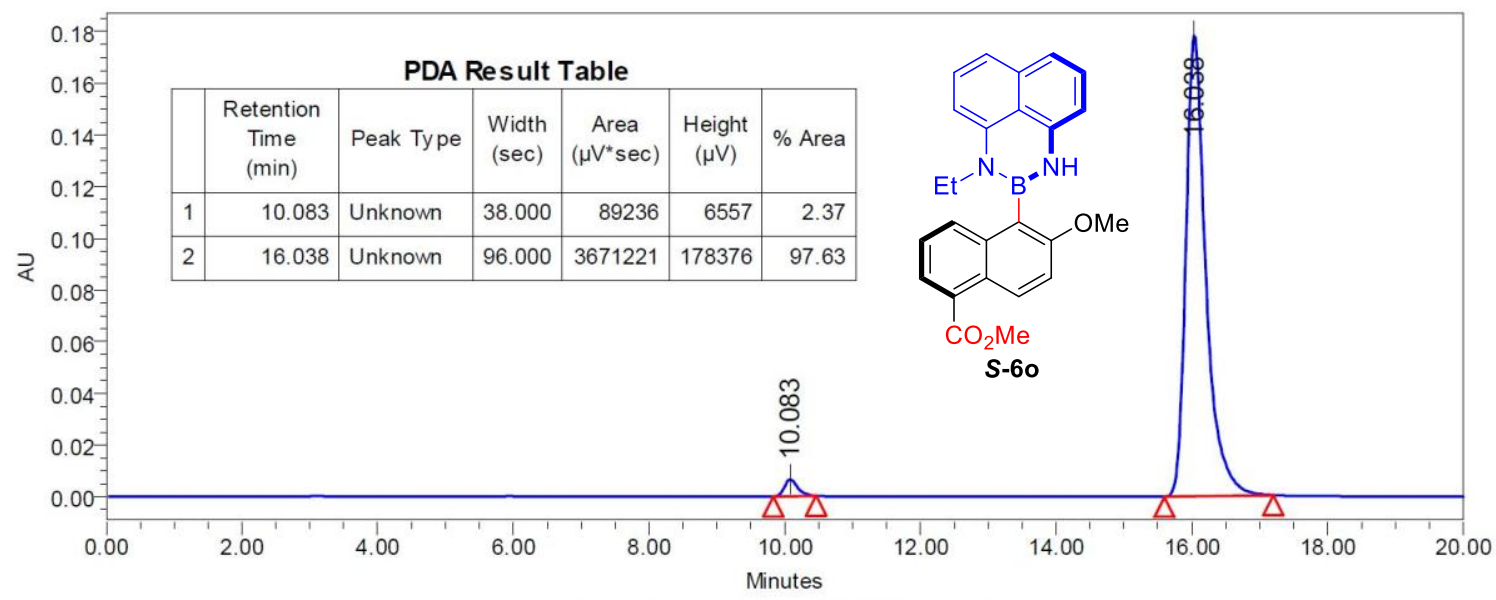

Channel Description W2489 ChA 254nm

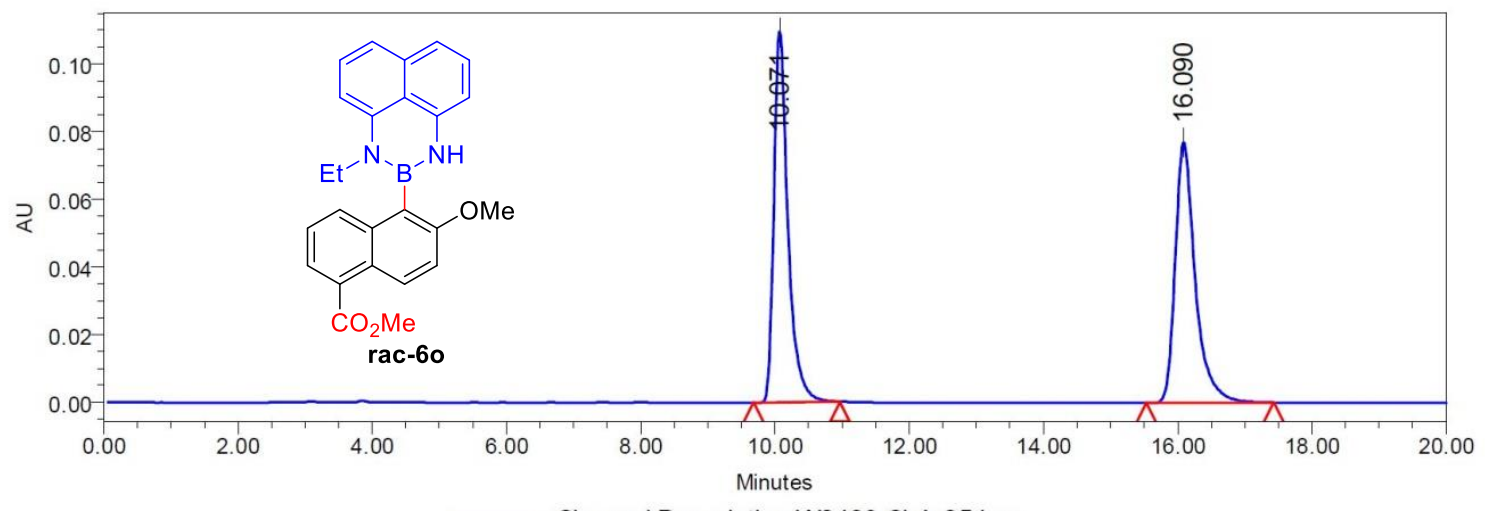

Channel Description W2489 ChA 254nm

PDA Result Table

\begin{tabular}{|c|c|l|r|r|r|r|}
\hline & $\begin{array}{c}\text { Retention } \\
\text { Time } \\
(\mathrm{min})\end{array}$ & Peak Type & $\begin{array}{c}\text { Width } \\
(\mathrm{sec})\end{array}$ & $\begin{array}{c}\text { Area } \\
\left(\mu \mathrm{V}^{*} \mathrm{sec}\right)\end{array}$ & $\begin{array}{c}\text { Height } \\
(\mu \mathrm{V})\end{array}$ & $\%$ Area \\
\hline 1 & 10.071 & Unknown & 77.000 & 1589872 & 109682 & 49.82 \\
\hline 2 & 16.090 & Unknown & 114.000 & 1601324 & 77078 & 50.18 \\
\hline
\end{tabular}




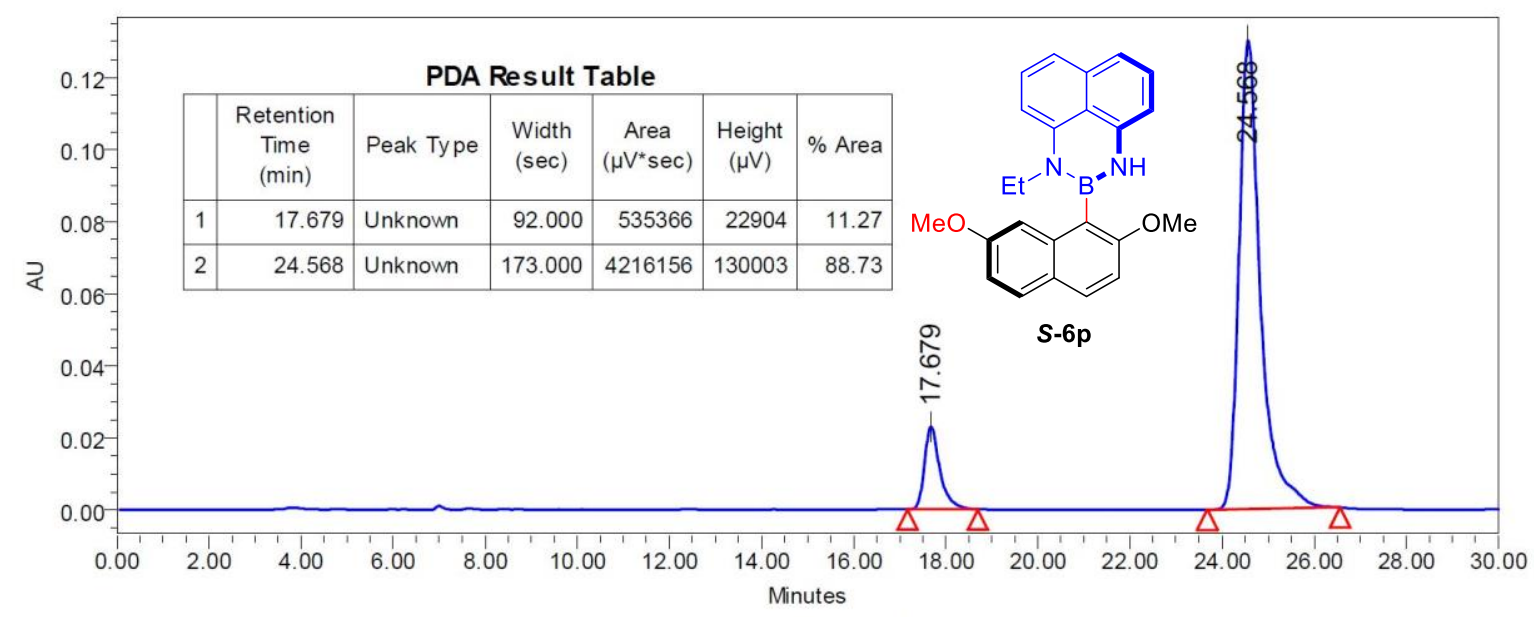

Channel Description W2489 ChA 254nm

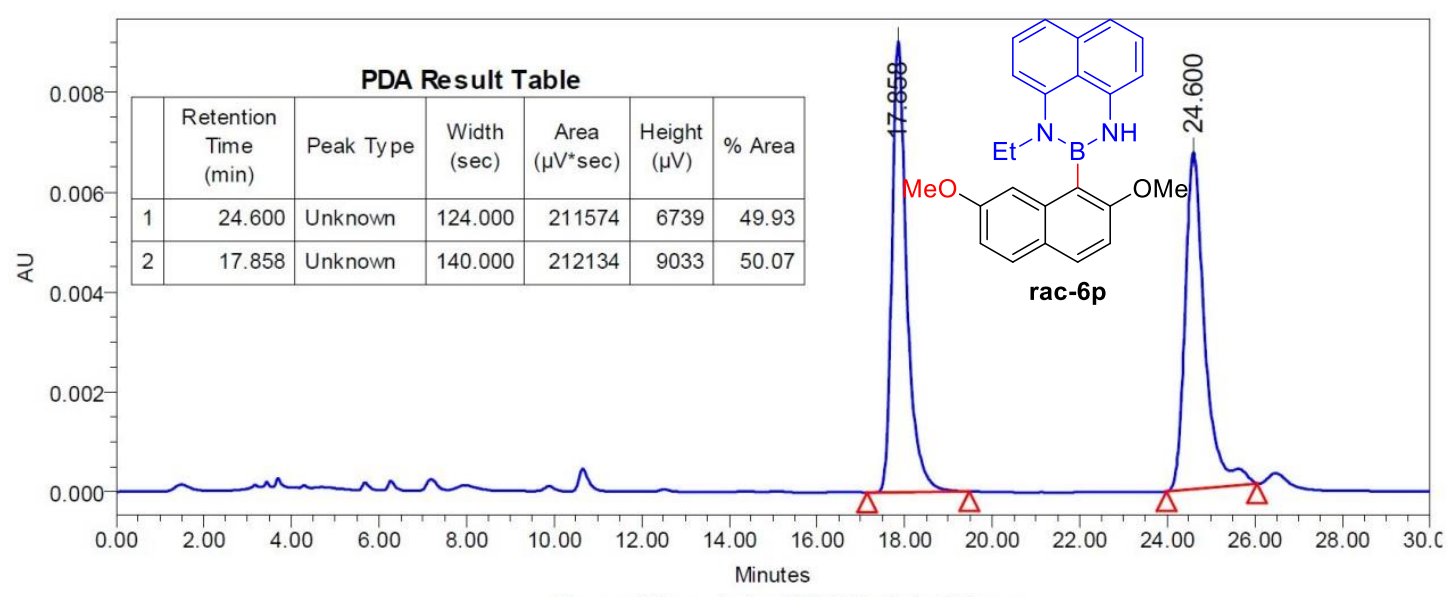

Channel Description W2489 ChA 254nm 


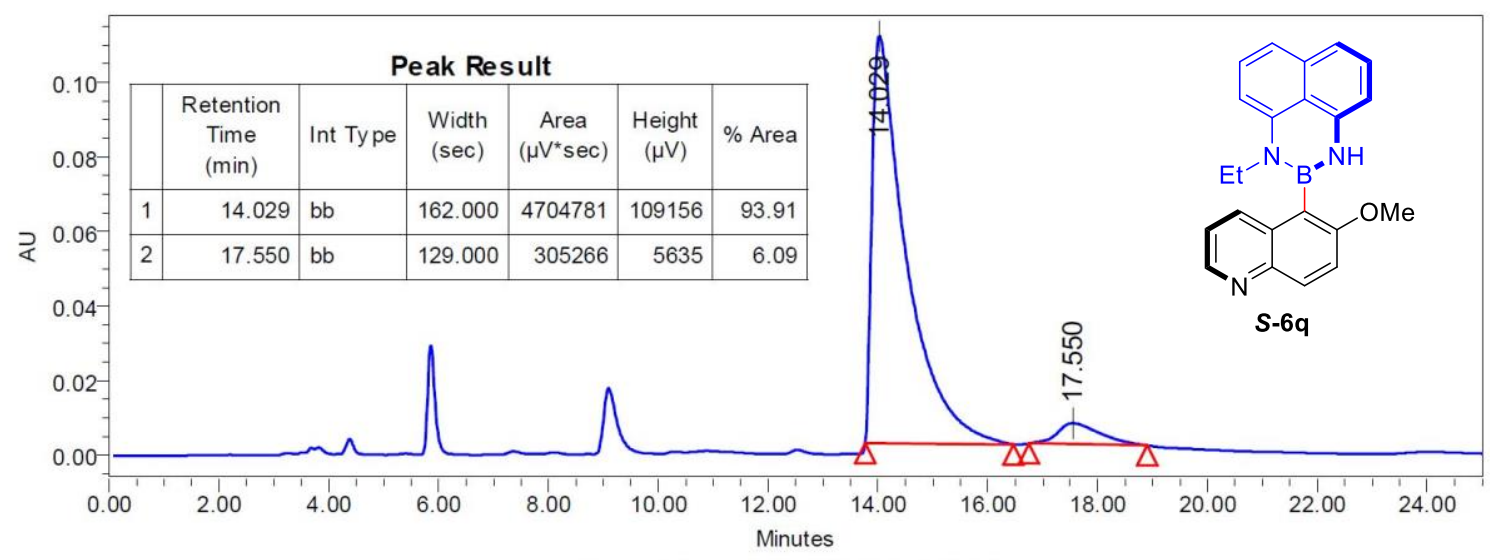

Channel Description W2489 ChA 254nm

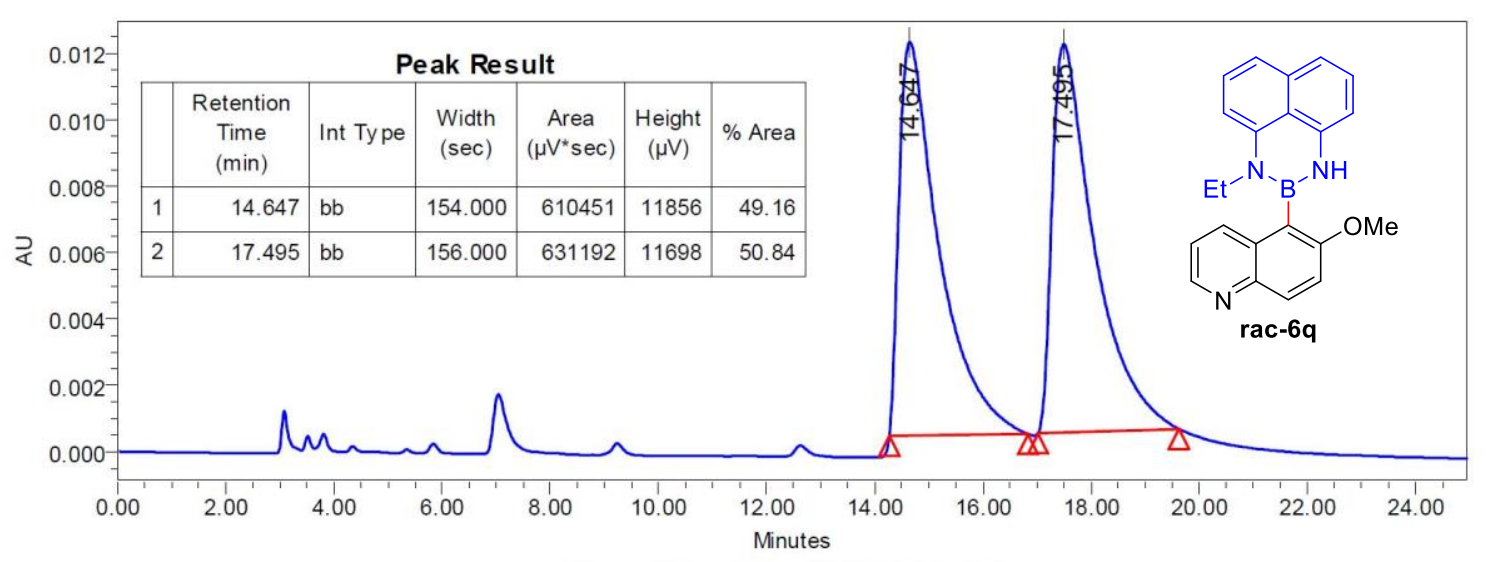

Channel Description W2489 ChA 254nm 

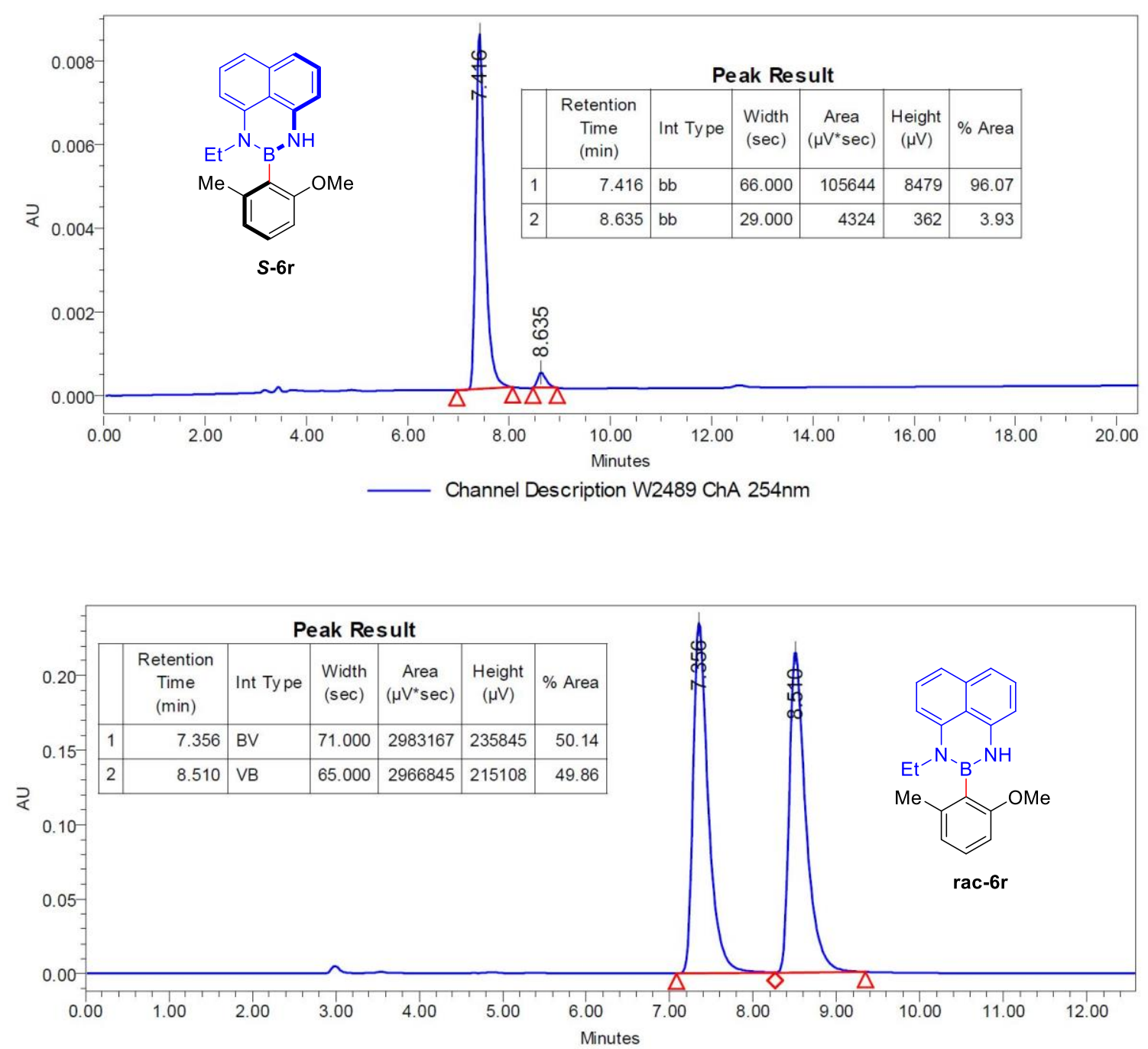

Channel Description W2489 ChA 254nm 


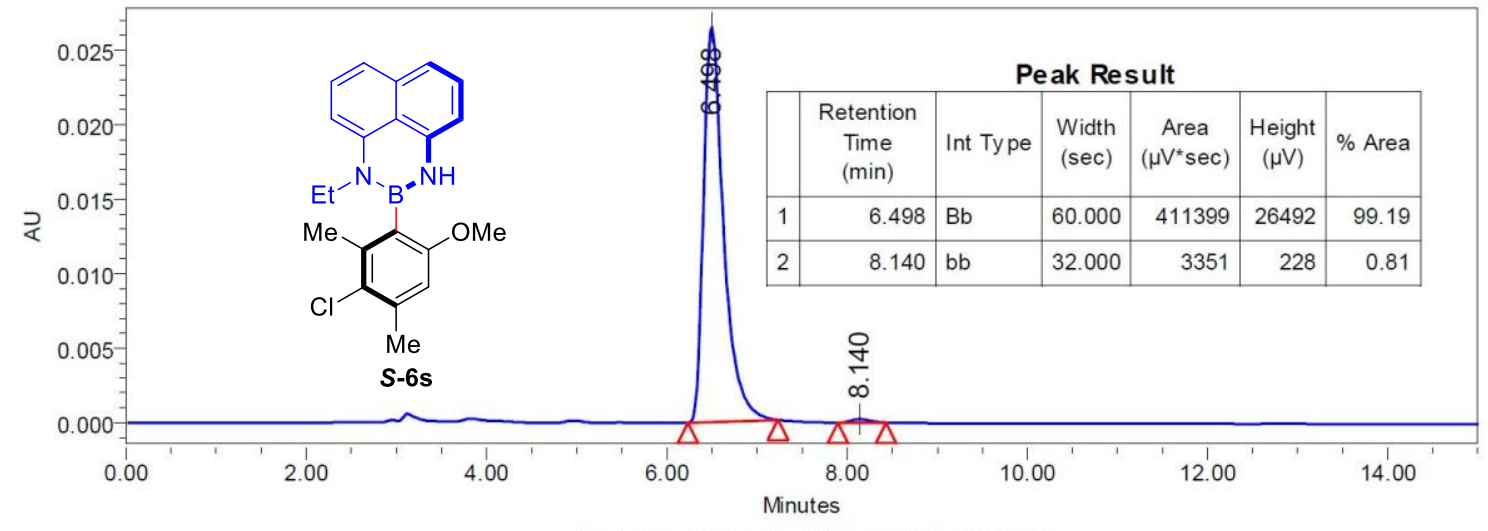

Channel Description W2489 ChA 254nm

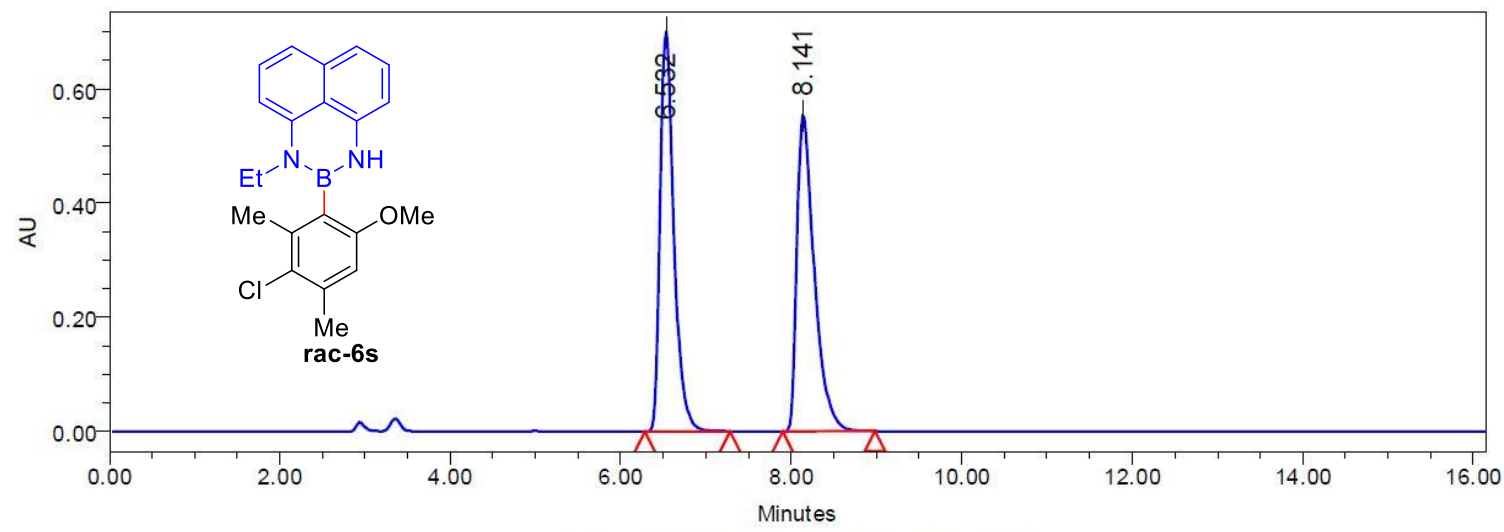

Channel Description W2489 ChA 254nm

Peak Result
\begin{tabular}{|c|c|l|l|l|l|l|}
\hline & $\begin{array}{c}\text { Retention } \\
\text { Time } \\
(\mathrm{min})\end{array}$ & Int Type & $\begin{array}{c}\text { Width } \\
(\mathrm{sec})\end{array}$ & $\begin{array}{c}\text { Area } \\
\left(\mu \mathrm{V}^{*} \mathrm{sec}\right)\end{array}$ & $\begin{array}{c}\text { Height } \\
(\mu \mathrm{V})\end{array}$ & $\%$ Area \\
\hline 1 & 6.532 & BB & 60.000 & 7850650 & 699957 & 49.95 \\
\hline 2 & 8.141 & BB & 65.000 & 7867914 & 556175 & 50.05 \\
\hline
\end{tabular}




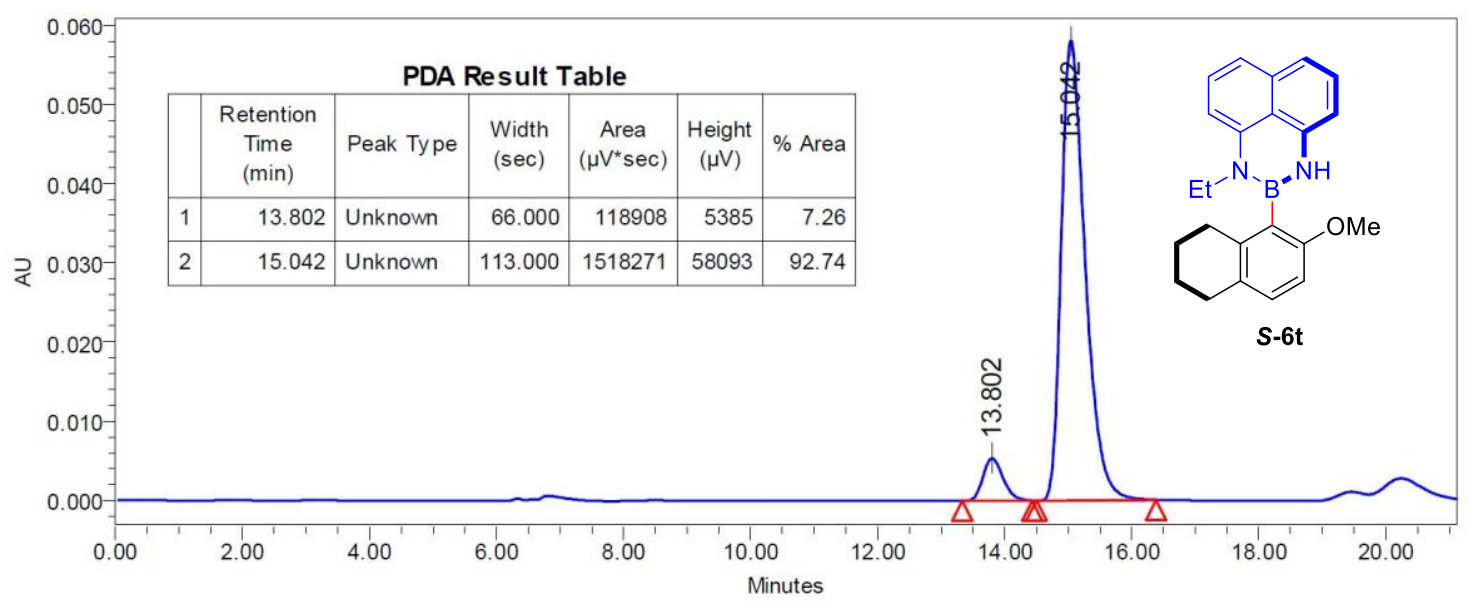

Channel Description W2489 ChA 254nm

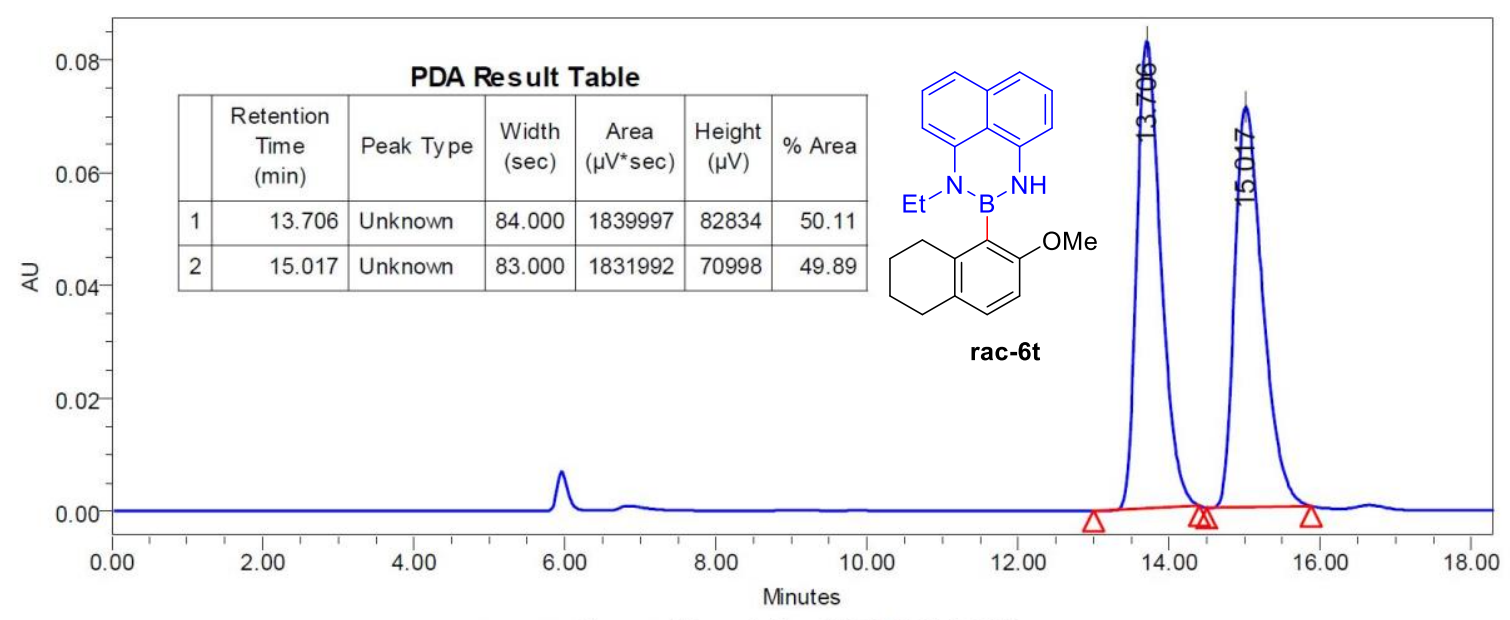

Channel Description W2489 ChA 254nm 


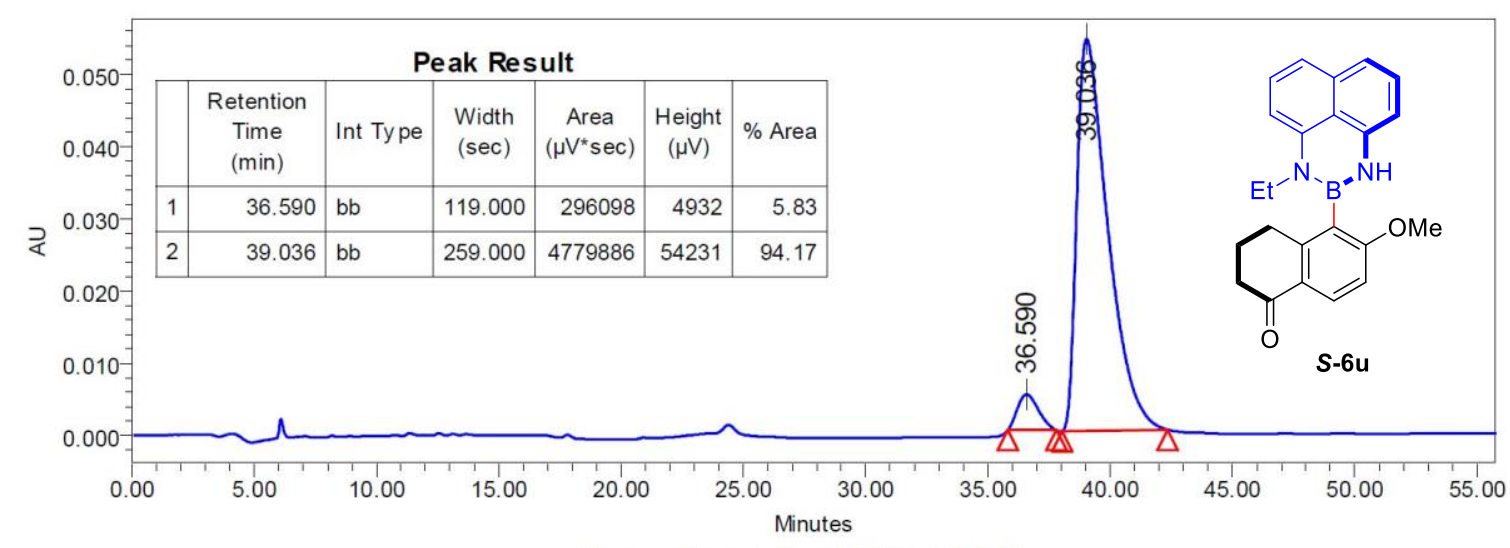

Channel Description W2489 ChA 254nm

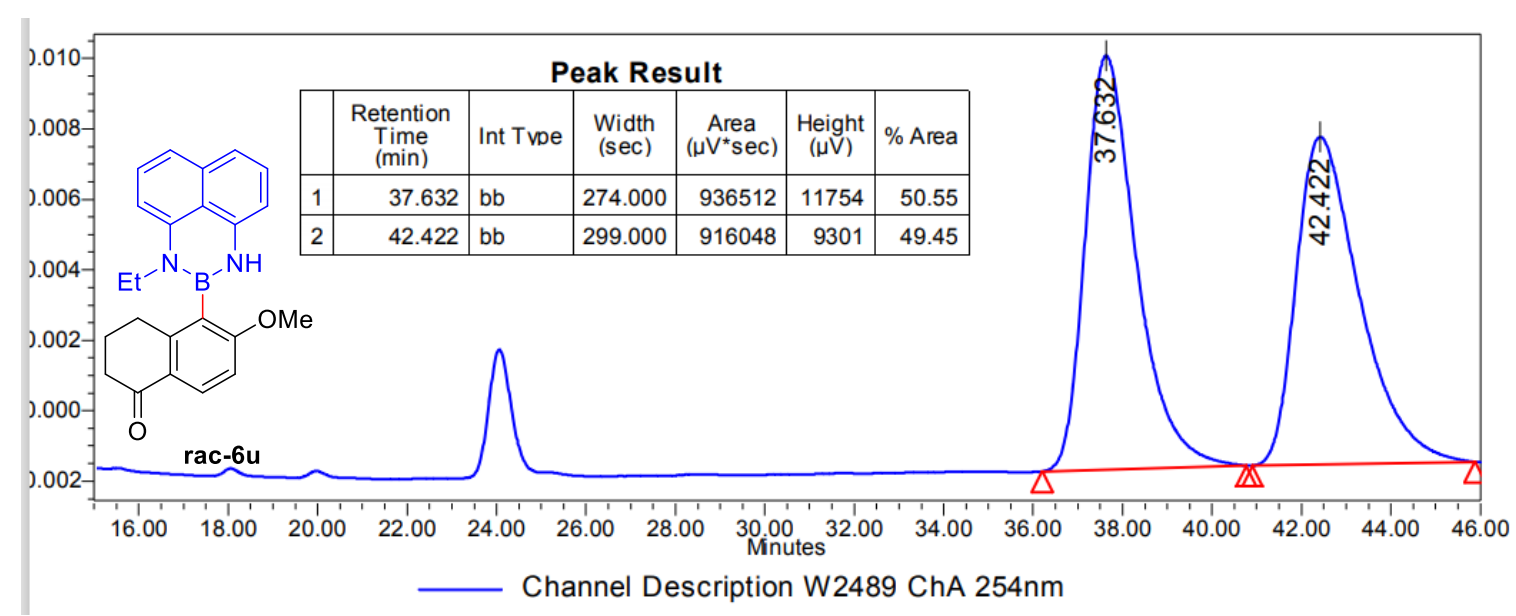



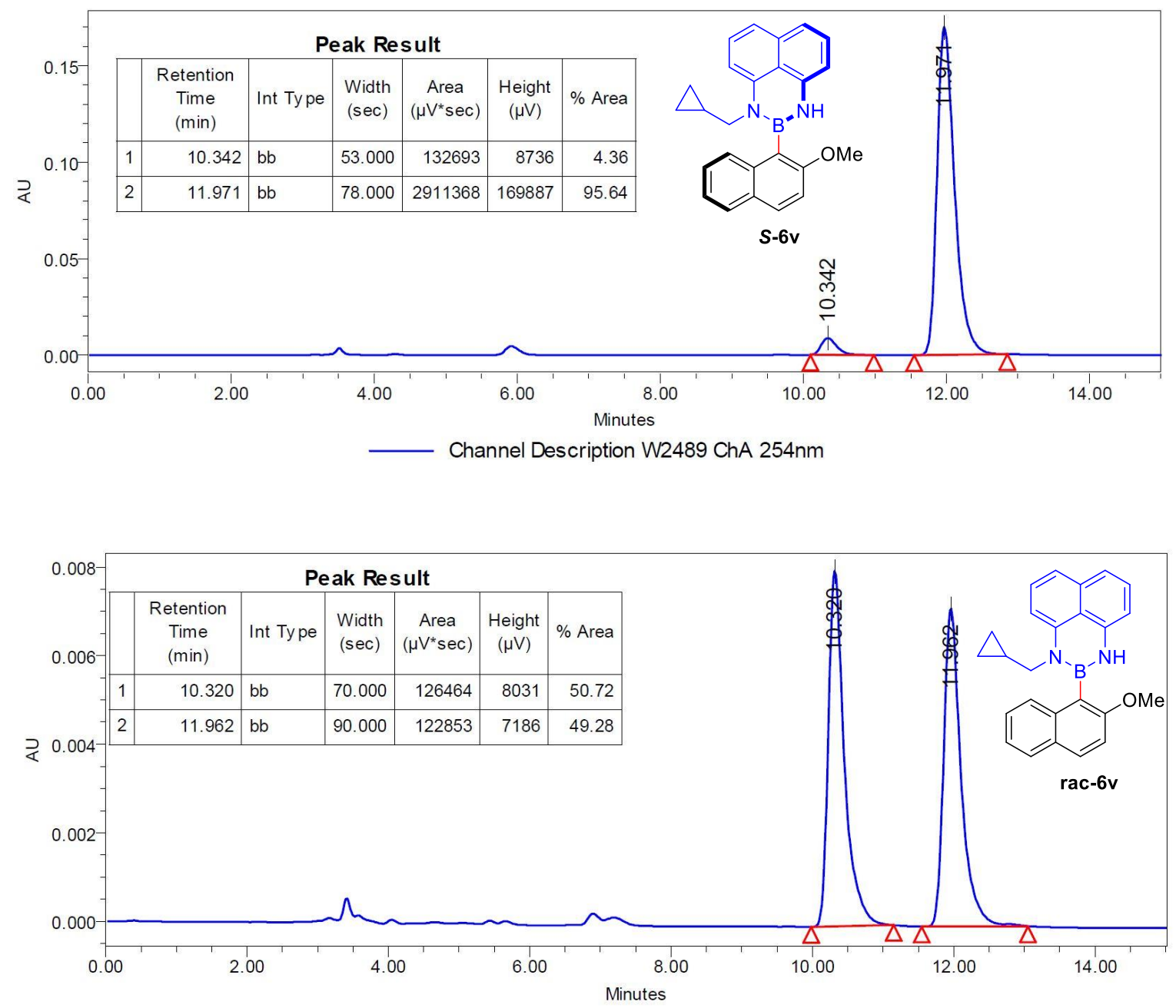

Channel Description W2489 ChA 254nm 


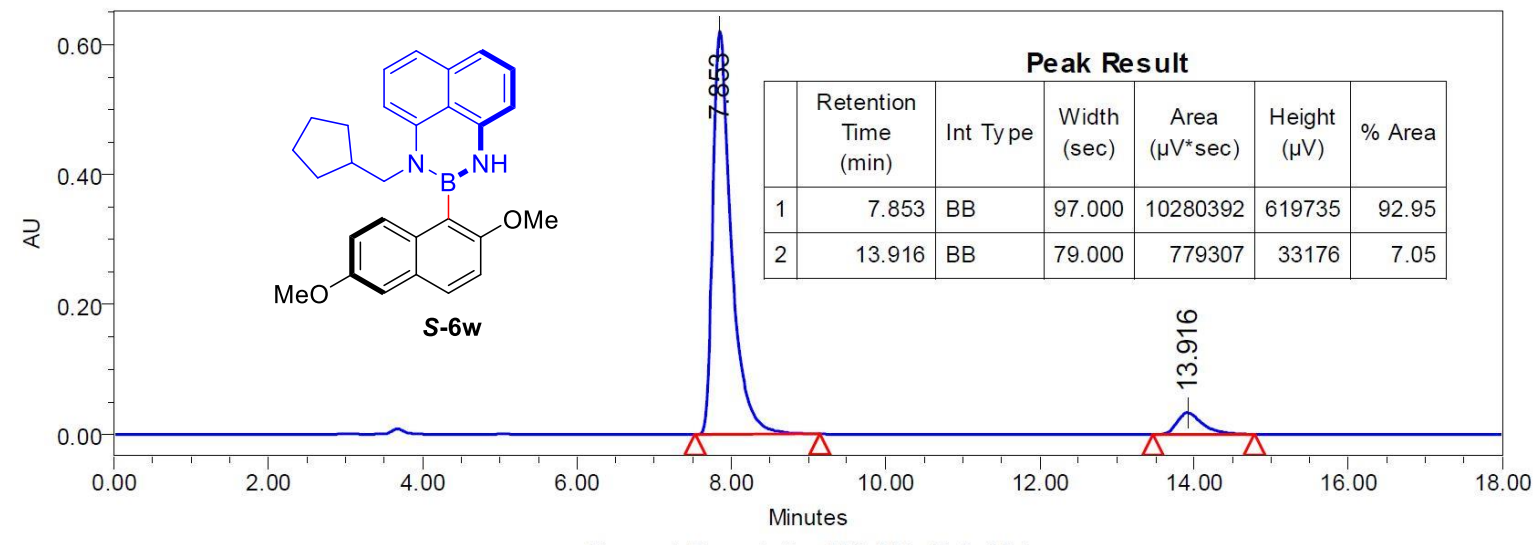

Channel Description W2489 ChA 254nm

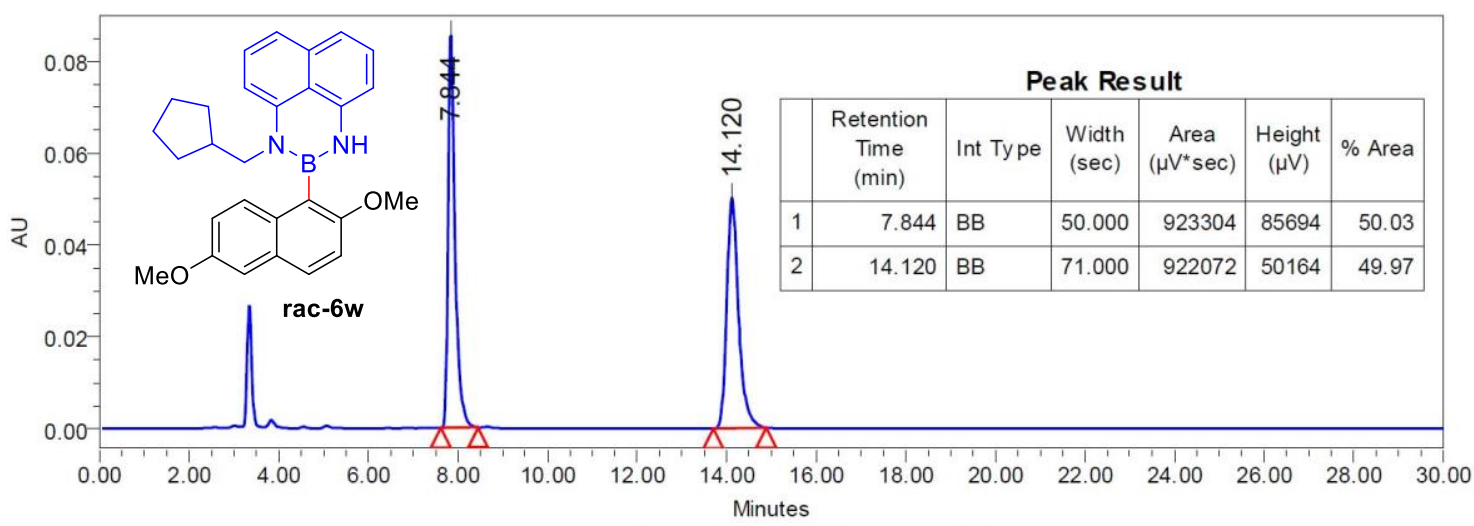

Channel Description W2489 ChA 254nm 


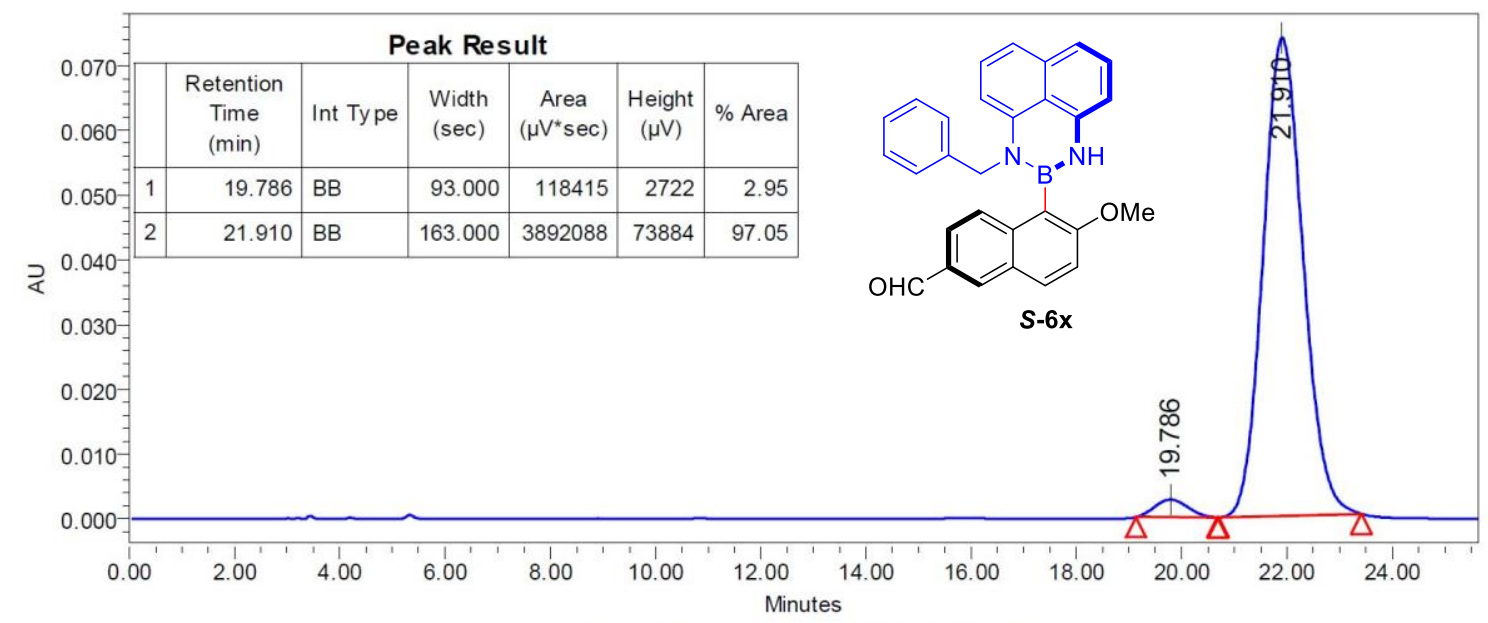

Channel Description W2489 ChA 254nm

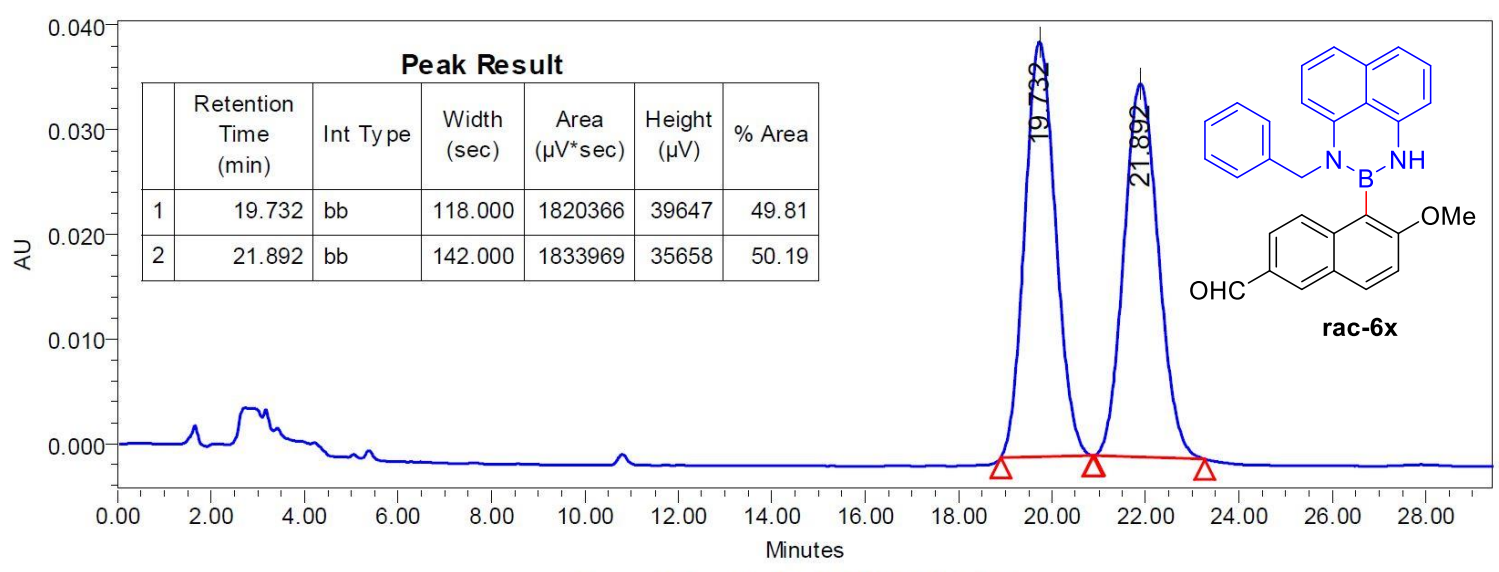

Channel Description W2489 ChA 254nm 


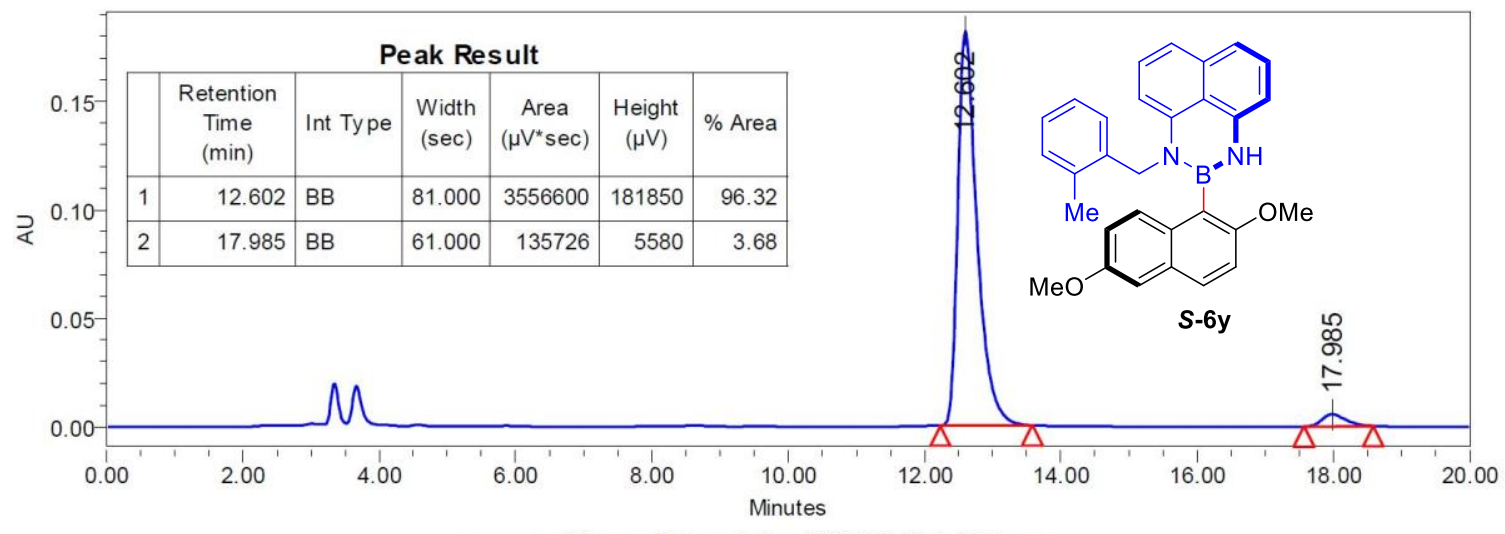

Channel Description W2489 ChA 254nm

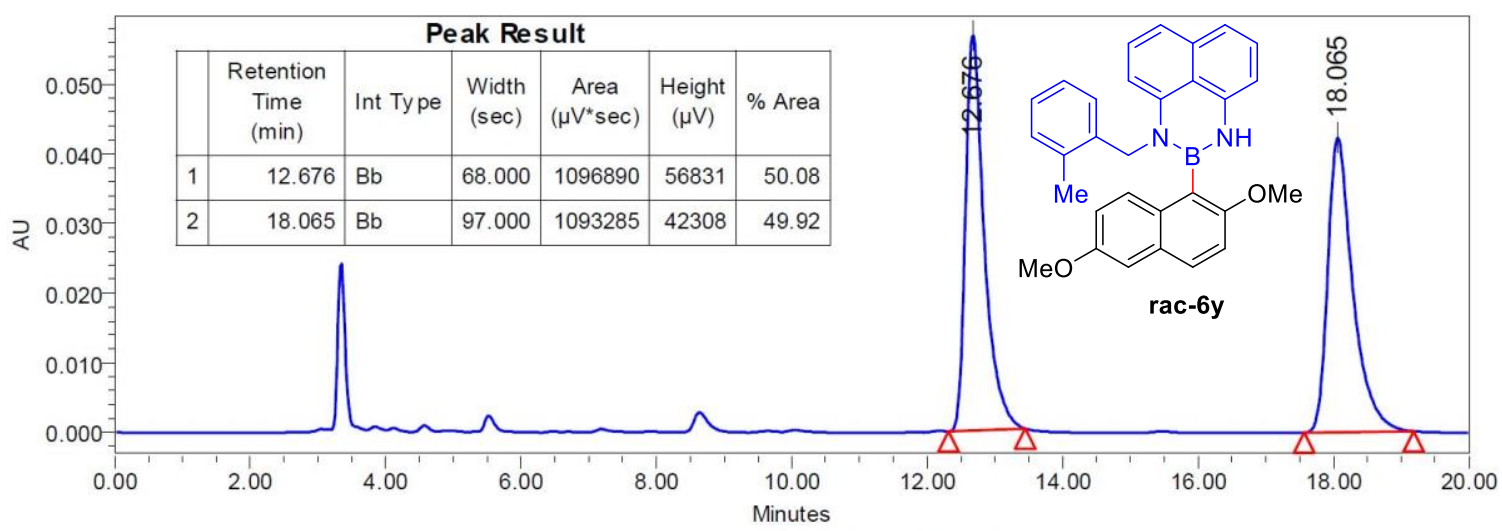

Channel Description W2489 ChA 254nm 

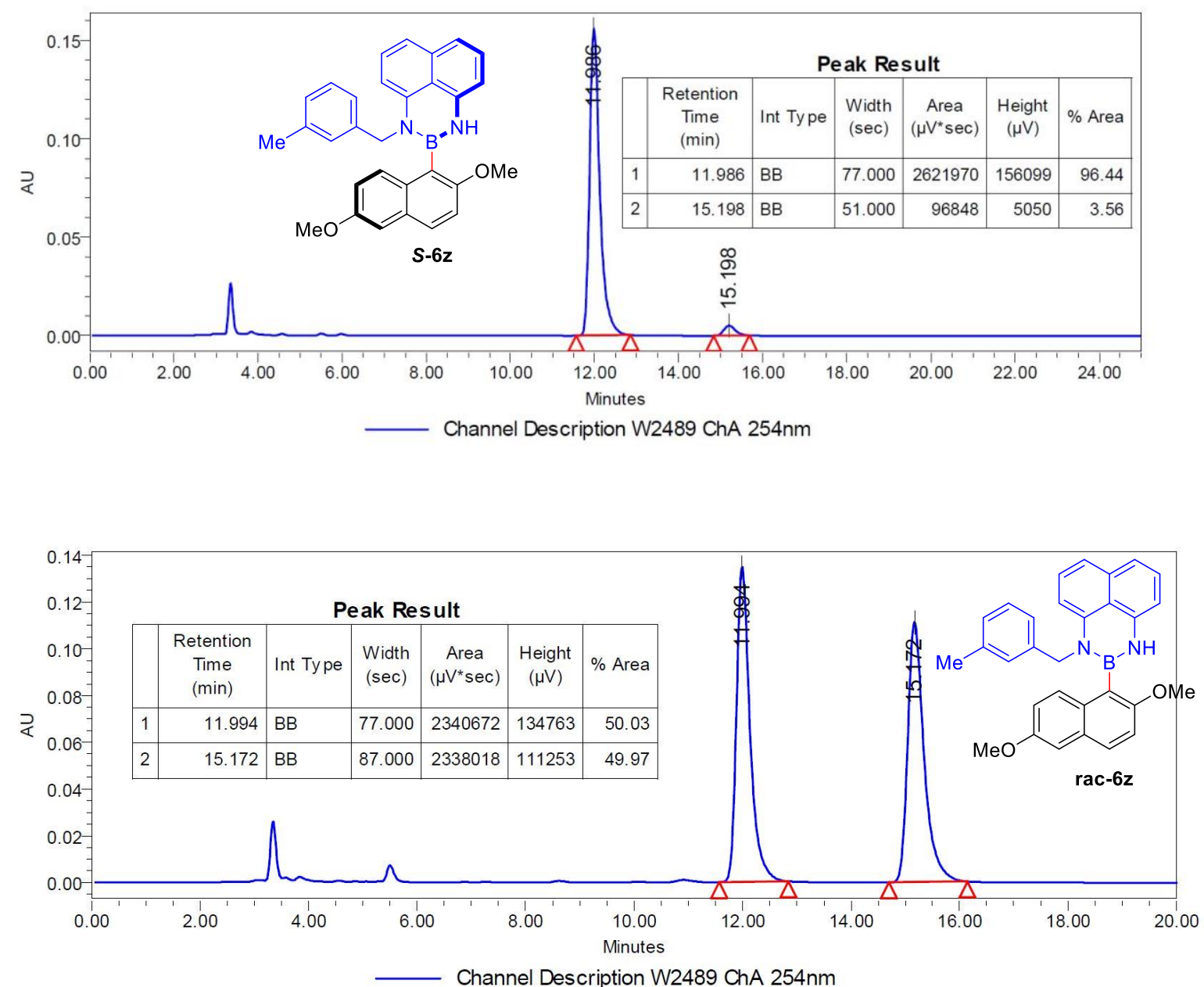

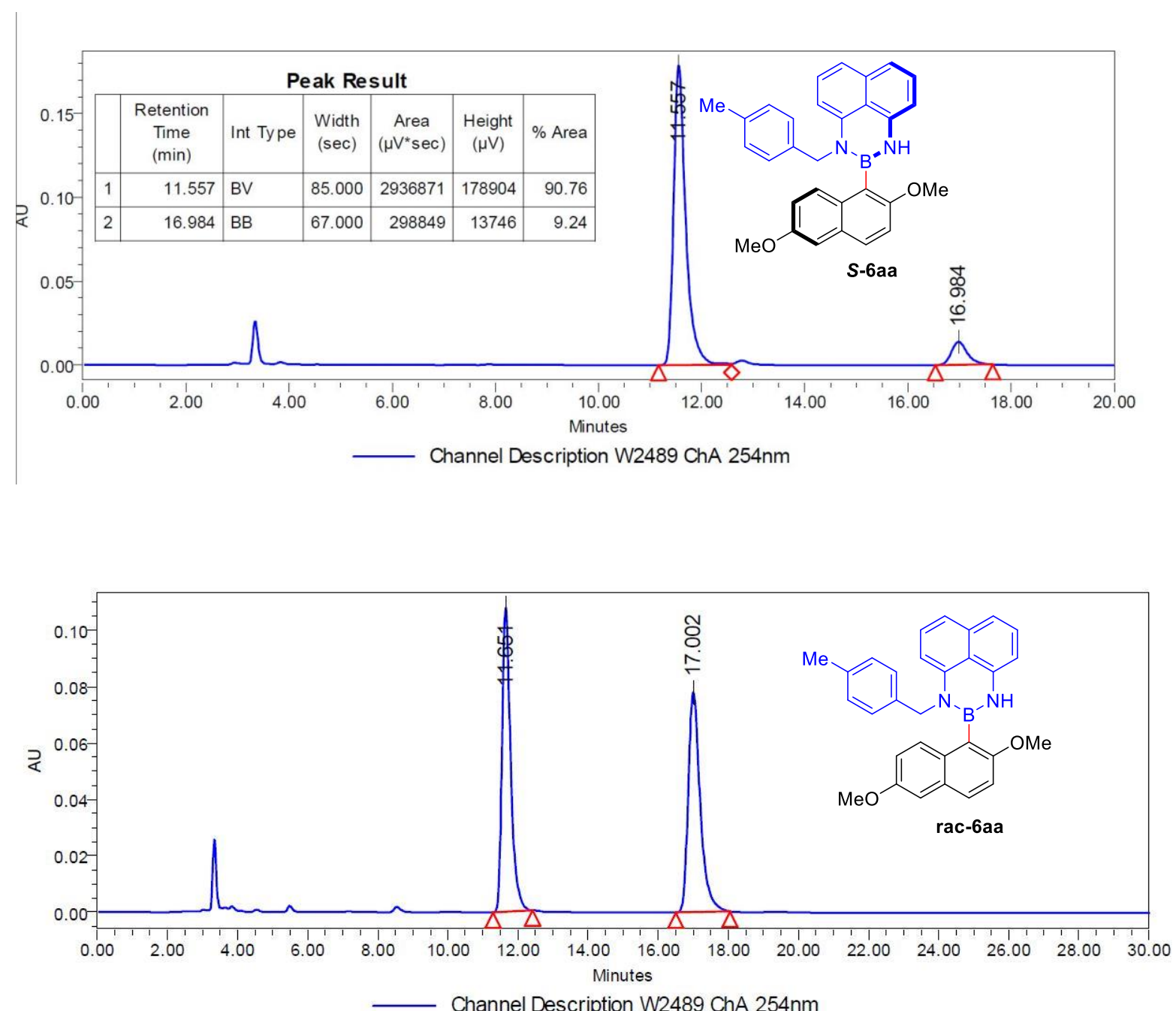

Peak Result

\begin{tabular}{|c|c|l|c|c|c|c|}
\hline & $\begin{array}{c}\text { Retention } \\
\text { Time } \\
(\mathrm{min})\end{array}$ & Int Type & $\begin{array}{c}\text { Width } \\
(\mathrm{sec})\end{array}$ & $\begin{array}{c}\text { Area } \\
\left(\mu \mathrm{V}^{*} \mathrm{sec}\right)\end{array}$ & $\begin{array}{c}\text { Height } \\
(\mu \mathrm{V})\end{array}$ & $\%$ Area \\
\hline 1 & 11.651 & $\mathrm{Bb}$ & 68.000 & 1845889 & 107827 & 49.90 \\
\hline 2 & 17.002 & $\mathrm{BB}$ & 93.000 & 1853275 & 78086 & 50.10 \\
\hline
\end{tabular}



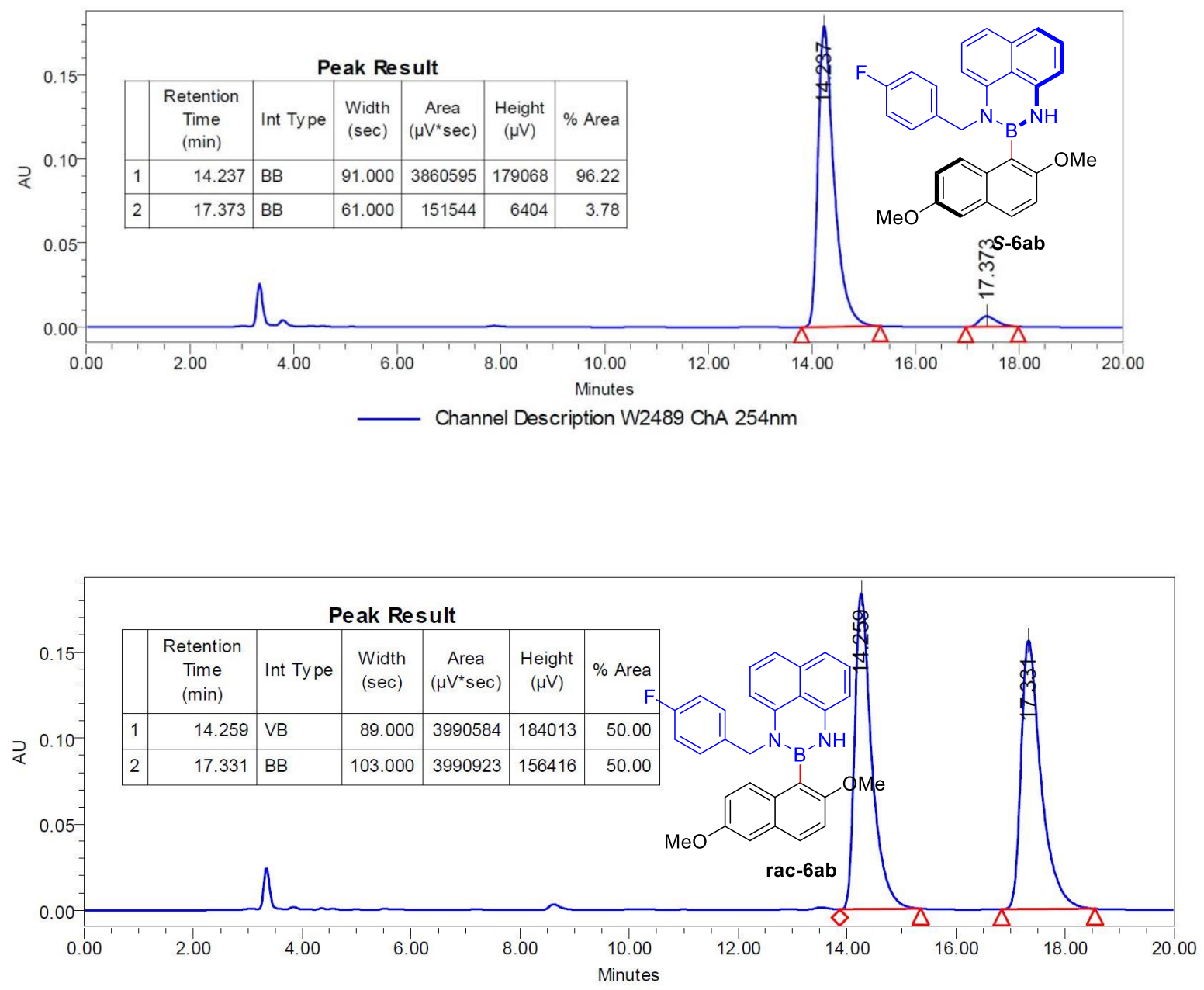

Channel Description W2489 ChA 254nm 


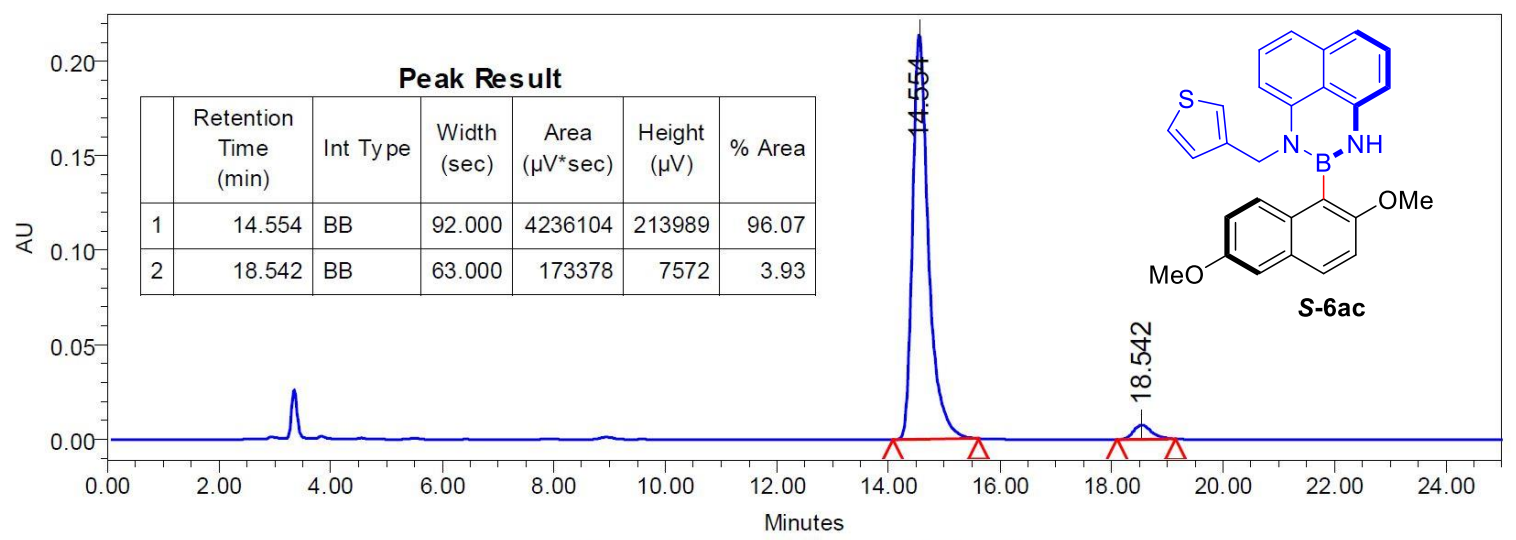

Channel Description W2489 ChA 254nm

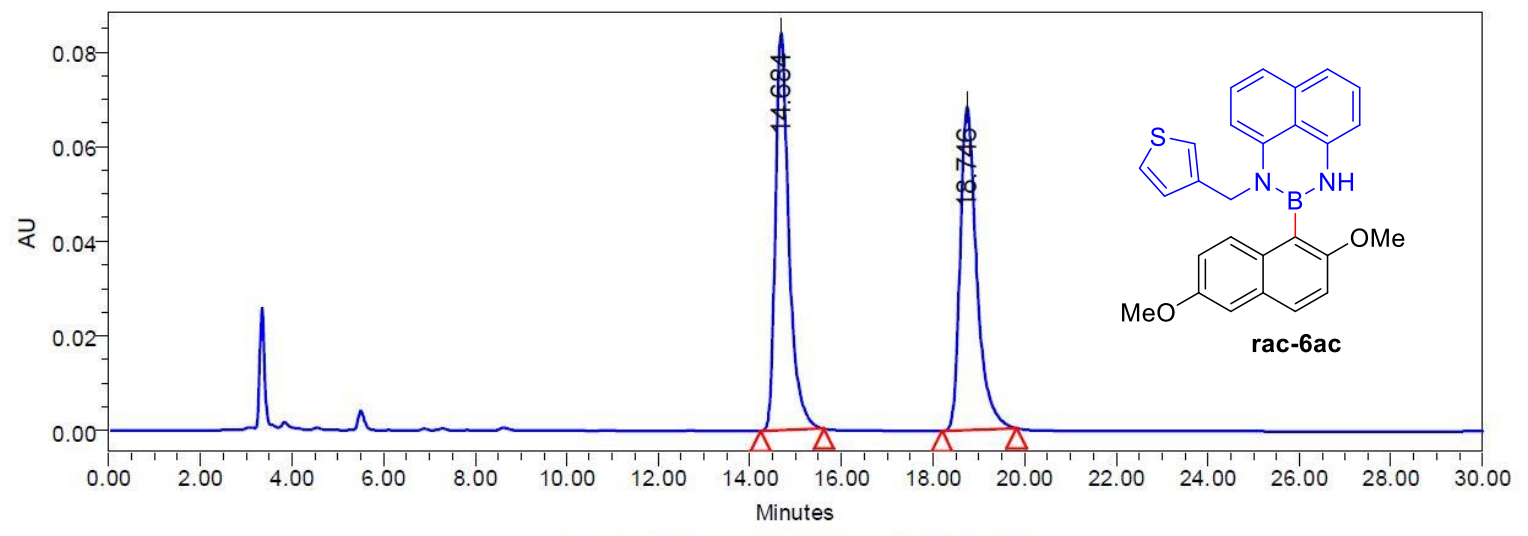

Channel Description W2489 ChA 254nm

Peak Result

\begin{tabular}{|c|c|l|l|l|l|r|}
\hline & $\begin{array}{c}\text { Retention } \\
\text { Time } \\
(\mathrm{min})\end{array}$ & Int Type & $\begin{array}{c}\text { Width } \\
(\mathrm{sec})\end{array}$ & $\begin{array}{c}\text { Area } \\
\left(\mu \mathrm{V}^{*} \mathrm{sec}\right)\end{array}$ & $\begin{array}{c}\text { Height } \\
(\mu \mathrm{V})\end{array}$ & $\%$ Area \\
\hline 1 & 14.684 & BB & 83.000 & 1725121 & 83971 & 50.06 \\
\hline 2 & 18.746 & BB & 97.000 & 1721097 & 68303 & 49.94 \\
\hline
\end{tabular}




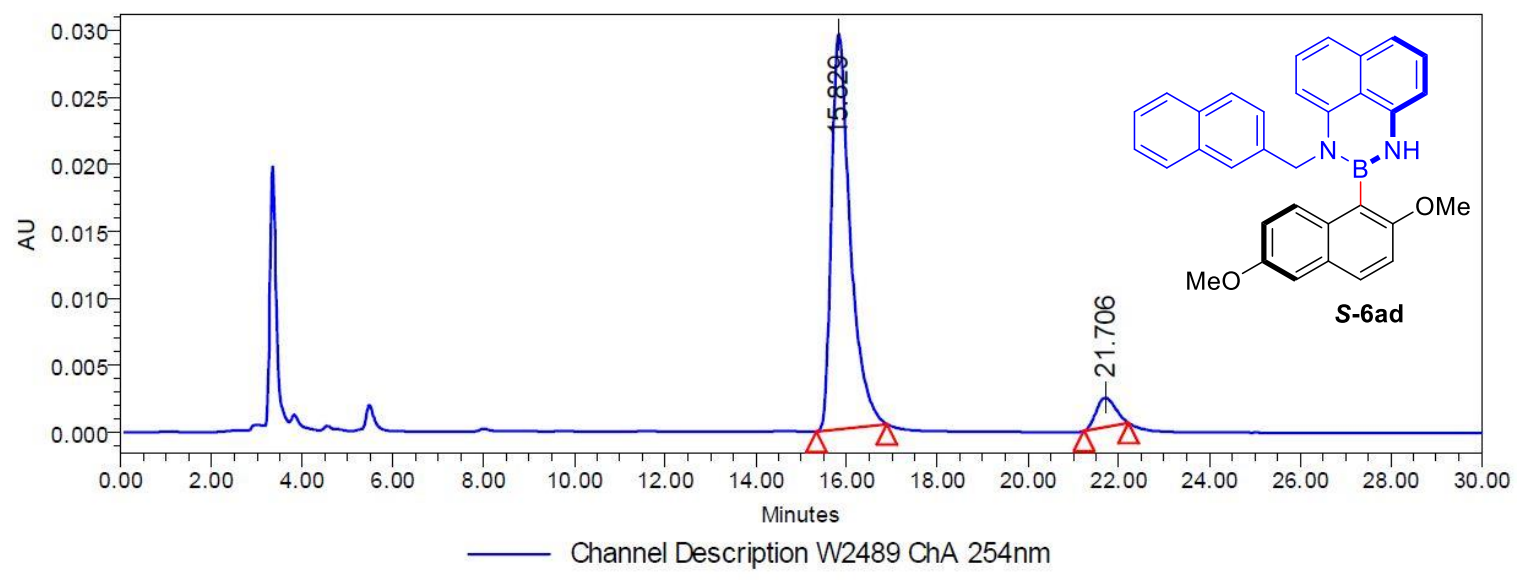

Peak Result

\begin{tabular}{|l|r|l|r|r|r|r|}
\hline & $\begin{array}{c}\text { Retention } \\
\text { Time } \\
(\mathrm{min})\end{array}$ & Int Ty pe & $\begin{array}{c}\text { Width } \\
(\mathrm{sec})\end{array}$ & $\begin{array}{c}\text { Area } \\
\left(\mu \mathrm{V}^{*} \mathrm{sec}\right)\end{array}$ & $\begin{array}{c}\text { Height } \\
(\mu \mathrm{V})\end{array}$ & $\%$ Area \\
\hline 1 & 15.829 & BB & 93.000 & 851531 & 29491 & 92.94 \\
\hline 2 & 21.706 & BB & 59.000 & 64697 & 2195 & 7.06 \\
\hline
\end{tabular}

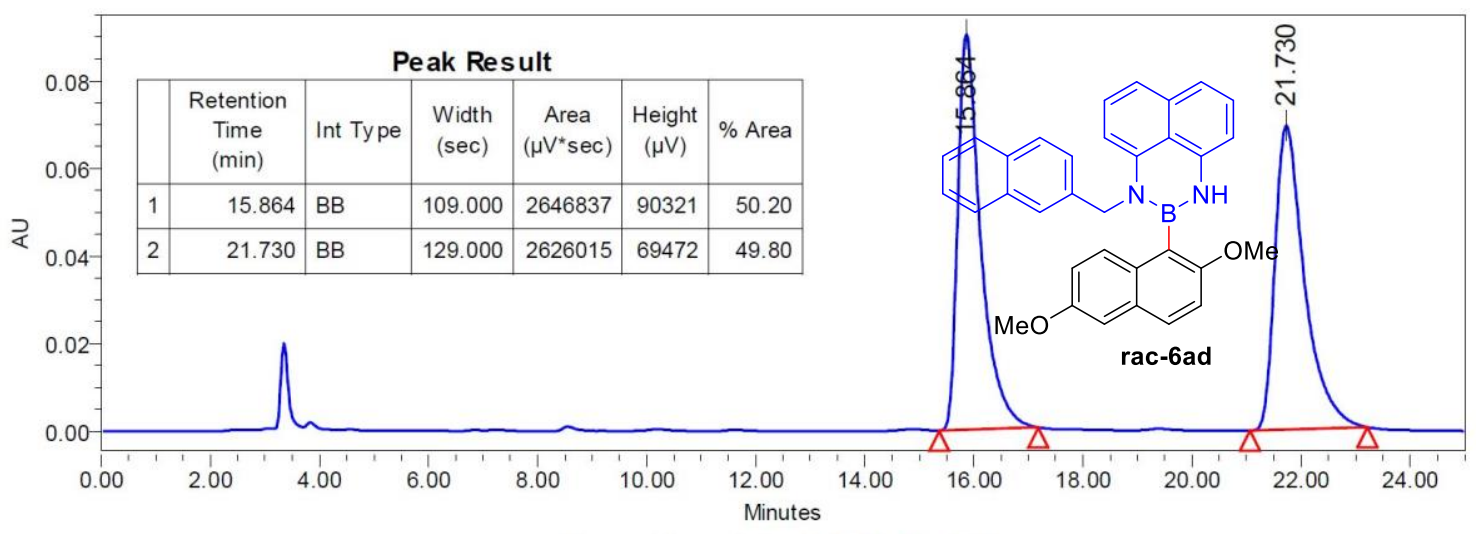

Channel Description W2489 ChA 254nm 


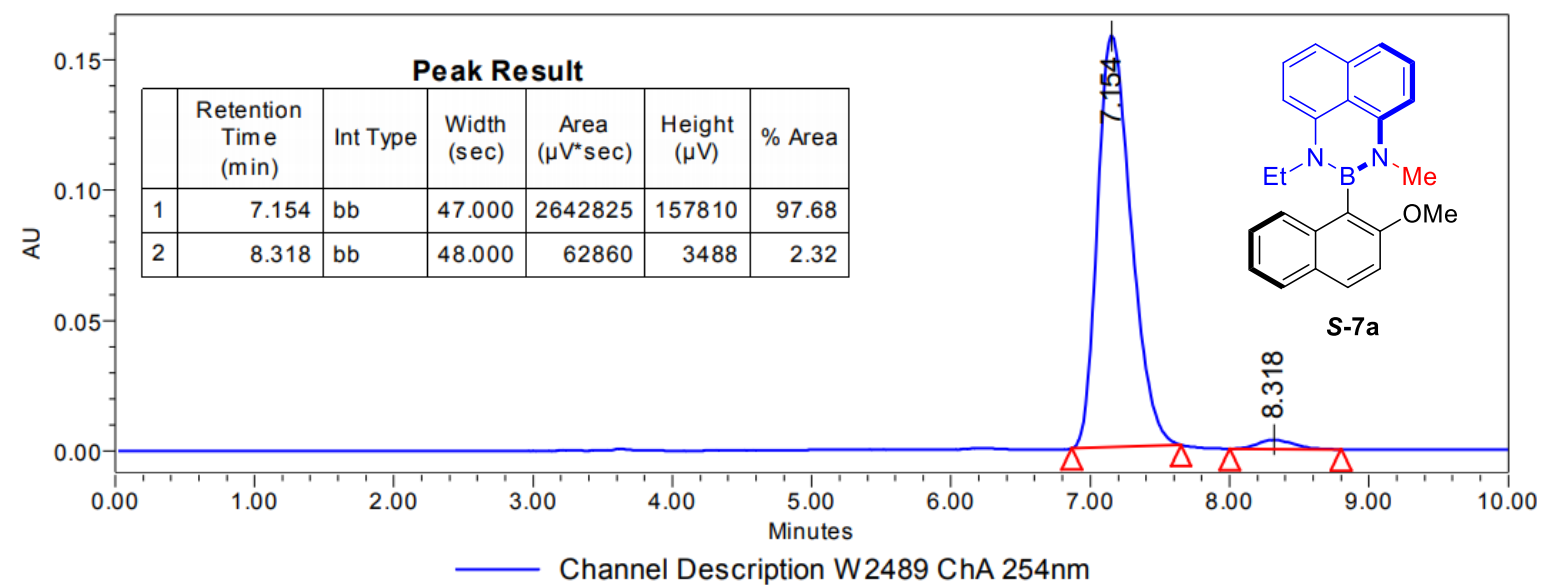

Channel Description W2489 ChA 254nm

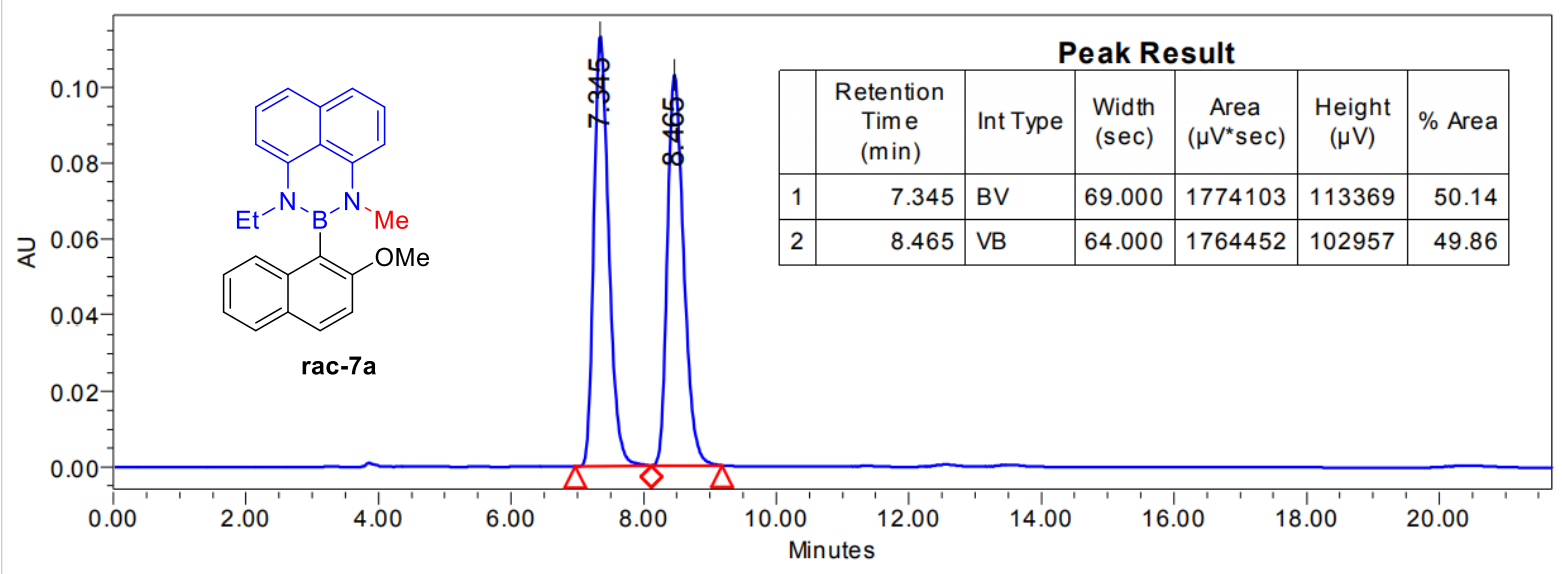

Channel Description W2489 ChA 254nm 
DAD1 D, Sig=230,4 Ref=360,100 (D:ICHEM32 I..ANIYK-7B-OD3-990105 2021-06-18 00-28-03IYK-7b-C-OD3-990105.D)
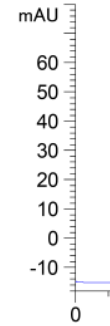

Signal 4: DAD1 D, Sig $=230,4$ Ref $=360,100$

Peak RetTime Type Width Area Height Area

$\#$ \# $\left[\begin{array}{lllll}\mathrm{min} & {[\mathrm{min}]} & {[\mathrm{mAU} * \mathrm{~s}]} & {[\mathrm{mAU}]} & \%\end{array}\right.$

-

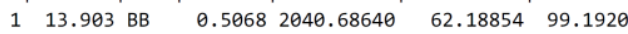

$2 \quad \begin{array}{llllll}2 & 15.730 \text { BB } \quad 0.4636 & 16.62400 & 4.75850 \mathrm{e}-1 & 0.8080\end{array}$

DAD1 D, Sig=230,4 Ref=360,100 (D:ICHEM32I...SANIYK-OD3-990105 2021-06-17 23-00-18IYK-7b-rac-OD3-990105.D)

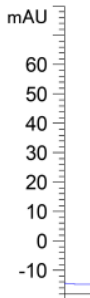

Signal 4: DAD1 D, Sig $=230,4$ Ref $=360,100$

Peak RetTime Type Width Area Height Area

[min] [mAU*s] [mAU]

-

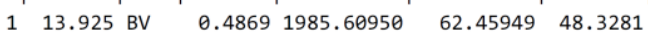

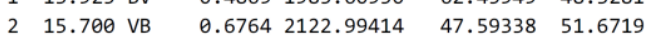

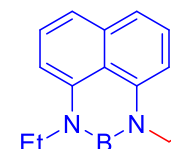

$\mathrm{Et}^{-} \mathrm{N}_{-\mathrm{B}^{-}} \mathrm{N} \sim$

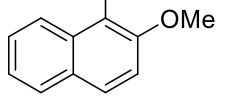

rac-7b

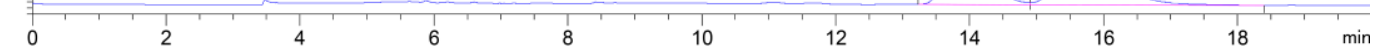




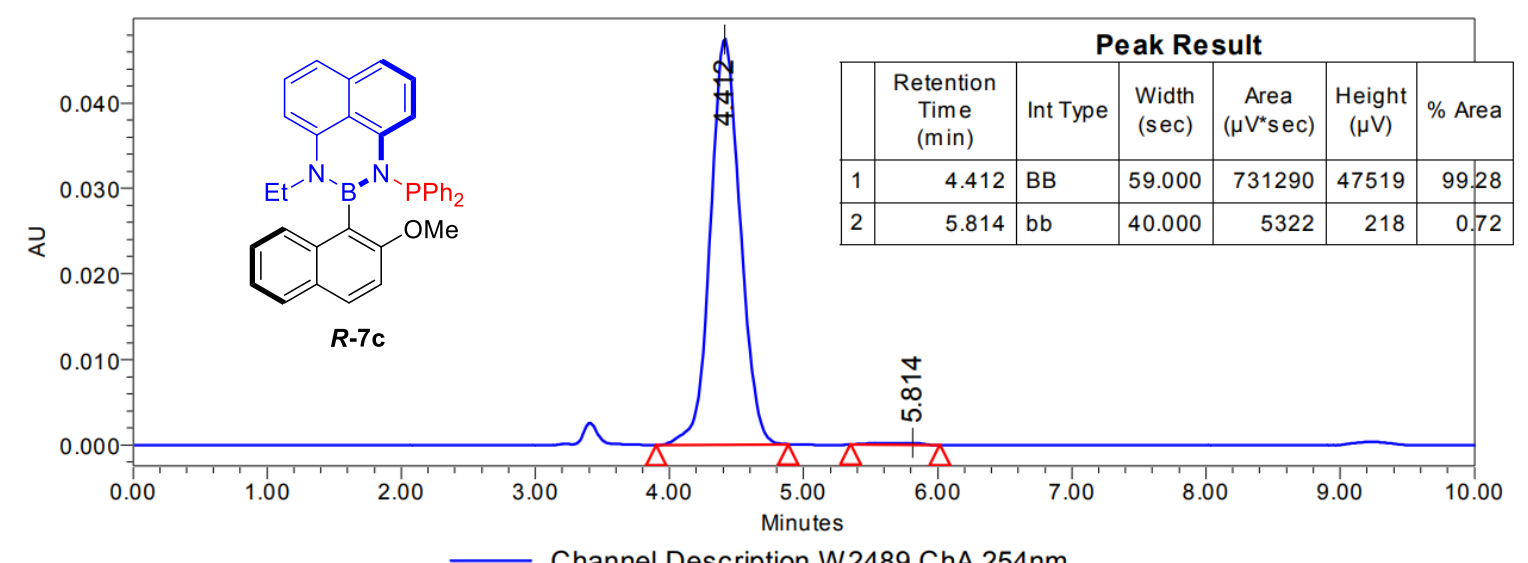

Channel Description W2489 ChA 254nm

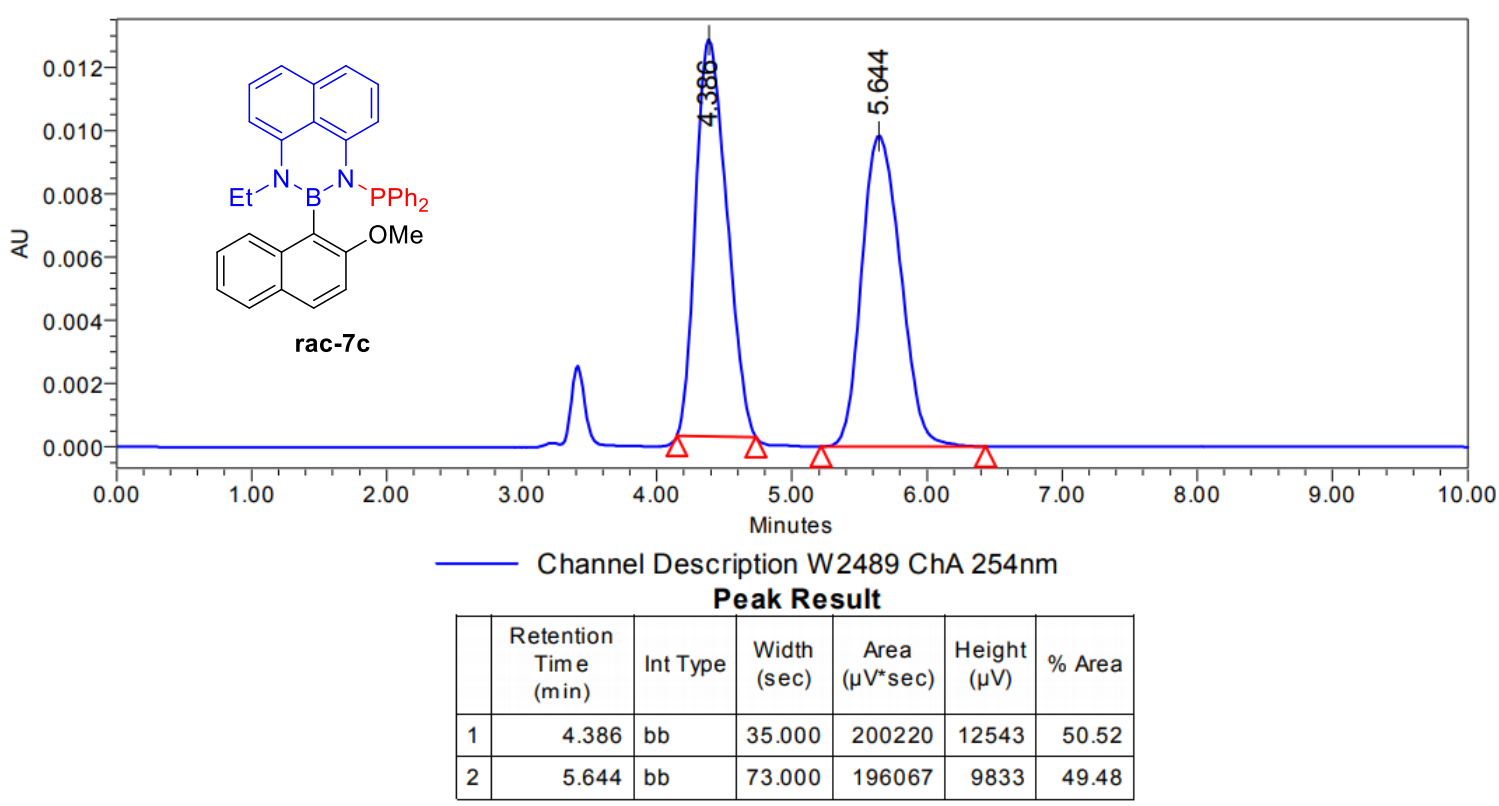



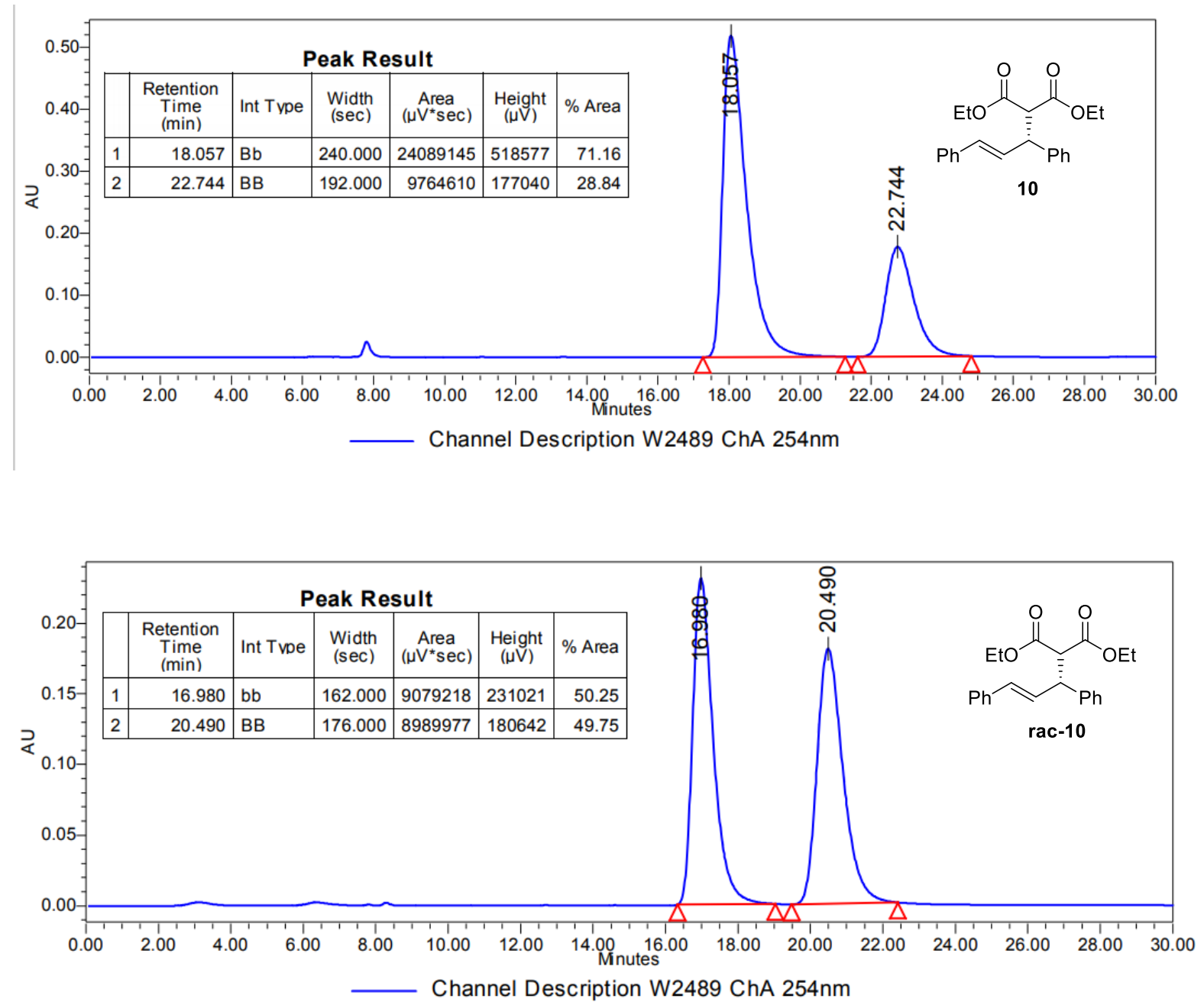